\title{
Developmental gene regulation by an ancient intercellular communication system in social amoebae
}

\author{
Dissertation \\ zur Erlangung des akademischen Grades \\ doctor rerum naturalium (Dr. rer. nat.)
}

vorgelegt dem Rat der Biologisch-Pharmazeutischen Fakultät der Friedrich-Schiller-Universität Jena

\author{
Asma Asghar \\ geboren am 08. Februar 1982 \\ aus Lahore, Pakistan
}

Jena, July 2012 
Dekan: Faculty of Pharmacy and Biology

1. Gutachter: Prof. Dr. Thomas Winckler, Friedrich-Schiller-Universität Jena, Germany 2. Gutachter: Prof. Dr. Christian Hertweck, Friedrich-Schiller-Universität Jena, Germany 3. Gutachter: Prof. Dr. Pauline Schaap, University of Dundee, Scotland

Tag der öffentlichen Verteidigung: 05. März 2013 


\title{
I dedicate this work
}

\author{
to \\ "My Loving Parents"
}

who taught me to recognize the value of biology and medicine from an early age 


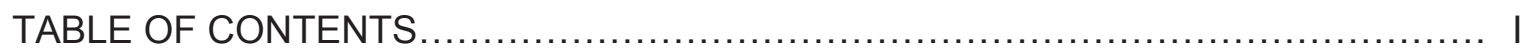

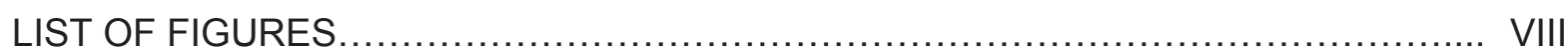

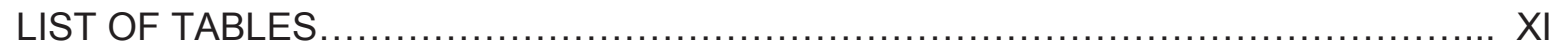

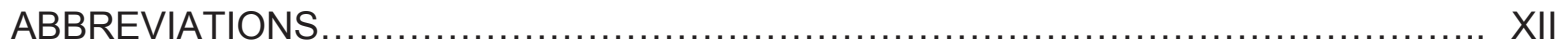

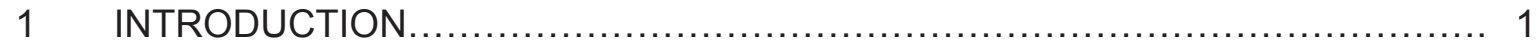

$1.1 \quad$ The social amoebae- Dictyostelids.................................... 1

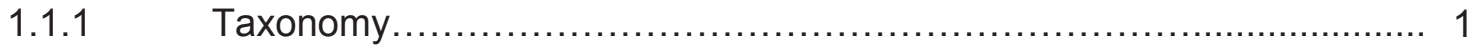

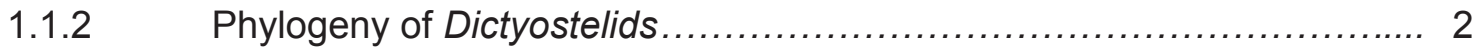

$1.2 \quad$ Communication modules in the life cycle of Dictyostelids................ 6

1.2.1 Communication at the Transition from Growth to Aggregation .............. 7

1.2.2 Receptors for cell communication in Dictyostelids....................... 9

1.2.3 Previous knowledge of cell-cell communication in D. discoideum......... 9

1.2.3.1 Secreted factors that regulate gene expression in Dictyostelids.......... 9

1.2.3.2 The Signalling pathways by which cAMP regulates expression of early

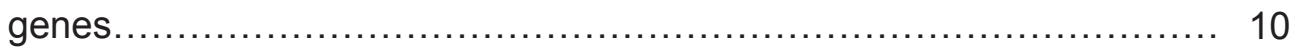

1.3 Significance of studying intercellular communication.................... 16

$1.4 \quad$ Advantages of social amoebae in researching cell-cell signalling ......... 16

1.5 Previous knowledge about the acrasin of Polysphondylium............... 18

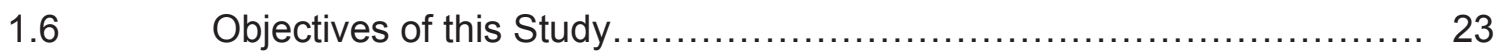

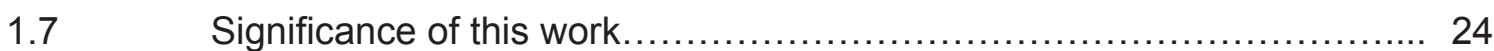

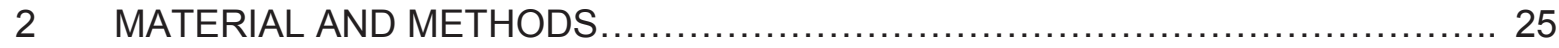

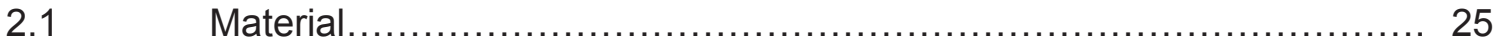

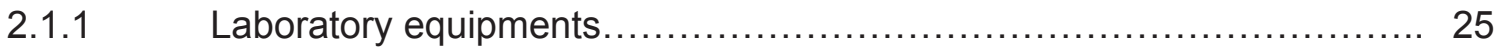

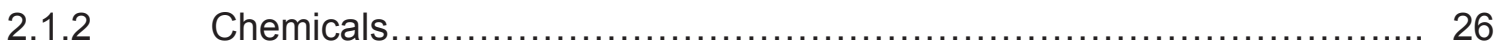

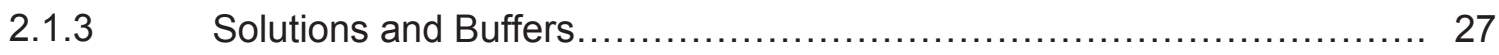

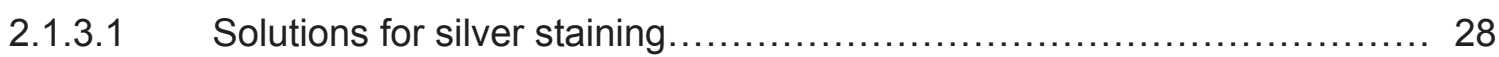

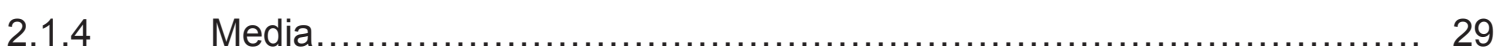

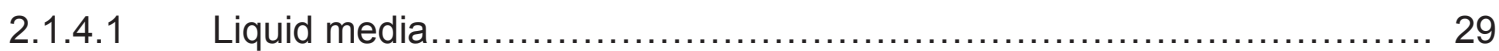

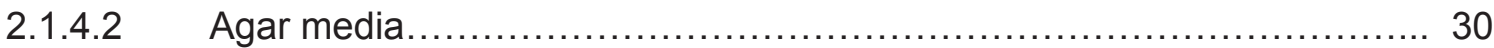

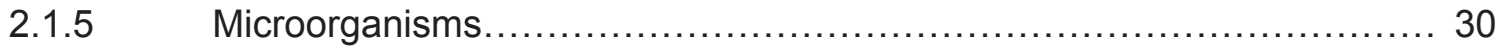

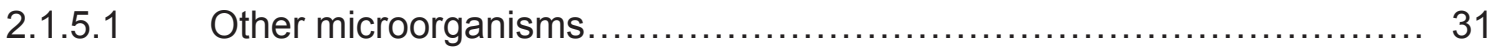

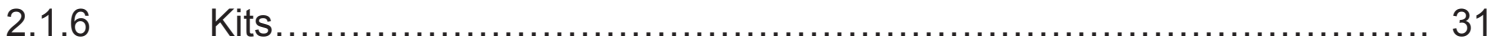

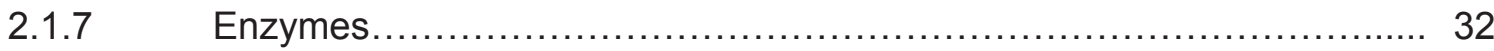




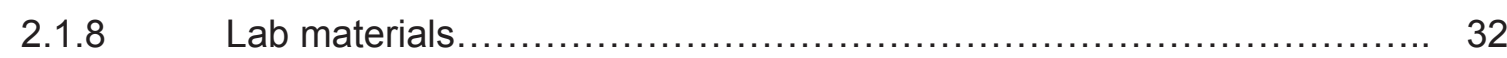

Size standards....... $\quad$ S.

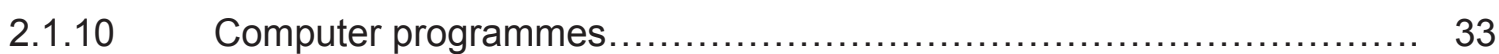

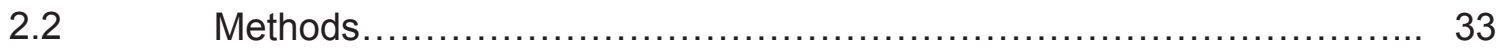

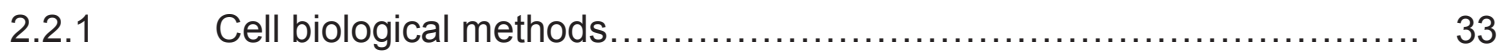

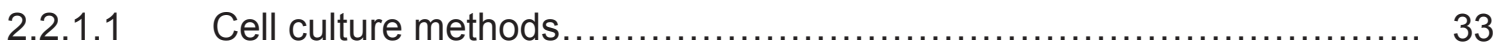

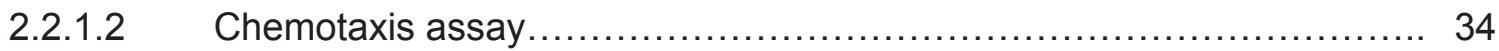

2.2.1.2.1 Preparation of hydrophobic agar and chemotaxis assay plates........... 34

2.2.1.3 Glorin pulsing experiments......................................... 34

2.2.1.4 Aggregation Analysis............................................ 35

2.2.1.4.1 Aggregation analysis under submerged conditions.................... 35

2.2.1.4.2 Aggregation analysis on phosphate-buffered agar plates................. 35

2.2.1.5 Development on phosphate agar to study aggregation stimulatory

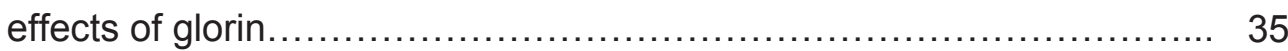

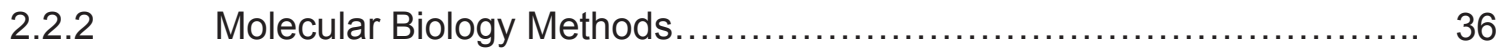

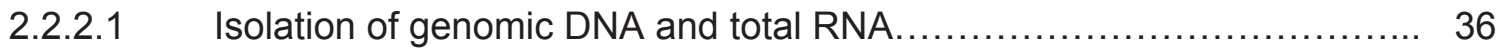

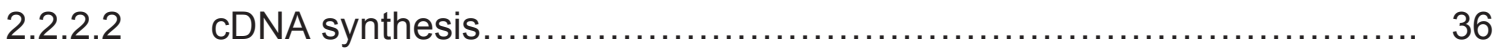

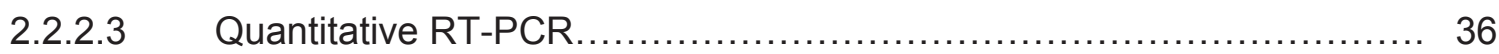

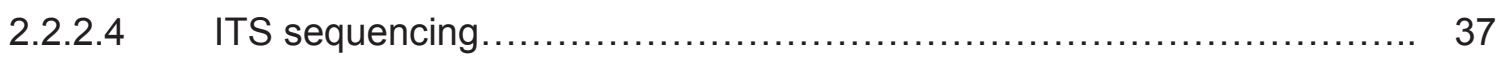

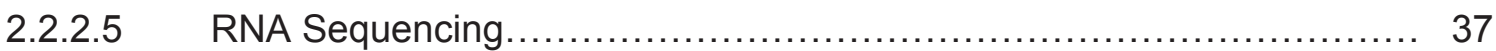

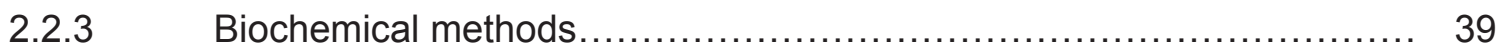

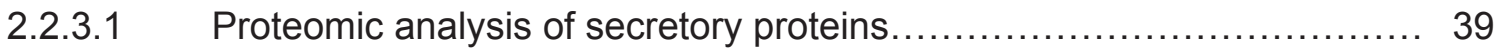

2.2.3.1.1 Preparation of extracellular proteins from aggregating $P$. pallidum........ 39

2.2.3.1.2 Concentration of collected proteins................................... 39

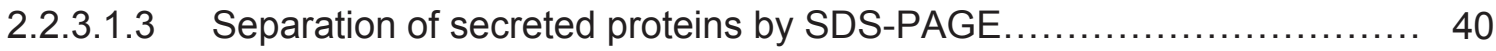

2.2.3.1.4 MS- compatible silver staining of polyacrylamide gel.................. 40

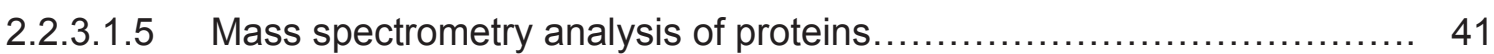

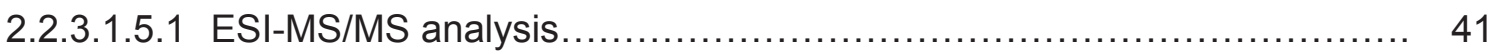

2.2.3.1.5.2 Database search to identify proteins..................................... 42

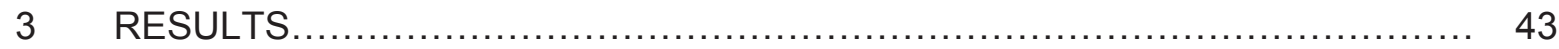

3.1 Development and validation of chemotaxis bioassay .................. 44

3.2 Chemotactic specificity of group 4 species............................. 46

3.3 Chemotactic specificity of $P$. violaceum lying at the edge of group $4 \ldots \ldots \quad 46$

3.3.1 Stage specificity in the response of $P$. violaceum amoebae to different chemotactic agents ................................................ 47 
3.4 Chemotaxis of social amoebae towards glorin is an ancient response.... 48

3.4.1 Chemotactic response of group 2 species to glorin.................... 48

3.4.1.1 Chemotactic specificity of $P$. pallidum PN500 cells.................... 48

3.4.1.2 Determination of optimal concentration of glorin required to obtain maximum chemotactic response of $P$. pallidum amoebae............... 49

3.4.1.3 Stage specificity in the response of $P$. pallidum PN500 cells to glorin...... 50

3.4.1.4 Delayed chemotactic response of $P$. pallidum amoebae to high

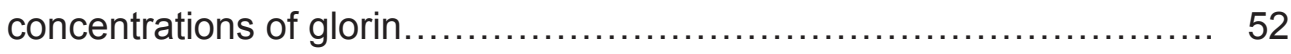

3.4.1.5 P. pallidum is a species complex............................... 53

3.4.1.6 Chemotactic response of different $P$. pallidum isolates and group 2 Dictyostelid species to glorin........................................ 56

3.4.2 Chemotactic response of group 1 species to glorin................... 58

3.5 Identification of the proteins secreted by aggregating $P$. pallidum amoebae: Fishing for putative glorinase .............................. 60

3.6 Genome-wide analysis of glorin modulated gene expression changes... 62

3.6.1 Starvation triggers dramatic changes in gene expression in $P$. pallidum

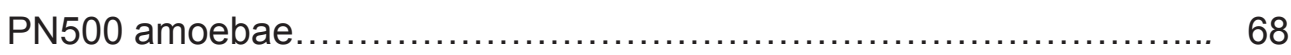

3.6.2 Glorin-induced developmental regulation of gene expression......... 71

3.6.2.1 Classification of glorin-induced developmental gene expression.......... 75

3.6.2.1.1 Class I: Genes stably induced by glorin ............................ 75

3.6.2.1.2 Class II: Genes transiently induced by glorin............................ 77

3.6.2.1.3 Class III: Genes induced by starvation, whereas glorin treatment further enhanced their expression.............................................. 79

3.6.2.1.4 Class IV: Genes induced by starvation, whereas glorin treatment

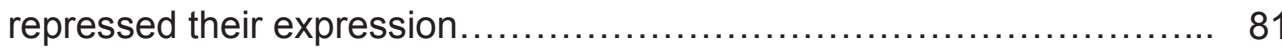

3.6.2.1.5 Class V: Genes repressed by starvation, whereas glorin treatment induced their expression.................................................. 83

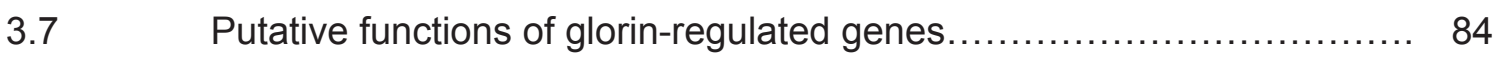

3.7.1 Annotation of GO terms to glorin-induced genes...................... 85

3.7.2 Annotation of GO terms to glorin-repressed genes.................... 90

3.8 Detailed kinetics of glorin-regulated gene expression.................. 91

3.8.1 Effect of signal modulation on glorin-regulated gene expression........... 91

3.8.1.1 Gene expression analysis under different concentrations of glorin........ 91

3.8.1.2 Gene expression analysis under different pulsing frequencies of glorin... 94

3.8.2 Expression kinetics of glorin-regulated genes during the first 5 hours of development in suspension cultures................................... 97 
3.8.3 Temporal expression pattern of glorin responsive genes during development in shaken suspensions.............................. 103

3.8.3.1 Class I: Genes stably induced by glorin in shaking cultures............... 104

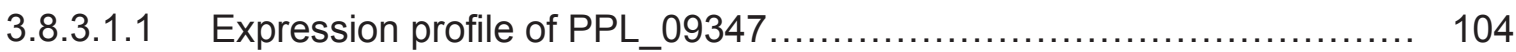

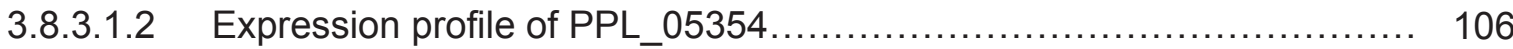

3.8.3.2 Class II: Genes induced by starvation, whereas exogenous glorin pulses result in their precocious expression............................... 107

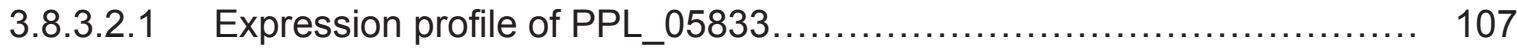

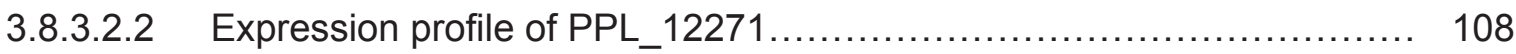

3.8.3.3 Class III: Gene induced by starvation, whereas glorin pulses repressed their expression during the early hours of development............... 110

3.8.3.3.1 Expression profile of PPL_07908 ...................................... 110

3.8.3.4 Class IV: Genes repressed by starvation, whereas glorin pulses induce their expression in shaking cultures.................................. 111

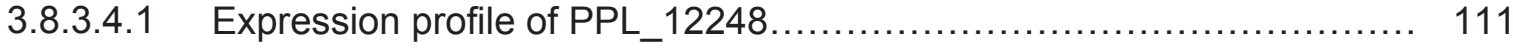

3.8.3.4.2 Expression profile of PPL_12249.................................. 112

3.8.4 Developmental time course of gene expression in cells starved on nonnutrient agar plates................................................... 114

3.8.4.1 Class I: Aggregation stage specific genes....................... 116

3.8.4.1.1 Developmental kinetics of PPL_09347 ........................... 116

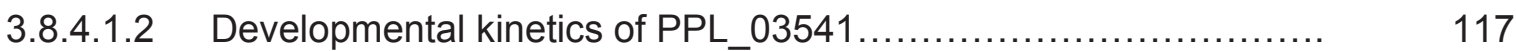

3.8.4.1.3 Developmental kinetics of PPL_06644 .......................... 118

3.8.4.2 Class II: Genes exhibiting characteristics of both 'early genes' and 'aggregation-stage genes' ............................................ 119

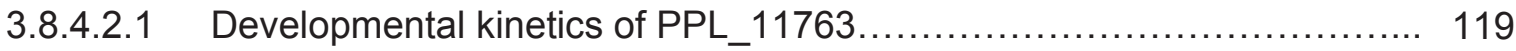

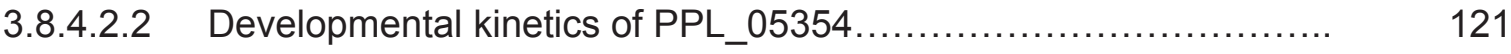

3.8.4.2.3 Developmental kinetics of PPL_00912.......................... 122

3.8.4.3 Class III: Genes nearly similarly expressed during the early stages of

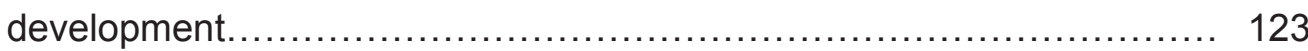

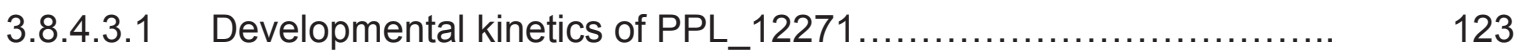

3.8.4.4 Class IV: Genes expressed at basal level during the early stages of

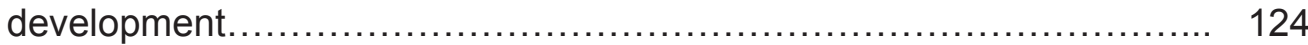

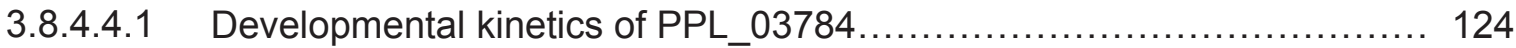

3.8.4.4.2 Developmental kinetics of PPL_05833 ............................. 125

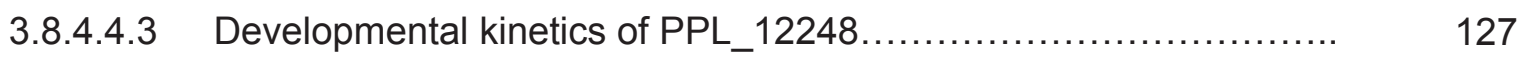

3.8.5 Developmental regulation of selective GPCRs.................... 129 
3.8.5.1 Developmental kinetics of GPCR genes induced by glorin.

3.8.5.1.1 Class I: GPCRs encoding genes expressed in growing amoebae, whose expression levels are enhanced upon starvation.

3.8.5.1.2 Class II: Starvation induced GPCRs encoding genes whose expression is augmented during aggregation.

3.8.5.1.3 Class III: GPCR encoding genes expressed specifically during aggregation.

3.9 Glorin signalling function independent of the cAMP signaling system 137

3.9.1 Expression patterns of components of cAMP signalling system are not affected by glorin stimulus........................................ 137

3.9.2 P. pallidum tas $A^{-} / \operatorname{tas} B^{-}$null mutant exhibits normal aggregation........... 138

3.10 Glorin elicits rapid changes in gene expression.......................... 140

3.10.1 Pre-starvation period is not necessary to observe glorin-induced gene expression

3.10.2 Glorin-regulated gene expression is not dependent on de novo protein synthesis

3.11 Glorin induces aggregation sensitivity in starving $P$. pallidum cells.

4.1 Glorin is an ancient extracellular messenger molecule used for intercellular communication in Dictyostelids.

4.2 Stage specific responses of $P$. pallidum PN500 cells to exogenous glorin

4.3 Glorin mediates rapid changes in gene expression during early development of $P$. pallidum. 153

4.4 Rapid turn-over of transcripts of glorin-induced genes. 154

4.5 Glorin-mediated gene repression. 155

4.6 Allosteric or covalent modification of existing proteins may mediate rapid effects of glorin.

4.7 Dose-response effects of glorin on gene induction. 159

4.8 General kinetics of gene induction in response to repetitive stimulation of $P$. pallidum PN500 cells with glorin remains the same.

4.9 Stimulation of $P$. pallidum PN500 cells with glorin induces precocious aggregation. 160

4.10 Pre-starvation is not needed to observe glorin-induced changes in gene expression 
4.11 Glorin-mediated changes in gene expression do not depend on de novo protein expression.

4.12 Possible molecular mechanisms by which glorin may modulate gene expression changes.

4.13 Differences between glorin-signalling system and cAMP acrasin system of $D$. discoideum 164

4.14 How did the glorin acrasin system evolve? 165

4.15 Synthesis, storage, and secretion of glorin. 167

4.16

Perspectives 168

4.16.1 Determination of mechanisms regulating glorin-induced gene expression 168

4.16.1.1 Does glorin stimulate de novo transcription?. 168

4.16.1.2 Possible post-transcriptional regulation of glorin-induced gene expression 169

4.16.2 Identification of glorin stimulus-inducible promoters by ChiP-on-chip. 170

4.16.3 Identification of cis-acting DNA elements that may be responsive to glorin signalling in the promoter region of glorin-regulated genes.

4.16.4 Identification of trans-acting factors interacting with promoters of glorininduced genes.

4.16.4.1 Gel mobility shift assays 172

4.16.5 Identification of glorinase and enzyme activity assay................... 173

4.16.6 Identification cell surface of glorin receptor............................ 174

4.16.7 Determination of the biological roles played by glorin-regulated genes.... 174

5 SUMMARY 175

6 ZUSAMMENFASSUNG. 177

Table A1: Summary of proteins identified in the buffer conditioned by aggregating P. pallidum PN500 amoebae. The list was assembled from two independent experiments. 205

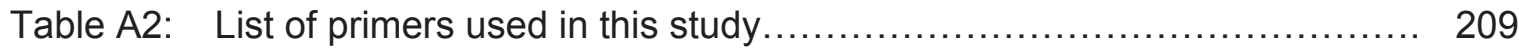

Table A3: Changes of gene expression in starving cells......................... 211

Table A4: List of genes regulated by glorin after $P$. pallidum PN500 cells prestarved for $1 \mathrm{hr}$ were stimulated with glorin for an additional $1 \mathrm{hr}$.

Table A5: List of genes regulated by glorin after $P$. pallidum PN500 cells 
prestarved for $1 \mathrm{hr}$ were stimulated with glorin for 2 additional hours..... 239

Table A6: Genes regulated by glorin after $1 \mathrm{hr}$ of pre-starvation plus $1 \mathrm{or} 2 \mathrm{hr}$ of glorin treatment.

Table A7: GO term analysis of genes differentially regulated after 1 hour of prestarvation plus $1 \mathrm{hr}$ of glorin treatment.

Table A8: GO term analysis of genes differentially regulated after 1 hour of pre-starvation plus 2 hours of glorin treatment.................... 247

Table A9: Complete List of GPCR genes upregulated by glorin ..................... 250

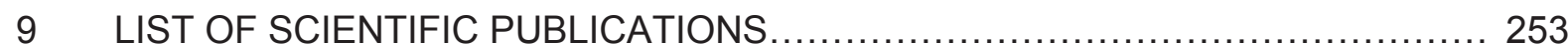

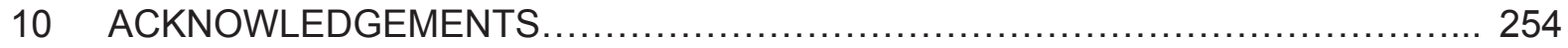

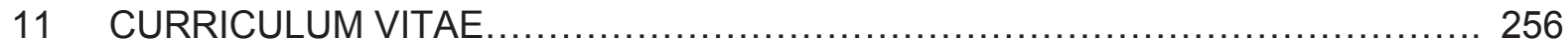

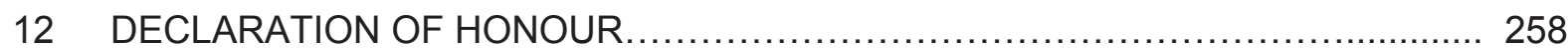




\section{LIST OF FIGURES}

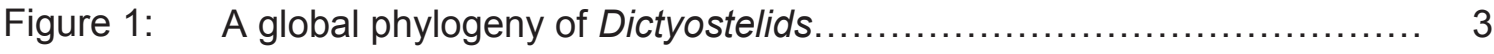

Figure 2: $\quad$ Fruiting body morphologies of different Dictyostelids species............ 4

Figure 3: $\quad$ Schematic illustration of the 24 hour life cycle of Dictyostelium ........... 7

Figure 4: Signal transduction pathways modulating chemotaxis and aggregation in Dictyostelium ....................................................... 11

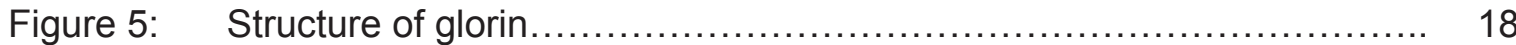

Figure 6: The chemical structures of glorin and the products of enzymatic

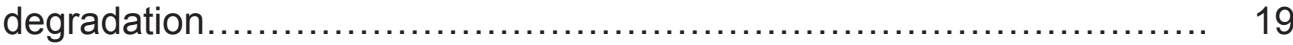

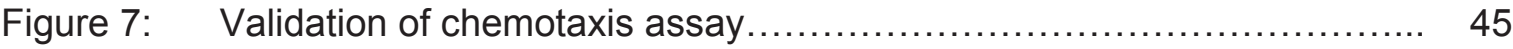

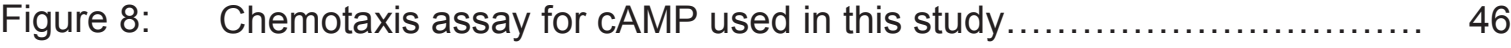

Figure 9: Chemotactic activity of starving $P$. violaceum amoebae to cAMP and

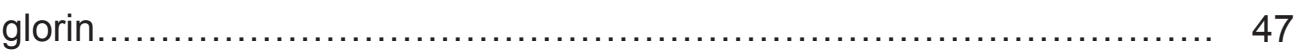

Figure 10: Chemotactic response to glorin by $P$. pallidum PN500 ..................... 49

Figure 11: Chemotactic response of $P$. pallidum PN500 amoebae to differing concentrations of glorin

Figure 12: Effects of varying starvation times on the ability of $P$. pallidum PN500 amoebae to respond to glorin.

Figure 13: Chemotactic response of starving P. pallidum PN500 amoebae to high

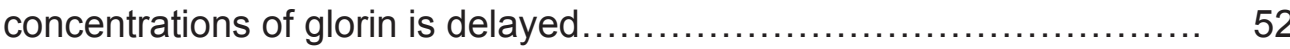

Figure 14: Fruiting body morphologies of different isolates of $P$. pallidum............ 54

Figure 15: Alignment of ITS sequences from P. pallidum isolates, namely, PN500, WS320, CK8, and H168 generated with CLUSTAL 2.0 ................. 56

Figure 16: Chemotactic response to glorin by group 2 species.................... 57

Figure 17: Chemotaxis to glorin by group 1 species.............................. 58

Figure 18: Experimental setup for collection of secreted proteins $\ldots \ldots \ldots \ldots \ldots \ldots \ldots \ldots \ldots$

Figure 19: $\quad$ Proteins secreted by aggregating $P$. pallidum PN500 cells............... 61

Figure 20: $\quad$ Aggregation of $P$. pallidum PN500 amoebae under buffer................ 64

Figure 21: Expression kinetics of two putative GPCR genes during pulse development.

Figure 22: Effect of starvation on global gene expression in starving $P$. pallidum cells

Figure 23: Correlation of RNA-seq data with real-time RT-PCR data 70 
Figure 24: Effects of exogenous glorin stimulation on global gene expression in $P$. pallidum PN500.

Figure 25: Correlation of RNA-seq data with real-time RT-PCR data................ 76

Figure 26: Correlation of RNA-seq data with real-time RT-PCR data............... 78

Figure 27: Correlation of RNA-seq data with real-time RT-PCR data............... 80

Figure 28: Correlation of RNA-seq data with real-time RT-PCR data............... 82

Figure 29: Correlation of RNA-seq data with real-time RT-PCR data............... 83

Figure 30: Glorin-induced gene expression in response to varying concentrations of

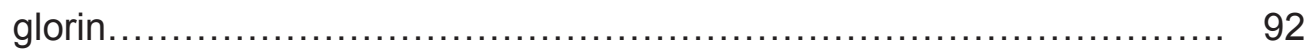

Figure 31: Glorin-induced gene expression in response to varying periods of exposure to glorin.

Figure 32: Glorin-induced changes in expression of PPL_09347 in response to varying concentrations and periods of exposure to glorin.

Figure 33: Glorin-induced changes in expression of PPL_05354 in response to varying concentrations and periods of exposure to glorin.

Figure 34: Glorin-induced changes in expression of PPL_05833 in response to varying concentrations and periods of exposure to glorin. 100

Figure 35: Glorin-induced changes in expression of PPL_12271 in response to varying concentrations and periods of exposure to glorin. 101

Figure 36: Glorin-induced changes in expression of PPL_07908 in response to varying concentrations and periods of exposure to glorin 102

Figure 37: Time course of glorin effects on PPL_09347 expression.... 105

Figure 38: Time course of glorin effects on PPL_05354 expression.................. 106

Figure 39: Time course of glorin effects on PPL_05833 expression.................. 108

Figure 40: Time course of glorin effects on PPL_12271 expression................. 109

Figure 41: Time course of glorin effects on PPL_07908 expression.................. 110

Figure 42: Time course of glorin effects on PPL_12248 expression.................. 111

Figure 43: Time course of glorin effects on PPL_12249 expression.................... 113

Figure 44: Prominent morphological states during growth-to-aggregation transition of starving $P$. pallidum PN500 amoebae. 115

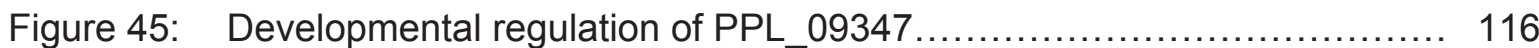

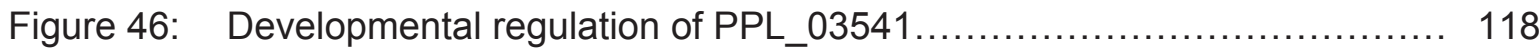

Figure 47: Developmental regulation of PPL_06644 $\ldots \ldots \ldots \ldots \ldots \ldots \ldots \ldots \ldots \ldots \ldots \ldots \ldots \ldots \ldots \ldots \ldots \ldots \ldots$

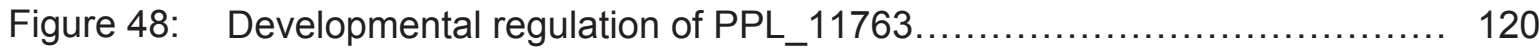

Figure 49: Developmental regulation of PPL_05354........................... 121

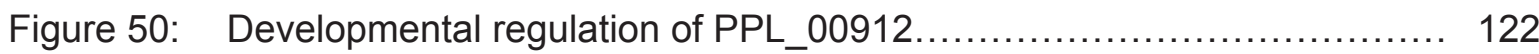




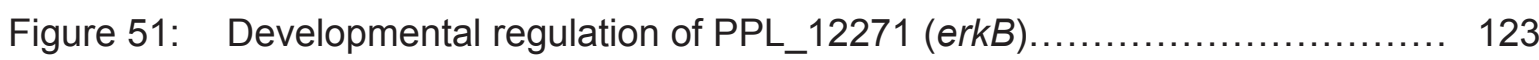

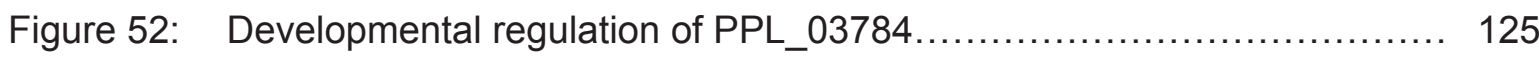

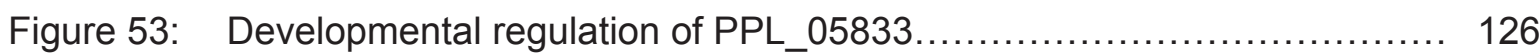

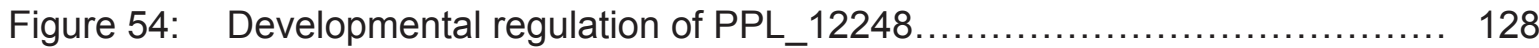

Figure 55: Developmental regulation of GPCR genes PPL_00902 \& PPL_05727... 131

Figure 56: Developmental regulation of GPCR genes PPL_04108 \& PPL_00855... 132

Figure 57: Developmental regulation of GPCR genes PPL_08454, PPL_08455 \&

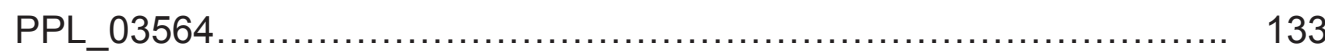

Figure 58: Expression kinetics of selected components of cAMP signalling in P. pallidum......................................................... 138

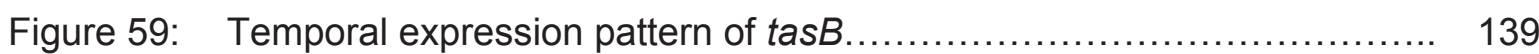

Figure 60: $\quad P$. pallidum PN500 tas $A^{-} / \operatorname{tas}^{-}$mutant exhibits normal chemotaxis

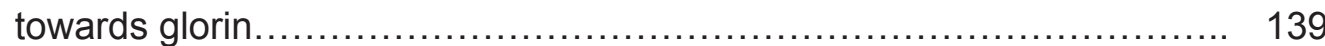

Figure 61: Time-course of PPL_09347 and PPL_05833 mRNA accumulation in untreated and glorin-treated cells.

Figure 62: Comparison of induction kinetics of PPL_09347 and PPL_05833 in unprestarved and prestarved cultures of $P$. pallidum PN500 cells following stimulation with exogenous glorin....................................... 143

Figure 63: Glorin-induced gene expression is insensitive to cycloheximide.......... 146

Figure 64: Developmental phenotypes of glorin treated and untreated cells.......... 148

Figure 65: A simplified phylogenetic tree summarizing glorin chemotaxis by social amoebae.

Figure 66: Chemical structure of glorin (left) and glutathione (right) 166 


\section{LIST OF TABLES}

Table 1: Chemotactic activity of aggregation competent amoebae from four groups of Dictyostelids tested towards glorin and cAMP................. 59

Table 2: $\quad$ Spearman correlation coefficients for all RNA-seq data sets.............. 68

Table 3: Absolute expression data of selected genes obtained by RNA-seq analysis.......

Table 4: $\quad$ Summary of glorin-induced genes in $P$. pallidum PN500.

Table 5: $\quad$ Summarized expression kinetics of chosen glorin-regulated genes in $P$. pallidum PN500 cells developed in shaking cultures (in the presence or absence of glorin) and on non-nutrient agar plates.... 


\section{ABBREVIATIONS}

\begin{tabular}{|c|c|}
\hline ACA & $\begin{array}{l}\text { Adenylyl cyclase A (aggregation stage adenylyl } \\
\text { cyclase) }\end{array}$ \\
\hline \multirow[t]{2}{*}{ AEBSF } & 4-(2-Aminoethyl)benzenesulfonyl fluoride \\
\hline & hydrochloride \\
\hline ARE-mRNA & $\mathrm{AU}$ rich elements containing mRNA \\
\hline ATCC & American Type Culture Collection \\
\hline $\mathrm{Bp}$ & base pair \\
\hline CbfA & C-module binding factor $A$ \\
\hline CF & counting factor \\
\hline cGMP & Cyclic Guanosine Monophosphate \\
\hline $\mathrm{CHX}$ & Cycloheximide \\
\hline CMF & conditioned medium factor \\
\hline cPIG & cAMP pulse-induced gene \\
\hline CRAC & Cytosolic Regulator of Adenylyl Cyclase \\
\hline DBS & Dictybase stock \\
\hline D. discoideum & Dictyostelium discoideum \\
\hline DdCAD & Dictyostelium discoideum cell adhesion molecule \\
\hline DdMEK1 & Dictyostelium discoideum MAP kinase kinase \\
\hline ePDE & Extracellular phosphodiesterase \\
\hline ESI-MS & Electrospray ionization mass spectrometry \\
\hline \multirow[t]{2}{*}{ ESI-MS/MS } & Electrospray Ionisation Tandem Mass \\
\hline & Spectrometry \\
\hline GAT & growth to aggregation transition \\
\hline GBF & G-box Binding Factor \\
\hline GbpC & cGMP-binding protein C \\
\hline GCA & Guanylyl cyclase A \\
\hline GCs & Guanylyl cyclases \\
\hline GDT & growth to development transition \\
\hline GEF & guanine nucleotide exchange factor \\
\hline GloRE & Glorin Response Element \\
\hline GPCRs & G-protein coupled receptors \\
\hline HGT & Horizontal gene transfer \\
\hline IP3 & Inositol trisphosphate \\
\hline
\end{tabular}




\begin{tabular}{|c|c|}
\hline ITS & Internal transcribed spacer \\
\hline JNK & C-Jun $\mathrm{N}$-terminal protein kinase \\
\hline $\mathrm{kDa}$ & kilo Dalton \\
\hline L & Litre \\
\hline MAPK & mitogen-activated protein kinase \\
\hline MAPKKK & $\begin{array}{l}\text { MAP kinase kinase kinase also called MAP3K or } \\
\text { MEKK }\end{array}$ \\
\hline $\mathrm{ml}$ & millilitre \\
\hline$M Q$ & $\begin{array}{l}\text { Milli } Q \text {, Distilled water fed through a special ion } \\
\text { exchange cartridge to increase its purity }\end{array}$ \\
\hline MW & Molecular weight \\
\hline oligo (dT) & oligodesoxythymidine \\
\hline PAGE & Polyacrylamide gel electrophoresis \\
\hline PDE & phosphodiesterase \\
\hline PDI & Phosphodiesterase Inhibitor \\
\hline $\mathrm{PH}$ & pleckstrin homology \\
\hline PKA & protein kinase $\mathrm{A}$ \\
\hline PKA-C & Catalytic subunit of pretein kinase $A$ \\
\hline PKA-R & Regulatory subunit of pretein kinase $A$ \\
\hline PKB & protein kinase $B, A k t$ \\
\hline PKC & Protein kinase $\mathrm{C}$ \\
\hline PI3K & Phosphatidylinositol 3-kinase \\
\hline PIP2 & Phosphatidylinositol $(3,4)$-bisphosphate \\
\hline PIP3 & Phosphatidylinositol $(3,4,5)$-trisphosphate \\
\hline PLC & Phospholipase C \\
\hline $\mathrm{Pp}$ & Polysphondylium pallidum \\
\hline P. pallidum & Polysphondylium pallidum \\
\hline PPM & parts per million \\
\hline PSF & prestarvation factor \\
\hline Ras & Rat Sarcoma (A family of proteins) \\
\hline Ras-GEF & Ras- Guanine nucleotide exchange factor \\
\hline $\operatorname{Reg} A$ & Intracellular phosphodiesterase \\
\hline RIP3 & Ras-interacting protein \\
\hline RNA-seq & RNA sequencing \\
\hline Rpm & Revolutions per minute \\
\hline RT-PCR & Reverse transcriptase polymerase chain reaction \\
\hline
\end{tabular}


SACGB

SDS

sGC

SNP

UTR
Social Amoebas Comparative Genome Browser sodium dodecyl sulfate

soluble guanylyl cyclase

single nucleotide polymorphism

Untranslated region 


\section{Introduction}

The social amoebas, or Dictyostelids, are an excellent system to study complex communication between cells in organizing multicellular development, since they can still alternate a sophisticated programme of multicellular morphogenesis with a unicellular feeding lifestyle. Development of multicellularity requires the intricate cell-to-cell communication and coordinated regulation of numerous genes (Iranfar et al. 2003; Van Driessche et al. 2002; Williams 2006). In the present work, roles of a peptide-based cellcell communication system in Dictyostelids will be studied. To introduce the theme, an overview is given of the Dictyostelids life history, taxonomic position, and the complex intercellular communication required at all stages of their life cycle. Then a special insight is given into the mechanism of cell-cell communication at the transition from growth to aggregation. The cAMP communication system operating at aggregation stage of $D$. discoideum is briefly introduced. The current state of research on peptide mediated cellcell communication among Dictyostelids is presented and finally aims of this study and its importance are discussed.

\subsection{The social amoebae- Dictyostelids}

The Dictyostelids, which are called cellular slime molds, are a major group of unicellular haploid soil-dwelling microorganisms that hover at the borderline of true multicellularity (Romeralo et al. 2011a; Schaap et al. 2006). While they spend most of their life cycle as solitary amoebae, upon starvation Dictyostelids can aggregate to form a multicellular fruiting body that consists of dead stalk cells and live spores (Kalla et al. 2011). The entire process of development is coordinated by extensive intercellular communication through the production and in response to chemoattractants.

\subsubsection{Taxonomy}

For long time, since their discovery in 1869 by Brefeld (Brefeld 1869), Dictyostelids were considered to be "lower fungi" based on their superficial resemblance to fungi. Dictyostelids are also called "myxamoebae" due to the fact that single cells look like amoebae and move and feed in an amoeboid manner. In traditional systematics, all amoeba-like protists that constructed spore-bearing fruiting bodies were assigned to class Myxomycota of division Mycetozoa in the kingdom of fungi. The Myxomycota were 
subdivided into the Protostelids, Myxogastrids, and the Acrasiomycetes. Acrasiomycetes included two subclasses: the Acrasids and the Dictyostelids. The Acrasids are significantly different from the Dictyostelids in the morphology of individual amoebas and aggregates and lack of cellulose in spore-bearing stalk tubes (Raper 1984; Bonner 1982).

Modern taxonomy based on a combination of cellular and molecular sequence data has revealed six supergroups of eukaryote organisms (excavates, Rhizaria, Chromalveolates, Plantae, Amoebozoa and Opisthokonts) that in turn may comprise just two superclades: unikonts (Amoebozoa, Opisthokonts) and bikonts (the other supergroups; Minge et al. 2009; Richards \& Cavalier-Smith 2005; Keeling et al. 2005; Keeling 2004; Simpson \& Roger 2004; Baldauf 2003; Stechmann \& Cavalier-Smith 2003). Dictyostelids are the members of the supergroup Amoebozoa (consisting mainly of unicellular amoeba-like-organisms) that forms a sister clade to the Opisthokonts, the group containing fungi and animals, having diverged more recently than the plants (Schaap 2011b; Baldauf et al. 2000; Baldauf \& Doolittle 1997). Obviously, morphologybased classification does not represent the genetic similarity between different groups of organisms (Schaap 2011b).

\subsubsection{Phylogeny of Dictyostelids}

Traditionally, classification of Dictyostelids has been based on their most notable trait, fruiting body morphology (Hagiwara 1989; Raper 1984). Based on this trend, three genera of Dictyostelids were defined: Dictyostelium (Brefeld 1869) with cellular stalks and mostly unbranched or rarely laterally branched fruiting bodies; Polysphondylium (Raper 1984) with regularly spaced whorls of lateral branches on cellular stalks, and Acytostelium (Raper 1956) which forms fruiting bodies with acellular stalks composed of cellulose.

A cladistic study by Swanson and co-workers (Swanson et al. 2002) proposed that traditional fruiting body morphology based classification of Dictyostelids is deeply flawed. Later, this suggestion was validated by the first molecular phylogeny based on alphatubulin and SSU (18S) rDNA sequences (Romeralo et al. 2007; Schaap et al. 2006). Molecular phylogeny constructed by Schaap et al. (2006; Figure 1) included nearly all of the species known at that time (most than 100 isolates of Dictyostelids) into four major groups. Group 1 consists of morphologically distinct Dictyostelium species, all of which 
have small spores bearing fruiting bodies. Group 2 named "Heterostelids" includes representatives from all three of the traditional genera, accommodating all pale-colored species of Polysphondylium (Figure 2), at least two species of Dictyostelium, and all species of Acytostelium.

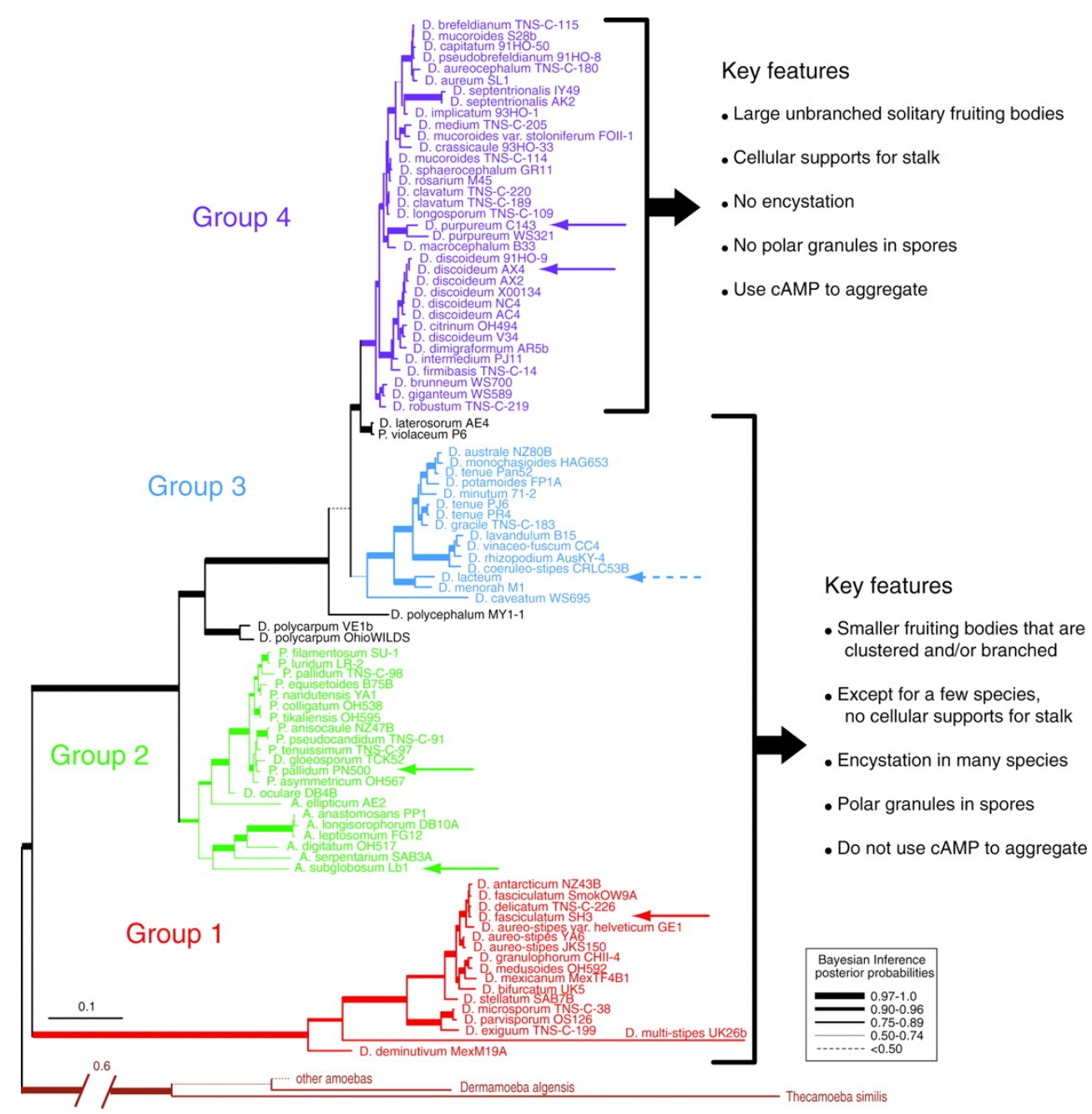

Figure 1: A global phylogeny of Dictyostelids. Most known species of social amoebas were subdivided into four major groups based on phylogenetic analysis of conserved SSU rDNA sequences from 1655 aligned positions using Bayesian inference. Coloured arrows refer to species with completely sequenced genomes. The family tree of social amoebas is rooted on SSU rDNA sequences of closely related solitary amoebas. (Modified from Schaap 2011a; Schaap et al. 2006)

Group 3 defined as "Rhizostelids" comprises a mixture of Dictyostelium species with rootlike support structures for their fruiting bodies and also includes the cannibalistic species, Dictyostelium caveatum. Group 4 is the most species-rich group of all major Dictyostelid groups composed almost exclusively of modern Dictyostelium species but 
also combines a clade of two violet-colored species from two independent traditional genera, Polysphondylium violaceum and Dictyostelium laterosorum (Schaap et al. 2006).
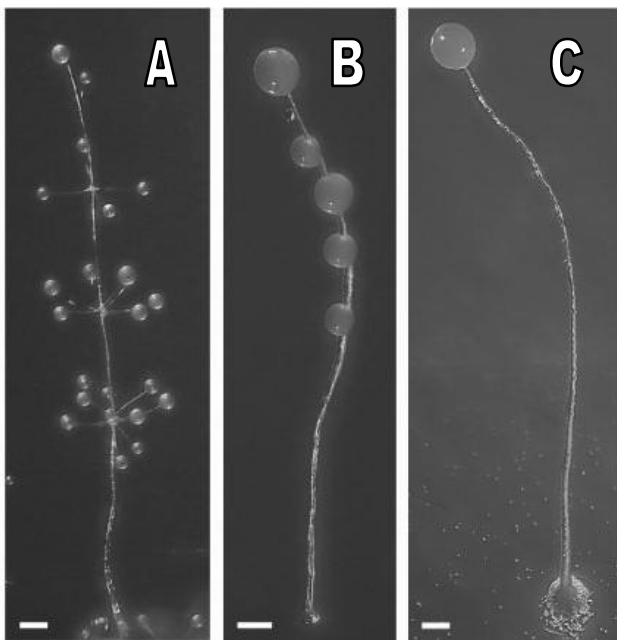

Figure 2: Fruiting body morphologies of different Dictyostelids species. A: Polysphondylium pallidum (group 2). B: Dictyostelium rosarium (group 4). C: Dictyostelium discoideum (group 4). (Modified from Schaap 2007)

Group 4 includes the best characterized Dictyostelid species D. discoideum (Figure 2), that is prominent as the simple eukaryotic model organism used to study important processes in cell biology, such as general principles for cell-to-cell communication, signal transduction, chemotaxis, cytoskeletal organization during cell motility, vesicle trafficking and phagocytosis (Schaap 2011a; Swaney et al. 2010; Cosson and Soldati 2008). The D. discoideum genome contains numerous orthologs of genes responsible for diseases in humans and has therefore become a popular model organism in biomedical research to explore the molecular basis of various human diseases, as well as the mechanism of drug action (Schaap et al. 2011a; Annesley \& Fisher 2009; Williams et al. 2006; Eichinger et al. 2005). Studies in this organism are contributing to understand the correlation between regulated cell movement and controlled cell differentiation that gives rise to the shape and pattern during multicellular development (Schaap 2011a; Weijer 2009; Williams 2006; Kimmel \& Firtel 2004).

Interestingly, none of the four molecularly defined groups are consistent with the traditional genera and none of the traditional genera are monophyletic excluding Acytostelium (Schaap et al. 2006). Moreover, first global SSU rDNA phylogeny of Dictyostelids revealed three lineages without clear affinity to any major group: $D$. 
polycarpum (2 isolates), $D$. polycephalum (one isolate) and $D$. laterosorum $+P$. violaceum (2 isolates: Schaap et al. 2006).

The molecular phylogeny of social amoebae also proposed enormous molecular depth roughly comparable to that of animals but significantly greater than that of fungi. While original dictyostelid molecular phylogeny (Schaap et al. 2006) was based on slowly evolving SSU rDNA, it could not resolve majority of branches within the four groups. Romeralo et al. (2010) determined complete sequence data of more rapidly evolving internal transcribed spacer (ITS) region of rDNA for almost all species in the first molecular phylogeny that in combination with previous data of SSU rDNA sequences successfully resolved nearly all species level relationships within each of the four major groups. Five "species complexes" consisting of cryptic species (i.e. morphologically similar but genetically quite distinct species) were identified dispersed across the dictyostelid phylogeny (Romeralo et al. 2010; Mehdiabadi et al. 2009). Romeralo and colleagues (Romeralo et al. 2011b) reported 18S ribosomal RNA gene sequences data of new isolates identified by the Mycetozoan Global Biodiversity Survey in the past four years. Analyses of these data showed at least 50 new species and some more isolates of already characterized species scattered across the phylogentic tree, breaking up many previously isolated long branches. The resulting highly extended tree now includes eight major groups instead of the formerly recognised four (Romeralo et al. 2011c; Romeralo et al. 2011b; Schaap et al. 2006). Three major groups in new highly resolved phylogeny (Romeralo et al. 2011b) correspond to "lineages" that included only one or two sequences in the first molecular phylogeny (Schaap et al. 2006), and two groups originate from a firmly supported deep split in Group 2 (Romeralo et al. 2011b). The new groups are referred as the "polycarpum", "polycephalum" and "violaceum" complexes (Romeralo et al. 2011b) in order to maintain the original group numbering scheme as presented by Schaap et al. (2006) until formal names can be ascribed (Romeralo et al. 2011b: Schaap et al. 2006). The new species also broaden the known morphological variance of the four major groups defined by Schaap et al. (2006), challenging nearly all previously suggested deep morphological patterns (Romeralo et al. 2011b; Schaap et al. 2006).

Currently two positions are suggested for the root of Dictyostelids, either positioning Group 1 as the earliest lineage that diverged from a common ancestor (root 1, Figure 1, Schaap et al. 2006) or bifurcating the taxon approximately equally along the branch connecting Groups $1+2$ and Groups $3+4$ (root 2; Romeralo et al. 2011b; Heidel \& 
Glöckner 2008; Schaap et al. 2006). Depending upon the outgroup used, analyses of SSU rDNA and alpha-tubulin present either alternative without concrete statistical support (Schaap et al. 2006), however, phylogenetic analysis of mitochondrial genes (Heidel \& Glöckner 2008) strongly supported root 2.

In addition to the genome of the model D. discoideum (Eichinger et al. 2005), the genomes of Dictyostelium fasciculatum (group 1 species) and $P$. pallidum (group 2) are now entirely sequenced (Heidel et al. 2011). Draft sequence data for Dictyostelium purpureum (group 4; Sucgang et al. 2011) are also accessible and the genome of a group 3 species Dictyostelium lacteum is presently being assembled. Genome sequence of Acytostelium subglobosum is also available now (http://acytodb.biol.tsukuba.ac.jp/cgibin/info.cgi?page=1; unpublished).

\subsection{Communication modules in the life cycle of Dictyostelids}

Dictyostelids start life as individual amoeboid cells that feed on bacteria in decaying vegetation (Schaap 2011b). As cells grow and divide, they have the ability to sense the gradients of metabolites secreted by bacteria they feed on. With an increase in population, cells start to behave cooperatively and secrete quorum-sensing factors to track population size. When the switch to enter development is made, intricate intercellular communication through the coordinated secretion of the chemoattractant governs the number and directionality of cells to aggregate into multicellular mounds (Schaap 2011b). The top of mound continues to emit chemoattractant pulses and is pushed upwards by the displacement of cells beneath (Schapp 2011b; Siegert \& Weijer, 1995). The emerging multicellular structure of cells then falls over and is now called the "slug". Within this structure, cells respond to and trigger cues that differentiate the clonal population into the specific cell types (Mahadeo \& Parent 2006). The best fed amoebae prepare to become dormant spore (Schaap 2011b), whereas the rest are destined to form different supporting regions of the terminal fruiting body (Figure 3 ). The slug migrates as a more or less coherent unit, with a head region searching environmental conditions favourable for the formation of fruiting bodies (Kessin 2001). The slug stage proceeds to a process called culmination, which involves the terminal differentiation of the multicelluar organism to build fruiting body (Figure 3). 


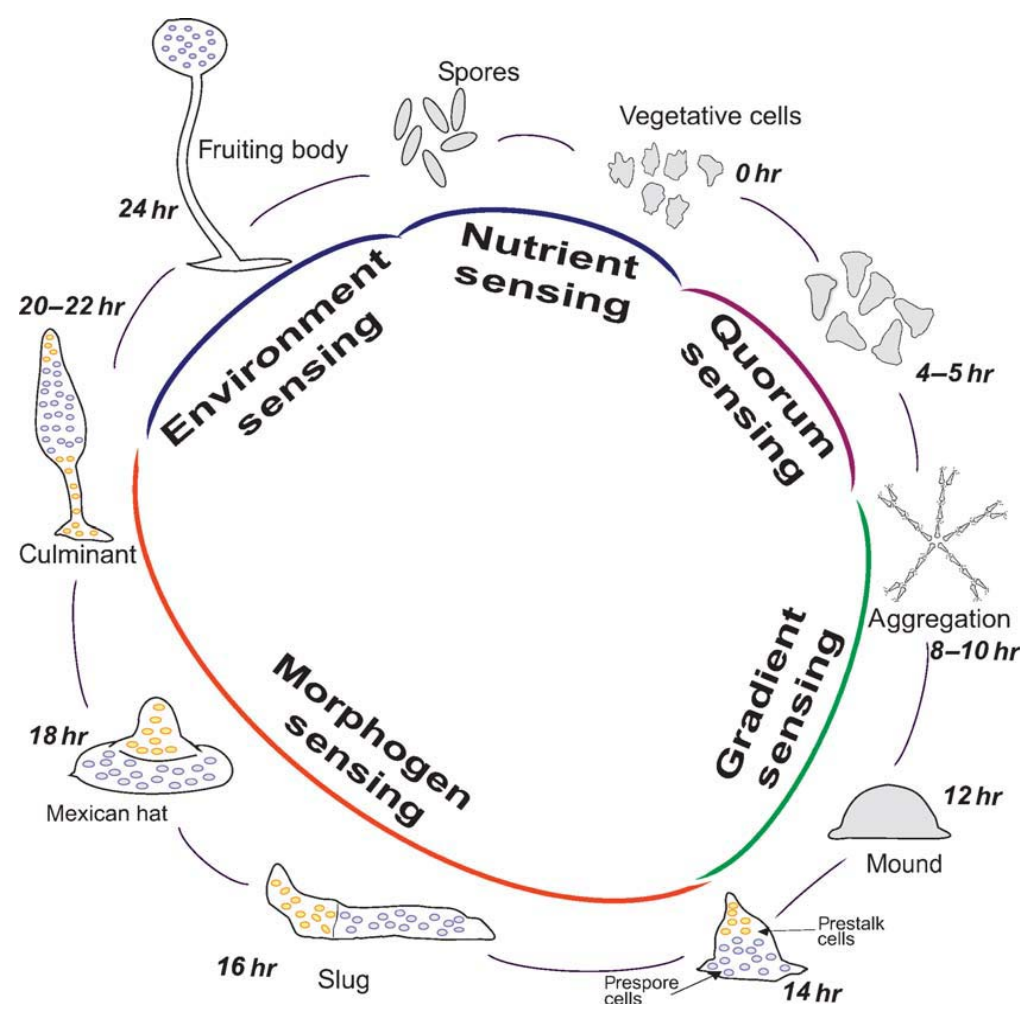

Figure 3: Schematic illustration of the $\mathbf{2 4}$ hour life cycle of Dictyostelium divided into five signalling modules. Time after starvation (hours) is marked next to each stage of development. Complex cell-cell communication is required at all these developmental check points (Mahadeo \& Parent 2006).

Depending on the species, fruiting bodies differ widely in morphology but essentially consist of one or more stalks, composed of vacuolated dead cells bearing aloft one or more distinct spore masses in a variety of arrangements (Raper 1984). The spores have the ability to sense the environment (Kishi et al. 1998) and cues from each other to control the decision to germinate (Mahadeo \& Parent 2006). Once the germination process has begun, spores can again communicate with each other to release amoebae in a synchronous fashion (Cotter et al. 2000).

\subsubsection{Communication at the Transition from Growth to Aggregation}

The life cycle of Dictyostelids is marked by two completely independent phases: growth and development. During growth period unicellular amoebae feed on bacteria and multiply by binary fission. Upon starvation, cells stop dividing and initiate a differentiation programme leading to the erection of a fruiting body (Kessin 2001; Romeralo et al. 
2011b; Raper 1940; Bonner 1967). The transition between these two phases occurs through a process of aggregation, during which the single amoebae gather to form a multicellular mound which subsequently differentiates into a migrating slug. The wellcoordinated activity of a large number of cells engaged in aggregation is mediated by extensive intercellular communication that involves the production of concentration gradients of diffusible chemoattractant molecules known as "acrasins", and the reading of the gradient at closely determined intervals of time (Konijn et al. 1967; Bonner 1947). Because traditionally Dictyostelids were placed together with Acrasids in the subdivision Acrasiomycetes of class Myxomycota, the term 'acrasin' was proposed by John Bonner to define " a type of substance consisting either of one or numerous compounds which is responsible for stimulating and directing aggregation in certain members of the Acrasiales" (Bonner 1947).

The 'acrasins' underlying aggregation in some Dictyostelid species have been identified. Model species $D$. discoideum and other investigated group four species use cAMP as acrasin (Schaap et al. 2006; Konijn et al.1969; Barkley 1969; Konijn et al. 1968; Konijn et al. 1967), Dictyostelium minutum utilizes folic acid derivative (De Wit \& Konijn 1983), Dictyostelium lacteum a pterin derivative (Van Haastert et al. 1982) and Polysphondylium violaceum and Dictyostelium caveatum use a modified dipeptide glorin (Shimomura et al. 1982; Waddell 1982b) to aggregate. A fascinating aspect of $D$. discoideum and other group 4 species development is that secreted cAMP is used both as a chemoattractant to coordinate aggregation and as a morphogen in organizing the construction of fruiting bodies. In comparison, in group 1-3 Dictyostelids, extracellular cAMP exerts its effects during postaggregative stages as a morphogen while the aggregative chemoattractant may be different.

For successful aggregation, starving cells must develop a number of specialized properties in the pre-aggregation phase which separates the growth and aggregation phases (Kessin 2001). In a starving population, a few autonomous cells must appear occasionally that start to release acrasin spontaneously, thereby initiating the coordinated activity of surrounding cells (Shaffer 1962). The amoebae must be able to detect the acrasin and respond by moving up its gradient towards increasing concentration. After being stimulated by acrasin, the starving amoebae must develop the capacity to synthesize and release the same by themselves, thus propagating the acrasin signal to neighboring cells. Also, cells must acquire the ability to become refractory to further acrasin stimuli for a short period of time (Brzostowski \& Kimmel 2006; 
Kessin 2001; Cohen \& Robertson 1971; Gerisch 1968; Shaffer 1957b). Cells must also be capable to remove acrasin locally by acrasinase activity so that acrasin concentration in the immediate vicinity of cells is insufficient to re-excite them as they come out of the refractory period (Shaffer 1957a). After individual starving cells have developed aggregation competence, a coordinated wave of activity begins at the autonomous cell resulting in secretion of acrasin that marks the beginning of the aggregation phase.

\subsubsection{Receptors for cell communication in Dictyostelids}

Social amoebae use different chemical signals for cell-cell communication during all phases of their life cycle. Accordingly, these organisms express stage-specific cellsurface receptors that distinctively bind the signal molecules, thereby discriminating between similar compounds in the soup of competing molecules. During growth phase, Dictyostelids can sense the folates emitting bacterial food, using highly specific folate receptors on their cell surface membranes (Pan et al. 1972; Wurster \& Butz 1980; Van Driel 1981; Tillinghast \& Newell 1984). When the food source is exhausted, amoebae develop different receptors to detect small communicating molecules, i.e. 'acrasins' that coordinate aggregation of amoebae to form a multicellular organism. The molecules that mediate aggregation, and the receptors involved, differ from species to species.

The convoluted mechanism of intercellular communication intervening aggregation is well determined in the model organism $D$. discoideum that uses cAMP as aggregative signalling molecule, i.e. acrasin, but the details of multicellular development are largely unknown in other species (Bonner 2009; Alvarez-Curto et al. 2005; Kessin 2001).

\subsubsection{Previous knowledge of cell-cell communication in D. discoideum}

\subsubsection{Secreted factors that regulate gene expression in Dictyostelids}

In $D$. discoideum, growth to aggregation transition is initially regulated by quorum sensing signal molecules including prestarvation factor (PSF), conditioned medium factor (CMF), and counting factor (CF). PSF, a glycoprotein (Mahadeo and Parent 2006; Clarke et al. 1988; Burns et al. 1981) is constantly secreted by growing cells to monitor their cell density relative to that of their bacterial food source. When the ratio of PSF relative to that of bacteria rises above a certain threshold, cells stop growing and PSF initiates to 'prime' the cells for the upcoming process of aggregation by inducing the expression of 
several early developmental genes (Schaap 2011a; Clarke \& Gomer 1995). First genes induced by PSF include those encoding discoidins and lectins, proteins that play a role in cytoskeletal organization and cell morphology during aggregation (Rathi \& Clarke 1992; Rathi et al. 1991). One of the critical signalling pathways modulating the growth to aggregation transition involves the activation of the serine/threonine kinase YakA by the PSF response pathway (Schaap 2011b; Souza et al. 1998). Activation of YakA leads to the phosphorylation and inhibition of PufA, a negative regulator of translation. In feeding cells of $D$. discoideum, PufA blocks the translation of mRNA encoding PkaC, the catalytic subunit of pretein kinase A (PKA) by binding to the 3' untranslated region (UTR) of PkaC mRNA (Schaap 2011b; Souza et al. 1999). PkaC modulates the timing of early developmental events by regulating the expression of vital CAMP signaling proteins such as CAR1 and ACA (Anjard et al. 1992; Mann et al. 1992). PKA activity is not required for growth, but it is a compulsion for the transition from growth to aggregation (Schaap 2011a; Schulkes \& Schaap 1995; Simon et al. 1989). At the onset of starvation, secretion of PSF declines rapidly (Rathi et al. 1991). A second glycoprotein, CMF (conditioned medium factor), is secreted during starvation and induces initial expression of genes whose products are required for cAMP relay system, which is necessary for the cells to aggregate (Yuen et al. 1995; van Haastert et al. 1996; Brazill et al. 1998). CMF acts by binding to a developmentally regulated cell surface CMF receptor (Jain \& Gomer 1994). PSF- and CMF-induced genes include adenylyl cyclase $A$ ' $a c a A$ ' responsible for synthesizing CAMP; CAMP receptor ' $C A R 1$ ' to detect CAMP during aggregation and the extracellular phosphodiesterase 'PdsA' for hydrolyzing cAMP (Schulkes \& Schaap 1995). cAR1, PdsA and ACA together with PKA and RegA, an intracellular cAMP phosphodiesterase, form complex biochemical connections that produce cAMP pulses in an oscillatory manner (Schaap 2011a; Iranfar et al. 2003; Laub \& Loomis 1998).

\subsubsection{The Signalling pathways by which cAMP regulates expression of early genes}

Initially a few amoebae behaving as the aggregation centres start to emit periodic cAMP pulses, which are detected by the surrounding amoebae via cell surface cAMP receptors (Goldbeter 2006; Kimmel \& Parent 2003; Halloy et al. 1998; Waddell 1982a). In D. discoideum, the extracellular cAMP signal is detected by four seven-transmembrane $G$ protein-coupled receptors (GPCRs), designated cAR1-4. First cAMP receptor expressed during development is cAR1, followed sequentially by cAR3, cAR2, and then cAR4. The high affinity cAMP receptors CAR1 and CAR3 are required for aggregation (Johnson et al. 
1993, 1991; Saxe et al. 1991a; Sun \& Devreotes 1991; Klein et al. 1988), however, cAR1 is the principle mediator of the effects of extracellular cAMP during early development. In D. discoideum, heterotrimeric G-protein complexes may include 1 of 11 a subunits coupled to a single ßy subunit (Mahadeo \& Parent 2006; Zhang et al. 2001; Wu et al. 1995a; Lilly et al. 1993). cAR1, the aggregation specific receptor is coupled to Ga2ßY complex (Kumagai et al. 1991).

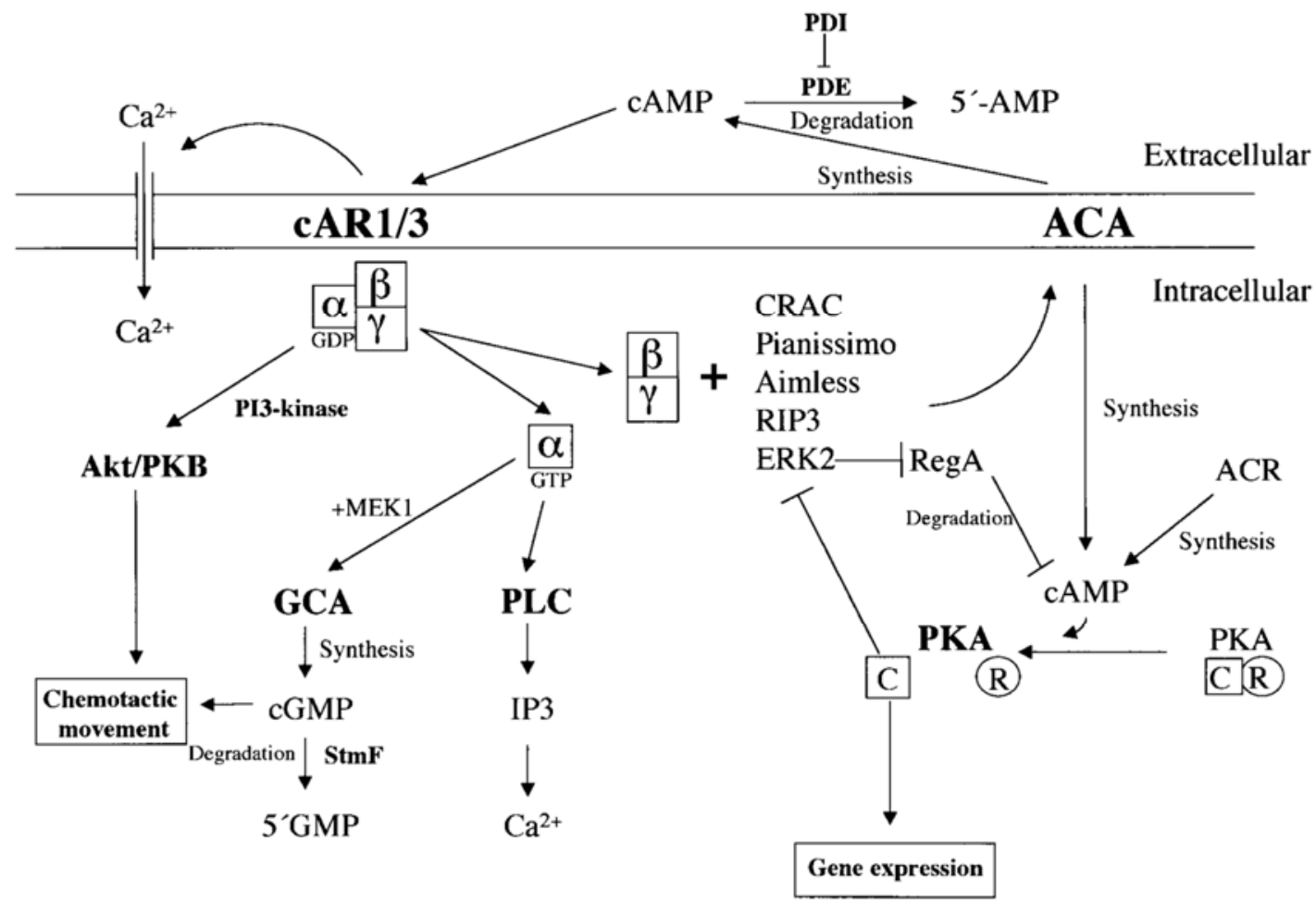

Figure 4: Signal transduction pathways modulating chemotaxis and aggregation in Dictyostelium (Escalante \& Vicente 2000)

cAMP binding to the cAR1 transduces signal into the cells via G-protein-dependent and independent pathways that elicit several responses associated with chemotaxis and aggregation. G-protein independent effects include calcium influx (Milne et al. 1995), tyrosine phosphorylation of the mitogen-activated protein kinase ERK2 (Brzostowski \& Kimmel 2006; Maeda et al. 1996; Segall et al. 1995), receptor phosphorylation and post aggregative gene expression mediated by the G-box binding factor (GBF) transcription factor (Escalante \& Vicente 2000; Schnitzler et al. 1995). 
Interaction of CAMP with CAR1 initiates G-protein dependent cascade of events with the exchange of GDP for GTP in the Ga2 subunit resulting in its dissociation from ßy subunit (Figure 4). Rapid and transient activation of Ras proteins, including RasG, in response to chemoattractant stimulation results in activation of PI3K (Phosphatidylinositol 3-kinase) that phosphorylates PIP2 (Phosphatidylinositol (3,4)-bisphosphate) to form PIP3 (Phosphatidylinositol (3,4,5)-trisphosphate; Sasaki et al. 2004).

The accumulation of PIP3 in the plasma membrane allows for proteins with pleckstrin homology $(\mathrm{PH})$ domains to be recruited to the plasma membrane and subsequently activated. PKB (protein kinase B, Akt) and CRAC (cytosolic regulator of adenylyl cyclase) are $\mathrm{PH}$ domain containing proteins that become activated upon CAMP stimulation (Funamoto et al. 2001; Meili et al. 2000; Lilly \& Devreotes, 1994). One of the most critical responses regulated by cAR1 is the activation of the 12-transmembrane adenylyl cyclase (ACA) mediated by the By subunit complex (Wu et al. 1995a) and two cytoplasmic proteins: CRAC (Cytosolic Regulator of Adenylyl Cyclase) and Pianissimo (Insall et al. 1994; Chen et al. 1997). As shown in Figure 4, CRAC is transiently translocated to the membrane (Comer et al. 2005) within seconds after cAMP binding to receptor resulting in activation of ACA leading to the production and secretion of CAMP (Roos et al. 1975). This CAMP-induced CAMP secretion, also called CAMP relay, is critical for the outward propagation of the CAMP signal throughout the cell population. Other components necessary for receptor mediated activation of ACA include MAP Kinase ERK2 (Segall et al. 1995), Aimless, a Ras-guanine nucleotide exchange factor (GEF; Insall et al. 1996) and a novel Ras-interacting protein, RIP3 (Lee et al. 1999). To secrete cAMP in an oscillatory manner, the cAMP relay pathway must adapt rapidly (Parent \& Devreotes, 1996) so that the adenylyl cyclase (ACA) can not be further activated until extracellular CAMP has been degraded by a secreted or membrane bound phosphodiesterase (PDE; McMains et al. 2008; Palsson et al. 1997; Hall et al. 1993; Franke et al. 1991; Podgorski et al. 1988; Roos et al. 1975; Gerisch et al. 1972). The cells recover sensitivity in a few minutes and these cycles of refractory and responsive states regulate the pulses of extracellular cAMP emitted with a periodicity of 6-7 minutes for several hours (Maeda et al. 2004). The activity of the extracellular phosphodiesterase (ePDE) is regulated by an ePDE inhibitor called PDI (Riedel et al. 1972). The diffusible PDI is secreted as cells arrive at the stationary growth phase (Franke et al. 1991; Riedel et al. 1972). Expression of $\mathrm{PDI}$ is regulated by the levels of extracellular CAMP, thus cells can control the activity of ePDE by monitoring cAMP concentrations (Franke et al.1991). 
It is remarkable that CAMP is not only the intercellular communication agent in Dictyostelium but it serves also as an intracellular secondary messenger modulating different developmental signalling pathways. A fraction of the cAMP produced by the ACA accumulates within the cell to activate the cAMP-dependent protein kinase A (PKA), the main transducer of intracellular cAMP. PKA is a highly conserved protein kinase that affects activity of a variety of proteins by phosphorylation (Loomis 1998). This ubiquitous enzyme is held in an inactive form by association of the catalytic subunit (PKAC) with an inhibitory regulatory subunit (PKA-R; Funamoto et al. 2003; Mann et al. 1997; Mann et al. 1994; Hopper et al. 1993a; Harwood et al. 1992a; Mann et al. 1992; Burki et al. 1991; Firtel \& Chapman 1990; Veron et al. 1988; Abe \& Yanagisawa 1983). When the intracellular CAMP binds the regulatory subunit of PKA, the catalytic subunit is released, causing differential expression of developmentally regulated genes (Funamoto et al. 2003).

The mitogen-activated protein kinases (MAPKs) are a family of highly conserved serine/threonine kinases activated upon phosphorylation of both the threonine and tyrosine residue in the conserved TXY motif and are associated with many types of signalling pathways (Hadwiger \& Nguyen 2011). D. discoideum ERK2 (extracellular signal related kinase 2) is a MAP kinase that is rapidly and transiently activated by extracellular CAMP acting through cAR1, involving, in part, phosphorylation by Ras proteins and/or an unidentified ERK2 kinase (Kimmel et al. 2004; Kimmel \& Parent 2003; Wang \& Segall 1998; Maeda \& Firtel 1997; Aubry et al. 1997; Kosaka \& Pears 1997; Knetsch et al. 1996). ERK2 inhibits activity of intracellular RegA by phosphorylating it at $\mathrm{Thr}^{676}$, thereby increasing intracellular cAMP levels, which, in turn, activate PKA (Hadwiger \& Nguyen 2011; McMains et al. 2008; Brzostowski \& Kimmel 2006; Maeda et al. 2004). As cAMP accumulates, it is also secreted, thus recruiting neighboring cells to contribute to cAMP signal relay during aggregation (Maeda et al. 2004). Another putative ERK2 substrate, EPPA has been identified as being necessary for chemotaxis to cAMP and intracellular production of cAMP (Chen \& Segall 2006). It has been shown by different research groups that ERK2 functions at independent stages during Dictyostelium development: during aggregation, ERK2 is required for the cAMP signal relay and normal chemotactic response but is not essential for aggregation-stage, CAMP pulse-induced gene expression, or for the expression of postaggregative genes. However, during multicellular development, ERK2 is required for morphogenesis and cell-type-specific gene expression (Sawai et al. 2005; Maeda et al. 2004; Zhang et al. 2003; Gaskins et al. 1996; Segall et al. 1995). 
Previously, Loomis and colleagues developed a computational oscillatory loop model by using only six components: cAR1, ACA, ERK2, PKA, PDE and RegA (Maeda et al. 2004; Laub \& Loomis 1998). In short, upon ligand binding, CAR1 activates both ACA and ERK2 leading to secretion of cAMP outside the cell and accumulation of cAMP inside. Extracellular PDE destroys cAMP outside the cell, while activation of PKA inside the cell blocks ACA and ERK2 activity, allowing RegA to degrade the intracellularly accumulated cAMP. This model also proposed that activated PKA may either directly or indirectly phosphorylate the receptor cAR1 causing loss of ligand binding. When the levels of intracellular cAMP are declined sufficiently by the activity of RegA, PKA is inhibited and protein phosphatases return cAR1 to its basal state and the whole process repeats thereby generating oscillations (Maeda et al. 2004; Escalante \& Vicente 2000; Laub \& Loomis 1998). However, erkB ${ }^{-}$and regA- mutants exhibit wave patterns suggestive of the spontaneous oscillations during Dictyostelium aggregation, therefore, Sawai et al. (2005) consummated that periodic CAMP signaling required the activity of the secreted CAMP phosphodiesterase for ligand clearing and not an intracellular feedback loop regulated by PKA (Sawai et al. 2005). Recently Brzostowski and Kimmel (2006) showed that pathways regulating ERK2 inhibition work independent of PKA activation (Brzostowski \& Kimmel 2006).

cGMP is synthesized via guanylyl cyclases (Wedel \& Garbers 2001) and regulate enzyme activities mainly via cGMP-dependent protein kinases (Roelofs \& Van Haastert 2002; Lohmann et al. 1997). Dictyostelium contains two genes that encode for unusual guanylyl cyclase (GC) enzymes, guanylyl cyclase A (GCA; expressed mainly during growth and multicellular development; Roelofs et al. 2001a) and soluble guanylyl cyclase (sGC; expressed largely during cell aggregation; Veltman et al. 2005; Roelofs \& Van Haastert 2002; Roelofs et al. 2001b). Extracellular cAMP binding to cAR1 activates both GCs via heterotrimeric G-proteins, leading to the transient accumulation of cGMP (Roelofs \& Van Haastert 2002; Bosgraaf et al. 2002). In Dictyostelium, cGMP is implicated as one of the second messengers for chemotaxis (Veltman et al. 2005; van Haastert \& Kuwayama 1997) that modulates the phosphorylation and localization of myosin II by binding to $\mathrm{GbpC}$, a high affinity cGMP-binding protein. GbpC contains Ras, MAPKKK and Ras-GEF domains that transduce the cGMP signal, but may also function to receive other input signals (Bosgraaf et al. 2002; de la Roche \& Cote 2001). Previously Ma et al. (1997) suggested that a MAP kinase kinase DdMEK1 activity is also necessary for CAMP-mediated guanylyl cyclase activation throughout the aggregation in Dictyostelium; nevertheless, DdMEK1 does not regulate ERK2 suggesting the presence 
of independent MAP kinase cascades involved in aggregation (Sobko et al. 2002; Escalante \& Vicente 2000; Ma et al.1997).

Other responses elicited by extracellular cAMP binding to CAR1 include the elevation of $\mathrm{IP}_{3}$ levels via PLC activation mediated by heterotrimeric G-proteins (Bominaar \& Haastert 1994); PLC independent IP3 formation interceded by receptor-stimulated $\mathrm{Ca}^{2+}$ influx (Van Dijken et al. 1997) and activation of the protein kinase Akt/PKB necessary for sensing and responding to the chemoattractant gradient during aggregation (Escalante \& Vicente 2000; Meili et al. 1999).

Signal relay and chemotactic movement of several hundred thousand amoeboid cells towards cAMP source ultimately result in aggregation (Schaap 2011b; Konijn et al. 1967). Initially, the cells aggregate individually, but as time progresses, they form bifurcating streams by establishing head-to-tail contacts and continue to move towards the aggregation centres, eventually coalescing into hemispherical aggregates; the mounds, containing about $10^{5}$ cells (Weijer 2004). Once aggregation is complete, the top of the mound keeps on secreting cAMP that result in continued cell movement towards the top, causing emergence of a tip (Schaap 2011a; Siegert \& Weijer 1995). Tipped mound further elongates and develops into a migrating slug which proceeds through culmination to generate a fruiting body (Bonner \& Lamont 2005).

Particularly relevant to this dissertation is that cAMP as an intercellular communication agent plays an important role not only in the chemotactic movement towards aggregation centres but also in the developmental regulation of gene expression, both responses being mediated via cell surface cAMP receptors. During aggregation, extracellular CAMP pulses at $\mathrm{nM}$ concentrations induce optimal expression of a number of aggregationspecific genes (Kessin 1988; Williams 1988; Mann \& Firtel 1987; Sun \& Devreotes 1991; Mann et al. 1988; Kimmel 1987; Chisholm et al. 1987; Noegel et al. 1986) while other genes, induced by different signals immediately with the onset of starvation, become repressed by the external cAMP signal. These differential gene expression changes cause all cells to become rapidly competent for aggregation (Schaap et al. 2011a; Bagorda et al. 2009; Maeda et al. 2004, Aubry \& Firtel 1999; Mann et al. 1997; Schulkes \& Schaap 1995; Gerisch et al. 1975). Moreover, to prevent the loss of directional information and gene expression resulting from saturation of the receptors due to excessive external cAMP, Dictyostelium cells use an intricate signal removal system 
consisting of secreted and membrane-bound phosphodiesterases to degrade the extracellular cAMP (Gerisch et al. 1972; Roos et al. 1975).

Interestingly, $D$. discoideum cells express many developmental genes while suspended in buffer if they are stimulated with pulses of $30 \mathrm{nM}$ cAMP at 6-min periods for several hours followed by high concentrations of cAMP to mimic conditions encountered on solid supports (Iranfar et al. 2003). Under these conditions, the cells are spatially homogeneous and express developmental genes more synchronously than when developing on solid supports where there are local variations in cell density. Additionally, this setting provides the opportunity to address the effects of cAMP pulses on gene expression more directly.

\subsection{Significance of studying intercellular communication}

Intercellular communication modulates a variety of biological processes in a diverse array of organisms. In mammals, precise cell-to-cell communication coordinating directed cell migration gives shape and form to developing embryos and generates many connections and interactions between the cells of nervous system during development (Dormann \& Weijer, 2003). Later in life, excessive intercellular communication promotes tissue maintenance and repair, hormonal action, wound healing and directional migration of cells of our immune system from the bloodstream towards sites of infection ( $\mathrm{Wu} \& \mathrm{Lin}$ 2011; Wang et al. 2011; Eccles 2004; Martin \& Parkhurst, 2004). Impaired cell-cell communication is the basis for several pathological conditions including atherosclerosis, cancer metastasis, asthma, and arthritis (Braunersreuther \& Mach 2006; Mrass \& Weninger 2006; Eccles 2005; Charo \& Taubman 2004; Trusolino \& Comoglio 2002). Further insight into the molecular mechanism of cell-cell communication underlying directional sensing and cell migration is crucial for the development of treatments for these disorders, as well as in understanding normal biological processes.

\subsection{Advantages of social amoebae in researching cell-cell signalling}

Dictyostelids are a large ancient group of unicellular eukaryotes with an unusual multicellular stage in their life cycle (Romeralo et al. 2011c). Because of the simplicity of their multicellular form and rapid life cycle of just 24 hours, researching Dictyostelids can provide useful insight into the adaptive evolution of social behaviour and multicellularity (Heidel et al. 2011). A crucial part of exploring how Dictyostelids are able to form 
multicellular bodies from their unicellular components is to understand how single cells communicate with each other in aggregation and differentiation processes. Thus Dictyostelids represent an ideal eukaryotic model system for studying basic principles of cell-cell communication (Das et al. 2011).

The factors that make Dictyostelids attractive as model organisms include their distinctive growth and developmental stages, relatively short and haploid life cycle and ease of growth. All known Dictyostelids species can be efficiently and cheaply cultured in the laboratory on bacterial lawns on agar, and many species can be grown as mass cultures in liquid media comprising glucose, peptone, and a defined mixture of vitamins and aminoacids (Schaap 2011a). It considerably simplifies the isolation and purification of cellular products for biochemical analysis and proteomics (Schaap 2011a). Complete genome sequence, about $30 \mathrm{Mb}$ in size, of a few species representing three major groups (Heidel et al. 2011; Sucgang et al. 2011; Eichinger et al. 2005) are accessible which provide a valuable resource for the comparison of genes between species and the identification of new genes. Sequencing projects have revealed that Dictyostelids genomes contain many genes that are homologous to those in higher eukaryotes and are missing in other model organisms such as Saccharomyces cerevisiae (Heidel et al. 2011; Goldburg et al. 2006; Eichinger et al. 2005). Orthologs of many genes involved in human disease are found in the genome of $D$. discoideum and have been the subject of functional studies (Myre et al. 2011; Williams 2010; Eichinger et al. 2005). In future, major use of this organism seems to be in biomedical research to understand gene function and pathological mechanisms in a variety of human disorders.

Both $D$. discoideum and the group 2 species $P$. pallidum are amenable to a broad range of molecular and genetic approaches (Schaap 2011a) including random plasmid insertional mutagenesis (REMI) which facilitates the identification of mutated genes, gene disruption by homologous recombination, RNA interference (RNAi) and antisense RNAs, episomal and integrating expression plasmids, and many other techniques.

Previous work with $D$. discoideum has provided a greater insight into the components of complex intercellular communication mechanism regulating chemotaxis to cAMP during aggregation. The seven-transmembrane receptors to detect chemoattractants, components of the heterotrimeric G-proteins and many downstream signalling pathways are highly conserved between $D$. discoideum and mammalian neutrophils despite of their large evolutionary divergence (Wang et al. 2011; Insall 2010; Swaney et al. 2010; Parent 
2004). This conservation not only signifies that these pathways are evolutionarily ancient, but also that research in Dictyostelids might be applicable to the human immune system.

Though $D$. discoideum research has contributed enormously to our exisiting knowledge of eukaryotic cell-cell communication, much work lies ahead to tackle the complexity of chemoattractant induced signalling networks regulating changes in patterns of gene expression to coordinate cell behaviour during aggregation. Therefore, researching effects mediated by acrasins other than cAMP can extend the current understanding of developmental signalling.

\subsection{Previous knowledge about the acrasin of Polysphondylium}

The polyphyletic genus Polysphondylium owes its individuality to the shape of the fruiting body, distinguished by the presence of whorls of side branches. Molecular phylogeny of Dictyostelids has placed all known Polysphondylium species into the ancient group 2B except Polysphondylium violaceum which is more related to the most derived group 4 Dictyostelids (Romeralo et al. 2011b; Romeralo et al. 2010; Schaap et al. 2006). It was known since the work of Shaffer (Shaffer 1953) that the acrasin of $P$. violaceum was different from that of the larger species of Dictyostelium. In 1976, Bonner and co-workers provided the first positive evidence that aggregation competent $P$. violaceum amoebae used an unusual dipeptide as acrasin that was later identified as $\mathrm{N}$-propionyl- $\gamma$-Lglutamyl-L-ornithine- $\delta$-lactam-ethylester, known as glorin (Figure 5; Shimomura et al. 1982; Wurster et al. 1976). Activity of glorin was found to be maximal during aggregation that declined to insignificant levels at the onset of culmination (De Wit et al. 1988).

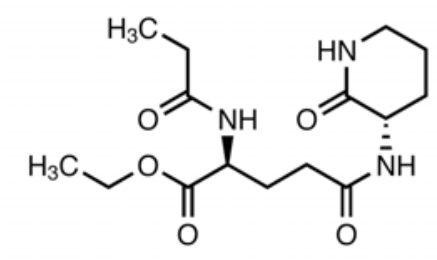

Figure 5: Structure of glorin 
In addition to Polysphondylium violaceum, Dictyostelium caveatum (a group 3 species) and Polysphondylium pallidum belonging to ancient group 2 were also reported to repond chemotactically to glorin (Waddell 1982b; Shimomura et al. 1982; Wurster et al. 1976).

In $P$. violaceum, glorin signal transduction system is demonstrated to have many similarities with CAMP and folic acid transduction systems in social amoebae. Occurrence of secreted and membrane-bound acrasinases that inactivate the glorin communication signal was proposed in aggregating $P$. violaceum amoebae (Kopachik 1990; Shimomura et al. 1982; Wurster et al. 1976). It was shown that degradation of glorin by intact cells occurs rapidly (half-time $2 \mathrm{~min}$ ) in a two-step process (De Wit et al. 1988). First step of this process involves hydrolyzing amide bond of the $\delta$-lactam ring while in the second step slower cleavage of the peptide bond between propionic acid and glutamic acid takes place (Figure 6).

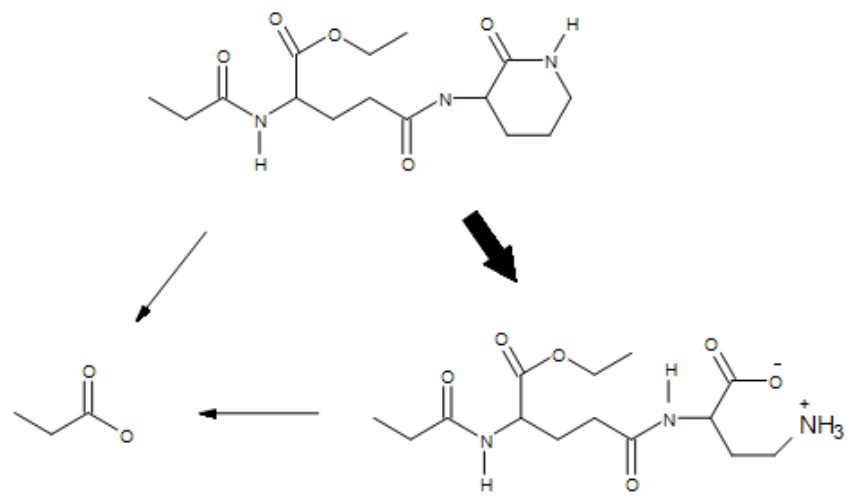

Figure 6: The chemical structures of glorin and the products of enzymatic degradation. (Modified from De Wit et al. 1988).

Two-step glorin degradation may favour reuse of the building blocks. It was demonstrated that glorin degradation occurs mainly by cleavage of $\delta$-lactam. Chemically synthesized $\delta$-lactam-cleaved glorin, corresponding to the first product of glorin degradation, was found to be chemotactically inactive, because this product is not able to bind to receptor (De Wit et al. 1988). A synthetic glorin derivative in which propionic acid group was absent possessed weak chemotactic activity. Degradation of glorin by extracellular medium takes place in a similar manner as by the intact cells but at a relatively low rate (half-time $45 \mathrm{~min}$ ). It was shown that crude homogenates also possessed some glorin inactivation activity. Cell surface glorinase activity was observed 
to peak during aggregation, followed by a rapid decrease. It was noticed that cell-surface bound glorinase activity was developmentally regulated that reached its maximum during aggregation (De Wit et al. 1988).

Further studies to investigate the first steps in the signal transduction towards glorin suggested that in $P$. violaceum that this unusual dipeptide acts by binding to cell-surface G-protein coupled receptors (De Wit et al. 1988). Authors illustrated a functional interplay between cell-surface receptors and signal-transducing G-proteins by showing that binding of glorin to cell-surface receptors of aggregation-competent $P$. violaceum amoebae is modulated by guanine nucleotides, and contrarily glorin stimulus mediates the binding of GTP to cell membranes (De Wit et al. 1988). It was demonstrated that vegetative stage $P$. violaceum amoebae exposed sufficient number of cell-surface glorin receptors that increased slightly during aggregation, declined at later stages of development and reduced to undetectable levels during culmination (De Wit et al. 1988). The affinity of cell-surface glorin receptors exhibited great variations in the early hours of development but was stabilized throughout the aggregation. The receptor binding kinetic studies indicated the presence of two kinetically distinct receptors (De Wit et al. 1988). The same authors showed that extracellular glorin stimulus induces cGMP accumulation, a cellular response that is developmentally regulated in $P$. violaceum, peaks during aggregation and reduces to minimum at the onset of culmination (De Wit et al. 1988).

Oscillatory aggregation has been reported in $P$. violaceum (De Wit et al. 1988), but surprisingly De Wit and colleagues could not detect glorin-stimulated glorin secretion. Authors observed that glorin-induced CAMP accumulation and glorin signal relay was also absent in aggregation-competent $P$. violaceum amoebae (De Wit et al. 1988); therefore, they proposed that glorin is not a primary oscillator and glorin secretion during aggregation may be modulated by an unknown oscillator.

Previously, Francis (1965) suggested that in P. pallidum only special founder cells are capable to initiate centres in a population of starving amoebae (Francis 1965). These single cells round off and actively secrete acrasin attracting amoebae in the immediate vicinity (Konijn 1972). Such founder cells were reported to appear occasionally among aggregation sensitive amoebae of $P$. violaceum (Shaffer 1961). Francis (1965) suspected absence of any signal relay in $P$. pallidum during aggregation because he could not observe pulses of oriented cell movement among the responding amoebae (Francis 1965). P. pallidum cells tends to form small sized aggregates compared to large 
species D. discoideum, therefore, Francis (1965) suggested that the concentration of acrasin in the small centres guiding formation of aggregates in $P$. pallidum may not be high enough to elicit the acrasin relay in responding amoebae (Francis 1965). Later, Jones (1976) reported that aggregation fields of $P$. pallidum amoebae display centrifugally propagated waves of excitation that are not systematically spaced (Jones 1976). In comparison, $P$. violaceum exhibited regularly spaced waves with an interval decreasing from 4 to 2.5 minutes (Jones 1976). In $P$. pallidum and $P$. violaceum wave velocities were observed to be $22 \mu \mathrm{m} / \mathrm{min}$ and $28 \mu \mathrm{m} / \mathrm{min}$ respectively (Jones 1976). Based on the observation that starving amoebae of $P$. pallidum aggregate in converging streams, he suggested that a relaying mechanism may mediate aggregation as in $D$. discoideum (Jones 1976).

It was shown by Newth \& Hanna (1982) that $P$. violaceum amoebae developed in liquid suspensions gained increased ability to respond to exogenous glorin shortly after starvation before cells have acquired aggregation competency (Newth \& Hanna 1982). Later, Will Kopachik (Kopachik 1990) reported some changes in protein synthesis when starving $P$. violaceum amoebae were stimulated with exogenous glorin in shaking cultures. The author showed that exogenous glorin treatment could not affect protein synthesis patterns in vegetative stage amoebae, whereas with progression of starvation, $P$. violaceum cells acquired increased sensitivity to glorin (Kopachik 1990). Furthermore, Kopachik suggested that in developing $P$. violaceum amoebae, glorin effects occur mainly during the early hours of development while sensitivity to glorin and secretion of glorin by starving cells declines rapidly once aggregation stage is over (Kopachik 1990).

Recently, Funamoto and co-workers (Funamoto et al. 2003) showed that $P$. pallidum cells transformed with dominant negative regulatory subunit $(\mathrm{Rm}$; that can not bind cAMP) of PKA from Dictyostelium exhibit aggregation defects, whereas the overexpression of Dictyostelium $\mathrm{PkaC}$ in $P$. pallidum results in precocious development; demonstrating that a biochemically similar PKA mechanism works in Dictyostelium and Polysphondylium (Funamoto et al. 2003). The same authors proposed that PKA-requiring and a non-PKA requiring intracellular signalling pathways may regulate early developmental genes in $P$. pallidum. However, so far, glorin-mediated changes in gene expression have not been studied.

To date, little is known about the secretome of $P$. pallidum at aggregation stage of development. The availability of valuable data of $P$. pallidum PN500 genome sequence 
and the advanced proteomics technology provides the foundation for the analysis of extracellular proteins secreted by aggregating $P$. pallidum cells. 


\subsection{Objectives of this Study}

Glorin is a peptide chemoattractant used by Polysphondylium violaceum amoebae for cell-cell communication to coordinate the process of aggregation (Shimomura et al. 1982; Wurster et al. 1976). An ancient group 2 species Polysphondylium pallidum also exhibits chemotactic activity towards glorin (Shimomura et al. 1982). The present thesis is focused on exploring glorin-based cell-cell communication among the Dictyostelids. For functional genomic studies, $P$. pallidum isolate PN500 is employed owing to its genetic tractability (Heidel et al. 2011).

Recently, molecular phylogeny data (Schaap et al. 2006) suggested that glorin-based cell-cell communication might be quite wide-spread among the Dictyostelids. Based on this hypothesis, in the present research work the roots of glorin-mediated communication are being traced in the Dictyostelids phylogeny and the question will be addressed how common this peptide-based communication is in the phylogenetic descent of the social amoebae. A proteomics approach will be used to search for the putative glorin-degrading enzyme by analyzing the secretory proteome of aggregating $P$. pallidum PN500 amoebae.

P. pallidum PN500 is an emerging model organism with full experimental and genetic amenability (Schaap 2011a). It could be interesting to study if glorin-based intercellular communication also executes intracellular communication, i.e. signal transduction via binding to its cognate receptor. This possibility will be examined in the present study by researching whether glorin communication induces differential gene expression in aggregation competent $P$. pallidum amoebae. For these investigations, Illumina sequencing technology will be applied, taking advantage of the fully sequenced and annotated reference genome of $P$. pallidum PN500 (Heidel et al. 2011). Genome-wide analysis will be performed to determine changes in total cellular mRNA levels in response to the stimulation of $P$. pallidum cells with exogenous glorin. Changes in global gene expression in response to starvation will also be investigated in parallel. Interaction between glorin communication system and the post-aggregation cAMP signalling system of $P$. pallidum PN500 will also be scrutinized. A critical question to address is whether glorin-induced gene expression would require de novo protein synthesis. Finally, this study will characterize the developmental effects of 'glorin-stimulated cell signalling' in starving $P$. pallidum cells. 


\subsection{Significance of this work}

The research work presented in this dissertation holds considerable promise. Firstly, it provides intriguing information about the origins of peptide communication in Dictyostelids. Secondly, it exquisitely compares signalling induced by two different acrasin systems (i.e. cAMP system and the glorin system) at the transition from growth to aggregation. $D$. discoideum that uses CAMP as aggregative chemoattractant and $P$. pallidum, which is presumed to use glorin as acrasin during aggregation, are similar in basic patterns of their life histories but biochemical relationship between both is utterly vague. If glorin is the acrasin of $P$. pallidum, it is expected to exert similar effects in organizing aggregation as CAMP does in $D$. discoideum. The fact that CAMP binding to the cell-surface receptors initiates distinct signaling pathways leading to differential changes in gene expression (that regulate diverse cellular functions during aggregation) prompted us to do comparative signal-receptor physiology with glorin. In this thesis, recent data on developmental roles of glorin mediated cell-cell signalling is presented with a particular emphasis on gene regulation by this vital cell behaviour that is suspected to coordinate aggregation in P. pallidum.

It has been reported that $P$. pallidum also secretes CAMP (Jones \& Robertson 1976; Konijn et al. 1968) which has been shown by Schaap and colleagues to be involved in post-aggregation differentiation events in this species (Kawabe et al. 2009; Alvarez-Curto et al. 2005). The assumption that glorin mediates aggregation in $P$. pallidum suggests a clear chemical distinction between the chemotactic system coordinating aggregation and cAMP differentiation system. This project provides the initial insight into the topic whether post-aggregation cAMP system is induced by aggregation-specific glorin system or both systems work independently in the development of $P$. pallidum.

Structurally, glorin is an interesting molecule, it is an exceptional dipeptide and like so many peptide attractants of leukocytes, it is also terminally blocked. Though $\mathrm{N}$ formylmethionyl peptides used for leukocyte chemotaxis do not attract $P$. violaceum amoebae that use glorin as chemotactic agent, but the basic molecular similarity is striking despite the difference in specificity (Shimomura et al. 1982; Devreotes \& Zigmond 1988). Researching signalling pathways induced by this unique molecule may provide further insight into signal transduction networks that coordinate immune cell functions. 


\section{Material and Methods}

\subsection{Material}

\subsubsection{Laboratory equipments}

Autoclave:

DNA electrophoresis:

Protein electrophoresis:

Gel documentation:

Microscope:

PCR machine:

$\mathrm{pH}$ meter:

Real-time Thermocycler:

Laminar Flow Hood (LAF):

UV/VIS-Spectrometer:

Vortexer:

Weighing machine:

Analytical weighing machine:

Water bath:

Cell counter:

Rotary evaporator:
Varioklav Dampfsterilisator $(\mathrm{H}+\mathrm{P}$

Labortechnik)

Hiclave ${ }^{\circledR}$ HV-85L (HMC)

PerfectBlue Gelsystem Mini (Peqlab)

PerfectBlue Doublegel system Twin M

(Peqlab)

Gel Jet Imager 2004 (Intas)

Binokular-Mikroskop SZX9 (Olympus)

Mikroskop CXX 41 (Olympus)

Lichtquelle U-RFL-T (Olympus)

Kamera Evolution ${ }^{\circledR}$

MP COLOR (Media Cybernetics)

Primus 96 advanced (Peqlab)

pH-Meter pH 526 MultiCal $^{\circledR}$ (WTW)

Mx3000P (Stratagene)

MRF-B (Steag)

BioPhotometer (Eppendorf)

DU ${ }^{\circledR} 640$ Spectrophotometer (Beckman)

VF2 (Janke \& Kunkel, IKA Labortechnik)

E2000D, BA 6100 und PT 310 (Sartorius)

A $210 P, B P 210 P$ und MC $210 P$

(Sartorius)

Seradest SD 2800 (SERAL

Reinstwassersysteme)

Serapur Delta (SERAL

Reinstwassersysteme)

CASY-Zellzähler Modell DT (Innovatis,

Schärfe System)

Concentrator 5301 (Eppendorf) 
Centrifuge machines

Gel dryer:

Freeze dryer
Centrifuge 5415 D (Eppendorf)

Centrifuge 5810R (Eppendorf)

J2-MC (Beckman)

Labofuge 400R (Heraeus)

Multifuge 1 L-R (Heraeus)

Megafuge 1.0R (Heraeus)

Gel Dryer Model 543 (Bio-Rad)

VIRTIS

\subsubsection{Chemicals}

\begin{tabular}{|c|c|}
\hline Substance & Manufacturer \\
\hline \multirow[t]{2}{*}{ Glorin } & Phoenix Pharmaceuticals \\
\hline & (Burlingame CA, USA) \\
\hline Acrylamide & Merck \\
\hline Agar-Agar & Roth \\
\hline Agarose (peqGold Universal-Agarose) & Peqlab \\
\hline APS & Roth \\
\hline ATP & Roth \\
\hline Bacto-Pepton & BD Biosciences \\
\hline Bromophenolblue & Roth \\
\hline BSA (Restriktionshydrolyse) & New England Biolabs \\
\hline Calcium chloride dihydrate & Roth \\
\hline cAMP & Roth \\
\hline Chlorophorm/lsoamylalcohol & Roth \\
\hline DMSO & Roth \\
\hline dNTPs & Roth \\
\hline D-Glucose-Monohydrat & Roth \\
\hline EDTA & Roth \\
\hline Ethidiumbromide & Roth \\
\hline Glycerin $86 \%$ & Roth \\
\hline Glycin & Roth \\
\hline Yeast extract & Serva \\
\hline Potassium acetate & Roth \\
\hline Potassium chloride & Roth \\
\hline
\end{tabular}




\begin{tabular}{|c|c|}
\hline Potassium -di-hydrogen phosphate & Prolabo \\
\hline Di-potassium hydrogen phosphate & Fluka \\
\hline Laemmli-Puffer & Sigma \\
\hline Magnesiumchlorid-Hexahydrate & Merck \\
\hline$\beta$-Mercaptoethanol & Ferak, Berlin \\
\hline Sodiumacetate trihydrat & Roth \\
\hline Sodium chloride & Roth \\
\hline Sodium carbonate & Roth \\
\hline Sodium dihydrogen carbonate & Roth \\
\hline Disodium hydrogen phosphate dihydrate & Roth \\
\hline Disodium hydrogen phosphate anhydrous & Roth \\
\hline $\begin{array}{l}\text { Phenol-Chloroform-Isoamylalkohol } \\
(25: 24: 1)\end{array}$ & Roth \\
\hline Collecting gel buffer & $\mathrm{BDH}$ \\
\hline Seperating gel buffer & Applichem \\
\hline SDS & Roth \\
\hline TEMED & Roth \\
\hline Tris & Roth \\
\hline Tween 20 & Roth \\
\hline Cycloheximide & Sigma \\
\hline Silver nitrate & Roth \\
\hline Sodium thiosulphate pentahydrate & Roth \\
\hline Formaldehyde $37 \%$ & Roth \\
\hline Methanol & Roth \\
\hline Acetic acid & Roth \\
\hline
\end{tabular}

\subsubsection{Solutions and Buffers}

\section{Solution/Buffer}

\section{Composition}

$\begin{array}{lll}\text { 1X Low TE }(\mathrm{pH} \mathrm{8}): & \text { Tris- } \mathrm{HCl} & 10 \mathrm{mM} \\ & \text { EDTA } & 1 \mathrm{mM} \\ & & \\ \text { PBS 10X }(\mathrm{pH} 7.2): & \mathrm{Na}_{2} \mathrm{HPO}_{4} & 80 \mathrm{mM} \\ & \mathrm{KH}_{2} \mathrm{PO}_{4} & 15 \mathrm{mM}\end{array}$




$\begin{array}{ll}\mathrm{KCl} & 25 \mathrm{mM} \\ \mathrm{NaCl} & 1.4 \mathrm{M}\end{array}$

\begin{tabular}{|c|c|c|}
\hline \multirow[t]{2}{*}{ Phosphate buffer $50 \mathrm{X}(\mathrm{pH} 7)$ : } & $\mathrm{Na}_{2} \mathrm{PO}_{4}$ & $100 \mathrm{mM}$ \\
\hline & $\mathrm{KH}_{2} \mathrm{PO}_{4}$ & $735 \mathrm{mM}$ \\
\hline \multirow[t]{3}{*}{ TBE 10X (pH 8.3): } & Tris & $1 \mathrm{M}$ \\
\hline & EDTA & $20 \mathrm{mM}$ \\
\hline & $\mathrm{H}_{3} \mathrm{BO}_{3}$ & $0.5 \mathrm{M}$ \\
\hline \multirow[t]{2}{*}{ Tris-HCl (pH 8): } & Tris & $1 \mathrm{M}$ \\
\hline & \multicolumn{2}{|c|}{$\mathrm{pH}$ value set to 8.0 with $\mathrm{HCl}$} \\
\hline RNase A solution: & $1 \mathrm{mg} / \mathrm{ml}$ in Milli-Q w & \\
\hline \multirow[t]{5}{*}{ SDS running buffer (10X): } & Tris & $60.4 \mathrm{~g}$ \\
\hline & Glycin & $288 \mathrm{~g}$ \\
\hline & SDS & $20 \mathrm{~g}$ \\
\hline & 2 litre & Milli-Q \\
\hline & & water \\
\hline \multirow[t]{6}{*}{ Laemmli buffer (2X): } & SDS & $4 \%$ \\
\hline & glycerol & $20 \%$ \\
\hline & 2-mercaptoethanol & $10 \%$ \\
\hline & bromphenol blue & $0.004 \%$ \\
\hline & Tris $\mathrm{HCl}$ & $0.125 \mathrm{M}$ \\
\hline & \multicolumn{2}{|l|}{ pH approx. 6.8 . } \\
\hline
\end{tabular}

\subsubsection{Solutions for silver staining}

Following solutions were prepared for silver staining:

Fixation solution: Mixed together $450 \mathrm{ml}$ methanol, 50 $\mathrm{ml}$ acetic acid and $500 \mathrm{ml}$ water. The solution was stored at room 
temperature for at least 1 month.

Rinsing solution:

Sensitization solution:

Silver solution:

Development solution:

Stop solution:

\subsubsection{Media}

\subsubsection{Liquid media}

\section{Medium}

1/5 SM medium:
Distilled water $1 \mathrm{~L}$.

$\begin{array}{llll}\text { Dissolved } & 0.2 & \mathrm{gm} & \text { sodium }\end{array}$ thiosulphate pentahydrate in $1 \mathrm{~L}$ water. This solution was prepared fresh.

Dissolved $1 \mathrm{~g}$ silver nitrate in $1 \mathrm{~L}$ water. This solution was prepared fresh.

Dissolved $20 \mathrm{~g}$ sodium carbonate in $1 \mathrm{~L}$ water followed by addition of $1.11 \mathrm{ml}$ of $37 \%$ formaldehyde. This solution was prepared fresh.

Mixed together $10 \mathrm{ml}$ acetic acid and $990 \mathrm{ml}$ water. This solution was stored in room temperature for at least 3 months 
Autoclaving of above medium was followed by addition of $3 \mathrm{ml}$ of $40 \%$ glucose and 0.48 $\mathrm{ml}$ of $1 \mathrm{M} \mathrm{MgSO}_{4} \cdot 6 \mathrm{H}_{2} \mathrm{O}$

\subsubsection{Agar media}

\section{Medium}

1/5 SM-Agar:
Agar

1/5 SM medium

\section{Composition}

$12 \mathrm{~g}$

quantity sufficient to make $1 \mathrm{~L}$

Autoclaving of above medium was followed by addition of $3 \mathrm{ml}$ of $40 \%$ glucose and 0.48 $\mathrm{ml}$ of $1 \mathrm{M} \mathrm{MgSO}_{4} \cdot 6 \mathrm{H}_{2} \mathrm{O}$

\subsubsection{Microorganisms}

The following species were used in this study, and DBS strain numbers from the Dictyostelium Stock Center (http://dictybase.org/StockCenter/StockCenter.html) are given.

\section{Dictyostelid species}

D. discoideum NC4

D. giganteum WS589

D. sphaerocephalum GR11

D. oculare DB4B holotype

D. gloeosporum TC-52

D. fasciculatum $\mathrm{SH} 3$

D. bifurcatum UK58

D. aureo-stipes JKS5150

D. parvisporum OS126

D. deminutivum $\mathrm{M} 19 \mathrm{~A}$

D. microsporum Hagiwara 143
Source

DBS0304666

DBS0235820

DBS0235889

DBS0235852

DBS0235825

DBS0235810

DBS0235731

DBS0235725

DBS0235853

DBS0235744

DBS0235841 
P. pallidum PN500

DBS0236807

P. pallidum PN500 tas $A^{-} /$tas $^{-}$

DBS0306505

(Kawabe et al. 2009)

P. pallidum CK8

DBS0236805

P. tenuissimum TNS-C-97

DBS0266526

P. tikaliensis OH595 holotype

DBS0238791

P. Iuridum LR-2

DBS0236804

P. pseudo-candidum Hagi-66-TNS-C-91

DBS0235858

$P$. asymmetricum $\mathrm{OH} 567$ holotype

DBS0235724

A. ellipticum AE2 holotype

DBS0235447

A. leptosonum FG12A

DBS0235449

A. subglobosum LB1

DBS0235452

P. violaceum

ATCC34156

P. pallidum WS320

ATCC44843

P. pallidum $\mathrm{H} 168$

a gift from M. Romeralo

(University of Uppsala)

\subsubsection{Other microorganisms}

Klebsiella planticola (Source: Dicty stock centre)

\subsubsection{Kits}

Kit

Brilliant ${ }^{\circledR}$ II SYBR ${ }^{\circledR}$ Green qRT-PCR Kit (2- Stratagene

Step)

DNeasy ${ }^{\circledR}$ Tissue Kit

Omniscript Reverse Transcription Kit

QIAquick $^{\circledR}$ Gel Extraction Kit

QIAquick ${ }^{\circledR}$ PCR Purification Kit

RNase-Free DNase-Set

RNeasy ${ }^{\circledR}$ Mini Kit

Taq DNA Polymerase Kit
Qiagen

Qiagen

Qiagen

Qiagen

Qiagen

Qiagen

5' prime 
Jena Bioscience

\subsubsection{Enzymes}

\section{Enzyme}

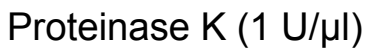

Restriktionsendonukleasen $(4-100 \mathrm{U} / \mu \mathrm{l})$ RNase A (2 U/Ml)

\section{Manufacturer/ Supplier}

Fermentas

New England BioLabs

New England BioLabs

\subsubsection{Lab materials}

\section{Lab materials}

$1 \mu \mathrm{M}$ pore size cell culture Inserts

Multiwe ${ }^{\mathrm{TM}} 6$ well plate for tissue culturing

Protein LoBind Tube, $1.5 \mathrm{ml}$

Petridishes $(5 \mathrm{ml}, 10 \mathrm{ml})$

Pipette tips, unsterile

\subsubsection{Size standards}

\section{Markers}

DNA size standard pUC19 BsiSI (Hpall)

DNA size standard $ү D N A / P s t l$

DNA size standard $0,1 \mathrm{~kb}$

Molecular weight marker for SDS-PAGE

(Page Ruler)

MagicMark ${ }^{\mathrm{TM}}$ for SDS-PAGE

\section{Manufacturer/ Supplier}

Becton Dickinson and company, USA

Becton Dickinson and company, USA

Eppendorf, USA

Greiner, Nunc

Greiner

\section{Manufacturer/ Supplier}

Jena Bioscience

Fermentas

New England BioLabs

Fermentas

Invitrogen 


\subsubsection{Computer programmes}

Gene Ontology Analysis Tool (GOAT) für R (Xu und Shaulsky 2005) über BioConductor, http://www.bioconductor.org/

Gene Expression Omnibus, http://www.ncbi.nlm.nih.gov/geo/

Gene Ontology Project (GO), http://www.geneontology.org/

Dictybase (Chisholm et al. 2006), http://dictybase.org/

dictyExpress (Rot et al. 2009), http://www.ailab.si/dictyexpress/run/

Protparam (Gasteiger et al. 2005) über Expasy,

http://www.expasy.org/tools/protparam.html

ClustalX 2.0.11 (Thomson et al. 1994, Larkin et al. 2007),

http://www.clustal.org/download/

Microsoft $\circledast$ Office Excel $\circledast 2003$ und 2007 (Microsoft Corporation)

pDRAW 1.1, http://www.acaclone.com/

MaxPro ${ }^{\text {TM }}$ QPCR Software (Stratagene)

QCapture Pro 5.0.1.26 (QImaging)

ImageJ 1.42 (http://rsbweb.nih.gov/ij/)

\subsection{Methods}

\subsubsection{Cell biological methods}

\subsubsection{Cell culture methods}

Dictyostelids were obtained from the Dictyostelium Stock Center (http://dictybase.org/StockCenter/StockCenter.html). Cells were cultured in association with Klebsiella planticola on $1 / 5 \mathrm{SM}$ agar plates at $21 \mathrm{C}^{0}$ as described by Raper (Raper 1984). After about 45 hours of incubation, the vegetatively growing amoebae were harvested from the agar plates using cold $17 \mathrm{mM}$ phosphate buffer ( $\mathrm{pH}$ 6.2). Cells were washed free of bacteria by repeated washing with phosphate buffer $\left(\mathrm{pH} \mathrm{6.2).} 2 \times 10^{7}\right.$ cells were harvested by centrifugation and pellet was immediately stored at $-80{ }^{\circ} \mathrm{C}$ for total RNA extraction or preparation of genomic DNA. 


\subsubsection{Chemotaxis assay}

\subsection{Preparation of hydrophobic agar and chemotaxis assay plates} (Ennis \& Sussman 1958; Konijn \& Raper 1961)

To prepare hydrophobic agar plates, agar was washed repeatedly with deionised water, suspended in $17 \mathrm{mM}$ phosphate buffer ( $\mathrm{pH}$ 6.2)., dissolved by boiling and allowed to gelate. The concentration of the agar was lowered to $1 \%$ that permits amoebae to move outside the boundaries of the drop to increasing concentration gradients of acrasin after cells degrade chemoattractant in their vicinity. $1 \mathrm{ml}$ of molten agar was poured in each petri plate $(5 \mathrm{~cm})$, agitated to allow even spreading and allowed to gelate as thin agar surface.

Synthetic glorin was obtained from Phoenix Pharmaceuticals (Burlingame CA, USA), dissolved in $17 \mathrm{mM}$ phosphate buffer $(\mathrm{pH}$ 6.2) and stored as a $3 \mathrm{mM}$ stock solution at $20{ }^{\circ} \mathrm{C}$. Chemotaxis assays were carried out following the protocol established by Shimomura et al. (Shimomura et al. 1982). Growing cells were washed to remove bacteria, adjusted to a concentration of $2 \times 10^{6}$ cells $/ \mathrm{ml}$ with $17 \mathrm{mM}$ phosphate buffer $(\mathrm{pH}$ 6.0) and shaken for $1-5$ hours at $120 \mathrm{rpm}$ at $22{ }^{\circ} \mathrm{C}$ to induce aggregation competence. Cells were then concentrated by centrifugation to $2 \times 10^{8} \mathrm{cells} / \mathrm{ml}$ and $10 \mu \mathrm{l}$ drops of cell suspension were deposited on $1 \%$ hydrophobic phosphate agar plates containing either no acrasin or different concentrations of glorin or cAMP. In the absence of acrasin in the agar, cells stayed inside the boundaries of drops over the complete period of observation. Instead, if acrasin was embedded in the agar, extracellular acrasinase degraded the acrasin within the drop, thereby generating local acrasin gradients that caused cells to move out of the margins of the drop over a certain distance. Pictures were generally taken 3 hours after placing drops of the cells on agar surface.

\subsubsection{Glorin pulsing experiments}

Vegetatively growing $P$. pallidum cells were collected, washed, and resuspended in 17 $\mathrm{mM}$ phosphate buffer at a concentration of $2 \times 10^{7}$ cells $/ \mathrm{ml}$. A pellet of $2 \times 10^{7}$ cells was stored immediately after washing at $-80^{\circ} \mathrm{C}$ that served as a reference for differential gene expression in developing cells. Suspension cultures of amoebae were then shaken at $120 \mathrm{rpm}$ at $22^{\circ} \mathrm{C}$. Unless otherwise specified, $P$. pallidum PN500 cells were first 
prestarved for 1 hour and then treated with $1 \mu \mathrm{M}$ glorin at 10-, 20-, or 30-minute intervals for an additional 2 to 8 hours (depending on the nature of experiment). Parallel cultures were left untreated. Generally, $2 \times 10^{7}$ cells were collected by centrifugation 30 minutes after each pulse, pelleted and stored at $-80^{\circ} \mathrm{C}$ for subsequent RNA extraction.

\subsubsection{Aggregation Analysis}

\subsection{Aggregation analysis under submerged conditions}

Vegetative P. pallidum PN500 amoebae grown in association with Klebsiella planticola were harvested and washed free of bacteria four times in ice-cold $17 \mathrm{mM}$ phosphate buffer ( $\mathrm{pH}$ 6.2). The cells were then resuspended in phosphate buffer at a density of $8 \times 10^{6} \mathrm{cell} / \mathrm{ml}$ and $4 \mathrm{ml}$ of this suspension was plated in monolayer on a $50-\mathrm{mm}$ petri dish. Images were captured after cells have settled to the bottom of petri plates $(0 \mathrm{hr})$ and when they start to aggregate.

\subsection{Aggregation analysis on phosphate-buffered agar plates}

$P$. pallidum PN500 amoebae were washed free of bacteria and adjusted to $2 \times 10^{7} \mathrm{cells} / \mathrm{ml}$ in cold $17 \mathrm{mM}$ phosphate buffer $(\mathrm{pH}$ 6.2). Cells were then evenly distributed as monolayers on $50 \mathrm{~mm}$ phosphate-buffered agar plates at a density of $8 \times 10^{5} \mathrm{cells} / \mathrm{cm}^{2}$ for development. The plates were air dried for 20 minutes and any excess of liquid was carefully removed without disturbing the cell layer. Cells were then allowed to develop at $22^{\circ} \mathrm{C}$. Different stages of development were observed using a stereomicroscope and images were captured at indicated time points. Cells were washed off the surface of agar plates at different stages of development, $2 \times 10^{7}$ cells were pelleted by centrifugation and stored at $-80^{\circ} \mathrm{C}$ for preparation of total RNA.

\subsubsection{Development on phosphate agar to study aggregation stimulatory effects of glorin}

To examine effects of glorin treatment on aggregation capacity of $P$. pallidum PN500, the suspension culture of amoebae was first prestarved for 1 hour and then distributed into three flasks (volume of the flask was 4-5 times that of the suspension) and shaken at $120 \mathrm{rpm}$. One flask was used for each of the following conditions: (i) no treatment; (ii) 
pulsed glorin: pulses of glorin given every 10 minutes to a final concentration of $1 \mu \mathrm{M}$ for 2 hours; (iii) pulsed glorin: pulses of glorin given every 10 minutes to a final concentration of $100 \mathrm{nM}$ for 2 hours. Cells were then washed, concentrated to $2 \times 10^{8} \mathrm{cell} / \mathrm{s} / \mathrm{ml}$ by centrifugation and plated on non-nutrient agar plates at concentration of $8 \times 10^{5} \mathrm{cells} / \mathrm{cm}^{2}$. Photographs were taken when cells reached aggregation stage.

\subsubsection{Molecular Biology Methods}

\subsubsection{Isolation of genomic DNA and total RNA}

For isolation of total RNA or genomic DNA, a cell pellet of $2 \times 10^{7}$ cells was used, which has been stored overnight at $-80{ }^{\circ} \mathrm{C}$. The isolation of gDNA was performed using the DNeasy Tissue $\mathrm{Kit}^{\circledR}$ (Qiagen) using 'Animal cells' protocol. For RNA isolation, the RNeasy ${ }^{\circledR}$ Mini Kit (Qiagen) was used.

\subsubsection{2 cDNA synthesis}

The synthesis of cDNA was carried out from $2 \mu$ of total RNA having concentration of $250 \mathrm{ng} / \mu \mathrm{l}$ using the Omniscript ${ }^{\circledR}$ Reverse Transcription Kit (Qiagen) and an oligo (dT)18primer.

\subsubsection{Quantitative RT-PCR}

For the comparative expression analysis of genes, the quantitative real-time polymerase chain reaction (qRT-PCR) was performed to complementary DNAs. Gene expression levels were standardized to the gene encoding glyceraldehyde-3-phosphate dehydrogenase (gpdA, SACGB accession number PPL_12017). The gpdA gene was amplified using primers gpdA-01 and gpdA-02 that yielded a 218 bp PCR product from genomic DNA and a 146 bp fragment from cDNA, respectively, thereby facilitating determination of genomic DNA contaminations in cDNA preparations by conventional RT-PCR prior to real-time RT-PCR runs. The real-time quantification was performed on a Mx3000P (Stratagene) using the Taq-polymerase kit (5' primer or Jena Bioscience) together with the Fluorescent dye EvaGreen ${ }^{\circledR}$. A 1:10 dilution of cDNA (synthesized as described in 2.2.2.2) was prepared and $1 \mu$ of this dilution was used in the amplification reaction mixture alongwith $300 \mathrm{nM}$ final concentration of primers was used for the 
amplification reactions. A list of primers used is given in Appendix Table A2. An initial denaturing step at $95^{\circ} \mathrm{C}$ for 10 minutes was followed by the PCR for 40 cycles at $95^{\circ} \mathrm{C}$ for $30 \mathrm{~s}, 58^{\circ} \mathrm{C}$ for $30 \mathrm{~s}$ and $72^{\circ} \mathrm{C}$ for $30 \mathrm{~s}$. Subsequently, the melting curves of the resulting products were analyzed and gene expression regulation was determined by the method of Pfaffl (2001). All measurements were carried out in at least three independent experiments.

\subsubsection{ITS sequencing (Romeralo et al. 2010)}

A 1000 base pair (bp) fragment of ITS rDNA was amplified by PCR from genomic DNA of four isolates of $P$. pallidum using $P$. pallidum specific primers described by Romeralo et al. (Romeralo et al. 2007). The PCR program was composed of 5 minutes at $95^{\circ} \mathrm{C}$, followed by 30 cycles of 1 minute at $94{ }^{\circ} \mathrm{C}, 1$ minute at $50{ }^{\circ} \mathrm{C}$, and 2 minutes at $72{ }^{\circ} \mathrm{C}$, and a final elongation step of 10 minutes at $72{ }^{\circ} \mathrm{C}$. PCR products were then separated on $1 \%$ agarose gels. DNA bands excised from the gel were extracted using the QIAquick gel extraction kit. The extracted DNA was sequenced on ABI 373 sequencing machine using the same primers as were used for amplification. ITS sequences from $P$. pallidum isolates, namely, PN500, WS320, CK8, and H168 were aligned using ClustalX 2.0.11 programme. ITS sequences of $P$. pallidum PN500 (HQ213802), $P$. pallidum WS320 (HQ213803), P. pallidum H168 (HQ213801) and P. pallidum CK8 (HQ213800 were deposited in GenBank.

\subsubsection{RNA Sequencing}

P. pallidum PN500 genome sequencing project was only recently published (Heidel et al. 2011), therefore, facility of commercial DNA microarrays was not available. When the project presented in this dissertation was started, we had access to unpublished sequencing data of $P$. pallidum PN500 genome by courtesy of Dr. Gernot Glöckner, Fritz Lipmann Institute, Jena, Germany. To study genome-wide gene expression changes at the early stages of development, we used high-throughput Illumina RNA sequencing (RNA-seq) technology because this method produces a quantitative, digital information of all the transcripts in a given sample together with increased dynamic range and better sensitivity in contrast to hybridization based microarrays (Wang et al. 2009).

P. pallidum PN500 amoebae were harvested at the vegetative stage, washed free of bacteria and resuspended at a density of $2 \times 10^{7} \mathrm{cells} / \mathrm{ml}$ in cold $17 \mathrm{mM}$ phosphate buffer. $2 \times 10^{7}$ cells were stored as a pellet at $-80^{\circ} \mathrm{C}$ for total RNA prepareation. This pellet served 
as "vegetative control" in RNA-seq analysis. Development was then initiated by starving amoebae for 1 hour in a suspension culture that was rotated at $120 \mathrm{rpm}$ on a platform shaker at $22^{\circ} \mathrm{C}$. After this initial treatment, culture was divided into two parts. To examine the effects of exogenous glorin on gene expression, glorin was added at a final concentration of $1 \mu \mathrm{M}$ at 30 -min intervals for total 2 hours whereas other culture received no glorin addition and served as "control". $2 \times 10^{7}$ cells were pelleted for total RNA preparation after 1 and 2 hours of glorin treatment i.e. after 2 or 4 pulses of glorin respectively. Untreated cells were harvested at the same time points. Cells were generally harvested 30 minutes after a glorin pulse was delivered.

Transcriptome-wide expression levels were determined using Illumina/Solexa nextgeneration sequencing (Bentley et al. 2008). For library preparation an amount of $5 \mu \mathrm{g}$ total RNA per sample was processed using Illumina's mRNA-seq sample prep kit following the manufacturer's instructions. The libraries were sequenced using a Genome Analyzer (GAllx) in a single read approach with 76 cycles resulting in reads with a length of 76 nucleotides. Reads were mapped to the $P$. pallidum reference transcriptome downloaded from the Social Amoeba Comparative Genome Browser (SACGB; http://sacgb.fli-leibniz.de/cgi/index.pl) using the Illumina-supplied tool ELAND (Bentley et al. 2008) with standard parameters. Expression values were calculated by counting the number of unique mappable reads per transcript and normalizing these to the total number of mappable reads and length of the respective transcript (adapted from Mortazavi et al. 2008). This resulted in RPKM values (Reads Per Kilobase transcript and Million mappable reads) representing the expression level of the respective transcript. RNA-seq was performed using RNA preparations from two independent experiments. Average RPKM values derived from both experiements were used to calculate differences in gene expression. However, genes were considered as being glorindependently expressed only if differences in RPKM values in glorin-treated versus untreated cells were greater than 3-fold in each of the two individual RNA-seq experiments. The RNA-seq data have been deposited in the Gene Expression Omnibus data base under accession number GSE24911. 


\subsubsection{Biochemical methods}

\subsubsection{Proteomic analysis of secretory proteins}

\subsection{Preparation of extracellular proteins from aggregating Polysphondylium pallidum}

To collect secreted proteins, wild-type $P$. pallidum cells were cultured in association with Klebsiella planticola on $1 / 5 \mathrm{SM}$ plates at $21 \mathrm{C}^{0}$ (Raper 1984). After about 45 hours of incubation, the vegetative amoebae were harvested from the agar plates using cold 17 $\mathrm{mM}$ phosphate buffer ( $\mathrm{pH} \mathrm{6.2)}$ and freed of bacteria by 4 times centrifugation at $800 \mathrm{rpm}$ in phosphate buffer. The final cell pellet was resuspended in $17 \mathrm{mM}$ phosphate buffer at a concentration of $10^{7} \mathrm{cells} / \mathrm{ml}$. Cells $\left(10^{7}\right)$ were then pipetted on to a Type $3531021 \mathrm{~mm}$ pore-sized polyethylene terephthalate membrane six-well format cell-culture insert (Becton Dickinson, Franklin Lakes, NJ, USA). After $10 \mathrm{~min}$, the cells had settled on, and attached to, the membrane, and the buffer was gently removed from the insert. Cold 17 $\mathrm{mM}$ phosphate buffer containing a protease inhibitor cocktail (one tablet was used for 10 $\mathrm{ml}$ buffer), AEBSF to a final concentration of $0.25 \mathrm{mM}$ and $5 \mathrm{mM}$ EDTA was added into the wells of a six-well plate. The inserts with cells were then placed in the wells. The volume of buffer in the wells was accommodated to just touch the membrane of the insert to keep the membrane and cells moist. Amoebae developed normally, with cells aggregation starting at 4 hour and branching streams formation at 8 hours (data not shown). After 8 hours of development at $21 \mathrm{C}^{0}$, the conditioned starvation buffer in the wells was collected in $2 \mathrm{ml}$ eppendorf tubes ( $1 \mathrm{ml}$ conditioned buffer per tube) and the samples were stored at $-80 \mathrm{C}^{0}$.

\subsection{Concentration of collected proteins}

Frozen conditioned starvation buffer samples ( $1 \mathrm{ml}$ sample per tube) were lyophilized and resulting powder in each tube was resuspended in $50 \mu \mathrm{l}$ of deionized water. Samples were then immediately boiled with $50 \mu \mathrm{l}$ of $2 \times$ Laemmli buffer for $5 \mathrm{~min}$ at 95 ${ }^{\circ} \mathrm{C}$ to denature proteins and subsequently centrifuged for $10 \mathrm{~min}$ at $11000 \mathrm{xg}$ in a table centrifuge (5415 D Eppendorf) to remove the large particles. Supernatant was collected and used for protein analysis by SDS-PAGE. 


\subsection{Separation of secreted proteins by SDS-PAGE}

Proteins in the sample were separated by electrophoresis on $12.5 \%$ SDS-PAGE gel according to Laemmli (1970). Seperation was done at 110- 160 Volt for 2 hours at room temperature using the $1 \times$ SDS buffer as running buffer. For protein MW estimation, $6 \mu$ of molecular weight marker in a range of 15 to $170 \mathrm{kDa}$ (Page Ruler, Fermentas) was run alongside of the gel. Composition of gel was as follows:

\begin{tabular}{|c|c|c|}
\hline \multirow[t]{5}{*}{ Seperating gel (12.5\%): } & $\mathrm{H} 2 \mathrm{O}$ & $9.2 \mathrm{ml}$ \\
\hline & Seperating gel buffer (4 x; pH 8.8) & $7 \mathrm{ml}$ \\
\hline & Acrylamid (30 \%) & $11.7 \mathrm{ml}$ \\
\hline & TEMED & $30 \mu \mathrm{l}$ \\
\hline & APS & $90 \mu \mathrm{l}$ \\
\hline \multirow[t]{5}{*}{ Stacking gel: } & $\mathrm{H} 2 \mathrm{O}$ & $7.7 \mathrm{ml}$ \\
\hline & Stacking gel buffer (10 x; pH 6.8) & $1 \mathrm{ml}$ \\
\hline & Acrylamid (30 \%) & $1.3 \mathrm{ml}$ \\
\hline & TEMED & $15 \mu \mathrm{l}$ \\
\hline & APS & $30 \mu \mathrm{l}$ \\
\hline \multirow[t]{3}{*}{ SDS-Running buffer $(10 \mathrm{x})$ : } & Tris & $0.25 \mathrm{M}$ \\
\hline & Glycin & $2 \mathrm{M}$ \\
\hline & SDS & $1 \%$ \\
\hline
\end{tabular}

\subsection{MS- compatible silver staining of polyacrylamide gel}

After electrophoresis, proteins were visualized by silver staining of polyacrylamide gel as described by Shevchenko et al. (1996). This staining method is mass spectrometry compatible; has no influence on peptide elution from the gel and doesn't interrupt ionization in a mass spectrometer. The limit of detection of this method is $1-5 \mathrm{ng}$ of protein per band. This technique is based on the affinity of the proteins for a cation i.e. silver. SDS also has a high affinity for silver and must be removed prior to staining by a fixation step.

After a gel run, the gel was placed in a glass tray containing $300 \mathrm{ml}$ of fixation solution. The tray was shaken gently on a platform shaker for about 1 hour. After fixation, the gel 
was rinsed 3 times for 30 minutes with $300 \mathrm{ml}$ of $\mathrm{MQ}$ water on a platform shaker. Gel was then sensitized with $300 \mathrm{ml}$ sensitizing solution for exactly 1.5 minutes. Rinsed the gel twice for 1 minute with plenty of $M Q$ water and then stained with $300 \mathrm{ml}$ silver solution for 30 minutes. Rinsed the gel twice for 1 minute with water, followed by development in $300 \mathrm{ml}$ of "development solution" by shaking it gently until the bands acquired the desired intensity. It took almost 2-5 minutes. Development was stopped by shaking the gel gently for 5 minutes in stop solution. Care was taken to add the stopper solution slightly before the desired stain intensity was reached to get clear background. Gel was then stored in stop solution at $4{ }^{\circ} \mathrm{C}$ overnight. The next day gel was rinsed with water 2 times for $10 \mathrm{~min}$ and images were captured by scanning silver stained gel.

\subsection{Mass spectrometry analysis of proteins}

To identify the secreted proteins, bands were excised from the silver stained gel, cut into smaller pieces $(1 \times 1 \mathrm{~mm})$ and sent to the mass spectrometry and proteomics core facility in the laboratory of Dr. Karl-Heinz Gührs at Leibniz Institute for Age Research, Fritz Lipmann Institute (FLI) Jena. The sample preparation procedure was based on the paper of Shevchenko et al. (1996) with some modifications.

\subsection{ESI-MS/MS analysis}

The analyses were performed by ESI-MS/MS with an LTQ Orbitrap XL instrument (Thermo Scientific) in nanospray mode. The mass spectrometer was online coupled to an HPLC (Easy nanoLC (Proxeon, Odense, Danmark)). Before analysis the sample was dissolved in $0.1 \%$ formic acid containing $5 \%$ acetonitrile. An aliquot of $10 \mu \mathrm{l}$ of the sample was subjected to nano HPLC at a flow rate of $250 \mathrm{nl} / \mathrm{min}$ using an Easy nanoLC (Proxeon, Odense, Danmark) equipped with a $(5 \times 0.3) \mathrm{mm}$ Zorbax trap column (Agilent, Waldbronn, Germany) and a $10 \mathrm{~cm} \times 75 \mu \mathrm{m}$ analytical column (NanoSeparations, Nieuwkoop, Netherlands). Separation of the components was achieved by application of a gradient from $5 \%$ to $80 \%$ acetonitrile in $0.1 \%$ formic acid. The efflux was directly sprayed into the orifice of a LTQ Orbitrap XL mass spectrometer (Thermo Scientific, Dreieich, Germany) and mass spectra were recorded. The evaluation of the spectra was performed by Xcalibur (Thermo Scientific, Dreieich, Germany). 


\subsection{Database search to identify proteins}

The selected peptide spectra were used to search for protein candidates in the fasta database of $P$. pallidum, which was generated by ORF identification within the sequenced genome of this organism (Heidel et al. 2011; http://sacgb.flileibniz.de/cgi/index.pl) by using MASCOT (Matrix Science) software programs. The identified proteins were visualized by the software packages ProteomeDiscoverer (Thermo Scientific) and Scaffold (Proteome Software, Portland, USA). The final protein list contains only entries which are characterized by at least two independent peptides with MASCOT peptide scores above 35. Initial search parameters were set as follows: fragment tolerance: 0.80 Da (monoisotopic); parent tolerance: 10.0 PPM (monoisotopic); fixed modifications: +57 on $C$ (carbamidomethyl); variable modifications: -14 on $\mathrm{C}$ (carbamyl), +16 on $\mathrm{M}$ (oxidation); maximum missed cleavages: 2; digestion enzyme: trypsin. Regular injections of $20 \mathrm{fmol}$ of a BSA standard were used to check the current machine state. If 50 to $70 \%$ of the protein was covered by identified peptides, machine status was taken satisfactory. Limit of sensitivity was in the range of 1 to $20 \mathrm{fmol}$ protein per sample injection. 


\section{Results}

Chemotaxis is an intriguing biological phenomenon that plays significant role during the life cycle of Dictyostelids. In the vegetative stage the amebae have to locate their bacterial food in the soil they dwell. At this time the amebae exhibit chemotaxis to folic acid and pterin (Liao \& Kimmel 2009; Pan et al. 1972; Pan et al. 1975), both of which are secreted by bacteria, and serve as cues for nutrient localization. Cyclic AMP secreted by E.coli has also been shown to attract vegetative amoebae of Dictyostelium and Polysphondylium species and was suggested as efficient food-seeking mechanism (Samuel 1961; Konijn 1961; Konijn et al. 1967; Konijn 1969; Konijn 1972). When food source is exhausted, the amoebae gain the ability to establish a complex cell-cell communication network and aggregate to initiate social phase of their life cycle. In 1902, Olive and Potts proposed the existence of a chemical mediator of aggregation in Dictyostelids. Later, Bonner (1947) reported that starving amoebae release diffusible chemical agents, named 'acrasins' that are capable to orient and attract neighbouring amoebae. It has been suggested that there might be at least 8 different chemical substances prevailed as acrasins among the Dictyostelids (Bonner 1982). Recently, molecular phylogeny of Dictyostelids classified more than 100 species of social amoebae into four major groups (Schaap et al. 2006). The well-studied Group 4 species, Dictyostelium discoideum, employs the chemoattractant cAMP as communication signal to organize aggregation (Konijn et al. 1967). Some other group 4 species, such as $D$. mucoroides, $D$. purpureum, and $D$. rosarium have also been reported to use cAMP as acrasin (Bonner 2009; Schaap et al. 2006; Konijn 1973; Bonner et al. 1969; Konijn et al. 1967; Shaffer 1953). Group 3 species D. lacteum uses pterin as aggregation chemoattractant (Van Haastert et al. 1982), whereas D. minutum employs a derivative of folic acid (De Wit \& Konijn 1983) as acrasin. A modified dipeptide glorin (N-propionyl- $\gamma$-Lglutamyl-L-ornithine- $\delta$-lactam-ethylester) is used as cell-cell communication agent to mediate aggregation in $P$. violaceum (Shimomura et al. 1982), a species placed at the bottom of group 4 in the molecular phylogeny of Dictyostelids (Schaap et al. 2006). It was reported that $P$. pallidum, a group 2 species, was also responsive to glorin (Shimomura et al. 1982) but no detailed description of related experiments was available. $D$. caveatum (group 3 species) was described to show activity towards the acrasin of $P$. violaceum (Waddell 1982b). These previous studies provided an indication that chemical language of glorin might be widely used among the Dictyostelids. 
Primary objective of this study was to explore how wide-spread glorin-based cell-cell communication is in the phylogenetic history of Dictyostelids. To address this question, commercially available glorin was used as an acrasin (or chemoattractant) and chemotactic sensitivity of a collection of dictyostelid species was evaluated using a bioassay described under section 3.1 .

\subsection{Development and validation of chemotaxis bioassay}

Konijn (1961, 1968, 1969, 1972) developed an effective small population assay to test chemotaxis of Dictyostelium and Polysphondylium species to cAMP. This assay makes use of aggregation competent cells that are highly sensitive to acrasin gradients. Futrelle et al. (1982) showed that starving amoebae start to gain chemotactic competence within an hour after the initiation of starvation that increases gradually, such that cells acquire full competence when aggregation begins (Futrelle et al. 1982). Small population assay was later modified by Shimomura and colleagues to determine the chemotactic activity of $P$. violaceum to glorin that was collected from aggregating amoebae of this species (Shimomura et al. 1982). It has been shown that aggregation competent $P$. violaceum cells possess high number of cell surface receptors for the detection of the extracellular glorin and are capable to degrade dipeptide chemoattractant by secreting or exposing glorin degradation enzymes (De Wit et al. 1988).

Small population assay requires a hydrophobic agar surface of low rigidity. To prepare hydrophobic agar plates, agar is washed repeatedly with deionised water, suspended in phosphate buffer, dissolved by boiling and allowed to gelate. The concentration of the agar is adjusted to $1 \%$ that allows amoebae to move outside the boundaries of the drop to increasing concentration gradients of acrasin after cells have degraded chemoattractant in their vicinity by the activity of acrasinases (Konijn 1972). The chemoattractants are added to the agar before pouring plates.

In the modified small population assay (Shimomura et al. 1982), small drops of aggregation-competent amoebae are deposited on a hydrophobic agar surface incorporating acrasin of choice, such that all cells remain within the boundary of the drop. Since the developing amoebae secrete acrasinase in their instant vicinity, any acrasin in that region is removed. If there were initially an even concentration of acrasin in the agar, there will now be much less or none within and surrounding the drop of amoebae, 
resulting in an increasing concentration gradient from the centre of drop outwards. This gradient causes the orientation of the amoebae beyond the margins of the drop displaying chemotactic sensitivity of cells to the acrasin. An optimal chemotaxis response can be obtained in this assay only when cells are at the right stage of aggregation competence that lasts only for a very restricted period of time; once starving cells cross this short aggregation-sensitivity peak, they move back to the new centres that have developed within the drop and start to secrete their own acrasin (Shimomura et al. 1982). Direction of movement of cells is reversed to the centres formed in the original drop because acrasin gradient is reversed (Futrelle et al. 1982). The success of the bioassay, then, is dependent on the ability of cells to detect extracellular acrasin and release a stable extracellular enzyme 'acrasinase' which destroys the attractant; properties that are essential for chemotaxis during the normal development of the social amoebae.

To perform chemotaxis assays in this study, the revised small population assay (Shimomura et al. 1982) was adapted. To induce aggregation competence, cells were starved for 3-4 hours in shaken suspensions as suggested by Browning and colleagues (Browning et al. 1995). This treatment is reported to trigger a spectrum of molecular and biochemical changes that result in the loss of sensitivity to vegetative-stage folate, while inducing responsiveness to aggregation-specific chemoattractant (Browning et al. 1995). This assay was first verified by investigating the chemotactic activity of $P$. violaceum to its known acrasins i.e. glorin (Figure 7). Source of glorin used in this assay was the commercial glorin. Different concentrations of glorin were tested (data not shown) and optimal chemotactic response was detected with $1 \mu \mathrm{M}$ glorin (final concentration; Figure 7).

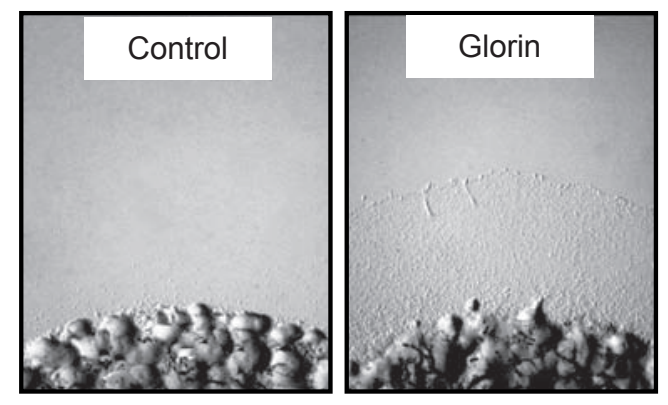

Figure 7: Validation of chemotaxis assay. P. violaceum cells developing in the absence (left picture) or presence of $1 \mu \mathrm{M}$ of glorin final concentration (right pictures). In each experiment, cells were starved in slow shaking buffer suspensions for $4 \mathrm{hrs}$ prior to plating as $10 \mu \mathrm{l}$ drops containing $2 \times 10^{5}$ cells on hydrophobic agar. In control plates containing no acrasin, cells remained confined inside the boundaries of the drop. If acrasin was embedded in the agar, amoebae crossed the 
margins of the drop in all directions and drop was completely dispersed. Edges of drops were photographed 3 hours after plating to document outward movement of cells from the boundaries of drop.

\subsection{Chemotactic specificity of group 4 species}

Acrasin of the well-known group 4 species $D$. discoideum is known to be cAMP. Chemotaxis assay adapted in this study was further validated by evaluating response of D. discoideum NC4 to CAMP (Figure 8). Different concentrations of cAMP were tested (data not shown) and optimal chemotactic response was noticed with $100 \mu \mathrm{M}$ final concentration of CAMP (Figure 8).

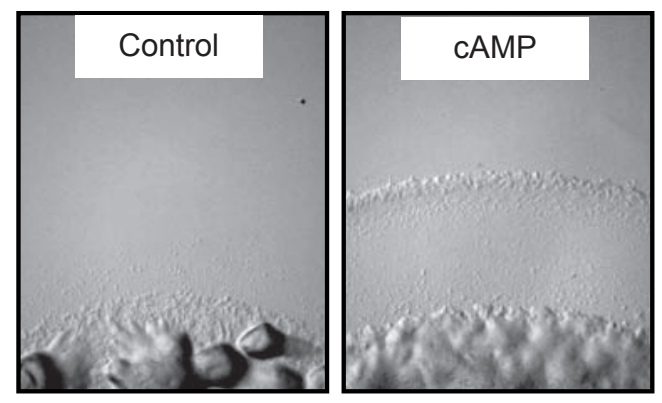

Figure 8: Chemotaxis assay for cAMP used in this study. D. discoideum NC4 cells were deposited on hydrophobic agar plates containing either no CAMP (left picture) or $100 \mu \mathrm{M}$ of cAMP (right picture). In each experiment, cells were starved in slow shaking buffer suspensions for 4 hrs prior to plating as $10 \mu \mathrm{l}$ drops containing $2 \times 10^{6}$ cells on hydrophobic agar. In control plates containing no acrasin, cells remained confined inside the boundaries of the drop. If acrasin was embedded in the agar, amoebae crossed the margins of the drop in all directions and drop was completely dispersed. Edges of drops were photographed 3 hours after plating to document outward movement of cells from the boundaries of drop.

Under similar conditions, response of five other group 4 species was evaluated towards cAMP and glorin. All tested group 4 species: $D$. sphaerocephalum, $D$. giganteum, $D$. dimigraforum, $D$. firmibasis and $D$. intermedium reacted well to cAMP but showed no response to glorin. These data are summarized in Table 1.

\subsection{Chemotactic specificity of $P$. violaceum lying at the edge of group 4}

The violet coloured $P$. violaceum is distinctly placed at the base of group 4 or in group 3 plus 4 (Figure 1; Schaap et al. 2006). Group 4 species use cAMP as chemotactic aggregant, whereas in $P$. violaceum, dipeptide glorin mediates aggregation. Chemotaxis 
of $P$. violaceum amoebae to glorin was reported by some groups (Shimomura et al. 1982; Wurster et al. 1976) and verified in this study (Figure 7 \& 9).

\subsubsection{Stage specificity in the response of $P$. violaceum amoebae to different chemotactic agents}

Previously, it was reported that folic acid causes a strong chemotactic response in the early preaggregation cells of $P$. violaceum (Wurster et al. 1978). This study showed that post-vegetative cells starved for 1 hour in shaken suspensions were sufficiently active to CAMP (Figure 9). Sensitivity to cAMP decreased with increase in starvation as shown by a minimal response to $100 \mu \mathrm{M}$ cAMP by $P$. violaceum amoebae starved for 3 hours.

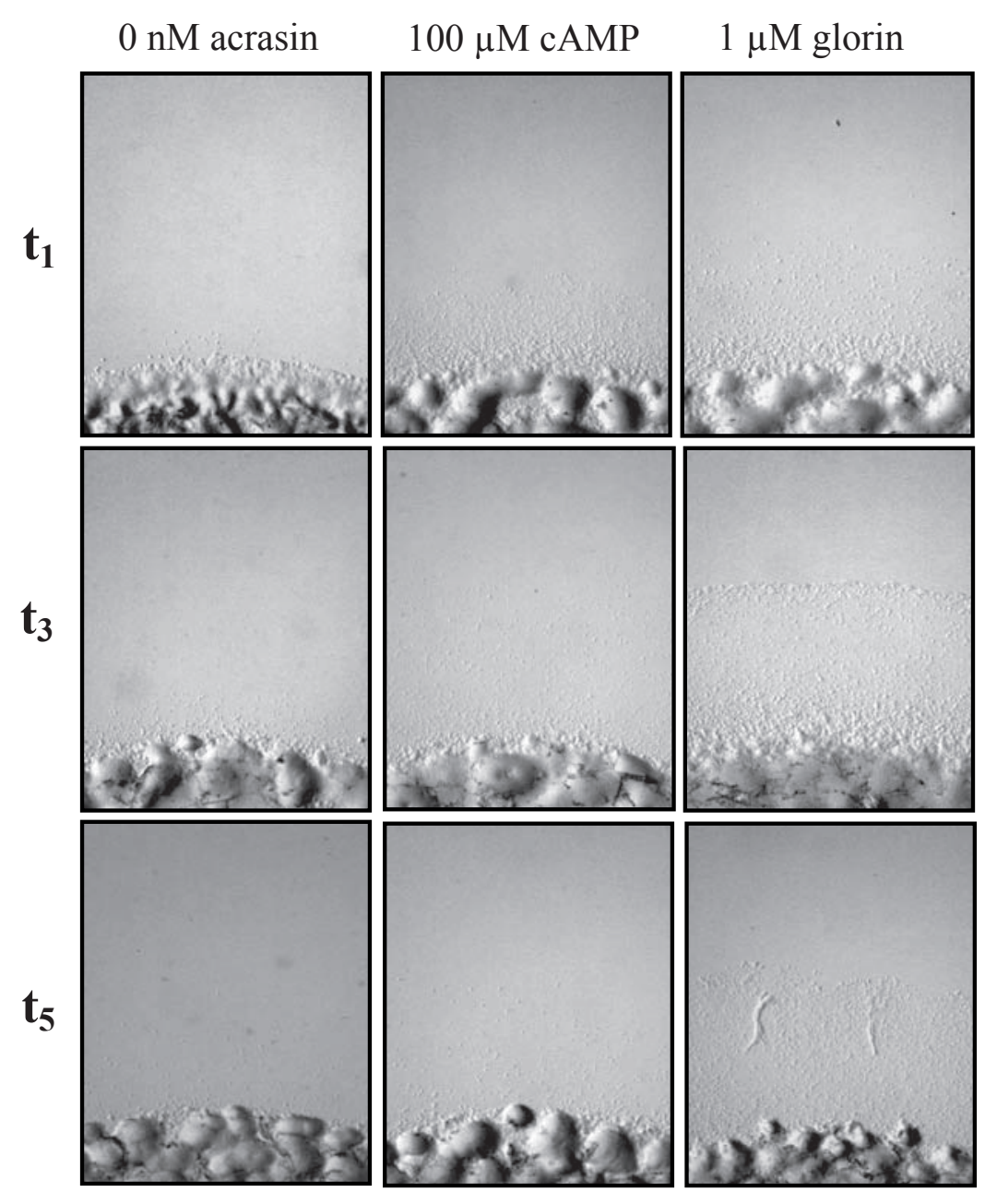

Figure 9: Chemotactic activity of starving $P$. violaceum amoebae to cAMP and glorin. Cells were starved for 1,3 and 5 hours $\left(t_{1}, t_{3}, t_{5}\right)$ in shaking cultures before $10 \mu$ drops containing $2 \times 10^{6}$ cells were placed on phosphate agar plates prepared with either $100 \mu \mathrm{M}$ cAMP or $1 \mu \mathrm{M}$ final 
concentration of glorin. Plates containing $0 \mathrm{nM}$ acrasin represented "controls without acrasin". Chemotaxis towards glorin was monitored every 30 minutes using an Olympus steromicroscope and pictures were taken 3 hours after plating.

Cells starved for 1 hour were adequately active to glorin, while aggregation competence induced by starving cells for 3 or 5 hours in suspensions provoked cells to display greater chemotaxis towards glorin. When cells were plated from cultures starved for 5 hours, a maximal response to glorin was observed where $P$. violaceum amoebae moved out of drop in a dense front with some streams. These results are in agreement with the observations made by Jones and Robertson (1976), which showed that $P$. violaceum amoebae are weakly attracted to a microelectrode releasing $100 \mu \mathrm{M}$ or $1 \mathrm{mM}$ cAMP (Jones and Robertson 1976). Owing to the phylogenetic position of $P$. violaceum at the bottom of group 4, the weak chemotactic response of starving amoebae of this species to cAMP may represent development of initial biochemical steps towards switching on the use of CAMP to coordinate aggregation that is characteristic of group 4 species.

\subsection{Chemotaxis of social amoebae towards glorin is an ancient response}

\subsubsection{Chemotactic response of group 2 species to glorin}

\subsubsection{Chemotactic specificity of $P$. pallidum PN500 cells}

Previously, P. pallidum amoebae have been described to be chemotactically reactive to glorin (Shimomura et al. 1982; Wurster et al. 1976). A group 2 species designated as $P$. pallidum PN500 is emerging as an eminent eukaryotic model system to study fundamental problems in cell and developmental biology because it is accessible to various genetic and biochemical approaches, and imaging techniques (Schaap 2011a). Additionally, recent availability of the genome sequence of $P$. pallidum PN500 (Heidel et al. 2011) has opened exceptional possibilities to identify developmentally relevant genes. The ultimate objective of this project was to examine glorin-mediated regulation of gene expression employing $P$. pallidum PN500 as test species. Therefore, as a first step, chemotactic response of $P$. pallidum PN500 towards glorin was investigated. This study demonstrated that $P$. pallidum PN500 amoebae exhibit marked chemotactic orientation and directional locomotion of cells towards glorin but no response was noticed towards cAMP (Figure 10). 

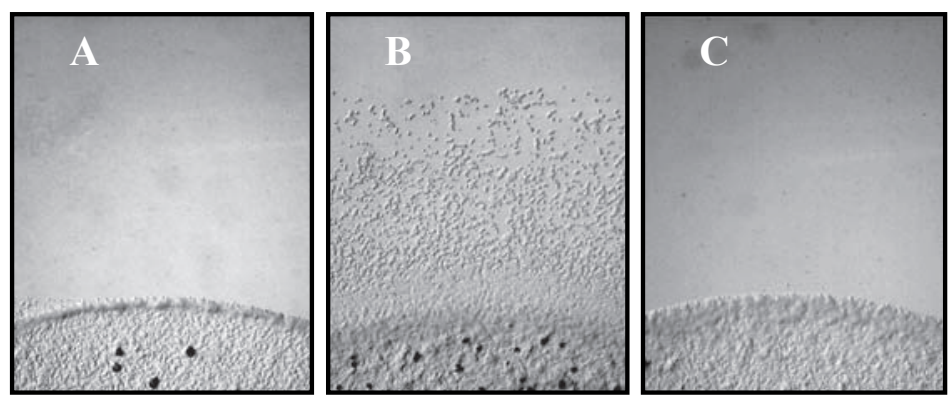

Figure 10: Chemotactic response to glorin by P. pallidum PN500. Cells were starved for 3 hours in shaking cultures before $10 \mu \mathrm{l}$ drops containing $2 \times 10^{6}$ cells were spotted on phosphate agar plates prepared without (picture A), with $1 \mu \mathrm{M}$ final concentration of glorin (picture $\mathbf{B}$ ) or with $100 \mu \mathrm{M}$ cAMP (picture C). Chemotaxis towards glorin was monitored every 30 minutes using an Olympus steromicroscope and pictures were taken 3 hours after plating. $P$. violaceum was used as a positive control (data not shown).

These data provide an indication that developing $P$. pallidum PN500 amoebae are capable to detect glorin, degrade it and move up a spatial gradient of chemoattractant demonstrating that glorin may be used as acrasin by P. pallidum PN500 to coordinate aggregation; therefore, it could be interesting to examine further cellular response to extracellular glorin using $P$. pallidum PN500 as model species.

\subsubsection{Determination of optimal concentration of glorin required to obtain maximum chemotactic response of $P$. pallidum amoebae}

In order to determine optimal concentrations of glorin required for the radial chemotaxis assays, $P$. pallidum PN500 'vegetative stage cells' and 'cells starved for 3 hours' were deposited in form of drops on thin agar plates containing a range of concentrations of glorin. Depending upon the chemoattractant concentration used, cells responded to varying degrees by moving out of the drop to a certain distance. Patterns of response were recorded approximately 3 hours after plating (Figure 11). In these experiments, $P$. pallidum aggregation-competent amoebae migrated 'as individuals' on plates containing 10-100 nM glorin, while cells migrated outward 'as aggregates' in response to $1 \mu \mathrm{M}$ glorin (Figure 11). These results indicate that $100 \mathrm{nM}$ (final concentration) is the optimal concentration of glorin required to observe maximal chemotaxis response of $P$. pallidum PN500 cells (Figure 11). Nevertheless, prominent chemotactic response was detected using even higher concentration of glorin, i.e. $1 \mu \mathrm{M}$ (Figure 11). 

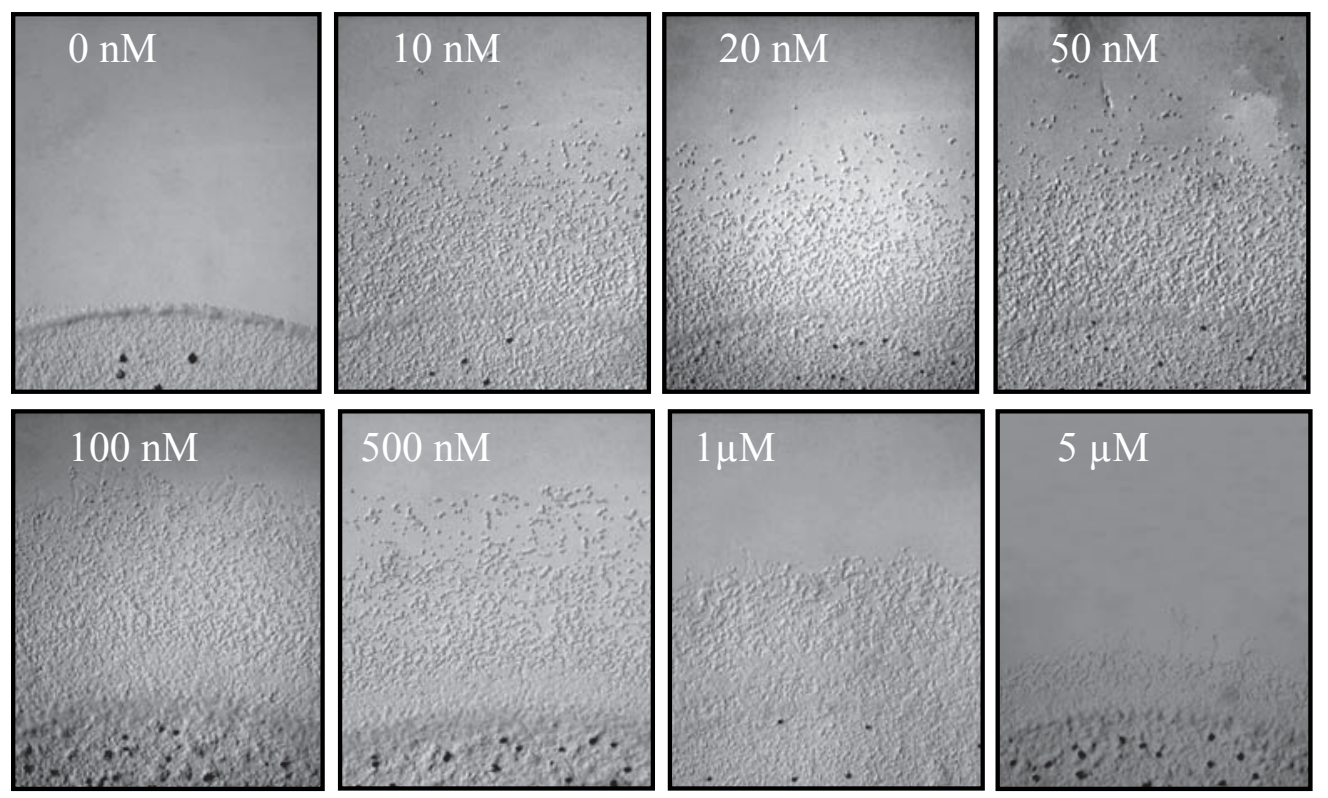

Figure 11: Chemotactic response of $P$. pallidum PN500 amoebae to differing concentrations of glorin. Cells were starved for 3 hours in shaking cultures before $10 \mu l$ drops containing $2 \times 10^{6}$ cells were placed on phosphate agar plates prepared with $0 \mathrm{nM}, 10 \mathrm{nM}, 20 \mathrm{nM}$, $50 \mathrm{nM}, 100 \mathrm{nM}, 500 \mathrm{nM}, 1 \mu \mathrm{M}$ and $5 \mu \mathrm{M}$ final concentration of glorin. Chemotaxis towards glorin was monitored every 30 minutes using an Olympus steromicroscope and pictures were taken 3 hours after plating.

\subsubsection{Stage specificity in the response of $P$. pallidum PN500 cells to glorin}

These experiments were designed to trace changes in the sensitivity of the amoebae as they "age" in shaking cultures. Vegetative stage P. pallidum PN500 cells (indicated by $\mathbf{t}_{0}$; Figure 12) deposited on hydrophobic phosphate agar plates showed a very weak response to $10 \mathrm{nM}$ glorin and were insensitive to higher concentrations of acrasin (Figure 12). Effects of varying starvation times (from 0 - to 4-hours) on the ability of cells to respond to glorin were then assessed. Amoebae starved for 1 hour (indicated by $\mathbf{t}_{1}$ ) were moderately more reactive to low concentrations of glorin (Figure 12). The progression of starvation led to an enhanced sensitivity of the responding amoebae, and as a result orientation of $P$. pallidum PN500 cells to the gradients of glorin increased. Cells starved for 2 hrs (data not shown) were adequately active to glorin, while there was a sudden rise in the sensitivity of cells starting at about 3 hours of starvation (indicated by $\mathbf{t}_{3}$; Figure 12). It was observed that cells developed for 3 hours in shaking suspensions were at the appropriate stage of aggregation competence and responded maximally on chemotaxis assay plates containing optimal concentration of glorin (i.e. $100 \mathrm{nM}$ glorin as shown in Figure 11 \& Figure 12). When amoebae starved in shaking cultures for 4 hours (indicated 
by $\mathbf{t}_{4}$ ) were deposited on hydrophobic agar, chemotactic efficiency of the responding cells was comparatively reduced (Figure 12). These data support stage-specificty in the response of $P$. pallidum $\mathrm{PN} 500$ cells to glorin.

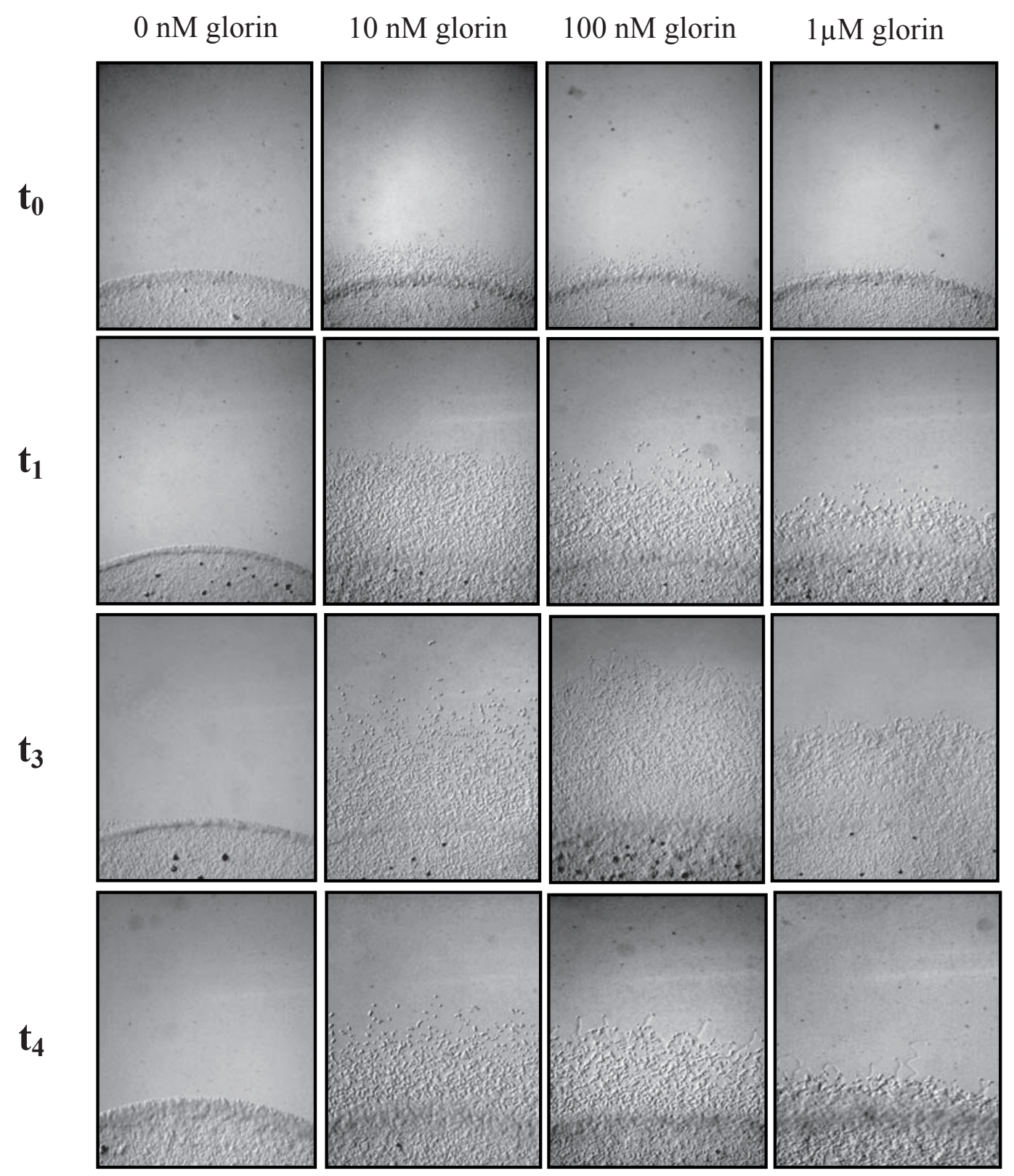

Figure 12: Effects of varying starvation times on the ability of $P$. pallidum PN500 amoebae to respond to glorin. Cells were starved for $1-4$ hours $\left(t_{1}, t_{2}, t_{3}, t_{4}\right)$ in shaking cultures before $10 \mu \mathrm{l}$ drops containing $2 \times 10^{6}$ cells were placed on phosphate agar plates prepared with $0 \mathrm{nM}, 10 \mathrm{nM}$, $100 \mathrm{nM}$ and $1 \mu \mathrm{M}$ final concentration of glorin. Chemotaxis towards glorin was monitored every 30 minutes using an Olympus steromicroscope and pictures were taken 3 hours after plating. 


\subsubsection{Delayed chemotactic response of $P$. pallidum amoebae to high concentrations of glorin}

When sensitivity of aggregation-competent $P$. pallidum PN500 cells was tested to higher concentrations of glorin, i.e. 10-20 $\mu \mathrm{M}$ glorin, it was noticed that starving amoebae could sense a higher concentration of attractant, however, they responded by relatively slow outward migration from the margins of the drop and did not move a greater distance away from the drop during the first few hours (indicated by $\mathbf{t}_{1} \& \mathbf{t}_{3}$ in Figure 13) after being plated on hydrophobic agar surface.

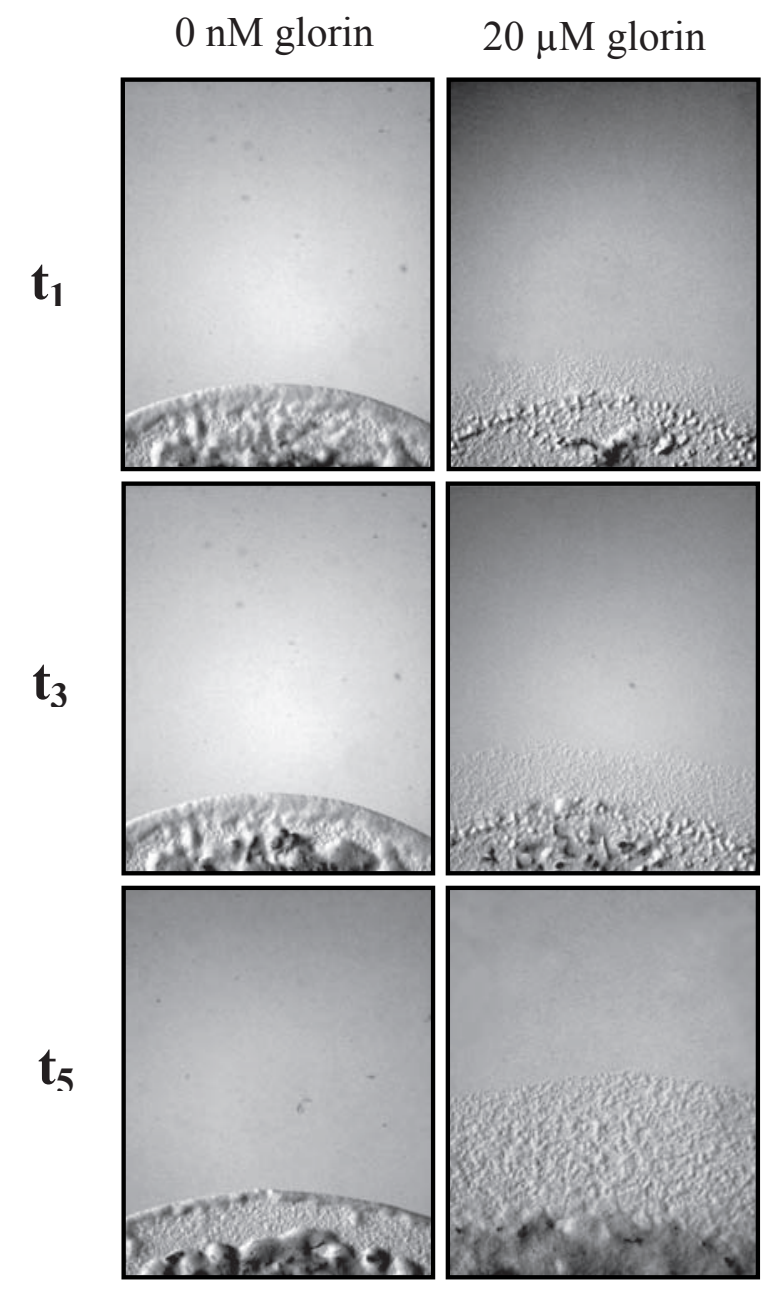

Figure 13: Chemotactic response of starving $P$. pallidum PN500 amoebae to high concentrations of glorin is delayed. Cells were starved for 3 hours in shaking cultures before $10 \mu \mathrm{l}$ drops containing $2 \times 10^{6}$ cells were placed on phosphate agar plates prepared with $0 \mathrm{nM}$ or $20 \mu \mathrm{M}$ final concentration of glorin. Chemotaxis towards glorin was monitored every 30 minutes using an Olympus steromicroscope and pictures were taken at 1,3 and 5 hours $\left(t_{1}, t_{3}, t_{5}\right)$ after plating. Slow migration of amoebae outside the borders of the drop was seen within 1 hour of placing the drop on agar surface, later this outward movement ceased considerably and the 
optimal response was recorded at 5 hour of plating. Hence chemotaxis to excess of glorin was delayed by a few hours. Interestingly, in response to higher glorin concentrations, amoebae moved out in a dense front. Similar observations were made when glorin was tested at a concentration of $10 \mu \mathrm{M}$.

It seemed clear that the chemotactic response of cells to the excess of glorin was delayed by 2-3 hours (compare results depicted in Figure 12 and Figure 13 at $\mathbf{t}_{3}$ for cells starved for 3 hours before being deposited on agar). As shown in Figure 12, cells starved in shaking suspensions for 3 hours exhibited significant chemotactic response to 'lower' concentrations of attractant when plated on agar plates and amoebae could travel a considerable distance away from the boundaries of drop (Figure 12). Similar observations were made by De Wit \& Konijn (1983), which showed that the chemotactic response of $D$. minutum to high concentrations of folic acid was delayed (De Wit \& Konijn, 1983). This phenomenon may indicate that Dictyostelids exhibit adaptation during chemotactic migration, undergoing consecutive phases of desensitization and resensitization in the presence of increasing concentrations of acrasins. Such adaptive behaviour allows the social amoebae to reversibly adjust their sensitivities over a wide range of concentrations of the chemoattractant; an essential feature for long-range chemotaxis.

\subsubsection{5 $P$. pallidum is a species complex}

P. pallidum has been considered as a 'species complex' (Romeralo et al. 2011b; Romeralo et al. 2010; Kawakami \& Hagiwara 2008; Schaap et al. 2006; Raper 1984) containing several morphologically similar species that are characterized by white sorocarps carrying elliptical spores and bearing sorophores without lengthened terminal segments (Hagiwara 1989, 1982). This species complex encompasses Polysphondylium pallidum Olive (Olive 1901) and its related species Polysphondylium album Olive (Olive 1901). P. album Olive is distinguished from $P$. pallidum primarily in having larger sori, prostrate sorocarps and larger number of branches per whorl (Kawakami \& Hagiwara, 2008). A recent morphological reconsideration of $P$. pallidum species complex by Kawakami and Hagiwara (2008) proposed that $P$. pallidum isolate PN500 (Figure 14) should be renamed as $P$. album, that was placed by Raper together with $P$. pallidum (Kawakami \& Hagiwara 2008; Raper 1984). P. pallidum isolate PN500 (Figure 14) is characterized by round to clavate sorophore bases, typically ovoid and shorter tip cells and exhibit higher whorl index values (Kawakami \& Hagiwara, 2008). However, the isolate P. pallidum CK8 (Figure 14) is reported to have lower whorl index values, typically subulate and longer tip cells and clavate sorophore bases (Kawakami and Hagiwara, 
2008). Other isolates, such as P. pallidum H168 (reported by Romeralo et al. 2010) and P. pallidum WS320 (Figure 14) are clearly demarcated by a combination of the shape of sorophore base, the number of branches per whorl, and the shape and length of tip cell. In the presented research work, ITS DNA sequencing (Romeralo et al. 2010) was used to show that $P$. pallidum strain WS320 is closely related to the isolate PN500 (placed in subclade 2B1; Romeralo et al. 2010) and both may therefore be designated as $P$. album isolates (Figure 15). It is further showed that $P$. pallidum CK8 (Kawakami \& Hagiwara 2008 ) is closely related to $\mathrm{H} 168$ isolate (placed in subclade 2B2; Romeralo et al. 2010), suggesting that they should be recognised as $P$. pallidum sensu strictu (Figure 15). When compared, the ITS sequences of isolates WS320/PN500 (group 2B1) and CK8/H168 (group 2B2) revealed low conservation, except in the 5.8 rRNA gene sequence, manifesting the relative phylogenetic distance between different isolates of the P. pallidum species complex (Figure 15; approved by Romeralo M. and Baldauf S.). It is not yet approved by the community that isolates, such as PN500 or WS320 will be renamed $P$. album, therefore, these strains are ascribed as $P$. pallidum in this study.

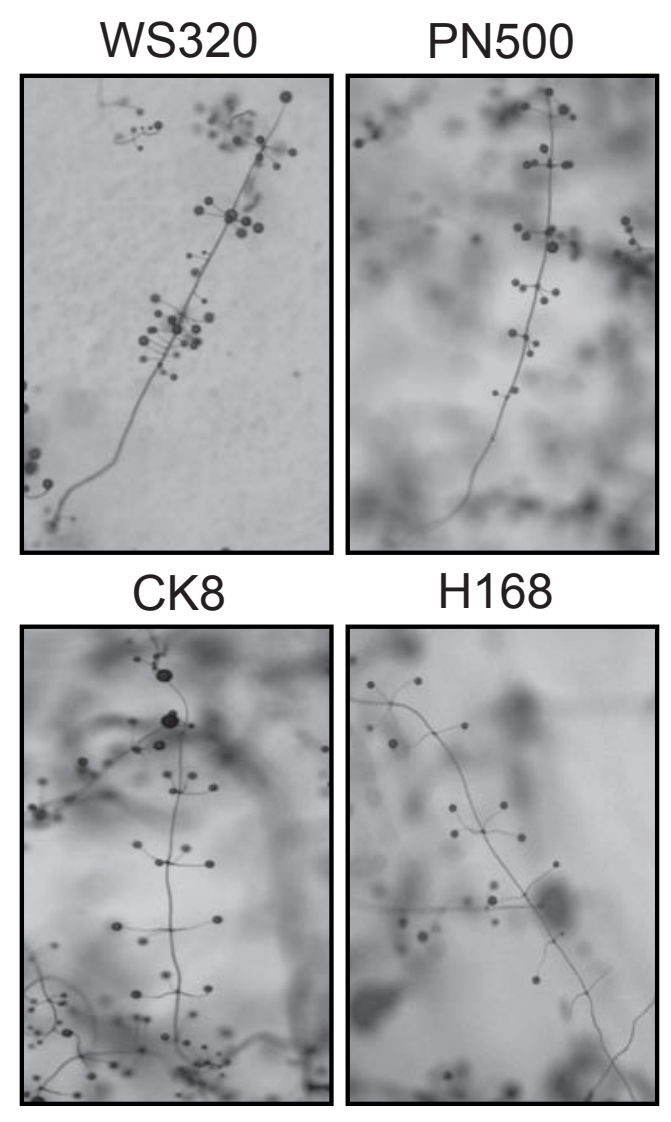

Figure 14: Fruiting body morphologies of different isolates of $P$. pallidum. Fruiting bodies are adorned with regular whorls of side branches. P. pallidum WS320 (upper left); $P$. pallidum PN500 (upper right); P. pallidum CK8 (lower left); P. pallidum H168 (lower right). 
PN500 WS 320

CK8 $\mathrm{H} 168$

PN500 WS 320 CK8 H1 68

TAAAAAAACGAACTCAAAAGGTGTATTAAGAAGCTTCGGTTTCATTTTACACCGCTCTTT TAAAAAA - CGAACTCAAAAGGTGTATTAAGAAGCTTCGGTTTCATTTTACACCGCTCTTT CATAAAAAAGAACTCAAAAAAAAAAGTTATTTATTTAACTTTTGCCTAATTT - - TTTTA CATAAAAAAGAACTCAAAAAAAAAAGTTATTTATTTAACTTTTGCCTAATTT- - TTTTA

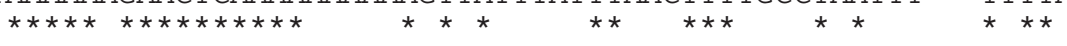

GCTAAAAATTCTTCGGAATTTAAGCTTA - - GAGTATCTCAAAAAA- - ACTTATTTCGTA GCTAAAAATTCTTCGGAATTTAAGCTTA - - GAGTATCTCAAAAAAAAACTTATTTCGTA ATTTGGTATTCATATTAAATTAAATTAATTTGGGCATCTCTCTCAAA-ATTTATTTCGTA ATTTGGTATTCATATTAAATTAAATTAATTTGGGCATCTCTCTCAAA-ATTTATTTCGTA $* \quad * * * * * * * * * * * * * * * * * * * \quad * * * * * * * * * * * *$

TGTCAAATTCAATGTAGTTGTCTGATAGTCTAATGACATCCTCGGATGTTCCAATTCTTG TGTCAAATTCAATGTAGTTGTCTGATAGTCTAATGACATCTTCGGATGTTCCAATTCTTG TGTCAAATTAATTGTAATTGTCTAA- - - - - - GATGTTTCCACTCTGTGGAAATATTA TGTCAAATTAATTGTAATTGTCTAA- - - - - - GATGTTTCCACTCTGTGGAAATATTA

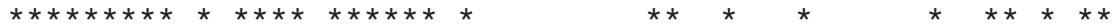

AAGTCAATGATTTTTAGTAAGCATAAATGGTGGATACCTCGGCTCCTAAATCGATGAAGA AAGTCAATGATTTTTAGTAAGCATAAATGGTGGATACCTCGGCTCCTAAATCGATGAAGA AAGTCAATGATTTTTAGTAAGCATAAATGGTGGATACCTCGGCTCCTAAATCGATGAAGA AAGTCAATGATTTTTAGTAAGCATAAATGGTGGATACCTCGGCTCCTAAATCGATGAAGA

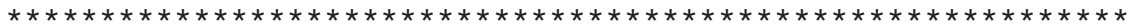

CCGTAGCAAACTGCGATAAGTCACTTGAATTGCAGACTACTGTGAAAGTCGAACTGTTGA CCGTAGCAAACTGCGATAAGTCACTTGAATTGCAGACTACTGTGAAAGTCGAACTGTTGA CCGTAGCAAACTGCGATAAGTCACTCGAATTGCAAACTACTGTGAAAGTCGAAATGTTGA CCGTAGCAAACTGCGATAAGTCACTCGAATTGCAGACTACTGTGAAAGTCGAAATGTTGA $* * * * * * * * * * * * * * * * * * * * * * * * * * * * * * * * * * * * * * * * * * * * * * * * * * * * * * * * *$

ACGCACATGATGATATTGATTCTTCACGGAGTTAAATATCACACTTGGTTGAGAGTCGCA ACGCACATGATGATATTGATTCTTCACGGAGTTAAATATCACACTTGGTTGAGAGTCGCA ACGCACATGATGACATTGACTCCCCC- - GAGTTAAATGTCACACTTGGTTGAGAGTCGCA ACGCACATGATGACATTGACTCCCCC- - GAGTTAAATGTCACACTTGGTTGAGAGTCGCA $* * * * * * * * * * * * * * * * * * * * * * * * * * * * * * * * * * * * * * * * * * * * * * * * * * *$ TCTCATTATCATTTACCAATAGTTTTTTTTTCGAAAAAGCTATTGGCAAAGTATTGGGAA TCTCATTATCATTTACCAATAGTTTTTTTT-CGAAAAAGCTATTGGCAAAGTATTGGGAA TCTCATTATCATTTCTTAATTCTATT- - - - AAGAAAGTATTGGTAAAGATTTCTTAG TCTCATTATCATTTCTTAATTCTATT------AAGAAAGTATTGGTAAAGATTTCTTAG

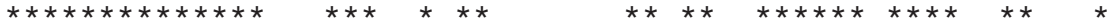

AGTTTTCTATGACAATTCGGGATTACTTTCTTTAAATATTAAAACATTGTCAGGTATGAA AGTTTTCTATGACAATTCGGGATTACTTTCTTTAAATATTAAAACATTGTCAGGTATGAA A- - - - TATTACA-TTCGGAAT- - CTTCACTTAAATATAAAAAAAAAAAGTAATATCAG A- - - - TATTACA-TTCGGAAT - CTTCACTTAAATATAAAAAAAAAAAGTAATATCAG * $\quad * * * * * * * * * * * * * * * * \quad * * * * * * * * * * * * * \quad * * * *$

AATTAGTTGTCTCACGACTTCTAATATCCAATAGATTCAAAATTATTTAATTGGTTTCCA AATTAGTTGTCTCACGACTTCTAATATCCAATAGATTCAAAATTATTTAATTGGTTTCCA GTACAATTCCAATAAGAAACAAAATCGTTTTCCAATTTGT - TTCCGCAAGGAAACAGCA GTACAATTCCAATAAGAAACAAAATCGTTTTCCAATTTGT- - TTCCGCAAGGAAACAGCA

$$
* * * \quad * * * \quad * * * \quad * * * \quad * * \quad * * \quad * *
$$

GTTTACTGGAGTGGCCAGTTGGATTTTTTAAAGTCTTTGTTCGTTTCGACTAAATTTTTA GTTTACTGGAGTGGCCAGTTGGATTTTTTAAAGTCTTTGTTCGTTTCGACTAAATTTTTA ATTGAATAATTTTTTTGTTATAAATTTTTAACAA - TTATTGATTTCGATTCAATTTTT ATTGAATAATTTTTTTGTTATAAATTTTTAACAA - TTATTGATTTCGATTCAATTTTT$* * * * * * * * * * * * * * * * * * * * * * * * * * * * * *$

GTGGAACGGGATTGGACTGAAGCATTTGCTTCTTTTTCATATAAAAACTTTAATTGGTTA GTGGAACGGGATTGGACTGAAGCATTTGCTTCTTTTTCATATAAAAGCTTTAATTGGTTA GTGAGAAAATTTCGGATAGTACGAAGTTTTGATCGTTAAT-TCAAGGATGGGATTTACAA GTGAGAAAATTTCGGATAGTACGAAGTTTTGATCGTTAAT - TCAAGGATGGGATTTACAA $* * * * * * * * * * * * * * * * * * * * * * * * * *$

AACCCAGTAACTATAGTTGATAAATCGACTTAGCTATTAATGAAAGTTTTAAACATCAAA AACCCAGTAACTATAGTTGATAAATCGACTTAGCTATTAATGAAAGTTTTAAACATCAAA AATCTTA-AACTGGTAATGAAAAATTAA - - AACTATCAAT - - - - - TTGAAAGATTGCC AATCTTA-AACTGGTAATGAAAAATTAA- - AACTATCAAT- - - - TTGAAAGATTGCC
$* * * * * * * * * * * * * * * * * * *$

TTGAAAAGTCTAACTTTCCAACGTTTAGAATTAGTAGTTTCTGACGCGTCTTTTGTGTAG TTGAAAAGTCTAACTTTCCAACGTTTAGAATTAGTAGTTTCTGACGCGTCTTTTGTGTAG TCGCGTGATTT - - TTTAAAATGTTTTAAATTAGTAATTATCG-TATGTTTTT- - - - - G 


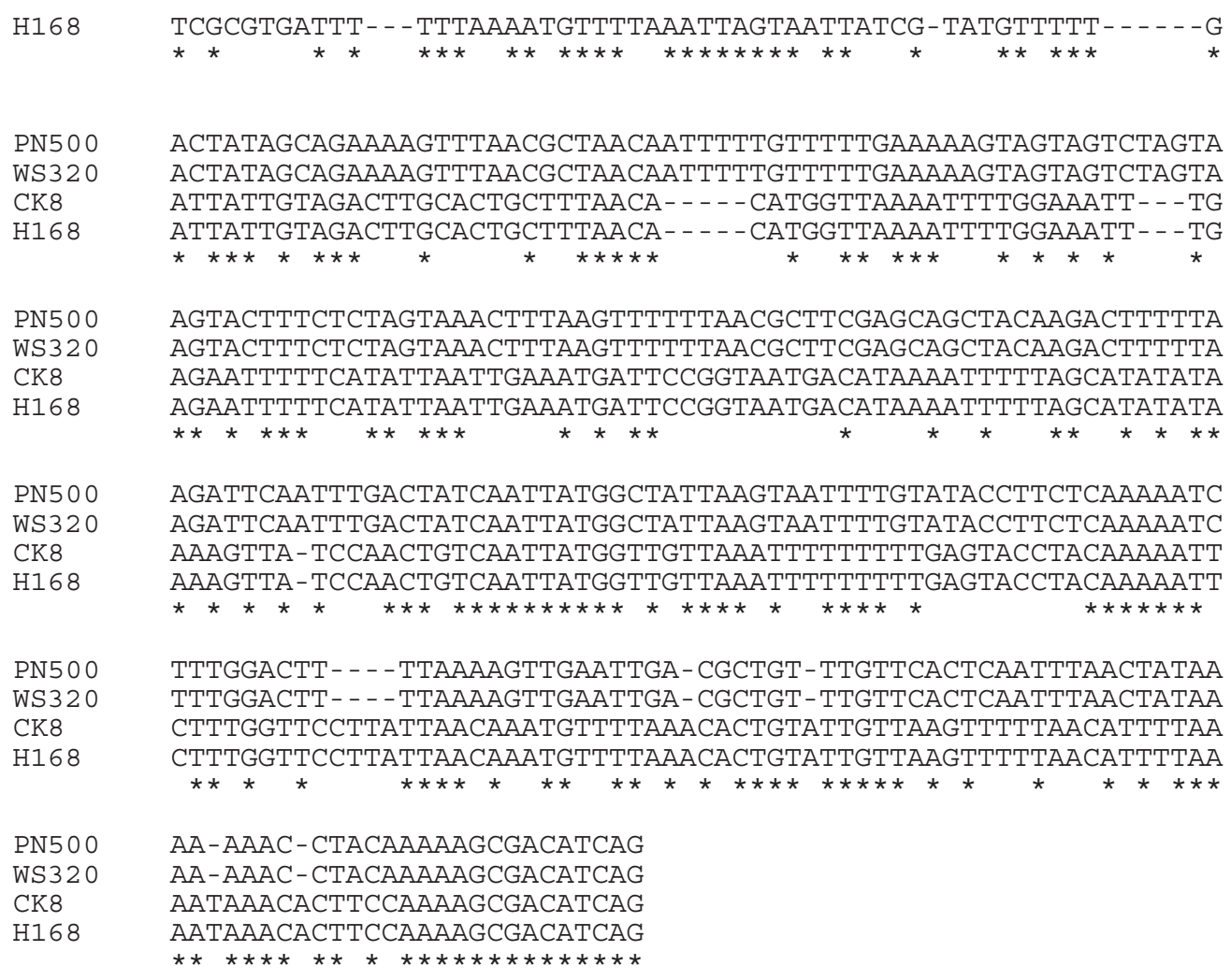

Figure 15: Alignment of ITS sequences from $P$. pallidum isolates, namely, PN500, WS320, CK8, and H168 generated with CLUSTAL 2.0. Nucleotide positions that are identical in all four sequences are indicated by stars. The 5.8 rRNA gene is illustrated in blue letters.

\subsubsection{Chemotactic response of different $P$. pallidum isolates and group 2 Dictyostelid species to glorin}

Next chemotactic response of different $P$. pallidum isolates, such as WS320, CK8 and $\mathrm{H} 168$ to glorin was tested. In these experiments, all $P$. pallidum isolates reacted equitably well to glorin. Eight other group 2 species were assayed for their responsiveness to glorin. The results are summated in Figure 16 and Table 1. It was observed that the group 2B1 polysphondylids $P$. pseudocandidum, $P$. asymmetricum, $P$. tenuissimum and the group 2B2 species $P$. tikaliensis and $P$. luridum were chemotactically active to glorin. Qualitatively, these polysphondylids reacted to glorin as efficiently as $P$. pallidum isolates did. Two Dictyostelium species placed in group 2, D. gloeosporum and D. oculare also responded to glorin equally well (Figure 16), while Acytostelium species were not found positive in this assay (Table 1). None of the tested group 2 species responded to cAMP (Table 1). These data indicate that glorin-based cell-cell communication is a common property of early diverged group 2 species. 

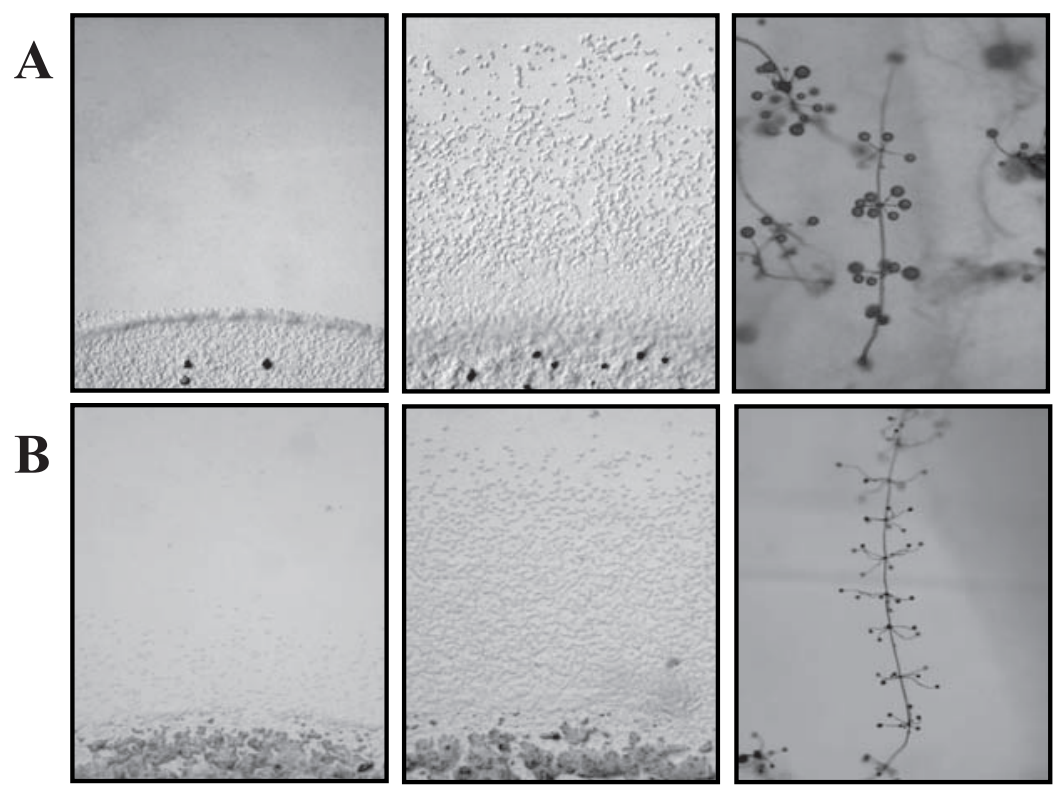

C
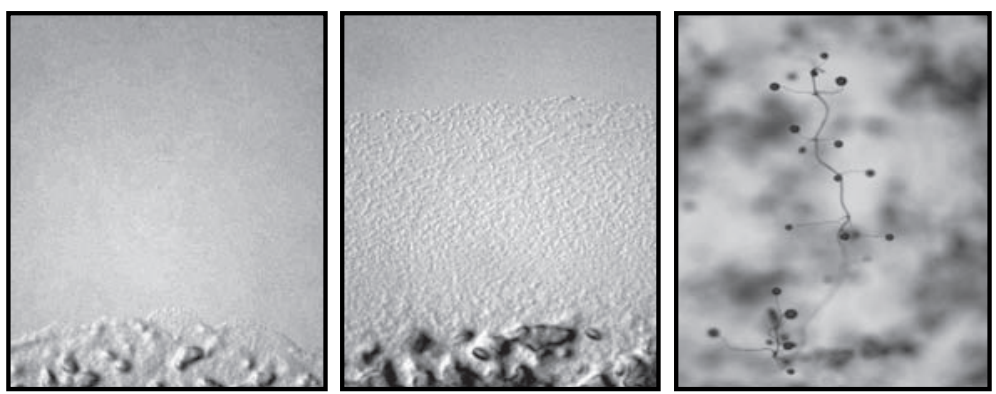

D
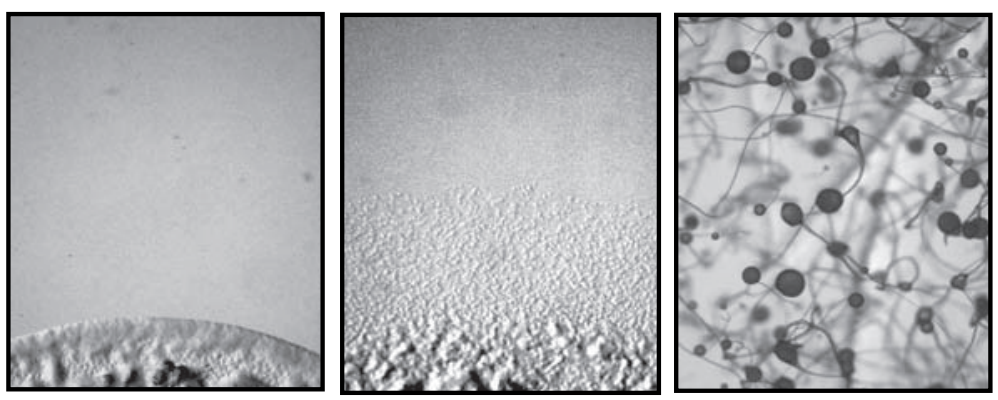

Figure 16: Chemotactic response to glorin by group 2 species. Cells were starved for 3 hours in shaking suspensions before $10 \mu \mathrm{l}$ drops containing $2 \times 10^{5}$ cells were placed on hydrophobic agar without (left pictures) or with $1 \mu \mathrm{M}$ glorin (middle pictures). Shape of fruiting bodies of each species is shown (right pictures). Chemotaxis assay pictures were taken 3 hours after plating. A: P. pallidum PN500; B: P. tenuissimum; C: P. asymmetricum; D: Dictyostelium gloeosporum. P. violaceum was used as positive control (data not shown).

\subsubsection{Chemotactic response of group 1 species to glorin}

Glorin communication was further explored more deeply into the phylogenetic history of social amoebae. It was found that group 1 species $D$. fasciculatum, $D$. parvisporum, $D$. aureo-stipes, $D$. microsporum and $D$. bifurcatum exhibited pronounced chemotaxis to glorin (Figure 17). It was convincing data to believe that ancient group 1 species 
generally employ glorin as extracellular signalling molecule. Group 1 species were not reactive to CAMP in these chemotaxis assays (Table 1).

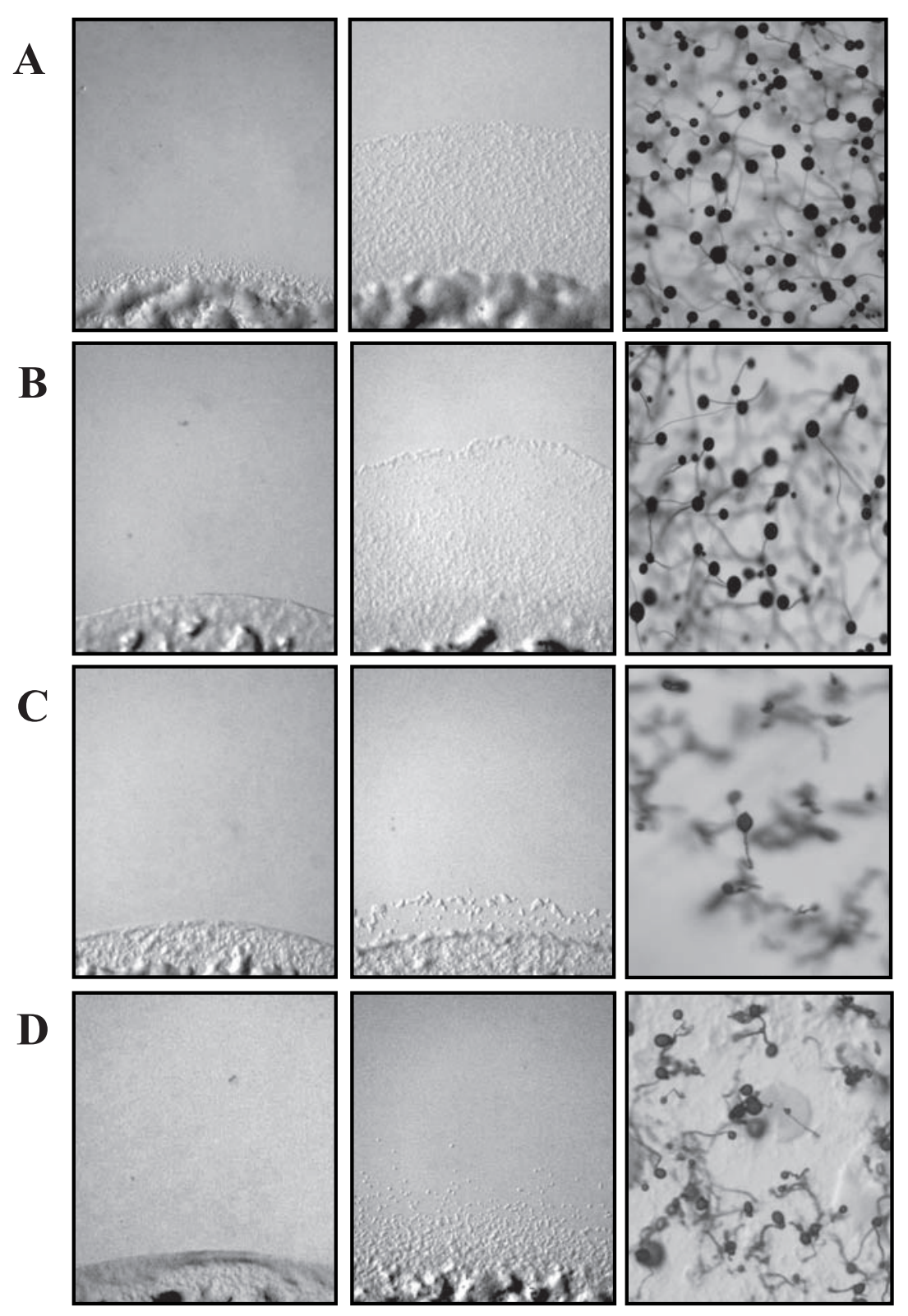

Figure 17: Chemotaxis to glorin by group 1 species. Cells were starved for 3 hours in shaking suspensions before $10 \mu \mathrm{l}$ drops containing $2 \times 10^{5}$ cells were deposited on hydrophobic agar prepared without (left pictures) or with $1 \mu \mathrm{M}$ glorin (middle pictures). Shape of fruiting bodies of each species is shown (right pictures). Chemotaxis assay pictures were taken 3 hours after plating. A: Dictyostelium fasciculatum; B: D. aureo-stipes; C: D. parvisporum; D: D. microsporum. 


\begin{tabular}{|l|c|c|c|}
\hline Species & $\begin{array}{c}\text { Dictyostelids } \\
\text { group }^{\mathbf{a}}\end{array}$ & $\begin{array}{c}\text { Activity to } \\
\text { cAMP }\end{array}$ & $\begin{array}{c}\text { Activity to } \\
\text { glorin }\end{array}$ \\
\hline Dictyostelium discoideum NC4 & 4 & + & - \\
\hline Dictyostelium discoideum AX2 & 4 & + & - \\
\hline Dictyostelium sphaerocephalum & 4 & + & - \\
\hline Dictyostelium giganteum & 4 & + & - \\
\hline Dictyostelium dimigraforum & 4 & + & - \\
\hline Dictyostelium firmibasis & 4 & + & - \\
\hline Dictyostelium intermedium & 4 & + & - \\
\hline Polysphondylium violaceum & $(4)$ & + & + \\
\hline P.pallidum WS320 & 2B1 & - & + \\
\hline P. pallidum PN500 & 2B1 & - & + \\
\hline Polysphondylium tenuissimum & 2B1 & - & + \\
\hline Polysphondylium asymmetricum & 2B1 & - & + \\
\hline Dictyostelium gloeosporum & 2B1 & - & + \\
\hline P. pallidum H168 & 2B2 & - & + \\
\hline P.pallidum CK8 & 2B2 & - & + \\
\hline Polysphondylium luridum & 2B2 & - & + \\
\hline Polysphondylium tikaliensis & 2B2 & - & + \\
\hline Dictyostelium oculare & 2B & - & - \\
\hline Acytostelium ellipticum & 2B & - & - \\
\hline Acytostelium subglobosum & 2A & - & + \\
\hline Dictyostelium fasciculatum & 1 & - & + \\
\hline Dictyostelium aureostipes & 1 & - & + \\
\hline Dictyostelium parvisporum & 1 & - & + \\
\hline Dictyostelium microsporum & 1 & - & + \\
\hline Dictyostelium bifurcatum & 1 & - & + \\
\hline & & & + \\
\hline
\end{tabular}

Table 1: Chemotactic activity of aggregation competent amoebae from four groups of Dictyostelids was tested towards glorin and cAMP. Chemotaxis tests were carried out twice for each species. "+" indicates positive chemotactic response. "-"stands for no response.

${ }^{a}$ Group description based on Schaap et al. (2006) and Romeralo et al. (2010).

In short, these data demonstrate that glorin-based cell-to-cell signalling is the oldest form of intercellular communication used at the transition from growth to multicellular development of social amoebae. 


\subsection{Identification of the proteins secreted by aggregating $P$. pallidum amoebae: Fishing for putative glorinase}

If glorin is the acrasin of $P$. pallidum PN500, then a glorin degrading enzyme must also be secreted in the medium during aggregation of cells to keep extracellular levels of glorin within bounds. In related species $D$. discoideum, during incubation of amoebae under buffered, a rise in the amount of extracellular cyclic AMP phosphodiesterase has been observed, which reaches a peak at the time of aggregation and then declines (Noce et al. 1983; Malkinson \& Ashworth 1973; Riedel et al. 1973; Chassy 1972; Gerisch et al. 1972; Chang 1968). Based on these previous reports, it was interesting to search for a glorin degrading enzyme in the medium conditioned by aggregating $P$. pallidum PN500 amoebae to get further insight into glorin communication system. Studies with $P$. violaceum have demonstrated the occurance of extracellular and membrane-bound enzyme(s) capable of inactivating glorin (De Wit et al. 1988; Wurster et al. 1976). It has been reported that inactivation of glorin signal occurs mainly by rapid cleavage of the $\delta$ lactam bond, followed by a slower cleavage of the peptide bond between propionic acid and glutamic acid (Figure 6; De Wit et al. 1988). The same authors showed that considerable degradation of glorin occurs when this dipeptide compound is incubated with extracellular medium of aggregating $P$. violaceum amoebae (De Wit et al. 1988). In the presented research work, the 'secretome' of aggregating P. pallidum PN500 cells was studied in an attempt to identify a putative hydrolase that would degrade glorin possibly by opening $\delta$-lactam ring.

To collect secreted proteins, vegetatively growing $P$. pallidum PN500 cells were harvested from culture plates using cold $17 \mathrm{mM}$ phosphate buffer $(\mathrm{pH} 6.2)$ and freed of bacteria by centrifugation at $800 \mathrm{rpm}$ in phosphate buffer. The final cell pellet was resuspended in phosphate buffer at a concentration of $10^{7}$ cells $/ \mathrm{ml}$. Cells $\left(10^{7}\right)$ were then pipetted on to Type $3531021 \mathrm{~mm}$ pore-sized polyethylene terephthalate membrane sixwell format cell-culture inserts (Figure 18). Within 10 minutes, when the cells had settled on, and attached to the membrane, the buffer was gently removed from the insert. Cold phosphate buffer containing a protease inhibitor cocktail, AEBSF to a final concentration of $0.25 \mathrm{mM}$ and $5 \mathrm{mM}$ EDTA was added into the wells of a six-well plate followed by the placement of the inserts with cells in the wells (Figure 18). The volume of phosphate buffer in the wells was adjusted to just touch the membrane of the insert to keep the membrane and the cells moist. Amoebae developed normally at $21^{\circ} \mathrm{C}$, with cells aggregation starting at 4 hour and branching streams formation at 8 hours (data not 
shown). When amoebae were actively forming aggregation streams on the surface of inserts, the conditioned starvation buffer in the wells was collected in $2 \mathrm{ml}$ eppendorf tubes and stored at $-80^{\circ} \mathrm{C}$. Frozen conditioned starvation buffer samples were lyophilized and resulting powder in each tube was resuspended in $50 \mu$ l of deionized water. Samples were then immediately boiled with $50 \mu$ of $2 \times$ Laemmli buffer for $5 \mathrm{~min}$ at $95^{\circ} \mathrm{C}$ to denature proteins and subsequently centrifuged for $10 \mathrm{~min}$ at $11000 \mathrm{xg}$ to remove the large particles. Supernatant was collected and used for protein analysis. Proteins were then separated on polyacrylamide gel on the basis of size. Silver-staining of the gel revealed the presence of numerous proteins (Figure 19). Protein bands were excised and proteomic analyses of secreted proteins were performed.

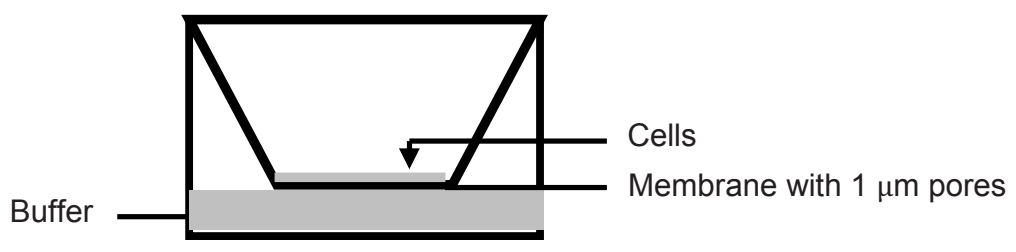

Figure 18: Experimental setup for collection of secreted proteins. $P$. pallidum PN500 cells were placed on a porous membrane in contact with buffer. Amoebae developed normally and aggregation streams could be seen at 8 hours (data not shown). During this period, proteins were secreted into the buffer.

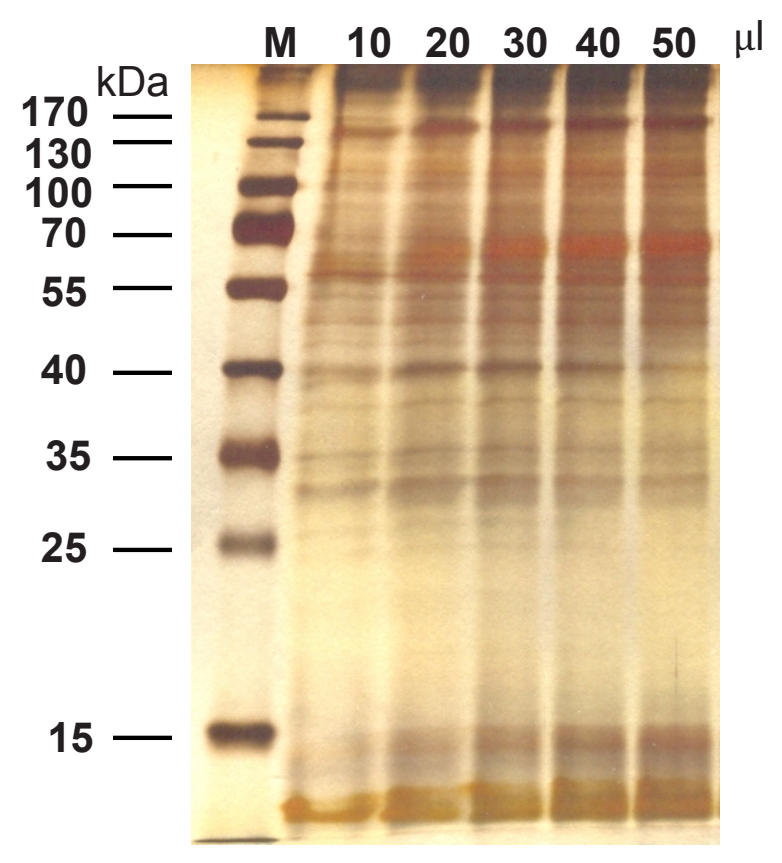

Figure 19: Proteins secreted by aggregating $P$. pallidum PN500 cells. Conditioned buffer was lyophilized and secreted proteins were subjected to separation by electrophoresis on a SDS- 
polyacrylamide gel, which was then silver stained. Different volumes of the same protein solution were loaded in each well of $12.5 \%$ SDS-PAGE gel to analyze protein band patterns. From left to right, lane1, molecular mass marker (Page Ruler), lane 2, 3, 4, 5, 6 contains $10 \mu \mathrm{l}, 20 \mu \mathrm{l}, 30 \mu \mathrm{l}, 40$ $\mu \mathrm{l}$ and $50 \mu \mathrm{l}$ of concentrated protein solution respectively. A number of protein bands on silver stained SDS-PAGE were rather diffused; this might be because of the heterogeneity of the secreted proteins owing to glycosylation.

A total 97 different proteins were identified including a variety of peptidases and hydrolases. Though, great care was taken to avoid any treatments likely to cause cell lysis, yet presence of some intracellular proteins was identified. The detection of internal proteins in secretome studies due to cell lysis is an inherent aspect of secretome proteomics. A complete list of the proteins identified in two independent experiments is shown in (Appendix Table A1). Same protein species were identified in many different bands (data not shown), revealing that many proteins were digested by proteases during the 8 hours of incubation and sample preparation despite the presence of protease inhibitors.

Most prominent proteins identified by ESI tandem mass spectrometry included members of glycoside hydrolase (GH) families $5,18,20,25,27,35$ and 39, peptidase families C26, C1A, C53 and S28, gamma-glutamyl hydrolase, glycosyl hydrolase family chitinase, dipeptidyl-peptidase III, beta-N acetylhexosaminidase, putative alpha-Nacetylgalactosaminidase, beta-galactosidase, alpha-glucosidase, beta-xylosidase-like protein, alpha-mannosidase, metallopeptidase, cathepsin Z-like protein and counting factor associated protein. Peptides crorresponding to cathepsin L-like proteinase, discoidin I and acetylornithine deacetylase were also detected. Many of the identified proteins belonged to the category of carbohydrate metabolism. Twenty proteins identified from $P$. pallidum PN500 database were 'hypothetical proteins' with unknown functions. For such proteins, homology searches were performed using the BlastP program against all non-redundant protein sequences available at the National Centre for Biotechnology Information database (http://www.ncbi.nlm.nih.gov/blast). However, no putative function could be assigned to these hypothetical proteins. These hypothetical proteins, if unique, may represent good targets for future biochemical studies.

In this study, any putative lactamase could not be detected in the conditioned buffer. It is possible that the lactam hydrolase protein was present, but not in sufficient quantity for detection via applied proteomics, or there is a possibility that it could not be detected as a result of extensive post-translational modifications. After two attempts, more efforts to search for a putative 'lactam opening hydrolase' as glorinase candidate or biochemical 
characterization of the identified peptidases were not possible because of time limitation. More research therefore needs to be done to identify/define glorinase.

\subsection{Genome-wide analysis of glorin modulated gene expression changes}

A distinct feature of development of $P$. pallidum is an aggregative transition from a unicellular to a multicellular phase. The outcome of the chemotaxis assays (described under Section 3.4.1.1; Figure 10) using glorin as chemoattractant clearly indicated that $P$. pallidum PN500 cells possess all necessary biochemical machinery required to use glorin as an acrasin. These findings prompted us to address the question whether glorin regulates gene expression changes at the transition from growth to aggregation; similar to the role that chemoattractant cAMP plays in $D$. discoideum during chemotactic aggregation (Winckler et al. 2004; Iranfar et al. 2003; Mann et al. 1997; Schulkes \& Schaap 1995; Pitt et al. 1993; Mann \& Firtel 1987). Previously Will Kopachik (Kopachik 1990 ) reported effects of glorin on protein synthesis in starving $P$. violaceum amoebae. In his experiments, beginning 1 hour after starvation, $P$. violaceum cells were exposed to glorin ( $1 \mu \mathrm{M}$ final concentration, at 30 minute intervals) for 2 to 7 hours. It was demonstrated that within 2 hours of the beginning of starvation, the amoebae displayed two fold increase in sensitivity to glorin. It was found that starving $P$. violaceum cells exhibit 'some changes' in protein synthesis when exposed to glorin for only 2 hours, whereas 'prominent changes' were observed when cells were treated with glorin for 7 hours (Kopachik 1990). These previous studies further incited us to investigate the putative role of glorin in modulating changes in gene expression patterns of developing $P$. pallidum PN500 cells. To approach this question, we were able to take advantage of recently completed genome sequence of $P$. pallidum PN500 (Heidel et al. 2011), while employing Illumina high-throughput RNA sequencing (RNA-seq) technology for analyzing global changes in gene expression.

Glorin-regulated gene expression changes have not been investigated before; therefore, no valuable information was available about optimal conditions to monitor transcriptional changes in response to externally added glorin. Results of the chemotaxis experiments employing $P$. pallidum PN500 as test species (described under Section 3.4.1.3; Figure 12) indicated that amoebae developed in shaking suspension for 1 hour were sufficiently responsive to glorin and gained maximum sensitivity to chemoattractant glorin within next 2-3 hours of development (Figure 12). Also it was noticed that $P$. pallidum amoebae start to aggregate within 3-4 hours of incubation under buffer in petri dishes (Figure 20). 

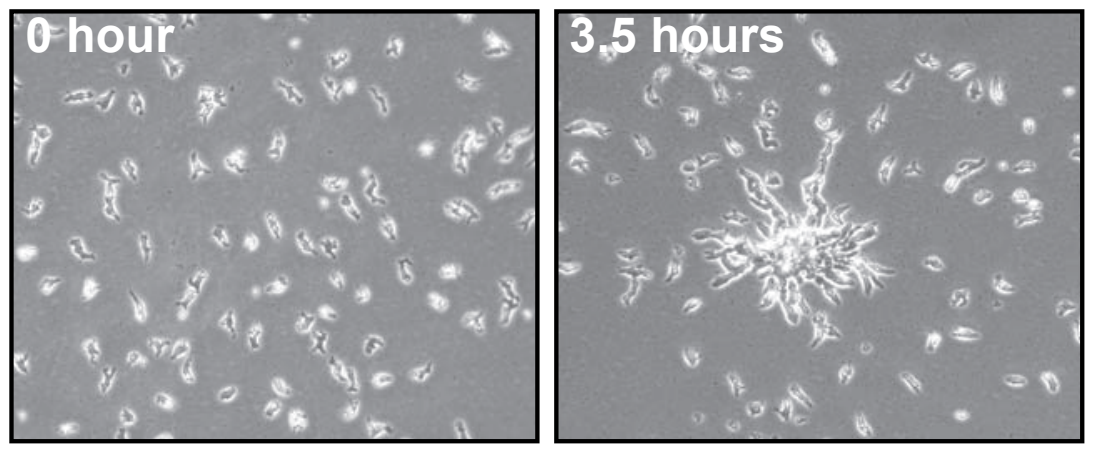

Figure 20: Aggregation of $P$. pallidum PN500 amoebae under buffer. $3 \times 10^{7}$ cells were starved submerged under a thin layer of phosphate buffer in a $50-\mathrm{mm}$ petri dish and monitored for aggregation by differential interference contrast microscopy. Images were taken at 0 hour (left picture) and when cells started to aggregate (right picture).

Previously, it was demonstrated that in starving $P$. violaceum cells glorin acts by binding to cell-surface G-protein coupled receptors (De Wit et al. 1988). The same authors reported that $P$. violaceum amoebae developed in shaken suspensions exposed greater number of glorin receptors compared to the cells developed on agar surface (De Wit et al. 1988). It was proposed that cells starved in suspension cultures gain maximum sensitivity to glorin because amoebae are not able to aggregate under these conditions and development is arrested in the preaggregation phase (De Wit et al. 1988).

Predominant role of G-protein coupled receptors (GPCRs) in Dictyostelids is chemoattractant sensing (Heidel et al. 2011). GPCRs activate intracellular responses by interacting with heterotrimeric G-proteins. In order to study glorin-induced changes in gene expression patterns of developing $P$. pallidum PN500 amoebae, it was important to identify some genes whose expression was differentially regulated by glorin. However, not many examples of developmentally regulated genes were available for $P$. pallidum PN500. When experiments presented in Section 3.6 of this study were initiated, genome sequence of $P$. pallidum PN500 was not yet published but a list of genes was available to us that may encode G-protein coupled receptor proteins in this species (courtesy of Dr. Gernot Glöckner, Fritz-Lippman Institute Jena, Germany). 25 GPCR genes were randomly selected from the available list (data not shown) and subjected to preliminary analysis of glorin-regulated gene expression.

In this study, initially the protocol devised by Kopachik (Kopachik 1990) was followed to stimulate $P$. pallidum amoebae with glorin. $P$. pallidum PN500 cells grown in association with Klebsiella planticola were harvested at vegetatively growing stage, washed free of 
bacteria and resuspended in phosphate buffer at cell density $2 \times 10^{7} \mathrm{cells} / \mathrm{ml}$. A pellet of $2 \times 10^{7}$ cells was immediately stored at $-80^{\circ} \mathrm{C}$ and served as 'growing cells control for subsequent gene expression analysis. Cell suspension of $P$. pallidum PN500 amoebae was then divided into two parts. One suspension culture was first prestarved for 1 hour to initiate development, and then treated with periodic additions of $1 \mu \mathrm{M}$ final concentration of glorin at 30-min intervals for up to 8 additional hours to assess the time-course effects of exogenous glorin on the expression of selected putative GPCR genes. In this experiment, 8 hour time scale was chosen to identify the period when the greatest increase in mRNA levels could be detected. Other culture was maintained in the absence of added glorin for the time period. Suspension cultures (at high cell density $2 \times 10^{7}$ cells $/ \mathrm{ml}$ ) were shaken at low speed i.e. $100 \mathrm{rpm}$ because it may permit cell-cell interactions. Starting at second hour of starvation, cell samples were taken from glorin treated and untreated cultures every hour for total 8 hours to extract total RNA. cDNAs were prepared and expression of chosen putative GPCR genes was analyzed using realtime RT-PCR.

It was taken into account that the final concentration of glorin, i.e. $1 \mu \mathrm{M}$ applied at 30minute intervals, used to study glorin-regulated gene expression in suspension cultures of starving $P$. pallidum PN500 cells was higher than the concentration (i.e. $100 \mathrm{nM}$ ) found to show optimal response in chemotaxis assays on agar surface for the same cells (Figure 11). This difference could be justified by the fact that for $P$. violcauem cells developing in shaken suspensions, concentration for half maximal occupancy of the major glorin receptor is $200 \mathrm{nM}$ and only $80 \mathrm{nM}$ for cells aggregating on agar surface (De Wit et al. 1988). Moreover, it was shown that developing $P$. violaceum amoebae exhibit glorin degradation activity and do not amplify glorin signal in response to externally added glorin; activities that would lead to rapid reduction in initially high concentrations of glorin (De Wit et al. 1988). Thus, in this study, final concentration of glorin available to affect gene expression in P. pallidum PN500 cells developing in buffer suspensions might be relatively lower and within the range exploited by amoebae for chemotaxis.

Among all tested GPCR genes (data not shown), PPL_04108 was found to be highly induced by glorin at second hour of starvation when cells received glorin treatment for only one hour, i.e. after total 2 additions of glorin, whereas maximal increase in the expression of another GPCR encoding gene, PPL_00855, was noticed at third hour of starvation after stimulation of cells with glorin for total two hours (Figure 21). After these time points, expression of these genes generally declined to lower levels (Figure 21). 

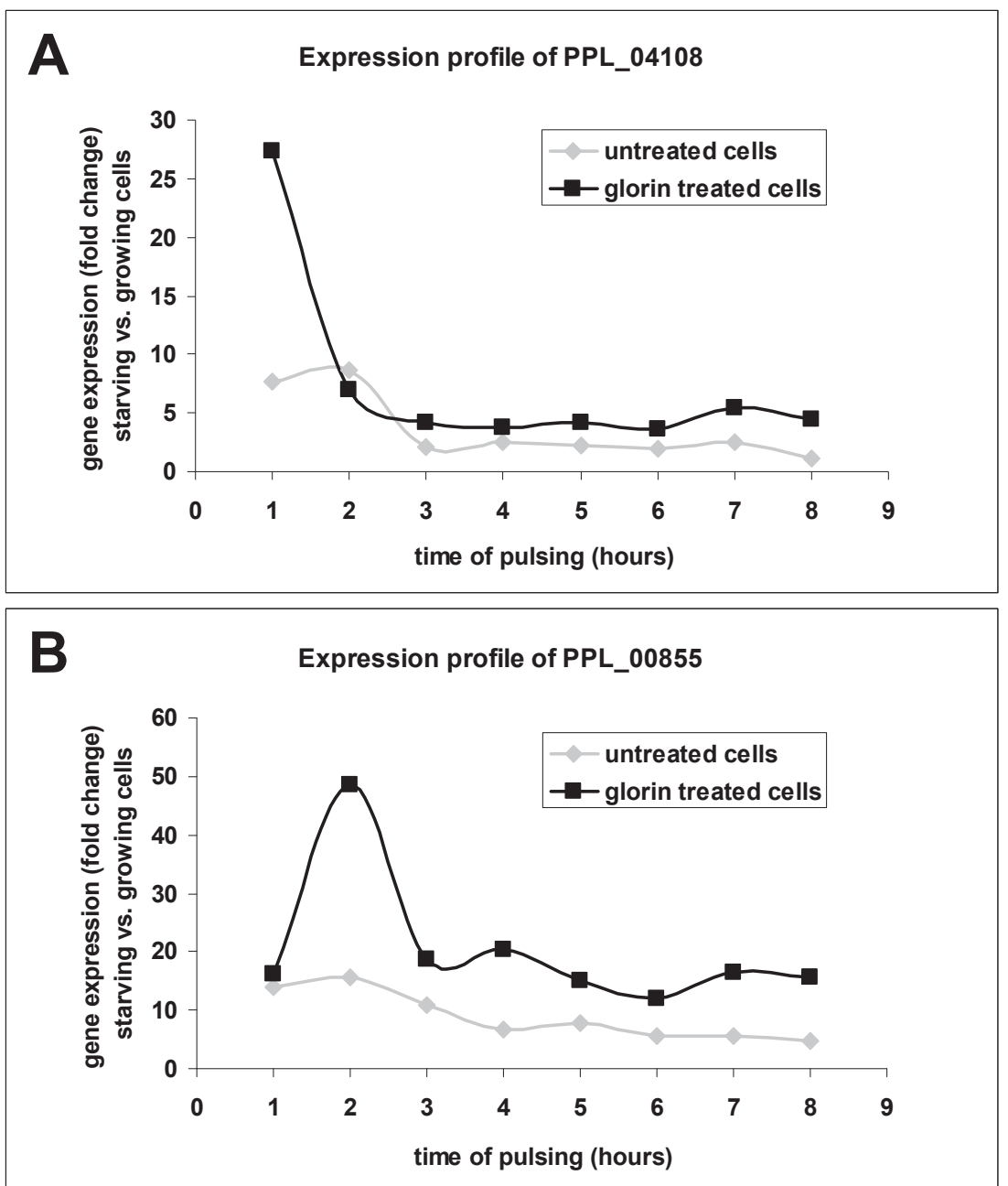

Figure 21: Expression kinetics of two putative GPCR genes during pulse development. $P$. pallidum PN500 cells were developed in shaken suspensions and given pulses of $1 \mu \mathrm{M}$ final concentration of glorin starting at 1 hour of starvation. At the indicated time points, gene expression levels were measured for PPL_04108 and PPL_00855 by quantitative RT-PCR. As a control, expression of house keeping gene gpdA was examined. Fold changes are shown. All data were compared to growing P. pallidum PN500 cells. Fold change was set to 1 where values $>1$ represent higher expression of the gene in starving cells than in growing cells. Mean of two independent experiments is presented.

Based on these preliminary data, it seemed interesting to study changes in the patterns of gene expression at 2 and 3 hours after initiation of development because during this time (known as interphase or pre-aggregation stage) cells may undergo many biochemical changes to prepare themselves for imminent aggregation. Therefore, it was decided to perform RNA-seq on cDNAs drived from cells treated with glorin for one and two hours (i.e. cells starved for total two and three hours) to study effects of glorin stimulation on developmentally regulated genes. 
For RNA-seq studies, samples of total RNA were prepared from growing cells (t0), cells developed for 2 hours without glorin treatment (t2), cells developed for 3 hours without glorin treatment $(\mathbf{t} \mathbf{3})$ and cells pre-starved for 1 hour and then stimulated with $1 \mu \mathrm{M}$ final concentration of glorin for 1 and 2 hours, respectively (t2+ and $\mathbf{t} 3+)$ in two independent biological replicates and analyzed by RNA-seq to obtain gene expression profiles from steady-state mRNAs (data deposited in the Gene Expression Omnibus (GEO) database under accession number GSE24911). In short, mRNA molecules were purified from total RNA using Illumina's mRNA-seq sample prep kit and cDNA libraries were sequenced on a high-throughput Illumina (GAllx) which generates about 35 million reads of 76 bases for each sample. These short reads were then aligned with the P. pallidum PN500 reference transcriptome (composed of the spliced sequences of all the transcripts annotated in the reference database) downloaded from the Social Amoeba Comparative Genome Browser (SACGB; http://sacgb.fli leibniz.de/cgi/index.pl). A total of 12,657 gene models (transcripts) are annotated from the genome of $P$. pallidum PN500. Approximately two third of the obtained reads mapped distinctively onto 12,657 annotated transcripts. Any read that could not be mapped to a unique sequence was not counted, thus eliminating repetitive elements. To estimate gene expression, each transcript was then quantified by calculating its RPKM (reads per kilobase of transcript per million mapped reads; Mortazavi et al. 2008). Using biological replicate I, 20,212,278 to $24,489,837$ mappable reads were obtained, whereas biological replicate II generated between 16,943,246 and 20,182,623 mappable reads. Spearman correlation coefficients were calculated for all data sets. The biological replicates were highly comparable; when comparing each experimental condition, Spearman correlation coefficients were $>0.95$, demonstrating a high quality of data (Table 2). While comparing data from growing and starving cells, Spearman correlation coefficients ranged from 0.88 to 0.98 (Table 2). This difference signifies global changes in gene expression at the transition from growth to development. 


\begin{tabular}{|c|c|c|c|c|c|c|c|c|c|c|}
\hline & $\begin{array}{c}\mathrm{t} 0 \\
\text { exp1 }\end{array}$ & $\begin{array}{c}\mathrm{t} 0 \\
\text { exp2 }\end{array}$ & $\begin{array}{c}\mathrm{t} 2 \\
\text { exp1 }\end{array}$ & $\begin{array}{c}\mathrm{t} 2 \\
\text { exp2 }\end{array}$ & $\begin{array}{c}\mathrm{t} 3 \\
\text { exp1 }\end{array}$ & $\begin{array}{c}\mathrm{t} 3 \\
\text { exp2 }\end{array}$ & $\begin{array}{c}\mathrm{t} 2+ \\
\text { exp1 }\end{array}$ & $\begin{array}{c}\mathrm{t} 2+ \\
\text { exp2 }\end{array}$ & $\begin{array}{c}\mathrm{t} 3+ \\
\text { exp1 }\end{array}$ & $\begin{array}{c}\mathrm{t} 3+ \\
\text { exp2 }\end{array}$ \\
\hline $\mathrm{t} 0 \exp 1$ & & 0.950 & 0.934 & 0.895 & 0.938 & 0.904 & 0.938 & 0.897 & 0.939 & 0.912 \\
\hline $\mathrm{t} 0 \exp 2$ & & & 0.880 & 0.922 & 0.899 & 0.928 & 0.900 & 0.928 & 0.885 & 0.925 \\
\hline $\mathrm{t} 2 \exp 1$ & & & & 0.953 & 0.984 & 0.949 & 0.979 & 0.938 & 0.977 & 0.949 \\
\hline $\mathrm{t} 2 \exp 2$ & & & & 0.958 & 0.988 & 0.954 & 0.983 & 0.935 & 0.974 \\
\hline $\mathrm{t} 3 \exp 1$ & & & & & 0.968 & 0.982 & 0.948 & 0.986 & 0.969 \\
\hline $\mathrm{t} 3 \exp 2$ & & & & & & 0.954 & 0.980 & 0.947 & 0.986 \\
\hline $\mathrm{t} 2+\exp 1$ & & & & & & & & 0.961 & 0.981 & 0.964 \\
\hline $\mathrm{t} 2+\operatorname{exp2}$ & & & & & & & & 0.934 & 0.978 \\
\hline $\mathrm{t} 3+\exp 1$ & & & & & & & & & & 0.965 \\
\hline $\mathrm{t} 3+\exp 2$ & & & & & & & & & \\
\hline
\end{tabular}

Table 2: Spearman correlation coefficients for all RNA-seq data sets. The time to refers to vegetatively growing cells. Cells were staved for 2 or 3 hours without glorin treatment (t2, t3) or pre-starved for 1 hour and treated with glorin for additional 1 or 2 hours (t2+, t3+). exp: experiments (biological replicates).

To assess the differential regulation of genes by starvation and glorin treatment, analyses focused only on those gene transcripts that changed in expression at least 3fold in each of the two biological replicates compared to a control condition to avoid noise in data.

\subsubsection{Starvation triggers dramatic changes in gene expression in $P$. pallidum PN500 amoebae}

To demarcate the effects of starvation from those of the glorin treatment, first, gene expression changes in response to starvation were studied using RNA-seq data from growing cells and from samples collected at 2 and 3 hours of starvation in buffer (with no glorin treatment). These analyses demonstrated that a considerable fraction of the genome of $P$. pallidum PN500 is represented as stage-specific transcripts. Many genes were found to be transcribed in vegetative cells, while others were transcribed at some time during early development. A number of transcripts found in cells at 3 hour of starvation were not found in vegetative cells, and many of the vegetative transcripts were no longer present by 3 hour of development (Appendix Table A3). 
While comparing growing cells with cells developed for 2 hours, a total of 12,628 genes were found to be transcribed (Figure 22). 1,299 gene transcripts were seen to be differentially regulated after 2 hours of starvation; 911 transcripts were up-regulated and 388 transcripts down-regulated. Analysis of data from growing cells and cells starved for 3 hours revealed that 680 transcripts were up-regulated and 376 were down-regulated (Figure 22; Appendix Table A3). When a comparison was made between the lists of gene transcripts differentially regulated at 2 and 3 hours of starvation, it was found that 553 of the up-regulated genes and 283 of the down-regulated genes matched between two lists (Appendix Table A3).

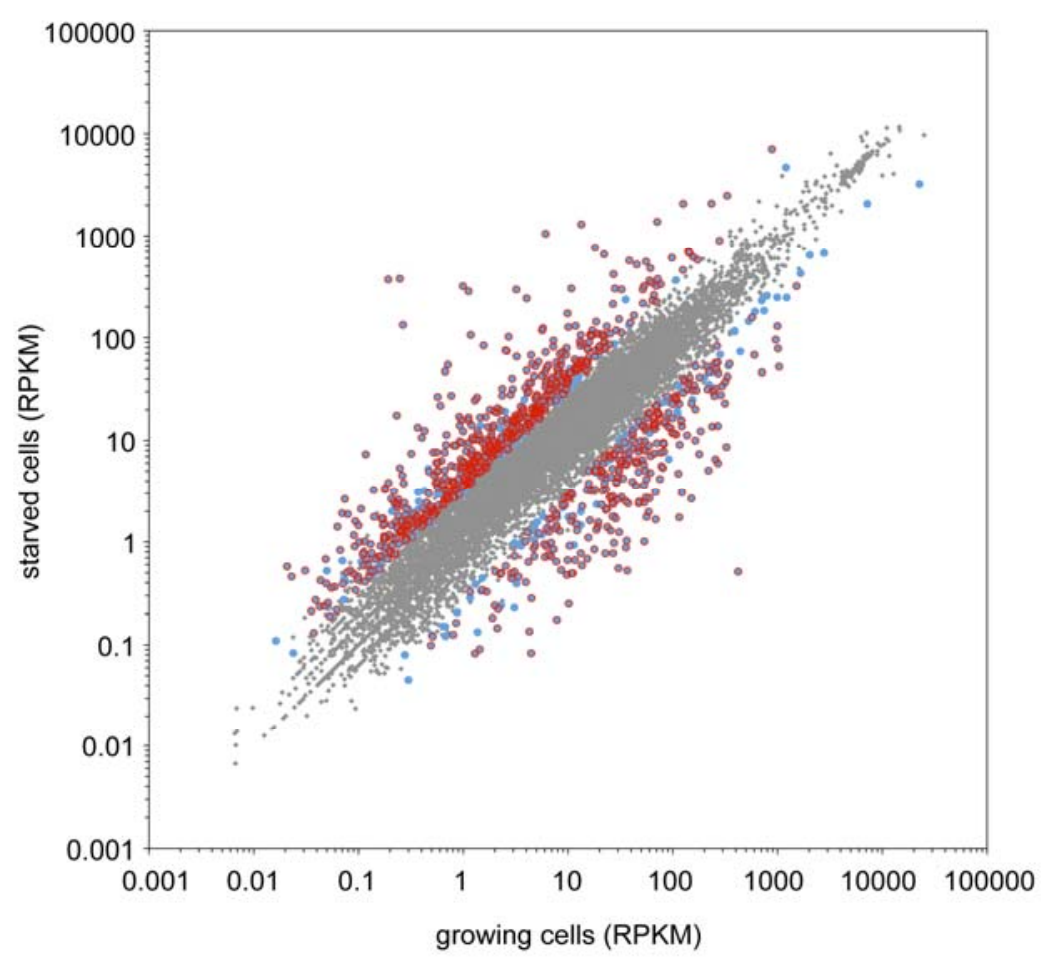

Figure 22: Effect of starvation on global gene expression in starving $P$. pallidum cells. A comparison is shown between steady-state levels of cellular transcripts (expressed as RPKM) in growing and starved cells for all 12,627 gene models (grey symbols). Genes that are regulated at least 3 -fold in cells starved for 2 hours are denoted with blue dots with red circles. 911 genes were up-regulated and 388 down-regulated after 2 hours of starvation. Blue dots indicate differentially expressed genes in cells starved for 3 hours; 680 genes were up-regulated and 376 were downregulated. Scatter blot presents the average result from two independent replications.

Starvation induced changes in expression of individual genes were highly reproducible when comparing RNA-seq data from both biological replicates (Table 2). The expression measurements obtained by RNA-seq analyses were further verified by quantitative RT- 
PCR. As shown by absolute expression data (Appendix Table A3), expression of many genes, of which PPL_02780 and PPL_02774 are clear examples, increased immediately upon starvation and continued to increase to high levels linearly during the first 3 hours of development. PPL_02780 and PPL_02774 were then selected for confirmation of RNAseq data using real-time RT-PCR. Quantitative RT-PCR results were in agreement with those obtained by RNA-seq analysis, thereby demonstrating validity of RNA-seq data (Figure 23).

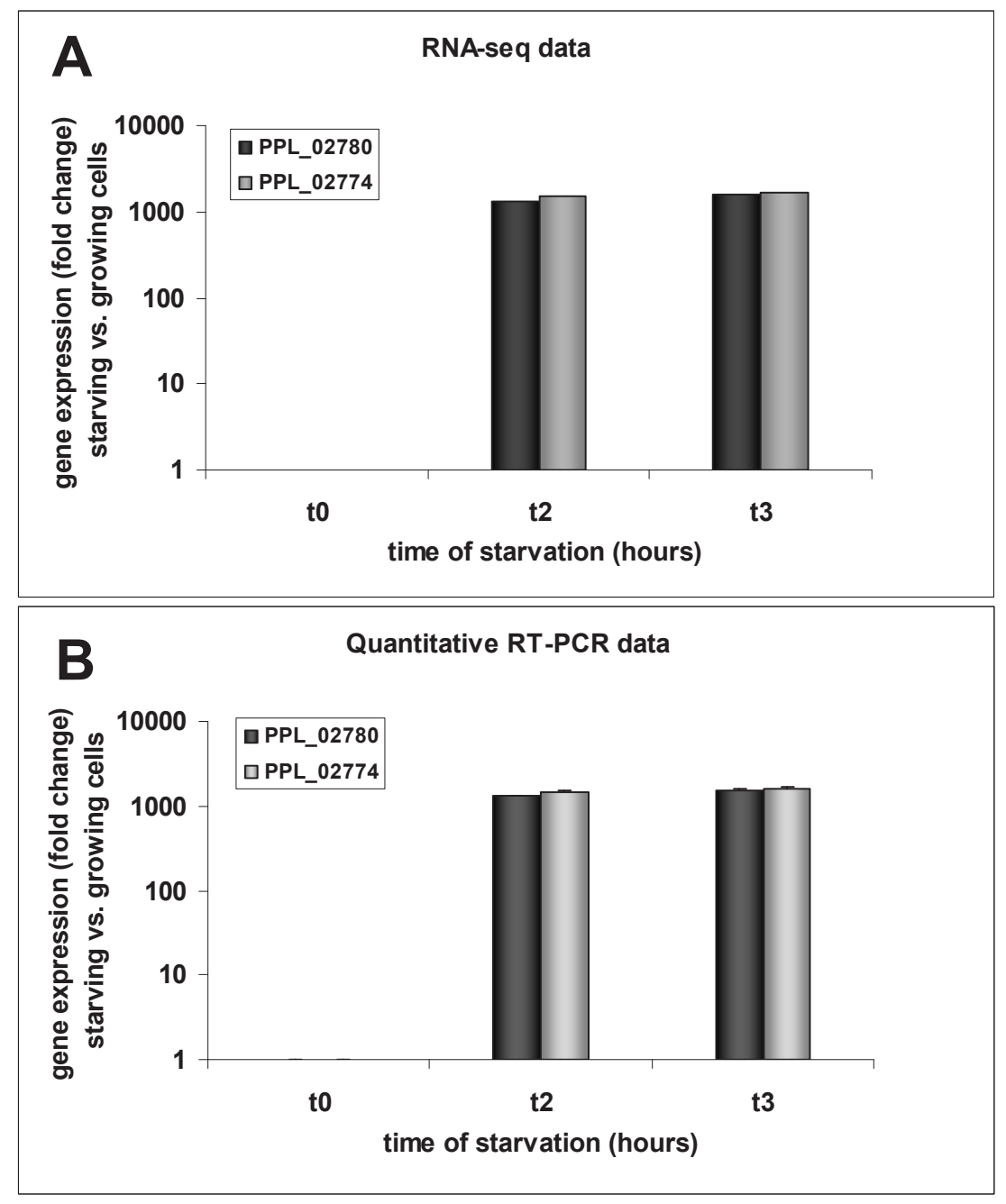

Figure 23: Correlation of RNA-seq data with real-time RT-PCR data. Fold changes are shown. All data were compared to growing P. pallidum PN500 amoebae (t0; fold change set to 1). Values $>1$ represent higher expression of the gene in starving cells than in growing cells. As a control, expression of house keeping gene gpdA was examined. Cells were starved in shaking cultures for 2 or 3 hours in the absence of exogenous glorin treatment (t2, t3). (A) Two model genes, indicated by the corresponding $P$. pallidum (PPL) gene numbers, were selected from RNA-seq data of first biological replicate to describe starvation induced gene expression. It should be noted that the results obtained from RNA-seq of second biological replicate were comparable to the results shown here. (B) Relative expression of representative genes (as shown in panel A) determined by real-time RT-PCR. cDNA used in this experiment were prepared from total RNA 
extracted from first biological replicate. Mean values of triplicate measurements of the same cDNA \pm SD were plotted. A high degree of correlation was observed between two methods demonstrating the effectiveness of RNA-seq approach.

Moreover, it was found that 837 genes diffrentially regulated by starvation (more than 3fold) after 2 hours of starvation were similarly regulated one hour later (Appendix Table A3). This set of genes is identified as 'starvation-responsive genes' and symbolizes the growth arrest of the cells. Genes down-regulated by starvation are possibly those that are expressed during vegetative growth and shut off at the onset of starvation. Whereas genes up-regulated by starvation represent 'developmentally induced genes'. RPKM values and fold changes for each individual gene regulated at 2 and 3 hours of development are available in Appendix Table A3.

Overall, these results indicate that the transition of $P$. pallidum PN500 amoebae from growth-to-starvation is accompanied by predominant changes in the gene expression patterns.

\subsubsection{Glorin-induced developmental regulation of gene expression}

After getting an insight into starvation triggered gene expression changes, it was interesting to investigate whether exogenous glorin can stimulate differential changes in the early developmental gene expression. To explore this question, gene expression data from cells starved for 2 hours without glorin treatment were compared with the data from cells that were pre-starved for 1 hour and then treated with $1 \mu \mathrm{M}$ final concentration of glorin at 30-minute intervals for an additional hour, meaning that only two pulses of glorin were applied. It was observed that stimulation of starving cells with glorin resulted in more than 3-fold differential expression of 115 gene transcripts, 70 of which were upregulated by 3 - to 57 -fold (Figure 24A). When gene expression profiles of cells starved for 3 hours were compared with gene expression data of cells pre-starved for 1 hour and treated with $1 \mu \mathrm{M}$ glorin every 30 minutes for 2 hours, a relatively different outcome was noticed (Figure 24B). In these analyses, 120 genes were differentially regulated more than 3-fold, but only 20 of them were up-regulated. Among these 20 genes, 11 were those that were induced more than 3 -fold after 1 hour of glorin treatment also.

The phenomenon that many genes differentially up-regulated more than 3 fold after 1 hour of glorin treatment exhibited abrupt changes in expression after 2 hours of stimulation with glorin, could be explained by comparing the absolute abundance and 
expression kinetics of these genes under starving conditions (in absence of glorin) as depicted in Appendix Table A4 \& A5.
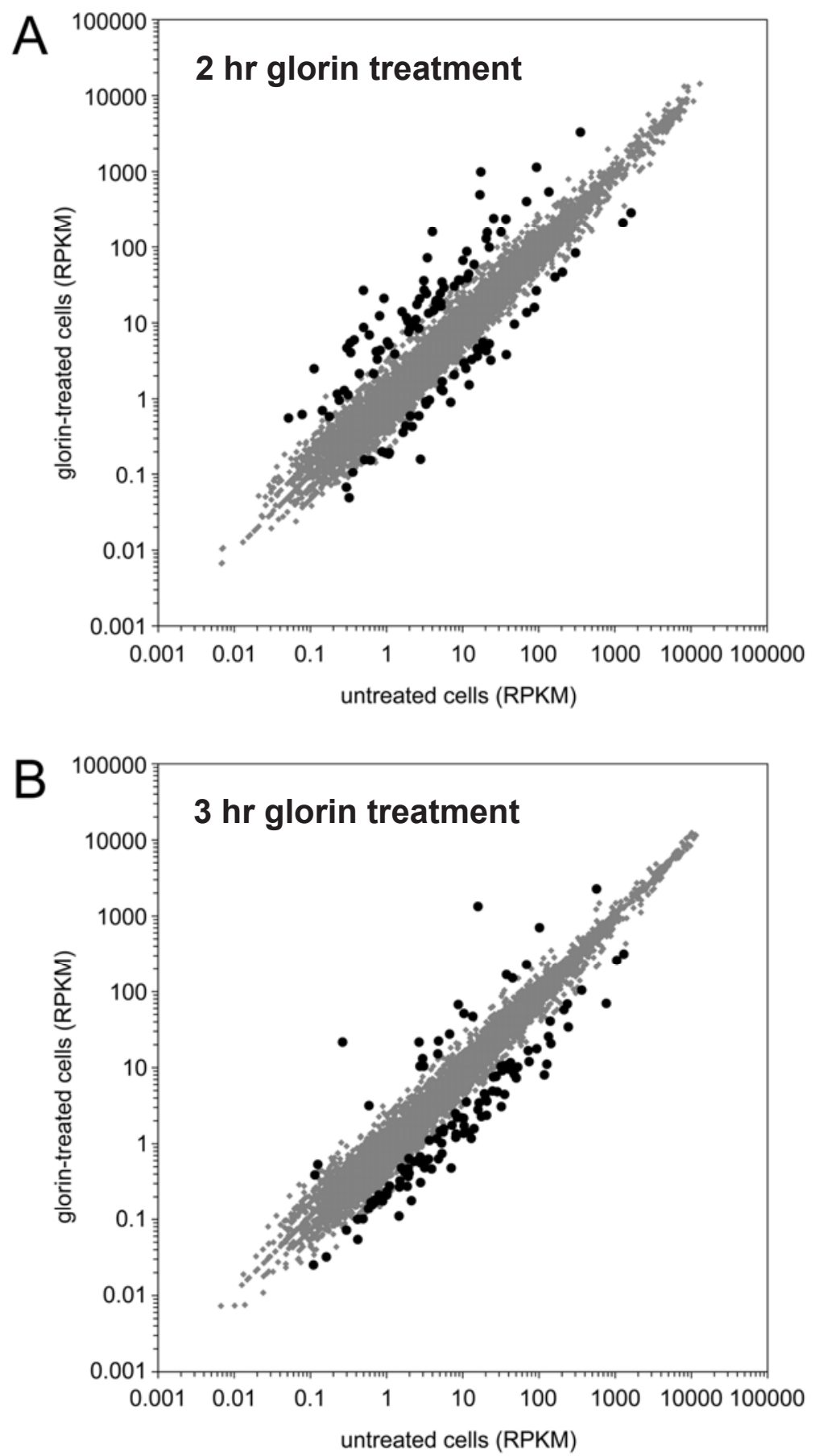

Figure 24: Effects of exogenous glorin stimulation on global gene expression in $P$. pallidum PN500. A comparison is shown between steady-state levels of cellular transcripts (expressed as RPKM) in P. pallidum PN500 cells treated with glorin for 1 hour (A) or 2 hours (B) and cells starved without glorin treatment for the time period. All 12,627 gene models are plotted (represented by grey symbols). Genes that were differentially regulated at least 3-fold after 1 or 2 hours of glorin treatment are indicated by black dots. Treatment of starving cells with glorin for 1 
hour resulted in differential expression of 115 genes, 70 of which were up-regulated. After 2 hours of stimulation with glorin, 120 genes were differentially regulated, but only 20 of them were upregulated more than 3 -fold. Scatter plot presents the average result from two independent replications.

A few genes, of which PPL_05354 and PPL_09347 (Table 3; Figure 25) are obvious representatives, exhibited very low basal expression in growing cells and retained low level expression constantly during the first hours of starvation. In comparison, the majority of other genes (43 out of 70 genes up-regulated more than 3 fold after 1 hour of glorin treatment) showed a moderate increase in expression at the growth-to-starvation transition, possibly because cells gain the ability to set up endogenous glorin signalling within first few hours of starvation, however, their expression was further enhanced by exogenous glorin pulses (examples in Table 3 \& Figure 27). Previously, it was shown that slow shaken high cell density cultures of $D$. discoideum were able to generate normal endogenous CAMP signals without exogenously supplied cAMP pulses (Kimmel \& Carlisle 1986).

While the expression levels of these genes increased with the progression of starvation, the effects of glorin treatment became relatively less significant. As a result, many genes that were differentially induced by glorin more than 3-fold after 1 hour of glorin treatment were up-regulated less than 3-fold one hour later even in the presence of glorin. This demonstrates that externally added glorin caused the induction of genes precociously compared to the natural conditions of development. RPKM values and fold changes for each individual gene regulated by glorin at 2 and 3 hours of development are presented in Table 3 \& Appendix Table A4, A5 and A6.

These data support the hypothesis that in starving P. pallidum PN500 amoebae, extracellular glorin binds to cell surface receptors, a signal is transduced intracellularly that leads to changes in developmental gene expression. 
Table 3: Absolute expression data of selected genes obtained by RNA-seq analysis. The time t0 refers to vegetatively growing cells. Cells were staved for 2 or 3 hours without glorin treatment ( $t 2, t 3)$ or pre-starved for 1 hour and treated with glorin for additional 1 or 2 hours (t2+, t3+).

\begin{tabular}{|c|c|c|c|c|c|c|c|c|c|c|c|}
\hline $\begin{array}{c}\text { Gene } \\
\text { Number }\end{array}$ & $\begin{array}{c}\text { t0 } \\
\text { RPKM }\end{array}$ & $\begin{array}{c}\text { t2 } \\
\text { RPKM }\end{array}$ & $\begin{array}{c}\text { t3 } \\
\text { RPKM }\end{array}$ & $\begin{array}{c}\text { t2+ } \\
\text { RPKM }\end{array}$ & $\begin{array}{r}\text { t3+ } \\
\text { RPKM }\end{array}$ & $\begin{array}{c}\text { t2/ to } \\
\text { fold } \\
\text { change }\end{array}$ & $\begin{array}{c}\text { t3/ to } \\
\text { fold } \\
\text { change }\end{array}$ & $\begin{array}{c}\text { t2+/ to } \\
\text { fold } \\
\text { change }\end{array}$ & $\begin{array}{c}\text { t3+/ to } \\
\text { fold } \\
\text { change }\end{array}$ & $\begin{array}{c}\text { t2+/ t2 } \\
\text { fold } \\
\text { change }\end{array}$ & $\begin{array}{c}\text { t3+/ t3 } \\
\text { fold } \\
\text { change }\end{array}$ \\
\hline PPL_05833 & 0.42 & 3.50 & 6.56 & 56.75 & 10.97 & 8.21 & 15.41 & 133.2 & 25.75 & 16.21 & 1.67 \\
\hline PPL_03784 & 0.16 & 0.74 & 0.79 & 3.35 & 1.30 & 4.51 & 4.84 & 20.40 & 7.96 & 4.51 & 1.64 \\
\hline PPL_00912 & 0.60 & 0.76 & 1.16 & 9.54 & 2.66 & 1.27 & 1.93 & 15.87 & 4.42 & 12.40 & 2.28 \\
\hline PPL_05195 & 3.71 & 42.36 & 84.83 & 9.46 & 5.42 & 11.39 & 22.81 & 2.54 & 1.45 & 0.22 & 0.06 \\
\hline PPL_05702 & 13.94 & 17.65 & 24.43 & 1.72 & 10.97 & 1.26 & 1.75 & 0.12 & 0.78 & 0.09 & 0.44 \\
\hline PPL_04459 & 18.55 & 8.22 & 4.62 & 12.50 & 22.54 & 0.44 & 0.24 & 0.67 & 1.21 & 1.52 & 4.87 \\
\hline PPL_03541 & 0.08 & 0.065 & 0.069 & 0.81 & 0.069 & 0.75 & 0.80 & 9.44 & 0.80 & 12.55 & 0.99 \\
\hline PPL_11763 & 0.62 & 0.86 & 0.92 & 11.40 & 2.71 & 1.38 & 1.49 & 18.31 & 4.36 & 13.18 & 2.92 \\
\hline PPL_02774 & 0.21 & 320.8 & 361.1 & 188.3 & 478.5 & 1488. & 1675. & 874.0 & 2220 & 0.58 & 1.32 \\
\hline PPL_07847 & 202.2 & 0.49 & 0.22 & 0.29 & 0.22 & 0.002 & 0.001 & 0.001 & 0.001 & 0.585 & 0.99 \\
\hline PPL_12271 & 31.53 & 10.77 & 25.60 & 105.4 & 26.19 & 0.34 & 0.81 & 3.34 & 0.83 & 9.78 & 1.02 \\
\hline PPL_12249 & 22.37 & 10.59 & 4.54 & 273.1 & 48.53 & 0.47 & 0.20 & 12.20 & 2.16 & 25.77 & 10.66 \\
\hline PPL_12248 & 4.23 & 1.93 & 1.48 & 68.90 & 10.42 & 0.45 & 0.35 & 16.26 & 2.46 & 35.62 & 7 \\
\hline PPL_09347 & 0.10 & 0.24 & 0.26 & 15.18 & 13.27 & 2.25 & 2.42 & 139.4 & 121.9 & 61.76 & 50.31 \\
\hline PPL_07908 & 0.12 & 105.0 & 97.31 & 22.54 & 19.10 & 854.4 & 791.1 & 183.2 & 155.3 & 0.21 & 0.19 \\
\hline PPL_05354 & 21.33 & 22.40 & 18.61 & 1141 & 1682 & 1.05 & 0.87 & 53.75 & 79.23 & 50.95 & 90.37 \\
\hline PPL_03564 & 6.05 & 3.12 & 4.13 & 27.04 & 7.01 & 0.51 & 0.68 & 4.46 & 1.15 & 8.65 & 1.69 \\
\hline PPL_08455 & 8.58 & 0.33 & 0.53 & 4.05 & 1.07 & 0.03 & 0.06 & 0.47 & 0.12 & 11.98 & 2.02 \\
\hline PPL_08454 & 0.30 & 0.37 & 0.45 & 5.96 & 1.20 & 1.23 & 1.5 & 19.86 & 4 & 15.86 & 2.66 \\
\hline PPL 00855 & 0.20 & 1.72 & 2.66 & 2.99 & 5.33 & 8.6 & 13.3 & 14.95 & 26.65 & 1.73 & 2.00 \\
\hline PPL_04108 & 1.83 & 6.79 & 7.58 & 22.55 & 7.04 & 3.71 & 4.14 & 12.32 & 3.84 & 3.32 & 0.92 \\
\hline PPL_05727 & 59.16 & 14.16 & 18.86 & 58.89 & 19.59 & 0.23 & 0.31 & 0.99 & 0.33 & 4.15 & 1.03 \\
\hline PPL_00902 & 66.97 & 70.05 & 96.22 & 192.5 & 148.7 & 1.04 & 1.43 & 2.87 & 2.22 & 2.74 & 1.54 \\
\hline PPL_06644 & 0.77 & 0.50 & 0.58 & 8.70 & 3.17 & 0.64 & 0.75 & 11.29 & 4.11 & 17.40 & 5.40 \\
\hline
\end{tabular}




\subsubsection{Classification of glorin-induced developmental gene expression}

For the convenience of data interpretation, genes differentially regulated by glorin were sorted into 5 classes on the basis of their expression kinetics. Instead of presenting the entire list of glorin-regulated genes in this section, some model genes were choosen to explain the glorin mediated modulation of gene expression.

\subsection{Class I: Genes stably induced by glorin}

RNA-seq data analyses indicated that glorin causes precocious and high-level induction of many genes (Table 3 \& Appendix Table A4, A5, and A6). Prominent examples of such genes are PPL_09347 and PPL_05354. As described in the absolute RNA-seq data of glorin-regulated genes (Table 3), in vegetative-stage cells and also at the beginning of development (2 and 3 hours of starvation), transcripts of PPL_09347 were barely detectable, whereas PPL_05354 was expressed at very low levels under these conditions. Marked induction in gene expression was observed in response to exogenous glorin as shown in Figure 25. 

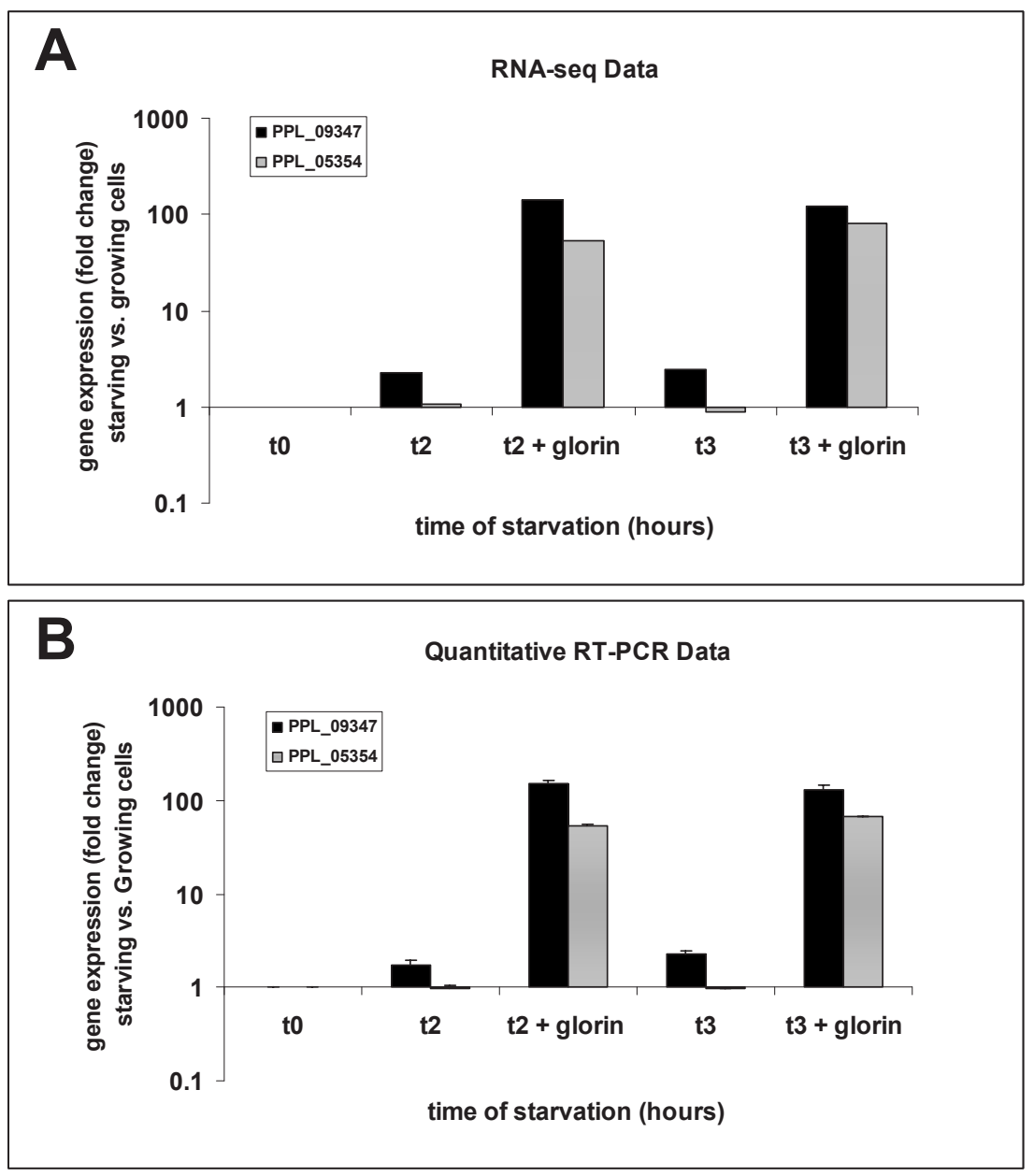

Figure 25: Correlation of RNA-seq data with real-time RT-PCR data. Fold changes are shown. All data were compared to growing P. pallidum PN500 amoebae (t0; fold change set to 1). Values $>1$ represent higher expression of the gene in starving cells than in growing cells. Values $<1$ show that expression of gene is lower in starving cells than in growing cells. As a control, expression of house keeping gene gpdA was examined. Cells were starved in shaking cultures for 2 or 3 hours in the absence of exogenous glorin treatment $(\mathbf{t} 2, \mathbf{t} 3)$ or for 1 hour followed by 1 hour or 2 hours of glorin treatment (t2+glorin, t3+glorin). (A) Two model genes, indicated by the corresponding $P$. pallidum (PPL) gene numbers, were selected from RNA-seq data of first biological replicate to describe glorin-induced gene expression. It should be noted that the results obtained from RNA-seq of the second biological replicate were comparable to the results shown here. (B) Relative expression of representative genes (as shown in panel A) determined by realtime RT-PCR. cDNA used in this experiment were prepared from total RNA extracted from first biological replicate. Mean values of triplicate measurements of the same CDNA \pm SD were plotted. A high degree of correlation was observed between two methods demonstrating the effectiveness of RNA-seq approach.

Transcripts of these genes accumulated rapidly in cells developed in buffer and treated with $1 \mu \mathrm{M}$ of glorin at 30 minute intervals for for 1 or 2 hours. Induction of expression of these genes by glorin was maintained at high levels over the course of observation (Figure 25). In cells treated with glorin for 1 and 2 hours, level of expression of PPL_09347 was found to be 53- and 82-fold higher, respectively, than in vegetative cells. 
PPL_05354 was induced 56- and 84-fold after 1 and 2 hours of glorin stimulation, respectively. This class of genes may exemplify 'aggregation stage specific genes'. The expression measurements obtained by RNA-seq data analyses were validated by quantitative RT-PCR (Figure 25).

\subsection{Class II: Genes transiently induced by glorin}

When P. pallidum PN500 cells were developed in buffer suspensions and stimulated with glorin, many genes showed a transient increase in expression relative to the expression in growing cells. PPL_11763, PPL_12271 and PPL_03541 were rapidly induced by glorin followed by a rapid turn over leading to a sharp decline in expression level at the next hour of glorin treatment (Table 3; Figure 26). Transcripts of PPL_11763 and PPL_03541 were not expressed at significant levels in vegetative cells (Table 3 ) and cells developed for 1 or 2 hours in the absence of glorin treatment. After 1 hour of glorin treatment expression of these genes increased to 22.66- and 22.2-fold, respectively, compared to vegetative cells. In comparison, PPL_12271 was moderately expressed in growing cells (Table 3), also at the beginning of development, i.e. 1 hour of starvation but further development without glorin pulses led to a small decrease in the transcript level (Table 3; Figure 26).

Pulsing with glorin for 1 hour significantly upregulated this gene to 7.68 fold compared to growing cells. A rapid decline in expression of all these genes was observed after 2 hours of glorin treatment (Figure 26). These glorin-induced genes may represent a class of 'developmentally induced aggregation specific genes' with the exception of PPL_12271 that may also play some role in growing cells because adequate level of transcripts of this gene were detected in vegetative cells (Table 3). Real-time RT-PCR analysis confirmed the expression levels obtained in RNA-seq experiments (Figure 26). 

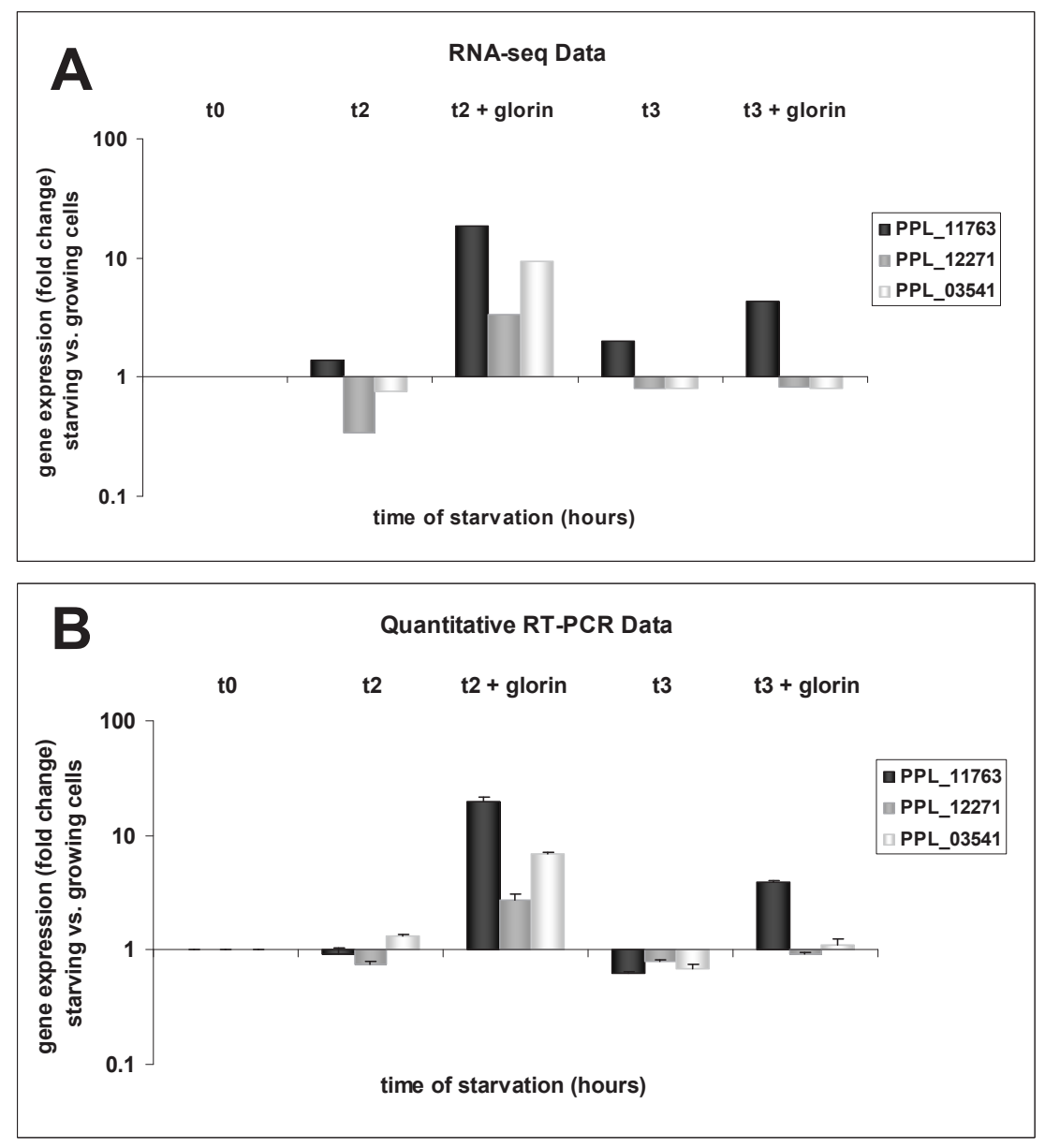

Figure 26: Correlation of RNA-seq data with real-time RT-PCR data. Fold changes are shown. All data were compared to growing P. pallidum PN500 amoebae (t0; fold change set to 1). Values $>1$ represent higher expression of the gene in starving cells than in growing cells. Values $<1$ show that expression of the gene is lower in starving cells than in growing cells. As a control, expression of house keeping gene gpdA was examined. Cells were starved in shaking cultures for 2 or 3 hours in the absence of exogenous glorin treatment $(\mathbf{t} 2, \mathbf{t} 3)$ or for 1 hour followed by 1 hour or 2 hours of glorin treatment (t2+glorin, t3+glorin). (A) Two model genes, indicated by the corresponding $P$. pallidum (PPL) gene numbers, were selected from RNA-seq data of first biological replicate to describe glorin-induced gene expression. It should be noted that the results obtained from RNA-seq of second biological replicate were comparable to the results shown here. (B) Relative expression of representative genes (as shown in panel A) determined by real-time RT-PCR. cDNA used in this experiment were prepared from total RNA extracted from first biological replicate. Mean values of triplicate measurements of the same CDNA \pm SD were plotted. A high degree of correlation was observed between two methods demonstrating the effectiveness of RNA-seq approach. 


\subsection{Class III: Genes induced by starvation, whereas glorin treatment further enhanced their expression}

For some other genes, such as PPL_05833, PPL_03784 and PPL_00912, only low levels of transcripts were detected at $0 \mathrm{hr}$ (Table 3 ). Starvation induced a moderate increase in the expression of PPL_05833 and PPL_03784 at 2 and 3 hours of development in buffer, whereas PPL_00912 was expressed at insignificant levels at 1 hour of starvation and only slightly upregulated at third hour of development in the absence of glorin pulses (Figure 27). When cells were exposed to glorin for 1 hour, transcripts of these genes accumulated significantly. PPL_05833, PPL_03784 and PPL_00912 were upregulated by 133-, 20- and 15-fold, respectively, compared to growing cells (Figure 27). Expression of PPL_03784 exhibited a low level of responsiveness to glorin. Comparatively reduced levels of expression were observed after 2 hours of glorin treatment indicating rapid turn over of these genes similar to class II genes. This class may symbolize 'early developmental genes required shortly during aggregation'. Products of these genes may be needed during the first few hours of development. 

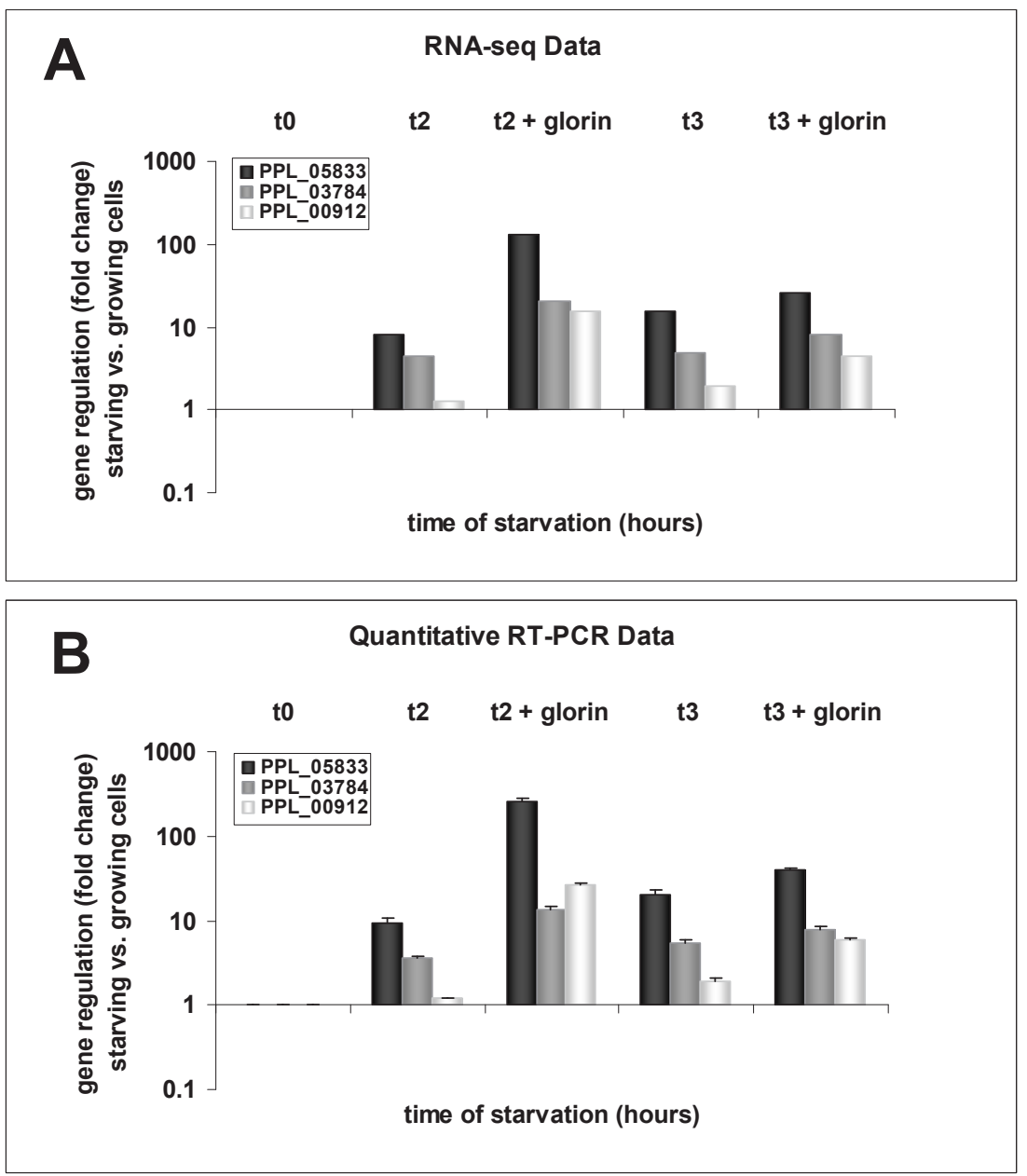

Figure 27: Correlation of RNA-seq data with real-time RT-PCR data. Fold changes are shown. All data were compared to growing P. pallidum PN500 amoebae (t0; fold change set to 1). Values $>1$ represent higher expression of the gene in starving cells than in growing cells. Values $<1$ show that expression of the gene is lower in starving cells than in growing cells. As a control, expression of house keeping gene gpdA was examined. Cells were starved in shaking cultures for 2 or 3 hours in the absence of exogenous glorin treatment $(\mathbf{t} 2, \mathbf{t} 3)$ or for 1 hour followed by 1 hour or 2 hours of glorin treatment (t2+glorin, $\mathbf{t} 3+$ glorin). (A) Two model genes, indicated by the corresponding P. pallidum (PPL) gene numbers, were selected from RNA-seq data of first biological replicate to describe glorin-induced gene expression. It should be noted that the results obtained from RNA-seq of second biological replicate were comparable to the results shown here. (B) Relative expression of representative genes (as shown in panel A) determined by real-time RT-PCR. cDNA used in this experiment were prepared from total RNA extracted from first biological replicate. Mean values of triplicate measurements of the same $C D N A \pm S D$ were plotted. A high degree of correlation was observed between two methods demonstrating the effectiveness of RNA-seq approach.

Induction of these genes by starvation indicates that some pre-starvation factor(s) may be involved in stimulating the expression of these genes. Glorin pulses further enhanced expression of this class of genes demonstrating that expression of these genes is controlled by a two-step regulatory pathway. 


\subsection{Class IV: Genes induced by starvation, whereas glorin treatment repressed their expression}

Messenger RNA of many genes, represented by PPL_07908, PPL_05702 and PPL_05195 started to accumulate immediately at the onset of development; indicating that these are 'starvation-induced' genes. In vegetative cells, negligible amount of transcripts of PPL_07908 was present (Table 3). Starvation led to 854- and 791-fold increase in expression of this gene compared to vegetative cells, at 2 and 3 hours of development (Figure 28). Administration of exogenous glorin pulses elicited a rapid decline of expression, such that only 22.54- and 19.10-fold expression (compared to growing cells) was detectable after glorin treatment for 1 and 2 hours, respectively. In comparison, some transcripts of PPL_05702 were detected in vegetative cells, whereas starvation slightly increased expression of this gene (Table 3 ).

PPL_05195 exhibited basal level expression in growing cells (Table 3). A moderate increase in transcripts of this gene was observed at 2 hour of development, followed by a high level expression one hour later. As shown in the Figure 28, expression of PPL_05195 increased by 11.39 - and 22.81 -fold at 2 and 3 hour of starvation. Stimulating cells with glorin dramatically decreased expression level of this gene to 2.54- and 1.45-fold after 1 and 2 hours of treatment. RNA-seq data for expression of these genes was confirmed by real-time RT-PCR as illustrated in Figure 28. 

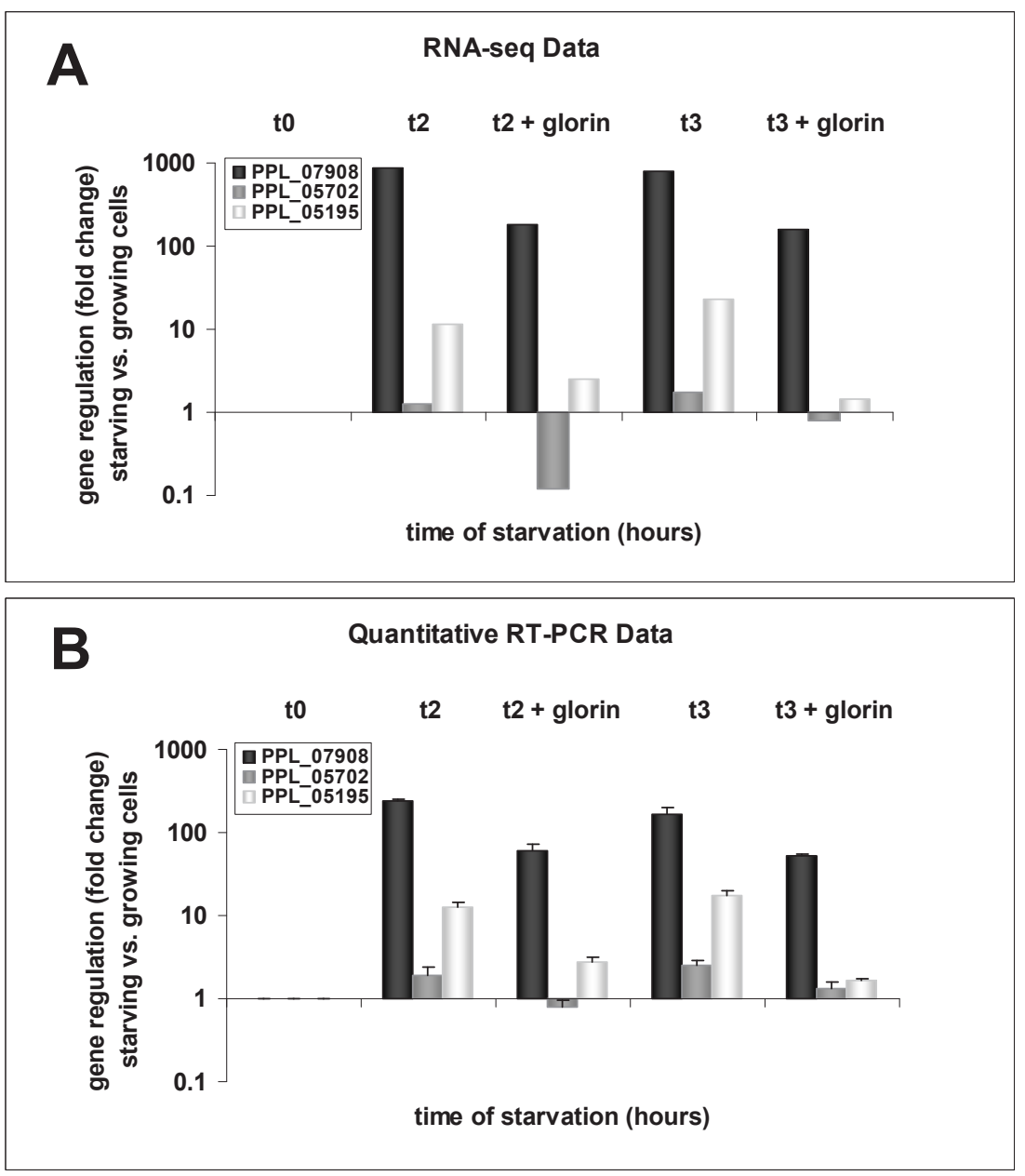

Figure 28: Correlation of RNA-seq data with real-time RT-PCR data. Fold changes are shown. All data were compared to growing P. pallidum PN500 amoebae (t0; fold change set to 1). Values $>1$ represent higher expression of the gene in starving cells than in growing cells. Values $<1$ show that expression of the gene is lower in starving cells than in growing cells. As a control, expression of house keeping gene gpdA was examined. Cells were starved in shaking cultures for 2 or 3 hours in the absence of exogenous glorin treatment $(\mathbf{t} 2, \mathbf{t} 3)$ or for 1 hour followed by 1 hour or 2 hours of glorin treatment (t2+glorin, $\mathbf{t} 3+$ glorin). (A) Two model genes, indicated by the corresponding $P$. pallidum (PPL) gene numbers, were selected from RNA-seq data of first biological replicate to describe glorin repressed gene expression. It should be noted that the results obtained from RNA-seq of second biological replicate were comparable to the results shown here. (B) Relative expression of representative genes (as shown in panel A) determined by real-time RT-PCR. cDNA used in this experiment were prepared from total RNA extracted from first biological replicate. Mean values of triplicate measurements of the same cDNA \pm SD were plotted. A high degree of correlation was observed between two methods demonstrating the effectiveness of RNA-seq approach.

These genes represent 'differentially expressed early genes' and repression of their expression by glorin indicates that products of these genes may function only in the early hours of starvation and may not be required for aggregation. These genes are 
considered to be negatively modulated by glorin pulses that may occur during the early development of $P$. pallidum to coordinate aggregation.

\subsection{Class V: Genes repressed by starvation, whereas glorin treatment induces their expression}

Expression of some other genes is negatively regulated during the early hours of development. Examples of such genes include PPL_12248 and PPL_12249. While considering absolute expression levels of these genes in growing cells of $P$. pallidum PN500, it was noticed that transcripts of PPL_12248 were present at low levels, whereas sufficient mRNA was detected for PPL_12249 (Table 3). With progression in development, transcripts of both genes declined in suspension developed cells.

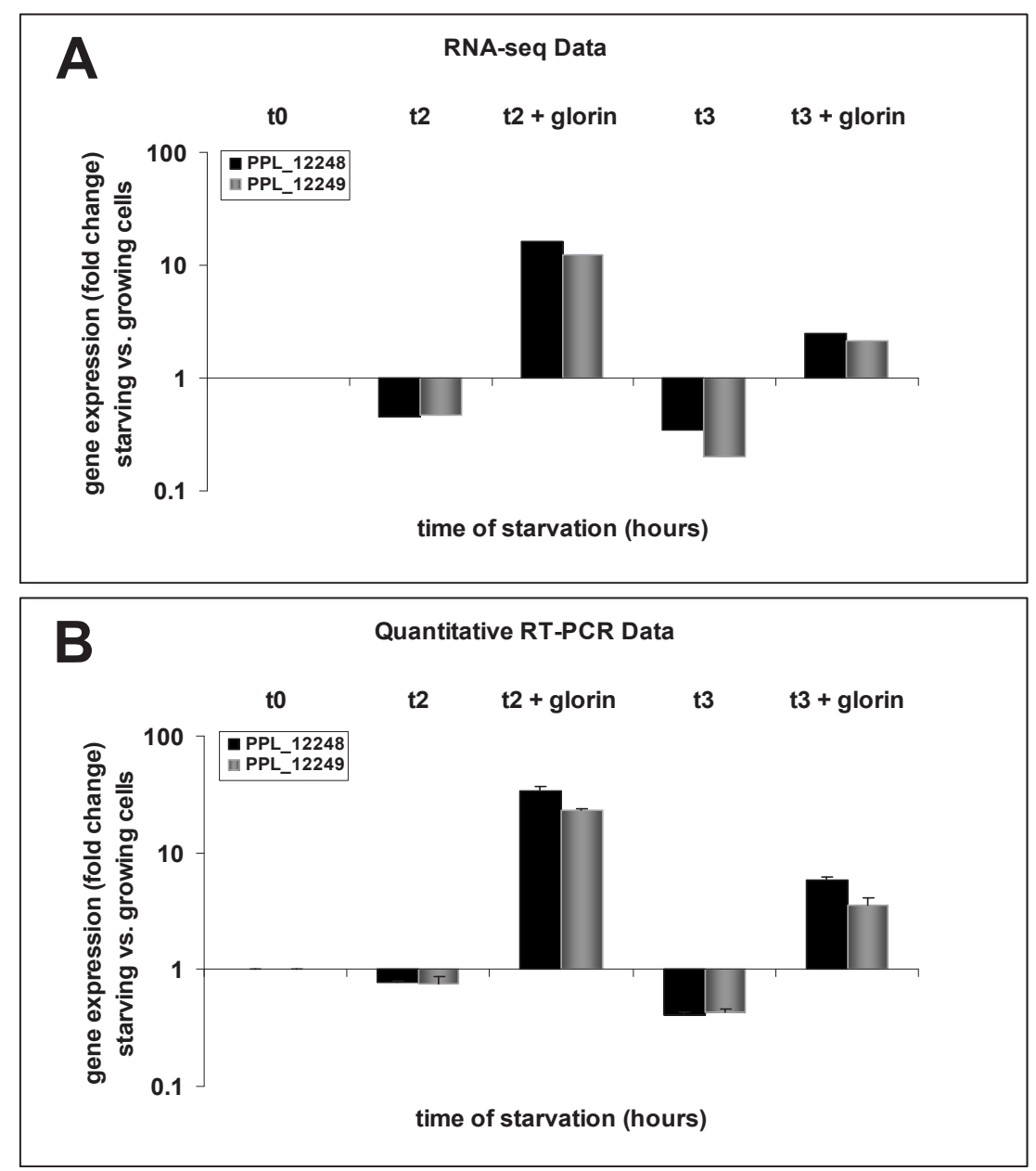

Figure 29: Correlation of RNA-seq data with real-time RT-PCR data. Fold changes are shown. All data were compared to growing $P$. pallidum PN500 amoebae (t0; fold change set to 1). Values $>1$ represent higher expression of the gene in starving cells than in growing cells. Values $<1$ 
show that expression of the gene is lower in starving cells than in growing cells. As a control, expression of house keeping gene gpdA was examined.Cells were starved in shaking cultures for 2 or 3 hours in the absence of exogenous glorin treatment $(\mathbf{t} 2, \mathbf{t} 3)$ or for 1 hour followed by 1 hour or 2 hours of glorin treatment (t2+glorin, $\mathbf{t} 3+$ glorin). (A) Two model genes, indicated by the corresponding $P$. pallidum (PPL) gene numbers, were selected from RNA-seq data of first biological replicate to describe glorin-induced gene expression. It should be noted that the results obtained from RNA-seq of second biological replicate were comparable to the results shown here. (B) Relative expression of representative genes (as shown in panel A) determined by real-time RT-PCR. cDNA used in this experiment were prepared from total RNA extracted from first biological replicate. Mean values of triplicate measurements of the same CDNA \pm SD were plotted. A high degree of correlation was observed between two methods demonstrating the effectiveness of RNA-seq approach.

Development of cells in the presence of glorin led to an increase in mRNA levels of both PPL_12248 and PPL_12249. After 1 hour of glorin treatment, PPL_12248 was 16.26fold induced compared to the growing cells, whereas PPL_12249 exhibited 12.20-fold increase in expression (Figure 29). Glorin-induced expression of both genes declined to low levels after stimulating cells with glorin for 2 hours indicating rapid turn over of these genes. RT-PCR data for validation of RNA-seq analyses of these genes is shown in Figure 29 that confirmed the RNA-seq findings.

PPL_12248 and PPL_12249 may represent 'vegetative stage genes' that turn off at the onset of development. Strong induction of these genes by glorin illustrates that products of these genes may also function in glorin signalling during aggregation of $P$. pallidum PN500 amoebae. Though these genes are transiently induced by glorin, they may play a crucial role if products of these genes are proteins required for only short period of time.

The results presented above clearly demonstrated that glorin may have two distinctly different effects during early development. The periodic addition of $1 \mu \mathrm{M}$ glorin every 30 min stimulates the rate of expression of many genes, while expression of several other genes is suppressed. RT-PCR data used to evaluate performance of RNA-seq analyses indicated reliability of the experimental methods used in this study. These experiments also validate the experimental conditions used to in this study to examine glorinregulated gene expression. Taken as a whole, studying differential gene expression changes mediated by glorin has contributed to the previous knowledge of cellular roles played by glorin.

\subsection{Putative functions of glorin-regulated genes}

To get insight into the possible roles of glorin responsive genes, putative molecular functions of these genes were searched based on homologies to annotated $D$. discoideum 
genes (Social Amoebas Comparative Genome Browser; http://sacgb.flileibniz.de/cgi/index.pl). A short list of gene ontology (GO) terms attributed to the glorininduced genes is presented in Table 4. Detailed information was obtained for genes significantly up- or down-regulated by glorin in three different organizing principles of GO, i.e. molecular function, biological process and cellular component ( $P$. pallidum database; http://sacgb.fli-leibniz.de/cgi/index.pl) and is depicted in Appendix Table A7 and A8.

\subsubsection{Annotation of GO terms to glorin-induced genes}

Interestingly, it was found that a considerable number of genes induced by glorin may encode proteins that can play important roles in transducing extracellular signals. For example, the list encompasses seven $\mathrm{G}$ protein-coupled receptors, upregulated by glorin to various degrees. Several putative serine/threonine kinases are induced by glorin, some of which are alpha kinases carrying the conserved " $\alpha$-kinase catalytic domain" as reported in $D$. discoideum myosin heavy chain kinases (MHCKs; Betapudi et al. 2005). The alpha-kinases family is a class of atypical protein kinases that exhibit low sequence similarity to conventional eukaryotic protein kinases. Some of these kinases contain von Willebrand factor A (vWFA) like motifs. The vWFA motif is found in a variety of intracellular proteins involved in various cellular functions, such as protein-protein interactions, DNA repair, transcription, ribosomal and membrane transport, and proteosomal functions (Betapudi et al. 2005; Whittaker and Hynes 2002). Some other proteins that include VWF domains take part in cell adhesion, signal transduction, migration, and pattern formation, engaging interaction with a variety of ligands (Colombatti et al. 1993). Extracellular signal-regulated kinase 2 (ErkB) is a protein involved in MAP kinase signal transduction pathways. D. discoideum erkB null mutants exhibit a decrease in motility and a severe chemotaxis defect towards cAMP gradient leading to impaired aggregation (Wang et al. 1998; Rodriguez et al. 2008). Stimulation of starving $P$. pallidum PN500 cells with glorin transiently upregulated expression of a gene designated as PPL_12271 that encodes putative ErkB protein. Glorin induced expression of genes encoding histidine kinases and tyrosine kinases-like transmembrane proteins that exhibit signal transduction activity across the cellular membrane.

A number of genes encoding small GTPases of Ras and Rho families were up-regulated by the glorin. Ras-superfamily small GTPases constitute components of signalling pathways connecting extracellular signals via transmembrane receptors to cytoplasmic or nuclear responses. Rho GTPases are involved in diverse signal transduction pathways, 
including organization of the actin cytoskeleton during cell migration, activation of certain MAP kinases and may also activate transcription factors. Glorin induces a gene encoding a 'Profilin I-like' actin binding protein that may be involved in the dynamic turnover and restructuring of the actin cytoskeleton during chemotaxis.

Genes encoding zinc-binding domain containing proteins are also upregulated by glorin. One of these proteins is a RING zinc-finger domain holding protein. Zinc fingers are relatively small protein motifs that bind one or more zinc ions to help stabilize their fold. Zinc fingers usually consist of multiple finger-like protrusions that bind to the target molecule, such as DNA, RNA, proteins, or small molecules. Zinc finger-containing proteins play key roles in gene transcription, mRNA trafficking, translation, protein folding, cytoskeleton organisation, cell adhesion, and chromatin remodelling. A RINGfinger domain is a protein structural domain of zinc finger type, whereas proteins containing a RING finger play vital roles in the ubiquitination pathway leading to degradation of proteins.

Glorin also induced expression of proteins involved in DNA metabolism, such as Deoxyribonuclease II that plays a major role in the degradation of nuclear DNA in cellular apoptosis during development. Glorin upregulated expression of genes encoding putative cytochrome P450 family proteins that are haem-containing mono-oxygenases, involved in oxidation-reduction processes. Enzymes of cytochrome P450 family play important role in the metabolism of compounds that control cell differentiation in $D$. discoideum (Gonzalez-Kristeller et al. 2008).

Glorin induced expression of many genes whose products are involved in carbohydrate metabolic processes. Examples include enzymes belonging to the glycoside hydrolase family 45 that exhibit endoglucanase activity, expansin-like proteins and cellulases. Expansins are unusual proteins that are believed to act as chemical grease and may allow cellulose fibrils to slide past one another when cellulose sheath is being assembled during the late aggregation stages. Similarly, cellulases seem to be involved in remodelling of slime sheath cellulose during morphogenesis (Sucgang et al. 2011).

Moreover, glorin induced genes encoding PA14 domain-containing proteins with homology to $D$. discoideum extracellular matrix proteins, such as EcmB. PA14 domain is a $\beta$-barrel structure recognized as a novel carbohydrate-binding module (Rigden et al. 2004). 
The mannose 6-phosphate receptor homology $(\mathrm{MRH})$ domain-containing family of proteins play key roles in the secretory pathway (Castonguay et al. 2011), whereas mannose 6-phosphate receptors (MPRs) play an essential role in lysosome biogenesis. These proteins (i.e. MPRs) bind newly synthesized Mannose-6-Phosphate-containing lysosomal hydrolases in the trans-Golgi network (TGN) and deliver them to the prelysosomal compartments (Castonguay et al. 2011).

Stimulation of cells with glorin induces expression of genes that may encode $A B C$ transporter proteins belonging to family A \& G. ATP-binding cassette (ABC) transporters are transmembrane proteins that make use of the energy of adenosine triphosphate (ATP) hydrolysis to transport various substrates across extra- and intracellular membranes, including metabolic products, lipids, toxins and drugs. Other genes induced by glorin may encode proteins displaying homology to glutamate-ammonia ligase involved in glutamine biosynthetic process, zymogen granule (pancreatic secretory granules) membrane glycoprotein, beta-lactamase-type transpeptidase fold containing protein, cellulose and chitin-binding domain containing proteins that exhibit carbohydratebinding activity, epidermal growth factor-like domain containing membrane-bound protein implemented in cell recognition and proteins involved in proteolysis, pathogenesis and transcription repression.

The concomitant upregulation of these genes during early development suggests their possible involvement in the glorin-mediated coordination of aggregation process. 


\begin{tabular}{|c|c|c|}
\hline Gene & \begin{tabular}{|c|}
$\begin{array}{c}\text { Expression } \\
\text { (fold } \\
\text { change) }\end{array}$ \\
\end{tabular} & Gene description \\
\hline \multicolumn{3}{|c|}{ Signal transduction } \\
\hline \multicolumn{3}{|c|}{ G-protein coupled receptor activity } \\
\hline PPL_08454 & 15.8 & $\begin{array}{l}\text { G-protein coupled receptor activity (family 3), metabotropic } \\
\text { glutamate receptor-like }\end{array}$ \\
\hline PPL_08455 & 11.9 & $\begin{array}{l}\text { G-protein coupled receptor activity (family } 3 \text { ), metabotropic } \\
\text { glutamate receptor-like }\end{array}$ \\
\hline PPL_03564 & 8.6 & $\begin{array}{l}\text { G-protein coupled receptor activity (family } 3 \text { ), metabotropic } \\
\text { glutamate receptor-like }\end{array}$ \\
\hline PPL_05727 & 4.1 & $\begin{array}{l}\text { G-protein coupled receptor activity (family } 3 \text { ), metabotropic } \\
\text { glutamate receptor-like }\end{array}$ \\
\hline PPL_04108 & 3.3 & $\begin{array}{l}\text { G-protein coupled receptor activity (family 3), metabotropic } \\
\text { glutamate receptor-like }\end{array}$ \\
\hline PPL_00902 & 2.7 & $\begin{array}{l}\text { G-protein coupled receptor activity (family 3), metabotropic } \\
\text { glutamate receptor-like }\end{array}$ \\
\hline PPL_00855 & 1.89 & $\begin{array}{l}\text { G-protein-coupled receptor (GPCR) family protein, frizzled and } \\
\text { smoothened-like protein }\end{array}$ \\
\hline \multicolumn{3}{|c|}{ protein serine/threonine kinase activity } \\
\hline PPL_12248 & 39.6 & protein serine/threonine kinase activity (alpha kinase superfamily) \\
\hline PPL_12249 & 29.2 & protein serine/threonine kinase activity (alpha kinase superfamily) \\
\hline PPL_12251 & 8.7 & $\begin{array}{l}\text { protein serine/threonine kinase activity (alpha kinase superfamily); } \\
\text { type A von Willebrand factor (VWFA) domain-containing protein }\end{array}$ \\
\hline PPL_12250 & 4.76 & protein serine/threonine kinase activity \\
\hline PPL_12271 & 7.7 & erkB, ERK subfamily protein kinase, extracellular response kinase \\
\hline \multicolumn{3}{|c|}{ Tyrosine kinase-like protein } \\
\hline PPL_00861 & 11.6 & tyrosine kinase-like protein \\
\hline \multicolumn{3}{|c|}{ Histidine kinase-like protein } \\
\hline PPL_04384 & 4.11 & $\begin{array}{l}\text { histidine kinase, protein kinase, signal transducer activity, } \\
\text { regulation of transcription }\end{array}$ \\
\hline \multicolumn{3}{|c|}{ Ras GTPases } \\
\hline PPL_07296 & 7.4 & $\begin{array}{l}\text { Ras GTPase domain-containing protein; type A von Willebrand } \\
\text { factor (VWFA) domain-containing protein }\end{array}$ \\
\hline \multicolumn{3}{|c|}{ Rho GTPases } \\
\hline PPL_05452 & 5.8 & Rho GTPase \\
\hline PPL_04393 & 3.17 & $\begin{array}{l}\text { GTP binding activity, Rho GTPase, small GTPase mediated } \\
\text { signal transduction }\end{array}$ \\
\hline \multicolumn{3}{|c|}{ Cytoskeleton organization } \\
\hline \multicolumn{3}{|c|}{ Actin cytoskeleton organization } \\
\hline PPL_09347 & 53.9 & profilin I, actin binding protein \\
\hline \multicolumn{3}{|c|}{ Putative extracellular matrix proteins } \\
\hline PPL_05354 & 56.9 & $\begin{array}{l}\text { putative extracellular matrix protein, PA14 domain-containing } \\
\text { protein }\end{array}$ \\
\hline PPL_12308 & 6.3 & $\begin{array}{l}\text { putative extracellular matrix protein, PA14 domain-containing } \\
\text { protein }\end{array}$ \\
\hline PPL_11304 & 2.8 & $\begin{array}{l}\text { putative extracellular matrix protein, PA14 domain-containing } \\
\text { protein }\end{array}$ \\
\hline \multirow{2}{*}{\multicolumn{3}{|c|}{$\begin{array}{l}\text { Transcription and translation } \\
\text { Zinc-binding domain-containing protein } \\
\end{array}$}} \\
\hline & & \\
\hline PPL_01619 & 3.32 & CMP/dCMP deaminase, zinc-binding domain-containing protein \\
\hline PPL_09669 & 4.37 & RING zinc finger-containing protein \\
\hline \multicolumn{3}{|c|}{ Secretory pathway } \\
\hline \multicolumn{3}{|c|}{ protein binding function } \\
\hline PPL_07812 & 15.4 & Mannose-6-phosphate receptor domain, protein binding function \\
\hline PPL_07800 & 5.48 & Mannose-6-phosphate receptor domain, protein binding function \\
\hline
\end{tabular}




\begin{tabular}{|c|c|c|}
\hline \multicolumn{3}{|l|}{ Transport } \\
\hline \multicolumn{3}{|c|}{ ABC transporter family proteins } \\
\hline PPL_07551 & 3.39 & ABC transporter A family protein \\
\hline PPL_07432 & 4.39 & ABC transporter $\mathrm{G}$ family protein, integral to membrane \\
\hline PPL_07551 & 3.49 & $\begin{array}{l}\text { ABC transporter A family protein, nucleoside-triphosphatase } \\
\text { activity }\end{array}$ \\
\hline \multicolumn{3}{|l|}{ Metabolism } \\
\hline \multicolumn{3}{|c|}{ DNA metabolic process } \\
\hline PPL_08854 & 2.84 & $\begin{array}{l}\text { similar to zymogen granule (pancreatic secretory granules) } \\
\text { membrane glycoprotein, deoxyribonuclease II activity }\end{array}$ \\
\hline PPL_05392 & 5.27 & deoxyribonuclease II activity, DNA metabolic process \\
\hline PPL_04328 & 3.40 & DNA metabolic process, deoxyribonuclease II activity \\
\hline \multicolumn{3}{|c|}{ Oxidoreductase activity } \\
\hline PPL_09729 & 4.19 & iron ion binding proteins, oxidoreductase activity \\
\hline PPL_04071 & 1.21 & electron carrier activity, iron ion binding activity \\
\hline \multicolumn{3}{|c|}{ Carbohydrate metabolic process } \\
\hline PPL_11763 & 22.6 & $\begin{array}{l}\text { expansin-like protein, Barwin-like endoglucanases, Glycoside } \\
\text { hydrolase family } 45 \text { protein }\end{array}$ \\
\hline \multicolumn{3}{|c|}{ Carbohydrate binding activity } \\
\hline \multicolumn{3}{|c|}{ Cellulose-binding domain containing protein } \\
\hline PPL_02674 & 2.7 & $\begin{array}{l}\text { Cellulose-binding domain containing protein, Carbohydrate } \\
\text { binding domain-containing protein, Stalk-specific protein }\end{array}$ \\
\hline PPL_05703 & 4.27 & $\begin{array}{l}\text { Cellulose-binding domain containing protein, Carbohydrate } \\
\text { binding domain-containing protein, extracellular region }\end{array}$ \\
\hline \multicolumn{3}{|c|}{ Cell recognition } \\
\hline \multicolumn{3}{|c|}{ Epidermal Growth Factor-like domain containing membrane-bound proteins } \\
\hline PPL_00062 & 3.3 & $\begin{array}{l}\text { Cell surface receptor, IPT/TIG Epidermal Growth Factor-like } \\
\text { domain containing membrane-bound proteins }\end{array}$ \\
\hline PPL_07302 & 4.90 & $\begin{array}{l}\text { Epidermal growth factor-like domain containing membrane-bound } \\
\text { protein, calcium ion binding activity, extracellular in location }\end{array}$ \\
\hline \multicolumn{3}{|c|}{ Miscellaneous } \\
\hline \multicolumn{3}{|c|}{ Transcription repressor activity } \\
\hline PPL_00612 & 3.95 & transcription repressor activity \\
\hline \multicolumn{3}{|l|}{ Proteolysis } \\
\hline PPL_05027 & 3.90 & $\begin{array}{l}\text { ubiquitin carboxyl-terminal hydrolase 2, Peptidase C19, ubiquitin } \\
\text { thiolesterase activity }\end{array}$ \\
\hline PPL_08725 & 3.66 & hydrolase activity, proteolysis \\
\hline \multicolumn{3}{|c|}{ Pathogenesis } \\
\hline PPL_10669 & 4.55 & Delta endotoxin insectocide, pathogenesis \\
\hline PPL_06262 & 3.80 & delta-Endotoxin insectocide \\
\hline \multicolumn{3}{|c|}{ Glutamate-ammonia ligase activity } \\
\hline PPL_04550 & 3.73 & $\begin{array}{l}\text { glutamate-ammonia ligase, glutamine synthetase type I, glutamine } \\
\text { biosynthetic process }\end{array}$ \\
\hline \multicolumn{3}{|c|}{ beta-lactamase-type transpeptidase fold containing protein } \\
\hline PPL_11763 & 22.6 & $\begin{array}{l}\text { beta-lactamase-type transpeptidase fold containing protein (based } \\
\text { on homology to } D \text {. discoideum protein DDB_0189589. }\end{array}$ \\
\hline \multicolumn{3}{|c|}{ Enhancing factor precursor } \\
\hline PPL_04587 & 7.6 & $\begin{array}{l}\text { Enhancing factor precursor, Chitin-binding domain containing } \\
\text { protein }\end{array}$ \\
\hline \multicolumn{3}{|c|}{ Immunoglobulin E-set domain-containing protein } \\
\hline PPL_04784 & 10.8 & $\begin{array}{l}\text { IPT/TIG domain-containing protein; immunoglobulin early-set } \\
\text { domain-containing protein }\end{array}$ \\
\hline PPL_04746 & 2.8 & $\begin{array}{l}\text { putative cell surface glycoprotein, IPT/TIG domain-containing } \\
\text { protein, Immunoglobulin early-set domain-containing protein }\end{array}$ \\
\hline \multicolumn{3}{|c|}{ Proteins with unknown function } \\
\hline PPL_03541 & 22.2 & unknown \\
\hline
\end{tabular}




\begin{tabular}{|l|c|l|}
\hline PPL_05833 & 20.9 & unknown \\
\hline PPL_06644 & 17.4 & unknown \\
\hline PPL_07811 & 16.3 & unknown \\
\hline PPL_00117 & 12.1 & unknown \\
\hline PPL_00912 & 15.2 & unknown \\
\hline PPL_10324 & 11.6 & unknown \\
\hline PPL_02621 & 9.3 & unknown \\
\hline PPL_02620 & 9.3 & unknown \\
\hline PPL_07801 & 8.0 & unknown \\
\hline PPL_07818 & 7.2 & unknown \\
\hline
\end{tabular}

Table 4: Summary of glorin-induced genes in P. pallidum PN500. ${ }^{\text {a }}$ Fold change calculated for gene expression in cells prestarved for 1 hour and then treated with glorin for an additional hour, compared to gene expression in cells starved for 2 hours in the absence of exogenous glorin.

\subsubsection{Annotation of GO terms to glorin-repressed genes}

GO term analysis of glorin-repressed genes could not provide much information as most of these genes have not yet been ascribed to a distinct function. Genes that were downregulated by glorin may encode proteins that may be involved in the regulation of transcription, carbohydrate and lipid metabolic processes, proteolysis, pathogenesis, steroid metabolic processes, oxidoreduction reactions, nucleosome assembly and transportation.

Glorin treatment repressed expression of some genes whose products may exhibit Omethyltransferase activity. Methyltransferases play important role in many vital cellular processes including signal transduction, chromatin regulation, gene silencing, and protein repair. Expression of a gene that may encode myb transcription factor domaincontaining protein was down-regulated by glorin. In $D$. discoideum Myb transcription factors play important role at culmination as activators of ancillary stalk differentiation (Tsujioka et al. 2007). Another gene repressed by glorin may encode a transcription factor including a homeodomain that binds DNA through a helix-turn-helix (HTH) structure.

Other glorin-repressed genes encode proteins with homology to cAMP-dependent protein kinase (PKA family protein kinase), winged helix-turn-helix transcription repressor, zinc ion binding proteins, and SecA protein that is a superfamily 2 helicase adapted to translocate proteins. GO annotation for some of the glorin-repressed genes is presented in Appendix Table A7 and A8. 


\subsection{Detailed kinetics of glorin-regulated gene expression}

\subsubsection{Effect of signal modulation on glorin-regulated gene expression}

Previously, Kopachik (1990) studied protein synthesis by developing P. violaceum amoebae in response to externally added glorin and showed that this reaction was concentration and time dependent (Kopachik 1990). The author reported that a higher concentration of glorin added for a longer time was most effective to stimulate synthesis of certain proteins (Kopachik 1990). Therefore, it was interesting to elucidate effects of different concentrations and pulsing frequencies of glorin on gene expression changes in P. pallidum PN500 amoebae.

\subsubsection{Gene expression analysis under different concentrations of glorin}

First, starving P. pallidum PN500 cells were stimulated with varying concentrations of glorin and changes in gene expression were examined.

P. pallidum PN500 cells cultured on bacterial lawns were harvested and starved by slow shaking in phosphate buffer at a cell density of $2 \times 10^{7}$ cells $/ \mathrm{ml}$ at $20^{\circ} \mathrm{C}$. Before inducing development, $2 \times 10^{7}$ vegetative stage cells were pelleted and stored at $-80^{\circ} \mathrm{C}$. This pellet of cells represented 'growing cells control' for total RNA extraction in subsequent gene expression analyses. After one hour of pre-starvation, culture was divided into 4 erlenmeyer flasks and cells were allowed to develop in shaking suspensions either in the presence or absence of glorin. Cells in each of three flasks were given glorin pulses at 10 $\mathrm{nM}, 100 \mathrm{nM}$ and $1 \mu \mathrm{M}$ final concentration, respectively, at 30-min intervals. Cells in one flask received no exogenous treatment (shown by ' $0 \mathrm{nM}$ glorin' symbol in Figure 30) and served as the 'control' for the time period. Cells $\left(2 \times 10^{7}\right)$ were harvested from all four cultures at $0.5,1$ and 2 hours after glorin treatment for isolation of total RNA to prepare cDNA for gene expression analysis using real-time RT-PCR. Cell samples were collected from 'untreated control' at the same time points. Model glorin-regulated genes, PPL_09347 and PPL_05354 that were noticed to be highly induced by glorin in RNA-seq analyses were chosen to study gene expression changes in response to varying concentrations of glorin applied at 30- minute intervals. Relative gene expression levels of both genes under all tested conditions were calculated and fold changes were determined compared to expression in growing cells. 
It was found that exposure of cells to $10 \mathrm{nM}$ glorin for the first 30 minutes (one pulse only) was sufficient to induce PPL_09347 to 30.50-fold, compared to growing cells, whereas further stimulation of cells with $10 \mathrm{nM}$ glorin added every 30 minutes for additional 1 and 2 hours increased expression of PPL_09347 by 39.26- and 45.62-fold, respectively (Figure 30A).

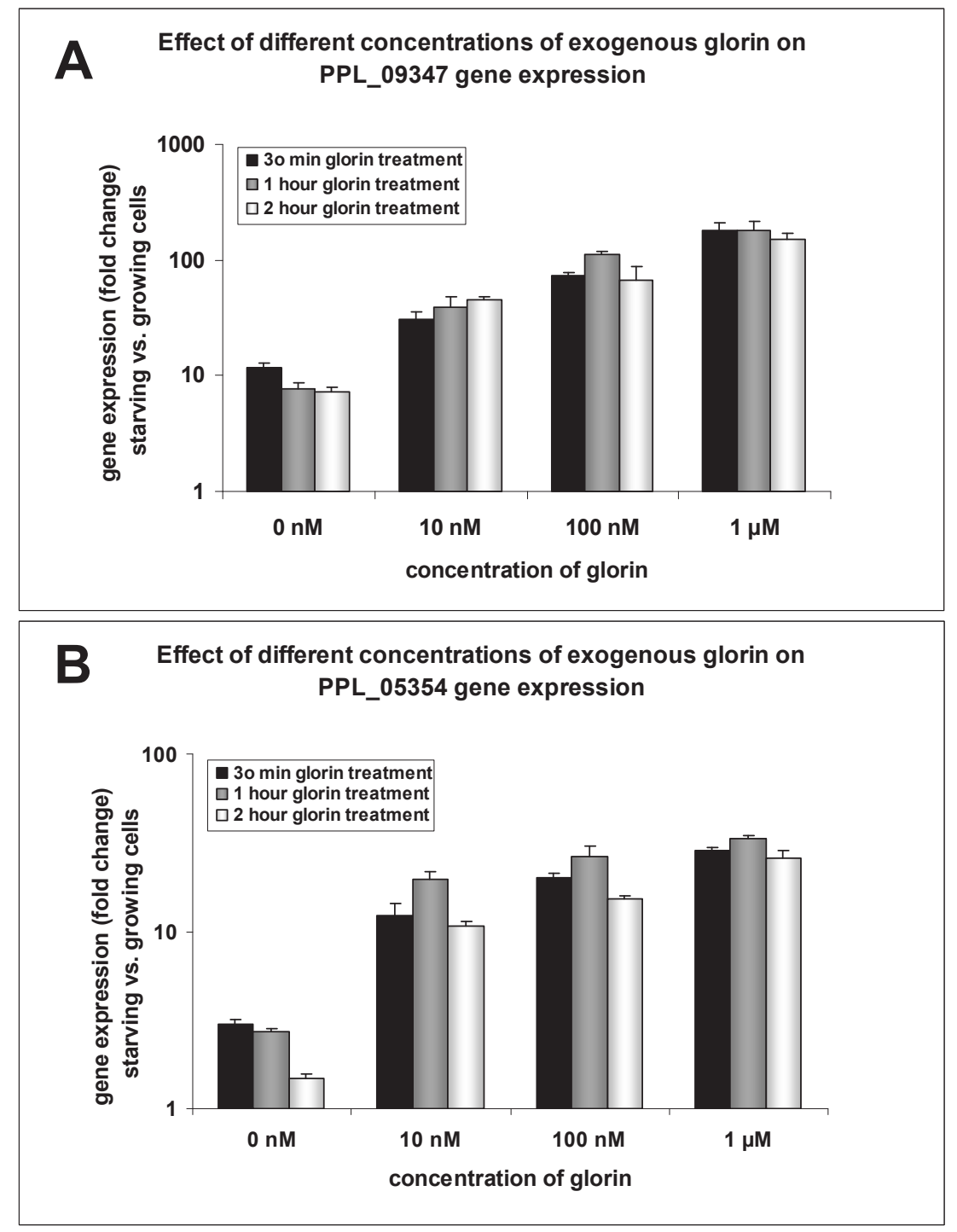

Figure 30: Glorin-induced gene expression in response to varying concentrations of glorin. P. pallidum PN500 cells were developed in shaking cultures for 3 hours in the presence or absence of exogenous glorin. In glorin treated cultures, beginning 1 hour after starvation, glorin was added at $10 \mathrm{nM}, 100 \mathrm{nM}$ or $1 \mu \mathrm{M}$ final concentration at 30-minute intervals. Untreated control is represented by $0 \mathrm{nM}$ glorin. Cell samples were collected after $30 \mathrm{~min}, 1$ and 2 hours of glorin treatment for total RNA extraction to analyse gene expression. Cells were generally harvested 30 minutes after a pulse of glorin was applied. Samples were collected from untreated cells at the same time points. Relative expression of model genes PPL_09347 (A) and PPL_05354 (B) was determined by real-time RT-PCR. As a control, expression of house keeping gene gpdA was examined. Fold changes are shown. All data were compared to growing $P$. 
pallidum PN500 cells. Fold change was set to 1 where values $>1$ represented higher expression of the gene in starving cells than in growing cells. Values $<1$ show that expression of gene is lower in starving cells than in growing cells. Mean values of duplicate measurements of the same $\mathrm{cDNA} \pm \mathrm{SD}$ were plotted.

Treatment of developing cells with $100 \mathrm{nM}$ glorin for 0.5-, 1- and 2-hours led to 72.49, 43.09- and 67.76-fold expression of PPL_09347. When $1 \mu \mathrm{M}$ glorin was added at 30 minutes intervals to cells pre-starved for 1 hours, 180.15-, 178.13- and 150.79-fold increased in gene expression of PPL_09347 was observed after 30 minutes, 1 and 2 hours of glorin treatment. It is interesting to note that only after first pulse of $10 \mathrm{nM}$, $100 \mathrm{nM}$, or $1 \mu \mathrm{M}$ glorin (final concentration), PPL_09347 was induced to 30.50-, 72.49and 180.15-fold respectively (Figure 30A). Overall, expression of PPL_09347 generally increased in response to glorin stimulation in the course of observation.

While analysing expression of model gene PPL_05354, it was noted that upon treatment of cells with $10 \mathrm{nM}$ glorin every 30 minutes for 0.5-, 1- and 2-hours, gene expression increased adequately by 12.31-, 19.84-, and 10.71-fold, respectively (Figure 30B). Exposure of cells to $100 \mathrm{nM}$ glorin for 30 minutes, 1 and 2 hours enhanced expression of PPL_05354 by 19.86, 26.12 and 15.15-fold, respectively. When glorin was added to starving cells at $1 \mu \mathrm{M}$ final concentration, 28.39-, 33.52- and 25.67-fold increase in mRNA levels was noticed after 0.5-, 1- and 2-hours of treatment, while comparing expression to that in growing cells (Figure 30B). An important observation was the slight decrease in expression of PPL_05354 at 3 hours of development under all tested conditions. This may indicate that expression of PPL_05354 reduces to varying degrees with progression of starvation when cells are developed in shaking cultures.

Contrary to the results obtained in RNA-seq analyses (Figure 25), PPL_09347 and PPL_05354 exhibited a considerable increase in expression in cells that were not treated with glorin (referred to $\mathrm{t} 2$ and $\mathrm{t} 3$ in Figure $25 \&$ untreated control ' 0 nM' in Figure 30). In experiment shown in Figure 25, untreated cells did not show significant expression of PPL_09347 and PPL_05354 genes. A possible interpretation of detecting low level expression of PPL_09347 and PPL_05354 in experiment depicted in Figure 30 could be that cells were harvested for this experiment when they were already starving on agar plates where they were grown in association with bacteria and most probably have already established some basic endogenous glorin signalling by themselves.

In short, it is shown that increasing concentrations of externally added glorin are associated with increased response in gene expression; maximum response in gene 
expression was obtained when cells were stimulated with $1 \mu \mathrm{M}$ glorin. Thus, these experiments further validated experimental conditions used for conducting RNA-seq analyses (described in Section 3.6). These data also provided an indication that glorin triggers rapid changes in gene expression.

\subsubsection{Gene expression analysis under different pulsing frequencies of glorin}

Physiological effects of many messengers of intercellular communication might be regulated by the frequency of their temporal variation (Li \& Goldbeter 1992; Knobil 1981). Such a frequency encoding may manifest more advantages than amplitude-modulated control (Rapp 1987). Frequency encoding of signals is exemplified by secretion of cAMP pulses in aggregating $D$. discoideum cells at periodicities of $\sim 6$ min (McMains et al. 2008; Goldbeter 2006). To date pulsatile glorin signalling has not been observed. Previously some researchers showed that starving $P$. violaceum exhibit oscillatory aggregation, but it was pointed out that glorin may not be the primary oscillator (De Wit et al. 1988). Similarly, some initial reports described the presence of centrifugally propagated waves of excitation in the aggregation fields of $P$. violaceum amoebae that were regularly spaced with an interval decreasing from 4 to 2.5 minutes, while in $P$. pallidum amoebae, these waves were not found to be systematically spaced (Jones 1976).

As described in the Section 3.8.1.1, $1 \mu \mathrm{M}$ final concentration of glorin was found to be optimal for studying glorin-induced gene expression. As a next step, gene expression changes in response to different pulsing frequencies of glorin were analyzed to understand how intervals between pulses may modulate gene expression.

P. pallidum PN500 cells were grown in association with bacteria and harvested after 48 hours of growth. Cells were washed and resuspended in phosphate buffer at a density of $2 \times 10^{7}$ cells $/ \mathrm{ml}$. A pellet of vegetative cells ( $2 \times 10^{7}$ amoebae) was collected for total RNA extraction for subsequent gene expression analyses. Amoebae were then starved in suspension culture for 1 hour by shaking at $100 \mathrm{rpm}$ at $22^{\circ} \mathrm{C}$ to induce development. After 1 hour pre-starvation, cell suspension was splitted into 4 parts. One culture designated as "untreated control" did not receive any exogenous treatment. Glorin (1 $\mu \mathrm{M}$ final concentration) was added for 2 hours to each of 3 other cultures at 10-, 20- and 30minute intervals under slow-shake conditions (i.e. $100 \mathrm{rpm}$ ). Control culture was developed similarly in the absence of externally added glorin. Cell samples were 
collected after 1 and 2 hours of glorin treatment to prepare total RNA for gene expression analysis. Samples were collected from 'untreated control' at the same time points.

To determine whether varying periods of exposure to glorin influenced gene expression, model genes PPL_09347 and PPL_05354 were subjected to quantitative RT-PCR analyses. Relative expression levels of both genes were determined under 3 different conditions of glorin treatment and fold changes were calculated compared to the expression in growing cells (Figure 31 ).

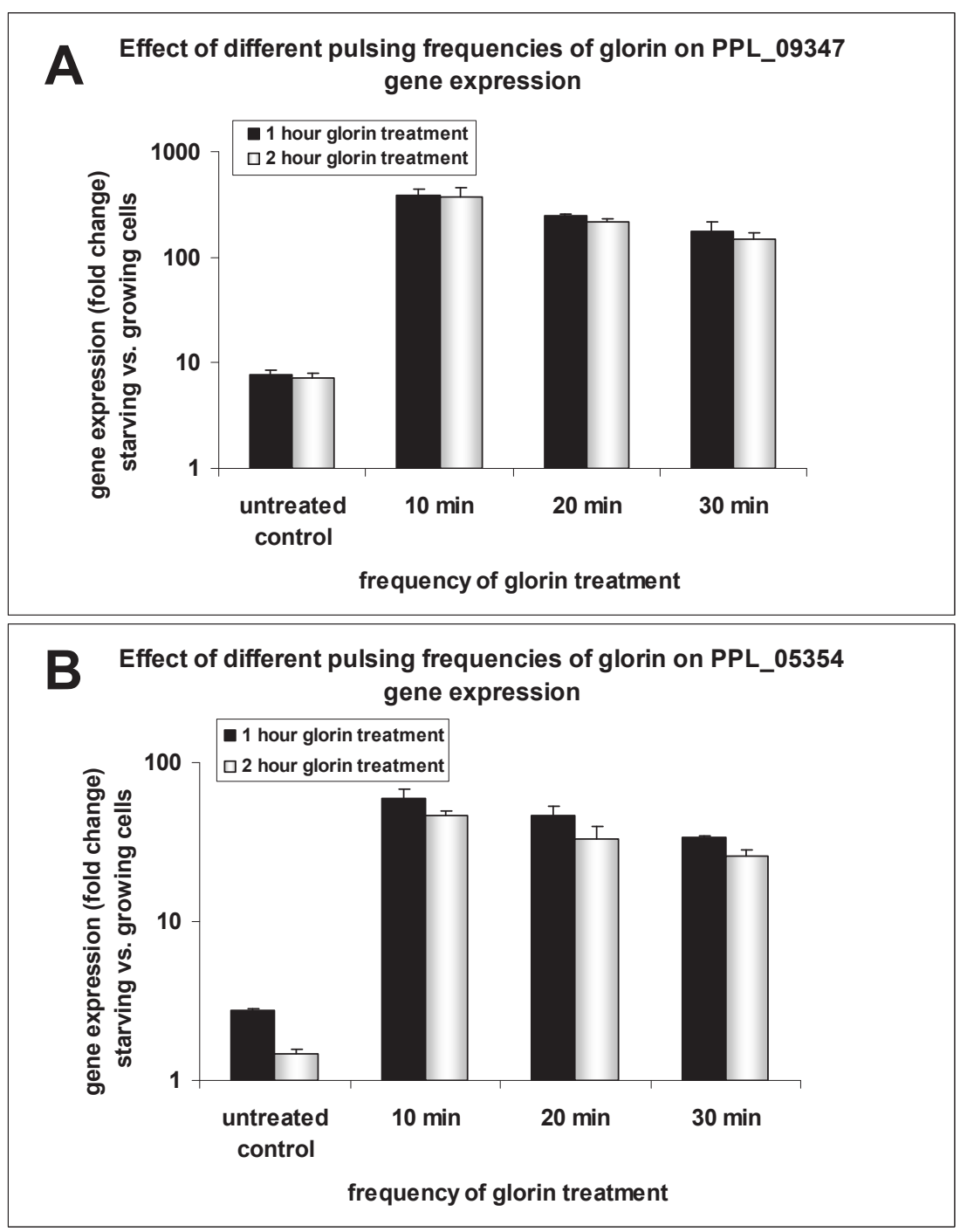

Figure 31: Glorin-induced gene expression in response to varying periods of exposure to glorin. P. pallidum PN500 cells were developed in shaking cultures for 3 hours in the presence or absence of exogenous glorin. In glorin treated cultures, beginning 1 hour after starvation, $1 \mu \mathrm{M}$ final concentration of glorin was added at 10-, 20- or 30-minute intervals. Untreated control is shown seperately. Cell samples were collected after 1 and 2 hours of glorin treatment for total RNA extraction to analyse gene expression. Cells were generally harvested 30 minutes after a 
pulse of glorin was applied. Samples were collected from untreated cells at the same time points. Relative expression of PPL_09347 (A) and PPL_05354 (B) was determined by real-time RTPCR. As a control, expression of house keeping gene gpdA was examined. Fold changes are shown. All data were compared to growing P. pallidum PN500 cells. Fold change was set to 1 where values $>1$ represent higher expression of the gene in starving cells than in growing cells. Values $<1$ show that expression of gene is lower in starving cells than in growing cells. Mean values of duplicate measurements of the same CDNA $\pm S D$ were plotted.

Real-time RT-PCR analysis showed that mRNA levels of PPL_09347 were highly upregulated by 385- and 378-fold after 1 and 2 hour of glorin treatment at 10 minute intervals (Figure 31A). When starving cells were treated with $1 \mu \mathrm{M}$ glorin at 20 minute intervals for 1 or 2 hours, PPL_09347 was induced to 249- and 212-fold, respectively, compared to growing cells. Adding glorin at 30 minute intervals led to 178 - and 150 -fold increase in expression of PPL_09347 at 1 and 2 hours (Figure 31A). For each pulsing condition tested, expression of PPL_09347 was similarly maintained at both hours of glorin treatment.

When gene expression data of PPL_05354 were analyzed, it was found that this gene was induced to 33.52- and 25.67-fold (compared to growing cells) when cells were pulsed with glorin at 30-minute intervals for 1 and 2 hour, respectively (Figure 31B). Significant level expression of this gene to 46.91- and 33.20-fold was noticed after pulsing cells with glorin at 20-minute intervals for 1 and 2 hours, respectively. Pronounced changes in gene expression levels were detected when cells received pulses of glorin at 10-minute intervals. 59.78- and 46.92-fold increase in expression of PPL_05354 was observed after 1 and 2 hours of exogenous glorin treatment, respectively (Figure 31B).

These data demonstrate that $1 \mu \mathrm{M}$ glorin (final concentration) induced model genes significantly when pulsed at 30-minute intervals, whereas comparatively higher levels of gene expression were obtained when glorin pulses were applied more frequently at 10minute intervals indicating that repetitive stimulation with glorin results in a stronger response. These results further support authenticity of pulsing conditions used for RNAseq analyses. It still remains to uncover how often pulses of glorin are secreted in aggregation fields of starving $P$. pallidum PN500 amoebae. 


\subsubsection{Expression kinetics of glorin-regulated genes during the first $\mathbf{5}$ hours of development in suspension cultures}

In the RNA-seq analysis and subsequent experiments, gene expression studies mainly focused on first 3 hours of starvation encompassing 2 hours of glorin treatment. After RNA-seq data analysis provided a list of glorin-regulated genes, and model genes were defined to study glorin effects on gene expression using real-time RT-PCR, it could be advantageous to investigate expression kinetics of glorin -induced and -repressed genes beyond 3 hours of starvation in the presence or absence of exogenous glorin using suspension cultures of $P$. pallidum PN500 amoebae.

Therefore, expression kinetics of selected glorin-regulated genes were determined during the first 5 hours of development under four different conditions. 5 hour duration was chosen in this experiment because it was assumed that within this time cells encounter a prominent shift from pre-aggregation to aggregation phase and may undergo major changes in gene expression patterns. $P$. pallidum PN500 amoebae were harvested in the late vegetative stage, washed to remove nutrients and suspended in phosphate buffer at a cells density of $2 \times 10^{7}$ cells $/ \mathrm{ml}$. The amoebae in suspension culture were pre-starved for 1 hour by slow shaking at $100 \mathrm{rpm}$ at $22^{\circ} \mathrm{C}$ to trigger development. After this initial treatment, cell suspension was equally divided into 5 parts. One culture of cells was not treated with glorin and acted as 'untreated control'. Each of 4 other cultures received four different glorin treatments for additional 4 hours. To one culture, $1 \mu \mathrm{M}$ of glorin was added at 10-minute intervals, whereas a second culture received $100 \mathrm{nM}$ (final concentration) glorin every 10 -minutes. The third culture was treated with $1 \mu \mathrm{M}$ (final concentration) glorin at 30-minute intervals, while fourth culture was pulsed with $100 \mathrm{nM}$ (final concentration) glorin at 30-minute intervals. All these cultures were then developed for 4 additional hours under slow shaking conditions. By this approach, the objective was to investigate how gene expression is modulated during development under suspension when starving cells are pulsed with low and high levels of glorin at varying intervals for 4 hours. $2 \times 10^{7}$ cells were harvested from glorin treated cultures after 0.5-, 1-, 2-, 3-, and 4hours of glorin treatment. Samples $\left(2 \times 10^{7}\right.$ cells per sample) were collected from untreated control culture at the same time points. Samples were generally collected 30 minutes after a pulse of glorin was applied. Total RNA was extracted to prepare cDNA for gene expression analysis using real-time RT-PCR. Relative gene expression of model genes PPL_09347, PPL_05354, PPL_05833, PPL_12271 and PPL_07908 was determined and fold changes were calculated compared to the expression in untreated cells. Four hours 
of glorin treatment represented total 5 hours of starvation ( 1 hour pre-starvation +4 hours glorin treatment).

Analyzing expression data of glorin induced gene PPL_09347 under above mentioned conditions revealed that both $1 \mu \mathrm{M}$ and $100 \mathrm{nM}$ concentrations of glorin were equally effective to induce high level gene expression when cells were pulsed frequently, i.e. at 10-minute intervals (Figure 32). $1 \mu \mathrm{M}$ glorin added every 30 minute also exhibited comparable induction of PPL_09347, whereas expression levels observed in response to $100 \mathrm{nM}$ glorin added at 30-minute intervals were relatively lower, signifying the requirement for higher concentrations of glorin to achieve optimal induction of expression (Figure 32). PPL_09347 retained high levels of expression at all 4 hours of glorin treatment.

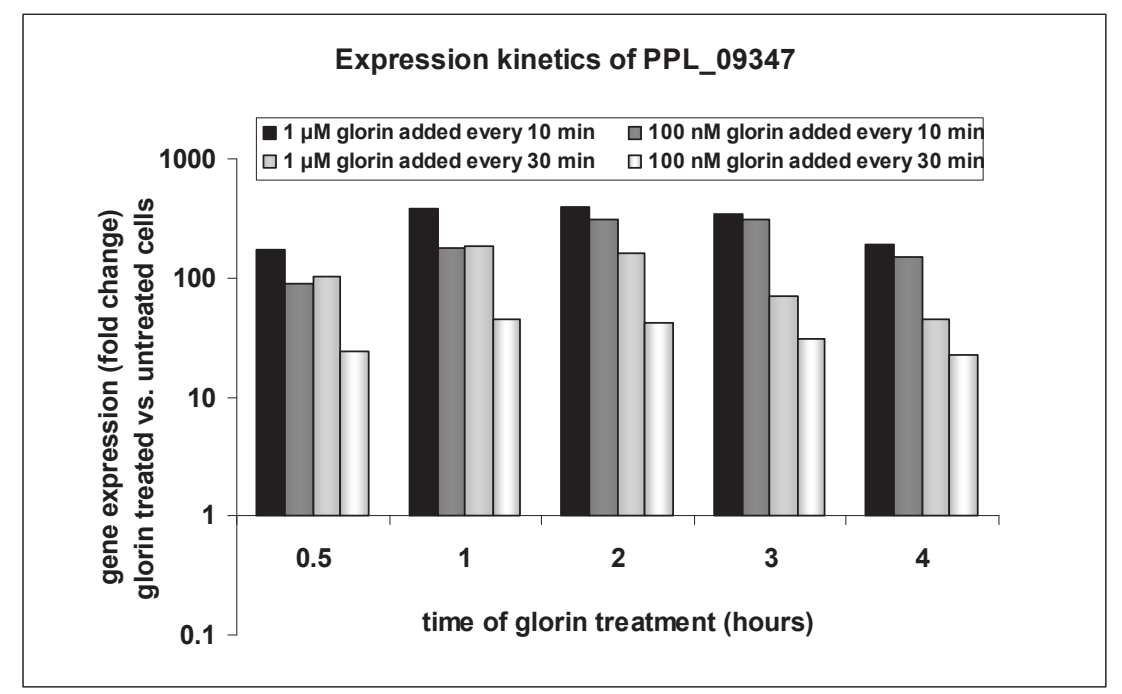

Figure 32: Glorin-induced changes in expression of PPL_09347 in response to varying concentrations and periods of exposure to glorin. P. pallidum PN500 were developed in shaking cultures for 5 hours in the presence or absence of exogenous glorin. In glorin treated cultures, beginning 1 hour after starvation, either $1 \mu \mathrm{M}$ or $100 \mathrm{nM}$ final concentration of glorin was added at 10 or 30 -minute intervals. Cell samples were collected after $0.5-, 1-, 2-, 3$ - and 4-hours of glorin treatment for total RNA extraction to analyse gene expression. Cells were generally harvested 30 minutes after a pulse of glorin was applied. Samples were collected from untreated cells at the same time points. As a control, expression of house keeping gene gpdA was examined. Relative expression of model genes PPL_09347 was determined by real-time RTPCR. Fold changes are shown. All data were compared to untreated P. pallidum PN500 cells. Fold change was set to 1 where values $>1$ represent higher expression of the gene in glorin treated cells than in untreated cells. Mean values of duplicate measurements of the same cDNA \pm SD were plotted. 4 hours of glorin treatment represented total 5 hours of starvation.

Another glorin-induced gene, PPL_05354, exhibited progressive increase in expression for the first 3 hours of glorin treatment, whereas expression levels declined drastically at $4^{\text {th }}$ hour (Figure 33). At $4^{\text {th }}$ hour of glorin treatment, low level expression of this gene was 
still detectable in cells pulsed frequently at 10-minute intervals with $1 \mu \mathrm{M}$ or $100 \mathrm{nM}$ glorin, whereas PPL_05354 showed clear down-regulation in cells treated with $1 \mu \mathrm{M}$ or $100 \mathrm{nM}$ glorin at 30-minute intervals (Figure 33). These data provide an understanding of kinetics of PPL_05354 in slow shaking cultures. There is a gradual increase in gene expression during the first 3 hours of glorin treatment followed by a steep reduction in mRNA levels. This rapid decline in expression may be explained by suggesting that starving cells may secrete glorin by themselves after a few hours of development and accumulation of glorin (exogenous glorin + glorin secreted by cells) may result in autonomous signalling, therefore, effects of externally added glorin appear less prominent.

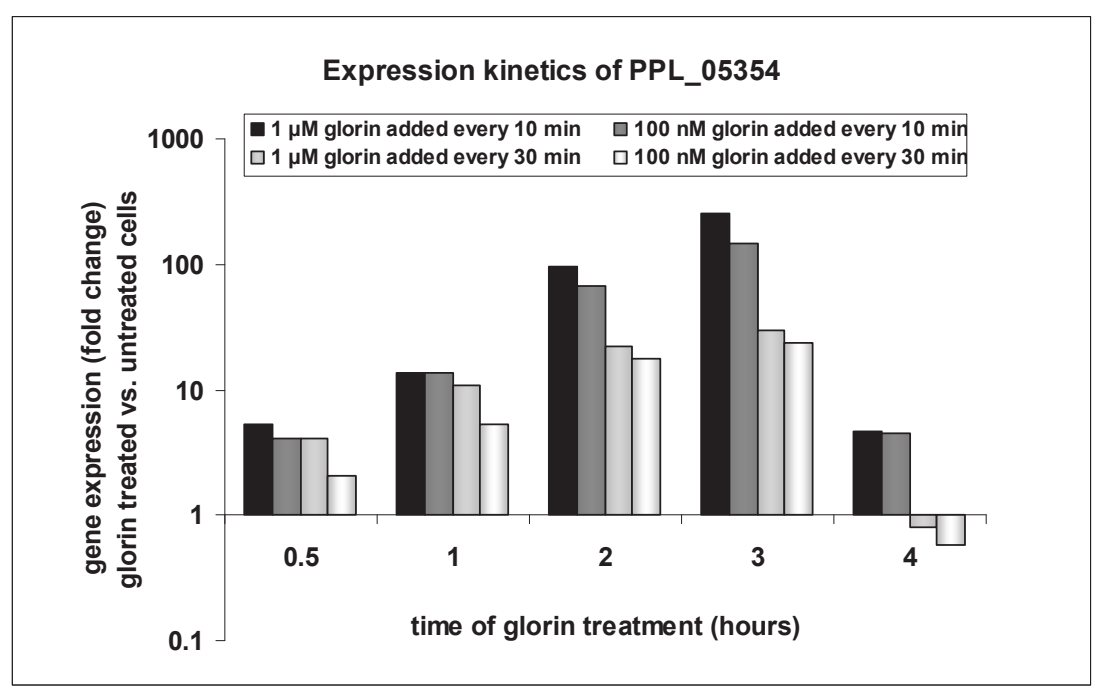

Figure 33: Glorin-induced changes in expression of PPL_05354 in response to varying concentrations and periods of exposure to glorin. $P$. pallidum PN500 were developed in shaking cultures for 5 hours in the presence or absence of exogenous glorin. In glorin treated cultures, beginning 1 hour after starvation, either $1 \mu \mathrm{M}$ or $100 \mathrm{nM}$ final concentration of glorin was added at 10 or 30-minute intervals. Cell samples were collected after 0.5-, 1-, 2-, 3- and 4-hours of glorin treatment for total RNA extraction to analyse gene expression. Cells were generally harvested 30 minutes after a pulse of glorin was applied. Samples were collected from untreated cells at the same time points. As a control, expression of house keeping gene gpdA was examined. Relative expression of model genes PPL_05354 was determined by real-time RT-PCR. Fold changes are shown. All data were compared to untreated P. pallidum PN500 cells. Fold change was set to 1 where values $>1$ represent higher expression of the gene in glorin treated cells than in untreated cells. Values $<1$ show that expression of gene is lower in glorin treated cells than in untreated cells. Mean values of duplicate measurements of the same cDNA \pm SD were plotted. 4 hours of glorin treatment represented total 5 hours of starvation.

PPL_05833 was another gene chosen from RNA-seq data of glorin-induced genes. This gene manifested very unique kinetics. It was highly induced within 30 minutes of glorin treatment, followed by progressive decline, such that only basal level expression was 
detectable after 4 hours of glorin treatment (Figure 34). These data were in agreement with the RNA-seq results which indicated that PPL_05833 exhibits rapid turn over and high-level gene expression is followed by a prompt decline. This phenomenon may be similarly explained as in case of PPL_05354 (Figure 33) by proposing that setting up of endogenous glorin signalling by starving cells may result in less significant responses to externally added glorin. Yet, a second possiblity is that product of PPL_05833 might be required only for very short period of time during aggregation, therefore expression of this gene declines quickly. However, it can not be ignored that mRNA of this gene might be unstable under shaking culture conditions leading to rapid decline in transcript levels. Induction of PPL_05833 in response to $1 \mu \mathrm{M}$ and $100 \mathrm{nM}$ glorin pulsed at 10-minute intervals was comparable and a high-level expression was detected under both of these conditions (Figure 34). Significant increase in gene expression was observed when cells were treated with $1 \mu \mathrm{M}$ glorin at 30-minute intervals. Messenger RNA of PPL_05833 accumulated to relatively lower levels in response to pulses of $100 \mathrm{nM}$ glorin delivered at 30 -minute intervals manifesting need of frequent pulsing with glorin to detect optimal gene expression responses.

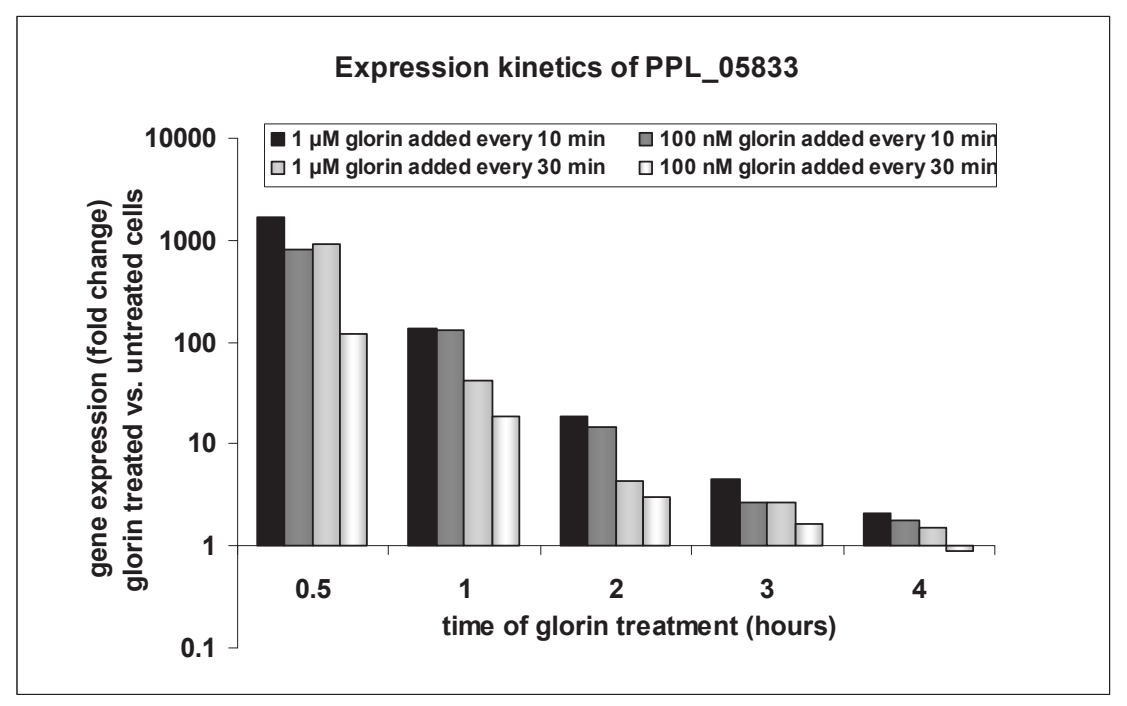

Figure 34: Glorin-induced changes in expression of PPL_05833 in response to varying concentrations and periods of exposure to glorin. P. pallidum PN500 were developed in shaking cultures for 5 hours in the presence or absence of exogenous glorin. In glorin treated cultures, beginning 1 hour after starvation, either $1 \mu \mathrm{M}$ or $100 \mathrm{nM}$ final concentration of glorin was added at 10 or 30-minute intervals. Cell samples were collected after 0.5-, 1-, 2-, 3- and 4-hours of glorin treatment for total RNA extraction to analyse gene expression. Cells were generally harvested 30 minutes after a pulse of glorin was applied. Samples were collected from untreated cells at the same time points. As a control, expression of house keeping gene gpdA was examined. Relative expression of model genes PPL_05833 was determined by real-time RT-PCR. Fold changes are shown. All data were compared to untreated P. pallidum PN500 cells. Fold change was set to 1 where values $>1$ represent higher expression of the gene in glorin treated cells than in untreated cells. Values $<1$ show that expression of gene is lower in glorin treated 
cells than in untreated cells. Mean values of duplicate measurements of the same cDNA \pm SD were plotted. 4 hours of glorin treatment represented total 5 hours of starvation.

PPL_12271 is another glorin-induced gene that encodes putative ErkB protein. This gene exhibited kinetics similar to PPL_05833 in this experiment. PPL_12271 was rapidly induced by glorin within 30-minute of glorin treatment, followed by a gradual decline, such that after 4 hours, expression of PPL_12271 droped to near background levels even in the presence of frequent glorin pulsing. These results confirm RNA-seq data that also indicated a rapid turn over of PPL_12271 (Figure 26 \& 35). Analogous to PPL_05833, the product of this gene seems to be required for short period during aggregation and may play important role in intracellular cell signalling. PPL_12271 was induced to almost comparable levels in response to $1 \mu \mathrm{M}$ and $100 \mathrm{nM}$ glorin pulsed at 10- minute intervals. $1 \mu \mathrm{M}$ glorin added every 30-minute showed similar response as displayed by pulses delivered more frequently. $100 \mathrm{nM}$ glorin pulsed at 30-minute intervals could induce PPL_12271 only at very low levels, pointing that induction of this gene may require high levels of glorin (Figure 35).

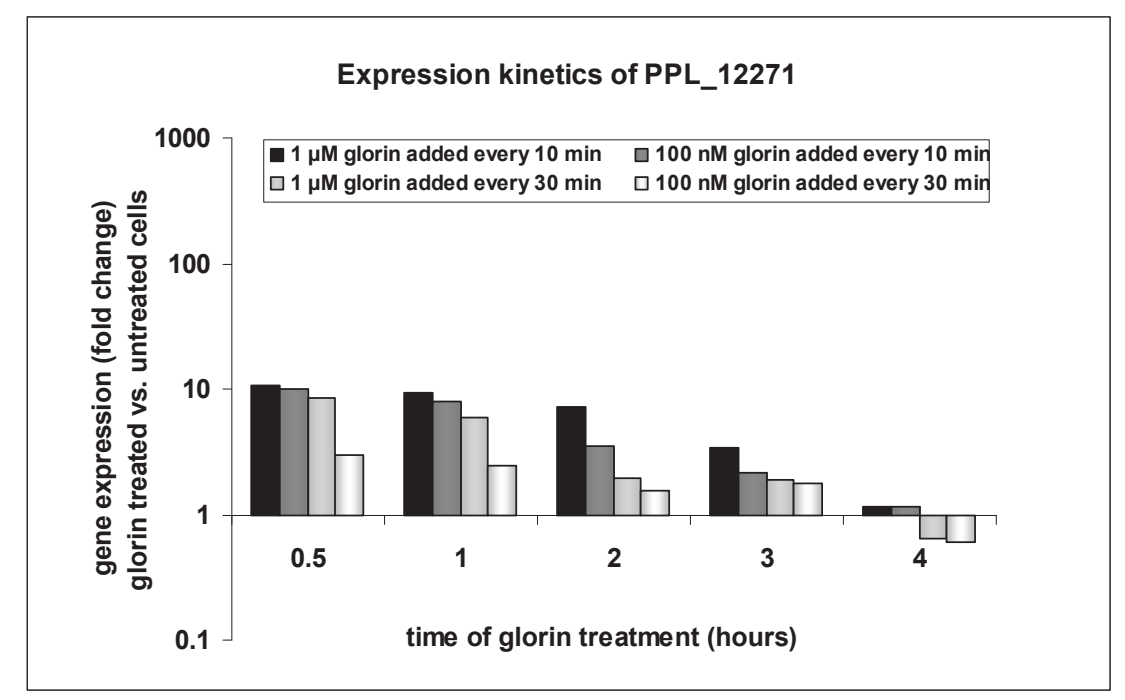

Figure 35: Glorin-induced changes in expression of PPL_12271 in response to varying concentrations and periods of exposure to glorin. P. pallidum PN500 were developed in shaking cultures for 5 hours in the presence or absence of exogenous glorin. In glorin treated cultures, beginning 1 hour after starvation, either $1 \mu \mathrm{M}$ or $100 \mathrm{nM}$ final concentration of glorin was added at 10 or 30-minute intervals. Cell samples were collected after 0.5-, 1-, 2-, 3- and 4-hours of glorin treatment for total RNA extraction to analyse gene expression. Cells were generally harvested 30 minutes after a pulse of glorin was applied. Samples were collected from untreated cells at the same time points. As a control, expression of house keeping gene gpdA was examined. Relative expression of model genes PPL_12271 was determined by real-time RT-PCR. Fold changes are shown. All data were compared to untreated P. pallidum PN500 cells. Fold change was set to 1 where values $>1$ represent higher expression of the gene in glorin treated cells than in untreated cells. Values $<1$ show that expression of gene is lower in glorin treated 
cells than in untreated cells. Mean values of duplicate measurements of the same cDNA \pm SD were plotted. 4 hours of glorin treatment represented total 5 hours of starvation.

An important comment to the data obtained with PPL_05833 and PPL_12271 is related to transient expression of these genes. RNA-seq data indicated that expression of many genes that were differentially up-regulated more than 3-fold was declined below the set threshold (3-fold expression) 1 hour later (Table 3). It was suspected that glorin levels may need to be elevated continually for the expression of this set of genes to be maintained. Therefore, when expression of some of these genes (Figure $34 \& 35$ ) was analyzed under frequent pulsing coniditons, it was found that even frequent exposure to glorin caused decline in gene expression. This provides evidence that transient changes in gene expression induced by glorin are an innate property of starving $P$. pallidum PN500 cells.

PPL_07908 is an example of a glorin-repressed gene. As indicated in figure 28, PPL_07908 is highly induced by starvation during the early hours of starvation but treatment with glorin rapidly suppresses expression of this gene. Expression data of PPL_07908 presented in Figure 36 confirmed the findings of RNA-seq analyses by showing that expression of this gene is strongly repressed by glorin during the early hours of development.

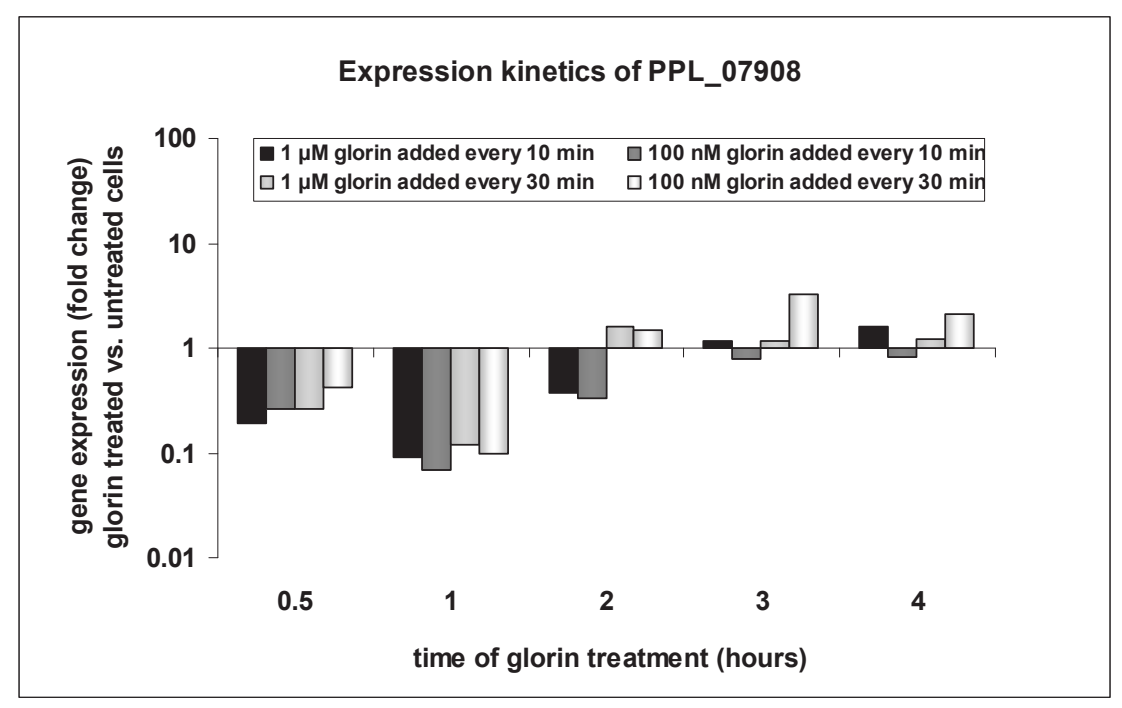

Figure 36: Glorin-induced changes in expression of PPL_07908 in response to varying concentrations and periods of exposure to glorin. P. pallidum PN500 were developed in shaking cultures for 5 hours in the presence or absence of exogenous glorin. In glorin treated cultures, beginning 1 hour after starvation, either $1 \mu \mathrm{M}$ or $100 \mathrm{nM}$ final concentration of glorin was added at 10 or 30-minute intervals. Cell samples were collected after 0.5-, 1-, 2-, 3- and 4-hours 
of glorin treatment for total RNA extraction to analyse gene expression. Cells were generally harvested 30 minutes after a pulse of glorin was applied. Samples were collected from untreated cells at the same time points. As a control, expression of house keeping gene gpdA was examined. Relative expression of model genes PPL_07908 was determined by real-time RT-PCR. Fold changes are shown. All data were compared to untreated P. pallidum PN500 cells. Fold change was set to 1 where values $>1$ represent higher expression of the gene in glorin treated cells than in untreated cells. Values $<1$ show that expression of gene is lower in glorin treated cells than in untreated cells. Mean values of duplicate measurements of the same cDNA $\pm S D$ were plotted. 4 hours of glorin treatment represented total 5 hours of starvation.

When cells were treated with $1 \mu \mathrm{M}$ or $100 \mathrm{nM}$ glorin (at 10 or 30 -minutes intervals) for 1 hour, expression of PPL_07908 declined to insignificant levels. As shown in Figure 36, expression of PPL_07908 remained downregulated in response to frequent pulsing of glorin ( $1 \mu \mathrm{M}$ or $100 \mathrm{nM}$ glorin pulsed at 10-minute intervals) for 4 hours, as indicated by gene expression levels after 2-, 3- and 4- hours of glorin treatment in Figure 36. However, when cells were pulsed with $100 \mathrm{nM}$ glorin at 30-minute intervals, low level expression of PPL_07908 was detected after 3 and 4 hours of treatment. These data may indicate that expression of PPL_07908 is repressed by high concentrations of glorin, whereas low level expression of this gene is recovered when intensity of glorin pulses is reduced.

Alternatively, these results demonstrate that to maintain down-regulation of PP_07908 for longer period, comparatively higher levels of glorin are required at shorter intervals. This gene may be an immediate early developmental gene induced by starvation and suppression of this gene by glorin indicates that products of this gene may not be necessary during aggregation.

\subsubsection{Temporal expression pattern of glorin responsive genes during development in shaken suspensions}

After determining relationships among glorin pulse concentration, pulsing interval and related gene expression changes, next the developmental kinetics of glorin responsive genes were determined in $P$. pallidum PN500 amoebae developed in shaken cultures over longer periods of time (i.e. more than 5 hours) to get an insight into how gene expression patterns develop when cells are starved in buffer suspensions. For this experiment, two different concentrations of glorin, i.e. $1 \mu \mathrm{M} \& 100 \mathrm{nM}$ were chosen and time-course effects of frequent glorin pulsing were examined on gene expression patterns of selected glorin-induced genes during the first 8 hours of development. 8 hour duration was selected to cover the complete period of early development in suspension cultures; however, chemotactic aggregation of cells is prevented under these conditions. 
P. pallidum PN500 cells were grown in association with bacteria, harvested in the late vegetative stage, washed and resuspended in phosphate buffer at a concentration of $2 \times 10^{7}$ cells $/ \mathrm{ml}$. A pellet of $2 \times 10^{7}$ cells was collected for total RNA isolation that represented 'growing cells control' for subsequent gene expression analyses. Cells in suspension cultures were pre-starved for 1 hour under slow shaking conditions (i.e. $100 \mathrm{rpm}$ ) at $22^{\circ} \mathrm{C}$ to initiate development. After 1 hour of starvation, cell suspension was divided into 3 parts. One culture served as 'untreated control' and did not receive glorin treatment. Second culture was treated with $1 \mu \mathrm{M}$ glorin every 10-minutes, whereas in third culture $100 \mathrm{nM}$ glorin was added at 10-minute intervals. These cultures were developed under shaking conditions at $22^{\circ} \mathrm{C}$ for 8 additional hours. Samples (each containing $2 \times 10^{7}$ cells) were collected from glorin-treated cultures starting at 0.5 hour of glorin treatment until total 8 hours for RNA extraction. Samples were collected from untreated cell cultures at the same time points. Cell samples were generally collected 30- minutes after a pulse of glorin was delivered. cDNA was syntheiszed and gene expression analyses were carried out using quantitative RT-PCR. Relative gene expressions of selected glorin-induced genes PPL_09347, PPL_05354, PPL_05833, PPL_12271 and a glorin repressed gene PPL_07908 were determined and fold changes were calculated compared to expression in growing cells. 8 hours of glorin treatment corresponded to 9 hours of starvation (1 hour pre-starvation +8 hours glorin treatment).

\subsubsection{Class I: Genes stably induced by glorin in shaking cultures}

\subsection{Expression profile of PPL_09347}

When expression kinetics of model gene PPL_09347 were analyzed in cells developed in the absence of glorin treatment, only basal level expression of this gene could be detected at 1.5 hour of development. Expression was then maintained at low levels for the next 4 hours (Figure 37). 


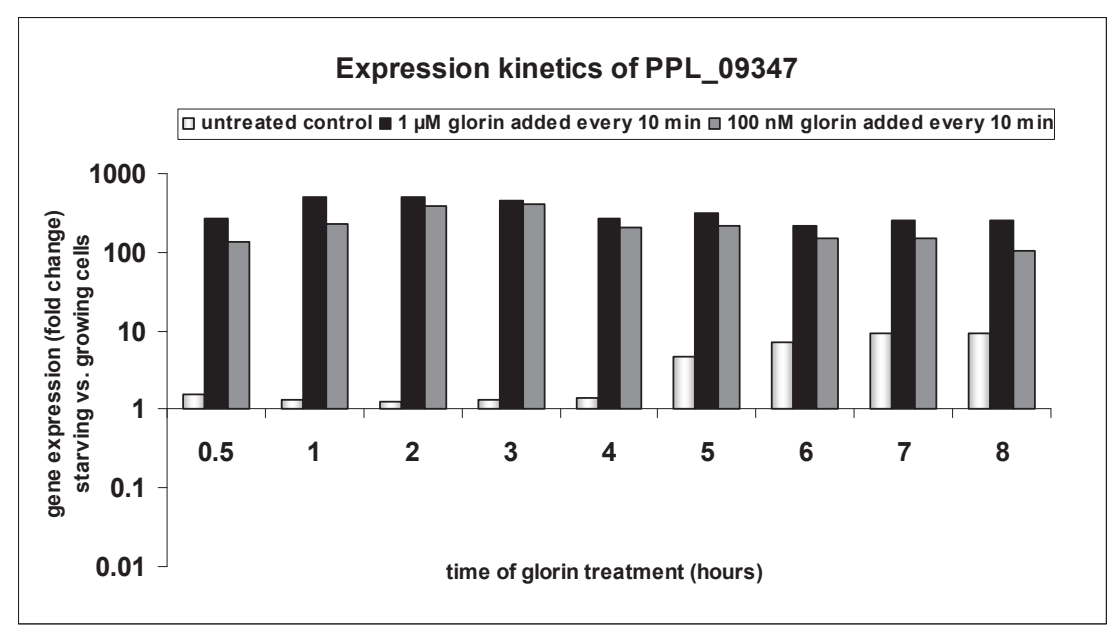

Figure 37: Time course of glorin effects on PPL_09347 expression. P. pallidum PN500 were developed in shaking buffer suspensions for 8 hours in the presence or absence of exogenous glorin. In glorin treated cultures, beginning 1 hour after starvation, either $1 \mu \mathrm{M}$ or $100 \mathrm{nM}$ final concentration of glorin was added at 10-minute intervals. Cell samples were collected at indicated time points after glorin treatment for total RNA extraction to analyse gene expression. Cells were generally harvested 30 minutes after a pulse of glorin was applied. Samples were collected from untreated cells at the same time points. As a control, expression of house keeping gene gpdA was examined. Relative expression of model genes PPL_09347 was determined by real-time RT-PCR. Fold changes are shown. All data were compared to growing $P$. pallidum PN500 cells. Fold change was set to 1 where values $>1$ represent higher expression of the gene in starving cells than in growing cells. Values $<1$ show that expression of gene is lower in starving cells than in growing cells. Mean values of duplicate measurements of the same cDNA \pm SD were plotted.

Levels of PPL_09347 mRNA started to rise in untreated cells after 5 hours of development and continued to increase gradually for the next hours of observation. These time points may correspond to aggregation time during normal development on agar. Only basal level expression of PPL_09347 during the first 4 hours of development may indicate that product of this gene is not needed in the first few hours of starvation.

When cells were pulsed with glorin every 10 minutes, mRNA of PPL_09347 accumulated rapidly within 30-minutes ( 0.5 hour of glorin treatment; Figure 37 ) and 265 - and 136.32fold induction was detected in response to $1 \mu \mathrm{M}$ and $100 \mathrm{nM}$ glorin pulses, respectively, delivered at 10-minute intervals. In the presence of glorin stimulus, high-level expression of PPL_09347 was maintained in the next 8 hours of observation. Gene induction in response to both concentrations of glorin used ( $1 \mu \mathrm{M} \& 100 \mathrm{nM})$ was comparable at all time points. These data indicate that PPL_09347 is constantly induced by glorin to high levels. These results also show that within a few hours of development in suspensions, 
cells may establish endogenous glorin signalling (referred to the expression kinetics of PPL_09347 in untreated control shown in Figure 37) and start to secrete glorin themselves; however, these signalling may not reach the same level as in glorin-pulsed cultures (Figure 37).

\subsection{Expression profile of PPL_05354}

When gene expression data collected from $P$. pallidum PN500 cells starved in shaking suspensions for 8 hours was analyzed, it was noticed that in the absence of glorin pulses, PPL_05354 exhibited nearly negligible expression during the first 6 hours of development in shaking suspensions. A slight increase in the expression was observed starting at 7 hours of starvation, while 3.03-, 5.5-, and 10.7-fold expression levels were detected at 7-, 8- and 9-hour of development, respectively (Figure 38). These results suggest that PPL_05354 is gradually induced in the course of development.

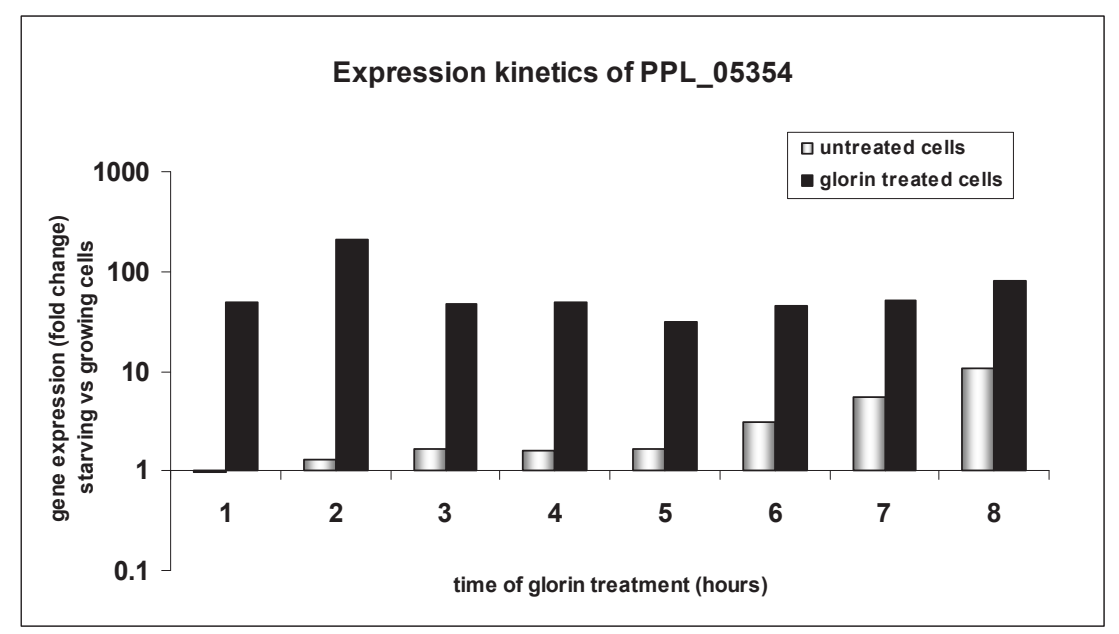

Figure 38: Time course of glorin effects on PPL_05354 expression. P. pallidum PN500 were developed in shaking buffer suspensions for 8 hours in the presence or absence of exogenous glorin. In glorin treated cultures, beginning 1 hour after starvation, $1 \mu \mathrm{M}$ final concentration of glorin was added at 30-minute intervals and cell samples were collected at indicated time points after glorin treatment for total RNA extraction to analyse gene expression. Cells were generally harvested 30 minutes after a pulse of glorin was applied. Samples were collected from untreated cells at the same time points. As a control, expression of house keeping gene gpdA was examined. Relative expression of model gene PPL_05354 was determined by real-time RT-PCR. Fold changes are shown. All data were compared to growing P. pallidum PN500 cells and fold change was set to 1 where values $>1$ represent higher expression of the gene in starving cells than in growing cells. Values $<1$ show that expression of gene is lower in starving cells than in growing cells. Mean values of duplicate measurements of the same cDNA \pm SD were plotted. 
When cells were treated with $1 \mu \mathrm{M}$ glorin at 30-minute intervals, PPL_05354 was induced rapidly, such that 48.5-fold expression (compared to growing cells) could be detected within 1 hour of glorin treatment (Figure 38). After stimulation of cells with glorin for 2 hours, this gene was further up-regulated to 210.83 -fold followed by a decline in expression to 47.17-, 48.5- and 31.12-fold at 3-, 4-, and 5-hours of glorin treatment, respectively (Figure 38). A slight increase in expression was noticed at 6- and 7-hours of glorin treatment, whereas 82.13-fold expression was detected in cells treated with glorin for 8 hours (Figure 38). Effects of pulsing $100 \mathrm{nM}$ glorin were not detected. These data indicate that PPL_05354 is rapidly induced by glorin to high levels and gene expression in response to exogenous glorin was stable over 8 hours of observation.

\subsubsection{Class II: Genes induced by starvation, whereas exogenous glorin pulses resulted in their precocious expression}

\subsection{Expression profile of PPL_05833}

Real-time RT-PCR data analyses showed that PPL_05833 was expressed to insignificant levels during the first 3 hours of development in shaking culture (Table 3; Figure 27). In response to starvation in suspension culture, a slight increase in the expression of PPL_05833 was observed starting at 4 hours of development, whereas 10.41-fold expression was detected at 7 hours of starvation (Figure 39). Expression was then maintained at approximately the same levels in the next hours of development (Figure 39). It may indicate that products of this gene are not required during the early hours of starvation. RNA-seq analyses predicted that PPL_05833 is a gene that is transiently induced by glorin (Figure 27). This experiment verified that PPL_05833 is rapidly induced by glorin to high levels within 30 minutes, follwed by a steep decline, such that after 2 hours of glorin treatment only a low level expression could be detected (Figure 39). 


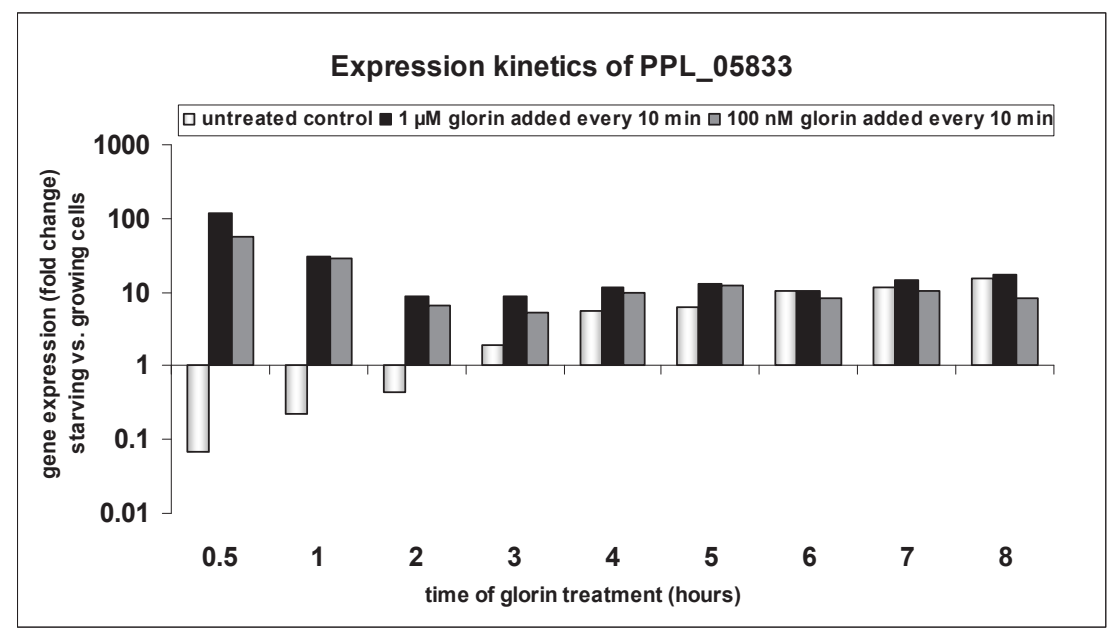

Figure 39: Time course of glorin effects on PPL_05833 expression. P. pallidum PN500 were developed in shaking buffer suspensions for 8 hours in the presence or absence of exogenous glorin. In glorin treated cultures, beginning 1 hour after starvation, either $1 \mu \mathrm{M}$ or $100 \mathrm{nM}$ final concentration of glorin was added at 10-minute intervals. Cell samples were collected at indicated time points after glorin treatment for total RNA extraction to analyse gene expression. Cells were generally harvested 30 minutes after a pulse of glorin was applied. Samples were collected from untreated cells at the same time points. As a control, expression of house keeping gene gpdA was examined. Relative expression of model genes PPL_05833 was determined by real-time RT-PCR. Fold changes are shown. All data were compared to growing $P$. pallidum PN500 cells. Fold change was set to 1 where values $>1$ represent higher expression of the gene in starving cells than in growing cells. Values $<1$ show that expression of gene is lower in starving cells than in growing cells. Mean values of duplicate measurements of the same cDNA \pm SD were plotted.

Effects of pulsing $1 \mu \mathrm{M}$ or $100 \mathrm{nM}$ glorin were comparable. These data validate observations made in previous section of this study (Section 3.8.2; Figure 34) that PPL_05833 is induced in response to glorin only for a short period, afterwards expression is retained at basal levels; supporting the suggestion that products of this gene may not be needed for long time during aggregation.

\subsection{Expression profile of PPL_12271}

PPL_12271 (erkB) is a gene that displayed kinetics similar to PPL_05833. In the absence of glorin pulses only very few transcripts of this gene were detected during the first 4 hours of development in suspension cultures. A low level expression was noticed at 5 hour of starvation that was maintained almost at the same levels for the next hours of development (Figure 40). 


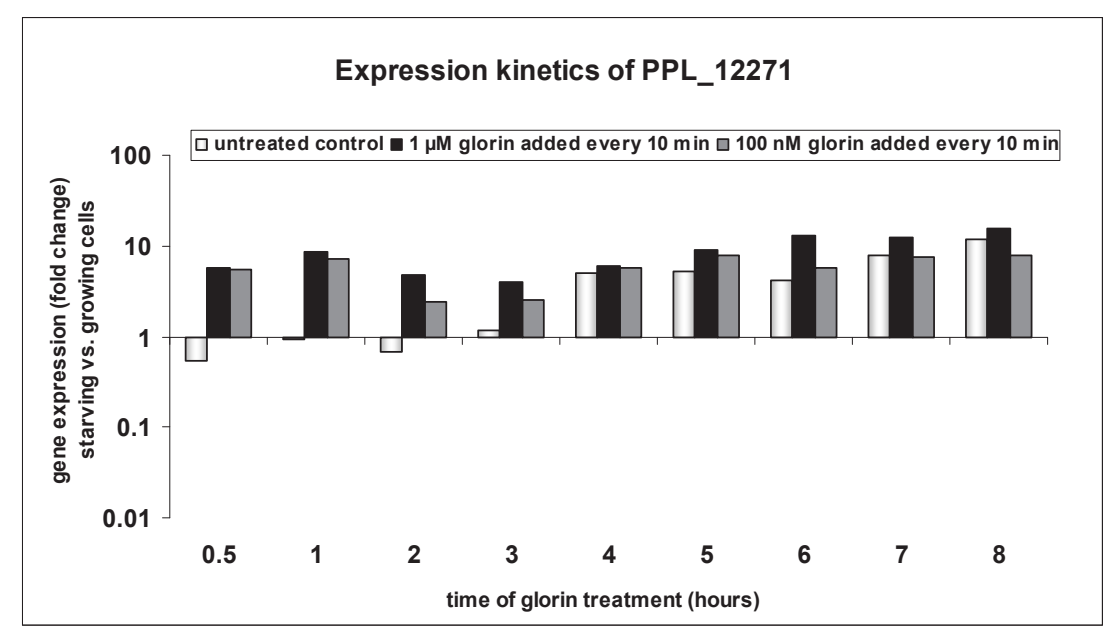

Figure 40: Time course of glorin effects on PPL_12271 expression. P. pallidum PN500 were developed in shaking buffer suspensions for 8 hours in the presence or absence of exogenous glorin. In glorin treated cultures, beginning 1 hour after starvation, either $1 \mu \mathrm{M}$ or $100 \mathrm{nM}$ final concentration of glorin was added at 10-minute intervals. Cell samples were collected at indicated time points after glorin treatment for total RNA extraction to analyse gene expression. Cells were generally harvested 30 minutes after a pulse of glorin was applied. Samples were collected from untreated cells at the same time points. As a control, expression of house keeping gene gpdA was examined. Relative expression of model genes PPL_12271 was determined by real-time RT-PCR. Fold changes are shown. All data were compared to growing $P$. pallidum PN500 cells. Fold change was set to 1 where values $>1$ represent higher expression of the gene in starving cells than in growing cells. Values $<1$ show that expression of gene is lower in starving cells than in growing cells. Mean values of duplicate measurements of the same cDNA \pm SD were plotted.

As shown in figure 40, PPL_12271 was induced within 30-minutes in the presence of 1 $\mu \mathrm{M}$ or $100 \mathrm{nM}$ pulses of glorin. Expression declined briefly at 2 and 3 hours of glorin treatment. A slight increase in expression of this gene was detected at 4 hour of stimulation with glorin that was maintained at low levels in the next hours. $1 \mu \mathrm{M}$ and 100 $\mathrm{nM}$ concentrations of glorin used for pulsing were almost equally effective. PPL_12271 exhibits rapid changes in expression during the early hours of starvation in response to glorin treatment (Figure $26 \& 40$ ); indicating that activity of this gene may be required only for short time during aggregation. 


\subsubsection{Class III: Gene induced by starvation, whereas glorin pulses repressed their expression during the early hours of development}

\subsection{Expression profile of PPL_07908}

PPL_07908 is a starvation induced early gene that was shown to be repressed by glorin treatment earlier in this study (Section 3.6.2.1.4 Figure 28; Figure 36).

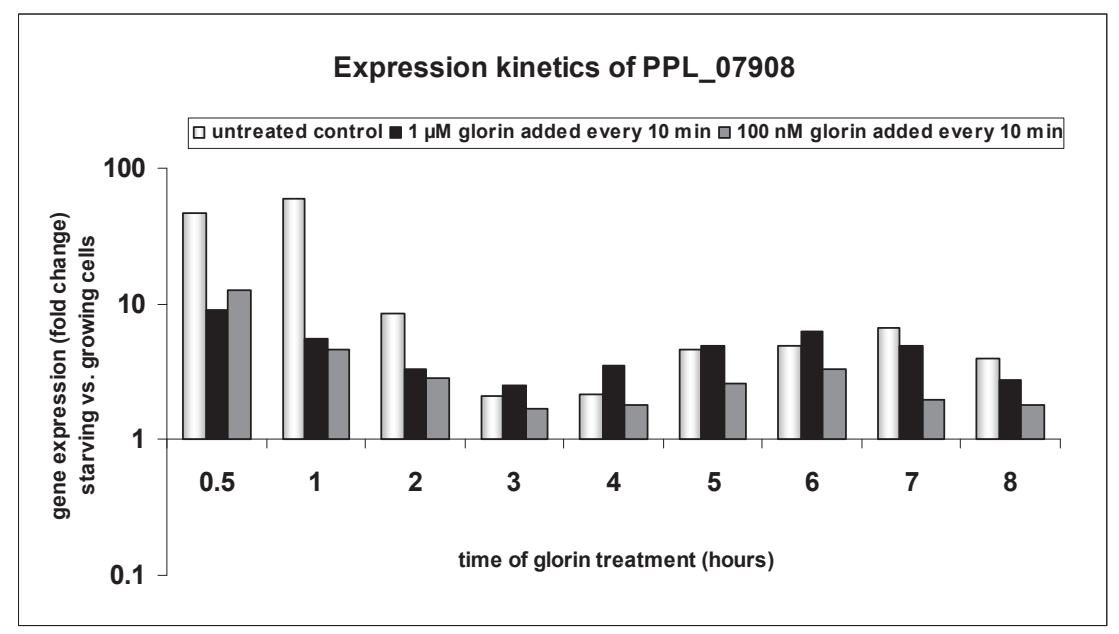

Figure 41: Time course of glorin effects on PPL_07908 expression. P. pallidum PN500 were developed in shaking buffer suspensions for 8 hours in the presence or absence of exogenous glorin. In glorin treated cultures, beginning 1 hour after starvation, either $1 \mu \mathrm{M}$ or $100 \mathrm{nM}$ final concentration of glorin was added at 10-minute intervals. Cell samples were collected at indicated time points after glorin treatment for total RNA extraction to analyse gene expression. Cells were generally harvested 30 minutes after a pulse of glorin was applied. Samples were collected from untreated cells at the same time points. As a control, expression of house keeping gene gpdA was examined. Relative expression of model genes PPL_07908 was determined by real-time RT-PCR. Fold changes are shown. All data were compared to growing $P$. pallidum PN500 cells. Fold change was set to 1 where values $>1$ represent higher expression of the gene in starving cells than in growing cells. Values $<1$ show that expression of gene is lower in starving cells than in growing cells. Mean values of duplicate measurements of the same cDNA \pm SD were plotted.

As shown in figure 41, this gene was rapidly induced in response to starvation in cells developed in shaking suspensions. High expression levels of this gene were noticed for first 2 hours of starvation followed by a steep decline, such that at 4 and 5 hours of development (in the absence of glorin), only basal level expression was detected. A slight increase in expression was observed beginning at 6 hours of starvation that was then maintained at the same level in the next hours. These data add to the suggestion 
that PPL_07908 is an early developmental gene required during the initial hours of starvation only.

Pulses of glorin delivered at 10-minutes intervals led to a rapid decline in the expression of PPL_07908, such that only basal level of expression was detected at 3 and 4 hours of glorin treatment. Afterwards (at 5 to 8 hours of glorin treatment) this gene was expressed at low levels; almost similar expression levels were detected both in glorin-treated and untreated cells at these time points (Figure 41). $1 \mu \mathrm{M}$ and $100 \mathrm{nM}$ glorin pulses were comparably effective during the first 3 hours of glorin treatment. Responses to both concentrations of glorin were very slightly different at later stages.

\subsubsection{Class IV: Genes repressed by starvation, whereas glorin pulses induce their expression in shaking cultures}

\subsection{Expression profile of PPL_12248}

As shown in Figure 29, RNA-seq data indicated that PPL_12248 is a gene that is repressed by starvation but glorin pulses induce its expression. In experiment presented in Figure 42, when P. pallidum PN500 amoebae were starved in shaking suspensions for 8 hours, continuous down-regulation of PPL_12248 was noticed over the whole time period confirming the results of RNA-seq analyses that this gene is negatively regulated by starvation (Figure 29 \& Figure 42).

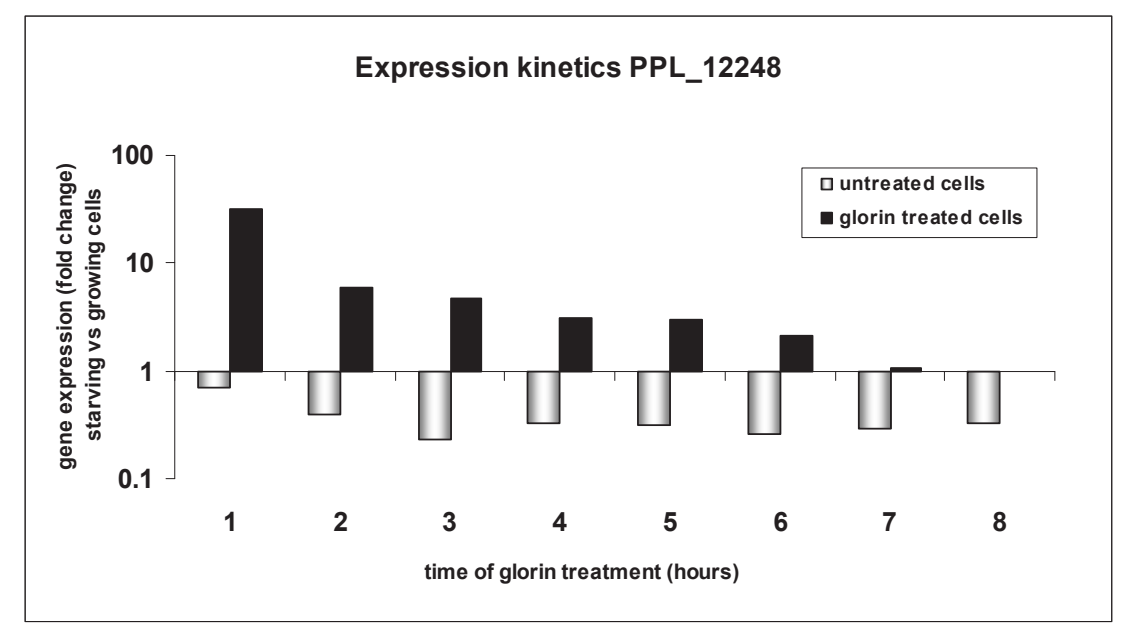

Figure 42: Time course of glorin effects on PPL_12248 expression. P. pallidum PN500 were developed in shaking buffer suspensions for 8 hours in the presence or absence of exogenous 
glorin. In glorin treated cultures, beginning 1 hour after starvation, $1 \mu \mathrm{M}$ final concentration of glorin was added at 30-minute intervals and cell samples were collected at indicated time points after glorin treatment for total RNA extraction to analyse gene expression. Cells were generally harvested 30 minutes after a pulse of glorin was applied. Samples were collected from untreated cells at the same time points. As a control, expression of house keeping gene gpdA was examined. Relative expression of model gene PPL_12248 was determined by real-time RT-PCR. Fold changes are shown. All data were compared to growing $P$. pallidum PN500 cells and fold change was set to 1 where values $>1$ represent higher expression of the gene in starving cells than in growing cells. Values $<1$ show that expression of gene is lower in starving cells than in growing cells. Mean values of duplicate measurements of the same cDNA \pm SD were plotted.

In comparison, when cells were pulsed with $1 \mu \mathrm{M}$ glorin at 30 -minute intervals, transient increase in expression was noticed. Within one hour of treatment with glorin, expression of this gene increased by 31.55 -fold. Expression decreased gradually thereafter, such that 5.93-, 4.65-, 3.13-, 3.03-, 2.12-, 1.06- and 1-fold expression was detected at 2-, 3-, 4-, 5-, 6-, 7- \& 8-hours of glorin treatment, respectively (Figure 42). Effects of pulsing 100 $\mathrm{nM}$ glorin were not studied. These data indicate that expression of PPL_12248 is highly induced by glorin for short time period, illustrating a possible short-term activity of this gene during aggregation.

\subsection{Expression profile of PPL_12249}

PPL_12249 is a gene that exhibits expression kinetics similar to PPL_12248. Upon starvation of $P$. pallidum PN500 amoebae in shaking cultures for 8 hours, a constant down-regulation of PPL_12249 gene expression was observed (Figure 43) demonstrating that this gene is developmentally repressed and may represent a 'growing stage gene'. While analyzing absolute expression levels of this gene (Table 3 ), it could be noticed that PPL_12249 transcripts were present in growing cells, however, at the onset of starvation number of transcripts declined confirming that development leads to down-regulation of this gene. 


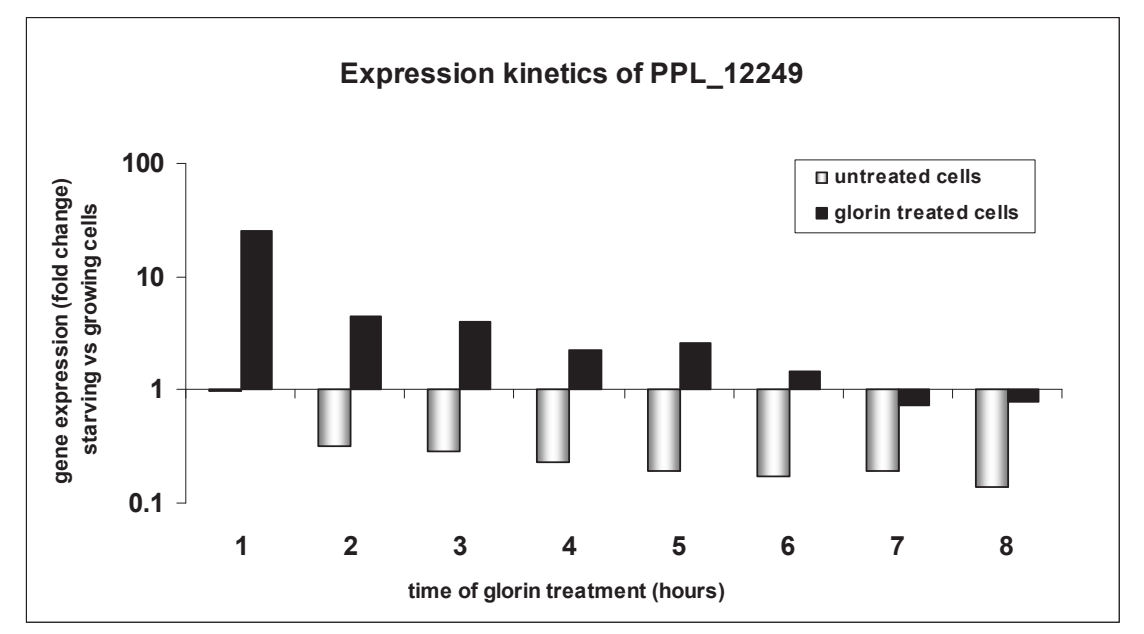

Figure 43: Time course of glorin effects on PPL_12249 expression. P. pallidum PN500 were developed in shaking buffer suspensions in the presence or absence of exogenous glorin. In glorin treated cultures, beginning 1 hour after starvation, $1 \mu \mathrm{M}$ final concentration of glorin was added at 30-minute intervals for an additional 8 hours and cell samples were collected at indicated time points for total RNA extraction to analyse gene expression. Cells were generally harvested 30 minutes after a pulse of glorin was applied. Samples were collected from untreated cells at the same time points. As a control, expression of house keeping gene gpdA was examined. Relative expression of model gene PPL_12249 was determined by real-time RT-PCR. Fold changes are shown. All data were compared to growing P. pallidum PN500 cells and fold change was set to 1 where values $>1$ represent higher expression of the gene in starving cells than in growing cells. Values $<1$ show that expression of gene is lower in starving cells than in growing cells. Mean values of duplicate measurements of the same cDNA \pm SD were plotted.

P. pallidum PN500 cells exhibited a high-level expression of PPL_12249 after 1 hour of glorin treatment $(1 \mu \mathrm{M}$ glorin delivered at 30-minute intervals) and 25.28-fold upregulation could be observed. Gene expression declined sharply at later time points but was still detectable until 6 hours of glorin treatment; expression was down-regulated afterwards. 4.5-, 3.97-, 2.28-, 2.56-, and 1.46-fold gene expression was detected at 2-, 3-, 4-, 5- and 6-hours of glorin treatment (Figure 43). Effects of pulsing $100 \mathrm{nM}$ glorin were not tested. Transient induction of PPL_12249 to high levels by exogenous glorin indicates that this gene may play a short-term but rather significant role in glorin signaling during aggregation. As shown in Figure 43, continuous supression of PPL_12249 by starvation over the course of observation (i.e. 8 hours) indicates that this gene may be a 'vegetative stage gene'. However, glorin treatment transiently induced expression of PPL_12249 to high levels; pointing to the possibility that momentary activity of this gene may be required during aggregation also though this gene may function mainly during growth phase of cells. 
Overall, these data suggest that suspension cultures provide an efficient setting to study glorin-induced changes in gene expression patterns in starving $P$. pallidum PN500 amoebae and cells successfully complete early developmental changes.

\subsubsection{Developmental time course of gene expression in cells starved on non-nutrient agar plates}

$P$. pallidum PN500 development is a highly synchronous process and is accompanied by a series of coordinated morphological and physiological changes. It starts with the transition from growth to aggregation that is induced by starvation. The major morphological change occurs when the amoebae begin to aggregate in response to communication with a diffusible chemoattractant that may be glorin as suggested by experiments performed in this study (described under Section 3.4.1.1 \& 3.6.2). The activity of cells and chemotaxis to acrasin mediate the aggregation of groups of cells into loose aggregates, followed by continuous strong streaming movements towards aggregation centres leading to the formation of tipped aggregates (Figure 44).

After studying developmental regulation of glorin-responsive genes in $P$. pallidum PN500 cells starved in shaking suspensions, the temporal expression patterns of selected glorin-induced genes were examined in cells developing on agar surface because development of amoebae on solid substrata is closer to the natural conditions than developing in a shaking culture. With this approach, the aim was to analyze distinct changes in gene expression patterns associated with morphological transitions and to determine which developmental event correspond to maximum increase in expression of glorin-regulated genes.

P. pallidum PN500 cells were grown in association with bacteria on 1/5 SM agar plates. When plates were clear, cells were collected, washed and resuspended in phosphate buffer at concentration of $2 \times 10^{7}$ cells $/ \mathrm{ml}$. A pellet of $2 \times 10^{7}$ vegetative stage cells was collected for total RNA extraction and served as 'growing cells control' for gene expression studies. Cells were spread as monolayers on non-nutrient agar plates at a density of $8 \times 10^{5} \mathrm{cells} / \mathrm{cm}^{2}$ for development. Cells $\left(2 \times 10^{7} /\right.$ sample) were harvested at 9 different stages of early development including initial 1-4 hours of starvation (the preaggregation stages) and distinct morphological states indicated by loose aggregates (5 hour), aggregates (6.5 hour), streaming (8.5 hour), late aggregates (10 hour) and mounds (13.5 hour) in Figure 44. 


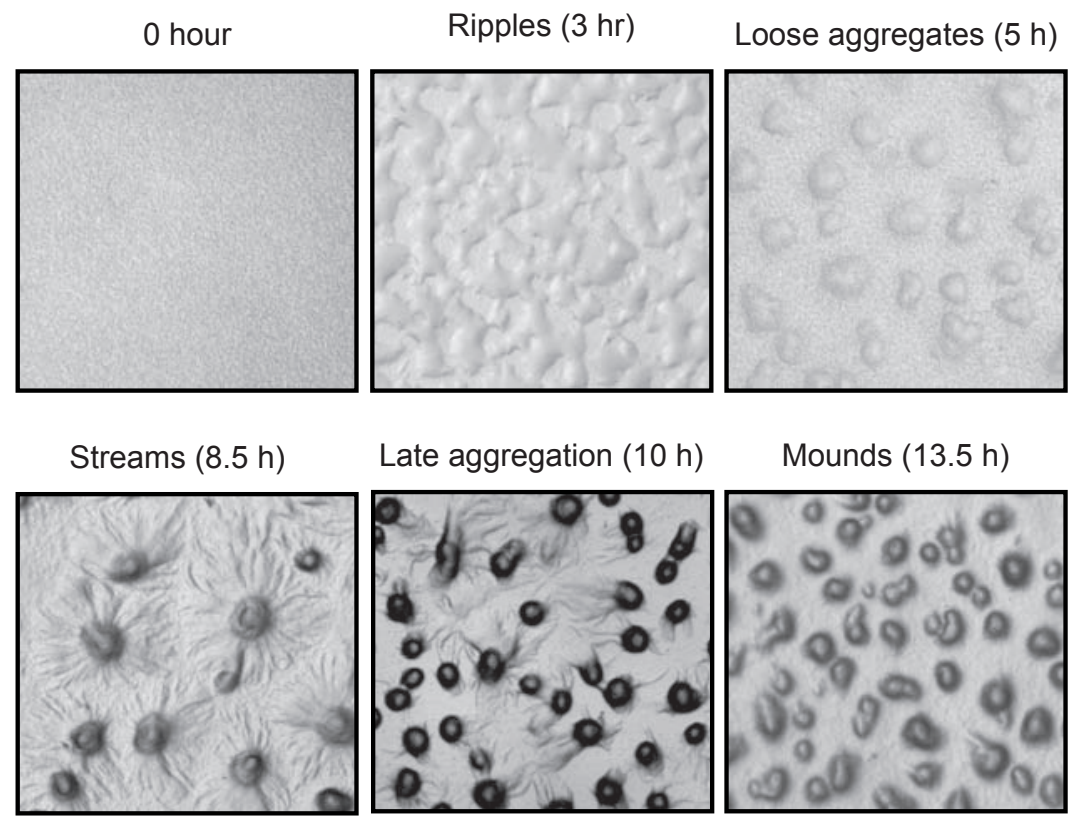

Figure 44: Prominent morphological states during growth-to-aggregation transition of starving $P$. pallidum PN500 amoebae. A top view of cells developing on non-nutrient agar is shown. No multicellular structures were visible at 0 hour. Ripples ( 3 hours), loose aggregates (5 hours), streams ( 8.5 hours), late aggregation (10 hours), and mounds (13.5 hours) are shown.

Using this strategy, the goal was to encompass all steps of growth-to-aggregation transition for analyzing temporal expression of glorin responsive genes. Total RNA was extracted from samples that were collected beginning at the onset of starvation until formation of mounds and temporal gene expression profiles were analyzed for selected glorin-regulated genes.

Genes including PPL_09347, PPL_05354, PPL_11763, PPL_00912, PPL_03541 and PPL_06644 were found to be developmentally regulated exhibiting maximum changes in gene expression between 4 and 8 hours of development; time points which correspond to the transition from unicellular to multicellular organization and are accompanied by most dramatic morphological changes in development of $P$. pallidum PN500. Genes such as PPL_12271 and PPL_ 05833 were expressed at low levels during the early hours of development, whereas PPL_12248 and PPL_03784 were expressed at basal levels approximately at all 9 developmental states that were monitored in this study. It has been suggested that individual group of genes expressed in the same manner may represent specialized patterns in the course of development (Loomis \& Shaulsky 2011). It is also proposed that a gene whose expression increases by at least four fold during 
development probably encodes a protein that is likely to provide selective advantage under one or another condition (Loomis \& Shaulsky 2011).

The results are interpreted on gene-by-gene basis in the following sections.

\subsubsection{Class I: Aggregation stage specific genes}

\subsection{Developmental kinetics of PPL_09347}

RNA-seq data indicated that bacterially grown vegetative-stage $P$. pallidum PN500 cells accumulate a low level of PPL_09347 mRNA (Table 3), however, as shown in Figure 45, development on agar led to a gradual increase of the mRNA level which reached a peak at 10 hour of development that corresponds to late aggregation stage (Figure 44). Within first 3 hours of development, only basal level expression could be detected. Expression levels increased thereafter, such that at 6.5 hours of development when aggregation was in process, 12.89-fold expression (compared to growing cells) could be detected that continued to increase during streaming. Maxmium level expression i.e. 30.27-fold was noticed with the formation of late aggregates, whereas expression levels declined to 22.16-fold when mounds were observed.

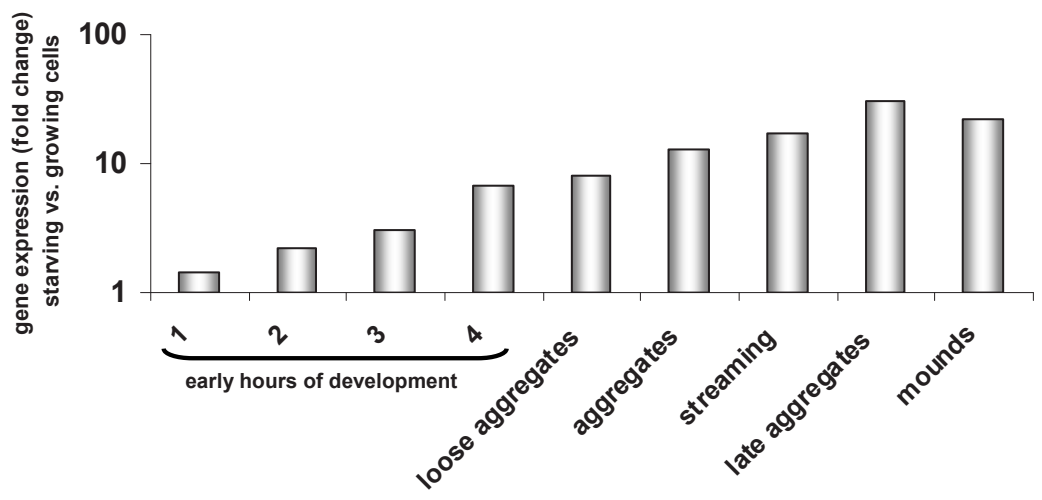

Figure 45: Developmental regulation of PPL_09347. P. pallidum PN500 cells grown in association with bacteria were harvested in the late vegetative stage, washed, and resuspended in phosphate buffer. Cells were then starved on phosphate agar plates at a density of $8 \times 10^{5} \mathrm{cells} / \mathrm{cm}^{2}$. Cell samples were collected at indicated developmental stages for total RNA extraction. Relative expression of model gene PPL_09347 was determined by real-time RT-PCR. As a control, expression of house keeping gene $g \bar{p} d A$ was examined. Fold changes are shown. All data were compared to growing P. pallidum PN500 cells. Fold change was set to 1 where values $>1$ represented higher expression of the gene in starving cells than in growing cells. Mean values of duplicate measurements of the same cDNA \pm SD were plotted. 
These data indicate that under natural conditions, the expression level of PPL_09347 starts to increase when cells shift from pre-aggregation to aggregation phase and continues to rise during streaming until formation of tight aggregates. These data confirm RNA-seq findings that also detected only basal level expression during the early hours of development (Figure 25). Under shaking culture conditions, in the absence of glorin, mRNA level of PPL_09347 started to rise at 6 hour of development and 9.18-fold expression was detected by 8 hour of development that was maintained at the same level in the next hour (Figure 37). No more increase in gene expression could be noticed under shaking conditions, indicating that normal level expression of this gene (as indicated in Figure 45) may require sufficient cell-cell contacts. Moreover, high level induction of this gene by glorin under shaking culture conditions suggested that this gene is developmentally regulated by glorin. On agar surface, continuous up-regulation of PPL_09347 during the entire aggregation process further validates the indication that cells might have fully established glorin signalling during aggregation phase leading to maximum level induction of PPL_09347.

\subsection{Developmental kinetics of PPL_03541}

In $P$. pallidum PN500 amoebae developed on phosphate agar plates, basal-level expression of PPL_03541 was detected during the first 3 hours of starvation. Moderate increase in expression of this gene was detected at 4- and 5-hour of development (Figure 46). Expression of this gene continued to rise, reached a peak in actively aggregating cells and was then maintained at moderate levels in cells forming streams and tight aggregates (Figure 46). Thus, in the early phase of development of $P$. pallidum, there seems to be a close temporal correlation between accumulation of mRNA of PPL_03541 and the appearance of aggregates. A slight decline in expression was noticed thereafter (Figure 46). Expression levels detected in cells starved for 1-, 2and 3-hours were 1.5-, 2.43- and 2.81-fold higher, respectively, than in growing cells. At 4- and 5-hour of development 5.36- and 7.95-fold increase in expression was observed, respectively (Figure 46). Compared to growing cells, this gene was 11.95-fold upregulated at 6.5 hour of development. During streaming and late aggregation stages of development 8.31- and 8.15-fold increase in expression was detected. PPL_03541 was 6.16-fold upregulated when cells had formed mounds (Figure 46). 


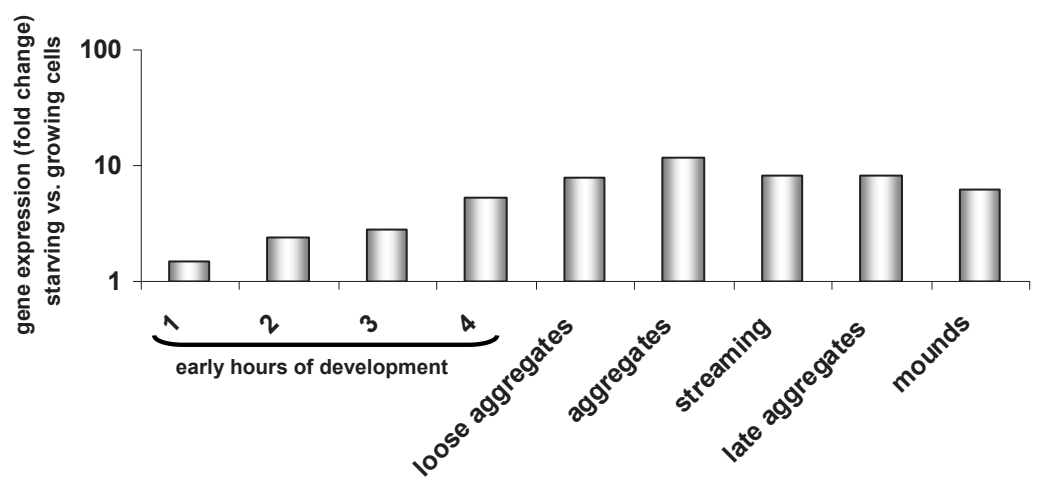

Figure 46: Developmental regulation of PPL_03541. P. pallidum PN500 cells grown in association with bacteria were harvested in the late vegetative stage, washed, and resuspended in phosphate buffer. Cells were then starved on phosphate agar plates at a density of $8 \times 10^{5} \mathrm{cells} / \mathrm{cm}^{2}$. Cell samples were collected at indicated developmental stages for total RNA extraction. Relative expression of model gene PPL_03541 was determined by real-time RT-PCR. As a control, expression of house keeping gene gp $d A$ was examined. Fold changes are shown. All data were compared to growing $P$. pallidum PN500 cells. Fold change was set to 1 where values $>1$ represented higher expression of the gene in starving cells than in growing cells. Mean values of duplicate measurements of the same CDNA \pm SD were plotted.

Absolute expression data obtained in RNA-seq experiment showed that mRNA of PPL_03541 was barely detectable in growing cells (Table 3). Negligible expression of this gene was detected in amoebae developed for 2- or 3-hours in shaking cultures (Table 3). However, when cells were treated with exogenous glorin for 1 hour, 9.44-fold higher expression was detected than in growing cells, whereas expression of PPL_03541 was downregulated after cells were treated with glorin for 2 hour demonstrating that PPL_03541 is developmentally induced by glorin for relatively short periods (Figure 26). Rapid decline of glorin-induced moderate increase in transcript levels of this gene in shaking cultures may result from instability of mRNA under these conditions. Maximal expression of PPL_03541 during aggregation may indicate that PPL_03541 plays a specific role during aggregation of $P$. pallidum PN500 amoebae.

\subsection{Developmental kinetics of PPL_06644}

When P. pallidum PN500 amoebae were developed on non-nutrient agar and expression profile of PPL_06644 was analyzed, it was noticed that this gene is developmentally induced. In the first 2 hours of starvation, only basal level expression was detected (1.1- \& 1.29-fold, respectively; Figure 47). With progression of starvation, expression of this gene increased gradually and reached a peak (23-fold higher than in 
growing cells; Figure 47) during the late aggregation stage. When cells have formed mounds, expression of PPL_06644 declined a little indicating that activity of this gene is required mainly during pre-aggregation and aggregation stages of development.

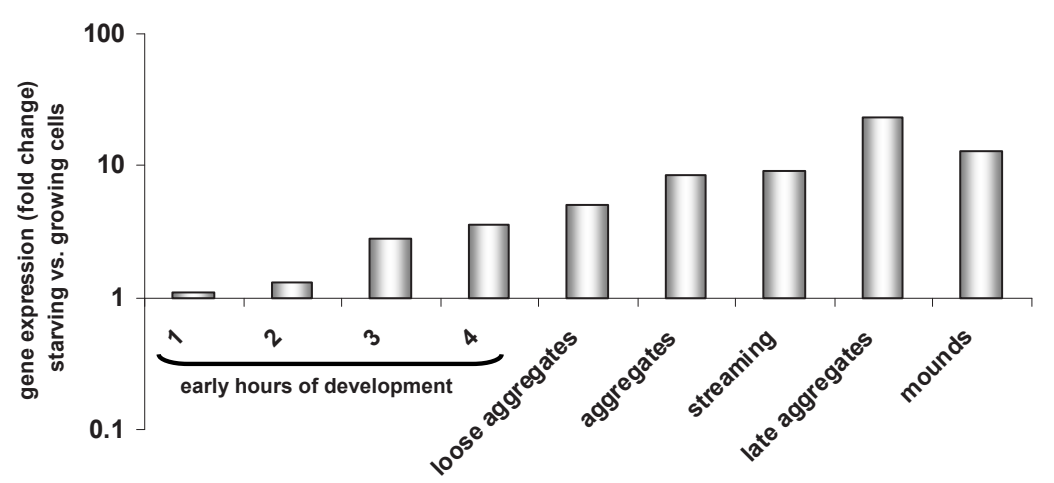

Figure 47: Developmental regulation of PPL_06644. P. pallidum PN500 cells grown in association with bacteria were harvested in the late vegetative stage, washed, and resuspended in phosphate buffer. Cells were then starved on phosphate agar plates at a density of $8 \times 10^{5} \mathrm{cells} / \mathrm{cm}^{2}$. Cell samples were collected at indicated developmental stages for total RNA extraction. Relative expression of model gene PPL_06644 was determined by real-time RT-PCR. As a control, expression of house keeping gene gpdA was examined. Fold changes are shown. All data were compared to growing $P$. pallidum PN500 cells. Fold change was set to 1 where values $>1$ represented higher expression of the gene in starving cells than in growing cells. Mean values of duplicate measurements of the same cDNA \pm SD were plotted.

RNA-seq data indicated that PPL_06644 is moderately induced by glorin (Table 3). If aggregating $P$. pallidum PN500 amoebae secrete glorin, then an induction of PPL_06644 is anticipated during aggregation phase of development and data presented in Figure 47 supports this assumption by indicating that PPL_06644 is upregulated throughout the aggregation process. These features show that PPL_06644 is an aggregation stage gene.

\subsubsection{Class II: Genes exhibiting characteristics of both 'early genes' and 'aggregation-stage genes'}

\subsection{Developmental kinetics of PPL_11763}

Previously, RNA-seq data indicated that PPL_11763 is expressed in growing cells to very low levels (Table 3 presenting absolute expression data). Also, it was noticed that expression of PPL_11763 is gradually induced by starvation, whereas glorin treatment 
leads to transient upregulation of this gene to high levels (Figure 26). When cells were washed free of bacteria and allowed to undergo normal development on phosphate agar plates, transcripts of PPL_11763 were detectable by $1 \mathrm{hr}$ of development, expression levels increased gradually, while maximal expression was detected at late aggregation stage until formation of mounds. Rapid accumulation of this gene to 2.56-, 7.31-, 8.22and 17.26-fold at 1-, 2-, 3- and 4-hours of development (time points corresponding to pre-aggrgeation period), respectively indicates that PPL_11763 is an early gene (Figure 48).

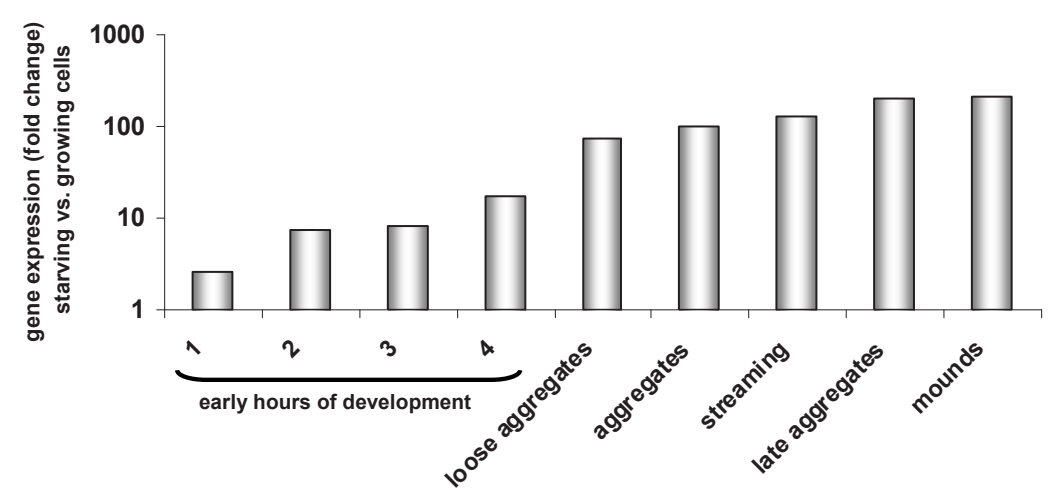

Figure 48: Developmental regulation of PPL_11763. P. pallidum PN500 cells grown in association with bacteria were harvested in the late vegetative stage, washed, and resuspended in phosphate buffer. Cells were then starved on phosphate agar plates at a density of $8 \times 10^{5} \mathrm{cells} / \mathrm{cm}^{2}$. Cell samples were collected at indicated developmental stages for total RNA extraction. Relative expression of model gene PPL_11763 was determined by real-time RT-PCR. As a control, expression of house keeping gene gpdA was examined. Fold changes are shown. All data were compared to growing $P$. pallidum PN500 cells. Fold change was set to 1 where values $>1$ represented higher expression of the gene in starving cells than in growing cells. Mean values of duplicate measurements of the same cDNA \pm SD were plotted.

Further increase in expression levels of this gene was noticed during the whole process of aggregation. At early aggregation stage 74.54-fold expression of PPL_11763 was detected that increased to 129.78-fold in actively streaming cells (Figure 48). Expression of this gene extends beyond the aggregation stage and a high level of transcript persists in the postaggregation stages; 200.85- and 210.83-fold higher expression of PPL_11763 was dectected at late aggregation and mound stage, repectively (Figure 48). These data support the indication provided by RNA-seq analyses that PPL_11763 displays characteristics of both early gene and aggregation-stage gene. Overall, these results and RNA-seq data may indicate that PPL_11763 is regulated both by starvation and glorin. Rapid turn over of glorin-induced moderate-level increase in the amounts of PPL_11763 
transcripts in shaking cultures may result from instability of mRNA of this gene under these conditions.

\subsection{Developmental kinetics of PPL_05354}

While analyzing expression pattern of PPL_05354 at various times of development, it was found that this gene was 4.19-fold upregulated after 1 hour of starvation compared to growing cells (Figure 49). PPL_05354 was further expressed moderately by 10.33and 12.99-fold at 2- and 3-hours of development suggesting that this gene is induced by starvation during the early hours of development (Figure 49).

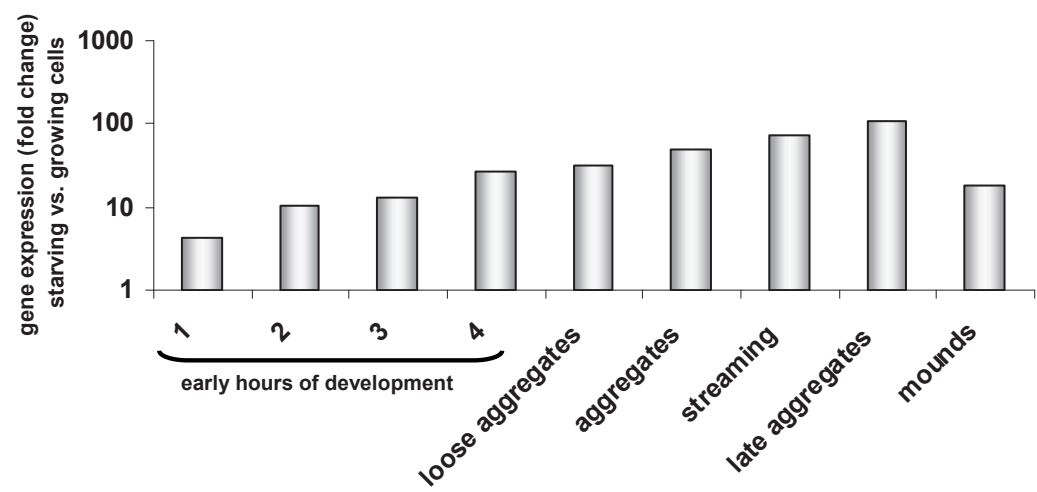

Figure 49: Developmental regulation of PPL_05354. P. pallidum PN500 cells grown in association with bacteria were harvested in the late vegetative stage, washed, and resuspended in phosphate buffer. Cells were then starved on phosphate agar plates at a density of $8 \times 10^{5} \mathrm{cells} / \mathrm{cm}^{2}$. Cell samples were collected at indicated developmental stages for total RNA extraction. Relative expression of model gene PPL_05354 was determined by real-time RT-PCR. As a control, expression of house keeping gene gp $d A$ was examined. Fold changes are shown. All data were compared to growing $P$. pallidum PN500 cells. Fold change was set to 1 where values $>1$ represented higher expression of the gene in starving cells than in growing cells. Mean values of duplicate measurements of the same cDNA \pm SD were plotted.

Expression levels increased to 33.22-fold at 5 hour of development; time corresponding to early aggregation stage. A continuous rise in expression was observed at next hours of development, such that 71.25- and 106.15-fold upregulation could be detected at streaming and late aggregation stages (Figure 49). High level expression of PPL_05354 during the developmental hours corresponding to aggregation illustrate that this gene may be additionally modulated by glorin secreted by aggregating cells. Expression of PPL_05354 then declined at mound stage indicating that activity of this gene is moderately required during pre-aggregation stages, optimally needed in the course of aggregation and once aggregates have formed, effects of PPL_05354 decline. These 
data suggest that PPL_05354 is a gene that exhibits features of both early gene and aggregation-stage gene. These results are in agreement with RNA-seq analysis that showed the presence of adequate number of transcripts of PPL_05354 in amoebae that were developed for 2 and 3 hour (Table 3 ) and demonstrated that this gene is additionally strongly induced by glorin (Figure 25). Moreover, RNA-seq data favours the assumption that activity of PPL_05354 might be required during the growth phase also because sufficient transcripts of this gene were detected in growing cells (Table 3 ).

\subsection{Developmental kinetics of PPL_00912}

Accumulation of PPL_00912 was determined during the first 14 hours of development of $P$. pallidum PN500 amoebae on non-nutrient agar that indicated a progressive increase in expression levels of this gene (Figure 50). At 1 and 2 hours of development 1.49- and 4.62-fold expression was noticed, respectively. Transcript levels increased to 7.48- and 10.5-fold in cells starved for 3 and 4 hours of development, respectively (Figure 50).

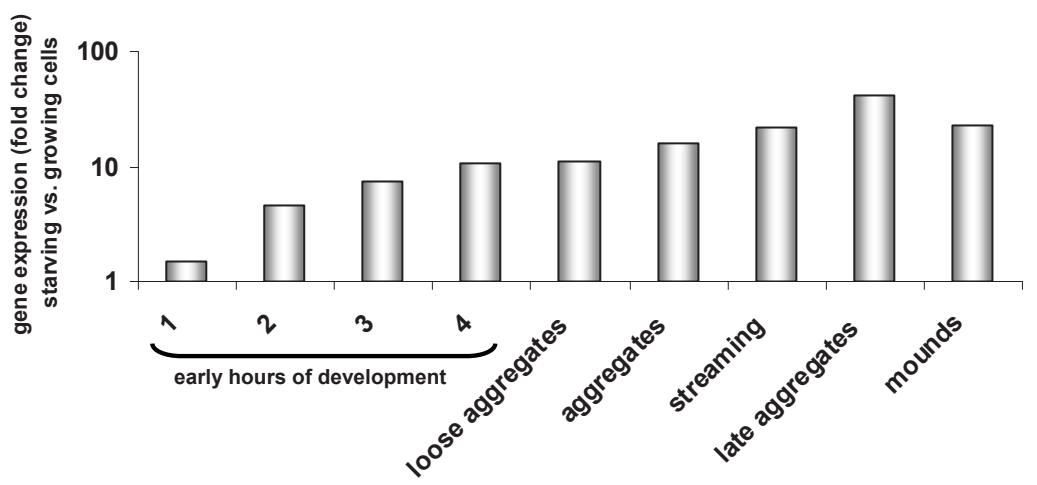

Figure 50: Developmental regulation of PPL_00912. P. pallidum PN500 cells grown in association with bacteria were harvested in the late vegetative stage, washed, and resuspended in phosphate buffer. Cells were then starved on phosphate agar plates at a density of $8 \times 10^{5} \mathrm{cells} / \mathrm{cm}^{2}$. Cell samples were collected at indicated developmental stages for total RNA extraction. Relative expression of model gene PPL_00912 was determined by real-time RT-PCR. As a control, expression of house keeping gene gpdA was examined. Fold changes are shown. All data were compared to growing P. pallidum PN500 cells. Fold change was set to 1 where values $>1$ represented higher expression of the gene in starving cells than in growing cells. Mean values of duplicate measurements of the same cDNA \pm SD were plotted.

Expression of this gene increased step-by-step during early aggregation and streaming stages, peaking at late aggregation stage where 41.18-fold expression was detected, whereas gene expression declined to relatively low-levels at mound stage (Figure 50). These data further confirm the findings of RNA-seq analyses suggesting that 
PPL_00912 is a starvation-induced gene, while expression of this gene is further enhanced by glorin signalling (Figure 27). Therefore, in parallel with PPL_11763 and PPL_05354, PPL_00912 also possesses characteristics of both early developmental gene and aggregation-stage gene.

\subsubsection{Class III: Genes nearly similarly expressed during the early stages of development}

\subsection{Developmental kinetics of PPL_12271 (erkB)}

PPL_12271 is a gene with distinct kinetics (Figure 51). RNA-seq analyses indicated that this gene is transiently up-regulated by glorin (Figure 26). Analysing absolute expression data of PPL_12271 showed that sufficient transcripts of this gene were present in growing cells and cells starved for 1 hour, while transcript number declined slightly at 2 hours of development (Table 3). When P. pallidum PN500 cells were developed on agar surface, 4.11-fold higher expression was detected in cells starved for 1 hour than in growing cells (Figure 51).

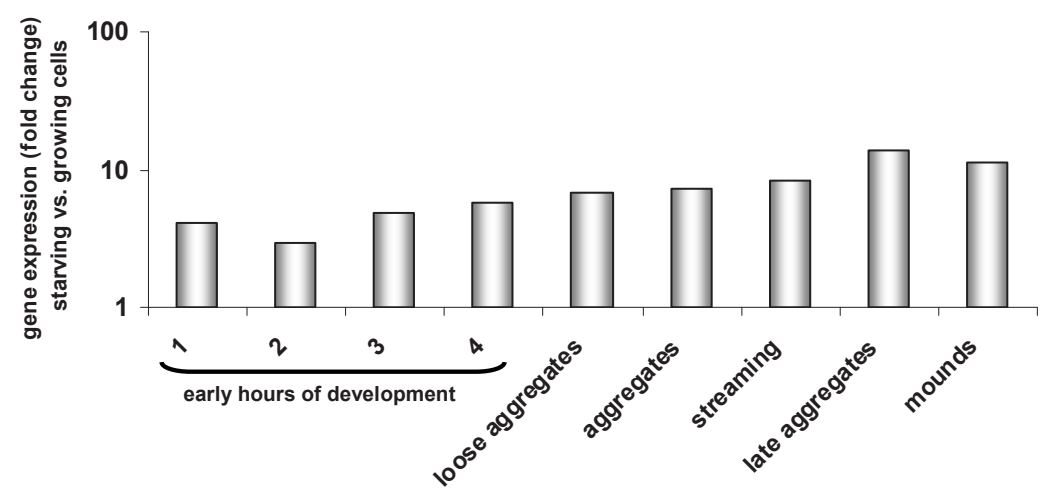

Figure 51: Developmental regulation of PPL_12271 (erkB). P. pallidum PN500 cells grown in association with bacteria were harvested in the late vegetative stage, washed, and resuspended in phosphate buffer. Cells were then starved on phosphate agar plates at a density of $8 \times 10^{5} \mathrm{cells} / \mathrm{cm}^{2}$. Cell samples were collected at indicated developmental stages for total RNA extraction. Relative expression of model gene PPL_12271 was determined by real-time RT-PCR. As a control, expression of house keeping gene gp $d A$ was examined. Fold changes are shown. All data were compared to growing $P$. pallidum PN500 cells. Fold change was set to 1 where values $>1$ represented higher expression of the gene in starving cells than in growing cells. Mean values of duplicate measurements of the same CDNA \pm SD were plotted.

Expression decreased to 2.98-fold at 2 hour of development. Expression levels started to rise afterwards, such that 6.88- and 8.28-fold expression was detected in cells forming 
early aggregates and streams, respectively. Expression reached a peak level (13.73fold) by 10 hours of development when tight aggregates of cells appeared (Figure 51). At mound stage, 11.15-fold expression was noticed. These data indicate that PPL_12271 is expressed in cells at moderate levels during all the stages of early development, whereas expression levels are slightly enhanced during aggregation supporting moderate level induction of this gene by glorin. Sufficient expression of PPL_12271 in growing cells and at different stages of development may demonstrate important role played by this gene during growth and development.

\subsubsection{Class IV: Genes expressed at basal level during the early stages of development}

\subsection{Developmental kinetics of PPL_03784}

When expression profile of PPL_03784 was analyzed in cells developing on non-nutrient agar plates, a low level expression of this gene was noticed during the first 4 hours of starvation. A small increase in expression of PPL_03784 was detected between 5 and 10 hour of development (Figure 52). At about 5 hour of development on agar surface, formation of early aggregates is observed followed by aggregation streams at 8.5 hour (Figure 44). Thus, a slight increase in expression of PPL_03784 within 5 to 10 hours of development might be connected to aggregation phase of cells. Transcripts levels of PPL_03784 appeared to peak at late aggregation stage when 7.11-fold higher expression was detected than in growing cells. Expression declined slightly at the mound stage (Figure 52). Gene expression data of PPL_03784 obtained in RNA-seq experiment indicated that PPL_03784 is a developmentally induced gene and treatment of starving cells with exogenous glorin further increases expression of this gene (Figure 27). Analyses of absolute expression data showed that this gene is insignificantly expressed in growing cells, whereas starvation led to an increase in number of transcripts of this gene at 2 and 3 hours of development (Table 3); results that correlate with the findings depicted in Figure 52. Moreover, RNA-seq data indicated that PPL_03784 is transiently up-regulated by glorin and 20 -fold increase in expression was detected in response to treatment with exogenous glorin for 1 hour that declined to 7.96 -fold after cells were treated with glorin for total 2 hours in shaking cultures (Table 3; Figure 27). 


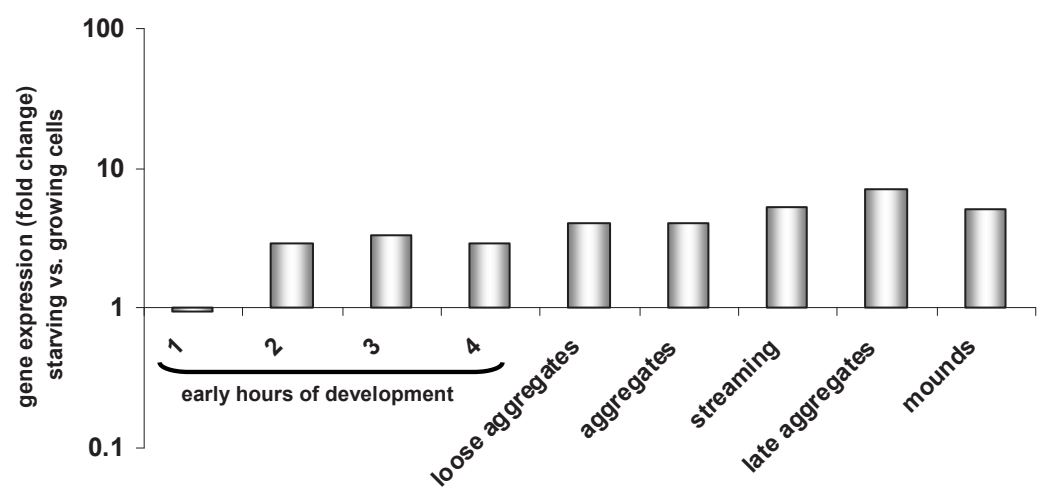

Figure 52: Developmental regulation of PPL_03784. P. pallidum PN500 cells grown in association with bacteria were harvested in the late vegetative stage, washed, and resuspended in phosphate buffer. Cells were then starved on phosphate agar plates at a density of $8 \times 10^{5} \mathrm{cells} / \mathrm{cm}^{2}$. Cell samples were collected at indicated developmental stages for total RNA extraction. Relative expression of model gene PPL_03784 was determined by real-time RT-PCR. As a control, expression of house keeping gene gp $d A$ was examined. Fold changes are shown. All data were compared to growing $P$. pallidum PN500 cells. Fold change in growing cells was defined as 1 where values $>1$ represent higher expression of the gene in starving cells than in growing cells. Values $<1$ show that expression of gene is lower in starving cells than in growing cells. Mean values of duplicate measurements of the same cDNA \pm SD were plotted.

However, cells developed on agar surface exhibited maximal expression of PPL_03784 at late aggregation stage when 7.11-fold increase in expression was detected (Figure 52). The difference between induction of expression of PPL_03784 in cells developed in shaking cultures in the presence of glorin and those starved on agar surface can be explained by assuming that aggregating amoebae of $P$. pallidum PN500 excrete glorin, nevertheless, transient changes in the expression of PPL_03784 in response to secreted glorin were not much prominent. This phenomenon may point to the difference in the behaviour of cells in shaking cultures and on agar surface. Another explanation could be that rapid and transient nature of gene expression changes did not allow for detection of short-lived, high-level upregulation of PPL_03784 by secreted glorin within the time points when cell samples were collected for gene expression analysis. These data suggest that PPL_03784 may play critical but short-term role during aggregation of $P$. pallidum PN500 amoebae.

\subsection{Developmental kinetics of PPL_05833}

PPL_05833 is a gene that exhibits expression profile almost similar to that of PPL_03784. When amoebae were starved on phosphate agar plates for 1 hour, PPL_05833 displayed low level of expression and compared to growing cells, only 1.23- 
fold increase in expression was detected (Figure 53). Expression of this gene increased to 3.05-, 3.58-, 4.14-fold at 2-, 3- and 4-hour of development, respectively (Figure 53). During aggregation, a slight increase in expression of PPL_05833 was detected. Messenger RNA complementary to PPL_05833 peaked at 10 hour of development and 7.51-fold higher expression of this gene was noted in cells forming tight aggregates than in growing cells (Figure 53). At mound stage, expression declined slightly. Moderate level expression of this gene at almost all stages of early development indicates that PPL_05833 may perform important biological role during the initial stages of development of $P$. pallidum PN500 amoebae.

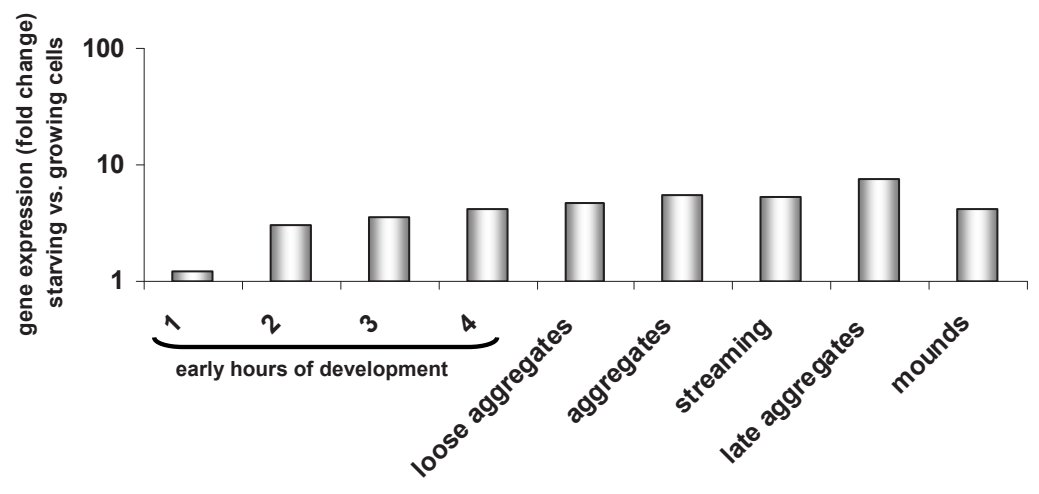

Figure 53: Developmental regulation of PPL_05833. P. pallidum PN500 cells grown in association with bacteria were harvested in the late vegetative stage, washed, and resuspended in phosphate buffer. Cells were then starved on phosphate agar plates at a density of $8 \times 10^{5} \mathrm{cells} / \mathrm{cm}^{2}$. Cell samples were collected at indicated developmental stages for total RNA extraction. Relative expression of model gene PPL_05833 was determined by real-time RT-PCR. As a control, expression of house keeping gene gpdA was examined. Fold changes are shown. All data were compared to growing $P$. pallidum PN500 cells. Fold change was set to 1 where values $>1$ represented higher expression of the gene in starving cells than in growing cells. Mean values of duplicate measurements of the same CDNA \pm SD were plotted.

In RNA-seq experiment, when absolute expression data of PPL_05833 was analyzed (Table 3), it was found that this gene is insignificantly expressed in vegetative cells. RNAseq analysis further showed that when cells are developed in shaking cultures, PPL_05833 is induced by starvation, while stimulation of starving amoebae with exogenous glorin further increases expression of this gene transiently. 133.2-fold increase in expression of PPL_05833 was induced in response to stimulation with glorin for 1 hour, while only 25.75-fold increase in expression could be detected after 2 hours of treatment with glorin (Figure $27 \mathrm{~A}$ ). When data obtained with cells developed on nonnutrient agar are compared with results from cells starved in shaking cultures for the same time period, it is noticed that expression of PPL_05833 is developmentally 
regulated in both cases (Figure $39 \& 53$ ). On the other hand, high-level but transient induction of PPL_05833 was detected when cells developing in shaking suspensions were treated with glorin (Figure $27 \& 39$ ). Speculating that aggregating amoebae of $P$. pallidum PN500 secrete glorin, a high-level expression of PPL_05833 is expected in cells undergoing aggregation on agar surface. However, data obtained from cells developed on non-nutrient agar do not provide such an indication and PPL_05833 is only moderately expressed during aggregation (Figure 53). These differences can be attributed to the rapid and transient induction of PPL_05833 by glorin. It is quite possible that this gene is induced to high-levels in P. pallidum PN500 cells developing on agar surface in response to glorin secreted by aggregating cells but such a change might be so fast and short-term that could not be detected in any of the cell samples collected at those specific stages of development shown in Figure 44. However, shaking cultures are not comparable to the natural conditions of development of amoebae and $1 \mu \mathrm{M}$ glorin used to stimulate cells at 10- or 30-minute intervals is not representative of natural pulsing frequency of glorin secreted by starving amoebae during aggregation. Therefore, different sensitivities of cells developing in shaking cultures or on agar surface may also explain differences in gene induction levels in response to externally added or secreted glorin.

\subsection{Developmental kinetics of PPL_12248}

Analyses of gene expression data obtained from cells developed on phosphate agar plates showed that PPL_12248 is expressed at basal levels throughout the early development (Figure 54). At 1-, 2-, 3- and 4-hour of development, 1.99-, 2.18-, 1.56-, and 1.37 -fold higher expression (than in growing cells) was detected, respectively (Figure 54). At the onset of aggregation expression of this gene increased to $2.25 \mathrm{fold}$, was maintained at 2.44-fold during streaming and reduced to 1.09-fold at mound stage (Figure 54). 


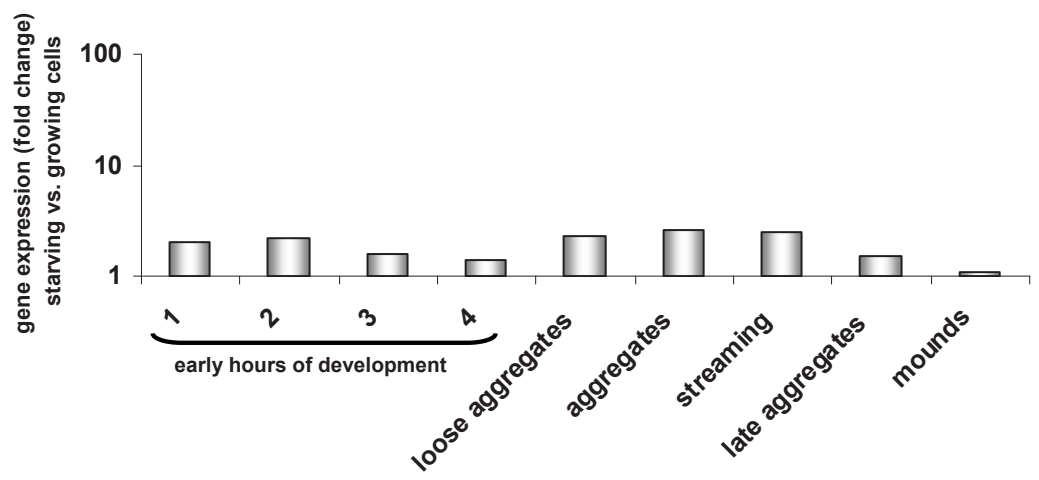

Figure 54: Developmental regulation of PPL_12248. $P$. pallidum PN500 cells grown in association with bacteria were harvested in the late vegetative stage, washed, and resuspended in phosphate buffer. Cells were then starved on phosphate agar plates at a density of $8 \times 10^{5} \mathrm{cells} / \mathrm{cm}^{2}$. Cell samples were collected at indicated developmental stages for total RNA extraction. Relative expression of model gene PPL_12248 was determined by real-time RT-PCR. As a control, expression of house keeping gene gpdA was examined. Fold changes are shown. All data were compared to growing $P$. pallidum PN500 cells. Fold change was set to 1 where values $>1$ represented higher expression of the gene in starving cells than in growing cells. Mean values of duplicate measurements of the same cDNA \pm SD were plotted.

RNA-seq data showed that some transcripts of PPL_12248 were present in vegetative cells that declined at the onset of starvation (Table 3), indicating that PPL_12248 may be a 'growth-stage-specific gene' that is repressed by starvation. However, it was shown that stimulation of suspension developed P. pallidum PN500 cells with exogenous glorin led to significant level induction of expression of PPL_12248 (Figure 29), such that after 1 hour of exposure to glorin 35.62-fold increase in gene expression could be noticed compared to expression in growing cells, whereas only 7-fold expression was detected in cells treated with glorin for 2 hours; illustrating rapid and transient induction of PPL_12248 in response to glorin (Figure 29). When P. pallidum PN500 cells were developed for 8 hours in shaking cultures in the absence of glorin, a continuous downregulation of this gene was detected, however, transient and high-level induction of PPL_12248 was noticed in the presence of exogenous glorin (Figure 42). When amoebae are developed on non-nutrient agar, the pattern of PPL_12248 gene expression is somewhat different from that observed in cells developed in shaking cultures. Though PPL_12248 is constantly down-regulated under shaking culture conditions, yet it exhibits a very low level expression in cells developing on agar surface (Figure 42 \& 54); indicating that cell-cell contacts may be required for low-level expression of this gene. PPL_12248 shows characteristics of vegetative-stage gene but is rapidly induced by glorin. This distinct kinetics of expression of PPL_12248 may demonstrate that glorin is detected by growing cells also and signal is transduced to 
downstream effectors that leads to gene expression changes during growth phase. Other justification could be that PPL_12248 is rapidly induced to moderate- or high-levels in response to glorin secreted by cells aggregating on agar surface but induction might be short-lived and increased levels of expression decline back to basal level in a short period of time, therefore, could not be detected within the sampling time points as shown in Figure 54. Transient induction of PPL_12248 by glorin may explain short-term activity of this gene during aggregation of $P$. pallidum PN500 amoebae.

\subsubsection{Developmental regulation of selective GPCRs}

GPCRs are seven-transmembrane proteins that transduce extracellular signals inside the cell by activating heterotrimeric G-proteins that couple receptors to effector proteins inside the cell to trigger physiological responses (Sucgang et al. 2011). Previous studies indicated that glorin acts by binding to cell surface G-protein coupled receptors (De Wit et al. 1988). It was demonstrated that aggregating amoebae of $P$. violaceum possess cell surface receptors for the detection of the extracellular glorin (De Wit et al. 1988). However, it was shown that vegetatively growing amoebae of $P$. violaceum also expose adequate number of glorin receptors on their cell surface that increases slightly during aggregation (De Wit et al. 1988). In D. discoideum expression of cAR1 is induced by pulses of extracellular cAMP during aggregation (Mu et al. 1998; Firtel 1995; Loomis 1996; Louis et al. 1993; Saxe et al. 1991a, b; Klein et al. 1988, 1987; Kimmel 1987). RNA-seq analyses conducted in this study indicated that in $P$. pallidum PN500 a number of GPCRs were positively regulated by glorin to varying degrees (Table 4). It was suspected that gene encoding glorin receptor(s) may be among the GPCR encoding genes differentially regulated by external glorin; similar to the induction of aggregation stage cAR 1 by extracellular pulses of cAMP in $D$. discoideum. It was therefore interesting to analyze developmental kinetics of selected glorin-regulated GPCR encoding genes during the early hours of development of $P$. pallidum PN500 cells. These experiments were designed to recognize GPCR genes that were expressed in growing cells and their expression was slightly enhanced during the process of aggregation. GPCR gene(s) exhibiting such kinetics might represent candidate glorin receptor gene(s).

P. pallidum PN500 cells grown in association with bacteria were harvested in the late vegetative stage, washed, and resuspended in phosphate buffer. Cells were then starved on phosphate agar plates at a density of $8 \times 10^{5} \mathrm{cells} / \mathrm{cm}^{2}$. Cell samples were collected at 9 
different developmental stages for total RNA extraction (Figure 44) and developmental kinetics of selected glorin-induced GPCR encoding genes were analyzed.

\subsubsection{Developmental kinetics of GPCR genes induced by glorin}

Developmentally regulated GPCR genes were divided into 3 major classes based on their respective kinetics.

\subsection{Class I: GPCR encoding genes expressed in growing amoebae, whose expression levels are enhanced upon starvation}

RNA-seq data demonstrated that PPL_00902 and PPL_05727 were two distinct GPCR genes whose transcripts were moderately expressed in vegetative cells, whereas stimulation of cells with glorin for 1 hour led to 2.74 - and 4.15 -fold increase in expression of PPL_00902 and PPL_05727, respectively (Table 3). When P. pallidum PN500 amoebae were developed on agar, starvation moderately enhanced expression of PPL_00902, such that at all stages of development analyzed in this experiment, PPL_00902 was almost similarly regulated (Figure 55). Expression of PPL_05727 was also enhanced in response to starvation but kinetics was different compared to PPL_00902. PPL_05727 exhibited low-level increase in expression during the first 4 hours of starvation (Figure 55). At the onset of aggregation, expression of this gene further increased and was maintained at high-levels during the whole process of aggregation (Figure 55). PPL_00902 and PPL_05727 represent ideal candidate genes that may encode glorin receptors because both of these genes are expressed moderately in growing cells and their expression is further enhanced with progression of starvation. 

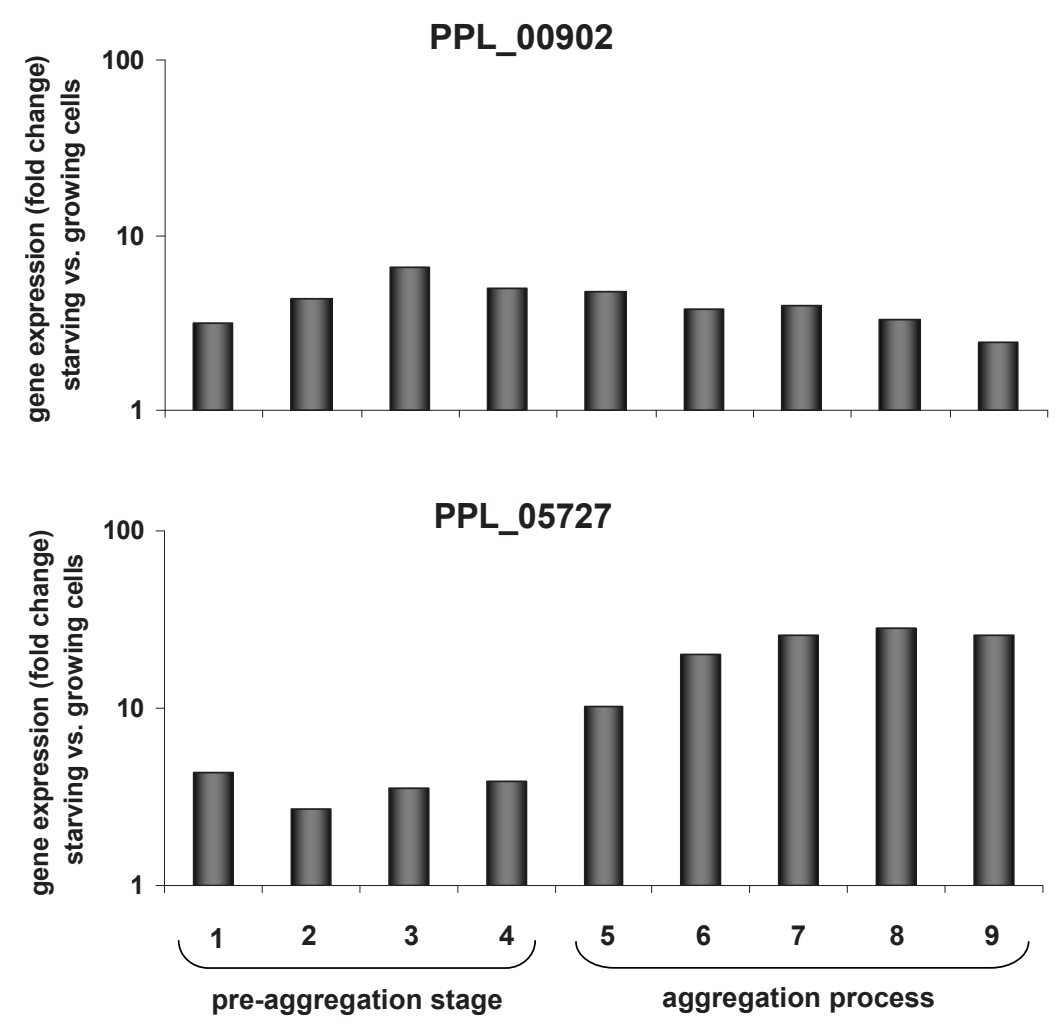

Figure 55: Developmental regulation of GPCR genes PPL_00902 \& PPL_05727. P. pallidum PN500 cells were starved on non-nutrient agar. Cell samples were collected at 9 different stages of early development including initial 1-4 hours of starvation (the pre-aggregation stage) and distinct morphological states indicated by number 5 (loose aggregates), 6 (aggregates), 7 (streaming), 8 (late aggregates) and 9 (mounds) for total RNA extraction. Relative expression of PPL_00902 and PPL_05727 was determined by real-time RT-PCR. As a control, expression of house keeping gene gpdA was examined. Fold changes are shown. All data were compared to growing $P$. pallidum PN500 cells. Fold change was set to 1 where values $>1$ represented higher expression of the gene in starving cells than in growing cells. Mean values of duplicate measurements of the same cDNA \pm SD were plotted.

\subsection{Class II: Starvation induced GPCRs encoding genes whose expression is augmented during aggregation}

Certain GPCR genes, of which PPL_ 04108 and PPL_00855 are obvious examples, exhibited negligible expression in growing cells (referred to absolute gene expression data presented in Table 3). Starvation caused a gradual increase in expression of PPL_04108 and a maximal level expression was observed during aggregation that decreased to low-levels after tight aggregates have formed (Figure 56). PPL_00855 exhibited comparatively different kinetics. This gene was moderately expressed at 1 hour of starvation, followed by a gradual decrease in expression until 4 hour of 
development on agar (Figure 56). A moderate increase in the expression of PPL_00855 was then noticed throughout the process of aggregation (Figure 56). These genes are selectively induced in response to starvation and may play vital roles during aggregation.
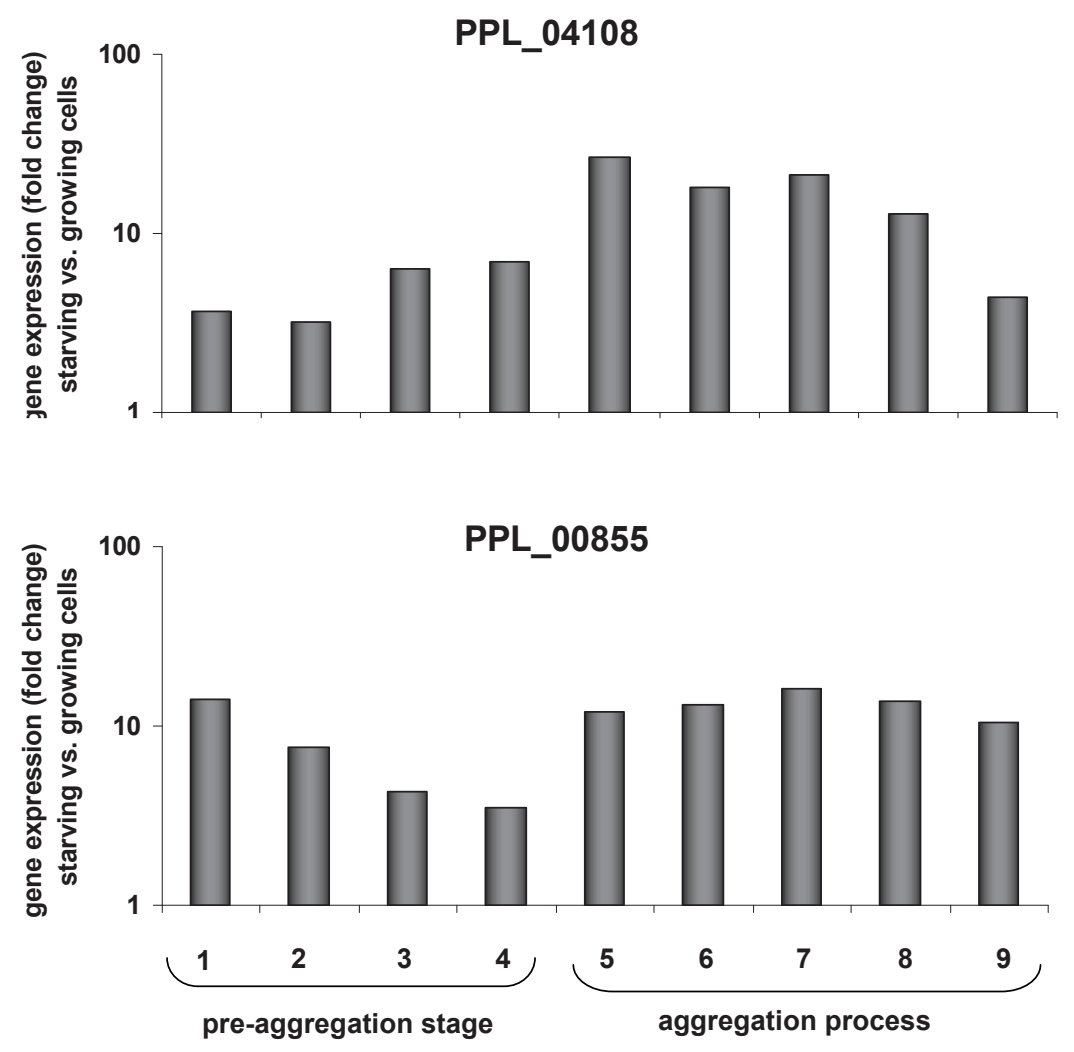

Figure 56: Developmental regulation of GPCR genes PPL_04108 \& PPL_00855. P. pallidum PN500 cells were starved on non-nutrient agar. Cell samples were collected at 9 different stages of early development including initial 1-4 hours of starvation (the pre-aggregation stage) and distinct morphological states indicated by number 5 (loose aggregates), 6 (aggregates), 7 (streaming), 8 (late aggregates) and 9 (mounds) for total RNA extraction. Relative expression of PPL_04108 and PPL_00855 was determined by real-time RT-PCR. As a control, expression of house keeping gene gpdA was examined. Fold changes are shown. All data were compared to growing $P$. pallidum PN500 cells. Fold change was set to 1 where values $>1$ represented higher expression of the gene in starving cells than in growing cells. Mean values of duplicate measurements of the same cDNA \pm SD were plotted.

\subsection{Class III: GPCR encoding genes expressed specifically during aggregation}

Some GPCR genes, such as PPL_08454, PPL_08455, and PPL_03564 were characteristically induced at the onset of aggregation and their expression was maintained at moderate levels during aggregation (Figure 57). Expression of these genes 
was insignificant in growing cells (Table 3). Products of these genes may play essential roles during aggregation.
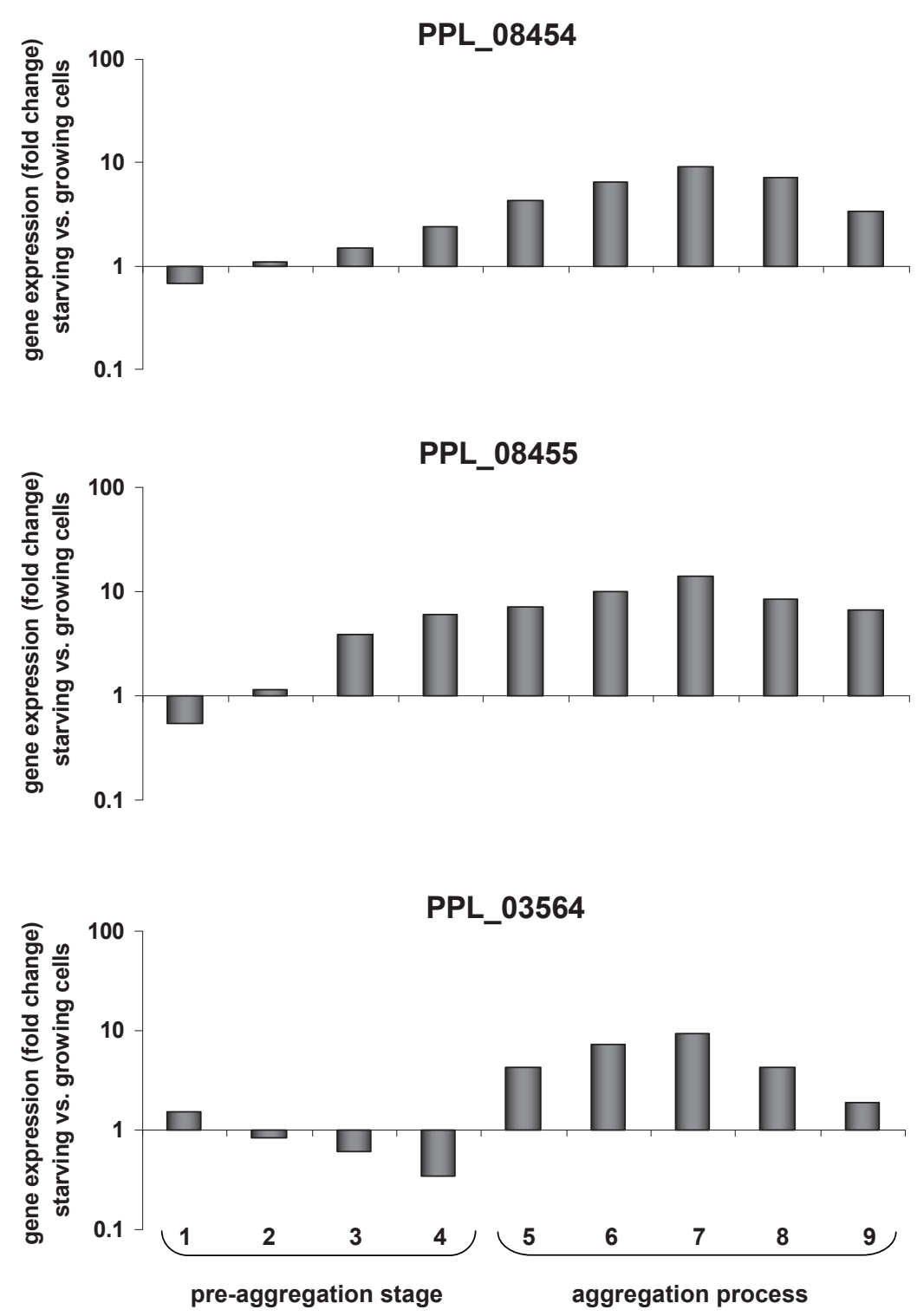

Figure 57: Developmental regulation of GPCR genes PPL_08454, PPL_08455 \& PPL_03564. P. pallidum PN500 cells were starved on non-nutrient agar. Cell samples were collected at 9 different stages of early development including initial 1-4 hours of starvation (the pre-aggregation stage) and distinct morphological states indicated by number 5 (loose aggregates), 6 (aggregates), 7 (streaming), 8 (late aggregates) and 9 (mounds) for total RNA extraction. Relative expression of PPL_08454, PPL_08455 and PPL_03564 was determined by real-time RT-PCR. As a control, expression of house keeping gene gpdA was examined. Fold changes are shown. All data were compared to growing P. pallidum PN500 cells. Fold change was set to 1 where values $>1$ represent higher expression of the gene in starving cells than in growing cells. Values $<1$ show that expression of gene is lower in starving cells than in growing cells. Mean values of duplicate measurements of the same $c D N A \pm S D$ were plotted. 
Expression kinetics of selected glorin-regulated genes in P. pallidum PN500 cells

\begin{tabular}{|c|c|c|c|}
\hline \multirow[t]{2}{*}{ Gene Name } & \multicolumn{2}{|c|}{$\begin{array}{c}\text { Developed in shaken suspensions for } 8 \\
\text { hours }\end{array}$} & \multirow{2}{*}{$\begin{array}{l}\text { Developed on non-nutrient } \\
\text { agar plates until formation of } \\
\text { mounds }\end{array}$} \\
\hline & $\begin{array}{c}\text { Effects of } \\
\text { starvation only }\end{array}$ & $\begin{array}{l}\text { Effects of glorin } \\
\text { treatment }\end{array}$ & \\
\hline PPL_09347 & $\begin{array}{l}\text { Only slightly } \\
\text { induced by } \\
\text { starvation during the } \\
\text { first } 4 \text { hours of } \\
\text { development, } \\
\text { moderately induced } \\
\text { afterwards }\end{array}$ & $\begin{array}{l}\text { Stably induced by } \\
\text { glorin to high- } \\
\text { levels }\end{array}$ & $\begin{array}{l}\text { Expression increased gradually } \\
\text { during the early hours of } \\
\text { development, highly expressed } \\
\text { during aggregation }\end{array}$ \\
\hline PPL_06644 & $\begin{array}{l}\text { Insignificant } \\
\text { expression during } \\
\text { the first few hours of } \\
\text { development }\end{array}$ & $\begin{array}{l}\text { Transiently } \\
\text { induced by glorin, } \\
\text { followed by a } \\
\text { rapid decline in } \\
\text { expression }\end{array}$ & $\begin{array}{l}\text { Expression increased gradually } \\
\text { during the early hours of } \\
\text { development, highly expressed } \\
\text { during aggregation }\end{array}$ \\
\hline PPL_05357 & $\begin{array}{l}\text { Only slightly } \\
\text { induced by } \\
\text { starvation during the } \\
\text { first } 4 \text { hours of } \\
\text { development, } \\
\text { moderately induced } \\
\text { afterwards }\end{array}$ & $\begin{array}{l}\text { Stably induced by } \\
\text { glorin to high- } \\
\text { levels }\end{array}$ & $\begin{array}{l}\text { Expression increased gradually } \\
\text { during the early hours of } \\
\text { development, highly expressed } \\
\text { during aggregation }\end{array}$ \\
\hline PPL_11763 & $\begin{array}{l}\text { Expressed at basal } \\
\text { levels during the } \\
\text { first } 3 \text { hours of } \\
\text { development }\end{array}$ & $\begin{array}{l}\text { Transiently } \\
\text { induced by glorin } \\
\text { to high levels, } \\
\text { followed by a } \\
\text { rapid decline in } \\
\text { expression }\end{array}$ & $\begin{array}{l}\text { Expression increased gradually } \\
\text { during the early hours of } \\
\text { development, highly expressed } \\
\text { during aggregation }\end{array}$ \\
\hline PPL_12271 & $\begin{array}{l}\text { Expressed at basal } \\
\text { levels during the } \\
\text { first } 3 \text { hours of } \\
\text { development, } \\
\text { gradual increase of } \\
\text { expression to } \\
\text { moderate levels is } \\
\text { observed afterwards }\end{array}$ & $\begin{array}{l}\text { Transiently } \\
\text { induced by glorin, } \\
\text { followed by a } \\
\text { rapid decline in } \\
\text { expression }\end{array}$ & $\begin{array}{l}\text { Basal level expression for the } \\
\text { first } 2 \text { hours. Expressed at low } \\
\text { levels during the later stages of } \\
\text { development investigated with } \\
\text { a slight increase during } \\
\text { aggrgeation }\end{array}$ \\
\hline PPL_03541 & $\begin{array}{l}\text { Expressed at basal } \\
\text { levels during the } \\
\text { first } 3 \text { hours of } \\
\text { development }\end{array}$ & $\begin{array}{l}\text { Transiently } \\
\text { induced by glorin } \\
\text { to moderate } \\
\text { levels, followed by } \\
\text { a rapid decline in } \\
\text { expression levels }\end{array}$ & $\begin{array}{l}\text { Expression increased gradually } \\
\text { during the early hours of } \\
\text { development, moderately } \\
\text { expressed during aggregation }\end{array}$ \\
\hline PPL_05833 & $\begin{array}{l}\text { Gradually induced } \\
\text { by starvation to } \\
\text { moderate levels }\end{array}$ & $\begin{array}{l}\text { Glorin treatment } \\
\text { further enhanced } \\
\text { its expression } \\
\text { rapidly, followed } \\
\text { by a decline in } \\
\text { expression. }\end{array}$ & $\begin{array}{l}\text { Basal level expression in the } \\
\text { first hour of starvation. } \\
\text { Expressed at low levels during } \\
\text { the later stages of development } \\
\text { investigated (starting from } 2^{\text {nd }} \\
\text { hour of starvation until mound } \\
\text { formation) }\end{array}$ \\
\hline
\end{tabular}




\begin{tabular}{|c|c|c|c|}
\hline PPL_03784 & $\begin{array}{l}\text { Induced by } \\
\text { starvation }\end{array}$ & $\begin{array}{l}\text { Glorin treatment } \\
\text { further enhanced } \\
\text { its expression } \\
\text { rapidly, followed } \\
\text { by a gradual } \\
\text { decline in } \\
\text { expression }\end{array}$ & $\begin{array}{l}\text { Basal level expression in the } \\
\text { first hour of starvation. } \\
\text { Expressed at low levels during } \\
\text { the later stages of development } \\
\text { investigated (starting from } 2^{\text {nd }} \\
\text { hour of starvation until mound } \\
\text { formation) }\end{array}$ \\
\hline PPL_00912 & $\begin{array}{l}\text { Induced by } \\
\text { starvation }\end{array}$ & $\begin{array}{l}\text { Glorin treatment } \\
\text { further enhanced } \\
\text { its expression, } \\
\text { follwed by a } \\
\text { gradual decline in } \\
\text { expression }\end{array}$ & $\begin{array}{l}\text { Expression increased gradually } \\
\text { during the early hours of } \\
\text { development, highly expressed } \\
\text { during aggregation }\end{array}$ \\
\hline PPL_07908 & $\begin{array}{l}\text { Highly induced by } \\
\text { starvation during the } \\
\text { first } 2-3 \text { hours of } \\
\text { starvation followed } \\
\text { by a rapid decline at } \\
4-5 \text { hours of } \\
\text { development. } \\
\text { Afterwards, } \\
\text { expression } \\
\text { increased gradually } \\
\text { to moderate levels }\end{array}$ & $\begin{array}{l}\text { Glorin treatment } \\
\text { repressed its } \\
\text { expression during } \\
\text { the first 2-3 hours } \\
\text { of development }\end{array}$ & Not detected \\
\hline PPL_05702 & $\begin{array}{l}\text { Induced by } \\
\text { starvation }\end{array}$ & $\begin{array}{l}\text { Glorin treatment } \\
\text { repressed its } \\
\text { expression }\end{array}$ & Not detected \\
\hline PPL_05195 & $\begin{array}{l}\text { Induced by } \\
\text { starvation }\end{array}$ & $\begin{array}{l}\text { Glorin treatment } \\
\text { repressed its } \\
\text { expression }\end{array}$ & Not detected \\
\hline PPL_12248 & $\begin{array}{l}\text { Repressed by } \\
\text { starvation }\end{array}$ & $\begin{array}{l}\text { Stimulation of } \\
\text { cells with glorin } \\
\text { induced its } \\
\text { expression to high } \\
\text { levels for the first } \\
\text { hour of treatment, } \\
\text { followed by a } \\
\text { gradual decrease } \\
\text { in expression }\end{array}$ & $\begin{array}{l}\text { Expressed at basal levels } \\
\text { during all the stages of } \\
\text { development investigated } \\
\text { (Figure 44) }\end{array}$ \\
\hline PPL_12249 & $\begin{array}{l}\text { Repressed by } \\
\text { starvation }\end{array}$ & $\begin{array}{l}\text { Stimulation of } \\
\text { cells with glorin } \\
\text { induced its } \\
\text { expression to high } \\
\text { levels for the first } \\
\text { hour of treatment, } \\
\text { followed by a } \\
\text { gradual decrease } \\
\text { in expression }\end{array}$ & $\begin{array}{l}\text { Expressed at basal levels } \\
\text { during all the stages of } \\
\text { development investigated }\end{array}$ \\
\hline PPL_00902 & $\begin{array}{l}\text { Expressed in } \\
\text { growing amoebae, } \\
\text { induced by } \\
\text { starvation }\end{array}$ & $\begin{array}{l}\text { Expression } \\
\text { enhanced by } \\
\text { glorin }\end{array}$ & $\begin{array}{l}\text { Expressed in growing } \\
\text { amoebae, expression } \\
\text { enhanced to moderate levels } \\
\text { during the early stages of } \\
\text { development }\end{array}$ \\
\hline PPL_05727 & $\begin{array}{l}\text { Expressed in } \\
\text { growing amoebae, } \\
\text { induced by } \\
\text { starvation to } \\
\text { moderate levels }\end{array}$ & $\begin{array}{l}\text { Expression } \\
\text { enhanced by } \\
\text { glorin to moderate } \\
\text { levels }\end{array}$ & $\begin{array}{l}\text { Expressed in growing } \\
\text { amoebae, expression } \\
\text { enhanced gradually and } \\
\text { reached a maximum during } \\
\text { aggregation }\end{array}$ \\
\hline
\end{tabular}




\begin{tabular}{|l|l|l|l|}
\hline PPL_04108 & $\begin{array}{l}\text { Induced by } \\
\text { starvation to } \\
\text { moderate levels }\end{array}$ & $\begin{array}{l}\text { Expression } \\
\text { enhanced by } \\
\text { glorin to moderate } \\
\text { levels }\end{array}$ & $\begin{array}{l}\text { Moderately expressed during } \\
\text { the early hours of development, } \\
\text { expression enhanced gradually } \\
\text { and reached a maximum during } \\
\text { aggregation }\end{array}$ \\
\hline PPL_00855 & $\begin{array}{l}\text { Induced by } \\
\text { starvation to low } \\
\text { levels }\end{array}$ & $\begin{array}{l}\text { Expression } \\
\text { enhanced by } \\
\text { glorin to low } \\
\text { levels }\end{array}$ & $\begin{array}{l}\text { Moderately expressed during } \\
\text { the early hours of development, } \\
\text { expression enhanced gradually } \\
\text { and reached a maximum during } \\
\text { aggregation }\end{array}$ \\
\hline PPL_08454 & $\begin{array}{l}\text { Induced by } \\
\text { starvation to low } \\
\text { levels }\end{array}$ & $\begin{array}{l}\text { Rapidly induced } \\
\text { by glorin to } \\
\text { moderate levels, } \\
\text { followed by a } \\
\text { rapid decline }\end{array}$ & $\begin{array}{l}\text { Expression enhanced gradually } \\
\text { during the early hours of } \\
\text { development and reached a } \\
\text { maximum during aggregation }\end{array}$ \\
\hline PPL_08455 & $\begin{array}{l}\text { Induced by } \\
\text { starvation to low } \\
\text { levels }\end{array}$ & $\begin{array}{l}\text { Rapidly induced } \\
\text { by glorin to } \\
\text { moderate levels, } \\
\text { followed by a } \\
\text { rapid decline }\end{array}$ & $\begin{array}{l}\text { Expression enhanced gradually } \\
\text { during the early hours of } \\
\text { development and reached a } \\
\text { maximum during aggregation }\end{array}$ \\
\hline PPL_03564 & $\begin{array}{l}\text { Induced by } \\
\text { levels } \\
\text { larvation to low }\end{array}$ & $\begin{array}{l}\text { apdly induced } \\
\text { moderate levels, } \\
\text { followed by a } \\
\text { rapid decline }\end{array}$ & $\begin{array}{l}\text { Expression enhanced gradually } \\
\text { during the early hours of } \\
\text { development and reached a } \\
\text { maximum during aggregation }\end{array}$ \\
\hline
\end{tabular}

Table 5: Summarized expression kinetics of chosen glorin-regulated genes in $P$. pallidum PN500 cells developed in shaking cultures (in the presence or absence of glorin) and on non-nutrient agar plates. 


\subsection{Glorin signalling function independent of the cAMP signaling system}

Cyclic AMP signalling is used to organize the post-aggregative stages of development throughout the Dictyostelids phylogeny (Schaap 2011a; Kawabe et al. 2009; AlvarezCurto et al. 2005). However, in younger group 4 species, secreted cAMP acts both as a chemoattractant to mediate aggregation of starving amoebae and as a developmental signal to trigger pre-spore differentiation (Schaap 2011a; Sucgang et al. 2011; Schaap 2007). It has been shown that cAMP is secreted by starving $P$. pallidum amoebae (Konijn et al. 1968) and a pdsA gene (phosphodiesterase $A$ ) showing low affinity for cAMP is expressed in $P$. pallidum PN500 cells during growth, aggregation and post-aggregation stages (Kawabe et al. 2012). However, the 'acrasin of aggregation' in this species is not cAMP (Shaffer 1962; Jones \& Robertson 1976; Shimomura et al. 1982), therefore, it is assumed that the low-affinity phosphodiesterase may not be efficacious to generate steep cAMP gradients during aggregation (Kawabe et al. 2012). After aggregation has completed, there is a major reorganization of the control of development in $P$. pallidum where intercellular communication within the multicellular slug exploits cAMP as chemotactic agent for the organization of post-aggregative morphogenesis. Therefore, it was interesting to study whether there is any cross-talk between glorin and CAMP, i.e. chemoattractant coordinating post-aggregation stages of $P$. pallidum development.

\subsubsection{Expression patterns of components of cAMP signalling system are not affected by glorin stimulus}

First, effects of stimulating starving P. pallidum PN500 cells with glorin were studied on expression patterns of chosen cAMP signalling system genes.

P. pallidum PN500 amoebae were harvested from bacterial growth plates, washed free of bacteria and resuspended in phosphate buffer at cell density $2 \times 10^{7}$ cells $/ \mathrm{ml}$. A pellet of $2 \times 10^{7}$ cells served as 'vegetative-stage growing cells control' for subsequent gene expression analysis. Cell suspension was then equally divided into two flasks. One suspension culture of $P$. pallidum PN500 amoebae was prestarved for 1 hour, and then treated with periodic additions of $1 \mu \mathrm{M}$ glorin at 30-min intervals for up to 2 additional hours to determine the influence of externally added glorin on the expression pattern of selected genes known to be involved in cAMP signalling (Figure 58). Other culture was 
maintained in the absence of added glorin for the time period. Cells samples were taken from glorin treated and untreated cultures at 2- and 3-hours of development for total RNA extraction to analyse changes in gene expression. Expression patterns of adenylyl cyclase A (acaA2; PPL_01657), phosphodiesterase A (pdsA; PPL_10234), cAMPdependent protein kinase $\mathrm{C}$ ( $p k a C$; PPL_05049), and a homolog of cAMP receptor gene named tas $B$ are shown in Figure 58. It was noted that stimulation of starving $P$. pallidum PN500 amoebae with glorin has no obvious influence on the expression patterns of all tested genes involved in cAMP signalling demonstrating that glorin signalling may not augument post-aggregation cAMP signalling.

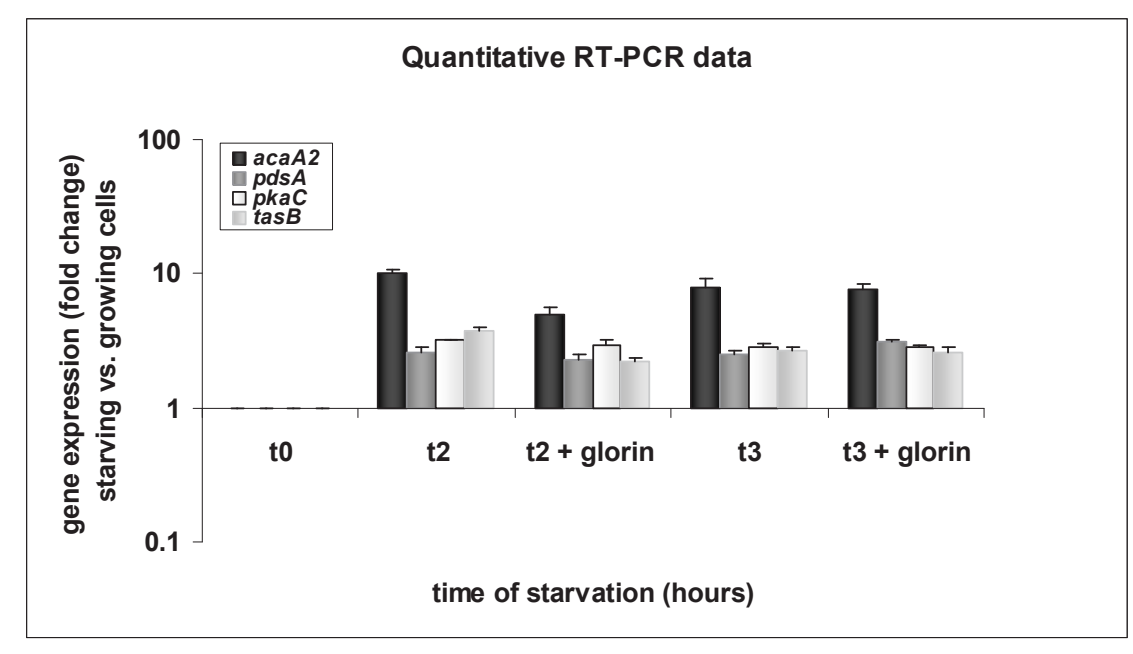

Figure 58: Expression kinetics of selected components of cAMP signalling in $P$. pallidum. Fold changes are shown. All data were compared to growing P. pallidum PN500 amoebae (t0; fold change set to 1). Values $>1$ represented higher expression of the gene in starving cells than in growing cells. As a control, expression of house keeping gene gpdA was examined. Cells were starved in shaking cultures for 2 or 3 hours in the absence of exogenous glorin treatment $(\mathbf{t} 2, \mathbf{t} 3)$ or for 1 hour followed by 1 hour or 2 hours of glorin treatment (t2+glorin, $\mathbf{t} 3+$ glorin). Relative expression of representative genes involved in cAMP signalling was determined by real-time RTPCR. Mean values of triplicate measurements of the same CDNA \pm SD were plotted.

\subsubsection{P. pallidum tas $A^{\top} /$ tas $B^{-}$null mutant exhibits normal aggregation}

$P$. pallidum PN500 genome encodes two serpentine receptor genes named tas $A$ and $\operatorname{tas} B$ that are highly homologous to $D$. discoideum cyclic AMP receptor genes. tas $A$ is expressed strictly only after aggregates have formed, whereas tas $B$ is expressed moderately during pre-aggregation and aggregation stages, while peak level expression is exhibited when aggregation has completed (Kawabe et al. 2009; Figure 59). In P. 
pallidum PN500, tasB plays key role in normal fruiting body morphogenesis working together with tasA.

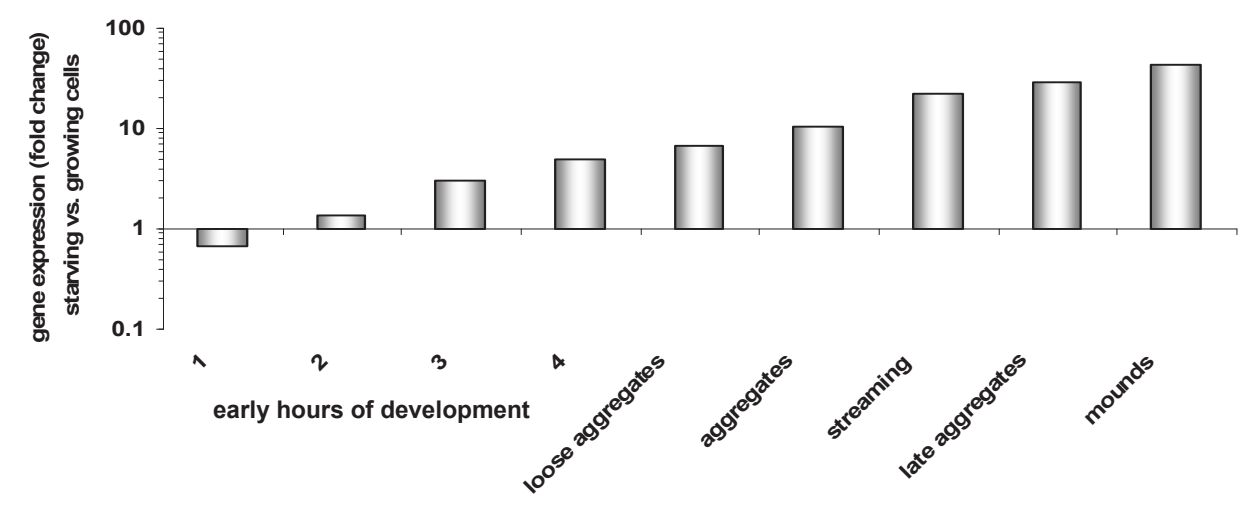

Figure 59: Temporal expression pattern of tasB. P. pallidum PN500 cells grown in association with bacteria were harvested in the late vegetative stage, washed, and resuspended in phosphate buffer. Cells were then starved on phosphate agar plates at a density of $8 \times 10^{5} \mathrm{cells} / \mathrm{cm}^{2}$. Cell samples were collected at indicated developmental stages for total RNA extraction. Numbers 1, 2, $3, \& 4$ represent first four hours of development. Relative expression of TasB was determined by real-time RT-PCR. As a control, expression of house keeping gene gpdA was examined. Fold changes are shown. All data were compared to growing P. pallidum PN500 cells where fold change was set to 1 and values $>1$ represented higher expression of the gene in starving cells than in growing cells. Mean values of duplicate measurements of the same cDNA \pm SD were plotted.

The tas $A$-tas $B^{-}$mutant exhibits astringent developmental aberrations; aggregation is normal, but after that instead of normal fruiting body formation, only small club-shaped structures are formed that are composed of thick lumpy stalks with spore heads containing cyst like cells rather than elliptical spores characteristic of normal fruiting bodies (Kawabe et al. 2009).

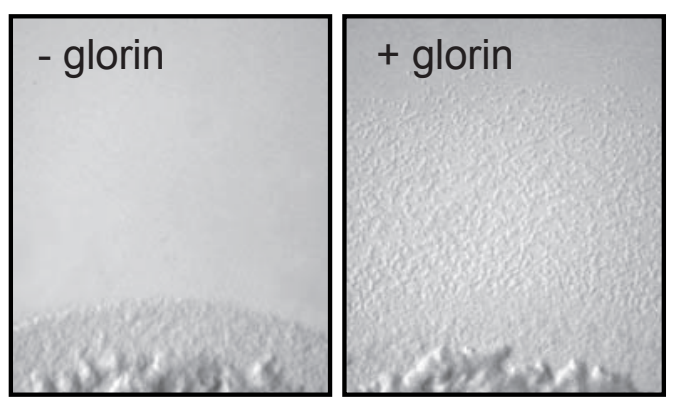

Figure 60: $P$. pallidum PN500 tas $A^{-1} /$ tas $B^{-}$mutant exhibits normal chemotaxis towards glorin. Cells were starved in slow shaking buffer suspensions for 3 hours before $10 \mu$ drops containing $2 \times 10^{5}$ cells were plated on agar without (left picture) or with $1 \mu \mathrm{M}$ final concentration of glorin (right picture). Pictures were taken 3 hours after plating. 
Using chemotaxis assay adapted in this study (as described in Section 3.1), it was shown that tasA-tasB- mutant displayed normal chemotaxis towards glorin (Figure 60) that is assumed as aggregative chemoattractant of $P$. pallidum. These results suggest that a functional cAMP signalling system is not invloved in mediating aggregation of $P$. pallidum amoebae.

Taken together, these limited data lead to speculate that in $P$. pallidum PN500 acrasin mediating aggregation and CAMP coordinating post-aggregation stages of development may function independent of each other. To get further insight into possible cross-talk between aggregation-specific glorin communication system and post-aggregative cAMP signalling, a future line of work would be to study glorin-regulated gene expression in $P$. pallidum PN500 mutants lacking individual components of cAMP signalling system.

\subsection{Glorin elicits rapid changes in gene expression}

Experiments conducted earlier in this study (Section 3.8.3) showed that when $P$. pallidum PN500 amoebae pre-starved for 1 hour were stimulated with glorin, expression of model genes PPL_09347 and PPL_05833 was either increased to high-levels (Figure 37 \& 39) or decreased by high degree (expression kinetics of PPL_07908 shown in Figure 41) within 30 minutes of exposure to glorin. This phenomenon indicated that glorin induces rapid changes in gene expression in starving cells. It was therefore intriguing to characterize more deeply how fast the molecular response to glorin stimulation could be. In this experiment, collection of cell samples (for total RNA extraction) was narrowed down to 5 or 10 minutes of glorin treatment to evaluate rapidity of response.

P. pallidum PN500 amoebae grown in association with bacteria were harvested at late vegetative stage, washed free of bacteria and resuspended in phosphate buffer at concentration of $2 \times 10^{7} \mathrm{cells} / \mathrm{ml}$. A pellet of $2 \times 10^{7}$ cells was stored at $-80^{\circ} \mathrm{C}$ that served as 'growing cells control' for successive gene expression analysis. Suspension of amoebae was pre-starved for 1 hour to initiate development and then equally divided into 2 flasks. Culture in one flask received $1 \mu \mathrm{M}$ glorin pulses at 10-minute intervals for an additional one hour, whereas culture in second flask received no exogenous treatment. Cell samples were collected after 5-, 10-, 30- and 60-minutes of glorin treatment to assess how rapidly mRNA of model genes (PPL_09347 \& PPL_05833; genes shown to be highly induced by glorin) accumulated in response to stimulation with exogenous glorin. 
Samples were collected from untreated culture at the same time points. Total RNA was extracted to prepare cDNA for gene expression analysis using real-time RT-PCR. Relative gene expression of model genes PPL_09347 \& PPL_05833 was determined in glorin-treated and untreated cells (Figure 61). Fold changes were calculated compared to expression in growing cells. As a control, expression of house keeping gene gpdA was examined.
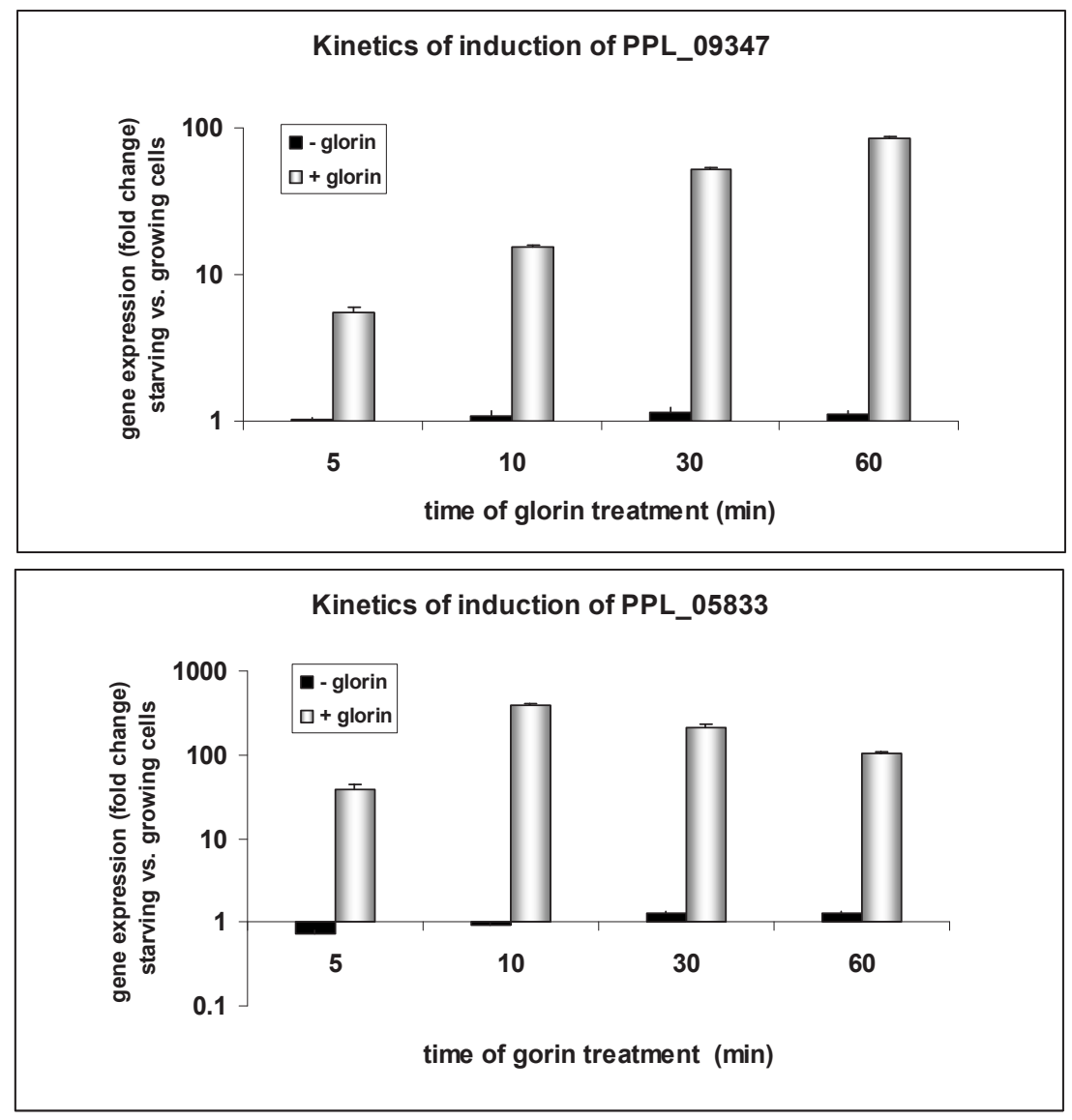

Figure 61: Time-course of PPL_09347 and PPL_05833 mRNA accumulation in untreated and glorin-treated cells. P. pallidum PN500 amoebae were pre-starved for 1 hour followed by treatment with $1 \mu \mathrm{M}$ glorin at 10-min intervals for an additional 1 hour. Cell samples were collected after 5, 10, $30 \& 60$ minutes of glorin treatment. Samples harvested after $5 \& 10$ minutes of glorin treatment received only one pulse of glorin, whereas those collected at $30 \& 60$ minutes received $3 \& 6$ pulses of glorin, respectively. Cell samples were harvested from untreated cells at the same time points. Relative expression of PPL_09347 and PPL_05833 was analyzed using real-time RTPCR. Fold changes are shown. All data were compared to growing $P$. pallidum PN500 cells where fold change was set to 1 and values $>1$ represent higher expression of the gene in starving cells than in growing cells. Values $<1$ show that expression of gene is lower in starving cells than in growing cells. Mean values of triplicate measurements of the same cDNA \pm SD were plotted.

It was noticed that the molecular response to glorin stimulation was very fast; within 5 minutes after addition of glorin, mRNAs of both model genes started to accumulate, 
however, maximal levels of mRNA accumulation were found at different time points for PPL_09347 and PPL_05833 (Figure 61). Within 5 minute of glorin treatment, PPL_05833 and PPL_09347 were induced to 38- and 5.44-fold, respectively, compared to growing cells. PPL_09347 exhibited maximal mRNA level after 1 hour of glorin treatment, whereas mRNA of PPL_05833 accumulated to highest levels after 10 minutes of glorin treatment (Figure 61).

As shown in Figure 61, transcripts of PPL_05833 exhibited a gradual decline at 30- and 60-minutes of glorin treatment indicating that mRNA of PPL_05833 is relatively unstable. The non-conformity of the kinetics of PPL_09347 and PPL_05833 gene responses suggests the possibility that variations may exist in the mechanisms of regulation of different glorin-induced genes.

\subsubsection{Pre-starvation period is not necessary to observe glorin-induced gene expression}

In all experiments aimed to investigate glorin-regulated gene expression, $P$. pallidum PN500 cells were pre-starved for 1 hour prior to exposure to glorin. However, rapidity of response to glorin treatment (Figure 61) suggested that de novo protein synthesis may not be required to observe glorin mediated changes in gene expression in $P$. pallidum PN500 amoebae. This hypothesis prompted us to investigate responses to exogenous glorin in cells that were freshly washed free of bacteria, without any pre-starvation period, to understand whether or not starvation is necessary to observe glorin effects.

P. pallidum PN500 cells were harvested from bacterial growth plates at late vegetative stage, washed free of bacteria and resuspeneded in phosphate buffer at concentration of $2 \times 10^{7}$ cells $/ \mathrm{ml}$. This suspension was equally divided into 3 flasks. Culture in one flask was immediately pulsed with $1 \mu \mathrm{M}$ glorin at 10-minute intervals for 1 hour without any prestarvation period. Cells in flask 2 were pre-starved for 1 hour prior to receiving glorin pulses every 10-minute for an additional hour. Culture in flask 3 did not receive any external treatment and served as 'untreated control' for the time period. Cell samples (each containing $2 \times 10^{7}$ cells) were harvested from both un-prestarved and prestarved cultures after 10-, 30-, and 60-minute of glorin treatment for total RNA preparation. Samples were harvested from 'untreated' control culture at the same time points. Quantitative real-time PCR was employed to compare induction kinetics of PPL_09347 and PPL_05833 in un-prestarved and pre-starved cells (Figure 62). 

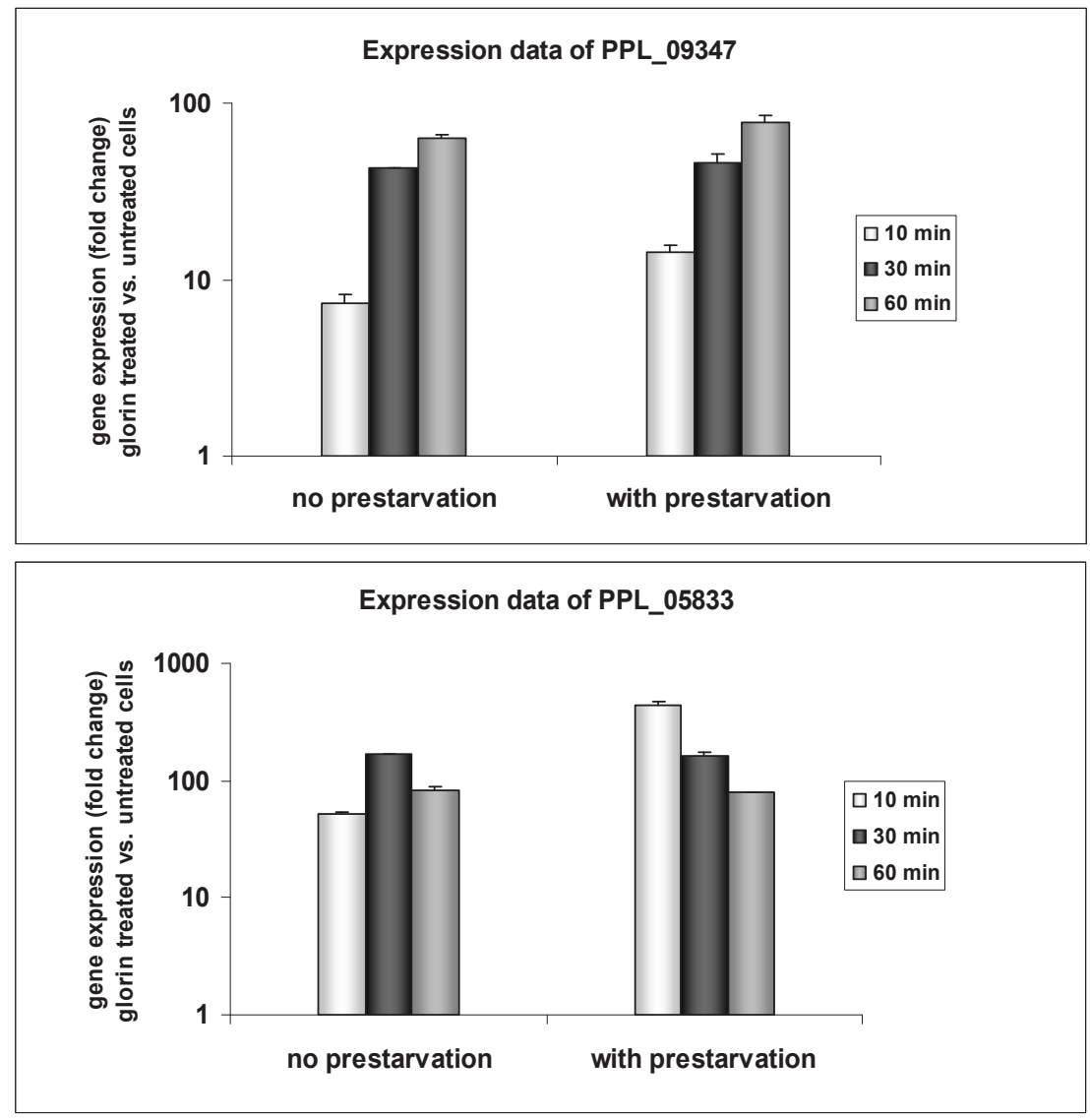

Figure 62: Comparison of induction kinetics of PPL_09347 and PPL_05833 in un-prestarved and prestarved cultures of $P$. pallidum PN500 cells following stimulation with exogenous glorin. Growing P. pallidum PN500 were harvested, washed to remove bacteria and resuspended in phosphate buffer at a density of $2 \times 10^{7} \mathrm{cell} / \mathrm{s} / \mathrm{ml}$. Amoebae were then developed in shaking cultures in the presence or absence of glorin. Cells in 'no prestarvation culture' were immediately treated with $1 \mu \mathrm{M}$ glorin at 10-minute intervals for 1 hour without any pre-starvation period. Culture labelled as 'with pre-starvation' was first starved for one hour before receiving glorin $(1 \mu \mathrm{M})$ treatment every 10 minute for an additional hour. A control culture was maintained in the absence of glorin treatment. Cell samples were collected after 10-, 30- and 60-minutes of glorin treatment for total RNA extraction to analyse gene expression. Samples were collected from untreated cells at the same time points. As a control, expression of house keeping gene gpdA was examined. Relative expression of model genes PPL_09347 and PPL_05833 was determined by real-time RT-PCR. Fold changes are shown. All data were compared to untreated P. pallidum PN500 cells where fold change was set to 1 and values $>1$ represented higher expression of the gene in glorin treated cells than in untreatedcells. Mean values of triplicate measurements of the same CDNA \pm SD were plotted.

As shown in Figure 62, induction of PPL_09347 in response to exogenous glorin was comparable in both un-prestarved and $1 \mathrm{hr}$ pre-starved cultures. PPL_05833 was also induced equally well in both cultures; however, an observed difference was comparatively higher induction of PPL_05833 after 10 minutes of glorin treatment in cells 
that were pre-starved (Figure 62), indicating that starvation may increase sensitivity of cells towards initial exposure to glorin. Nevertheless, responses observed for PPL_05833 after 30- \& 60-minutes of glorin treatment were consistent in both un-prestarved and prestarved cells (Figure 62). Collectively, these data show that starvation is not required to observe glorin-regulated gene expression and supported the idea that prior protein sysnthesis may not required to see glorin responses. These results suggest that all components of an intracellular glorin signalling cascade are letently present in growing $P$. pallidum PN500 amoebae.

\subsubsection{Glorin-regulated gene expression is not dependent on de novo protein synthesis}

To verify the possibility that protein expression is not required for detection of gene regulatory effects of glorin, vegetative-stage $P$. pallidum PN500 cells were developed in shaking cultures in the presence or absence of cycloheximide that inhibits the elongation step of protein synthesis, followed by treatment with glorin. This approach was designed to assess the sensitivity of 'glorin-induced differential changes in gene expression' to the cycloheximide that is known to inhibit the synthesis of proteins in related species $D$. discoideum (Clotworthy \& Traynor 2006). In order to examine the sensitivity of $P$. pallidum PN500 cells to cycloheximide, a suspension culture of these cells $\left(2 \times 10^{7} \mathrm{cells} / \mathrm{ml}\right)$ was treated with $2 \mathrm{mM}$ cycloheximide for 2 hours. A second culture was run under similar conditions in the absence of any external treatment (no cycloheximide added) for the time period and served as 'control'. Cycloheximide treated and untreated cells were then plated for development on non-nutrient agar plates at a density of $8 \times 10^{5} \mathrm{cells} / \mathrm{cm}^{2}$ at $21^{\circ} \mathrm{C}$. It was observed that cycloheximide-treated cells exhibited 12 hours delay in development compared to untreated cells (data not shown), indicating that $P$. pallidum PN500 cells are sensitive to cycloheximide in the same manner as are the $D$. discoideum amoebae.

Growing $P$. pallidum PN500 cells were harvested, washed and suspended in phoshphate buffer at cell density of $2 \times 10^{7}$ cells $/ \mathrm{ml}$. A pellet of $2 \times 10^{7}$ cells was stored at $80^{\circ} \mathrm{C}$ and served as 'growing cells control' for consecutive gene expression analysis. Cell suspension was equally splitted to 4 flasks. Culture in flask 1 did not receive any external treatment (- cycloheximide - glorin) and acted as control for the time period. Cells in flask 2 were incubated with $2 \mathrm{mM}$ cycloheximide immediately at the onset of starvation for 30 minutes and then maintained in the absence of glorin treatment for an 
additional 60 minutes (+ cycloheximide - glorin); this culture was prepared to detect effects of only cycloheximide treatment. Culture 3 was pre-starved for 30-minutes in the absence of cycloheximide treatment followed by exposure to $1 \mu \mathrm{M}$ glorin at 10 -minute intervals for 60 minutes (- cycloheximide + glorin); this culture was prepared to observe the effects of only glorin treatment. Flask 4 culture was incubated with $2 \mathrm{mM}$ cycloheximide instantly at the onset of starvation for 30 minutes prior to the addition of 1 $\mu \mathrm{M}$ glorin every 10 minute for an additional 60 minutes (+ cycloheximide + glorin); this culture was prepared to examine gene regulatory effects of glorin in cells treated with cycloheximide. All cultures were developed under slow shaking conditions (i.e. 100 rpm). Cell samples were collected from glorin treated cultures (flask $3 \& 4$ ) after 10-, 30-, and 60-minutes of exposure to glorin. Samples were harvested from culture $1 \& 2$ (not treated with glorin) at the same time points. Total RNA was extracted and quantitative RT-PCR was employed to study induction kinetics of two model genes PPL_09347 and PPL_05833 in untreated cells (i.e. control) and cells treated either with glorin or cycloheximide or both glorin and cycloheximide. All data were compared to gene expression in growing cells (Figure 63). 

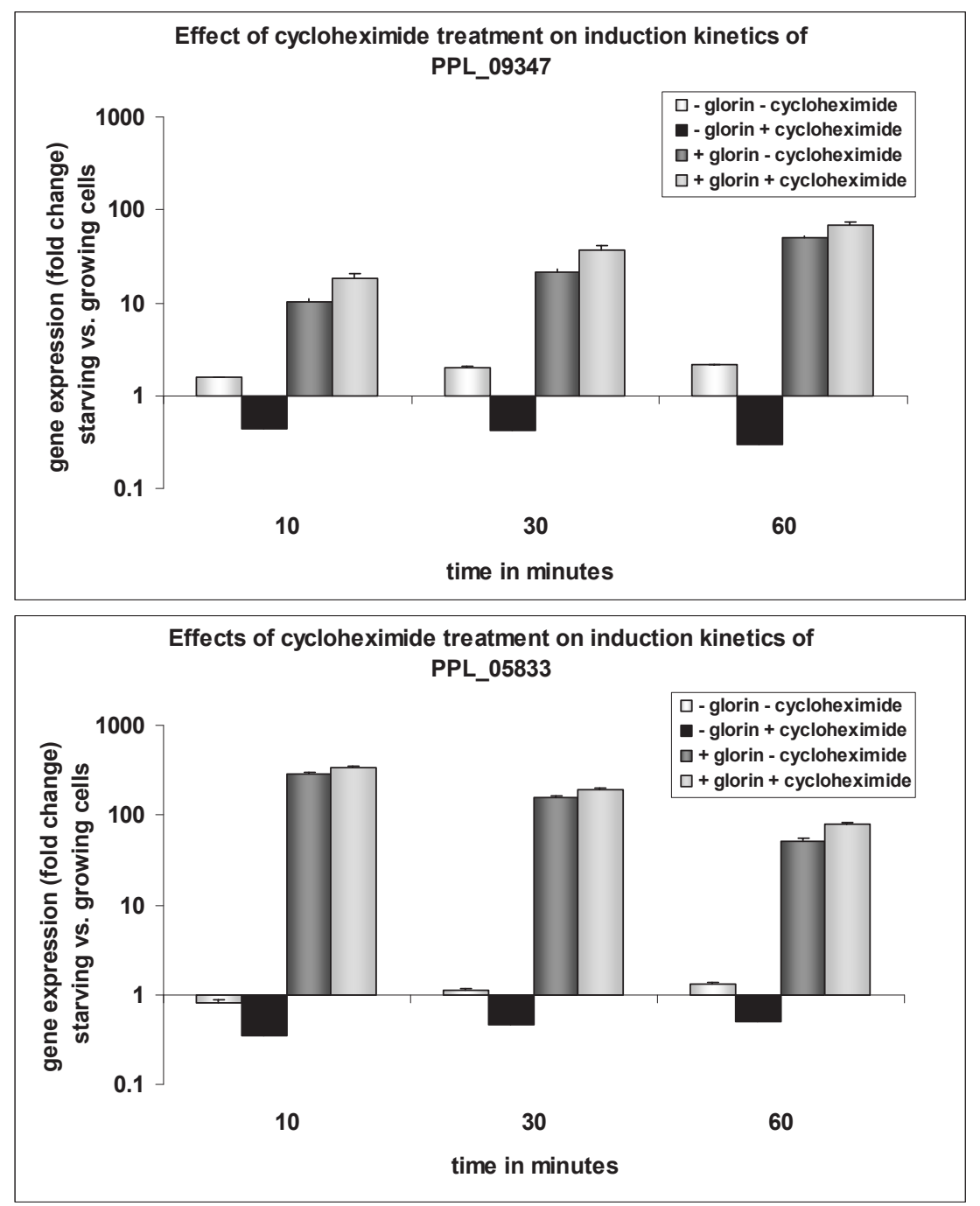

Figure 63: Glorin-induced gene expression is insensitive to cycloheximide. $P$. pallidum PN500 cells were harvested from bacterial growth plates, washed and resuspended in phosphate buffer. The cell suspension was splitted into 4 flasks, each of which received one of the following treatments: (i) no additions (ii) $2 \mathrm{mM}$ cycloheximide for 30 minutes immediately at the onset of starvation (iii) pre-starved for 30 minutes followed by addition of $1 \mu \mathrm{M}$ pulses of glorin delivered at 10-min intervals for an additional 60 minutes (iv) pre-treatment with $2 \mathrm{mM}$ cycloheximide for 30 minutes prior to addition of $1 \mu \mathrm{M}$ glorin every 10- minutes for an additional 60 minutes. From glorin treated cultures, cells were sampled after 10, $30 \& 60$ minutes of exposure to glorin. From untreated or only cycloheximide treated cultures, cell samples were collected at the same time points. Total RNA was extracted ans relative expression of model genes PPL_09347 and PPL_05833 was determined by real-time RT-PCR. As a control, expression of house keeping gene gpdA was examined. Fold changes are shown. All data were compared to growing $P$. pallidum PN500 cells with fold change set to 1 where values $>1$ represented higher expression of the gene in glorin treated cells than in growing cells. Values $<1$ represented lower expression of the gene in glorin treated cells than in growing cells. Mean values of triplicate measurements of the same $\mathrm{CDNA} \pm \mathrm{SD}$ were plotted. 
It was found that in control cells (shaken in the absence of added glorin and cycloheximide), PPL_09347 was expressed to low levels, whereas PPL_05833 exhibited only basal level expression (Figure 63). A short incubation of cells in cycloheximide (in the absence of glorin treatment) led to down-regulation of both genes, indicating that even slight induction of model genes in response to starvation was inhibited by cycloheximide (demonstrating that starvation-induced expression of PPL_09347 and PPL_05833 may require de novo protein synthesis). However, cells treated only with glorin showed precocious induction of both PPL_09347 and PPL_05833 (Figure 63). Interestingly, it was observed that amoebae pre-treated with cycloheximide retained their ability to respond to externally added glorin. Cultures shaken in the presence of cycloheximide showed no detectable changes in glorin-induced gene expression, instead expression of PPL_09347 and PPL_05833 was somewhat enhanced in cells treated with both cycloheximide and glorin. This phenomenon may reflect that cycloheximide stabilizes mRNA signal that would otherwise be degraded at this time in development. These data indicate that glorin-induced changes in gene expression do not require de novo protein synthesis.

\subsection{Glorin induces aggregation sensitivity in starving $P$. pallidum cells}

Aggregation is the first step in the transition from growth to development of Dictyostelids. Polysphondylium amoebae aggregate towards collecting centres that would develop spontaneously (Shaffer 1957b). Upon starvation, a few of the randomly moving amoebae come to rest and start acting as centres on which the others converge. Such centres are known to secrete acrasin (Bonner 1949). Sensitivity to acrasin begins to develop slowly throughout the population as centres appear.

In the present work, it is shown that exposure of $P$. pallidum PN500 cells to glorin prepared starving cells for aggregation precociously, compared with control cells that were not treated with glorin. To examine stimulatory effects of glorin on aggregation capacity of amoebae, vegetatively growing $P$. pallidum PN500 cells were harvested, resuspended in phosphate buffer at a cell density of $2 \times 10^{7}$ cells $/ \mathrm{ml}$, and pre-starved for 1 hour. Suspension of amoebae was then divided into three parts. One culture was pulsed with $100 \mathrm{nM}$ glorin (final concentration) at 10 minute intervals for 2 hours. A second culture was treated with $1 \mu \mathrm{M}$ glorin (final concentration) added every 10-minutes for 2 hours. A control culture was run under similar conditions where cells did not receive any 
exogenous treatment. Cells were then harvested by centrifugation and plated for development as monolayers on non-nutrient agar plates at a density of $8 \times 10^{5} \mathrm{cells} / \mathrm{cm}^{2}$.

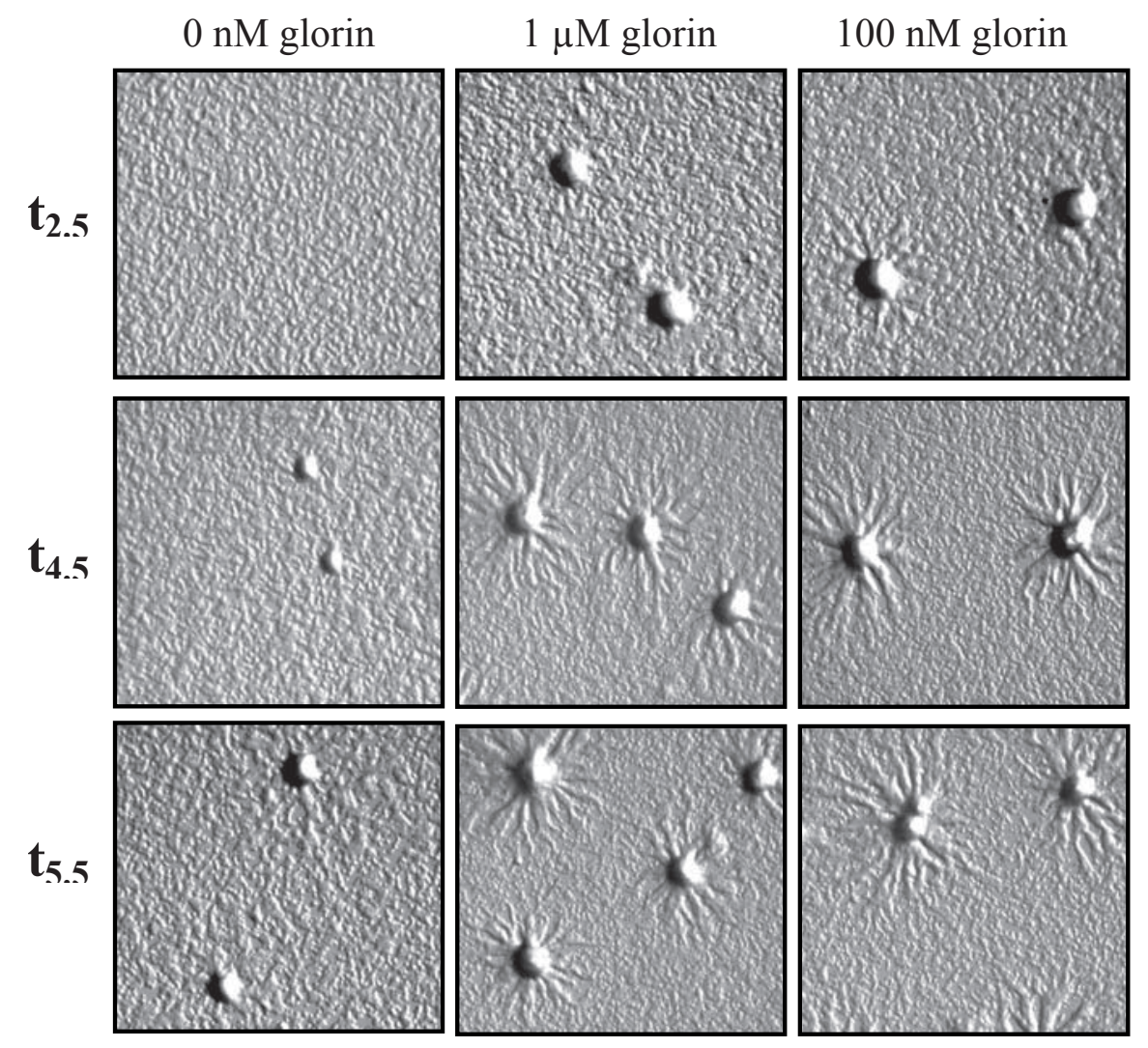

Figure 64: Developmental phenotypes of glorin treated and untreated cells. $P$. pallidum PN500 cells were first pre-starved for 1 hour in shaken suspensions and then stimulated with OnM, $1 \mu \mathrm{M}$ and $100 \mathrm{nM}$ glorin at 10-min intervals for 2 hours in three separate cultures. Amoebae were then harvested from shaking cultures and plated on non-nutrient agar plates and photographed when first signs of aggregation were seen in glorin-treated cells (middle and right pictures). Photographs were captured for untreated cells at the same time points, shown in the left picture. Cells were equally sensitive to both concentrations of glorin i.e. $1 \mu \mathrm{M}$ and $100 \mathrm{nM}$ applied as pulses. At 2.5 hours $\left(t_{2.5}\right)$ after plating, the centres have grown considerably in glorin stimulated cells, though still almost devoid of streams indicating that glorin stimulates rate of aggregation in starving cells. At the same time there was no sign of centre formation in untreated cells $\left(t_{2.5 ;}\right.$ left picture).

It was observed that glorin treatment sensitized cells and they gained ability to initiate centres precociously as compared to untreated cells. Appearance of some primary centres was noticed in glorin treated cells at approximately 2.5 hours $\left(\mathrm{t}_{2.5}\right)$ after plating, whereas at the same time point untreated cells showed no signs of centre formation (Figure 64). Amoebae stimulated with glorin exhibited streams of cells converging on the aggregation centres at 4.5 hour (Figure $64 ; \mathrm{t}_{4.5}$ ). In comparison, centres just started to appear in untreated cells at that time ( $t_{4.5}$ hour), implying that initial formation of aggregation centers was delayed by 2 hours (Figure 64). By 5.5 hours (Figure 64; $t_{5.5}$ ), 
some more aggregation centers were visible in untreated cells. The glorin-treated cells congregated more efficiently into aggregates, indicating that sensitizing cells with glorin switched aggregation competence to earlier time points. No pronounced difference in responsiveness of cells was observed with two different concentrations of glorin employed for pulsing (i.e. $100 \mathrm{nM}$ and $1 \mu \mathrm{M}$ final concentration of glorin). At 5.5 hours $\left(t_{5.5}\right)$ centres began to grow in untreated cells. These data suggest that glorin accelerates cell aggregation; most probably by precociously inducing expression of several aggregation specific genes. 


\section{Discussion}

One of the major transitons in evolution is the emergence of multicellular organisms from single cells (Mian \& Rose 2011). Multicellularity apparently evolved through clonal development (from a unicellular spore or zygote) or aggregation of scattered cells (Siu et al. 2011; Grosberg \& Strathmann 2007; Bonner 1998). Aggregative development of multicellularity takes place in a few groups of microorganisms, including myxobacteria, myxomycetes, and Dictyostelids (Siu et al. 2011; Grosberg \& Strathmann 2007; Bonner 2000). A fundamental requirement for aggregative multicellular organization is cell-cell communication that coordinates cellular behaviour. The unique life cycle of social amoebae shifting between unicellular and multicellular stages offers an ideal system to study complex process of intercellular communication (Chisholm \& Firtel 2004; Annesley \& Fisher 2009). These organisms live independently for most of their life cycles, but aggregate to become multicellular in response to starvation, constructing a fruiting body within 24 hours. So far, intercellular communication has been extensively studied in the model organism $D$. discoideum that uses cAMP language for cell-cell interaction to organize the process of aggregation.

Glorin is a peptide chemoattractant employed by $P$. violaceum amoebae for cell-cell communication to coordinate the process of aggregation. It was proposed that glorin mediated communication might be wide-spread among the Dictyostelids (Schaap et al. 2006). In the first part of this study, responses to glorin were studied in all 4 phylogenetic groups of Dictyostelids defined by Schaap et al. (2006) to identify evolutionary roots of peptide communication in Dictyostelids. In the second part of this research work, specific cellular responses to glorin such as glorin-mediated changes in gene expression patterns and developmental effects of glorin were studied in the genetically tractable species $P$. pallidum PN500.

\subsection{Glorin is an ancient extracellular messenger molecule used for intercellular communication in Dictyostelids}

$P$. violaceum amoebae start to secrete glorin when the aggregation process begins to stimulate surrounding cells of the same species that can sense glorin signal by specific cell surface receptors and move chemotactically toward glorin gradients generated by the glorinase activity of these cells (De Wit et al. 1988; Wurster et al. 1976). Thus, $P$. violaceum cells possess the requisite biochemical machinery to use glorin as acrasin. To 
explore how outspread glorin communication is among Dictyostelids, a modified small population chemotaxis assay was used (Section 3.1) and responses to glorin of a collection of species that span the dictyostelid phylogeny were examined (Table 1). All tested Polysphondylid species from early diverged group 2 reacted well to glorin in this study (Figure 16). Two dictyostelid taxa $D$. gloeosporum and $D$. oculare placed in group 2 were also equally responsive to glorin (Figure 16). This study further showed for the first time that group 1 Dictyostelids are chemotactically reactive to glorin in a very similar manner as the Polysphondylids (Figure 17). However, none of the tested Acytostelium species responded to glorin.

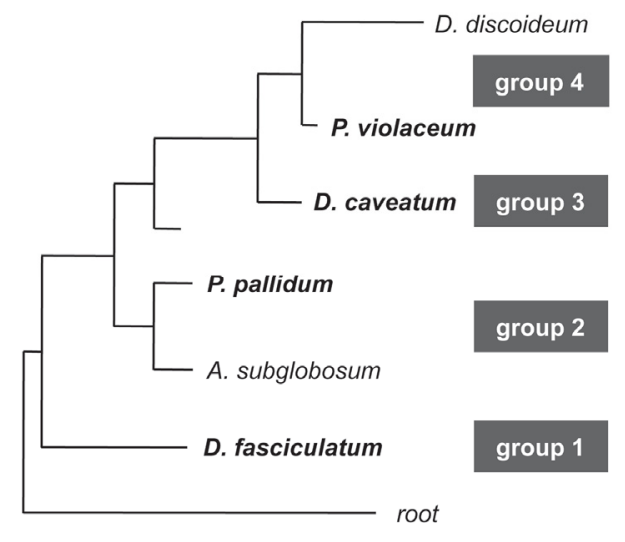

Figure 65: A simplified phylogenetic tree summarizing glorin chemotaxis by social amoebae. This tree is rooted according to (Schaap et al. 2006) and is not drawn to scale. The four major groups of social amoebae are illustrated by a single representative species investigated in this study (except for Dictyostelium caveatum, which was reported to respond to glorin by Waddell (1982). Species that show chemotaxis to glorin are indicated in bold.

Thus, this study uncovered that glorin-based cell-cell signalling is a common feature of species from the early diverging group 1 and 2 of Dictyostelids and therefore, the oldest form of intercellular communication used at the transition from growth to multicellular development of social amoebae. Previously, D. caveatum, belonging to group 3 in the molecular phylogeny of Dictyostelids (Schaap et al. 2006), has been noted to respond chemotactically to glorin (Waddell 1982b). Chemotactic aggregation of individual amoebae of $P$. violaceum, which is more related to the most derived group 4 species (Romeralo et al. 2011b; Schaap et al. 2006) than to other group 2 Polysphondylids, was shown to be mediated by glorin (Shimomura et al. 1982). Nevertheless, none of the tested group 4 species was reactive to glorin. Thus, a striking finding of this research work is that in the course of evolution, social amoebae either lost or abandoned the use 
of glorin as intercellular communication molecule in group 2 Acytostelia, some group 3 species and all group 4 Dictyostelids (Figure 65).

In some of these taxa, glorin-mediated cell-cell signalling was replaced by communication systems based on cellular metabolites such as cAMP and folate derivatives. The molecular phylogeny introduced by Schaap et al. (Schaap et al. 2006) anticipated that group 1 Dictyostelids preceded group 2 taxa, though an alternative root organizing group $1 / 2$ and $3 / 4$ in a paraphyletic clade cannot be ruled out. Complete nuclear genome sequences of $D$. discoideum, $P$. pallidum PN500, and D. fasciculatum (Heidel et al. 2011) and the mitochondrial genomes of these species (Heidel \& Glöckner 2008) encourage the latter possibility. Whatever the origin of Dictyostelids is, data presented in this dissertation strengthen the assumption that the unknown last common ancestor of all social amoebae used glorin to coordinate the transition from uni-to multicellularity.

Critical evaluation of the positive chemotactic response of group 1 and 2 species (except Acytostelids) to glorin suggests that the cells of these species are capable of sensing chemoattractant and detect its gradient. It is intriguing that sensitivity to glorin is highest just before aggregation of responding species. These facts support that glorin may be the acrasin of responding species. However, at this stage of research, chemotaxis of amoebae towards glorin does not identify this dipeptide as the chemical messenger responsible to organize aggregation. Therefore, future studies should focus on the isolation of glorin from aggregating amoebae of group 1 and 2 species to confirm the presumption that glorin is indeed the acrasin of these species. The identification of some components of the glorin signalling pathway, most importantly glorinase(s), cell surface glorin receptor and enzymes involved in glorin biosynthesis in the genetically amenable species $P$. pallidum PN500 may lead to further elucidation of the process of chemotaxis and cell aggregation in this species, whereas comparison with other genetically tractable dictyostelid species will provide interesting information about possible functions of glorinsignalling system genes in species that no longer use glorin as acrasin.

\subsection{Stage specific responses of $P$. pallidum PN500 cells to exogenous glorin}

In some experiments that were designed to study gene regulatory effects of glorin, considerable variations were noticed in the cellular responses to exogenous glorin when different batches of $P$. pallidum PN500 cells were compared with each other (compare expression levels of PPL_05833 after 2 hours of glorin treatment in Figure 27 \& Figure 
39). This phenomenon can be explained by the fact that, despite great care, the populations of cells used in these experiments were never completely synchronized. Insufficient synchronization results in substantial variability in the number of glorin receptors per cell (De Wit et al. 1988), therefore, responses to externally added glorin varied significantly between experiments. It was also observed that $P$. pallidum PN500 cells were sensitive to the effects of exogenous glorin only within a certain period during early hours of development, whereas responses decline once cells have crossed that sensitivity stage (data not shown); most probably because cells establish endogenous glorin signalling with progression in development, therefore responses to exogenous glorin diminish.

\subsection{Glorin mediates rapid changes in gene expression during early development of $P$. pallidum}

D. discoideum cells exhibit profound changes in transcription as they undergo growth- to aggregation transition (Iranfar et al. 2003; Van Driessche et al. 2002). Extracellular cAMP pulses not only act as a chemoattractant to organize aggregation but also to induce optimal expression of genes whose activity is required for the aggregation process, such as genes encoding aggregation-stage cAMP receptors, adenylate cyclase A, extracellular cAMP phosphodiesterase PdsA (Schaap 2011a; Alvarez-Curto 2001; Pitt et al. 1993; Darmon et al. 1975; Gerisch et al. 1975) and the cell adhesion molecule gp80 (Desbarats et al. 1992; ; Kumagai et al. 1991; Ma and Siu 1990; Mann \& Firtel 1989; Kumagai et al. 1989; Kamboj et al. 1989, 1988; Mann \& Firtel 1987; Noegel et al. 1986; Wong \& Siu 1986), whereas many preaggregation stage genes are repressed by cAMP pulses (Mann \& Firtel 1989).

In this study, using high-throughput RNA-seq technology it was shown that the treatment of $P$. pallidum PN500 cells with glorin led to dramatic changes in gene expression patterns during first few hours of starvation (Section 3.6.2; Figure 24). It was observed that glorin not only induced expression of a number of genes but also enhanced expression of many starvation-induced genes. However, several other genes were repressed in response to glorin treatment including some of the genes that were, otherwise, induced by starvation. Products of genes whose expression was induced or enhanced by glorin, might play important roles in the aggregation process. Search of the putative molecular functions for glorin-induced genes, based on homologies to annotated $D$. discoideum genes, indicated that many genes upregulated by glorin may play roles in 
signal transduction pathways, reorganization of actin cytoskeleton during chemotaxis, reprogramming of the metabolic machinery, gene transcription and protein translation, cell adhesion, degradation of nuclear DNA, secretory pathways, transportation of metabolic products, lipids and toxins across extra- and intracellular membranes, cell recognition and transcription repression (Table 4; section 3.7.1).

\subsection{Rapid turn-over of transcripts of glorin-induced genes}

RNA-seq analysis demonstrated that glorin-mediated induction/enhancement of expression of several genes was transient (Section 3.6.2). When shaken suspensions of $P$. pallidum PN500 cells were stimulated with glorin for 1 hour, a more than 3-fold differential expression of 115 genes, 70 of which were upregulated by 3 - to 57 -fold was observed. When cells were treated with glorin for an additional hour, 120 genes were differentially expressed, but only 20 of them were upregulated more than 3-fold (Section 3.6.2; Figure 24). It was noticed that 133-, 16- \& 12-fold increase in the mRNA levels of PPL_05833 (Figure 27), PPL_12248 and PPL_12249 (Figure 29), respectively, seen after 1 hour of glorin treatment was not maintained even if pulses of glorin were given frequently at short intervals (Figure 39; Figure 42; Figure 43). Consequently, transcript levels of these genes declined significantly after 2 hours of exposure to exogenous glorin. This behaviour indicates a rapid mRNA turn-over of glorin-induced gene transcripts. Studies suggest that in mammalian cells a large percentage of activationinduced transcripts have short mRNA half-lives (Raghavan \& Bohjanen 2004; Frevel et al. 2003; Tebo et al. 2003; Raghavan et al. 2002; Lam et al. 2001); demonstrating that cells have a coordinated strategy to rapidly modulate the mRNA levels of these genes (Lam et al. 2001). A similar situation was described for the regulation of prolactin gene transcription by thyrotropin-releasing hormone $(\mathrm{TRH})$ in the $\mathrm{GH} 4$ rat pituitary cell line, where mRNA levels started to decline within 1 hour after initial rapid induction of prolactin gene expression by TRH and further addition of thyrotropin-releasing hormone resulted in no additional response during this time (Saski et al. 1984; Murdoch et al. 1983). The 'burst-attenuation' kinetics of expression of genes in response to glorin may be due to a common molecular mechanism at the gene level. The inducibility of many of these genes may be due to transcriptional activation of their promoters and the rapid degradation of these transcripts permits their levels to rapidly drop when transcriptional activation is terminated. Assuming that glorin is the acrasin of $P$. pallidum, and then under natural conditions, expression of these genes might be stably induced/enhanced by glorin in the aggregation process, when products of these genes are actually required. This 
assumption was verified by examining developmental expression kinetics of a number of glorin-regulated genes in cells starved on agar surface. These experiments showed that most of the genes 'transiently upregulated by glorin under shaking conditions' were stably expressed in aggregating cells during development on agar (Figure $26 \& 46$, Figure $48 \& 26$, Figure $27 \& 50$; Figure $51 \& 26$; Figure $53 \& 27$; Expression kinetics of PPL_06644 depicted in Figure $47 \&$ Table 3); indicating that cell-cell contacts may be necessary for stable expression of these genes. Under artificial shaking culture conditions, externally applied glorin induced these genes or enhanced their expression precociously (before cells have gained aggregation competence); therefore at this time in development, transcripts of most of these genes were unstable and degraded promoting rapid turn-over of these genes. Recently, it was reported that mRNA decay plays an important role in regulating transient gene expression events in mammalian cells (Raghavan \& Bohjanen 2004). Another explanation of rapid decrease in certain mRNA abundances may be that excess of these transcripts are accumulated in mRNA-protein complexes at this stage of development; though mRNA sequestration has not generally been recognized as a major factor in the dynamics of mRNA abundance (Aragon et al. 2006). Yet another justification of the rapid changes in mRNA levels of many genes may be that increases in translation rates lead to decreases in transcript abundance (Aragon et al. 2006). Hence, by regulating mRNA turnover, cells can selectively and efficiently maintain or diminish their gene expression programme appropriate for a specific stage of development.

\subsection{Glorin-mediated gene repression}

When $P$. pallidum PN500 cells in shaken suspensions were stimulated with exogenous glorin for 1 hour, 45 genes were down-regulated between 3- and 17-fold (Figure 24; Section 3.6.2). However, after 2 hours of exposure to glorin, 100 genes were downregulated (Figure 24; Section 3.6.2). A reasonable justification of this difference may be the rapid kinetics of glorin effects. Similar phenomenon was observed in microarraybased studies of primary human T cells where almost 400 transcripts that were stable in resting $T$ cells became destabilised following $T$ cell receptor-mediated stimulation of cells and their steadystate levels were repressed (Raghavan et al. 2002). It was noticed that $80 \%$ of the genes down-regulated after two hours of glorin treatment were actually downregulated one hour earlier also, but they did not reach the 3-fold change threshold (Section 3.6.2). Stimulating $P$. pallidum cells with exogenous glorin starting at 1 hour of starvation was an unnatural experimental arrangement. Apparently, this approach 
interfered with the normal developmental cycle and the associated gene expression changes. In wild-type cells, under natural conditions, glorin may be secreted by cells at the onset of the aggregation process to mediate relevant gene expression changes including repression of early developmental genes, induction of aggregation-stage genes and enhancement of expression of many other starvation induced genes. However, when $P$. pallidum PN500 cells in shaking cultures were treated with $1 \mu \mathrm{M}$ glorin at 30 -minute intervals during very early hours of starvation (Section 3.6), normal physiological concentrations of acrasin and natural timings of its effects do not exist when correlated to wild type cells. Most probably, treating $P$. pallidum amoebae with glorin conditioned them in a way that either the starvation responses of the signalling competent cells were enhanced precociously or these responses were shifted to earlier time points in the developmental cycle. Therefore, repression of many genes observed in response to glorin treatment during early hours of starvation may be required for proceeding to aggregation under normal physiological conditions. Apparently, stimulation of cells with glorin triggered signals that altered the cellular mRNA degradation machinery, leading to specific transcript destabilisation. Recently, it was suggested that mRNA decay may be an important mechanism for gene repression in an activation-dependent manner (Raghavan \& Bohjanen 2004). A recent study by Frevel and colleagues (Frevel et al. 2003) suggested that p38 mitogen-activated protein kinase (MAPK) plays critical role in the regulation of the decay of a variety of transcripts in THP-1 monocytes. Annotation of GO terms to glorin-repressed genes was not very explanatory because many of these genes have not yet been assigned to any specific functions (Section 3.7.2). However, a number of genes repressed by glorin may encode products that are constituents of the metabolic machinery (Appendix Table A7 and A8). Future studies focusing on the identification and subcellular localization of the proteins encoded by glorin repressed genes will help to understand the functional roles of these genes.

\subsection{Allosteric or covalent modification of existing proteins may mediate rapid effects of glorin}

In this study, it is shown that mRNAs of model glorin-induced genes started to accumulate within 5 minute after the addition of glorin (Figure 61; Section 3.10). Similar rapid kinetics of induction has been reported for P-enolpyruvate carboxykinase gene transcription in H4IIE cells (differentiated rat liver cells) in response to stimulation with CAMP analogs (Sasaki et al. 1984); transcription of multiple genes after the addition of beta interferon to human fibroblasts or to HeLa cells (Larner et al. 1984); prolactin gene 
transcription in pituitary cells on exposure to cAMP (Preston et al. 1990; Sasaki et al. 1984; Murdoch \& Rosenfeld 1982b) and the yeast Saccharomyces cerevisiae responding to diverse environmental transitions (Aragon et al. 2006; Martinez et al. 2004; Newcomb et al. 2003).

Apparent rapid increase in steady-state mRNA levels of a number of genes in response to glorin treatment (Figure 24; Appendix Table A4, A5 \& A6) may result from a combination of de novo transcription, stabilization of mRNA, or potential release of extraction-resistant mature mRNA present in cells in a rapidly releasable form, possibly sequestered in protein complexes. Formation of such mRNA-protein complexes has been reported in yeast, mammalian and plant cells (Aragon et al. 2006; Kedersha \& Anderson 2002). Release of the sequestered mRNAs upon exposure of cells to glorin may be a mechanism that allows cells to respond to environmental conditions as quickly as possible, in preparation for the activation of transcription and translation.

Regulated mRNA turn over upon extracellular stimulation is a critical process in control of gene expression in eukaryotic cells. Recently, it has been shown that mRNA stability is modulated by signal transduction pathways involving phosphorylation events (Sinsimer et al. 2008; Knapinska et al. 2005; Shim \& Karin 2002). In mammalian cells several signalling pathways are involved in regulating the stability of mRNAs including activation of protein kinase $\mathrm{C}$, Phosphatidylinositol 3-kinase (PI3K), and the mitogen activated protein kinases, elevation of intracellular $\mathrm{Ca}^{2+}$, c-Jun $\mathrm{N}$-terminal protein kinase (JNK) and p38 (Shim \& Karin 2002). In D. discoideum YakA protein kinase regulates stabilization of pkaC mRNA by inhibiting the expression of PufA protein (Souza et al. 1999, 1998).

Seemingly, the rapidity of the effects of glorin, and the observation that de novo protein synthesis is not required may indicate that glorin is directly affecting transcription of 'glorin-regulated genes' and the signal generated by binding of glorin to cell surface receptors rapidly reaches the cell nucleus. A protein phosphorylation-dephosphorylation mechanism appears to be an obvious possibility that may require either the translocation of a protein kinase and/or protein phosphatase from the cytoplasm to the nucleus, or the translocation of a regulatory phosphoprotein to the nucleus, or the presence of a cAMPdependent protein kinase/protein phosphatase system in the nucleus. It has been shown that in mammalian cells hormones or neurotransmitters acting though G-protein coupled receptors activate second messenger pathways that in turn regulate the phosphorylation of specific nuclear proteins, leading to change in gene expression (De Cesare D \& 
Sassone-Corsi P 2000; Montminy 1997; Lalli \& Sassone-Corsi 1994; Sassone-Corsi 1994). Previously, it was proposed by Jungmann and colleagues (Jungmann et al. 1983) that isoproterenol induces a cAMP-dependent protein kinase to translocate into the nucleus of $\mathrm{C} 6$ glioma cells that results in the phosphorylation of histones and/or RNA polymerase II. If such a mechanism is involved then the substrate of phosphorylation is of principle importance. Rosenfeld and coworkers reported that stimulation of $\mathrm{GH} 4$ cells with CAMP or thyrotropin-releasing hormone leads to rapid phosphorylation of a chromatin-associated basic protein, and this modification occurs prior to the increase of prolactin gene transcription (Murdoch et al. 1983; Murdoch et al. 1982a; Murdoch \& Rosenfeld 1982b). It has been shown that addition of 8-CPT-cAMP to H4IIE rat pituitary cells causes full activation of protein kinase in less than 1 minute (Murdoch \& Rosenfeld 1982b), therefore, on kinetic grounds, protein kinase activation may be involved in mediating effects of extracellular glorin on gene expression. However, transcription induction mechanism may also involve proteins that possess domains containing intracellular CAMP inducible activities which are independent of direct phosphorylation by protein kinase $A(P K A)$.

Results of this research work pointed that stimulation of $P$. pallidum cells with glorin not only rapidly induced a number of genes but also predominantly 'enhanced' expression of many genes that were normally induced by starvation to very low levels (Section 3.6.2.1.3; Figure 27) during very early hours of development. If de novo transcription is involved in increasing transcript levels of glorin-regulated genes, then glorin-mediated transcriptional activation appears to be a highly ordered process. It is plausible that an inactive RNA polymerase II holoenzyme complex is constitutively positioned and maintained on the promoters of many genes that are rapidly induced in response to glorin stimulation. Therefore, $P$. pallidum cells appear to be programmed to increase the abundance of transcripts of these genes in response to glorin under any condition. This research work led us to propose that stimulation of cells with exogenous glorin triggers a cascade of intracellular phosphorylation events, which may lead to phosphorylation and subsequent translocation of a regulatory protein from the cytoplasm to the nucleus where it binds to sequence-specific enhancer elements in the promoter/enhancer regions of glorin-regulated genes. The assembled 'enhanceosome' may then recruit the transcriptional coactivator, which binds in a complex with the RNA polymerase II holoenzyme, allowing rapid transcriptional induction in response to glorin. It is suggested that the nuclear regulatory protein may be constitutively expressed but its function is acutely sensitive to glorin-mediated intracellular signalling cascade. During growth or late 
development, when glorin signalling does not occur, the trans-acting protein may be quiescent.

\subsection{Dose-response effects of glorin on gene induction}

To determine whether starving amoebae are capable of detecting differences in the strength of the glorin stimulation, mRNA levels of model glorin-induced genes were compared in cells that were subjected to increasing doses of glorin. Increased doses of exogenous glorin resulted in relative increased levels of transcripts of PPL_09347 and PPL_05354 genes (Figure 30). Therefore, the ability to detect the strength of stimulation and to transmit quantitatively variable signals is a feature expected of the machinery that perceives glorin stimulus. Moreover, the regulatory mechanism governing the expression of PPL_09347 and PPL_05354 does not seem to be an on-off switch, but must act proportionally to a quantitative signal.

\subsection{General kinetics of gene induction in response to repetitive stimulation of $\boldsymbol{P}$. pallidum PN500 cells with glorin remains the same}

Experiments aimed to determine the optimal glorin concentration and pulsing frequency required to observe maximal response by $P$. pallidum PN500 cells indicated that the general time-course of glorin activity was not dependent on the glorin concentration or pulsing frequency applied, only the level of induction was lower at reduced glorin concentrations and vice versa. When $P$. pallidum PN500 cells were treated with 10, 100, or $1000 \mathrm{nM}$ glorin at $30 \mathrm{~min}$ intervals, it was found that $10 \mathrm{nM}$ glorin was sufficient to induce expression of model genes PPL_09347 and PPL_05354 (Figure 30). Induction was significantly stronger at $100 \mathrm{nM}$, but most pronounced at $1000 \mathrm{nM}$ glorin (Figure 30). In order to evaluate whether the frequency of glorin pulses influenced the induction of model genes, when $1 \mu \mathrm{M}$ or $100 \mathrm{nM}$ of glorin was applied for 4 hours at 10 and 30 minute intervals, it was found that repetitive pulsing resulted only in a stronger response, whereas general kinetics of induction remained the same (Figure $32 \& 34$ ). Also it was noticed that even a single pulse of glorin could induce model genes like PPL_09347 and PPL_05833 to almost the same level as observed after repeated pulsing (Figure 32 \& 34). Generally, repetitive stimulation is supposed to result in a response that is stronger and more pronlonged and though amoebae are capable to respond to intermittent repititive stimulation, partial desensitization or habituation may occur. 


\subsection{Stimulation of $P$. pallidum PN500 cells with glorin induces precocious aggregation}

It was noticed that $P$. pallidum PN500 cells treated with glorin displayed accelerated aggregation (Figure 64). Apparently, exogenous glorin signal triggered a cascade of intracellular biochemical events that led to the precocious induction of a number of genes whose products may participate in the aggregation process while repressing expression of several other early developmental genes. Considerable number of glorin-induced genes may facilitate cell-cell interactions causing formation of many small aggregates earlier than under natural conditions. Correspondingly, previous studies reported precocious aggregation of $D$. discoideum cells that were treated with $30 \mathrm{nM}$ cAMP for 5 hours to mimic the normal oscillatory pulses of cAMP that occur during aggregation $(\mathrm{Mu}$ et al. 1998; Ma et al. 1997; Insall et al. 1996; Louis et al. 1993; Saxe et al. 1991a,b; Mann et al. 1988; Kimmel 1987; Mann \& Firtel 1987; Janssens \& van Haastert 1987; Devreotes 1982; Juliani et al. 1981; Darmon et al. 1975; Gerisch et al. 1975b; Gerisch 1968).

\subsection{Pre-starvation is not needed to observe glorin-induced changes in gene expression}

In vegetatively growing $D$. discoideum amoebae, the cAMP signalling system is expressed at very low levels. It has been known for some time that $D$. discoideum cells express many developmental genes while suspended in buffer and treated with pulses of cAMP at 6-min intervals for 2-6 hours (Iranfar et al. 2003; Louis et al. 1993; Pitt et al. 1993; Mann \& Firtel 1989; Kumagai et al. 1989; Mann \& Firtel 1987), reflecting that developmentally regulated synthesis of a protein(s) is required for induction of these genes. Contrarily, in P. pallidum PN500 amoebae all components of glorin signalling appear to exist in cells prior to the onset of starvation because even freshly washed cells, without any pre-starvation period, significantly responded to exogenous glorin pulses and accumulation of mRNA of model glorin-induced genes was observed within 10 minutes post treatment (Figure 62). This period of time does not appear sufficient enough for de novo expression of a regulatory protein or transcription factor that would then differentially regulate the observed target genes. 


\subsection{Glorin-mediated changes in gene expression do not depend on de novo protein expression}

Glorin-induced changes in gene expression were not found to be sensitive to cycloheximide treatment confirming that de novo protein synthesis is not required to observe glorin effects. Addition of cycloheximide to $P$. pallidum amoebae, immediately after washing away bacterial food, did not affect glorin-mediated gene expression validating that all proteins constituting intracellular glorin signalling cascade are latently present in growing cells (Figure 63). Rather, expression of model glorin-induced genes PPL_09347 and PPL_05833 actually slightly enhanced in cells treated with both cycloheximide and glorin (Figure 63), manifesting a stabilizing effect of cycloheximide on transcripts. Cycloheximide $(\mathrm{CHX})$ inhibits the elongation step of protein synthesis (Vazquez 1979), therefore, in $\mathrm{CHX}$ treated cells, ribosomes do not terminate normally and new ribosomes enter the polysome, ultimately saturating the mRNA. Since the stability of mRNA may depend upon the availability of sites for inactivation by nucleases, it seems probable that in the presence of cycloheximide, these sites are protected because in $\mathrm{CHX}$ treated cells, the mRNAs are bound to ribosomes that occupy these nuclease sensitive sites, rendering the mRNA stable (Kelly et al. 1987). However, starvation-mediated expression of these genes (in the absence of exogenous glorin) was sensitive to cycloheximide treatment (Figure 63), suggesting that induction of these genes in response to starvation may depend on protein expression. In short, these data indicate that the mechanism of glorin-mediated induction of genes pre-exists in growing cells and is distinct from the starvation induction mechanism for these genes.

\subsection{Possible molecular mechanisms by which glorin may modulate gene expression changes}

In D. discoideum, cAMP plays a dual role as it serves both as chemotactic agent to coordinate aggregation of starving amoebae and as secondary messenger to elicit signal transduction pathways. Binding of extracellular cAMP to cAR1 receptor leads to the activation of ACA and ERK2, which together promote a dramatic increase in intracellular cAMP that activates cAMP-dependent protein kinase (PKA). PKA inturn regulates the expression of aggregation-stage genes (Zhang et al. 2005; Iranfar et al. 2003; Mann et al. 1997; Schulkes \& Schaap 1995). Dictyostelium cAMP dependent protein kinase A (PKA) is a complex composed of an inhibitory regulatory subunit (PKA-R) and a catalytic subunit (PKA-C) (Funamoto et al. 2003; Mann et al. 1997; Mann et al. 1994; Hopper et 
al. 1993a; Harwood et al. 1992a; Mann et al. 1992; Burki et al. 1991; Firtel \& Chapman 1990). The primary target for intracellular CAMP is the regulatory subunit of PKA, which upon cAMP binding dissociates from the catalytic subunit that leads to its activation.

In $P$. pallidum, unlike $D$. discoideum, cAMP is not the chemoattractant mediating aggregation. However, occurrence of a developmentally regulated cAMP-dependent protein kinase has been reported in $P$. pallidum that is similar in its properties with PKA isolated from $D$. discoideum (Francis et al. 1984). Funamoto and colleagues (Funamoto et al. 2003) showed that Dictyostelium PKA subunits introduced into Polysphondylium amoebae were functional as signal components demonstrating that a biochemically similar PKA signalling pathway works in Polysphondylium. The authors showed that $P$. pallidum cells overexpressing dominant negative mutant of the regulatory subunit (that is unable to bind cAMP) from Dictyostelium, did not form typical aggregation streams, rather many small clumps were formed that eventually differentiated to small mounds and finally very small fruiting bodies were formed without branching of secondary sorogens (Funamoto et al. 2003). P. pallidum PN500 cells overexpressing the catalytic subunit (PKA-C) of Dictyostelium developed precociously and rapidly formed many small sized mounds. In $P$. pallidum, there is developementally regulated increase in the activity of cAMP-dependent protein kinase (PKA) before aggregates are formed (Funamoto et al. 2003) indicating that PKA may be required for the aggregation of $P$. pallidum.

D. discoideum ACA (Adenylyl cyclase $A$ ) is a development-specific membrance-bound enzyme activated by extracellular cAMP through cARs to synthesize cAMP in the aggregation process. Most of the synthesized CAMP is secreted to coordinate cell-cell communication, however, some cAMP is retained intracellularly and activates PKA. $D$. discoideum, acaA null mutants ( $a c a A^{-}$) exhibit defective chemotactic cAMP signal relay and inefficient aggregation (Galardi-Castilla et al. 2010; Alvarez-Curto et al. 2001; Pitt et al. 1993; Pitt et al. 1992). Glorin is supposed to be the acrasin of P. pallidum, therefore, normal aggregation in this species is assumed to require glorin-induced changes in gene expression. Recently, Schaap and colleagues (personal communication) showed that $P$. pallidum PN500 mutants lacking two putative aggregation-adenylyl cyclases were still able to aggregate, thereby demonstrating that increases in internal levels of cAMP may not be required to activate PKA for glorin-induced changes in gene expression. Alternatively, if intracellular cAMP regulates gene expression by triggering PKA activity, then constitutive PKA activity should be sufficient for full gene expression independent of 
cAMP synthesis. PKA-C is inhibited when associated with its regulatory subunit but gets activated when intracellular cAMP binds to the PKA-R. It was shown that in Dictyostelium the PKA-R and PKA-C subunits are only loosely connected (Pitt et al. 1993; De Gunzburg et al. 1984) and when PKA-C outnumbers the regulatory subunit, some of the constitutive and intracellular cAMP-independent PKA activity is speculated (Schaap 2011b; Pitt et al. 1993; Anjard et al. 1992). Another possibility is that P. pallidum PKA-C may be acutely sensitive to a cell surface glorin receptor-mediated intracellular signalling cascade and cells can produce an excess of C over R. Alternatively, PKA may be activated by agents other than cAMP (Pitt et al. 1993). Another explanation could be that cGMP induces some PKA activation because basal cGMP may be present at higher levels than cAMP (Pitt et al. 1993). In mammalian cells, it has been shown that cGMP regulates gene expression both at transcriptional and posttranscriptional levels (Pilz \& Casteel 2003). Moreover, in P. pallidum mutant cells lacking two potential aca genes, glorin-stimulation mediated increases in cGMP accumulation may be normal, leading to regular changes in gene expression. However, the presence of a third adenylyl cyclase enzyme is suspected in $P$. pallidum PN500 that may be responsible to provide intracellular cAMP for activation of PKA in the absence of other two ACA enzymes (Pauline Schaap; Personal communication).

Furthermore, it was shown that in P. pallidum there is a PKA-dependent and a non-PKAdependent intracellular signalling pathway regulating early developmental genes (Funamoto et al. 2003). Additionally, results presented in this thesis showed that exogenous glorin pulses have no influence on pkaC expression (Figure 58). However, expression of erkB (PPL_12271; also known as erk2) was enhanced by glorin treatment (Figure 26). In D. discoideum, PKA-requiring cAMP-pulse-induced gene (cPIG) expression requires ERK2, owing to the ability of ERK2 to inhibit RegA. However, ERK2 is also required for induction of non-PKA-requiring CPIG expression in D. discoideum, indicating that ERK2 regulates PKA-independent pulse-induced genes by a distinct mechanism. It is possible that in $P$. pallidum ERK2 may govern glorin-regulated gene expression by the direct phosphorylation and activation of a transcription factor or a nuclear regulatory protein; a role exhibited by MAP kinases in other systems ranging from yeasts to mammals (Whitmarsh \& Davis 1999). Knetsch and colleagues (Knetsch et al. 1996) showed that in D. discoideum roughly $1-2 \%$ of ERK2 translocates to the nucleus upon cAMP stimulation; however, a direct role of ERK2 in transcriptional regulation still remains to be established. Besides RegA, another putative substrate of ERK2 is EppA that is required for chemotaxis and cAMP production during early 
developmental stages in D. discoideum (Hadwiger \& Nguyen 2011; Chen \& Segall 2006). Nevertheless, EppA does not seem to serve as transcription factor for PKA-independent gene expression because of its cytosolic localization and absence of known DNA binding motifs. $D$. discoideum erkB- cells are impaired in development and fail to aggregate (Segall et al. 1995), possibly due to the failure to induce PKA-independent cAMP pulseinduced genes, which are critical for cAMP signalling.

A clear understanding of whether or not glorin induces changes in gene expression through a pathway independent of PKA activation, would require studying the gene expression changes in response to exogenous glorin in various mutants altered in PKA signalling, i.e. P. pallidum PN500 cells overexpressing PKA-C and PKA-Rm, cells lacking both aggregation-stage adenylyl cyclases, and the MAP kinase ERK2 (erkB') that exhibit lack of intracellular cAMP and PKA activity.

\subsection{Differences between glorin-signalling system and cAMP acrasin system of $D$. discoideum}

Although the glorin signalling system shares many parallels with the CAMP acrasin system of $D$. discoideum, there are also considerable differences. The cAMP signalling system is developmentally regulated in $D$. discoideum cells and cascades of protein synthesis are required to induce most of cAMP-dependent genes. The results presented in this dissertation suggest that in $P$. pallidum cells all basic elements of glorin-signalling are latently present in growing cells. Studies of the glorin signalling system in $P$. violaceum cells revealed that amoebae possess a considerable number of glorin receptors during the growth phase with only a slight increase during early aggregation (De Wit et al. 1988). Furthermore, $D$. discoideum cells secrete cAMP to the extracellular space in response to a cAMP stimulus, thereby relaying the signal into the surrounding region that causes amoebae to aggregate in stream pattern. Conversely, glorin-induced glorin secretion has not been detected in aggregating $P$. violaceum cells (De Wit et al. 1988), however, $P$. violaceum or $P$. pallidum amoebae join together to form moving streams of cells that converge at aggregation centres. Nevertheless, it still remains to be answered whether aggregation of $P$. violaceum or $P$. pallidum amoebae involves any kind of signal relay. It can not be ruled out that glorin stimulus may induce the export of a signal molecule other than glorin itself to propagate signal in neighbouring cells. Previous studies reported developmentally regulated, glorin-induced cGMP accumulation in $P$. violaceum cells that reached to its maximum during aggregation but declined at the onset 
of culmination (De Wit et al. 1988). Interestingly, it has been shown that cGMP, the secondary messenger, is vigorously exported from many mammalian cell types in quantities that exceed that of cAMP (Hofer \& Lefkimmiatis 2007; Andric et al. 2006; Sager 2004). So far, in mammalian cells, specific molecular receptors for cGMP have not yet been identified. However, in $D$. discoideum cells, at concentration of $10^{-4} \mathrm{M} \mathrm{cGMP}$ binds to the aggregation stage cAMP receptor (Mato et al. 1978). Later, Klein et al. (1985) mentioned that cyclic GMP can also behave as chemoattractant for $D$. discoideum cells at high concentrations and could trigger cell differentiation to aggregation competence similar to extracellular cAMP (Klein et al. 1985). In another study, when $D$. discoideum and $D$. lacteum cells were stimulated with their respective chemoattractants, $20 \%$ of the intracellularly accumulated cGMP was secreted (Van Haastert et al. 1983). Several biological roles of extracellular cGMP have been described for mammalian cells (Hofer \& Lefkimmiatis 2007; Sager 2004). However, in Dictyostelids, putative roles of extracellular cGMP in transferring information to neighbouring cells have not yet been identified.

Moreover, it can not be ignored that 'relay' may be required to propagate the signal extensively into the field of starving cells leading to aggregation of more cells at central collection points that may favour bigger aggregate size and therefore formation of the larger fruiting bodies that are characteristic of group 4 species. However, the formation of smaller fruiting bodies typical for group 2 species such as $P$. pallidum may be acquired without any signal relay.

\subsection{How did the glorin acrasin system evolve?}

A few hypotheses can be presented regarding evolution of glorin acrasin system. First attractive idea is that glorin may be secreted by some kind of bacteria and amoebae invented cell-surface receptors to detect glorin-producing bacteria as food. In the course of evolution, amoebae converted this food-seeking mechanism into an acrasin system to coordinate their own aggregation by inventing few more proteins. However, this assumption is confronted by the consideration that glorin appears to be a product of secondary metabolism, and then it seems rather pointless that social amoebae will adapt food-seeking system into an acrasin system because then amoebae would have required the invention of an entirely new biosynthetic machinery to synthesize glorin. A second possibility is that all acrasin system genes are closely linked on a chromosome in bacteria or some other soil microorganism, and that there has been a horizontal transfer 
of a segment of DNA containing this entire genetic material from bacteria to amoebae. It is now known that such transfers events are entirely possible and in $D$. discoideum 16 genes have been recognized as horizontal gene transfer (HGT) events (Sucgang et al. 2011).

Contrarily, Bonner (1982) hypothesized that an acrasin can be any small, readily diffusible molecule regularly synthesized and degraded in the cell, and with a receptor and other associated proteins already present (Bonner 1982). This idea seems quite reasonable because known acrasin systems in Dictyostelids, using cAMP, folic acid or pterins as aggregation mediators, employ existing cellular biochemical machinery to exploit these primary metabolites as acrasins. Interestingly, chemical structure of glorin shares some similarities with that of glutathione (GSH) as shown in Figure 66.

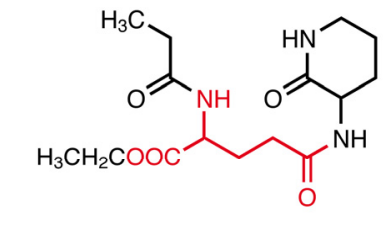

N-propionyl- $\gamma$-L-glutamyl-L-ornithine-

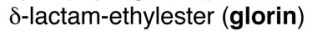

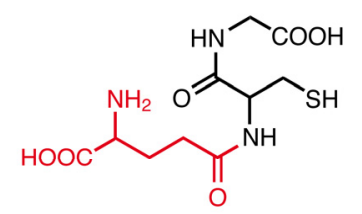

$\gamma$-L-glutamyl-L-cysteinyl-glycine (glutathione)

Figure 66: Chemical structure of glorin (left) and glutathione (right). Glorin is a dipeptide of glutamate and ornithine, in which amino group and a carboxyl group of the glutamate are blocked by a propionyl group and an ethyl ester, respectively. An amino group in the ornithine moiety is blocked by the formation of a lactam ring.

Glutathione is a ubiquitous tripeptide with an unusual peptide linkage between the amine group of cysteine (which is attached by normal peptide linkage to a glycine) and the carboxyl group of the glutamate side-chain. It plays critical roles in antioxidant defense, regulation of cellular events including gene expression, nutrient metabolism, DNA and protein synthesis, amino acid transport, enzyme activation, cell proliferation, apoptosis, and signal transduction (Kim et al. 2005; Wu et al. 2004). The glutathione biosynthetic machinery is found in some bacteria including cyanobacteria and proteobacteria (E.coli, Salmonella, Vibrio, Helicobacter). However, most eukaryotes synthesize glutathione, including Dictyostelids and humans. Some previous studies reported that the addition of glutathione to Amoeba proteus elicits pseudopod formation, activates chemotactic movement towards food source and promotes phagocytosis (Prusch \& Minek 1985) suggesting that glutathione-related substances released by certain bacteria or other 
microorganisms may act as chemical cues that help amoebae to recognize and locate a suitable prey organism.

In the cells of central nervous system, glutathione acting as a neuromodulator exerts its action by binding to glutamate receptors. Interestingly, several putative metabotropic glutamate receptors are encoded by $P$. pallidum PN500 genome (Heidel et al. 2011). Moreover, homologs of all genes encoding components of glutathione biosynthesis and degradation appear to be present in P. pallidum PN500 cells (Social Amoebas Comparative Genome Browser: http://sacgb.fli-leibniz.de/cgi/index.pl). Recently, it has been shown that glutathione is essential for Dictyostelium growth and development (Kim et al. 2005). It seems probable that glutathione is accumulated in Polysphondylium cells during the stress response and a suspected correlation between glutathione metabolism and glorin synthesis can not be entirely excluded. This hypothesis is supported by the fact that developmental cAMP signalling in the Dictyostelids has also evolved from stress response (Ritchie et al. 2008) and favours the concept that opportunistic exploitation of existing genes is a major phenomenon in the emergence of novel characters (Kawabe et al. 2012). If this is the case, then all the major components of an acrasin system seem to be encoded in the genome, and already present in the cell. Whether structural similarity between glorin and glutathione offers some meanings in the context of molecular functions of these two substances needs to be carefully investigated.

Nevertheless, previous studies showing that growing Polysphondylium amoebae possess almost as many glorin receptors on their cell surface as do aggregating cells (Kopachik 1990; De Wit et al. 1988) and the results presented in this dissertation provide a basis to assume that glorin may be a signal molecule used by ancient Dictyostelids not only to find food but also to coordinate the process of cell aggregation. Thus far, folic acid and pterins were recognized as chemoattractants with dual functions i.e., food detection and organization of aggregation (De Wit \& Konijn 1983; Van Haastert et al. 1982; Pan et al. 1975). The fact that food seeking and cell aggregation make use of same or related signalling molecules may illustrate an evolutionary connection between these two processes.

\subsection{Synthesis, storage, and secretion of glorin}

A recent review by Hook et al. (2010) discusses function of brain neuropeptides in mediating chemical cell-cell communications among neurons in the central nervous 
system (Hook et al. 2010). Nascent precursors of these peptide transmitters, known as proneuropeptides, are packaged within the newly formed secretory vesicles in the cell body. Proteolytic processing of the precursor protein occurs during axonal transport and maturation of the secretory vesicle (Hook et al. 2008). Mature processed neuropeptides are stored within secretory vesicles at the synapse where activity-dependent, regulated secretion of peptide transmitter occurs to mediate neurotransmission when peptidergic receptors are activated by neuropeptides (Hook et al. 2010). It may not be presumptuous to hypothesize that synthesis, storage and secretion of glorin in $P$. pallidum may follow a similar mechanism because glorin is an unusual peptide and its synthesis may involve complex biosynthetic steps. Moreover, it does not seem realistic that cells might have to synthesize and secrete glorin simultaneously; as soon as the process of aggregation begins. Furthermore, results presented in this dissertation suggest that all components of glorin signalling are present in the growing cells, therefore, it is reasonable to speculate that in $P$. pallidum cells, glorin is constitutively synthesized and accumulated into 'vacuolar membrane vesicles' or 'secretory granules'. Subsequently, at the onset of aggregation, intracellularly compartmented glorin is eliminated by exocytosis via vesicular secretory pathway. Similar 'storage-excretion mechanisms' are used by mammalian and yeast cells to eliminate a broad range of lipophilic toxins from the cytosol after their conjugation with glutathione (Li et al. 1996).

\subsection{Perspectives}

\subsubsection{Determination of mechanisms regulating glorin-induced gene expression}

\subsubsection{Does glorin stimulate de novo transcription?}

To investigate whether the stimulation of gene expression by glorin pulses is at the transcriptional level, following 3 approaches are suggested:

1. A combination of actinomycin $\mathbf{D}$ and daunomycin rapidly inhibits synthesis of mRNA in $D$. discoideum (Firtel et al. 1973). Same drugs may be tested in $P$. pallidum PN500 to examine whether de novo transcription is responsible for the rapid increase in transcript abundance in response to glorin treatment. 
2. In vitro Nuclear run-on assays: $P$. pallidum PN500 cells can be developed in liquid cultures with or without exogenous glorin pulses for 2 hours, followed by isolation of transcriptionally active nuclei from cells at $0 \mathrm{hr}$ and $2 \mathrm{hr}$. Nuclear run-on assays will be carried out as described by Nellen et al. (1987) involving incubation of standard reaction mixtures containing reaction buffer, each of ATP, GTP, CTP and $\left[\alpha^{32} P\right]-U T P$, RNAase Guard and isolated nuclei at room temperature for 30 minutes. Labelled RNA can then be collected, denatured at $80^{\circ} \mathrm{C}$ and placed on ice. Samples of plasmid DNA containing sequences corresponding to respective cDNA probes of representative glorin-induced genes (PPL_09347 or PPL_05833) will be linearized and applied onto Hybond-N nylon membrane using a slot blot apparatus. Pre-hybridization procedure will be followed by hybridization using labelled RNA samples. The membrane will be air dried and exposed to X-ray film. To estimate the relative rate of transcription, autoradiogram can be quantified and values will be normalized to the intensity of the actin band after background subtraction. The intensities of signals obtained will give indication of increase in nuclear transcription. Transcription rate will be compared with the rate of increase in cytoplasmic mRNA levels.

3. Alpha-amanitin treatment: Nuclear run-on experiments can be performed in the presence and absence of $50 \mu \mathrm{M}$ alpha-amanitin (RNA polymerase II inhibitor) to determine whether transcription of glorin-regulated genes is catalyzed by RNA polymerase II.

\subsubsection{Possible post-transcriptional regulation of glorin-induced gene expression}

Signal transduction pathways influence the stability of specific transcripts by rapidly modulating the function of RNA binding proteins that regulate mRNA decay. Possibly, mRNA of many genes that are efficiently induced by glorin are synthesized in the cells from the very beginning of development but these transcripts do not accumulate because they may be very unstable until aggregation process starts. However, glorin signalling may lead to potential stabilisation of these specific transcripts, thereby enhancing their level rapidly.

Polyadenylation of message is one of the post-transcriptional mechanisms that contribute to the stabilization of mRNA. Rapid polyadenylation is reported to occur during Xenopus oocyte development as well as during the dorsal ventral patterning of the Drosophila embryo (Richter 1999). To test the hypothesis that transcripts may be present 
in isolated total RNA but lack poly(A)+ tails and increased mRNA abundance could be a result of rapid polyadenylation, quantitative RT-PCR analysis can be performed on CDNA samples synthesized using oligo-dT (that would not prime cDNA synthesized from nonadenylated transcripts) or random hexamer primers followed by comparison of fold changes between hexamer-primed and oligo-dT-primed cDNAs.

To determine whether partial transcripts were present in isolated total RNA, primer pairs that would amplify small fragments from either $5^{\prime}$ or $3^{\prime}$ ends of transcripts of model glorin-induced genes PPL_09347 or PPL_05833 can be used and the differences in fold changes between the $5^{\prime}$ or $3^{\prime}$ end of any of the two transcripts can be measured.

To examine whether $m R N A$ are present as intact messages bound to proteins in $P$. pallidum PN500 amoebae, cell-free lysates can be incubated with different proteases such as trypsin, proteinase $\mathrm{K}$, or Qiagen protease prior to protein precipitation during RNA extraction. Subsequently, the release of transcripts by protease treatment can be detected by real-time RT-PCR.

In short, an insight into the mechanisms by which glorin regulates gene expression changes may provide an understanding of the modulation of gene expression by other chemotactic facors in eukaryotes.

\subsubsection{Identification of glorin stimulus-inducible promoters by ChiP-on-chip}

Recently it was reported that kinases (including PKA and ERKs) are physically associated with promoters they regulate (Pokholok et al. 2006). Glorin-induced gene expression may be regulated directly by ERK2 or PKA or both. Therefore, ChiP-on-chip technique that combines chromatin immunoprecipitation ("ChIP") with microarray technology ("chip"), using antibodies against ERK2 and PKA may indicate a set of glorinstimulus induced promoters. If the available antibodies are not compatible with chromatin immunoprecipitation, tagged PKA and ERK2 would be an appropriate alternative. The resulting group of similarly regulated promoters can then be used to recognize critical DNA binding elements and may facilitate to determine the factors that regulate the glorinstimulus inducible promoters. 


\subsubsection{Identification of cis-acting DNA elements that may be responsive to glorin signalling in the promoter region of glorin-regulated genes}

To understand molecular basis for the developmental regulation of early gene expression by glorin, it would be interesting to analyze promoter of a model glorin-induced gene such as PPL_09347. For this purpose, reporter-gene constructs will be prepared by fusing promoter ( 1000bp-1500bp upstream of transcription start site) of PPL_09347 with a transcription unit that can express a reporter protein such as luciferase. This strategy will help to determine whether this promoter region can regulate the expression of a reporter gene in the same manner as it does for the endogenous gene (i.e. PPL_09347) in response to glorin stimulation. If reporter gene responds to glorin signal in a similar way, it will indicate that this promoter fragment contains glorin response activities and can be used to further define glorin responsive elements. To determine the major glorin response element within the PPL_09347 prototype promoter region, sequential deletions will be made in this region using either PCR amplification or existing restriction enzyme sites. The deletion constructs containing 5'-flanking sequences of PPL_09347 starting at different positions will be cloned into expression vectors and used to transform $P$. pallidum PN500 cells. Transformants will be selected, pooled and assayed for luciferase activity in response to glorin pulsing. Other techniques that can be used to analyze promoter regions include replacing the full length promoter of PPL_09347 with truncations and site-directed mutations. These approaches will help to identify a 'sequence element' within promoter region or transcriptional enhancer region upstream of the PPL_09347 promoter that may be necessary for glorin-induced gene expression. Once recognized, the consensus sequence will be searched in the promoters of other glorin-regulated genes.

\subsubsection{Identification of trans-acting factors interacting with promoters of glorin-induced genes}

The largest changes in transcription are intervened by variations in the levels or activities of transcription factors. Some transcription factors have been identified in $D$. discoideum (Williams 2006; Eichinger et al. 2005) that are required for the cells to progress through development such as CbfA (C-module binding factor $A$; necessary for aggregation; Winckler et al. 2004), G-box Binding Factor (GBF; necessary for post-aggregation development due to its critical role in expression of post-aggregative genes; Schnitzler et al. 1994), Myb proteins (that play important role at culmination; Tsujioka et al. 2007) and 
DNG-1. However, transcription factors directly involved in cAMP-pulse induced gene expression in $D$. discoideum have not yet been recognized. Also, no PKA-activated transcription factor has been identified that could be responsible for cAMP pulse-induced PKA-dependent gene expression. Therefore, investigation of tran-acting transcription factors and their specific cis-acting binding sites is of utmost importance for understanding glorin-modulated developmental responses in P. pallidum.

In order to understand whether a transcription factor is involved in glorin-regulated gene expression, promoter regions (upstream of the transcriptional start site) of $25 P$. pallidum PN500 genes, highly upregulated by glorin, were searched to identify a specific nucleotide sequence element common to all these genes where a sequence-specific glorin-activated transcription factor could bind. Surprisingly, no obvious sequence motif was found that was common to all genes. Some C-rich boxes were found, but they also appeared in genes not regulated by glorin, indicating that glorin signalling may not directly activate a transcription factor to regulate gene expression, rather a nuclear factor(s) could be triggered by glorin signalling. It is likely that a sequence-specific protein interaction with a transcriptional enhancer is involved in the glorin-dependent regulation of gene expression. Nevertheless, chromatin structure and histone modification also exert effects on gene expression.

4.16.4.1 Gel mobility shift assays: It would be interesting to research for the nuclear protein that may interact with specific sequences within promoter of glorin-regulated genes to modulate glorin-mediated gene expression. Gel mobility shift assays can be performed using the DNA fragment of the 5'-flanking sequence of model glorin-induced gene such as PPL_09347. DNA fragment will be obtained by PCR and labelled for the band shift assay. Nuclear extracts, prepared from $P$. pallidum cells developed for 2 hours in the presence or absence of glorin pulsing will be incubated with the labelled fragements. DNA-protein complexes will be separated by native polyacrylamide gel electrophoresis and mobilities of DNA fragments can be visualized by autoradiography. It would be expected that probes form a specific band with nuclear factor(s) resulting in shifting of the nuclear proteins. Since the PPL_09347 is induced by glorin pulses, it will be of interest to determine whether the shifted nuclear factor is also affected by glorin pulses. Any possible increase in the nuclear factor would suggest that glorin-modulated induction of the PPL_09347 gene could in part be through a direct increase in the amount of the specific trans activating factor or through DNA-binding function of such a factor. Identification of any DNA-protein interaction will be followed by affinity purification 
of interacting protein from nucleoprotein complexes. Once a specific region in the 5 'flanking sequence of selected glorin responsive gene is identified as "putative glorin response element", it would be interesting to create mutations in a few bases and analyze whether nuclear protein has stringent nucleotide sequence requirement for binding. For this purpose, gel mobility shift assays can be carried out using both wild type and mutant forms of "glorin response elements". Future work using DNA methylation interference assays will help to further pinpoint specific bases that are involved in protein binding.

\subsubsection{Identification of glorinase and enzyme activity assay}

Unexpectedly, proteomics experiments conducted in this research work could not detect any putative 'lactamase' protein (that could open ornithine lactam ring of glorin to degrade the signal) in the extracellular filtrates of $P$. pallidum PN500 cells, suggesting that the experimental strategy should be modified. In order to identify glorinase, an alternative approach should include comparison of proteins secreted by wild type $P$. pallidum PN500 cells with that of glorin-treated cells because glorin may directly or indirectly induce the gene encoding glorinase. Proteins that are observed in the extracellular filtrate of glorin treated cells but not in that of untreated cells may include putative glorinase protein. As a next step, biochemical characterization of the glorinase candidate proteins using synthetic glorin as substrate will provide information about enzyme specificity.

An alternative strategy to identify glorinase may include following steps: (1) fractionation of extracellular proteins secreted by $P$. pallidum PN500 cells by ultrafiltration, (2) Determination of glorin degrading activity of all fractions, (3) Further characterization of highly active fraction to purify "active factor" using chromatographic methods, (4) Concentration of highly active fraction using ultrafiltraion, (5) Fractionation of highly active fraction on a gel filtration column and activity testing, (6) Concentration of active fraction eluted from gel filteration column, using ultrafiltration, (7) Fractionation of active fraction on anion exchange column, (8) Seperation of different proteins in the active fraction eluted from anion exchange column by "analytical hydrophobic interaction exchange column", (9) Fractionation of active fractions obtained from anion exchange column on a native polyacrylamide gel, (10) Further fractionation of active fractions obtained from native polyacrylamide gel on a denaturing SDS polyacrylamide gel to determine $\mathrm{N}$-terminal sequence of proteins, (11) Expression of identified proteins in 
bacteria, (12) Glorinase activity testing by incubating putative glorinase protein with different concentrations of glorin, followed by HPLC analysis of degradation products.

\subsubsection{Identification cell surface of glorin receptor}

Previously, it was reported that glorin act by binding to cell-surface G-protein coupled receptor (De Wit et al. 1988). Same authors showed that growing Polysphondylium amoebae possess adequate number of glorin receptors that increase slightly in aggregating cells (Kopachik 1990; De Wit et al. 1988). Results presented in this thesis point to two putative G-protein coupled receptor genes, i.e. PPL_00902 and PPL_05727 that are expressed in growing cells, whereas their expression levels are moderately enhanced in aggregating cells. In $D$. discoideum, aggregation stage cAR1 receptor is induced by nanomolar cAMP pulses (Mu et al. 1998; Firtel 1995; Loomis 1996; Louis et al. 1993; Saxe et al. 1991a, b; Klein et al. 1988, 1987; Kimmel 1987). RNA-seq analysis indicated that both of these genes are induced in response to glorin stimulation. Therefore, these two genes may be potential candidates for glorin receptor and future research should focus on studying their functional roles by generating knock-out mutants in $P$. pallidum PN500. Other approach to identify glorin receptor will make use of 'affinity labelling' technique using a derivative of glorin that can form a covalent linkage once bound to the receptor. Alternatively, REMI mutants of $P$. pallidum can be generated, and FACS (fluorescence activated cell sorting) analysis can be performed to sort out mutants defective in binding fluorescent derivatives of glorin.

\subsubsection{Determination of the biological roles played by glorin-regulated genes}

Future studies should focus to unravel the functions of glorin-regulated genes by generating knock-out mutations in the genes of interest, followed by determination of the cellular processes which have been disrupted or compromised in such mutants. Products of the genes differentially modulated by glorin may be involved not only in the reception and transduction of extracellular glorin signal but also in the synthesis and degradation of this dipeptide chemoattractant. 


\section{Summary}

The exclusive life cycle of social amoebae with the frequent shifting between uni- and multicellular stages offers an ideal system to study the complex process of intercellular communication. Dictyostelids live as single cells for most of their life time, but under unfavourable conditions they can aggregate to form multicellular fruiting bodies that hold dormant spores. A fundamental requisite for aggregative multicellular development is cell-cell communication. A variety of chemical signals including CAMP, folic acid, pterins and glorin are used by Dictyostelids to mediate cell-cell communication in the process of aggregation.

The aggregative chemoattractant glorin ( $\mathrm{N}$-propionyl-y-L-glutamyl-L-ornithine- $\delta$-lactam ethyl ester) was originally isolated from Polysphondylium violaceum. In this study, a chemotaxis assay was established to study responses of social amoabae to glorin. Chemotaxis of cells to glorin was found in species from all four major phylogenetic groups of Dictyostelids. Previously, some aspects of glorin signalling were studied in the species $P$. violaceum, but the putative role of glorin in mediating changes in gene expression remained unexplored. In this study, effects of glorin as a modulator of gene regulation in the early multicellular development were studied in the species Polysphondylium pallidum PN500 whose genome has recently been sequenced and that is chemotactically responsive to glorin. Using Illumina RNA sequencing technology it was shown that glorin mediates rapid changes in gene expression in early development of $P$. pallidum. The optimal glorin concentration and the pulsing frequency required to observe maximal response by $P$. pallidum PN500 was determined to be $1 \mu \mathrm{M}$ glorin applied at $10-$ minute intervals. However, glorin effects on gene expression were roughly independent of the glorin concentration applied; only the level of induction was higher at high glorin concentrations used whereas patterns of expression remained unchanged under a variety of pulsing conditions. Glorin-induced genes started to accumulate within 5 minutes of glorin treatment and pre-starvation was not required to detect glorin-induced gene expression, indicating that all basic components of glorin signalling are already present in a latent state in growing amoebae. Developmental kinetics of selected glorininduced genes showed that most of the glorin-induced genes were maximally expressed at the aggregation stage in the development of $P$. pallidum cells. $P$. pallidum cells treated with glorin exhibited accelerated aggregation when plated for development on agar, most probably because glorin induced precocious expression of aggregation specific genes. 
These results imply that glorin is the most ancient intercellular communication molecule that mediates not only chemotactic aggregation but also prepares cells to postaggregative development by inducing genes required for intra- and intercellular signaling in the multicellular organism. Interestingly, however, the glorin-based communication during aggregation has been replaced several times by other communication systems during dictyostelid evolution. 


\section{Zusammenfassung}

Der exklusive Lebenszyklus "sozialer Amöben", der häufig zwischen ein- und mehrzelligen Stadien wechselt, bietet ein ideales System für das Studium komplexer Prozesse der interzellulären Kommunikation. Soziale Amöben leben als einzellige Organismen, können aber unter ungünstigen Umweltbedingungen vielzellige Fruchtkörper mit ausdauernden Sporen bilden. Eine Vielzahl chemischer Signale, einschließlich zyklischem AMP, Folsäure, Pterine-Derivaten und Glorin werden von sozialen Amöben verwendet, um die Zell-Zell-Kommunikation während der Aggregation zu vermitteln.

Das chemotaktisch wirkende Molekül Glorin (N-Propionyl-y-L-glutamyl-L-ornithin- $\delta$ lactam-ethylester) wurde ursprünglich aus Polysphondylium violaceum isoliert. In dieser Studie wurde zunächst ein Chemotaxis-Test entwickelt, der es erlaubt, die Reaktion von Zellen auf Glorin zu untersuchen. Es wurde gezeigt, dass Chemotaxis auf Glorin in sozialen Amöben aller vier phylogenetisch verwandter Gruppen der sozialen Amöben vorkommt. Einige Aspekte der Glorin-basierten interzellulären Kommunikation wurden früher in $P$. violaceum studiert, allerdings blieb die mögliche Rolle von Glorin in der Regulation der Genexpression weitgehend unerforscht. In dieser Studie wurden Effekte von Glorin auf die Genexpression in der frühen Entwicklung der Spezies Polysphondylium pallidum untersucht, dessen Genom kürzlich sequenziert wurde und die eine chemotaktische Reaktion von Glorin zeigt. Mittels Illumina RNA-Sequenzierung wurde gezeigt, dass Glorin rasche Veränderungen in der Genexpression in der frühen Entwicklung von $P$. pallidum bewirkt. Es wurde gefunden, dass die optimale Konzentration und Pulsfrequenz zur Stimulation der Genexpression bei exogener Zugabe von Glorin zu hungernden Zellen bei $1 \mu \mathrm{M}$ Glorin und 10-Minuten-Intervallen lag. Der zeitliche Verlauf der Glorin-Effekte auf die Genexpression war allerdings weitestgehend unabhängig von der Glorin-Konzentration. Die Expression Glorininduzierter Gene wurde bereits innerhalb von fünf Minuten nach Glorin-Zugabe beobachtet und war unabhängig von einer vorherigen Hungerphase der Amöben, was darauf hindeutet, dass das Glorin-basierte Kommunikationssystem schon in wachsenden Zellen latent etabliert ist. Ferner wurde gezeigt, dass die meisten der Glorin-induzierten Gene während der Aggregationsphase in der Entwicklung von P. pallidum maximal experimiert werden. Mit Glorin behandelte $P$. pallidum Zellen zeigten eine beschleunigte Aggregation, was möglicherweise als Folge einer verfrühten Expression Glorininduzierter Gene unter den experimentellen Bedingungen zurückzuführen war. 
Die erzielten Ergebnisse lassen vermuten, dass Glorin das evolutionär ältereste interzelluläre Kommunikationsmolekül innerhalb der sozialen Amöben darstellt. Dabei wirkt Glorin nicht nur als chemotaktisches Signalmolekül, sondern konditioniert die Zellen auch für die Postaggragtionsphase, indem es Gene reguliert, die im multizellulären organismus für die intra- und interzelluläre Kommunikation benötigt werden. Bemerkenswerterweise wurde das während der Aggregationsphase aktive Glorinbasierte Kommunikation allerdings in verschiedenen Spezies erfolgreich durch andere Kommunikationssysteme ersetzt. 


\section{References}

Abe K. and Yanagisawa K. (1983) A new class of rapid developing mutants in Dictyostelium discoideum: implications for cyclic AMP metabolism and cell differentiation. Dev Biol. 95:200-210.

Alton T.H. and Lodish H.F. (1977) Translational control of protein synthesis during the early stages of differentiation of the slime mold Dictyostelium discoideum. Cell. 12(1):301-10.

Alvarez-Curto E, Meima E. M, Schaap P (2001) Expression and role of adenylyl cyclases during late development in Dictyostelium discoideum. Int. J. Dev. Biol. 45 (S1): S147-S148

Alvarez-Curto E, Rozen D, Ritchie A, Fouquet C, Baldauf SL, Schaap P. (2005) Evolutionary origin of cAMP-based chemoattraction in the social amoebae. Proc Nat Acad Sci USA. 102:63856390.

Alvarez-Curto E., Saran S., Meima M., Zobel J., Scott C. \& Schaap P. (2007) cAMP production by adenylyl cyclase $\mathrm{G}$ induces prespore differentiation in Dictyostelium slugs. Development. 134:959-966.

Andric SA, Kostic TS, Stojilkovic SS. (2006) Contribution of multidrug resistance protein MRP5 in control of cyclic guanosine 5'- monophosphate intracellular signaling in anterior pituitary cells. Endocrinology 147: 3435-3445.

Anjard C., Pinaud S., Kay R.R. and Reymond C.D. (1992). Overexpression of DdPK2 protein kinase causes rapid development and affects the intracellular cAMP pathway of Dictyostelium discoideum. Development. 115:785-790.

Anjard C., Soderbom F., and Loomis W.F. (2001). Requirements for the adenylyl cyclases in the development of Dictyostelium. Development. 128: 3649-3654.

Anjard C. \& Loomis W.F. (2008) Cytokinins induce sporulation in Dictyostelium. Development. 135:819-827.

Annesley S.J. and Fisher P.R. (2009) Dictyostelium discoideum--a model for many reasons. Mol Cell Biochem. 329(1-2):73-91.

Aragon AD, Quiñones GA, Thomas EV, Roy S, Werner-Washburne M. (2006) Release of extraction-resistant mRNA in stationary phase Saccharomyces cerevisiae produces a massive increase in transcript abundance in response to stress. Genome Biol. 7(2):R9 (doi:10.1186/gb2006-7-2-r9)

Aubry L., Maeda M., Insall R., Devreotes P.N., Firtel R.A. (1997) The Dictyostelium mitogen activated protein kinase ERK2 is regulated by Ras and cAMP-dependent protein kinase (PKA) and mediates PKA function. J Biol Chem. 272(7):3883-6.

Aubry L. \& Firtel R.A. (1999). Integration of signaling networds that regulate Dictyostelium differentiation. Annu. Rev. Cell Dev. Biol. 15:469-517.

Bagorda A., Das S., Rericha E.C., Chen D., Davidson J., Parent C.A. (2009) Real-time measurements of cAMP production in live Dictyostelium cells. J Cell Sci. 122(Pt 21):3907-14.

Baldauf, S. L. \& Doolittle, W. F. (1997). Origin and evolution of the slime molds (mycetozoa). Proc. Natl Acad. Sci. USA. 94:12007-12012.

Baldauf, S. L., Roger, A. J., Wenk-Siefert, I. \& Doolittle, W. F. (2000). A kingdom-level phylogeny of eukaryotes based on combined protein data. Science. 290:972-977. 
Baldauf, S. L. (2003) The deep roots of eukaryotes. Science 300:1703-1706.

Ball J.B, Craik D.J, Alewood P.F, Morrison S, Andrews P.R, Nicholls I.A (1989) Synthesis and conformational analysis of the slime-mould acrasin glorin. Aust J Chem. 42:2171-2180

Barkley D.S. (1969) Adenosine-3',5'-phosphate: identification as acrasin in a species of cellular slime mold. Science. 165(3898):1133-4.

Benabentos R, Hirose S, Sucgang R, Curk T, Katoh M, Ostrowski E.A, Strassmann J.E, Queller D.C, Zupan B, Shaulsky G, Kuspa A. (2009) Polymorphic members of the lag gene family mediate kin discrimination in Dictyostelium. Curr Biol. 19(7):567-72.

Bentley DR, Balasubramanian S, Swerdlow HP, Smith GP, Milton J, Brown CG, Hall KP, Evers DJ, Barnes CL, Bignell HR, Boutell JM, Bryant J, Carter RJ, Keira Cheetham R, Cox AJ, Ellis DJ, Flatbush MR, Gormley NA, Humphray SJ, Irving LJ, Karbelashvili MS, Kirk SM, Li H, Liu X, Maisinger KS, Murray LJ, Obradovic B, Ost T, Parkinson ML, Pratt MR, Rasolonjatovo IM, Reed MT, Rigatti R, Rodighiero C, Ross MT, Sabot A, Sankar SV, Scally A, Schroth GP, Smith ME, Smith VP, Spiridou A, Torrance PE, Tzonev SS, Vermaas EH, Walter K, Wu X, Zhang L, Alam MD, Anastasi C, Aniebo IC, Bailey DM, Bancarz IR, Banerjee S, Barbour SG, Baybayan PA, Benoit VA, Benson KF, Bevis C, Black PJ, Boodhun A, Brennan JS, Bridgham JA, Brown RC, Brown AA, Buermann DH, Bundu AA, Burrows JC, Carter NP, Castillo N, Chiara E Catenazzi M, Chang S, Neil Cooley R, Crake NR, Dada OO, Diakoumakos KD, Dominguez-Fernandez B, Earnshaw DJ, Egbujor UC, Elmore DW, Etchin SS, Ewan MR, Fedurco M, Fraser LJ, Fuentes Fajardo KV, Scott Furey W, George D, Gietzen KJ, Goddard CP, Golda GS, Granieri PA, Green DE, Gustafson DL, Hansen NF, Harnish K, Haudenschild CD, Heyer NI, Hims MM, Ho JT, Horgan AM, Hoschler K, Hurwitz S, Ivanov DV, Johnson MQ, James T, Huw Jones TA, Kang GD, Kerelska TH, Kersey AD, Khrebtukova I, Kindwall AP, Kingsbury Z, Kokko-Gonzales PI, Kumar A, Laurent MA, Lawley CT, Lee SE, Lee X, Liao AK, Loch JA, Lok M, Luo S, Mammen RM, Martin JW, McCauley PG, McNitt P, Mehta P, Moon KW, Mullens JW, Newington T, Ning Z, Ling Ng B, Novo SM, O'Neill MJ, Osborne MA, Osnowski A, Ostadan O, Paraschos LL, Pickering L, Pike AC, Pike AC, Chris Pinkard D, Pliskin DP, Podhasky J, Quijano VJ, Raczy C, Rae VH, Rawlings SR, Chiva Rodriguez A, Roe PM, Rogers J, Rogert Bacigalupo MC, Romanov N, Romieu A, Roth RK, Rourke NJ, Ruediger ST, Rusman E, Sanches-Kuiper RM, Schenker MR, Seoane JM, Shaw RJ, Shiver MK, Short SW, Sizto NL, Sluis JP, Smith MA, Ernest Sohna Sohna J, Spence EJ, Stevens K, Sutton N, Szajkowski L, Tregidgo CL, Turcatti G, Vandevondele S, Verhovsky Y, Virk SM, Wakelin S, Walcott GC, Wang J, Worsley GJ, Yan J, Yau L, Zuerlein M, Rogers J, Mullikin JC, Hurles ME, McCooke NJ, West JS, Oaks FL, Lundberg PL, Klenerman D, Durbin R, Smith AJ. (2008) Accurate whole human genome sequencing using reversible terminator chemistry. Nature. 456(7218):53-9.

Betapudi V, Mason C, Licate L, Egelhoff T.T. (2005) Identification and characterization of a novel alpha-kinase with a von Willebrand factor A-like motif localized to the contractile vacuole and Golgi complex in Dictyostelium discoideum. Mol Biol Cell. 16(5):2248-62.

Bominaar A.A. \& Van Haastert P.J. (1994) Phospholipase C in Dictyostelium discoideum. Identification of stimulatory and inhibitory surface receptors and G-proteins. Biochem J. 297:18993.

Bonner J.T. (1947) Evidence for the formation of cell aggregates by chemotaxis in the development of the slime mold Dictyostelium discoideum. J Exp Zool. 106:1- 26

Bonner J.T. (1949) The demonstration of acrasin in the later stages of the development of the slime mold Dictyostelium discoideum. J Exp Zool. 110(2):259-71.

Bonner J.T. (1967). The Cellular Slime Molds. Princeton University Press. 
Bonner J.T, Barkley D.S, Hall EM, Konijn T.M, Mason J.W, O'Keefe G III, Wolfe P.B. (1969) Acrasin, Acrasinase, and the sensitivity to acrasin in Dictyostelium discoideum. Dev Biol. 20(1):72-87.

Bonner J.T. (1982) Evolutionary strategies and developmental constraints in the cellular slime molds. American Naturalist. 119:530-552.

Bonner J.T. (1998). The origins of multicellularity. Integrative Biology. 1-1: 27-36.

Bonner JT. (2000). First Signals: The Evolution of Multicellular Development. Princeton, NJ: Princeton Univ. Press. 146 pp.

Bonner, J.T. and Lamont, D. S. (2005). Behavior of cellular slime molds in the soil. Mycologia. 97:178-184.

Bonner J.T. (2009) The Social Amoebae: The Biology of Cellular Slime Molds Princeton University Press, Princeton, N.J.

Bosgraaf L., Russcher H., Smith J.L., Wessels D., Soll D.R., Van Haastert P.J. (2002) A novel cGMP signalling pathway mediating myosin phosphorylation and chemotaxis in Dictyostelium. EMBO J. 21(17):4560-70.

Bozzaro S, Gerisch G. (1978) Contact sites in aggregating cells of Polysphondylium pallidum. J Mol Biol. 120(2):265-79.

Braunersreuther V. \& Mach F. (2006) Leukocyte recruitment in atherosclerosis: potential targets for therapeutic approaches? Cell Mol. Life Sci. 63:2079-88.

Brazill D.T., Lindsey D.F., Bishop J.D., Gomer R.H. (1998) Cell density sensing mediated by a G protein-coupled receptor activating phospholipase C. J. Biol. Chem. 273:8161-8168.

Brefeld JO. (1869) Dictyostelium mucoroides. Ein neuer organismus aus der Verwandschaft der Myxomiceten. Abh Senckenb Naturf Ges. 7:85-107.

Brenner S, Prösch S, Schenke-Layland K, Riese U, Gausmann U, Platzer C. (2003) cAMPinduced Interleukin-10 promoter activation depends on CCAAT/enhancer-binding protein expression and monocytic differentiation. J Biol Chem. 278(8):5597-604.

Brook M, Sully G, Clark A.R, Saklatvala J. (2000) Regulation of tumour necrosis factor alpha mRNA stability by the mitogen-activated protein kinase p38 signalling cascade. FEBS Lett. 483(1):57-61.

Browning D.D, The T, O'Day D.H. (1995) Comparative analysis of chemotaxis in Dictyostelium using a radial bioassay method: protein tyrosine kinase activity is required for chemotaxis to folate but not to cAMP. Cell Signal. 7(5):481-9.

Brzostowski J.A. \& Kimmel A.R. (2006) Nonadaptive regulation of ERK2 in Dictyostelium: implications for mechanisms of cAMP relay. Mol Biol Cell. 10:4220-7.

Burki E., Anjard C., Scholder J.C. and Reymond C.D. (1991) Isolation of two genes encoding putative protein kinases regulated during Dictyostelium discoideum development. Gene. 102:5765.

Burns R.A., Livi G.P., Dimond R.L. (1981) Regulation and secretion of early developmentally controlled enzymes during axenic growth in Dictyostelium discoideum. Dev Biol. 84(2):407-16. 
Castonguay A.C, Olson L.J, Dahms N.M (2011) Mannose 6-phosphate receptor homology $(\mathrm{MRH})$ domain-containing lectins in the secretory pathway. Biochim Biophys Acta. 1810(9):81526.

Chang Y.Y. (1968) Cyclic 3',5'-adenosine monophosphate phosphodiesterase produced by the slime mold Dictyostelium discoideum. Science. 161(3836):57-9.

Charo I.F. \& Taubman M.B. (2004) Chemokines in the pathogenesis of vascular disease. Circ. Res. 95:858-866.

Chassy B.M. (1972) Cyclic nucleotide phosphodiesterase in Dictyostelium discoideum: interconversion of two enzyme forms. Science. 175(4025):1016-8.

Chen M.Y., Long Y., Devreotes P.N. (1997). A novel cytosolic regulator, pianissimo, is required for chemoattractant receptor and $G$ protein-mediated activation of the 12 transmembrane domain adenylyl cyclase in Dictyostelium. Genes Dev. 11: 3218-3231.

Chen S. \& Segall J.E. (2006) EppA, a putative substrate of DdERK2, regulates cyclic AMP relay and chemotaxis in Dictyostelium discoideum. Eukaryot Cell. 5:1136-46.

Chisholm R.L, Hopkinson S, Lodish H.F. (1987) Superinduction of the Dictyostelium discoideum cell surface cAMP receptor by pulses of cAMP. Proc Natl Acad Sci U S A. 84(4):10304.

Chisholm R.L, Firtel R.A. (2004) Insights into morphogenesis from a simple developmental system. Nat Rev Mol Cell Biol. 5: 531-541.

Chisholm RL, Gaudet P, Just EM, Pilcher KE, Fey P, Merchant SN, Kibbe WA. (2006) dictyBase, the model organism database for Dictyostelium discoideum. Nucleic Acids Res. 34(Database issue):D423-7.

Clarke M., Yang J., and Kayman S.C. (1988). Analysis of the prestarvation response in growing cells of Dictyostelium discoideum. Dev. Genet. 9(4-5):315-326.

Clarke M., Dominguez N., Yuen I.S., Gomer R.H. (1992). Growing and starving Dictyostelium cells produce distinct density-sensing factors. Dev Biol. 152(2):403-6.

Clarke M. and Gomer R.H. (1995) PSF and CMF, autocrine factors that regulate gene expression during growth and early development of Dictyostelium. Experientia 51:1124-1134.

Cloonan N., Forrest A.R., Kolle G., Gardiner B.B., Faulkner G.J., Brown M.K., Taylor D.F., Steptoe A.L., Wani S., Bethel G., Robertson A.J., Perkins A.C., Bruce S.J., Lee C.C., Ranade S.S., Peckham H.E., Manning J.M., McKernan K.J., Grimmond S.M. (2008) Stem cell transcriptome profiling via massive-scale mRNA sequencing. Nat Methods. 5(7):613-9.

Clotworthy M. \& Traynor D. (2006) On the effects of cycloheximide on cell motility and polarisation in Dictyostelium discoideum. BMC Cell Biol. 7:5.

Cohen M.H. \& Robertson A. (1971) Wave propagation in the early stages of aggregation of the cellular slime molds. J. theor. Biol. 31:101-118.

Cohen M.H. \& Robertson A. (1972) Differentiation for aggregation in the cellular slime molds. In Cell Differentiation (ed. R. Harris, P. Allin \& D. Viza), pp. 35-45. Copenhagen: Munksgaard.

Colombatti A, Bonaldo P, Doliana R. (1993) Type A modules: interacting domains found in several non-fibrillar collagens and in other extracellular matrix proteins. Matrix. 13(4):297-306. 
Comb M, Birnberg NC, Seasholtz A, Herbert E, Goodman HM. (1986) A cyclic AMP- and phorbol ester-inducible DNA element. Nature. 323(6086):353-6.

Comer F.I., Lippincott C.K., Masbad J.J., Parent C.A. (2005) The PI3K-mediated activation of CRAC independently regulates adenylyl cyclase activation and chemotaxis. Current Biology. 15(2):134-9.

Cosson P, Soldati T. (2008) Eat, kill or die: when amoeba meets bacteria. Curr Opin Microbiol. 11(3):271-6.

Cotter, D. A., Mahadeo, D. C., Cervi, D. N., Kishi, Y., Gale, K., Sands, T., and Sameshima, M. (2000). Environmental regulation of pathways controlling sporulation, dormancy and germination utilizes bacterial like signaling complexes in Dictyostelium discoideum. Protist. 151(2):111-126.

Crowley, W. F., Jr., and Hofler J. G., editors. 1987. The Episodic Secretion of Hormones. Churchill Livingstone, New York. 518 pp.

Darmon M, Brachet P, Da Silva L.H. (1975). Chemotactic signals induce cell differentiation in Dictyostelium discoideum. Proc. Natl. Acad. Sci. USA 72:3163-3166.

Das S, Rericha E.C, Bagorda A, Parent C.A. (2011) Direct biochemical measurements of signal relay during Dictyostelium development. J Biol Chem. 286(44):38649-58.

De Cesare D, Sassone-Corsi P. (2000) Transcriptional regulation by cyclic AMP-responsive factors. Prog Nucleic Acid Res Mol Biol. 64:343-69.

De Gunzburg J., Part D., Guiso N., Veron M. (1984) An unusual adenosine cyclic 3',5'phosphate-dependent protein kinase from Dictyostelium discoideum. Biochemistry. 23(17): 38053812.

De Wit, R.J.W. \& Konijn, T.M. (1983) Identification of the acrasin of Dictyostelium minutum as a derivative of folic acid. Cell Differ. 12:205-210.

De Wit R.J.W., van Bemmelen M.X.P., Penning L.C., Pinas J.E., Calandra T.D., Bonner J.T. (1988) Studies of cell-surface glorin receptors, glorin degradation, and glorin-induced cellular responses during development of Polysphondylium violaceum. Exp Cell Res. 179:332-343

de la Roche M.A. \& Côté GP. (2001) Regulation of Dictyostelium myosin I and II. Biochim Biophys Acta. 1525(3):245-61.

Desbarats L., Lam T.Y., Wong L.M., Siu C.H. (1992) Identification of a unique cAMP-response element in the gene encoding the cell adhesion molecule gp80 in Dictyostelium discoideum. J Biol Chem. 267(27):19655-64.

Devreotes P.N. (1982). In "The Development of Dictyostelium discoideum." (W. F. Loomis, Ed.), pp. 117-168. Academic Press, NY.

Devreotes P.N., Zigmond S.H. (1988) Chemotaxis in eukaryotic cells: a focus on leukocytes and Dictyostelium. Annu Rev Cell Biol. 4:649-86.

Devreotes P.N. (1994) G protein-linked signaling pathways control the developmental program of Dictyostelium. Neuron.12:235-241.

Dormann D. and Weijer C.J. (2003) Chemotactic cell movement during development. Curr Opin Genet Dev. 13: 358-364. 
Eccles S.A. (2004) Parallels in invasion and angiogenesis provide pivotal points for therapeutic intervention. Int. J. Dev. Biol. 48:583-598.

Eccles, S.A. (2005) Targeting key steps in metastatic tumour progression. Curr. Opin. Genet. Dev. 15:77-86.

Eichinger L, Pachebat JA, Glöckner G, Rajandream MA, Sucgang R, Berriman M, Song J, Olsen R, Szafranski K, Xu Q, Tunggal B, Kummerfeld S, Madera M, Konfortov BA, Rivero F, Bankier AT, Lehmann R, Hamlin N, Davies R, Gaudet P, Fey P, Pilcher K, Chen G, Saunders D, Sodergren E, Davis P, Kerhornou A, Nie X, Hall N, Anjard C, Hemphill L, Bason N, Farbrother P, Desany B, Just E, Morio T, Rost R, Churcher C, Cooper J, Haydock S, van Driessche N, Cronin A, Goodhead I, Muzny D, Mourier T, Pain A, Lu M, Harper D, Lindsay R, Hauser H, James K, Quiles M, Madan Babu M, Saito T, Buchrieser C, Wardroper A, Felder M, Thangavelu M, Johnson D, Knights A, Loulseged H, Mungall K, Oliver K, Price C, Quail MA, Urushihara H, Hernandez J, Rabbinowitsch E, Steffen D, Sanders M, Ma J, Kohara Y, Sharp S, Simmonds M, Spiegler S, Tivey A, Sugano S, White B, Walker D, Woodward J, Winckler T, Tanaka Y, Shaulsky G, Schleicher M, Weinstock G, Rosenthal A, Cox EC, Chisholm RL, Gibbs R, Loomis WF, Platzer M, Kay RR, Williams J, Dear PH, Noegel AA, Barrell B, Kuspa A. (2005) The genome of the social amoeba Dictyostelium discoideum. Nature. 5; 435(7038):43-57.

Ennis H.L., Sussman M. (1958) The initiator cell for slime mold aggregation. Proc Natl Acad Sci U S A. 44(5):401-11.

Escalante R, Vicente J.J. (2000) Dictyostelium discoideum: a model system for differentiation and patterning. Int J Dev Biol. 44(8):819-35.

Europe-Finner G.N, Newell P.C. (1985) Inositol 1,4,5-trisphosphate induces cyclic GMP formation in Dictyostelium discoideum. Biochem Biophys Res Commun. 130(3):1115-22.

Europe-Finner G.N, Newell P.C. (1986) Inositol 1,4,5-triphosphate induces calcium release from a non- mitochondrial pool in amoebae of Dictyostelium. Biochim Biophys Acta. 887(3):335-40.

Europe-Finner G.N, Newell P.C. (1987) GTP analogues stimulate inositol trisphosphate formation transiently in Dictyostelium. J Cell Sci. 87 ( Pt 4):513-8.

Faure M, Franke J, Hall A.L, Podgorski G.J, Kessin R.H. (1990). The cyclic nucleotide phosphodiesterase gene of Dictyostelium discoideum contains three promoters specific for growth, aggregation, and late development. Mol. Cell. Biol. 10:1921-1930.

Firtel RA, Baxter L, Lodish HF. (1973) Actinomycin D and the regulation of enzyme biosynthesis during development of Dictyostelium discoideum. J Mol Biol. 79(2):315-27.

Firtel, R.A. and Chapman, A.L. (1990) A role for cAMP-dependent protein kinase A in early Dictyostelium development. Genes Dev. 4:18-28.

Firtel R.A. (1995) Integration of signaling information in controlling cell-fate decisions in Dictyostelium. Genes Dev. 9(12):1427-44.

Franca-Koh J, Devreotes P.N. (2004) Moving forward: mechanisms of chemoattractant gradient sensing. Physiology (Bethesda). 19:300-8.

Francis D. (1965) Acrasin and the development of Polysphondylium pallidum. Dev Biol. 12(3):329-46. 
Francis D, Majerfeld I.H, Kakinuma S, Leichtling B.H, Rickenberg H.V. (1984) An increase of cAMP-dependent protein kinase during development in Polysphondylium pallidum. Dev Biol. 106(2):478-84.

Franke J., Faure M., Wu L., Hall A.L., Podgorski G.J., and Kessin R.H. (1991). Cyclic nucleotide phosphodiesterase of Dictyostelium discoideum and its glycoprotein inhibitor: Structure and expression of their genes. Dev. Genet. 12(1-2):104-112.

Frevel M.A, Bakheet T, Silva A.M, Hissong J.G, Khabar K.S, Williams B.R. (2003) p38 Mitogen-activated protein kinase-dependent and -independent signaling of mRNA stability of AUrich element-containing transcripts. Mol Cell Biol. 23(2):425-36.

Funamoto S., Milan K., Meili R., Firtel R.A. (2001) Role of phosphatidylinositol 3' kinase and a downstream pleckstrin homology domain-containing protein in controlling chemotaxis in Dictyostelium. J Cell Biol. 153(4): 795-810.

Funamoto S, Anjard C, Nellen W, Ochiai H. (2003) cAMP-dependent protein kinase regulates Polysphondylium pallidum development. Differentiation. 71(1):51-61.

Futrelle R.P, Traut J, McKee W.G. (1982) Cell behavior in Dictyostelium discoideum: preaggregation response to localized cyclic AMP pulses. J Cell Biol. 92(3):807-21.

Galardi-Castilla M, Garciandía A, Suarez T, Sastre L. (2010) The Dictyostelium discoideum acaA gene is transcribed from alternative promoters during aggregation and multicellular development. PLoS One. 5(10):e13286.

Gaskins C., Clark A.M., Aubry L., Segall J.E., Firtel R.A. (1996) The Dictyostelium MAP kinase ERK2 regulates multiple, independent developmental pathways. Genes Dev. 10(1):118-28.

Gasteiger E, Hoogland C, Gattiker A, Duvaud S, Wilkins MR, et al. (2005) Protein Identification and Analysis Tools on the ExPASy Server. In: John M. Walker (ed): The Proteomics Protocols Handbook. Humana Press pp 571-607.

Gerisch G. (1968). Cell aggregation and differentiation in Dictyostelium. Curr. Top. dev. Biol. 3:157-197.

Gerisch G., Malchow D., Riedel V., Muller E., and Every M. (1972) Cyclic AMP phosphodiesterase and its inhibitor in slime mould development. Nature-New Biol. 235(55): 9092.

Gerisch G., Fromm H., Huesgen A. \& Wick U. (1975) Control of cell-contact sites by cyclic AMP pulses in differentiating Dictyostelium cells. Nature 255, 547-549.

Gerisch G., Huesgen A., and Malchow D. (1975b). Genetic control of cell differentiation and aggregation in Diotyostelium: The role of cyclic AMP pulses. In "Proceedings of the Tenth FEBS Meeting."pp. 257-267.

Gerisch G., Malchow D., Roos W., Wick U. (1979). Oscillations of cyclic nucleotide concentrations in relation to the excitability of Dictyostelium cells. J. Exp. Biol. 81:33-47.

Gerisch G. (1987) Cyclic AMP and other signals controlling cell development and differentiation in Dictyostelium. Annu Rev Biochem. 56:853-79.

Goldbeter A. (2006) Oscillations and waves of cyclic AMP in Dictyostelium: a prototype for spatio-temporal organization and pulsatile intercellular communication. Bull Math Biol. 68(5):1095109. 
Goldberg J.M., Manning G., Liu A., Fey P., Pilcher K.E., Xu Y., Smith J.L. (2006) The dictyostelium kinome--analysis of the protein kinases from a simple model organism. PLoS Genet. 2(3):e38.

Gomer R.H, Datta S, Mehdy M, Crowley T, Sivertsen A, Nellen W, Reymond C, Mann S, Firtel R.A. (1985) Regulation of cell-type-specific gene expression in Dictyostelium. Cold Spring Harb Symp Quant Biol. 50:801-12.

Gonzalez-Kristeller D.C, Farage L, Fiorini L.C, Loomis W.F, da Silva A.M. (2008) The P450 oxidoreductase, RedA, controls development beyond the mound stage in Dictyostelium discoideum. BMC Dev Biol. 8:8.

Grant W.N, Williams K.L (1983) Monoclonal antibody characterisation of slime sheath: the extracellular matrix of Dictyostelium discoideum. EMBO J. 2(6):935-40.

Grant CM, Collinson LP, Roe JH, Dawes IW. (1996) Yeast glutathione reductase is required for protection against oxidative stress and is a target gene for yAP-1 transcriptional regulation. $\mathrm{Mol}$ Microbiol. 21(1):171-9.

Grosberg R.K., Strathmann R.R. (2007) The Evolution of Multicellularity: A Minor Major Transition? Annu. Rev. Ecol. Evol. Syst. 38:621-54.

Hadwiger J.A. \& Nguyen H.N. (2011) MAPKs in development: insights from Dictyostelium signaling pathways. Biomol Concepts. 2(1-2):39-46.

Hagiwara H. (1982). Altitudinal distribution of dictyostelid cellular slime molds in the Gosainkund region of Nepal. In: Otani Y., ed. Reports on the cryptogamic study in Nepal. Tokyo: National Science Museum. p 105-117.

Hagiwara H. (1989) The Taxonomic Study of Japanese Dictyostelid Cellular Slime Molds National Science Museum, Tokyo.

Halloy J, Lauzeral J, Goldbeter A. (1998) Modeling oscillations and waves of CAMP in Dictyostelium discoideum cells. Biophys Chem. 72(1-2):9-19.

Hall A.L., Franke J., Faure M., Kessin R.H. (1993). The role of the cyclic nucleotide phosphodiesterase of Dictyostelium discoideum during growth, aggregation, and morphogenesis: overexpression and localization studies with the separate promoters of the pde. Dev. Biol. 157: 73-84.

Harwood A.J., Hopper N.A., Simon M.N., Bouzid S., Veron M. and Williams J.G. (1992a) Multiple roles for cAMP-dependent protein kinase during Dictyostelium development. Dev Biol. 149:90-99.

Harwood A.J., Hopper N.A., Simon M.N., Driscoll D.M., Veron M. \& Williams J.G. (1992b). Culmination in Dictyostelium is regulated by the cAMP-dependent protein kinase. Cell. 69: 615624.

Heidel A.J., Glöckner G. (2008) Mitochondrial genome evolution in the social amoebae. Mol Biol Evol. 25(7):1440-50.

Heidel A.J., Lawal H.M., Felder M., Schilde C., Helps N.R., Tunggal B., Rivero F., John U., Schleicher M., Eichinger L., Platzer M., Noegel A.A., Schaap P., Glöckner G. (2011) Phylogeny-wide analysis of social amoeba genomes highlights ancient origins for complex intercellular communication. Genome Res. 21(11):1882-91. 
Hirose S, Benabentos R, Ho H.I, Kuspa A, Shaulsky G. (2011) Self-recognition in social amoebae is mediated by allelic pairs of tiger genes. Science. 333(6041):467-70.

Hofer AM, Lefkimmiatis K. (2007) Extracellular calcium and cAMP: second messengers as "third messengers"? Physiology (Bethesda). 22:320-7.

Hook V, Funkelstein L, Lu D, Bark S, Wegrzyn J, Hwang SR. (2008) Proteases for processing proneuropeptides into peptide neurotransmitters and hormones. Annu Rev Pharmacol Toxicol. 48:393-423.

Hook V, Bark S, Gupta N, Lortie M, Lu WD, Bandeira N, Funkelstein L, Wegrzyn J, O'Connor DT, Pevzner P. (2010) Neuropeptidomic components generated by proteomic functions in secretory vesicles for cell-cell communication. AAPS J. 12(4):635-45.

Hopper N.A., Anjard C., Reymond C.D. and Williams J.G. (1993a) Induction of terminal differentiation of Dictyostelium by cAMP-dependent protein kinase and opposing effects of intracellular and extracellular cAMP on stalk cell differentiation. Development. 119:147-154.

Hopper N.A., Harwood A.J., Bouzid S., Ve'ron M. \& Williams J.G. (1993b). Activation of the prespore and spore cell pathway of Dictyostelium differentiation by CAMP-dependent protein kinase and evidence for its upstream regulation by ammonia. EMBO J. 12:2459-2466.

Insall R., Kay R.R. (1990) A specific DIF binding protein in Dictyostelium. EMBO J. 9(10):3323-8.

Insall R., Kuspa A., Lilly P.J., Shaulsky G., Levin L.R., Loomis W.F., Devreotes P. (1994). CRAC, a cytosolic protein containing a pleckstrin homology domain, is required for receptor and $G$ protein-mediated activation of adenylyl cyclase in Dictyostelium. J. Cell Biol. 126:1537-1545.

Insall R.H., Borleis J., Devreotes P.N. (1996). The aimless RasGEF is required for processing of chemotactic signals through G-protein-coupled receptors in Dictyostelium. Curr. Biol. 6: 719-729.

Insall, R.H. (2010). Understanding eukaryotic chemotaxis: a pseudopod-centred view. Nat. Rev. Mol. Cell Biol. 11:453-458.

Iranfar N, Fuller D, Loomis WF. (2003) Genome-wide expression analyses of gene regulation during early development of Dictyostelium discoideum. Eukaryot Cell. 2(4):664-70.

Jain R., Gomer R.H. (1994) A developmentally regulated cell surface receptor for a densitysensing factor in Dictyostelium. J Biol Chem. 269(12):9128-36.

Janssens P.M, Van Haastert P.J. (1987) Molecular basis of transmembrane signal transduction in Dictyostelium discoideum. Microbiol Rev. 51(4):396-418.

Janáky R, Ogita K, Pasqualotto BA, Bains JS, Oja SS, Yoneda Y, Shaw CA. (1999) Glutathione and signal transduction in the mammalian CNS. J Neurochem. 73(3):889-902.

Jia M.H, Larossa R.A, Lee J.M, Rafalski A, Derose E, Gonye G, Xue Z. (2000) Global expression profiling of yeast treated with an inhibitor of amino acid biosynthesis, sulfometuron methyl. Physiol Genomics. 3(2):83-92.

Jin T, Amzel M, Devreotes PN, Wu L. (1998) Selection of gbeta subunits with point mutations that fail to activate specific signaling pathways in vivo: dissecting cellular responses mediated by a heterotrimeric G protein in Dictyostelium discoideum. Mol Biol Cell. 9(10):2949-61.

Jones M.E. (1976) Aggregation in Polysphondylium. J Cell Sci. 22(1):35-40. 
Jones M.E, Robertson A. (1976) Cyclic adenosine monophosphate and the development of Polysphondylium. J Cell Sci. 22(1):41-7.

Johnson, R.L., Vaughan R.A., Caterina M.J., Van Haastert P.J. and Devreotes P.N. (1991) Overexpression of the cAMP receptor 1 in growing Dictyostelium cells. Biochemistry. 30(28: 6982-6986.

Johnson R.L., Saxe C.L., III Gollop R., Kimmel A.R., and Devreotes P.N.(1993) Identification and targeted gene disruption of cAR3, a cAMP receptor subtype expressed during multicellular stages of Dictyostelium development. Genes Dev. 7(2):273-282.

Juliani M.H, Brusca J, Klein C. (1981) cAMP regulation of cell differentiation in Dictyostelium discoideum and the role of the cAMP receptor. Dev Biol. 83(1):114-21.

Jungmann RA, Kelley DC, Miles MF, Milkowski DM. (1983) Cyclic AMP regulation of lactate dehydrogenase. Isoproterenol and N6,O2-dibutyryl cyclic amp increase the rate of transcription and change the stability of lactate dehydrogenase a subunit messenger RNA in rat C6 glioma cells. J Biol Chem. 258(8):5312-8.

Juvan P, Demsar J, Shaulsky G, Zupan B. (2005) GenePath: from mutations to genetic networks and back. Nucleic Acids Res. 33(Web Server issue):W749-52.

Kalla SE, Queller DC, Lasagni A, Strassmann JE. (2011) Kin discrimination and possible cryptic species in the social amoeba Polysphondylium violaceum. BMC Evol Biol. 11:31.

Kamboj R.K., Wong L.M., Lam T.Y., Siu C.H. (1988) Mapping of a cell-binding domain in the cell adhesion molecule gp80 of Dictyostelium discoideum. J Cell Biol. 107(5):1835-43.

Kamboj R.K., Gariepy J., Siu C.H. (1989) Identification of an octapeptide involved in homophilic interaction of the cell adhesion molecule gp80 of Dictyostelium discoideum. Cell. 59(4):615-25.

Kawabe Y, Morio T, James J.L, Prescott A.R, Tanaka Y, Schaap P. (2009) Activated cAMP receptors switch encystation into sporulation. Proc Natl Acad Sci U S A. 106(17):7089-94.

Kawabe Y, Weening KE, Marquay-Markiewicz J, Schaap P. (2012) Evolution of selforganisation in Dictyostelia by adaptation of a non-selective phosphodiesterase and a matrix component for regulated cAMP degradation. Development.139(7):1336-45.

Kawakami S, Hagiwara H. (2008) Polysphondylium multicystogenum sp. nov., a new dictyostelid species from Sierra Leone, West Africa. Mycologia. 100(2):347-51.

Kedersha N, Anderson P. (2002) Stress granules: sites of mRNA triage that regulate mRNA stability and translatability. Biochem Soc Trans. 30:963-969.

Keeling, P. (2004) A brief history of plastids and their hosts. Protist. 155:3-7.

Keeling, P. J., Burger, G., Durnford, D. G., Lang, B. F., Lee, R. W., Pearlman, R. E., Roger, A. J. \& Gray, M. W. (2005) The tree of eukaryotes. Trends. Ecol. Evol. 20:670-676.

Kelly R, Shaw DR, Ennis HL. (1987) Role of protein synthesis in decay and accumulation of mRNA during spore germination in the cellular slime mold Dictyostelium discoideum. Mol Cell Biol. 7(2):799-805.

Kessin R.H. (1988) Genetics of early Dictyostelium discoideum development. Microbiol Rev. 52(1):29-49. 
Kessin R.H. (2001) Dictyostelium: evolution, cell biology, and the development of multicellularity Cambridge University Press, Cambridge.

Kessin R.H. (2010) Two different genomes that produce the same result. Genome Biol. 11(4):114.

Kim, H. J., W. T. Chang, M. Meima, J. D. Gross, and P. Schaap. (1998). A novel adenylyl cyclase detected in rapidly developing mutants of Dictyostelium. J. Biol. Chem. 273:3085930862.

Kim BJ, Choi CH, Lee CH, Jeong SY, Kim JS, Kim BY, Yim HS, Kang SO. (2005) Glutathione is required for growth and prespore cell differentiation in Dictyostelium. Dev Biol. 284(2):387-98.

Kimmel A.R, Carlisle B. (1986) A gene expressed in undifferentiated vegetative Dictyostelium is repressed by developmental pulses of CAMP and reinduced during dedifferentiation. Proc Natl Acad Sci U S A. 83(8):2506-10.

Kimmel A.R. (1987) Different molecular mechanisms for cAMP regulation of gene expression during Dictyostelium development. Dev Biol. 122(1):163-71.

Kimmel A.R. \& Parent C.A. (2003) The signal to move: D. discoideum go orienteering. Science. 300:1525-1527.

Kimmel A.R., Parent C.A., Gough N.R. (2004) Teaching resources. Spatial and temporal dynamics of signaling components involved in the control of chemotaxis in Dictyostelium discoideum. Sci STKE. 234:tr3.

Kimmel AR, Firtel RA. (2004) Breaking symmetries: regulation of Dictyostelium development through chemoattractant and morphogen signal-response. Curr Opin Genet Dev. 14(5):540-9.

Kishi, Y., Clements, C., Mahadeo, D. C., Cotter, D. A., and Sameshima, M. (1998) High levels of actin tyrosine phosphorylation: Correlation with the dormant state of Dictyostelium spores. J. Cell Sci. 111(Pt. 19):2923-2932.

Klein C, Lubs-Haukeness J, Simons S. (1985) cAMP induces a rapid and reversible modification of the chemotactic receptor in Dictyostelium discoideum. J Cell Biol. 100(3):715-20.

Klein P, Vaughan R, Borleis J, Devreotes P. (1987) The surface cyclic AMP receptor in Dictyostelium. Levels of ligand-induced phosphorylation, solubilization, identification of primary transcript, and developmental regulation of expression. J Biol Chem. 262(1):358-64.

Klein P., Theibert A., and Devreotes P. (1988). Identification and ligand induced modification of the cAMP receptor in Dictyostelium. Methods Enzymol. 159:267-278.

Knapinska, A. M., P. Irizarry-Barreto, S. Adusumalli, I. Androulakis, and G. Brewer. (2005). Molecular mechanisms regulating mRNA stability: physiological and pathological significance. Curr. Genomics. 6471-486.

Knetsch M.L., Epskamp S.J., Schenk P.W., Wang Y., Segall J.E., Snaar-Jagalska B.E. (1996). Dual role of cAMP and involvement of both G-proteins and ras in regulation of ERK2 in Dictyostelium discoideum. EMBO J. 15(13):3361-8.

Knobil E. (1981). Patterns of hormonal signals and hormone action. N. Engl. J. Med. 305:15821583.

Kon T, Adachi H, Sutoh K. (2000) amiB, a novel gene required for the growth/differentiation transition in Dictyostelium. Genes Cells. 5(1):43-55. 
Konijn T.M. (1961) Cell aggregation in Dictyostelium discoideum. PhD Thesis. University of Wisconsin. Madison. Wisconsin.

Konijn T.M. Raper K.B. (1961) Cell aggregation in Dictyostelium discoideum. Develop. Biol. 3: 725-756.

Konijn T.M, Van De Meene J.G., Bonner J.T., Barkley D.S. (1967) The acrasin activity of adenosine-3',5'-cyclic phosphate. Proc Natl Acad Sci U S A. 58(3):1152-4.

Konijn T.M. (1968) Chemotaxis in the cellular slime molds. II. The effect of cell density. Biol Bull. 134(2):298-304.

Konijn T.M, Barkley D.S., Chang Y.Y., Bonner J.T. (1968) Cyclic AMP: A naturally occurring acrasin in the cellular slime molds. The American Naturalist. 102:225-233.

Konijn T.M., Chang Y.Y., Bonner J.T. (1969) Synthesis of cyclic AMP in Dictyostelium discoideum and Polysphondylium pallidum. Nature. 224(5225):1211-2.

Konijn T.M. (1969) Effect of bacteria on chemotaxis in the cellular slime molds. J Bacteriol. 99(2):503-9.

Konijn T.M. (1972) Cyclic AMP as a first messenger. Advances in Cyclic Nucleotide Research. 1:17-31

Konijn T. M. (1973) in Proceedings of the Tenth International Congress of Microbiology, Mexico City, Mexico, ed. Perez-Mirauete, A. (Plenum Press, New York), pp. 48-61.

Kopachik W. (1990) Glorin-regulated protein synthesis in Polysphondylium violaceum. Exp Cell Res. 186:394-397

Kosaka C. \& Pears C.J. (1997) Chemoattractants induce tyrosine phosphorylation of ERK2 in Dictyostelium discoideum by diverse signalling pathways. Biochem. J. 324(Pt 1):347-352.

Kumagai A, Pupillo M, Gundersen R, Miake-Lye R, Devreotes P.N., Firtel R.A. (1989) Regulation and function of G-alpha protein subunits in Dictyostelium. Cell. 57: 265-275.

Kumagai A., Hadwiger J.A., Pupillo M., Firtel R.A. (1991) Molecular genetic analysis of two G alpha protein subunits in Dictyostelium. J Biol Chem. 266(2):1220-8.

Lacombe M.L, Podgorski G.J, Franke J, Kessin R.H. (1986) Molecular cloning and developmental expression of the cyclic nucleotide phosphodiesterase gene of Dictyostelium discoideum. J Biol Chem. 261(36):16811-7.

Laemmli U.K. (1970) Cleavage of structural proteins during the assembly of the head of bacteriophage T4. Nature. 227(5259):680-5.

Lalli E., Sassone-Corsi P. (1994) Signal transduction and gene regulation: the nuclear response to cAMP. J Biol Chem. 269(26):17359-62.

Lam L.T, Pickeral O.K, Peng A.C, Rosenwald A, Hurt E.M, Giltnane J.M, Averett L.M, Zhao H, Davis R.E, Sathyamoorthy M, Wahl L.M, Harris E.D, Mikovits J.A, Monks A.P, Hollingshead M.G, Sausville E.A, Staudt L.M. (2001) Genomic-scale measurement of mRNA turnover and the mechanisms of action of the anti-cancer drug flavopiridol. Genome Biol. 2(10): RESEARCH0041. 
Larkin MA, Blackshields G, Brown NP, Chenna R, McGettigan PA, McWilliam H, Valentin F, Wallace IM, Wilm A, Lopez R, Thompson JD, Gibson TJ, Higgins DG. (2007) Clustal W and Clustal X version 2.0. Bioinformatics. 23(21):2947-8.

Larner AC, Jonak G, Cheng YS, Korant B, Knight E, Darnell JE Jr. (1984) Transcriptional induction of two genes in human cells by beta interferon. Proc Natl Acad Sci U S A. 81(21):67337.

Lasa M, Brook M, Saklatvala J, Clark A.R. (2001) Dexamethasone destabilizes cyclooxygenase 2 mRNA by inhibiting mitogen-activated protein kinase p38. Mol Cell Biol. 21(3):771-80.

Laub M.T. \& Loomis W.F. (1998) A molecular network that produces spontaneous oscillations in excitable cells of Dictyostelium. Mol. Biol. Cell. 9:3521-3532.

Lee S., Parent C.A., Insall R., Firtel R.A. (1999). A novel Ras-interacting Protein Required for Chemotaxis and Cyclic Adenosine Monophosphate Signal Relay in Dictyostelium. Mol. Biol. Cell. 10: $2829-2845$.

Leng G. (1988). Editor; Pulsatility in Neuroendocrine Systems. CRC Press, Boca Raton, Florida. $261 \mathrm{pp}$.

Li Y, Goldbeter A. (1992) Pulsatile signaling in intercellular communication. Periodic stimuli are more efficient than random or chaotic signals in a model based on receptor desensitization. Biophys J. 61(1):161-71.

Li ZS, Zhao Y, Rea PA. (1995) Magnesium Adenosine 5[prime]-Triphosphate-Energized Transport of Glutathione-S-Conjugates by Plant Vacuolar Membrane Vesicles. Plant Physiol. 107(4):1257-1268.

Li ZS, Szczypka M, Lu YP, Thiele DJ, Rea PA. (1996) The yeast cadmium factor protein (YCF1) is a vacuolar glutathione S-conjugate pump. J Biol Chem. 271(11):6509-17.

Liao X.H, Kimmel A.R. (2009) Biochemical responses to chemoattractants in Dictyostelium: ligand-receptor interactions and downstream kinase activation. Methods Mol Biol. 571:271-81.

Lilly P., Wu L., Welker D. L., and Devreotes P.N. (1993). A G-protein beta-subunit is essential for Dictyostelium development. Genes Dev. 7(6): 986-995.

Lilly P.J. \& Devreotes P.N. (1994) Identification of CRAC, a cytosolic regulator required for guanine nucleotide stimulation of adenylyl cyclase in Dictyostelium. J Biol Chem. 269(19):141239.

Lohmann S.M., Vaandrager A.B., Smolenski A., Walter U., and De Jonge H.R. (1997) Distinct and specific functions of cGMP-dependent protein kinases. Trends Biochem. Sci. 22:307-312

Loomis W.F. (1996). Genetic networks that regulate development in Dictyostelium cells. Micro. Rev. 60:135-150.

Loomis, W. F., Shaulsky G., and Kuspa A. (1998). Molecular networks that regulate development, p. 201-211. In D. Beysens and G. Forgacs (ed.), Dynamical systems in physics and biology. Springer-Verlag, Paris, France.

Loomis W.F. (1998) Role of PKA in the timing of developmental events in Dictyostelium cells. Microbiol Mol Biol Rev. 62(3):684-94.

Loomis W.F. \& Shaulsky G. (2011) Developmental changes in transcriptional profiles. Dev Growth Differ. 53(4):567-75. 
Louis J.M., Saxe C.L. III, Kimmel A.R. (1993). Two transmembrane signaling mechanisms control expression of the cAMP receptor gene CAR1 during Dictyostelium development. Proc. Natl. Acad. Sci. USA. 90:5969-5973.

Ma P.C., Siu C.H. (1990) A pharmacologically distinct cyclic AMP receptor is responsible for the regulation of gp80 expression in Dictyostelium discoideum. Mol Cell Biol. 10(7):3297-306.

Ma H, Gamper M, Parent C, Firtel R.A. (1997) The Dictyostelium MAP kinase kinase DdMEK1 regulates chemotaxis and is essential for chemoattractant-mediated activation of guanylyl cyclase. EMBO J. 16(14):4317-32.

MacArthur S, Li X.Y, Li J, Brown J.B, Chu H.C, Zeng L, Grondona B.P, Hechmer A, Simirenko L, Keränen S.V, Knowles D.W, Stapleton M, Bickel P, Biggin M.D, Eisen M.B. (2009) Developmental roles of 21 Drosophila transcription factors are determined by quantitative differences in binding to an overlapping set of thousands of genomic regions. Genome Biol. 10(7):R80.

Maeda M, Aubry L, Insall R, Gaskins C, Devreotes P.N., Firtel R.A. (1996). Seven helix chemoattractant receptors transiently stimulate mitogenactivated protein kinase in DictyosteliumRole of heterotrimeric G proteins. J. Biol. Chem. 271:3351-3354.

Maeda M., Firtel R.A. (1997) Activation of the mitogen-activated protein kinase ERK2 by the chemoattractant folic acid in Dictyostelium. J Biol Chem. 272(38):23690-5.

Maeda M., Lu S., Shaulsky G., Miyazaki Y., Kuwayama H., Tanaka Y., Kuspa A., Loomis W.F. (2004) Periodic signaling controlled by an oscillatory circuit that includes protein kinases ERK2 and PKA. Science. 304(5672):875-8.

Mahadeo D.C., Parent C.A. (2006) Signal relay during the life cycle of Dictyostelium. Current Topics in Developmental Biology. 73:115-40.

Mahtani K.R, Brook M, Dean J.L, Sully G, Saklatvala J, Clark A.R. (2001) Mitogen-activated protein kinase p38 controls the expression and posttranslational modification of tristetraprolin, a regulator of tumor necrosis factor alpha mRNA stability. Mol Cell Biol. 21(19):6461-9.

Malkinson A.M, Ashworth J.M. (1973) Adenosine 3':5'-cyclic monophosphate concentrations and phosphodiesterase activities during axenic growth and differentiation of cells of the cellular slime mould Dictyostelium discoideum. Biochem J. 134(1):311-9.

Mangiarotti G, Giorda R, Ceccarelli A, Perlo C. (1985). mRNA stabilization controls the expression of a class of developmentally regulated genes in Dictyostelium discoideum. Proc Natl Acad Sci U S A. 82(17):5786-90.

Mann S.K., Firtel R.A. (1987) Cyclic AMP regulation of early gene expression in Dictyostelium discoideum: mediation via the cell surface cyclic AMP receptor. Mol Cell Biol. 7(1):458-69.

Mann S.K, Pinko C, Firtel R.A. (1988) cAMP regulation of early gene expression in signal transduction mutants of Dictyostelium. Dev Biol. 130(1):294-303.

Mann S.K., Firtel R.A. (1989) Two-phase regulatory pathway controls cAMP receptor-mediated expression of early genes in Dictyostelium. Proc Natl Acad Sci U S A. 86(6):1924-8.

Mann S.K., Firtel R.A. (1991) A developmentally regulated, putative serine/threonine protein kinase is essential for development in Dictyostelium. Mech Dev. 35(2):89-101. 
Mann S.K. O., Yonemoto W.M., Taylor S.S. and Firtel R.A. (1992) DdPK3, which plays essential roles during Dictyostelium development, encodes the catalytic subunit of cAMPdependent protein kinase. Proc. Natl. Acad. Sci. USA. 89:10701-10705.

Mann, S.K.O., Richardson, D.L., Lee, S., Kimmel, A.R. and Firtel, R.A. (1994) Expression of cAMP-dependent protein kinase in prespore cells is sufficient induce spore cell differentiation in Dictyostelium. Proc Natl Acad Sci USA. 91:10561-10565.

Mann S.K.O, Brown J.M., Briscoe C., Parent C., Pitt G., Devreotes P. and Firtel R.A. (1997). Role of cAMP-dependent protein kinase in controlling aggregation and post-aggregative development in Dictyostelium. Dev. Biol. 183:208-221.

Martin P., \& Parkhurst S.M. (2004) Parallels between tissue repair and embryo morphogenesis. Development. 131:3021-3034.

Martinez MJ, Roy S, Archuletta AB, Wentzell PD, Anna-Arriola SS, Rodriguez AL, Aragon AD, Quiñones GA, Allen C, Werner-Washburne M. (2004) Genomic analysis of stationaryphase and exit in Saccharomyces cerevisiae: gene expression and identification of novel essential genes. Mol Biol Cell. 15:5295-305.

Mato JM, Jastorff B, Morr M, Konijn TM. (1978) A model for cyclic AMP-chemoreceptor interaction in Dictyostelium discoideum. Biochim Biophys Acta. 544(2):309-14.

Mayanagi T, Amagai A, Maeda Y. (2005) DNG1, a Dictyostelium homologue of tumor suppressor ING1 regulates differentiation of Dictyostelium cells. Cell Mol Life Sci. 62(15):1734-43.

McMains V.C., Liao X.H., Kimmel A.R. (2008) Oscillatory signaling and network responses during the development of Dictyostelium discoideum. Ageing Res Rev. 234-48. Epub 2008 May 4.

McMullen P.D., Morimoto R.I., Amaral L.A. (2010) Physically grounded approach for estimating gene expression from microarray data. Proc Natl Acad Sci USA. 107(31):13690-5.

Mehdiabadi N.J., Kronforst M.R., Queller D.C., Strassmann JE (2009) Phylogeny, reproductive isolation and kin recognition in the social amoeba, Dictyostelium purpureum. Evolution. 63(2):542548.

Mehdy M.C, Firtel R.A. (1985) A secreted factor and cyclic AMP jointly regulate cell-type-specific gene expression in Dictyostelium discoideum. Mol Cell Biol. 5(4):705-13.

Meili R., Ellsworth C., Firtel R.A. (2000) A novel Akt/PKB-related kinase is essential for morphogenesis in Dictyostelium. Curr Biol. 10(12):708-17.

Meili R., Ellsworth C., Lee S., Reddy T.B., Ma H., Firtel R.A. (1999) Chemoattractant-mediated transient activation and membrane localization of Akt/PKB is required for efficient chemotaxis to cAMP in Dictyostelium. EMBO J. 18(8):2092-105.

Mian I.S, Rose C. (2011) Communication theory and multicellular biology. Integr Biol (Camb). 3(4):350-67.

Milne J.L., Wu L., Caterina M.J., Devreotes P.N. (1995) Seven helix cAMP receptors stimulate $\mathrm{Ca} 2+$ entry in the absence of functional $\mathrm{G}$ proteins in Dictyostelium. J. Biol. Chem. 270:59265931.

Minge MA, Silberman JD, Orr RJ, Cavalier-Smith T, Shalchian-Tabrizi K, Burki F, Skjaeveland A, Jakobsen KS. (2009) Evolutionary position of breviate amoebae and the primary eukaryote divergence. Proc Biol Sci. 276(1657):597-604. 
Mohr S, Bakal C, Perrimon N. (2010) Genomic screening with RNAi: results and challenges. Annu Rev Biochem. 79:37-64.

Montminy MR, Sevarino KA, Wagner JA, Mandel G, Goodman RH. (1986) Identification of a cyclic-AMP-responsive element within the rat somatostatin gene. Proc Natl Acad Sci U S A. 83(18):6682-6.

Montminy M. (1997) Transcriptional regulation by cyclic AMP. Annu Rev Biochem. 66:807-22.

Mortazavi A, Williams B.A, McClue K, Schaeffer L, Wold B. (2008) Mapping and quantifying mammalian transcriptomes by RNA-Seq. Nat Methods. 5:621-628.

Mrass P. \& Weninger W. (2006) Immune cell migration as a means to control immune privilege: lessons from the CNS and tumors. Immunol Rev. 213:195-212.

Mu X, Lee B, Louis J.M, Kimmel A.R. (1998) Sequence-specific protein interaction with a transcriptional enhancer involved in the autoregulated expression of cAMP receptor 1 in Dictyostelium. Development. 125(18):3689-98.

Mu X, Spanos SA, Shiloach J, Kimmel A. (2001) CRTF is a novel transcription factor that regulates multiple stages of Dictyostelium development. Development. 128(13):2569-79.

Mullens I.A, Franke J, Kappes D.J, Kessin R.H. (1984) Developmental regulation of the cyclicnucleotide-phosphodiesterase mRNA of Dictyostelium discoideum. Analysis by cell-free translation and immunoprecipitation. Eur J Biochem. 142(2):409-15.

Murdoch GH, Potter E, Nicolaisen AK, Evans RM, Rosenfeld MG. (1982a) Epidermal growth factor rapidly stimulates prolactin gene transcription. Nature. 300(5888):192-4.

Murdoch GH, Rosenfeld MG. (1982b) Eukaryotic transcriptional regulation and chromatinassociated protein phosphorylation by cyclic AMP. Science. 218(4579):1315-7.

Murdoch G.H, Franco R, Evans R.M, Rosenfeld M.G. (1983) Polypeptide hormone regulation of gene expression. Thyrotropin-releasing hormone rapidly stimulates both transcription of the prolactin gene and the phosphorylation of a specific nuclear protein. J Biol Chem. 258(24):1532935.

Myre M.A, Lumsden A.L, Thompson M.N, Wasco W, Macdonald M.E, Gusella J.F. (2011) Deficiency of huntingtin has pleiotropic effects in the social amoeba Dictyostelium discoideum. PLoS Genet. 2011 Apr;7(4):e1002052. Epub 2011 Apr 28.

Natarajan K, Meyer M.R, Jackson B.M, Slade D, Roberts C, Hinnebusch A.G, Marton M.J. (2001) Transcriptional profiling shows that Gcn4p is a master regulator of gene expression during amino acid starvation in yeast. Mol Cell Biol. 21(13):4347-68.

Nellen W, Datta S, Reymond C, Sivertsen A, Mann S, Crowley T, Firtel RA. (1987) Molecular biology in Dictyostelium: tools and applications. Methods Cell Biol. 28:67-100.

Newcomb LL, Diderich JA, Slattery MG, Heideman W. (2003) Glucose regulation of Saccharomyces cerevisiae cell cycle genes. Eukaryot Cell. 2(1):143-9.

Newth C.K., Hanna M.H. (1984) Chemotactic response of wild-type and aggregation-defective mutants of Polysphondylium violaceum. Differentiation. 28: 94-100

Noce T, Okamoto K, Takeuchi I. (1983) Purification and characterization of the extracellular cyclic AMP phosphodiesterase of Dictyostelium discoideum. J Biochem. 93(1):37-45. 
Noegel A, Gerisch G, Stadler J, Westphal M. (1986). Complete sequence and transcript regulation of a cell adhesion protein from aggregating Dictyostelium cells. EMBO J. 5(7):1473-6.

Noegel A.A, Blau-Wasser R, Sultana H, Müller R, Israel L, Schleicher M, Patel H, Weijer C.J. (2004) The cyclase-associated protein CAP as regulator of cell polarity and cAMP signaling in Dictyostelium. Mol Biol Cell. 15(2):934-45.

Nowrousian M. (2010) Next-generation sequencing techniques for eukaryotic microorganisms: sequencing-based solutions to biological problems. Eukaryot Cell. 9(9):1300-10.

Olive E.W (1901) A preliminary enumeration of the sorophoreae. Proc Am Acad Arts Sci. 37:333344

Olive, E.W. (1902) Monograph of the Acrasieae. Proc. Boston Soc. Nat. Hist. 30: 451-5I3-.pl.5-8.

Otsuka H, Van Haastert PJ. (1998) A novel Myb homolog initiates Dictyostelium development by induction of adenylyl cyclase expression. Genes Dev. 12(11):1738-48.

Palatnik C.M., Storti R.V., Jacobson A. (1979). Fractionation and functional analysis of newly synthesized and decaying messenger RNAs from vegetative cells of Dictyostelium discoideum. $J$ Mol Biol. 128(3):371-95.

Palatnik C.M., Storti R.V., Capone A.K., Jacobson A. (1980) Messenger RNA stability in Dictyostelium discoideum: does poly(A) have a regulatory role? J Mol Biol. 141(2):99-118.

Palsson E., Lee K.J., Goldstein R.E., Franke J., Kessin R.H., and Cox E. C. (1997). Selection for spiral waves in the social amoebae Dictyostelium. Proc. Natl. Acad. Sci. USA 94(25):1371913723.

Pan P, Hall E.M, Bonner J.T. (1972) Folic acid as second chemotactic substance in the cellular slime moulds. Nat New Biol. 237(75):181-2.

Pan P, Hall E.M, Bonner J.T. (1975) Determination of the active portion of the folic acid molecule in cellular slime mold chemotaxis. J Bacteriol. 122(1):185-91

Parent C.A. \& Devreotes P.N. (1996). Molecular genetics of signal transduction in Dictyostelium. Annu. Rev. Biochem. 65:411-440.

Parent C. A. and Devreotes P. N. (1999). A cell's sense of direction. Science. 284:765-770.

Parent C.A. (2004). Making all the right moves: chemotaxis in neutrophils and Dictyostelium. Curr. Opin. Cell Biol. 16:4-13.

Parikh A., Miranda E.R., Katoh-Kurasawa M., Fuller D., Rot G., Zagar L., Curk T., Sucgang R., Chen R., Zupan B., Loomis W.F., Kuspa A., Shaulsky G. (2010) Conserved developmental transcriptomes in evolutionarily divergent species. Genome Biol. 11(3):R35.

Pfaffl MW. (2001) A new mathematical model for relative quantification in real-time RT PCR. Nucleic Acids Res. 29(9):e45.

Pilz RB, Casteel DE. (2003) Regulation of gene expression by cyclic GMP. Circ Res. 93(11):1034-46.

Pitt G.S, Milona N, Borleis J, Lin K.C, Reed R.R, Devreotes P.N. (1992) Structurally distinct and stage-specific adenylyl cyclase genes play different roles in Dictyostelium development. Cell. 69: 305-315. 
Pitt G.S, Brandt R, Lin K.C, Devreotes P.N, Schaap P. (1993) Extracellular cAMP is sufficient to restore developmental gene expression and morphogenesis in Dictyostelium cells lacking the aggregation adenylyl cyclase (ACA). Genes Dev. 7(11):2172-80.

Podgorski G.J., Faure M., Franke J., Kessin R.H. (1988). The cyclic nucleotide phosphodiesterase of Dictyostelium discoideum: the structure of the gene and its regulation and role in development. Dev. Genet. 9:267-278.

Pokholok DK, Zeitlinger J, Hannett NM, Reynolds DB, Young RA. (2006) Activated signal transduction kinases frequently occupy target genes. Science. 313(5786):533-6.

Potts G. (1902) Zur Physiologie des Dictyostezium mucoroides. Flora. 91: 281-347.

Primpke G, lassonidou V, Nellen W, Wetterauer B. (2000) Role of cAMP-dependent protein kinase during growth and early development of Dictyostelium discoideum. Dev Biol. 221(1):10111.

Preston GM, Billis WM, White BA. (1990) Transcriptional and posttranscriptional regulation of the rat prolactin gene by calcium. Mol Cell Biol. 10(2):442-8.

Prusch R.D and Minek D.R. (1985) Chemical stimulation of phagocytosis in Amoeba proteus and the influence of external calcium. Cell Tissue Res. 242:557-564

Radonjic M, Andrau JC, Lijnzaad P, Kemmeren P, Kockelkorn TT, van Leenen D, van Berkum NL, Holstege FC. (2005) Genome-wide analyses reveal RNA polymerase II located upstream of genes poised for rapid response upon $S$. cerevisiae stationary phase exit. Mol Cell. 18(2):171-83.

Raghavan A, Ogilvie R.L., Reilly C., Abelson M.L., Raghavan S., Vasdewani J., Krathwohl M., Bohjanen P.R. (2002) Genome-wide analysis of mRNA decay in resting and activated primary human T lymphocytes. Nucleic Acids Res. 30(24):5529-38.

Raghavan A., Bohjanen P.R. (2004) Microarray-based analyses of mRNA decay in the regulation of mammalian gene expression. Brief Funct Genomic Proteomic. 3(2):112-24.

Raper K.B. (1940). Pseudoplasmodium formation and organization in Dictyostelium discoideum. jf. Elisha Mitchell scient. Soc. 56:241-282.

Raper K.B, Thom C (1941). Interspecies mixtures of Dictyosteliaceae. Am J Bot. 28:69-78

Raper KB. (1956) Factors affecting growth and differentiation in simple slime molds. Mycologia. 48:169-205.

Raper KB. (1984) The dictyostelids Princeton University Press, Princeton.

Rapp PE. (1987) Why are so many biological systems periodic? Prog Neurobiol. 29(3):261-273.

Rathi A, Kayman SC, Clarke M. (1991). Induction of gene expression in Dictyostelium by prestarvation factor, a factor secreted by growing cells. Dev. Genet. 12:82 87.

Rathi A. and Clarke M. (1992). Expression of early developmental genes in Dictyostelium discoideum is initiated during exponential growth by an autocrine dependent mechanism. Mech. Devel. 36: 173-182.

Reymond C.D., Schaap P., Véron M., Williams J.G. (1995) Dual role of cAMP during Dictyostelium development. Experientia. 51(12):1166-74. 
Richards, T. A. \& Cavalier-Smith, T. (2005) Myosin domain evolution and the primary divergence of eukaryotes. Nature. 436:1113-1118.

Richter JD (1999) Cytoplasmic polyadenylation in development and beyond. Microbiol Mol Biol Rev. 63:446-456.

Riedel V., Gerisch G. (1968) Regulation of extracellular cyclic-AMP-phosphodiesterase activity during development of Dictyostelium discoideum. Biochem Biophys Res Commun. 42(1):119-24.

Riedel V., Malchow D., Gerisch G., and Nagele B. (1972). Cyclic AMP phosphodiesterase interaction with its inhibitor of the slime mold, Dictyostelium discoideum. Biochem. Biophys. Res. Commun. 46(1):279-287.

Riedel V, Gerisch G, Müller E, Beug H. (1973) Defective cyclic adenosine-3', 5'-phosphatephosphodiesterase regulation in morphogenetic mutants of Dictyostelium discoideum. J Mol Biol. 74(4):573-85.

Rigden D.J, Mello L.V, Galperin M.Y. (2004) The PA14 domain, a conserved all-beta domain in bacterial toxins, enzymes, adhesins and signaling molecules. Trends Biochem Sci. 29(7):335-9.

Ritchie A. V., Van Es S., Fouquet C. \& Schaap P. (2008). From drought sensing to developmental control: evolution of cyclic AMP signaling in social amoebas. Mol. Biol. Evol. 25:2109-2118.

Robinson M.D., Oshlack A. (2010) A scaling normalization method for differential expression analysis of RNA-seq data. Genome Biol. 11(3):R25.

Rodriguez M, Kim B, Lee N.S, Veeranki S, Kim L. (2008) MPL1, a novel phosphatase with leucine-rich repeats, is essential for proper ERK2 phosphorylation and cell motility. Eukaryot Cell. 7(6):958-66.

Roelofs J, Snippe H, Kleineidam RG, Van Haastert PJ. (2001a) Guanylate cyclase in Dictyostelium discoideum with the topology of mammalian adenylate cyclase. Biochem J. 354:697-706.

Roelofs J., Meima M., Schaap P., and Van Haastert P.J.M. (2001b) The Dictyostelium homologue of mammalian soluble adenylyl cyclase encodes a guanylyl cyclase. EMBO J. 20: 4341-4348.

Roelofs J., Loovers H.M., Van Haastert P.J. (2001c) GTPgammaS regulation of a 12transmembrane guanylyl cyclase is retained after mutation to an adenylyl cyclase. $J$ Biol Chem. 276(44):40740-5.

Roelofs J. \& Van Haastert P.J. (2002) Characterization of two unusual guanylyl cyclases from dictyostelium. J Biol Chem. 277(11):9167-74.

Romeralo M, Escalante R, Sastre L, Lado C. (2007) Molecular systematics of dictyostelids: 5.8S ribosomal DNA and internal transcribed spacer region analyses. Eukaryotic Cell. 6(1):110-116.

Romeralo M, Spiegel FW, Baldauf S. (2010) A Fully Resolved Phylogeny of the Social Amoebas (Dictyostelia) Based on Combined SSU and ITS rDNA Sequences. Protist. 161:539-548.

Romeralo M, Moya-Laraño J, Lado C. (2011a) Social amoebae: environmental factors influencing their distribution and diversity across south-western Europe. Microb Ecol. 61(1):15465. 
Romeralo M, Cavender JC, Landolt JC, Stephenson SL, Baldauf SL. (2011b) An expanded phylogeny of social amoebas (Dictyostelia) shows increasing diversity and new morphological patterns. BMC Evolutionary Biology. 11:84

Romeralo M, Escalante R, Baldauf SL. (2011c) Evolution and Diversity of Dictyostelid Social Amoebae. Protist. http://dx.doi.org/10.1016/j.protis.2011.09.004

Roos W., Nanjundiah V., Malchow D. \& Gerisch G. (1975). Amplification of cyclic-AMP signals in aggregating cells of Dictyostelium discoideum. FEBS Lett. 53:139-142.

Rot G, Parikh A, Curk T, Kuspa A, Shaulsky G, Zupan B. (2009) dictyExpress: a Dictyostelium discoideum gene expression database with an explorative data analysis web-based interface. BMC Bioinformatics. 10:265.

Sager G. (2004) Cyclic GMP transporters. Neurochem Int. 45: 865-873.

Samuel E.W. (1961) Orientation and rate of locomotion of individual amebas in the life cycle of the cellular slime mold Dictyostelium mucoroides. Dev Biol. 3:317-35

Saran S, Meima M.E, Alvarez-Curto E, Weening K.E, Rozen D.E. Schaap P. (2002) cAMP signaling in Dictyostelium. Complexity of cAMP synthesis, degradation and detection. J Muscle Res Cell Motil. 23: 793-802.

Sasaki K, Cripe T.P, Koch S.R, Andreone T.L, Petersen D.D, Beale E.G, Granner D.K. (1984) Multihormonal regulation of phosphoenolpyruvate carboxykinase gene transcription. The dominant role of insulin. J Biol Chem. 259(24):15242-51.

Sasaki A.T., Chun C., Takeda K., Firtel R.A. (2004) Localized Ras signaling at the leading edge regulates PI3K, cell polarity, and directional cell movement. J Cell Biol. 167(3):505-18.

Sassone-Corsi P. (1994) Goals for signal transduction pathways: linking up with transcriptional regulation. EMBO J. 13(20):4717-28.

Sawai, S., Thomason, P. A., and Cox, E. C. (2005). An autoregulatory circuit for long-range selforganization in Dictyostelium cell populations. Nature. 433:323-326.

Saxe C.L., III Johnson R., Devreotes P.N., and Kimmel A.R. (1991a). Multiple genes for cell surface cAMP receptors in Dictyostelium discoideum. Dev. Genet. 12(1-2:6-13.

Saxe C.L., Johnson R.L., Devreotes P.N., and Kimmel A.R. (1991b) Expression of a cAMP receptor gene of Dictyostelium and evidence for a multigene family. Genes Dev. 5:1-8.

Schaap P, Winckler T, Nelson M, Alvarez-Curto E, Elgie B, Hagiwara H, Cavender J, MilanoCurto A, Rozen DE, Dingermann T, Mutzel R, Baldauf SL (2006) Molecular phylogeny and evolution of morphology in the social amoebas. Science. 314:661-663

Schaap P. (2007) Evolution of size and pattern in the social amoebas. Bioessays. 29(7): 635644.

Schaap P. (2011a) Evolutionary crossroads in developmental biology: Dictyostelium discoideum. Development. 138(3):387-96.

Schaap P. (2011b) Evolution of developmental cyclic adenosine monophosphate signaling in the Dictyostelia from an amoebozoan stress response. Dev Growth Differ. 53(4):452-62. 
Schatzle J., Rathi A., Clarke M., Cardelli J.A. (1991) Developmental regulation of the alphamannosidase gene in Dictyostelium discoideum: control is at the level of transcription and is affected by cell density. Mol Cell Biol. 11(6):3339-47.

Schatzle J., Bush J., Cardelli J. (1992) Molecular cloning and characterization of the structural gene coding for the developmentally regulated lysosomal enzyme, alpha-mannosidase, in Dictyostelium discoideum. J Biol Chem. 267(6):4000-7.

Schatzle J., Bush J., Dharmawardhane S., Firtel R.A., Gomer R.H., Cardelli J. (1993) Characterization of the signal transduction pathways and cis-acting DNA sequence responsible for the transcriptional induction during growth and development of the lysosomal alphamannosidase gene in Dictyostelium discoideum. J Biol Chem. 268(26):19632-9.

Schulkes C. and Schaap P. (1995) cAMP-dependent protein kinase activity is essential for preaggegative gene expression in Dictyostelium. FEBS Lett. 368:381- 384.

Schnitzler G.R, Fischer W.H, Firtel R.A. (1994) Cloning and characterization of the G-box binding factor, an essential component of the developmental switch between early and late development in Dictyostelium. Genes Dev. 8(4):502-14.

Schnitzler G.R., Briscoe C, Brown J.M., Firtel R.A. (1995). Serpentine cAMP receptors may act through a G protein-independent pathway to induce postaggregative development in Dictyostelium. Cell. 81: 737-745.

Segall J. E., Kuspa A., Shaulsky G., Ecke M., Maeda M., Gaskins C., Firtel R. A., and Loomis W. F. (1995). A MAP kinase necessary for receptor-mediated activation of adenylyl cyclase in Dictyostelium. J. Cell Biol. 128:405-413.

Segall J. E., Kuspa A., Shaulsky G., Ecke M., Maeda M., Gaskins C., Firtel R. A., and Loomis W. F. (1995). A MAP kinase necessary for receptor-mediated activation of adenylyl cyclase in Dictyostelium. J. Cell Biol. 128(3):405-413.

Shaffer B.M. (1953) Aggregation in cellular slime moulds: in vitro isolation of acrasin. Nature. 1953 May 30;171(4361):975.

Shaffer B.M. (1957a) Aspects of aggregation in cellular slime molds. Am. Nat. 91, 19-35.

Shaffer B.M. (1957b) Properties of slime-mould amoebae of significance for aggregation. Q.JI microsc. Sci. 98:377-392.

Shaffer B.M. (1958) Integration in aggregating slime moulds. Quart. J. Microscop. Sci. 99: 103121

Shaffer B.M. (1961). The cells founding aggregation centers in the slime mould, Polysphondylium violaceum. J. Exptl. Biol. 38:833-849

Shaffer B.M. (1962). The Acrasina. Adv. Morphogen. 2, 109-182.

Shevchenko A., Wilm M., Vorm O., Mann M. (1996) Mass spectrometric sequencing of proteins silver-stained polyacrylamide gels. Anal Chem. 68(5):850-8.

Shim J, Karin M. (2002) The control of mRNA stability in response to extracellular stimuli. Mol Cells. 14(3):323-31.

Shimomura O., Suthers H.L.B, Bonner J.T. (1982) Chemical identity of the acrasin of the cellular slime mold Polysphondylium violaceum. Proc Natl Acad Sci USA. 79:7376-7379 
Siegert, F. \& Weijer, C. J. (1995). Spiral and concentric waves organize multicellular Dictyostelium mounds. Curr. Biol. 5:937-943.

Sies H. (1999) Glutathione and its role in cellular functions. Free Radic Biol Med. 27(9-10):91621.

Simon M.N., Driscoll D., Mutzel R., Part D., Williams J. \& Ve'ron, M. (1989) Overproduction of the regulatory subunit of the CAMP-dependent protein kinase blocks the differentiation of Dictyostelium discoideum. EMBO J. 8:2039-2043.

Simpson, A. G. B. \& Roger, A. J. (2004) The real 'kingdoms' of eukaryotes. Curr. Biol. 14:R693R696.

Sinsimer KS, Gratacós FM, Knapinska AM, Lu J, Krause CD, Wierzbowski AV, Maher LR, Scrudato S, Rivera YM, Gupta S, Turrin DK, De La Cruz MP, Pestka S, Brewer G. (2008) Chaperone Hsp27, a novel subunit of AUF1 protein complexes, functions in AU-rich elementmediated mRNA decay. Mol Cell Biol. 28(17):5223-37.

Siol O., Dingermann T., Winckler T. (2006) The C-module DNA-binding factor mediates expression of the Dictyostelium aggregation-specific adenylyl cyclase ACA. Eukaryot Cell. 5(4):658-64.

Siu C.H, Sriskanthadevan S, Wang J, Hou L, Chen G, Xu X, Thomson A, Yang C. (2011) Regulation of spatiotemporal expression of cell-cell adhesion molecules during development of Dictyostelium discoideum. Dev Growth Differ. 53(4):518-27.

Souza G.M., da Silva A.M. and Kuspa A. (1999). Starvation promotes Dictyostelium development by relieving PufA inhibition of PKA translation through the YakA kinase pathway. Development. 126:3263-3274.

Souza G.M., Lu S.J. and Kuspa A. (1998). YakA, a protein kinase required for the transition from growth to development in Dictyostelium. Development 125:2291-2302.

Sobko A., Ma H., Firtel R.A. (2002) Regulated SUMOylation and ubiquitination of DdMEK1 is required for proper chemotaxis. Dev Cell. 2(6):745-56.

Soderbom, F., C. Anjard, N. Iranfar, D. Fuller, and W. F. Loomis. 1999. An adenylyl cyclase that functions during late development of Dictyostelium. Development. 126:5463-5471.

Stechmann, A. \& Cavalier-Smith, T. (2003) The root of the eukaryote tree pinpointed. Curr. Biol. 13:R665-R666.

Strassmann J.E. (2010) In Dictyostelium, the social amoeba.Volume 1. Edited by: Breed MD, Moore J. Oxford: Academic Press;:13-519, Encyclopedia of Animal behavior.

Sucgang, R., G. Chen, W. Liu, R. Lindsay, J. Lu, D. Muzny, G. Shaulsky, W. Loomis, R. Gibbs, and A. Kuspa. (2003). Sequence and structure of the extrachromosomal palindrome encoding the ribosomal RNA genes in Dictyostelium. Nucleic Acids Res. 31:2361-2368.

Sucgang R, Kuo A, Tian X, Salerno W, Parikh A, Feasley CL, Dalin E, Tu H, Huang E, Barry K, Lindquist E, Shapiro H, Bruce D, Schmutz J, Salamov A, Fey P, Gaudet P, Anjard C, Babu MM, Basu S, Bushmanova Y, van der Wel H, Katoh-Kurasawa M, Dinh C, Coutinho PM, Saito T, Elias M, Schaap P, Kay RR, Henrissat B, Eichinger L, Rivero F, Putnam NH, West CM, Loomis WF, Chisholm RL, Shaulsky G, Strassmann JE, Queller DC, Kuspa A, Grigoriev IV. (2011) Comparative genomics of the social amoebae Dictyostelium discoideum and Dictyostelium purpureum. Genome Biol. 12(2):R20. 
Sultan M., Schulz M.H., Richard H., Magen A., Klingenhoff A., Scherf M., Seifert M., Borodina T., Soldatov A., Parkhomchuk D., Schmidt D., O'Keeffe S., Haas S., Vingron M., Lehrach H., Yaspo M.L. (2008) A global view of gene activity and alternative splicing by deep sequencing of the human transcriptome. Science. 321(5891):956-60.

Sun T.J. \& Devreotes P.N. (1991). Gene targeting of the aggregation stage cAMP receptor cAR1 in Dictyostelium. Genes Dev. 5(4):572-582.

Swaney K. F., Huang C.H. \& Devreotes P.N. (2010). Eukaryotic chemotaxis: a network of signaling pathways controls motility, directional sensing, and polarity. Annu. Rev. Biophys. 39:265-289.

Swanson AR, Spiegel FW, Cavender JC (2002) Taxonomy, slime molds, and the questions we ask. Mycologia. 94:968-979.

Tebo J, Der S, Frevel M, Khabar K.S, Williams B.R, Hamilton T.A. (2003) Heterogeneity in control of mRNA stability by AU-rich elements. J Biol Chem. 278(14):12085-93.

Thompson JD, Higgins DG, Gibson TJ. (1994) CLUSTAL W: improving the sensitivity of progressive multiple sequence alignment through sequence weighting, position-specific gap penalties and weight matrix choice. Nucleic Acids Res. 22(22):4673-80.

Tillinghast H.S. \& Newell P.C. (1984). Retention of folate receptors on the cytoskeleton of Dictyostelium during development. FEBS Lett. 176:325-330.

Tsujioka M, Zhukovskaya N, Yamada Y, Fukuzawa M, Ross S, Williams J.G. (2007) Dictyostelium Myb transcription factors function at culmination as activators of ancillary stalk differentiation. Eukaryot Cell. 6(3):568-70.

Trusolino L., Comoglio P.M. (2002) Scatter-factor and semaphorin receptors: cell signalling for invasive growth. Nat. Rev. Cancer. 2:289-300.

Van Dijken P., Bergsma J.C., Van Haastert P.J. (1997) Phospholipase-C-independent inositol 1,4,5-trisphosphate formation in Dictyostelium cells. Activation of a plasma-membrane-bound phosphatase by receptor-stimulated Ca2+ influx. Eur J Biochem. 244(1):113-9.

Van Driel R. (1981) Binding of the chemoattractant folic acid by Dictyostelium discoideum cells. Eur J Biochem. 115(2):391-5.

van Es S, Virdy K.J, Pitt G.S, Meima M, Sands T.W, Devreotes P.N, Cotter D.A, Schaap P. (1996) Adenylyl cyclase G, an osmosensor controlling germination of Dictyostelium spores. J Biol Chem. 271(39):23623-5.

Van Haastert P. J. M., De Wit R.J., Grijpma Y., Konijn T.M. (1982) Identification of a pterin as the acrasin of the cellular slime mold Dictyostelium lacteum. Proc Natl Acad Sci U S A. 79(20): 6270-6274.

Van Haastert P.J, De Wit R.J, Konijn T.M. (1982b) Antagonists of chemoattractants reveal separate receptors for cAMP, folic acid and pterin in Dictyostelium. Exp Cell Res. 140(2):453-6.

Van Haastert P.J, Campagne Van Lookeren M.M; Kesbeke F. (1983) Multiple degradation pathways of chemoattractant mediated cGMP accumulation in Dictyostelium. Biochemica et Biophysica Acta. 756:67-71

Van Haastert, P.J.M. \& Van der Heijden, P.R. (1983) J. Cell Biol. 96:347-353. 
Van Haastert P.J., Bishop J.D., Gomer R.H. (1996). The cell density factor CMF regulates the chemoattractant receptor cAR1 in Dictyostelium. J. Cell Biol. 134:1543-1549.

Van Haastert P.J. \& Kuwayama H. (1997) cGMP as second messenger during Dictyostelium chemotaxis. FEBS Lett. 410(1):25-8.

van Es S, Virdy K.J., Pitt G.S., Meima M., Sands T.W., Devreotes P.N., Cotter D.A., Schaap P. (1996) Adenylyl cyclise G, an osmosensor controlling germination of Dictyostelium spores. J. Biol. Chem. 271:23623-23625.

Van Driessche N, Shaw C, Katoh M, Morio T, Sucgang R, Ibarra M, Kuwayama H, Saito T, Urushihara H, Maeda M, Takeuchi I, Ochiai H, Eaton W, Tollett J, Halter J, Kuspa A, Tanaka Y, Shaulsky G. (2002) A transcriptional profile of multicellular development in Dictyostelium discoideum. Development. 129(7):1543-52.

Vazquez, D. 1979. Inhibition of protein biosynthesis, p. 155-159. Springer-Verlag KG, Berlin.

Veltman D.M., Roelofs J., Engel R., Visser A.J., Van Haastert P.J. (2005) Activation of soluble guanylyl cyclase at the leading edge during Dictyostelium chemotaxis. Mol Biol Cell. 16(2):976-83.

Veron M., Mutzel R., Lacombe M.L., Simon M.N. and Wallet V. (1988) cAMP-dependent protein kinase from Dictyostelium discoideum. Dev Genet. 9:247-258.

Waddell D.R. (1982a) The spatial pattern of aggregation centres in the cellular slime mould. $J$ Embryol Exp Morphol. 70:75-98.

Waddell D.R. (1982b) A predatory slime mould. Nature. 298:464-466

Wang N., Soderbom F., Anjard C., Shaulsky G. \& Loomis W. F. (1999) SDF-2 induction of terminal differentiation in Dictyostelium discoideum is mediated by the membrane-spanning sensor kinase DhkA. Mol. Cell. Biol. 19:4750-4756.

Wang Y, Liu J, Segall J.E. (1998) MAP kinase function in amoeboid chemotaxis. J Cell Sci. 111 ( Pt 3):373-83.

Wang Y. \& Segall J.E. (1998) The Dictyostelium MAP kinase DdERK2 functions as a cytosolic protein in complexes with its potential substrates in chemotactic signal transduction. Biochem Biophys Res Commun. 244(1):149-55.

Wang Y., Chen C.L., lijima M. (2011) Signaling mechanisms for chemotaxis. Dev Growth Differ. 53(4):495-502.

Wang Z., Gerstein M., Snyder M. (2009) RNA-Seq: a revolutionary tool for transcriptomics. Nat Rev Genet. 10(1):57-63.

Wasinger V.C., Cordwell S.J., Cerpa-Poljak A., Yan J.X., Gooley A.A., Wilkins M.R., Duncan M.W., Harris R., Williams K.L., Humphery-Smith I. (1995) Progress with gene-product mapping of the Mollicutes: Mycoplasma genitalium. Electrophoresis. 16(7):1090-4.

Wedel B., and Garbers D. (2001) The guanylyl cyclase family at Y2K. Annu. Rev. Physiol. 63:215-233.

Weijer C.J. (2004) Dictyostelium morphogenesis. Curr Opin Genet Dev. 14(4):392-8.

Weijer CJ. (2009) Collective cell migration in development. J Cell Sci. 122(Pt 18):3215-23. 
Whitmarsh A.J, Davis R.J (1999) Signal transduction by MAP kinases: regulation by phosphorylation-dependent switches. Sci STKE. (1):PE1.

Whittaker C.A, Hynes R.O. (2002) Distribution and evolution of von Willebrand/integrin A domains: widely dispersed domains with roles in cell adhesion and elsewhere. Mol Biol Cell. 13(10):3369-87.

Willard S.S. and Devreotes P.N. (2006) Signaling pathways mediating chemotaxis in the social amoeba, Dictyostelium discoideum. Eur J Cell Biol. 85(9-10):897-904.

Williams J.G. (1988) The role of diffusible molecules in regulating the cellular differentiation of Dictyostelium discoideum. Development. 103(1):1-16.

Williams J.G, Ceccarelli A., McRobbie S., Mahbubani H., Kay R.R., Early A., Berks M., Jermyn K.A. (1987) Direct induction of Dictyostelium prestalk gene expression by DIF provides evidence that DIF is a morphogen. Cell. 49(2):185-92.

Williams J.G. (1997). Prestalk and stalk cell heterogeneity in Dictyostelium. Dictyostelium- A model system for cell and developmental biology (Maeda, M., Inouye, K, and Takeuchi, I., eds), Universal Press, Inc., 293-304.

Williams J.G. (2006) Transcriptional regulation of Dictyostelium pattern formation. EMBO Rep. 7(7):694-8.

Williams J.G. (2010) Dictyostelium finds new roles to model. Genetics. 185(3):717-26.

Williams RS, Boeckeler K, Gräf R, Müller-Taubenberger A, Li Z, Isberg RR, Wessels D, Soll DR, Alexander H, Alexander S. (2006) Towards a molecular understanding of human diseases using Dictyostelium discoideum. Trends Mol Med. 12(9):415-24.

Winckler T, Iranfar N, Beck P, Jennes I, Siol O, Baik U, Loomis W.F, Dingermann T. (2004) CbfA, the C-module DNA-binding factor, plays an essential role in the initiation of Dictyostelium discoideum development. Eukaryot Cell. 3(5):1349-58.

Winzen R, Kracht M, Ritter B, Wilhelm A, Chen C.Y, Shyu A.B, Müller M, Gaestel M, Resch K, Holtmann H. (1999) The p38 MAP kinase pathway signals for cytokine-induced mRNA stabilization via MAP kinase-activated protein kinase 2 and an AU-rich region-targeted mechanism. EMBO J. 18(18):4969-80.

Wong L.M., Siu C-H. (1986). Cloning of cDNA for the contact site A glycoprotein of Dictyostelium discoideum. Proc Natl Acad Sci U S A. 83(12):4248-52.

Wu D. \& Lin F. (2011) Modeling cell gradient sensing and migration in competing chemoattractant fields. PLoS One. 6(4):e18805.

Wu G, Fang YZ, Yang S, Lupton JR, Turner ND. (2004) Glutathione metabolism and its implications for health. J Nutr. 134(3):489-92.

Wu L., Valkema R., Van Haastert P.J., and Devreotes P.N. (1995a). The G protein beta subunit is essential for multiple responses to chemoattractants in Dictyostelium. J. Cell Biol. 129(6):16671675.

Wu L., Franke J., Blanton R.L., Podgorski G.J., and Kessin R.H. (1995b). The phosphodiesterase secreted by prestalk cells is necessary for Dictyostelium morphogenesis. Dev. Biol. 167(1):1-8. 
Wurster B., Pan P., Tyan G.G., Bonner J.T. (1976) Preliminary characterization of the acrasin of the cellular slime mold Polysphondylium violaceum. Proc Natl Acad Sci USA. 73:795-799

Wurster B, Bozzaro S, Gerisch G. (1978) Cyclic GMP regulation and responses of Polysphondylium violaceum to chemoattractants. Cell Biol Int Rep. 2(1):61-9.

Wurster B, Butz U. (1980) Reversible binding of the chemoattractant folic acid to cells of Dictyostelium discoideum. Eur J Biochem. 109(2):613-8.

Xu Q, Shaulsky G. (2005) GOAT: An R Tool for Analysing Gene Ontologytrade mark Term Enrichment. Appl Bioinformatics. 4(4):281-3.

Yamamoto K.R, Alberts B.M. (1976) Steroid receptors: elements for modulation of eukaryotic transcription. Annu Rev Biochem. 45:721-46.

Yuen I.S., Jain R., Bishop J.D., Lindsey D.F., Deery W.J., Van Haastert P.J., Gomer R.H. (1995). A density-sensing factor regulates signal transduction in Dictyostelium. J. Cell. Biol. 129:1251-1262.

Zhang N., Long Y., and Devreotes P.N. (2001). Gy in Dictyostelium: Its role in localization of G6y to the membrane is required for chemotaxis in shallow gradients. Mol. Biol. Cell 12(10):3204-3213.

Zhang H., Heid P.J., Wessels D., Daniels K.J., Pham T., Loomis W.F., and Soll D.R. (2003). Constitutively active protein kinase A disrupts motility and chemotaxis in Dictyostelium discoideum. Eukaryot. Cell. 2:62-75.

Zhang M, Goswami M, Hereld D. (2005) Constitutively active G protein-coupled receptor mutants block Dictyostelium development. Mol Biol Cell. 16(2):562-72.

Zigmond S.H, Joyce M, Borleis J, Bokoch G.M, Devreotes P.N. (1997) Regulation of actin polymerization in cell-free systems by GTPYS and Cdc42. J Cell Biol. 138:363-374 


\section{Appendix}

Table A1: Summary of proteins identified in the buffer conditioned by aggregating $P$. pallidum PN500 amoebae. The list was assembled from two independent experiments.

\begin{tabular}{|c|c|c|}
\hline $\begin{array}{l}\text { Accession } \\
\text { Number }\end{array}$ & \begin{tabular}{|c|} 
Molecular \\
Weight, kDa
\end{tabular} & Putative protein function \\
\hline PPL_09146 & 214 & transmembrane protein \\
\hline PPL_07850 & 171 & $\begin{array}{l}\text { nagA, beta-N-acetylhexosaminidase, glycoside } \\
\text { hydrolase family } 20 \text { protein, beta-hexosaminidase }\end{array}$ \\
\hline PPL_06508 & 143 & hypothetical protein \\
\hline PPL_03654 & 137 & hypothetical protein \\
\hline PPL_03794 & 119 & manC, alpha-mannosidase \\
\hline PPL_01936 & 113 & manA, alpha-mannosidase \\
\hline PPL_04497 & 111 & plbD, phospholipase B-like protein \\
\hline PPL_09385 & 110 & unpredicted protein \\
\hline PPL_02389 & 109 & athl1, acid trehalase-like protein 1 \\
\hline PPL_03165 & 105 & Succinyl-diaminopimelate desuccinylase. \\
\hline PPL_10946 & 103 & aprA, PhoPQ-activated pathogenicity-related protein \\
\hline PPL_12272 & 103 & hypothetical protein \\
\hline PPL_04936 & 102 & $\begin{array}{l}\text { psaB, puromycin-sensitive aminopeptidase-like protein, } \\
\text { metallopeptidase }\end{array}$ \\
\hline PPL_11497 & 102 & discoidin I, A chain \\
\hline PPL_02349 & 99 & unpredicted protein \\
\hline PPL_02539 & 98 & cpnE, phospholipid-binding protein, copine E \\
\hline PPL_12255 & 96 & $\begin{array}{l}\text { aco1, putative iron regulatory protein, aconitate } \\
\text { hydratase, aconitase }\end{array}$ \\
\hline PPL_08085 & 96 & alpha-glucosidase \\
\hline PPL_01713 & 92 & abpC, gelation factor, actin binding protein, filamin \\
\hline PPL_07033 & 90 & dpp3-2, dipeptidyl-peptidase III \\
\hline PPL_04038 & 89 & unpredicted protein \\
\hline PPL_04711 & 88 & psiB, PA14 domain-containing protein \\
\hline PPL_02660 & 86 & glycoside hydrolase family 18 protein \\
\hline PPL_02430 & 82 & pyridoxal phosphate-dependent decarboxylase family \\
\hline
\end{tabular}




\begin{tabular}{|c|c|c|}
\hline & & protein \\
\hline PPL_05694 & 78 & $\begin{array}{l}\text { dcd2A, neutral/alkaline nonlysosomal ceramidase } \\
\text { family protein, acid ceramidase }\end{array}$ \\
\hline PPL_07741 & 77 & AY055590, peptidase C53 family protein \\
\hline PPL_08459 & 76 & $\begin{array}{l}\text { psiJ, PA14 domain-containing protein, discoidin- } \\
\text { inducing complex (DIC) protein }\end{array}$ \\
\hline PPL_05069 & 74 & $\begin{array}{l}\text { putative alpha- } \mathrm{N} \text {-acetylgalactosaminidase, glycoside } \\
\text { hydrolase family } 27 \text { protein }\end{array}$ \\
\hline PPL_08886 & 73 & hypothetical protein \\
\hline PPL_04575 & 70 & $\begin{array}{l}\text { glb1, glycoside hydrolase family } 35 \text { protein, beta- } \\
\text { galactosidase }\end{array}$ \\
\hline PPL_00324 & 69 & $\begin{array}{l}\text { mcfS, mitochondrial substrate carrier family protein, } \\
\text { putative mitochondrial carnitine/acylcarnitine } \\
\text { transporter }\end{array}$ \\
\hline PPL_00313 & 68 & Beta-amylase activity \\
\hline PPL_09281 & 66 & $\begin{array}{l}\text { putative cellulase, cellulose-binding domain-containing } \\
\text { protein, glycoside hydrolase family } 5 \text { protein }\end{array}$ \\
\hline PPL_11402 & 65 & hypothetical protein \\
\hline PPL_03772 & 64 & Physaropepsin. \\
\hline PPL_09152 & 64 & plbA, phospholipase B \\
\hline PPL_01789 & 64 & plbF, phospholipase B-like protein \\
\hline PPL_02993 & 63 & unpredicted protein \\
\hline PPL_08963 & 62 & hypothetical protein \\
\hline PPL_02984 & 61 & unpredicted protein \\
\hline PPL_08977 & 60 & hypothetical protein \\
\hline PPL_02985 & 59 & beta-N-acetylhexosaminidase \\
\hline PPL_04308 & 59 & probable chitinase \\
\hline PPL_06169 & 59 & $\begin{array}{l}\text { cfaD, counting factor associated protein, cathepsin L- } \\
\text { like proteinase, peptidase C1A family protein }\end{array}$ \\
\hline PPL_00444 & 58 & $\begin{array}{l}\text { P10901 Alpha-L-fucosidase precursor (EC 3.2.1.51) } \\
\text { (Alpha-L-fucoside fucohydrolase). }\end{array}$ \\
\hline PPL_10565 & 58 & $\begin{array}{l}\text { putative cholinesterase, carboxylesterase, type B } \\
\text { family protein }\end{array}$ \\
\hline PPL_09334 & 58 & hypothetical protein \\
\hline PPL_03598 & 57 & unpredicted protein \\
\hline
\end{tabular}




\begin{tabular}{|c|c|c|}
\hline PPL_01061 & 57 & hypothetical protein \\
\hline PPL_05757 & 57 & peptidase S10 family protein, serine carboxypeptidase \\
\hline PPL_12550 & 56 & amyA, putative alpha-amylase \\
\hline PPL_02110 & 55 & $\begin{array}{l}\text { beta-xylosidase-like protein, glycoside hydrolase family } \\
39 \text { protein }\end{array}$ \\
\hline PPL_09813 & 54 & hypothetical protein \\
\hline PPL_04364 & 54 & saposin B domain-containing protein \\
\hline PPL_02907 & 53 & glycosyl hydrolase family chitinase \\
\hline PPL_09525 & 52 & peptidase S28 family protein \\
\hline PPL_00337 & 52 & expl9, expansin-like protein \\
\hline PPL_02779 & 52 & cprA, cysteine proteinase 1 \\
\hline PPL_09452 & 51 & hypothetical protein \\
\hline PPL_02147 & 50 & pldZ, phospholipase D3 \\
\hline PPL_02789 & 49 & PPL_02789 \\
\hline PPL_02060 & 49 & hypothetical protein \\
\hline PPL_10948 & 48 & phospholipase D \\
\hline PPL_00750 & 48 & $\operatorname{argE}$, acetylornithine deacetylase \\
\hline PPL_02386 & 46 & hypothetical protein \\
\hline PPL_08292 & 43 & unpredicted protein \\
\hline PPL_05209 & 42 & ctsD, cathepsin D, preprocathepsin D \\
\hline PPL_06275 & 42 & $\begin{array}{l}\text { cyclophilin-type peptidylprolyl cis-trans isomerase } \\
\text { (PPlase) }\end{array}$ \\
\hline PPL_08791 & 42 & 3-carboxymuconate cyclase \\
\hline PPL_05734 & 41 & unpredicted protein \\
\hline PPL_08646 & 41 & sodB, superoxide dismutase \\
\hline PPL_01576 & 40 & $\begin{array}{l}\text { peptidase C1A family protein, papain family cysteine } \\
\text { protease, cathepsin Z-like protein }\end{array}$ \\
\hline PPL_03302 & 39 & hypothetical protein \\
\hline PPL_07364 & 38 & chitinase \\
\hline PPL_10376 & 37 & glycoside hydrolase family 25 protein \\
\hline PPL_05106 & 36 & $\begin{array}{l}\text { gghA, peptidase C26 family protein, gamma-glutamyl } \\
\text { hydrolase }\end{array}$ \\
\hline PPL_03967 & 34 & $\begin{array}{l}\text { ctsZ, cathepsin Z precursor, peptidase C1A family } \\
\text { protein, papain family cysteine protease }\end{array}$ \\
\hline PPL_03069 & 33 & hypothetical protein \\
\hline
\end{tabular}




\begin{tabular}{|l|l|l|}
\hline PPL_01142 & 28 & hypothetical protein \\
\hline PPL_04317 & 28 & $\begin{array}{l}\text { tpiA, triose phosphate isomerase, triosephosphate } \\
\text { isomerase }\end{array}$ \\
\hline PPL_01619 & 28 & $\begin{array}{l}\text { CMP/dCMP deaminase, zinc-binding domain- } \\
\text { containing protein }\end{array}$ \\
\hline PPL_07180 & 27 & $\begin{array}{l}\text { dscC-2, discoidin I, C chain and B chain, discoidin I, } \\
\text { beta chain, discoidin I, gamma chain }\end{array}$ \\
\hline PPL_11278 & 27 & glycoside hydrolase family 25 protein \\
\hline PPL_07647 & 26 & $\begin{array}{l}\text { cad3, putative calcium-dependent cell adhesion } \\
\text { molecule-3 }\end{array}$ \\
\hline PPL_12314 & 26 & P80 protein. \\
\hline PPL_01003 & 24 & hypothetical protein \\
\hline PPL_06311 & 23 & unpredicted protein \\
\hline PPL_02846 & 23 & hypothetical protein \\
\hline PPL_06421 & 22 & superoxide dismutase \\
\hline PPL_10113 & 20 & NLP/P60 domain-containing protein \\
\hline PPL_04524 & 18 & DD7-1, galactose-binding domain-containing protein \\
\hline PPL_02277 & 17 & gcvH1, glycine cleavage system H-protein \\
\hline PPL_03282 & 16 & cda, cytidine deaminase \\
\hline PPL_02795 & 15 & putative phospholipid transfer protein \\
\hline PPL_04564 & 15 & cyb5A, cytochrome b5 A \\
\hline PPL_01762 & 12 & cytC, cytochrome c \\
\hline PPL_00779 & 10 & cpiC, cystatin A3, putative cysteine protease inhibitor \\
\hline
\end{tabular}


Table A2: List of primers used in this study.

\begin{tabular}{|c|c|c|}
\hline $\begin{array}{l}\text { SACGB/GenBank } \\
\text { accession number }\end{array}$ & Primers $\left(5^{\prime} \rightarrow 3^{\prime}\right)$ & Product \\
\hline PPL_00912 & $\begin{array}{l}\text { GATACATCGATCCAAGAGATCCAGCTC } \\
\text { CAACAACATACTATCAGAATTATTCGG }\end{array}$ & $230 \mathrm{bp}$ \\
\hline PPL_03784 & $\begin{array}{l}\text { CCACTACACAATACCATTGAACCTAAC } \\
\text { GAAATTTCAACCATCTTATTGTGTTGG }\end{array}$ & $234 \mathrm{bp}$ \\
\hline PPL_05354 & $\begin{array}{l}\text { GTCAACACCACCACCGGACAATGTG } \\
\text { GTTTGGACATTACATTGGTCGTAGGTG }\end{array}$ & $236 \mathrm{bp}$ \\
\hline PPL_05833 & $\begin{array}{l}\text { CAAACCTCCACAAGAAGGCAGCTCGTC } \\
\text { CTGTCCAAGTTGGTCTCTCTGAAAGCG }\end{array}$ & 202 bp \\
\hline PPL_09347 & $\begin{array}{l}\text { GGTGTATGGGCATGTAGTCCAATAAAC } \\
\text { CAGTCTCCACCTTGTCTACCATAGATAC }\end{array}$ & 176 bp \\
\hline PPL_12248 & $\begin{array}{l}\text { GGCACCACAACCATTTTCTCAAGGAG } \\
\text { CCTCTGAGGTGATTGAAATCAAATGC }\end{array}$ & 192 bp \\
\hline PPL_12271 & $\begin{array}{l}\text { CCAACCGCCGAAGACATAGAAGCAGTG } \\
\text { CGCTCGACCGATGGTTCGTCGGCGGTG }\end{array}$ & 242 bp \\
\hline PPL_12370 & $\begin{array}{l}\text { CAAGTTCGACGTCTGGGGTGACACTG } \\
\text { GAGTGGAAGATGTTCGTTGATCTCATTG }\end{array}$ & $261 \mathrm{bp}$ \\
\hline PPL_01657, (acaA2) & $\begin{array}{l}\text { CCTCGAGAATGCAGACCTCAGCGCCAC } \\
\text { CCAGTGGTTTGGTAGTTGGTCTATTCC }\end{array}$ & $261 \mathrm{bp}$ \\
\hline $\begin{array}{l}\text { PPL_05049, } \\
\text { (pkaC) }\end{array}$ & $\begin{array}{l}\text { CTGCTGACAGAACACGTAGACTTGGTG } \\
\text { CACATTGGTTCCTCATCGTATTTCTC }\end{array}$ & 274 bp \\
\hline $\begin{array}{l}\mathrm{PPL} 10234 \\
(p d s A)\end{array}$ & $\begin{array}{l}\text { CAACAATCTCATTAATATTACATTCCC } \\
\text { CTTTTACCATACCTAACAGACCACCTTC }\end{array}$ & 218 bp \\
\hline EU797668 & $\begin{array}{l}\text { CTTGGTGTGTTGGATATTCGCGGTCATC } \\
\text { GTTGCGCTCGAGACGCTTCGCTGTGTC }\end{array}$ & 232 bp \\
\hline PPL_04108 & $\begin{array}{l}\text { CAGTGCAGCACGTAGTCCAATCGCTG } \\
\text { CATCGGAATCAAAGGTGGCGTGTATTG }\end{array}$ & $197 \mathrm{bp}$ \\
\hline PPL_00855 & $\begin{array}{l}\text { GTAACAAATATACATCATGATAATAGG } \\
\text { CATTCTTAAATAAGGTGGTCCTTTGAC }\end{array}$ & $225 \mathrm{bp}$ \\
\hline PPL_02780 & $\begin{array}{l}\text { CACCAGCTAACCCAGCAGCTGGTATCTG } \\
\text { CTTTACATTTATCTGCGTCTACTGGTGG }\end{array}$ & 198 bp \\
\hline PPL_02774 & $\begin{array}{l}\text { CCAGCAATCCTTGCCAAGGCGTAGAATG } \\
\text { AGTATCACAACAACGGTGTTCATAGATG }\end{array}$ & 236 bp \\
\hline PPL_11763 & $\begin{array}{l}\text { CTACTCAATACACACCGATGAGACGTAC } \\
\text { GTTGAGTGTTGGCAGATGAAGTAGTATC }\end{array}$ & 249 bp \\
\hline PPL_03541 & $\begin{array}{l}\text { GGATCAGTCTACAATGCTAATAGATCTC } \\
\text { CCATTCCATCAGTTGTTCTCCTTGACC }\end{array}$ & 216 bp \\
\hline PPL_07908 & $\begin{array}{l}\text { GATGGGGATGTTGGTGGTGTCAAGTTG } \\
\text { GAAGCAACAGCAGCAGTGATATCGAAC }\end{array}$ & 172 bp \\
\hline PPL_05702 & $\begin{array}{l}\text { CTGGAAGGGTGGTGTCGTTGATGGTTTG } \\
\text { CGGTTGGTGGGAAAACACCTGGGATGG }\end{array}$ & 242 bp \\
\hline PPL_05195 & $\begin{array}{l}\text { GGATCGTGTAAAATCTGGAGTTTCATTG } \\
\text { CTCCAACTCTAAGGTCCTTGAAACATCC }\end{array}$ & 206 bp \\
\hline
\end{tabular}




\begin{tabular}{|l|l|l|}
\hline PPL_12249 & $\begin{array}{l}\text { CAGCAATCCATTGTACATATGTAAGAAG } \\
\text { GGATATTGTGTTTGGTGATTAGTGG }\end{array}$ & $188 \mathrm{bp}$ \\
\hline PPL_06644 & $\begin{array}{l}\text { ATACCTTGGCATGGCCATACCTAAAGGG } \\
\text { GTGAACTTTGATGATACTTGAGTGCGT }\end{array}$ & $190 \mathrm{bp}$ \\
\hline PPL_00902 & $\begin{array}{l}\text { CATAGATCTCGAAGAGTTTGAAGAGAC } \\
\text { CTGTGGAAGACCGTCGTCTTCTACTGG }\end{array}$ & $232 \mathrm{bp}$ \\
\hline PPL_05727 & $\begin{array}{l}\text { CCACCTACGACTCACAAATCATTATC } \\
\text { CATTTCGACTGGACCCTTGGTTTGAC }\end{array}$ & $197 \mathrm{bp}$ \\
\hline PPL_08454 & $\begin{array}{l}\text { CCATGTTACTTCTCTTTGTACCAAAG } \\
\text { CATCACTATCAAATTGTGCATGGATAG }\end{array}$ & $219 \mathrm{bp}$ \\
\hline PPL_08455 & $\begin{array}{l}\text { TTTTCATTCCAAAATTCCTTCGTATTGC } \\
\text { GATTATTATCGGTTTGGTTGGACTTGG }\end{array}$ & $225 \mathrm{bp}$ \\
\hline PPL_03564 & $\begin{array}{l}\text { GTATCGTAGAGATCCCAGAGTTTAAC } \\
\text { CTCCAGAATTTTGGTACGAATAGAATG }\end{array}$ & $199 \mathrm{bp}$ \\
\hline ITS_Pp & $\begin{array}{l}\text { GAGGAAGGAGAAGTCGTAACAAGGTATC } \\
\text { GCTTACTGATATGCTTAAGTTCAGCGGG }\end{array}$ & $1000 \mathrm{bp}$ \\
\hline
\end{tabular}


Table A3: Changes of gene expression in starving cells (1292 genes).

P. pallidum PN500 cells were starved for 2 or $3 \mathrm{hr}$ without glorin. Listed are genes that were $>3$-fold regulated after $2 \mathrm{hr}$ of starvation. The same list is compared to gene expression one $\mathrm{hr}$ later (note that many genes are below the $>3$-fold criterion after $3 \mathrm{hr}$ ). Note that 837 genes of $1292(65 \%)$ genes are regulated $>3$-fold up or down after both 2 and 3 hours of starvation.

\begin{tabular}{|c|c|c|c|c|c|}
\hline Gene ID & $\begin{array}{l}\text { RPKM } \\
\text { to }\end{array}$ & $\begin{array}{l}\text { RPKM } \\
\text { t2 }\end{array}$ & $\begin{array}{c}\text { RPKM } \\
\text { t3 }\end{array}$ & $\begin{array}{c}\text { fold } \\
\text { change } \\
\text { t2/t0 }\end{array}$ & $\begin{array}{c}\text { fold } \\
\text { change } \\
\text { t3/t0 }\end{array}$ \\
\hline PPL_02774 & 0.192 & 283.820 & 370.858 & 1476.512 & 1929.304 \\
\hline PPL_02780 & 0.248 & 276.561 & 377.055 & 1114.751 & 1519.820 \\
\hline PPL_ 07908 & 0.266 & 163.753 & 133.324 & 615.725 & 501.309 \\
\hline PPL 09544 & 0.992 & 378.066 & 318.751 & 381.124 & 321.329 \\
\hline PPL_00254 & 1.122 & 399.002 & 284.051 & 355.517 & 253.094 \\
\hline PPL_00132 & 0.117 & 26.541 & 7.313 & 226.387 & 62.378 \\
\hline PPL_04306 & 6.108 & 1281.763 & 1049.866 & 209.856 & 171.889 \\
\hline PPL_03342 & 0.232 & 47.961 & 17.526 & 206.993 & 75.640 \\
\hline PPL 04307 & 13.385 & 1636.434 & 1296.892 & 122.256 & 96.889 \\
\hline PPL_02038 & 3.208 & 384.521 & 295.730 & 119.856 & 92.179 \\
\hline PPL_06550 & 1.178 & 107.991 & 106.252 & 91.667 & 90.191 \\
\hline PPL 04586 & 0.714 & 41.435 & 54.630 & 58.055 & 76.544 \\
\hline PPL_03576 & 0.074 & 4.289 & 2.647 & 57.953 & 35.767 \\
\hline PPL_02271 & 1.564 & 87.558 & 84.027 & 55.979 & 53.722 \\
\hline PPL_04882 & 0.768 & 42.031 & 27.333 & 54.751 & 35.605 \\
\hline PPL_10681 & 1.121 & 60.605 & 16.830 & 54.056 & 15.011 \\
\hline PPL 06523 & 0.139 & 7.506 & 3.256 & 53.823 & 23.348 \\
\hline PPL_02260 & 4.023 & 205.156 & 242.138 & 50.993 & 60.186 \\
\hline PPL_09188 & 1.292 & 65.583 & 24.588 & 50.771 & 19.034 \\
\hline PPL_06520 & 0.319 & 15.438 & 7.421 & 48.434 & 23.283 \\
\hline PPL 06519 & 0.367 & 17.686 & 13.363 & 48.168 & 36.395 \\
\hline PPL_04835 & 1.754 & 81.059 & 9.893 & 46.204 & 5.639 \\
\hline PPL_04774 & 0.070 & 3.131 & 1.921 & 44.838 & 27.517 \\
\hline PPL_09143 & 2.707 & 117.785 & 102.047 & 43.516 & 37.702 \\
\hline PPL_10531 & 0.021 & 0.874 & 0.577 & 41.972 & 27.724 \\
\hline PPL_03183 & 0.570 & 23.047 & 26.627 & 40.465 & 46.751 \\
\hline PPL_01039 & 22.087 & 832.893 & 656.853 & 37.709 & 29.739 \\
\hline PPL_09787 & 9.891 & 365.735 & 172.666 & 36.977 & 17.457 \\
\hline PPL_09781 & 0.275 & 9.622 & 2.429 & 35.027 & 8.843 \\
\hline PPL_03343 & 0.179 & 5.824 & 3.365 & 32.467 & 18.759 \\
\hline PPL_03929 & 0.262 & 8.315 & 4.518 & 31.733 & 17.243 \\
\hline PPL_03184 & 0.427 & 12.359 & 12.403 & 28.968 & 29.070 \\
\hline PPL_02069 & 27.146 & 781.723 & 418.791 & 28.797 & 15.427 \\
\hline PPL 08488 & 0.961 & 26.933 & 15.832 & 28.040 & 16.482 \\
\hline PPL_03638 & 18.118 & 499.623 & 764.683 & 27.577 & 42.206 \\
\hline PPL_01716 & 0.063 & 1.672 & 1.415 & 26.565 & 22.472 \\
\hline PPL_05982 & 0.950 & 24.665 & 6.570 & 25.969 & 6.917 \\
\hline PPL_ 02528 & 0.270 & 6.908 & 3.786 & 25.596 & 14.029 \\
\hline PPL 11771 & 2.542 & 64.853 & 75.165 & 25.513 & 29.570 \\
\hline
\end{tabular}




\begin{tabular}{|c|c|c|c|c|c|}
\hline PPL_01209 & 0.218 & 5.506 & 3.153 & 25.293 & 14.483 \\
\hline $\mathrm{PPL} 02367$ & 0.248 & 6.271 & 5.327 & 25.259 & 21.456 \\
\hline PPL_ 10757 & 1.354 & 33.574 & 26.288 & 24.791 & 19.411 \\
\hline PPL 08807 & 4.890 & 118.135 & 52.563 & 24.156 & 10.748 \\
\hline PPL 00578 & 0.692 & 16.604 & 11.319 & 24.001 & 16.361 \\
\hline PPL 09690 & 0.568 & 13.574 & 3.825 & 23.908 & 6.736 \\
\hline PPL_00953 & 0.605 & 13.556 & 21.897 & 22.405 & 36.192 \\
\hline PPL 02536 & 10.753 & 239.839 & 302.725 & 22.305 & 28.153 \\
\hline PPL_02150 & 1.066 & 23.485 & 7.819 & 22.039 & 7.338 \\
\hline PPL 11512 & 6.502 & 142.697 & 53.915 & 21.946 & 8.292 \\
\hline PPL 02247 & 0.558 & 12.169 & 7.675 & 21.815 & 13.758 \\
\hline PPL 04010 & 0.079 & 1.721 & 1.880 & 21.772 & 23.792 \\
\hline PPL 06900 & 4.443 & 94.314 & 67.822 & 21.230 & 15.267 \\
\hline PPL 09458 & 0.257 & 5.346 & 1.292 & 20.767 & 5.019 \\
\hline $\mathrm{PPL} 00731$ & 1.158 & 23.848 & 5.827 & 20.599 & 5.033 \\
\hline PPL_00716 & 0.209 & 4.128 & 1.089 & 19.739 & 5.207 \\
\hline PPL 11666 & 0.401 & 7.897 & 10.651 & 19.671 & 26.529 \\
\hline PPL_09745 & 0.023 & 0.441 & 0.460 & 19.183 & 20.008 \\
\hline PPL 06552 & 2.583 & 49.525 & 74.177 & 19.170 & 28.712 \\
\hline $\mathrm{PPL}_{-} 10845$ & 0.092 & 1.730 & 0.907 & 18.799 & 9.860 \\
\hline PPL 00538 & 71.055 & 1332.896 & 1376.547 & 18.759 & 19.373 \\
\hline PPL_00051 & 0.093 & 1.742 & 1.604 & 18.684 & 17.200 \\
\hline $\mathrm{PPL} 12476$ & 1.800 & 32.930 & 20.032 & 18.290 & 11.126 \\
\hline PPL_05902 & 0.672 & 12.190 & 47.110 & 18.149 & 70.139 \\
\hline PPL 00519 & 0.106 & 1.916 & 2.116 & 18.111 & 20.007 \\
\hline PPL_10560 & 0.814 & 14.563 & 8.957 & 17.892 & 11.005 \\
\hline PPL_02982 & 1.639 & 29.142 & 16.994 & 17.781 & 10.368 \\
\hline $\mathrm{PPL} 02248$ & 0.236 & 4.051 & 2.410 & 17.132 & 10.194 \\
\hline PPL_05194 & 0.164 & 2.799 & 0.722 & 17.092 & 4.411 \\
\hline PPL_10461 & 0.585 & 9.982 & 4.033 & 17.069 & 6.895 \\
\hline PPL_02657 & 0.664 & 11.100 & 5.052 & 16.721 & 7.610 \\
\hline PPL 09440 & 1.229 & 20.547 & 10.163 & 16.717 & 8.268 \\
\hline PPL_00372 & 0.115 & 1.908 & 1.014 & 16.662 & 8.854 \\
\hline PPL_00715 & 1.607 & 26.613 & 4.404 & 16.558 & 2.740 \\
\hline PPL_02781 & 1.125 & 18.197 & 15.720 & 16.179 & 13.976 \\
\hline PPL_04947 & 0.067 & 1.066 & 0.836 & 15.955 & 12.506 \\
\hline PPL_03840 & 0.665 & 10.611 & 7.605 & 15.950 & 11.433 \\
\hline PPL_04785 & 2.926 & 46.546 & 40.309 & 15.909 & 13.777 \\
\hline PPL_08268 & 0.924 & 14.542 & 14.015 & 15.743 & 15.173 \\
\hline PPL_08352 & 0.447 & 7.000 & 2.455 & 15.656 & 5.491 \\
\hline PPL_08805 & 3.236 & 50.069 & 65.538 & 15.474 & 20.255 \\
\hline PPL_04412 & 0.571 & 8.806 & 5.075 & 15.420 & 8.888 \\
\hline PPL_08689 & 12.015 & 183.535 & 57.631 & 15.275 & 4.797 \\
\hline PPL_04356 & 30.734 & 461.288 & 151.690 & 15.009 & 4.935 \\
\hline PPL 04193 & 0.914 & 13.682 & 17.098 & 14.972 & 18.710 \\
\hline PPL_07733 & 16.493 & 244.244 & 103.609 & 14.809 & 6.282 \\
\hline PPL_03785 & 0.031 & 0.440 & 0.527 & 14.225 & 17.009 \\
\hline $\mathrm{PPL}=11193$ & 38.447 & 545.655 & 569.936 & 14.193 & 14.824 \\
\hline $\mathrm{PPL} 02633$ & 45.259 & 629.361 & 521.113 & 13.906 & 11.514 \\
\hline PPL_09814 & 28.004 & 384.807 & 302.224 & 13.741 & 10.792 \\
\hline PPL_08361 & 4.737 & 64.947 & 42.283 & 13.711 & 8.926 \\
\hline PPL_06494 & 0.093 & 1.268 & 0.353 & 13.599 & 3.782 \\
\hline
\end{tabular}




\begin{tabular}{|c|c|c|c|c|c|}
\hline PPL_10714 & 2.830 & 38.377 & 27.991 & 13.560 & 9.890 \\
\hline PPL_00292 & 1.447 & 19.228 & 16.804 & 13.286 & 11.611 \\
\hline PPL_07200 & 0.071 & 0.938 & 0.391 & 13.193 & 5.501 \\
\hline PPL 10561 & 0.239 & 3.136 & 2.632 & 13.113 & 11.003 \\
\hline PPL_11798 & 0.471 & 6.120 & 4.932 & 13.001 & 10.477 \\
\hline PPL_05600 & 1.941 & 25.206 & 9.988 & 12.988 & 5.147 \\
\hline PPL_07885 & 0.193 & 2.508 & 3.828 & 12.979 & 19.815 \\
\hline PPL 02784 & 0.685 & 8.667 & 9.049 & 12.661 & 13.218 \\
\hline PPL_11792 & 1.977 & 24.917 & 19.288 & 12.602 & 9.755 \\
\hline PPL_05680 & 0.236 & 2.934 & 2.182 & 12.421 & 9.235 \\
\hline PPL_05195 & 5.658 & 69.666 & 117.677 & 12.313 & 20.798 \\
\hline PPL 04271 & 0.925 & 11.215 & 9.825 & 12.129 & 10.626 \\
\hline PPL_12532 & 0.850 & 10.303 & 8.909 & 12.117 & 10.477 \\
\hline PPL_07910 & 0.049 & 0.588 & 0.681 & 12.081 & 13.995 \\
\hline PPL_00405 & 5.411 & 64.608 & 17.936 & 11.939 & 3.314 \\
\hline PPL_04524 & 125.867 & 1498.588 & 2068.794 & 11.906 & 16.436 \\
\hline PPL_10555 & 0.171 & 2.039 & 1.710 & 11.896 & 9.975 \\
\hline PPL_09702 & 3.691 & 43.732 & 19.584 & 11.847 & 5.306 \\
\hline PPL_05932 & 20.783 & 245.908 & 106.781 & 11.832 & 5.138 \\
\hline PPL_02039 & 11.818 & 138.307 & 130.314 & 11.703 & 11.026 \\
\hline PPL_08429 & 6.523 & 76.312 & 30.830 & 11.698 & 4.726 \\
\hline PPL_08089 & 0.047 & 0.553 & 0.238 & 11.687 & 5.041 \\
\hline PPL_09134 & 0.564 & 6.579 & 4.926 & 11.669 & 8.737 \\
\hline PPL_11490 & 5.753 & 66.634 & 123.843 & 11.584 & 21.528 \\
\hline PPL_03571 & 13.594 & 151.313 & 33.721 & 11.131 & 2.481 \\
\hline PPL_07634 & 0.037 & 0.415 & 0.131 & 11.089 & 3.501 \\
\hline PPL_05961 & 1.223 & 13.366 & 10.703 & 10.930 & 8.752 \\
\hline PPL_12019 & 4.439 & 48.081 & 36.440 & 10.832 & 8.209 \\
\hline PPL_00052 & 6.104 & 65.793 & 72.431 & 10.779 & 11.866 \\
\hline PPL_01229 & 0.100 & 1.069 & 1.455 & 10.679 & 14.540 \\
\hline PPL_10932 & 0.090 & 0.964 & 0.488 & 10.653 & 5.390 \\
\hline PPL_05299 & 5.634 & 59.725 & 42.868 & 10.601 & 7.609 \\
\hline PPL_05174 & 9.682 & 102.295 & 79.247 & 10.565 & 8.185 \\
\hline PPL_02541 & 0.996 & 10.472 & 12.271 & 10.509 & 12.315 \\
\hline PPL_06374 & 0.427 & 4.462 & 1.642 & 10.440 & 3.841 \\
\hline PPL_02535 & 0.112 & 1.162 & 0.790 & 10.416 & 7.080 \\
\hline PPL_00367 & 0.574 & 5.946 & 6.097 & 10.351 & 10.613 \\
\hline PPL_08647 & 1.899 & 19.482 & 13.229 & 10.259 & 6.967 \\
\hline PPL_05601 & 0.415 & 4.232 & 2.143 & 10.186 & 5.159 \\
\hline PPL_03341 & 0.361 & 3.669 & 1.788 & 10.171 & 4.955 \\
\hline PPL_09762 & 2.965 & 30.096 & 31.752 & 10.151 & 10.710 \\
\hline PPL_04438 & 1.363 & 13.776 & 12.393 & 10.104 & 9.090 \\
\hline PPL_09662 & 0.130 & 1.310 & 0.954 & 10.037 & 7.309 \\
\hline PPL_01872 & 0.175 & 1.728 & 1.090 & 9.865 & 6.224 \\
\hline PPL_10462 & 0.285 & 2.793 & 2.134 & 9.815 & 7.499 \\
\hline PPL_07129 & 0.195 & 1.890 & 0.943 & 9.701 & 4.838 \\
\hline PPL_05405 & 4.540 & 43.865 & 55.887 & 9.661 & 12.309 \\
\hline PPL_12333 & 17.073 & 164.932 & 118.068 & 9.660 & 6.915 \\
\hline PPL_02846 & 330.946 & 3173.149 & 2483.372 & 9.588 & 7.504 \\
\hline PPL_08265 & 7.685 & 73.083 & 30.960 & 9.510 & 4.029 \\
\hline PPL_11833 & 2.260 & 21.361 & 19.688 & 9.451 & 8.711 \\
\hline PPL_01305 & 4.025 & 37.514 & 48.298 & 9.319 & 11.998 \\
\hline
\end{tabular}




\begin{tabular}{|c|c|c|c|c|c|}
\hline PPL_11559 & 1.196 & 11.108 & 14.145 & 9.288 & 11.827 \\
\hline PPL_10680 & 0.824 & 7.574 & 2.555 & 9.196 & 3.103 \\
\hline PPL_12442 & 1.723 & 15.811 & 13.618 & 9.175 & 7.903 \\
\hline PPL 06890 & 0.090 & 0.824 & 1.167 & 9.126 & 12.919 \\
\hline $\mathrm{PPL} 02234$ & 0.469 & 4.267 & 3.001 & 9.102 & 6.402 \\
\hline PPL 07203 & 10.070 & 90.752 & 126.865 & 9.012 & 12.598 \\
\hline PPL_06178 & 0.226 & 2.033 & 0.921 & 9.012 & 4.085 \\
\hline PPL 09437 & 0.207 & 1.866 & 1.462 & 9.004 & 7.055 \\
\hline PPL_06442 & 0.367 & 3.295 & 0.917 & 8.984 & 2.501 \\
\hline $\mathrm{PPL} 04766$ & 0.119 & 1.070 & 0.681 & 8.975 & 5.717 \\
\hline $\mathrm{PPL} 11065$ & 0.686 & 6.119 & 2.167 & 8.922 & 3.159 \\
\hline PPL_10945 & 13.243 & 116.379 & 106.186 & 8.788 & 8.018 \\
\hline PPL_05486 & 0.526 & 4.609 & 4.564 & 8.763 & 8.678 \\
\hline PPL 01952 & 0.233 & 2.038 & 1.410 & 8.737 & 6.046 \\
\hline PPL 07597 & 2.126 & 18.549 & 20.756 & 8.725 & 9.763 \\
\hline PPL_07057 & 6.080 & 52.754 & 33.352 & 8.677 & 5.486 \\
\hline PPL 09894 & 0.209 & 1.809 & 0.912 & 8.670 & 4.371 \\
\hline PPL_03107 & 0.148 & 1.274 & 0.679 & 8.633 & 4.603 \\
\hline $\mathrm{PPL} 12370$ & 0.259 & 2.229 & 1.335 & 8.612 & 5.156 \\
\hline PPL_01146 & 8.346 & 71.607 & 69.605 & 8.579 & 8.340 \\
\hline $\mathrm{PPL} 02281$ & 8.208 & 69.942 & 43.838 & 8.521 & 5.341 \\
\hline PPL_03752 & 0.240 & 2.044 & 1.803 & 8.513 & 7.510 \\
\hline PPL 11990 & 1.141 & 9.646 & 9.553 & 8.455 & 8.373 \\
\hline PPL_02628 & 5.275 & 44.349 & 11.568 & 8.408 & 2.193 \\
\hline PPL_00855 & 0.206 & 1.727 & 2.661 & 8.399 & 12.939 \\
\hline PPL_01117 & 0.843 & 7.078 & 5.524 & 8.392 & 6.550 \\
\hline PPL_08056 & 234.038 & 1949.188 & 2075.470 & 8.329 & 8.868 \\
\hline PPL_09695 & 55.525 & 461.751 & 555.589 & 8.316 & 10.006 \\
\hline PPL_09054 & 1.247 & 10.366 & 6.164 & 8.310 & 4.941 \\
\hline PPL_02950 & 0.956 & 7.883 & 5.335 & 8.243 & 5.578 \\
\hline PPL_05337 & 4.206 & 34.661 & 36.681 & 8.240 & 8.721 \\
\hline PPL_12554 & 0.353 & 2.898 & 1.042 & 8.219 & 2.955 \\
\hline PPL_05917 & 0.159 & 1.297 & 0.545 & 8.165 & 3.430 \\
\hline PPL_12216 & 0.129 & 1.051 & 0.516 & 8.155 & 4.000 \\
\hline PPL_09724 & 4.279 & 34.790 & 14.570 & 8.131 & 3.405 \\
\hline PPL_12381 & 98.103 & 796.042 & 607.292 & 8.114 & 6.190 \\
\hline PPL_00057 & 7.229 & 58.529 & 81.436 & 8.096 & 11.265 \\
\hline PPL_05394 & 0.049 & 0.399 & 0.394 & 8.094 & 8.003 \\
\hline PPL_00085 & 0.055 & 0.445 & 0.358 & 8.094 & 6.502 \\
\hline $\mathrm{PPL} 09260$ & 884.568 & 7150.300 & 7073.941 & 8.083 & 7.997 \\
\hline PPL_01361 & 0.122 & 0.984 & 0.603 & 8.064 & 4.942 \\
\hline PPL_03340 & 0.279 & 2.239 & 1.845 & 8.014 & 6.603 \\
\hline PPL_00396 & 0.767 & 6.094 & 5.643 & 7.949 & 7.362 \\
\hline $\mathrm{PPL}^{-} 10074$ & 16.208 & 128.557 & 106.539 & 7.931 & 6.573 \\
\hline $\mathrm{PPL} 12030$ & 0.039 & 0.307 & 0.272 & 7.892 & 7.000 \\
\hline PPL_04158 & 0.141 & 1.112 & 1.771 & 7.890 & 12.566 \\
\hline PPL_00368 & 1.949 & 15.377 & 14.102 & 7.889 & 7.235 \\
\hline PPL_04042 & 4.778 & 37.560 & 18.452 & 7.862 & 3.862 \\
\hline PPL 07388 & 0.196 & 1.542 & 1.175 & 7.858 & 5.986 \\
\hline PPL_10626 & 36.444 & 285.977 & 95.263 & 7.847 & 2.614 \\
\hline PPL_09786 & 0.257 & 2.013 & 1.265 & 7.837 & 4.924 \\
\hline PPL_01365 & 0.942 & 7.376 & 4.748 & 7.830 & 5.041 \\
\hline
\end{tabular}




\begin{tabular}{|c|c|c|c|c|c|}
\hline PPL_12169 & 0.133 & 1.039 & 0.783 & 7.813 & 5.890 \\
\hline $\mathrm{PPL} 06502$ & 0.660 & 5.126 & 3.279 & 7.764 & 4.966 \\
\hline PPL_10928 & 0.180 & 1.396 & 0.888 & 7.749 & 4.930 \\
\hline PPL_09010 & 0.446 & 3.446 & 2.016 & 7.722 & 4.518 \\
\hline $\mathrm{PPL} 00514$ & 7.253 & 55.967 & 93.525 & 7.716 & 12.894 \\
\hline PPL 06920 & 0.815 & 6.245 & 3.811 & 7.666 & 4.678 \\
\hline PPL_10158 & 0.228 & 1.749 & 1.578 & 7.663 & 6.910 \\
\hline $\mathrm{PPL} 03356$ & 0.043 & 0.325 & 0.235 & 7.628 & 5.501 \\
\hline $\mathrm{PPL} 11295$ & 0.084 & 0.639 & 0.545 & 7.628 & 6.504 \\
\hline PPL 10595 & 0.099 & 0.756 & 0.460 & 7.612 & 4.631 \\
\hline $\mathrm{PPL} 05113$ & 0.251 & 1.908 & 1.259 & 7.606 & 5.019 \\
\hline PPL 03339 & 19.950 & 151.101 & 34.276 & 7.574 & 1.718 \\
\hline PPL_06996 & 279.376 & 2114.808 & 895.883 & 7.570 & 3.207 \\
\hline PPL 12525 & 2.107 & 15.909 & 18.790 & 7.550 & 8.917 \\
\hline PPL_00378 & 0.103 & 0.770 & 0.302 & 7.476 & 2.935 \\
\hline PPL_06684 & 17.116 & 127.829 & 105.543 & 7.469 & 6.166 \\
\hline PPL 05364 & 0.950 & 7.053 & 1.266 & 7.427 & 1.334 \\
\hline $\mathrm{PPL} 10866$ & 0.070 & 0.513 & 0.496 & 7.367 & 7.131 \\
\hline $\mathrm{PPL} 11865$ & 0.179 & 1.313 & 3.297 & 7.332 & 18.418 \\
\hline $\mathrm{PPL}_{-} 11523$ & 1.314 & 9.633 & 4.671 & 7.329 & 3.554 \\
\hline PPL 08603 & 0.642 & 4.663 & 1.948 & 7.263 & 3.034 \\
\hline PPL_04124 & 0.171 & 1.240 & 1.457 & 7.235 & 8.499 \\
\hline PPL_10805 & 0.239 & 1.728 & 1.077 & 7.224 & 4.502 \\
\hline PPL_02839 & 0.112 & 0.811 & 0.590 & 7.221 & 5.251 \\
\hline $\mathrm{PPL} 12463$ & 11.569 & 83.530 & 61.498 & 7.220 & 5.316 \\
\hline PPL_02903 & 13.117 & 94.534 & 82.971 & 7.207 & 6.325 \\
\hline PPL_08043 & 9.078 & 65.116 & 44.484 & 7.173 & 4.900 \\
\hline $\mathrm{PPL} 07198$ & 0.256 & 1.828 & 0.577 & 7.152 & 2.260 \\
\hline PPL_06066 & 0.535 & 3.811 & 2.697 & 7.129 & 5.044 \\
\hline PPL_03605 & 0.224 & 1.596 & 0.758 & 7.128 & 3.387 \\
\hline PPL_05105 & 1.351 & 9.583 & 6.716 & 7.092 & 4.970 \\
\hline PPL_08395 & 53.105 & 375.800 & 319.215 & 7.077 & 6.011 \\
\hline PPL_01720 & 0.688 & 4.862 & 3.273 & 7.062 & 4.754 \\
\hline PPL_02300 & 7.782 & 54.832 & 57.910 & 7.046 & 7.442 \\
\hline PPL_11965 & 0.178 & 1.248 & 1.512 & 7.029 & 8.513 \\
\hline PPL_09575 & 0.233 & 1.632 & 2.327 & 6.998 & 9.977 \\
\hline PPL_01233 & 0.137 & 0.953 & 0.519 & 6.946 & 3.781 \\
\hline PPL_08054 & 1.189 & 8.258 & 5.379 & 6.946 & 4.524 \\
\hline PPL_06383 & 34.472 & 239.265 & 86.907 & 6.941 & 2.521 \\
\hline PPL_06907 & 2.068 & 14.350 & 5.793 & 6.938 & 2.801 \\
\hline PPL_11493 & 0.634 & 4.397 & 3.620 & 6.932 & 5.707 \\
\hline PPL_09869 & 0.355 & 2.444 & 1.621 & 6.892 & 4.570 \\
\hline PPL_00758 & 0.258 & 1.777 & 1.612 & 6.890 & 6.252 \\
\hline PPL_05298 & 6.908 & 47.586 & 39.069 & 6.889 & 5.656 \\
\hline PPL 05755 & 2.298 & 15.819 & 15.654 & 6.884 & 6.812 \\
\hline PPL_04321 & 0.619 & 4.245 & 6.315 & 6.859 & 10.204 \\
\hline PPL_08350 & 1.358 & 9.285 & 6.654 & 6.840 & 4.901 \\
\hline PPL_00136 & 8.855 & 60.501 & 72.238 & 6.832 & 8.158 \\
\hline PPL_02325 & 0.659 & 4.501 & 2.523 & 6.830 & 3.828 \\
\hline PPL_09364 & 3.135 & 21.376 & 17.554 & 6.818 & 5.599 \\
\hline PPL_06434 & 0.326 & 2.212 & 1.552 & 6.795 & 4.768 \\
\hline PPL_08476 & 0.967 & 6.559 & 9.544 & 6.781 & 9.867 \\
\hline
\end{tabular}




\begin{tabular}{|c|c|c|c|c|c|}
\hline PPL_03841 & 1.787 & 12.079 & 7.464 & 6.760 & 4.177 \\
\hline $\mathrm{PPL} 06230$ & 0.294 & 1.984 & 0.603 & 6.752 & 2.052 \\
\hline PPL_08480 & 1.456 & 9.739 & 2.553 & 6.689 & 1.753 \\
\hline PPL_08317 & 1.438 & 9.610 & 10.084 & 6.681 & 7.011 \\
\hline PPL 02939 & 0.327 & 2.178 & 1.169 & 6.666 & 3.578 \\
\hline PPL 05833 & 0.517 & 3.441 & 7.650 & 6.661 & 14.808 \\
\hline PPL_11240 & 0.545 & 3.629 & 3.823 & 6.659 & 7.014 \\
\hline $\mathrm{PPL} 11992$ & 1.867 & 12.425 & 7.119 & 6.654 & 3.813 \\
\hline PPL_05855 & 15.774 & 104.966 & 35.133 & 6.654 & 2.227 \\
\hline PPL 05877 & 1.547 & 10.275 & 9.417 & 6.643 & 6.088 \\
\hline PPL 07208 & 0.249 & 1.653 & 0.809 & 6.632 & 3.247 \\
\hline PPL 08967 & 8.548 & 56.651 & 58.923 & 6.627 & 6.893 \\
\hline PPL_00746 & 12.970 & 85.610 & 91.786 & 6.600 & 7.077 \\
\hline PPL 07879 & 6.250 & 41.191 & 38.970 & 6.590 & 6.235 \\
\hline PPL_07232 & 1.411 & 9.274 & 11.406 & 6.573 & 8.085 \\
\hline PPL_06848 & 1.855 & 12.158 & 10.753 & 6.554 & 5.797 \\
\hline PPL 05333 & 2.358 & 15.452 & 32.013 & 6.552 & 13.574 \\
\hline PPL_09816 & 10.305 & 67.316 & 45.287 & 6.533 & 4.395 \\
\hline $\mathrm{PPL} 02211$ & 0.715 & 4.661 & 3.949 & 6.515 & 5.520 \\
\hline PPL_04148 & 1.758 & 11.440 & 8.572 & 6.507 & 4.875 \\
\hline PPL 02377 & 2.949 & 19.184 & 15.533 & 6.506 & 5.267 \\
\hline PPL_08134 & 0.766 & 4.947 & 3.183 & 6.457 & 4.155 \\
\hline $\mathrm{PPL} 12462$ & 1.235 & 7.972 & 6.892 & 6.453 & 5.579 \\
\hline $\mathrm{PPL} 10943$ & 0.673 & 4.331 & 4.259 & 6.434 & 6.327 \\
\hline PPL 08309 & 22.049 & 141.724 & 100.292 & 6.428 & 4.549 \\
\hline PPL_07920 & 2.309 & 14.779 & 10.839 & 6.401 & 4.694 \\
\hline PPL_09924 & 0.270 & 1.729 & 1.399 & 6.399 & 5.177 \\
\hline $\mathrm{PPL} 08973$ & 0.441 & 2.821 & 1.856 & 6.396 & 4.209 \\
\hline PPL_06732 & 0.307 & 1.960 & 2.173 & 6.390 & 7.084 \\
\hline PPL_01566 & 0.292 & 1.864 & 1.111 & 6.379 & 3.802 \\
\hline $\mathrm{PPL}_{-} 11328$ & 0.802 & 5.106 & 3.200 & 6.366 & 3.990 \\
\hline PPL_04125 & 1.441 & 9.136 & 1.718 & 6.342 & 1.193 \\
\hline PPL_04413 & 0.252 & 1.584 & 1.192 & 6.294 & 4.736 \\
\hline PPL_07939 & 3.283 & 20.644 & 15.990 & 6.288 & 4.871 \\
\hline PPL_02856 & 39.361 & 247.447 & 140.397 & 6.287 & 3.567 \\
\hline PPL_00897 & 0.284 & 1.786 & 1.495 & 6.281 & 5.258 \\
\hline PPL_09743 & 0.927 & 5.816 & 4.445 & 6.277 & 4.797 \\
\hline PPL_02658 & 1.871 & 11.692 & 7.821 & 6.249 & 4.181 \\
\hline PPL_07084 & 20.861 & 130.291 & 63.179 & 6.246 & 3.029 \\
\hline PPL_07014 & 0.110 & 0.688 & 0.752 & 6.242 & 6.820 \\
\hline PPL_08856 & 1.181 & 7.364 & 7.291 & 6.238 & 6.176 \\
\hline PPL_07365 & 2.972 & 18.372 & 15.102 & 6.182 & 5.082 \\
\hline PPL_03471 & 0.488 & 3.014 & 2.263 & 6.180 & 4.640 \\
\hline PPL_07687 & 0.125 & 0.774 & 0.460 & 6.172 & 3.667 \\
\hline PPL 09878 & 1.641 & 10.114 & 7.001 & 6.163 & 4.266 \\
\hline PPL_00612 & 22.149 & 136.175 & 90.452 & 6.148 & 4.084 \\
\hline PPL_03291 & 0.254 & 1.562 & 0.668 & 6.139 & 2.625 \\
\hline PPL_10426 & 0.059 & 0.363 & 0.366 & 6.118 & 6.170 \\
\hline PPL_08861 & 28.815 & 176.050 & 118.088 & 6.110 & 4.098 \\
\hline PPL_02872 & 0.281 & 1.710 & 1.327 & 6.092 & 4.726 \\
\hline PPL_09865 & 0.035 & 0.216 & 0.213 & 6.091 & 6.001 \\
\hline PPL_08648 & 1.717 & 10.447 & 7.612 & 6.086 & 4.434 \\
\hline
\end{tabular}




\begin{tabular}{|c|c|c|c|c|c|}
\hline PPL_05017 & 0.317 & 1.926 & 0.869 & 6.077 & 2.741 \\
\hline PPL_09639 & 0.724 & 4.382 & 2.199 & 6.056 & 3.040 \\
\hline PPL 04565 & 4.225 & 25.543 & 30.510 & 6.046 & 7.222 \\
\hline PPL_08615 & 0.547 & 3.309 & 1.601 & 6.045 & 2.924 \\
\hline PPL_03292 & 49.089 & 296.287 & 133.761 & 6.036 & 2.725 \\
\hline PPL_08534 & 19.085 & 115.028 & 109.427 & 6.027 & 5.734 \\
\hline PPL_11597 & 93.622 & 563.686 & 258.143 & 6.021 & 2.757 \\
\hline PPL_00798 & 1.355 & 8.151 & 3.551 & 6.017 & 2.621 \\
\hline PPL 06696 & 0.261 & 1.565 & 1.255 & 5.993 & 4.805 \\
\hline PPL_00125 & 0.540 & 3.237 & 1.922 & 5.993 & 3.558 \\
\hline PPL_02062 & 0.339 & 2.032 & 1.882 & 5.990 & 5.548 \\
\hline PPL 06029 & 0.650 & 3.888 & 3.918 & 5.980 & 6.025 \\
\hline PPL_03338 & 2.686 & 16.033 & 5.367 & 5.970 & 1.998 \\
\hline PPL_04883 & 0.057 & 0.341 & 0.041 & 5.960 & 0.708 \\
\hline PPL_01778 & 1.855 & 11.042 & 11.679 & 5.953 & 6.296 \\
\hline PPL_10576 & 26.872 & 159.803 & 212.746 & 5.947 & 7.917 \\
\hline $\mathrm{PPL} 06627$ & 0.031 & 0.182 & 0.184 & 5.929 & 6.004 \\
\hline PPL_10847 & 0.176 & 1.041 & 1.142 & 5.929 & 6.503 \\
\hline PPL_08369 & 19.898 & 117.542 & 75.084 & 5.907 & 3.773 \\
\hline $\mathrm{PPL}_{-} 10608$ & 0.802 & 4.734 & 3.966 & 5.906 & 4.948 \\
\hline PPL_02583 & 11.528 & 67.815 & 29.029 & 5.883 & 2.518 \\
\hline PPL_12443 & 2.657 & 15.613 & 9.611 & 5.877 & 3.618 \\
\hline PPL_02037 & 0.541 & 3.178 & 1.941 & 5.871 & 3.586 \\
\hline PPL_11619 & 51.888 & 304.579 & 216.638 & 5.870 & 4.175 \\
\hline PPL_07775 & 3.112 & 18.261 & 16.341 & 5.868 & 5.251 \\
\hline PPL_05311 & 3.456 & 20.233 & 16.766 & 5.854 & 4.851 \\
\hline $\mathrm{PPL}=12310$ & 32.442 & 189.313 & 296.441 & 5.835 & 9.138 \\
\hline PPL_01693 & 0.387 & 2.252 & 1.612 & 5.823 & 4.168 \\
\hline PPL_10539 & 2.792 & 16.256 & 11.509 & 5.822 & 4.122 \\
\hline PPL_04390 & 1.417 & 8.251 & 4.860 & 5.821 & 3.429 \\
\hline PPL_02951 & 1.483 & 8.625 & 6.238 & 5.816 & 4.207 \\
\hline PPL_05679 & 1.153 & 6.701 & 5.021 & 5.812 & 4.355 \\
\hline PPL_10894 & 0.319 & 1.851 & 1.356 & 5.800 & 4.251 \\
\hline PPL_06106 & 5.038 & 29.174 & 28.070 & 5.791 & 5.572 \\
\hline PPL 12011 & 0.161 & 0.929 & 0.455 & 5.781 & 2.834 \\
\hline PPL_04588 & 3.543 & 20.471 & 10.460 & 5.778 & 2.953 \\
\hline PPL_10520 & 0.738 & 4.245 & 3.889 & 5.752 & 5.269 \\
\hline $\mathrm{PPL}^{-} 11400$ & 5.221 & 30.004 & 58.195 & 5.747 & 11.147 \\
\hline PPL_09106 & 1.006 & 5.774 & 5.454 & 5.740 & 5.422 \\
\hline PPL_00648 & 21.558 & 123.706 & 107.751 & 5.738 & 4.998 \\
\hline PPL_07447 & 0.204 & 1.167 & 0.407 & 5.727 & 2.000 \\
\hline PPL_10979 & 0.220 & 1.260 & 0.660 & 5.727 & 2.999 \\
\hline $\mathrm{PPL}_{-} 11349$ & 0.043 & 0.248 & 0.424 & 5.719 & 9.760 \\
\hline PPL 05265 & 0.204 & 1.155 & 0.764 & 5.671 & 3.748 \\
\hline PPL_09438 & 5.175 & 29.242 & 19.087 & 5.651 & 3.688 \\
\hline PPL_11135 & 23.500 & 132.614 & 35.603 & 5.643 & 1.515 \\
\hline PPL_06896 & 7.601 & 42.865 & 33.202 & 5.639 & 4.368 \\
\hline $\mathrm{PPL} 12151$ & 4.636 & 26.133 & 13.454 & 5.636 & 2.902 \\
\hline PPL_11401 & 3.915 & 22.049 & 32.889 & 5.632 & 8.401 \\
\hline PPL_00916 & 0.418 & 2.348 & 1.987 & 5.618 & 4.754 \\
\hline PPL_03314 & 59.867 & 335.345 & 363.527 & 5.602 & 6.072 \\
\hline PPL_08940 & 172.592 & 966.814 & 579.757 & 5.602 & 3.359 \\
\hline
\end{tabular}




\begin{tabular}{|c|c|c|c|c|c|}
\hline PPL_09436 & 13.699 & 76.439 & 88.597 & 5.580 & 6.467 \\
\hline PPL_01401 & 8.749 & 48.606 & 38.499 & 5.555 & 4.400 \\
\hline PPL_12175 & 0.276 & 1.533 & 0.516 & 5.545 & 1.865 \\
\hline PPL_10947 & 2.153 & 11.914 & 8.512 & 5.533 & 3.953 \\
\hline PPL_03860 & 51.752 & 286.178 & 296.263 & 5.530 & 5.725 \\
\hline PPL_04644 & 61.076 & 337.251 & 478.432 & 5.522 & 7.833 \\
\hline PPL_04006 & 0.444 & 2.449 & 2.358 & 5.516 & 5.312 \\
\hline PPL 06319 & 0.101 & 0.559 & 0.998 & 5.511 & 9.841 \\
\hline PPL 05987 & 0.584 & 3.209 & 2.308 & 5.496 & 3.953 \\
\hline PPL_02824 & 1.380 & 7.578 & 7.238 & 5.493 & 5.246 \\
\hline PPL_01133 & 0.696 & 3.822 & 2.780 & 5.488 & 3.992 \\
\hline PPL 04942 & 1.248 & 6.830 & 6.420 & 5.474 & 5.146 \\
\hline PPL_07240 & 15.983 & 87.297 & 90.310 & 5.462 & 5.650 \\
\hline PPL_09638 & 2.531 & 13.818 & 7.780 & 5.460 & 3.074 \\
\hline PPL_06018 & 1.410 & 7.681 & 6.878 & 5.447 & 4.878 \\
\hline PPL_04260 & 0.900 & 4.885 & 4.189 & 5.428 & 4.655 \\
\hline PPL_00087 & 0.668 & 3.617 & 2.639 & 5.417 & 3.953 \\
\hline PPL 06292 & 1.136 & 6.148 & 5.869 & 5.411 & 5.165 \\
\hline PPL_01786 & 13.140 & 71.061 & 53.788 & 5.408 & 4.093 \\
\hline PPL_07745 & 1.990 & 10.705 & 9.375 & 5.378 & 4.710 \\
\hline PPL_00053 & 6.075 & 32.581 & 46.736 & 5.363 & 7.694 \\
\hline PPL_02720 & 0.181 & 0.972 & 1.088 & 5.362 & 6.001 \\
\hline PPL_01419 & 0.569 & 3.051 & 3.416 & 5.362 & 6.003 \\
\hline PPL_03943 & 1.933 & 10.351 & 8.207 & 5.355 & 4.246 \\
\hline PPL_05153 & 12.187 & 65.094 & 48.529 & 5.341 & 3.982 \\
\hline PPL_09630 & 1.369 & 7.302 & 6.689 & 5.334 & 4.887 \\
\hline PPL_00137 & 0.162 & 0.862 & 0.917 & 5.328 & 5.670 \\
\hline PPL_10964 & 0.287 & 1.528 & 1.080 & 5.327 & 3.766 \\
\hline PPL_08494 & 4.871 & 25.927 & 26.136 & 5.322 & 5.365 \\
\hline PPL_06598 & 0.331 & 1.759 & 1.405 & 5.317 & 4.248 \\
\hline PPL_02138 & 1.615 & 8.587 & 8.274 & 5.316 & 5.122 \\
\hline PPL_02157 & 0.287 & 1.522 & 1.621 & 5.307 & 5.649 \\
\hline PPL_05651 & 1.015 & 5.369 & 3.698 & 5.290 & 3.644 \\
\hline PPL_00029 & 3.411 & 18.034 & 16.908 & 5.288 & 4.957 \\
\hline PPL_10059 & 3.599 & 19.018 & 4.442 & 5.285 & 1.234 \\
\hline PPL_04465 & 1.321 & 6.978 & 10.235 & 5.284 & 7.750 \\
\hline PPL 06134 & 0.498 & 2.632 & 2.085 & 5.279 & 4.182 \\
\hline PPL_10890 & 3.134 & 16.506 & 15.791 & 5.266 & 5.038 \\
\hline PPL_10516 & 0.069 & 0.362 & 0.241 & 5.261 & 3.501 \\
\hline PPL_03721 & 0.220 & 1.158 & 0.770 & 5.261 & 3.500 \\
\hline PPL_00545 & 2.334 & 12.266 & 6.622 & 5.256 & 2.837 \\
\hline PPL_11245 & 1.025 & 5.380 & 4.173 & 5.251 & 4.073 \\
\hline PPL_05469 & 5.755 & 30.140 & 12.514 & 5.237 & 2.175 \\
\hline PPL 03307 & 0.585 & 3.063 & 1.739 & 5.236 & 2.973 \\
\hline PPL_05305 & 0.201 & 1.050 & 0.720 & 5.234 & 3.593 \\
\hline PPL_09780 & 5.353 & 28.009 & 11.363 & 5.233 & 2.123 \\
\hline PPL_01943 & 2.653 & 13.850 & 11.059 & 5.220 & 4.168 \\
\hline PPL_09220 & 125.010 & 652.143 & 459.886 & 5.217 & 3.679 \\
\hline PPL_09658 & 0.278 & 1.448 & 1.057 & 5.207 & 3.802 \\
\hline PPL_12204 & 0.981 & 5.103 & 3.063 & 5.205 & 3.123 \\
\hline PPL_12089 & 3.261 & 16.970 & 6.420 & 5.204 & 1.969 \\
\hline PPL_03561 & 3.759 & 19.557 & 18.635 & 5.202 & 4.957 \\
\hline
\end{tabular}




\begin{tabular}{|c|c|c|c|c|c|}
\hline PPL_02558 & 156.082 & 811.930 & 618.048 & 5.202 & 3.960 \\
\hline $\mathrm{PPL} 08058$ & 0.079 & 0.413 & 0.163 & 5.200 & 2.048 \\
\hline PPL_04807 & 0.818 & 4.252 & 3.661 & 5.198 & 4.476 \\
\hline PPL_07011 & 0.530 & 2.744 & 1.932 & 5.179 & 3.647 \\
\hline PPL 08784 & 6.059 & 31.365 & 24.854 & 5.176 & 4.102 \\
\hline PPL 04534 & 1.205 & 6.234 & 8.013 & 5.173 & 6.650 \\
\hline PPL_02876 & 4.575 & 23.619 & 20.971 & 5.163 & 4.584 \\
\hline PPL 10787 & 0.065 & 0.338 & 0.262 & 5.160 & 4.000 \\
\hline $\mathrm{PPL}^{-} 10224$ & 45.657 & 235.423 & 99.682 & 5.156 & 2.183 \\
\hline PPL 06229 & 14.870 & 76.646 & 36.911 & 5.154 & 2.482 \\
\hline PPL 04874 & 0.156 & 0.805 & 0.723 & 5.152 & 4.629 \\
\hline PPL 09720 & 16.851 & 86.699 & 50.295 & 5.145 & 2.985 \\
\hline PPL_10573 & 0.228 & 1.169 & 1.077 & 5.130 & 4.725 \\
\hline PPL 06757 & 2.076 & 10.643 & 8.876 & 5.126 & 4.275 \\
\hline PPL_09185 & 9.731 & 49.779 & 37.118 & 5.115 & 3.814 \\
\hline PPL_10314 & 13.721 & 69.902 & 28.431 & 5.095 & 2.072 \\
\hline PPL 09719 & 9.205 & 46.861 & 27.383 & 5.091 & 2.975 \\
\hline $\mathrm{PPL} 12368$ & 13.994 & 71.220 & 52.134 & 5.089 & 3.725 \\
\hline PPL 09817 & 12.237 & 62.264 & 37.561 & 5.088 & 3.069 \\
\hline PPL_02685 & 9.522 & 48.375 & 39.918 & 5.080 & 4.192 \\
\hline $\mathrm{PPL} 12497$ & 1.458 & 7.402 & 4.548 & 5.076 & 3.119 \\
\hline PPL_06823 & 0.155 & 0.787 & 0.505 & 5.076 & 3.259 \\
\hline PPL_01948 & 1.121 & 5.678 & 4.510 & 5.066 & 4.024 \\
\hline PPL_03088 & 1.325 & 6.715 & 4.858 & 5.066 & 3.666 \\
\hline $\mathrm{PPL} 11510$ & 6.133 & 31.066 & 36.246 & 5.065 & 5.910 \\
\hline PPL_06044 & 6.263 & 31.669 & 51.009 & 5.057 & 8.145 \\
\hline PPL_00666 & 0.203 & 1.022 & 0.574 & 5.043 & 2.833 \\
\hline $\mathrm{PPL} 00700$ & 0.135 & 0.679 & 0.525 & 5.043 & 3.895 \\
\hline $\mathrm{PPL} 11334$ & 15.386 & 77.490 & 76.715 & 5.036 & 4.986 \\
\hline PPL_05323 & 0.414 & 2.084 & 1.694 & 5.029 & 4.088 \\
\hline PPL_09319 & 6.079 & 30.562 & 27.574 & 5.028 & 4.536 \\
\hline PPL_01699 & 32.299 & 161.921 & 112.480 & 5.013 & 3.482 \\
\hline PPL_03046 & 1.068 & 5.332 & 5.458 & 4.992 & 5.109 \\
\hline PPL_09653 & 4.910 & 24.481 & 20.921 & 4.986 & 4.261 \\
\hline PPL_12279 & 0.325 & 1.620 & 1.032 & 4.985 & 3.174 \\
\hline PPL_01733 & 0.561 & 2.788 & 2.037 & 4.969 & 3.631 \\
\hline PPL_02229 & 1.057 & 5.249 & 4.478 & 4.968 & 4.238 \\
\hline $\mathrm{PPL} 10519$ & 2.401 & 11.874 & 3.764 & 4.945 & 1.567 \\
\hline PPL_07239 & 3.755 & 18.562 & 20.736 & 4.943 & 5.522 \\
\hline PPL_10271 & 7.017 & 34.646 & 32.299 & 4.937 & 4.603 \\
\hline PPL_10588 & 114.068 & 562.760 & 271.753 & 4.934 & 2.382 \\
\hline PPL_01755 & 0.752 & 3.710 & 2.435 & 4.934 & 3.238 \\
\hline PPL_02456 & 0.858 & 4.228 & 3.312 & 4.928 & 3.861 \\
\hline $\mathrm{PPL} 111746$ & 1.182 & 5.818 & 5.496 & 4.921 & 4.649 \\
\hline PPL 01532 & 2.112 & 10.341 & 9.061 & 4.896 & 4.290 \\
\hline PPL_05115 & 3.152 & 15.372 & 10.696 & 4.878 & 3.394 \\
\hline PPL_09751 & 0.644 & 3.138 & 1.666 & 4.876 & 2.589 \\
\hline PPL_05632 & 2.160 & 10.531 & 7.474 & 4.876 & 3.461 \\
\hline $\mathrm{PPL} 08023$ & 1.090 & 5.312 & 3.918 & 4.872 & 3.593 \\
\hline $\mathrm{PPL}=11623$ & 0.275 & 1.340 & 0.746 & 4.864 & 2.706 \\
\hline PPL_01915 & 19.655 & 95.515 & 46.180 & 4.860 & 2.350 \\
\hline PPL_07250 & 0.650 & 3.160 & 1.896 & 4.859 & 2.915 \\
\hline
\end{tabular}




\begin{tabular}{|c|c|c|c|c|c|}
\hline PPL_06369 & 3.952 & 19.160 & 12.466 & 4.848 & 3.154 \\
\hline PPL_09863 & 2.351 & 11.392 & 27.442 & 4.845 & 11.672 \\
\hline PPL_07238 & 1.655 & 8.007 & 7.004 & 4.838 & 4.232 \\
\hline PPL_07880 & 0.120 & 0.583 & 0.249 & 4.837 & 2.069 \\
\hline $\mathrm{PPL} 11094$ & 0.100 & 0.482 & 0.191 & 4.835 & 1.918 \\
\hline PPL 10270 & 18.174 & 87.815 & 74.467 & 4.832 & 4.098 \\
\hline PPL_03912 & 1.636 & 7.890 & 6.019 & 4.823 & 3.679 \\
\hline PPL 04129 & 0.472 & 2.273 & 1.548 & 4.819 & 3.283 \\
\hline PPL_03025 & 11.770 & 56.577 & 51.093 & 4.807 & 4.341 \\
\hline PPL 05842 & 5.015 & 24.086 & 20.518 & 4.803 & 4.091 \\
\hline PPL 04971 & 1.201 & 5.764 & 5.371 & 4.801 & 4.473 \\
\hline PPL 03618 & 1.913 & 9.181 & 3.511 & 4.800 & 1.835 \\
\hline PPL_09426 & 0.083 & 0.400 & 0.625 & 4.796 & 7.505 \\
\hline PPL 03441 & 1.278 & 6.103 & 5.943 & 4.777 & 4.652 \\
\hline PPL_09624 & 0.716 & 3.419 & 2.819 & 4.776 & 3.938 \\
\hline PPL_07912 & 0.244 & 1.163 & 0.960 & 4.773 & 3.939 \\
\hline PPL 07541 & 0.433 & 2.055 & 1.805 & 4.744 & 4.166 \\
\hline PPL_09487 & 62.917 & 298.306 & 343.343 & 4.741 & 5.457 \\
\hline PPL 01134 & 1.809 & 8.575 & 7.419 & 4.739 & 4.100 \\
\hline PPL_07165 & 8.194 & 38.758 & 47.798 & 4.730 & 5.834 \\
\hline PPL 00056 & 6.561 & 30.940 & 27.562 & 4.716 & 4.201 \\
\hline PPL_08594 & 0.280 & 1.323 & 0.838 & 4.716 & 2.990 \\
\hline PPL_11997 & 2.248 & 10.595 & 9.187 & 4.713 & 4.087 \\
\hline PPL_00403 & 5.034 & 23.687 & 21.716 & 4.706 & 4.314 \\
\hline PPL 03582 & 3.386 & 15.911 & 18.236 & 4.700 & 5.386 \\
\hline PPL_11862 & 0.553 & 2.598 & 6.055 & 4.696 & 10.943 \\
\hline PPL_00878 & 0.958 & 4.500 & 2.381 & 4.696 & 2.485 \\
\hline $\mathrm{PPL} 10846$ & 0.051 & 0.240 & 0.256 & 4.695 & 5.002 \\
\hline PPL_06403 & 4.100 & 19.229 & 29.141 & 4.690 & 7.108 \\
\hline PPL_07697 & 0.807 & 3.782 & 2.781 & 4.687 & 3.446 \\
\hline PPL_03325 & 1.659 & 7.774 & 5.552 & 4.685 & 3.346 \\
\hline PPL_11042 & 0.398 & 1.864 & 1.417 & 4.681 & 3.558 \\
\hline PPL_02615 & 0.113 & 0.527 & 0.311 & 4.670 & 2.759 \\
\hline PPL_05849 & 0.453 & 2.112 & 1.168 & 4.659 & 2.576 \\
\hline PPL_06382 & 1.159 & 5.398 & 3.755 & 4.658 & 3.240 \\
\hline PPL_02460 & 1.805 & 8.401 & 8.792 & 4.655 & 4.872 \\
\hline PPL_03826 & 1.053 & 4.894 & 3.232 & 4.649 & 3.069 \\
\hline PPL_10809 & 0.116 & 0.538 & 0.410 & 4.642 & 3.540 \\
\hline PPL_01002 & 1.322 & 6.121 & 5.964 & 4.629 & 4.510 \\
\hline PPL 07045 & 1.707 & 7.890 & 7.624 & 4.622 & 4.466 \\
\hline PPL_10259 & 0.517 & 2.382 & 1.475 & 4.611 & 2.855 \\
\hline PPL_01703 & 0.241 & 1.110 & 0.720 & 4.609 & 2.989 \\
\hline PPL_11984 & 0.508 & 2.342 & 1.375 & 4.606 & 2.705 \\
\hline $\mathrm{PPL}^{\prime} 11893$ & 2.932 & 13.498 & 11.844 & 4.605 & 4.040 \\
\hline PPL 00175 & 1.285 & 5.918 & 4.851 & 4.605 & 3.775 \\
\hline PPL_09251 & 1.037 & 4.758 & 3.937 & 4.589 & 3.797 \\
\hline PPL_08051 & 1.658 & 7.598 & 8.374 & 4.582 & 5.050 \\
\hline $\mathrm{PPL}=11239$ & 3.168 & 14.488 & 12.751 & 4.574 & 4.026 \\
\hline PPL_03316 & 141.314 & 646.147 & 697.645 & 4.572 & 4.937 \\
\hline PPL_10637 & 7.131 & 32.605 & 41.265 & 4.572 & 5.786 \\
\hline PPL_01058 & 0.138 & 0.631 & 0.413 & 4.570 & 2.988 \\
\hline PPL_01295 & 2.165 & 9.894 & 8.230 & 4.570 & 3.802 \\
\hline
\end{tabular}




\begin{tabular}{|c|c|c|c|c|c|}
\hline PPL_00373 & 1.493 & 6.816 & 4.990 & 4.564 & 3.342 \\
\hline $\mathrm{PPL} 04630$ & 7.901 & 36.037 & 36.240 & 4.561 & 4.587 \\
\hline PPL_00383 & 0.296 & 1.351 & 1.439 & 4.561 & 4.858 \\
\hline $\mathrm{PPL} 11671$ & 0.172 & 0.784 & 0.401 & 4.560 & 2.333 \\
\hline PPL 04532 & 1.564 & 7.116 & 3.724 & 4.549 & 2.381 \\
\hline PPL 09289 & 0.824 & 3.746 & 2.389 & 4.546 & 2.900 \\
\hline PPL_06701 & 1.222 & 5.524 & 3.175 & 4.521 & 2.598 \\
\hline PPL 05180 & 19.950 & 89.928 & 54.053 & 4.508 & 2.709 \\
\hline $\mathrm{PPL} 10361$ & 2.059 & 9.254 & 8.856 & 4.495 & 4.301 \\
\hline PPL 06889 & 0.158 & 0.706 & 0.366 & 4.473 & 2.319 \\
\hline PPL 04993 & 0.969 & 4.329 & 6.129 & 4.467 & 6.324 \\
\hline PPL 05073 & 2.937 & 13.112 & 10.764 & 4.464 & 3.665 \\
\hline PPL_03617 & 8.618 & 38.465 & 14.580 & 4.463 & 1.692 \\
\hline $\mathrm{PPL} 05504$ & 6.054 & 27.013 & 20.172 & 4.462 & 3.332 \\
\hline PPL_09798 & 0.169 & 0.756 & 0.537 & 4.459 & 3.170 \\
\hline $\mathrm{PPL} 11700$ & 7.182 & 31.993 & 15.952 & 4.455 & 2.221 \\
\hline PPL 06982 & 6.002 & 26.722 & 19.734 & 4.452 & 3.288 \\
\hline PPL_06790 & 12.817 & 56.914 & 56.179 & 4.440 & 4.383 \\
\hline PPL 01263 & 2.894 & 12.798 & 10.303 & 4.423 & 3.561 \\
\hline PPL_07868 & 22.214 & 97.833 & 76.653 & 4.404 & 3.451 \\
\hline PPL 08018 & 7.613 & 33.522 & 35.597 & 4.403 & 4.676 \\
\hline PPL_02033 & 5.178 & 22.792 & 7.704 & 4.401 & 1.488 \\
\hline $\mathrm{PPL} 11972$ & 0.404 & 1.777 & 1.474 & 4.397 & 3.645 \\
\hline $\mathrm{PPL} 11273$ & 7.903 & 34.741 & 43.643 & 4.396 & 5.523 \\
\hline PPL 09904 & 2.015 & 8.853 & 5.838 & 4.394 & 2.897 \\
\hline PPL_12309 & 9.751 & 42.845 & 35.697 & 4.394 & 3.661 \\
\hline PPL_04374 & 0.053 & 0.234 & 0.188 & 4.392 & 3.533 \\
\hline $\mathrm{PPL} 04464$ & 0.210 & 0.923 & 0.914 & 4.391 & 4.348 \\
\hline $\mathrm{PPL} 12621$ & 0.228 & 0.998 & 0.720 & 4.383 & 3.161 \\
\hline PPL_11324 & 0.359 & 1.571 & 0.874 & 4.380 & 2.437 \\
\hline PPL_07124 & 72.274 & 316.065 & 86.672 & 4.373 & 1.199 \\
\hline PPL_07479 & 6.130 & 26.782 & 27.195 & 4.369 & 4.436 \\
\hline PPL_06125 & 2.703 & 11.781 & 8.381 & 4.358 & 3.100 \\
\hline PPL_01337 & 12.909 & 56.208 & 41.369 & 4.354 & 3.205 \\
\hline PPL_07157 & 15.520 & 67.452 & 46.283 & 4.346 & 2.982 \\
\hline PPL_05618 & 0.288 & 1.250 & 0.960 & 4.342 & 3.335 \\
\hline PPL_08699 & 8.049 & 34.907 & 25.605 & 4.337 & 3.181 \\
\hline PPL_09200 & 4.853 & 21.025 & 19.674 & 4.332 & 4.054 \\
\hline PPL_09815 & 2.307 & 9.960 & 7.533 & 4.318 & 3.266 \\
\hline PPL_10688 & 1.026 & 4.430 & 3.612 & 4.317 & 3.520 \\
\hline PPL_00856 & 0.165 & 0.711 & 1.704 & 4.316 & 10.346 \\
\hline PPL_11974 & 0.464 & 1.991 & 1.590 & 4.292 & 3.427 \\
\hline PPL_12065 & 0.349 & 1.496 & 1.046 & 4.290 & 3.001 \\
\hline PPL_01343 & 0.071 & 0.306 & 0.230 & 4.288 & 3.219 \\
\hline PPL 03778 & 6.834 & 29.263 & 23.889 & 4.282 & 3.495 \\
\hline PPL_09261 & 0.346 & 1.480 & 1.089 & 4.274 & 3.145 \\
\hline $\mathrm{PPL}^{-} 12472$ & 3.463 & 14.795 & 12.030 & 4.272 & 3.474 \\
\hline PPL_08313 & 3.936 & 16.810 & 13.414 & 4.271 & 3.408 \\
\hline PPL 07478 & 9.573 & 40.874 & 67.774 & 4.270 & 7.080 \\
\hline PPL_04912 & 25.357 & 108.108 & 109.686 & 4.264 & 4.326 \\
\hline PPL_06010 & 14.746 & 62.723 & 47.017 & 4.254 & 3.188 \\
\hline PPL_10463 & 0.358 & 1.523 & 0.636 & 4.253 & 1.776 \\
\hline
\end{tabular}




\begin{tabular}{|c|c|c|c|c|c|}
\hline PPL_10747 & 0.830 & 3.529 & 3.269 & 4.252 & 3.938 \\
\hline PPL_01493 & 1.607 & 6.833 & 5.466 & 4.251 & 3.401 \\
\hline PPL 10267 & 3.537 & 15.014 & 21.533 & 4.245 & 6.088 \\
\hline PPL_07009 & 2.601 & 11.042 & 6.658 & 4.245 & 2.560 \\
\hline PPL_02444 & 2.604 & 11.049 & 16.167 & 4.244 & 6.210 \\
\hline PPL_01540 & 10.816 & 45.905 & 8.712 & 4.244 & 0.806 \\
\hline PPL_00090 & 0.220 & 0.933 & 0.763 & 4.238 & 3.466 \\
\hline PPL_02316 & 13.347 & 56.508 & 23.270 & 4.234 & 1.743 \\
\hline PPL 04742 & 0.232 & 0.983 & 0.349 & 4.229 & 1.500 \\
\hline PPL_02948 & 1.180 & 4.989 & 4.304 & 4.227 & 3.647 \\
\hline PPL_05771 & 1.587 & 6.704 & 5.567 & 4.225 & 3.508 \\
\hline PPL 09222 & 9.921 & 41.900 & 38.199 & 4.223 & 3.850 \\
\hline PPL_03766 & 0.993 & 4.190 & 3.499 & 4.221 & 3.524 \\
\hline PPL_09327 & 0.522 & 2.203 & 1.811 & 4.220 & 3.469 \\
\hline PPL_09067 & 0.582 & 2.446 & 1.866 & 4.205 & 3.208 \\
\hline PPL_05159 & 2.697 & 11.317 & 7.840 & 4.197 & 2.907 \\
\hline $\mathrm{PPL} 11946$ & 0.515 & 2.160 & 2.016 & 4.195 & 3.916 \\
\hline PPL_04704 & 4.991 & 20.932 & 13.047 & 4.194 & 2.614 \\
\hline $\mathrm{PPL} 11906$ & 17.921 & 75.061 & 65.427 & 4.189 & 3.651 \\
\hline $\mathrm{PPL}_{-} 11069$ & 1.683 & 7.040 & 5.739 & 4.183 & 3.410 \\
\hline PPL_08477 & 7.825 & 32.677 & 50.321 & 4.176 & 6.431 \\
\hline PPL_02106 & 5.624 & 23.483 & 12.074 & 4.176 & 2.147 \\
\hline $\mathrm{PPL}^{-} 12471$ & 14.636 & 61.066 & 45.197 & 4.172 & 3.088 \\
\hline PPL_05462 & 142.513 & 594.355 & 679.916 & 4.171 & 4.771 \\
\hline PPL_10893 & 1.897 & 7.911 & 6.301 & 4.169 & 3.321 \\
\hline PPL_06760 & 0.778 & 3.243 & 3.978 & 4.169 & 5.115 \\
\hline PPL_09774 & 0.098 & 0.408 & 0.429 & 4.168 & 4.381 \\
\hline PPL_01657 & 0.093 & 0.388 & 0.220 & 4.159 & 2.354 \\
\hline PPL_02105 & 148.051 & 615.473 & 488.877 & 4.157 & 3.302 \\
\hline PPL_05760 & 6.582 & 27.317 & 31.378 & 4.150 & 4.767 \\
\hline PPL_05171 & 27.009 & 111.901 & 40.463 & 4.143 & 1.498 \\
\hline PPL_07774 & 0.372 & 1.539 & 1.506 & 4.142 & 4.053 \\
\hline PPL_06343 & 0.643 & 2.664 & 2.316 & 4.140 & 3.599 \\
\hline PPL_01978 & 1.666 & 6.890 & 5.374 & 4.135 & 3.225 \\
\hline PPL_03722 & 2.532 & 10.466 & 7.586 & 4.133 & 2.996 \\
\hline PPL_10628 & 1.384 & 5.719 & 4.823 & 4.133 & 3.486 \\
\hline PPL_00615 & 0.078 & 0.321 & 0.506 & 4.128 & 6.502 \\
\hline PPL_01920 & 2.079 & 8.579 & 6.627 & 4.127 & 3.188 \\
\hline PPL_00650 & 2.931 & 12.060 & 11.059 & 4.115 & 3.774 \\
\hline PPL_01609 & 2.278 & 9.364 & 6.076 & 4.111 & 2.668 \\
\hline PPL_05043 & 0.827 & 3.400 & 2.771 & 4.110 & 3.350 \\
\hline PPL_05593 & 1.960 & 8.038 & 7.534 & 4.101 & 3.844 \\
\hline PPL_02319 & 5.709 & 23.377 & 23.955 & 4.095 & 4.196 \\
\hline PPL 03563 & 0.369 & 1.510 & 1.396 & 4.091 & 3.782 \\
\hline PPL_09526 & 0.384 & 1.568 & 1.212 & 4.087 & 3.159 \\
\hline PPL_07630 & 1.055 & 4.304 & 5.420 & 4.081 & 5.139 \\
\hline PPL_11382 & 1.328 & 5.407 & 6.978 & 4.071 & 5.254 \\
\hline PPL 02487 & 4.123 & 16.772 & 13.595 & 4.068 & 3.297 \\
\hline PPL_09971 & 5.097 & 20.731 & 16.562 & 4.067 & 3.249 \\
\hline $\mathrm{PPL} 11099$ & 2.054 & 8.337 & 6.970 & 4.060 & 3.394 \\
\hline $\mathrm{PPL}=11778$ & 0.601 & 2.438 & 1.658 & 4.058 & 2.760 \\
\hline PPL_01610 & 5.309 & 21.526 & 19.624 & 4.055 & 3.696 \\
\hline
\end{tabular}




\begin{tabular}{|c|c|c|c|c|c|}
\hline PPL_04402 & 0.359 & 1.453 & 1.055 & 4.043 & 2.934 \\
\hline $\mathrm{PPL} 02947$ & 1.201 & 4.852 & 3.903 & 4.041 & 3.250 \\
\hline PPL_12586 & 0.141 & 0.571 & 0.384 & 4.036 & 2.717 \\
\hline PPL 12224 & 144.267 & 581.429 & 679.912 & 4.030 & 4.713 \\
\hline PPL 04955 & 0.414 & 1.667 & 1.488 & 4.029 & 3.595 \\
\hline PPL 00903 & 0.397 & 1.599 & 0.500 & 4.028 & 1.260 \\
\hline $\mathrm{PPL} 11598$ & 37.083 & 149.230 & 77.682 & 4.024 & 2.095 \\
\hline PPL 08197 & 0.311 & 1.249 & 1.626 & 4.021 & 5.234 \\
\hline $\mathrm{PPL} 10765$ & 27.920 & 112.175 & 52.052 & 4.018 & 1.864 \\
\hline PPL 11396 & 1.227 & 4.923 & 4.534 & 4.013 & 3.696 \\
\hline PPL 07389 & 1.111 & 4.457 & 2.893 & 4.013 & 2.605 \\
\hline PPL 01596 & 4.572 & 18.306 & 15.516 & 4.004 & 3.394 \\
\hline PPL_02478 & 5.575 & 22.309 & 11.744 & 4.002 & 2.107 \\
\hline PPL 03001 & 3.264 & 13.043 & 14.816 & 3.996 & 4.539 \\
\hline PPL_02736 & 1.355 & 5.416 & 3.658 & 3.996 & 2.699 \\
\hline PPL_10562 & 4.384 & 17.509 & 13.905 & 3.994 & 3.172 \\
\hline PPL 09637 & 2.307 & 9.200 & 3.662 & 3.989 & 1.588 \\
\hline PPL_05074 & 6.057 & 24.130 & 21.405 & 3.984 & 3.534 \\
\hline PPL 05491 & 0.274 & 1.091 & 0.441 & 3.981 & 1.609 \\
\hline PPL_04261 & 0.891 & 3.543 & 3.651 & 3.975 & 4.095 \\
\hline PPL 12367 & 5.009 & 19.911 & 18.489 & 3.975 & 3.691 \\
\hline PPL_06209 & 23.416 & 93.080 & 128.624 & 3.975 & 5.493 \\
\hline PPL_01093 & 11.988 & 47.614 & 49.035 & 3.972 & 4.090 \\
\hline PPL_04721 & 6.233 & 24.745 & 19.904 & 3.970 & 3.193 \\
\hline PPL 11894 & 15.465 & 61.370 & 58.128 & 3.968 & 3.759 \\
\hline PPL_07922 & 6.324 & 25.082 & 13.887 & 3.966 & 2.196 \\
\hline PPL_08310 & 2.438 & 9.658 & 7.919 & 3.961 & 3.248 \\
\hline $\mathrm{PPL} 12316$ & 0.591 & 2.339 & 2.222 & 3.957 & 3.760 \\
\hline PPL_00876 & 0.643 & 2.544 & 2.406 & 3.955 & 3.741 \\
\hline PPL_03476 & 40.402 & 159.610 & 56.185 & 3.951 & 1.391 \\
\hline $\mathrm{PPL}_{-} 11509$ & 4.065 & 16.051 & 14.303 & 3.949 & 3.519 \\
\hline PPL_07670 & 19.697 & 77.764 & 80.870 & 3.948 & 4.106 \\
\hline PPL_01573 & 5.419 & 21.391 & 19.540 & 3.947 & 3.606 \\
\hline PPL_06961 & 11.440 & 45.156 & 27.371 & 3.947 & 2.393 \\
\hline PPL_08052 & 0.315 & 1.239 & 0.858 & 3.939 & 2.728 \\
\hline PPL_05012 & 22.964 & 90.386 & 80.816 & 3.936 & 3.519 \\
\hline PPL_02046 & 18.554 & 72.891 & 143.320 & 3.929 & 7.724 \\
\hline PPL_09081 & 1.050 & 4.121 & 3.041 & 3.925 & 2.896 \\
\hline PPL_10119 & 3.380 & 13.264 & 10.193 & 3.925 & 3.016 \\
\hline PPL_03053 & 14.664 & 57.548 & 58.483 & 3.925 & 3.988 \\
\hline PPL_08668 & 12.032 & 47.213 & 16.197 & 3.924 & 1.346 \\
\hline PPL_04938 & 0.223 & 0.876 & 0.627 & 3.922 & 2.806 \\
\hline PPL_06153 & 0.939 & 3.682 & 1.813 & 3.920 & 1.930 \\
\hline PPL_06116 & 4.826 & 18.915 & 19.131 & 3.920 & 3.964 \\
\hline PPL 06393 & 0.305 & 1.193 & 1.346 & 3.915 & 4.417 \\
\hline PPL_08353 & 0.123 & 0.482 & 0.349 & 3.911 & 2.832 \\
\hline PPL_04418 & 0.486 & 1.899 & 2.234 & 3.911 & 4.601 \\
\hline PPL_01907 & 0.397 & 1.553 & 1.252 & 3.911 & 3.152 \\
\hline PPL 05998 & 2.106 & 8.232 & 8.296 & 3.908 & 3.938 \\
\hline PPL_05584 & 15.002 & 58.570 & 58.570 & 3.904 & 3.904 \\
\hline PPL_03971 & 1.135 & 4.427 & 4.010 & 3.901 & 3.534 \\
\hline PPL_07688 & 1.907 & 7.437 & 7.027 & 3.900 & 3.685 \\
\hline
\end{tabular}




\begin{tabular}{|c|c|c|c|c|c|}
\hline PPL_00229 & 0.319 & 1.246 & 1.282 & 3.900 & 4.012 \\
\hline $\mathrm{PPL} 12361$ & 19.627 & 76.501 & 55.975 & 3.898 & 2.852 \\
\hline PPL_03616 & 12.420 & 48.343 & 19.610 & 3.892 & 1.579 \\
\hline PPL_09169 & 0.858 & 3.339 & 2.113 & 3.892 & 2.463 \\
\hline PPL 10789 & 0.255 & 0.990 & 0.448 & 3.881 & 1.756 \\
\hline PPL 06318 & 2.723 & 10.565 & 12.107 & 3.880 & 4.446 \\
\hline $\mathrm{PPL} 01516$ & 5.148 & 19.971 & 16.715 & 3.880 & 3.247 \\
\hline $\mathrm{PPL} 08475$ & 1.633 & 6.333 & 10.241 & 3.879 & 6.272 \\
\hline PPL_02944 & 2.329 & 9.033 & 6.943 & 3.878 & 2.980 \\
\hline $\mathrm{PPL} 06311$ & 594.066 & 2302.449 & 1414.487 & 3.876 & 2.381 \\
\hline $\mathrm{PPL} 11519$ & 6.804 & 26.331 & 35.926 & 3.870 & 5.280 \\
\hline PPL 01274 & 5.208 & 20.137 & 16.022 & 3.866 & 3.076 \\
\hline PPL_07778 & 0.852 & 3.284 & 1.723 & 3.854 & 2.022 \\
\hline PPL 03028 & 0.390 & 1.502 & 0.192 & 3.854 & 0.493 \\
\hline PPL_08166 & 64.029 & 246.311 & 230.957 & 3.847 & 3.607 \\
\hline PPL_10268 & 2.233 & 8.585 & 6.751 & 3.844 & 3.023 \\
\hline PPL 05062 & 0.190 & 0.731 & 0.687 & 3.842 & 3.613 \\
\hline PPL_02171 & 0.327 & 1.255 & 1.122 & 3.840 & 3.434 \\
\hline PPL 03464 & 1.068 & 4.097 & 3.681 & 3.835 & 3.445 \\
\hline PPL_00065 & 1107.776 & 4243.324 & 3878.237 & 3.830 & 3.501 \\
\hline PPL 00720 & 0.062 & 0.236 & 0.219 & 3.827 & 3.537 \\
\hline PPL_00873 & 2.364 & 9.045 & 4.823 & 3.826 & 2.040 \\
\hline PPL_07890 & 0.219 & 0.839 & 2.461 & 3.825 & 11.220 \\
\hline $\mathrm{PPL} 12512$ & 1.111 & 4.247 & 3.119 & 3.824 & 2.808 \\
\hline PPL 08444 & 0.268 & 1.024 & 0.827 & 3.821 & 3.087 \\
\hline PPL_04396 & 0.423 & 1.616 & 1.269 & 3.820 & 3.000 \\
\hline PPL_08641 & 2.649 & 10.109 & 14.641 & 3.816 & 5.528 \\
\hline $\mathrm{PPL} 09663$ & 6.149 & 23.462 & 15.080 & 3.815 & 2.452 \\
\hline $\mathrm{PPL} 12435$ & 4.828 & 18.416 & 18.643 & 3.814 & 3.861 \\
\hline PPL_08745 & 2.327 & 8.870 & 10.274 & 3.811 & 4.415 \\
\hline PPL_03885 & 1.373 & 5.234 & 3.420 & 3.811 & 2.491 \\
\hline PPL_03784 & 0.270 & 1.026 & 1.176 & 3.806 & 4.363 \\
\hline PPL_12329 & 1.614 & 6.137 & 5.894 & 3.802 & 3.651 \\
\hline PPL_06761 & 9.633 & 36.558 & 41.699 & 3.795 & 4.329 \\
\hline PPL_03090 & 6.352 & 24.072 & 18.973 & 3.789 & 2.987 \\
\hline PPL_04162 & 0.813 & 3.078 & 3.363 & 3.787 & 4.137 \\
\hline PPL_07674 & 4.190 & 15.864 & 14.925 & 3.786 & 3.562 \\
\hline PPL_04223 & 0.633 & 2.391 & 2.160 & 3.780 & 3.416 \\
\hline PPL_00073 & 1.655 & 6.254 & 4.955 & 3.779 & 2.994 \\
\hline PPL_08640 & 2.595 & 9.788 & 8.234 & 3.772 & 3.173 \\
\hline PPL_09773 & 0.295 & 1.113 & 0.881 & 3.769 & 2.983 \\
\hline PPL_11397 & 0.155 & 0.585 & 0.357 & 3.767 & 2.299 \\
\hline PPL_06886 & 1.577 & 5.938 & 5.581 & 3.766 & 3.540 \\
\hline PPL_08645 & 15.975 & 60.154 & 54.364 & 3.765 & 3.403 \\
\hline PPL 08793 & 2.009 & 7.561 & 5.487 & 3.764 & 2.732 \\
\hline PPL_06691 & 2.689 & 10.122 & 11.747 & 3.764 & 4.368 \\
\hline PPL_00718 & 0.903 & 3.399 & 2.949 & 3.764 & 3.266 \\
\hline PPL_09537 & 2.028 & 7.623 & 3.277 & 3.758 & 1.616 \\
\hline PPL 09182 & 2.930 & 11.001 & 13.423 & 3.754 & 4.581 \\
\hline PPL_07149 & 0.702 & 2.635 & 2.684 & 3.751 & 3.822 \\
\hline $\mathrm{PPL} 11066$ & 9.245 & 34.669 & 22.027 & 3.750 & 2.382 \\
\hline PPL_01911 & 2.180 & 8.171 & 5.828 & 3.749 & 2.674 \\
\hline
\end{tabular}




\begin{tabular}{|c|c|c|c|c|c|}
\hline PPL_00922 & 0.071 & 0.267 & 0.225 & 3.734 & 3.149 \\
\hline $\mathrm{PPL} 08012$ & 0.073 & 0.272 & 0.183 & 3.734 & 2.520 \\
\hline PPL_00968 & 0.084 & 0.313 & 0.527 & 3.734 & 6.301 \\
\hline $\mathrm{PPL} 11820$ & 4.543 & 16.938 & 19.878 & 3.728 & 4.376 \\
\hline PPL 01257 & 1.603 & 5.968 & 4.721 & 3.724 & 2.946 \\
\hline PPL 07002 & 12.497 & 46.517 & 38.001 & 3.722 & 3.041 \\
\hline PPL_05003 & 8.928 & 33.198 & 18.415 & 3.718 & 2.063 \\
\hline PPL 07477 & 5.515 & 20.495 & 15.975 & 3.716 & 2.897 \\
\hline PPL_09742 & 8.958 & 33.281 & 28.305 & 3.715 & 3.160 \\
\hline PPL 02297 & 1.244 & 4.622 & 3.360 & 3.714 & 2.700 \\
\hline $\mathrm{PPL} 10709$ & 0.861 & 3.196 & 2.555 & 3.714 & 2.969 \\
\hline PPL 08108 & 4.380 & 16.256 & 9.102 & 3.712 & 2.078 \\
\hline PPL_04419 & 0.665 & 2.463 & 1.329 & 3.707 & 2.000 \\
\hline PPL 00961 & 1.149 & 4.259 & 3.030 & 3.707 & 2.637 \\
\hline PPL_04933 & 0.458 & 1.695 & 1.248 & 3.703 & 2.725 \\
\hline PPL_10815 & 11.563 & 42.722 & 47.855 & 3.695 & 4.139 \\
\hline PPL 10323 & 0.348 & 1.282 & 1.241 & 3.690 & 3.569 \\
\hline PPL_08589 & 0.285 & 1.052 & 0.881 & 3.689 & 3.089 \\
\hline $\mathrm{PPL} 12293$ & 7.592 & 28.002 & 24.701 & 3.688 & 3.254 \\
\hline $\mathrm{PPL}_{-} 11105$ & 56.649 & 208.873 & 173.776 & 3.687 & 3.068 \\
\hline PPL 02191 & 4.222 & 15.560 & 16.677 & 3.686 & 3.950 \\
\hline PPL_07932 & 8.235 & 30.350 & 33.562 & 3.686 & 4.076 \\
\hline PPL_07214 & 0.433 & 1.594 & 1.511 & 3.683 & 3.491 \\
\hline PPL_00656 & 0.618 & 2.275 & 1.651 & 3.683 & 2.673 \\
\hline PPL 06800 & 0.875 & 3.221 & 2.603 & 3.682 & 2.976 \\
\hline PPL_01192 & 7.375 & 27.131 & 20.464 & 3.679 & 2.775 \\
\hline $\mathrm{PPL} 10535$ & 24.831 & 91.272 & 85.423 & 3.676 & 3.440 \\
\hline $\mathrm{PPL} 02025$ & 1.948 & 7.157 & 5.094 & 3.674 & 2.615 \\
\hline PPL_06895 & 0.327 & 1.202 & 0.980 & 3.671 & 2.993 \\
\hline PPL_09472 & 0.094 & 0.344 & 0.340 & 3.671 & 3.624 \\
\hline PPL_01779 & 20.836 & 76.477 & 34.929 & 3.670 & 1.676 \\
\hline PPL_01045 & 10.686 & 39.181 & 45.935 & 3.667 & 4.299 \\
\hline PPL_07345 & 4.215 & 15.452 & 16.196 & 3.666 & 3.842 \\
\hline PPL_01811 & 7.500 & 27.487 & 19.715 & 3.665 & 2.629 \\
\hline PPL_08189 & 5.244 & 19.218 & 14.464 & 3.664 & 2.758 \\
\hline PPL_09150 & 2.467 & 9.032 & 4.876 & 3.661 & 1.976 \\
\hline PPL_02258 & 1.360 & 4.978 & 5.528 & 3.661 & 4.065 \\
\hline PPL_01821 & 0.777 & 2.841 & 2.103 & 3.658 & 2.708 \\
\hline $\mathrm{PPL}=12460$ & 3.289 & 12.024 & 11.270 & 3.656 & 3.427 \\
\hline PPL_08168 & 2.599 & 9.485 & 12.138 & 3.650 & 4.670 \\
\hline PPL_07223 & 37.207 & 135.788 & 50.895 & 3.650 & 1.368 \\
\hline PPL_01541 & 69.451 & 253.346 & 223.146 & 3.648 & 3.213 \\
\hline PPL_01662 & 5.043 & 18.383 & 26.757 & 3.646 & 5.306 \\
\hline PPL_06270 & 0.603 & 2.196 & 0.851 & 3.641 & 1.410 \\
\hline $\mathrm{PPL} 12185$ & 1.282 & 4.662 & 3.754 & 3.636 & 2.927 \\
\hline PPL_01858 & 71.886 & 261.378 & 376.187 & 3.636 & 5.233 \\
\hline PPL_02690 & 10.775 & 39.167 & 45.989 & 3.635 & 4.268 \\
\hline PPL_04019 & 2.748 & 9.989 & 8.255 & 3.635 & 3.004 \\
\hline PPL 04535 & 5.439 & 19.733 & 15.999 & 3.628 & 2.941 \\
\hline PPL_09202 & 0.279 & 1.011 & 1.039 & 3.626 & 3.728 \\
\hline PPL_02584 & 0.723 & 2.621 & 1.186 & 3.625 & 1.640 \\
\hline PPL_00819 & 3.439 & 12.461 & 11.126 & 3.624 & 3.236 \\
\hline
\end{tabular}




\begin{tabular}{|c|c|c|c|c|c|}
\hline PPL_02544 & 0.368 & 1.335 & 1.272 & 3.623 & 3.451 \\
\hline PPL_08862 & 0.291 & 1.053 & 0.688 & 3.620 & 2.366 \\
\hline PPL 00360 & 0.935 & 3.384 & 3.092 & 3.619 & 3.307 \\
\hline PPL_09365 & 1.435 & 5.191 & 4.900 & 3.617 & 3.414 \\
\hline PPL_07207 & 15.235 & 55.083 & 79.112 & 3.615 & 5.193 \\
\hline PPL_02027 & 2.849 & 10.291 & 8.844 & 3.612 & 3.104 \\
\hline PPL_09046 & 20.164 & 72.641 & 82.660 & 3.602 & 4.099 \\
\hline PPL_04213 & 5.601 & 20.168 & 13.144 & 3.601 & 2.347 \\
\hline PPL_02235 & 4.038 & 14.517 & 17.005 & 3.595 & 4.211 \\
\hline PPL_12390 & 9.496 & 34.144 & 31.312 & 3.595 & 3.297 \\
\hline PPL_03753 & 12.232 & 43.940 & 37.051 & 3.592 & 3.029 \\
\hline PPL 04781 & 16.158 & 57.965 & 31.210 & 3.587 & 1.932 \\
\hline PPL_12570 & 15.278 & 54.776 & 47.990 & 3.585 & 3.141 \\
\hline PPL_06074 & 0.110 & 0.395 & 0.300 & 3.581 & 2.715 \\
\hline PPL_05682 & 1.714 & 6.119 & 5.595 & 3.570 & 3.264 \\
\hline PPL_00316 & 9.698 & 34.620 & 30.484 & 3.570 & 3.143 \\
\hline PPL 01170 & 1.344 & 4.796 & 3.747 & 3.567 & 2.787 \\
\hline PPL_10693 & 0.517 & 1.844 & 0.970 & 3.564 & 1.875 \\
\hline PPL_03956 & 5.716 & 20.349 & 20.308 & 3.560 & 3.553 \\
\hline PPL_07557 & 2.739 & 9.746 & 2.833 & 3.559 & 1.034 \\
\hline PPL 00025 & 0.134 & 0.475 & 0.368 & 3.557 & 2.755 \\
\hline PPL_09053 & 0.735 & 2.613 & 2.631 & 3.557 & 3.582 \\
\hline PPL_05710 & 4.933 & 17.549 & 9.768 & 3.557 & 1.980 \\
\hline $\mathrm{PPL} 111119$ & 42.643 & 151.539 & 165.318 & 3.554 & 3.877 \\
\hline $\mathrm{PPL} 10154$ & 0.796 & 2.825 & 2.602 & 3.549 & 3.268 \\
\hline PPL_01560 & 12.677 & 44.959 & 50.166 & 3.546 & 3.957 \\
\hline PPL_05963 & 10.326 & 36.562 & 21.497 & 3.541 & 2.082 \\
\hline PPL_09131 & 7.843 & 27.740 & 22.732 & 3.537 & 2.898 \\
\hline $\mathrm{PPL} 111663$ & 0.082 & 0.289 & 0.353 & 3.533 & 4.314 \\
\hline PPL 03233 & 4.814 & 16.967 & 15.222 & 3.525 & 3.162 \\
\hline PPL_02952 & 3.618 & 12.736 & 12.974 & 3.520 & 3.586 \\
\hline PPL_08752 & 49.993 & 175.793 & 80.167 & 3.516 & 1.604 \\
\hline PPL_10299 & 1.551 & 5.451 & 2.618 & 3.514 & 1.687 \\
\hline PPL_09769 & 0.774 & 2.720 & 1.438 & 3.514 & 1.858 \\
\hline PPL 11208 & 15.705 & 54.972 & 63.982 & 3.500 & 4.074 \\
\hline PPL_04752 & 0.367 & 1.283 & 1.751 & 3.500 & 4.777 \\
\hline PPL_08836 & 0.197 & 0.688 & 0.825 & 3.486 & 4.183 \\
\hline PPL_05659 & 0.513 & 1.787 & 1.233 & 3.481 & 2.402 \\
\hline PPL 05029 & 1.144 & 3.968 & 5.244 & 3.469 & 4.584 \\
\hline PPL_07451 & 1.085 & 3.762 & 3.776 & 3.468 & 3.481 \\
\hline PPL_03774 & 1.534 & 5.314 & 4.174 & 3.463 & 2.720 \\
\hline PPL_04443 & 5.221 & 18.036 & 11.583 & 3.455 & 2.219 \\
\hline PPL_02786 & 440.372 & 1521.141 & 1132.616 & 3.454 & 2.572 \\
\hline PPL 09607 & 1.526 & 5.267 & 4.028 & 3.452 & 2.640 \\
\hline PPL_09448 & 46.686 & 161.064 & 80.356 & 3.450 & 1.721 \\
\hline PPL_10057 & 12.880 & 44.383 & 31.356 & 3.446 & 2.434 \\
\hline PPL 05502 & 6.182 & 21.301 & 15.724 & 3.446 & 2.544 \\
\hline PPL 07855 & 66.108 & 227.600 & 261.176 & 3.443 & 3.951 \\
\hline PPL_04697 & 1.044 & 3.592 & 2.073 & 3.440 & 1.986 \\
\hline PPL_05363 & 9.251 & 31.817 & 23.949 & 3.439 & 2.589 \\
\hline PPL_05546 & 1.036 & 3.561 & 1.955 & 3.437 & 1.886 \\
\hline PPL_07946 & 10.041 & 34.480 & 40.139 & 3.434 & 3.998 \\
\hline
\end{tabular}




\begin{tabular}{|c|c|c|c|c|c|}
\hline PPL_05453 & 2.215 & 7.603 & 6.404 & 3.432 & 2.891 \\
\hline PPL_07399 & 2.559 & 8.780 & 5.539 & 3.431 & 2.164 \\
\hline PPL 01064 & 3.762 & 12.893 & 5.355 & 3.427 & 1.423 \\
\hline PPL 03355 & 5.415 & 18.554 & 21.183 & 3.427 & 3.912 \\
\hline PPL_03224 & 0.537 & 1.840 & 1.324 & 3.426 & 2.465 \\
\hline PPL_03173 & 6.593 & 22.496 & 19.062 & 3.412 & 2.891 \\
\hline PPL_01845 & 0.739 & 2.521 & 1.968 & 3.411 & 2.663 \\
\hline PPL 09727 & 2.219 & 7.546 & 6.978 & 3.401 & 3.145 \\
\hline PPL_03951 & 7.031 & 23.840 & 28.073 & 3.391 & 3.993 \\
\hline PPL_06054 & 14.487 & 49.050 & 48.939 & 3.386 & 3.378 \\
\hline PPL_09170 & 0.913 & 3.087 & 2.334 & 3.380 & 2.556 \\
\hline PPL 05274 & 3.212 & 10.856 & 8.890 & 3.380 & 2.768 \\
\hline PPL_09462 & 0.704 & 2.374 & 1.898 & 3.372 & 2.695 \\
\hline $\mathrm{PPL} 11260$ & 39.498 & 132.789 & 140.239 & 3.362 & 3.551 \\
\hline PPL_09091 & 2.289 & 7.692 & 6.283 & 3.360 & 2.744 \\
\hline PPL_05357 & 2.354 & 7.902 & 6.870 & 3.357 & 2.918 \\
\hline $\mathrm{PPL} 11717$ & 36.916 & 123.183 & 78.201 & 3.337 & 2.118 \\
\hline PPL_10380 & 0.726 & 2.422 & 3.211 & 3.335 & 4.422 \\
\hline PPL_10721 & 81.588 & 271.376 & 220.033 & 3.326 & 2.697 \\
\hline $\mathrm{PPL}_{-} 11463$ & 6.736 & 22.312 & 15.094 & 3.312 & 2.241 \\
\hline PPL 03897 & 16.230 & 53.662 & 34.445 & 3.306 & 2.122 \\
\hline PPL_10120 & 0.536 & 1.770 & 1.228 & 3.305 & 2.292 \\
\hline PPL_02015 & 13.649 & 45.099 & 59.495 & 3.304 & 4.359 \\
\hline PPL_07406 & 0.738 & 2.439 & 2.645 & 3.304 & 3.583 \\
\hline PPL_02743 & 1.194 & 3.945 & 3.674 & 3.303 & 3.076 \\
\hline PPL 01512 & 1.314 & 4.339 & 3.013 & 3.302 & 2.293 \\
\hline PPL_01555 & 8.376 & 27.652 & 20.472 & 3.301 & 2.444 \\
\hline PPL_05439 & 3.546 & 11.689 & 8.321 & 3.297 & 2.347 \\
\hline PPL_03411 & 0.584 & 1.925 & 1.355 & 3.297 & 2.321 \\
\hline PPL 09477 & 0.589 & 1.942 & 1.382 & 3.297 & 2.346 \\
\hline PPL_01818 & 5.201 & 17.142 & 14.029 & 3.296 & 2.697 \\
\hline PPL_03163 & 1.666 & 5.487 & 4.397 & 3.294 & 2.640 \\
\hline PPL_04195 & 69.570 & 229.084 & 320.783 & 3.293 & 4.611 \\
\hline PPL_07143 & 3.425 & 11.273 & 13.508 & 3.291 & 3.944 \\
\hline PPL 10854 & 5.396 & 17.733 & 17.693 & 3.286 & 3.279 \\
\hline $\mathrm{PPL} 11168$ & 0.394 & 1.294 & 1.071 & 3.281 & 2.716 \\
\hline PPL_09290 & 10.537 & 34.538 & 21.737 & 3.278 & 2.063 \\
\hline PPL_07659 & 8.538 & 27.914 & 53.532 & 3.269 & 6.270 \\
\hline PPL 00182 & 13.161 & 42.973 & 27.071 & 3.265 & 2.057 \\
\hline PPL_00786 & 0.535 & 1.747 & 1.170 & 3.265 & 2.186 \\
\hline PPL_03112 & 1.734 & 5.660 & 6.148 & 3.265 & 3.546 \\
\hline PPL_04262 & 76.863 & 250.901 & 333.020 & 3.264 & 4.333 \\
\hline $\mathrm{PPL} 10652$ & 2.387 & 7.775 & 6.330 & 3.257 & 2.652 \\
\hline PPL 03188 & 4.456 & 14.511 & 10.165 & 3.256 & 2.281 \\
\hline PPL_01530 & 0.919 & 2.987 & 2.687 & 3.250 & 2.924 \\
\hline PPL_00277 & 12.384 & 40.204 & 22.967 & 3.246 & 1.855 \\
\hline PPL 08557 & 1.261 & 4.082 & 3.261 & 3.238 & 2.586 \\
\hline PPL 01525 & 1.205 & 3.898 & 3.065 & 3.236 & 2.544 \\
\hline PPL_01389 & 0.690 & 2.230 & 1.651 & 3.233 & 2.394 \\
\hline PPL_00356 & 3.644 & 11.740 & 8.842 & 3.222 & 2.426 \\
\hline PPL_07412 & 3.494 & 11.239 & 8.676 & 3.217 & 2.483 \\
\hline PPL_05829 & 20.288 & 65.245 & 53.788 & 3.216 & 2.651 \\
\hline
\end{tabular}




\begin{tabular}{|c|c|c|c|c|c|}
\hline PPL_06698 & 4.380 & 14.057 & 14.105 & 3.209 & 3.220 \\
\hline PPL_12565 & 5.409 & 17.346 & 16.474 & 3.207 & 3.046 \\
\hline PPL_05964 & 1.584 & 5.076 & 3.987 & 3.204 & 2.517 \\
\hline PPL_04797 & 11.319 & 36.232 & 23.351 & 3.201 & 2.063 \\
\hline PPL_10447 & 9.908 & 31.622 & 30.801 & 3.192 & 3.109 \\
\hline PPL_01065 & 1.683 & 5.372 & 3.710 & 3.192 & 2.204 \\
\hline PPL_05947 & 3.261 & 10.395 & 8.817 & 3.187 & 2.704 \\
\hline PPL 04346 & 0.287 & 0.915 & 0.774 & 3.187 & 2.696 \\
\hline PPL 09294 & 17.810 & 56.758 & 29.824 & 3.187 & 1.675 \\
\hline PPL_12364 & 0.418 & 1.332 & 0.816 & 3.184 & 1.950 \\
\hline PPL_00977 & 1.014 & 3.228 & 3.063 & 3.182 & 3.019 \\
\hline PPL_00649 & 23.584 & 74.911 & 61.803 & 3.176 & 2.621 \\
\hline PPL_09799 & 46.071 & 146.031 & 115.706 & 3.170 & 2.511 \\
\hline PPL_12416 & 2.904 & 9.151 & 6.503 & 3.151 & 2.239 \\
\hline PPL_00994 & 37.222 & 117.302 & 19.745 & 3.151 & 0.530 \\
\hline PPL_01834 & 0.769 & 2.413 & 2.025 & 3.139 & 2.634 \\
\hline PPL_10895 & 11.991 & 37.609 & 18.453 & 3.136 & 1.539 \\
\hline PPL_11686 & 0.683 & 2.141 & 1.726 & 3.133 & 2.525 \\
\hline PPL_04521 & 4.488 & 14.030 & 10.876 & 3.126 & 2.424 \\
\hline PPL_03562 & 2.614 & 8.148 & 10.465 & 3.117 & 4.003 \\
\hline PPL_09516 & 13.189 & 41.112 & 32.625 & 3.117 & 2.474 \\
\hline PPL_01420 & 1.504 & 4.679 & 3.016 & 3.112 & 2.005 \\
\hline PPL_09791 & 41.988 & 130.559 & 108.103 & 3.109 & 2.575 \\
\hline PPL_02940 & 1.169 & 3.625 & 3.370 & 3.102 & 2.884 \\
\hline PPL_01181 & 6.504 & 20.162 & 14.502 & 3.100 & 2.230 \\
\hline PPL_12303 & 9.050 & 27.999 & 28.869 & 3.094 & 3.190 \\
\hline PPL_00914 & 162.703 & 502.224 & 202.500 & 3.087 & 1.245 \\
\hline PPL_00728 & 2.388 & 7.365 & 5.853 & 3.085 & 2.451 \\
\hline PPL_05338 & 1.127 & 3.463 & 4.119 & 3.074 & 3.655 \\
\hline PPL_10801 & 2.124 & 6.498 & 6.696 & 3.060 & 3.153 \\
\hline PPL_08764 & 39.120 & 119.435 & 117.984 & 3.053 & 3.016 \\
\hline PPL_03179 & 1.023 & 3.108 & 6.158 & 3.040 & 6.022 \\
\hline PPL_11365 & 54.307 & 164.479 & 138.565 & 3.029 & 2.552 \\
\hline PPL_07859 & 122.218 & 39.799 & 77.368 & 0.326 & 0.633 \\
\hline PPL_00995 & 68.612 & 22.239 & 21.447 & 0.324 & 0.313 \\
\hline PPL_11728 & 65.731 & 21.219 & 15.186 & 0.323 & 0.231 \\
\hline PPL 12602 & 0.491 & 0.158 & 0.099 & 0.322 & 0.201 \\
\hline PPL_00871 & 1517.455 & 482.175 & 319.570 & 0.318 & 0.211 \\
\hline PPL_01136 & 30.778 & 9.774 & 8.057 & 0.318 & 0.262 \\
\hline PPL_09190 & 1094.476 & 345.486 & 319.642 & 0.316 & 0.292 \\
\hline PPL_06073 & 41.577 & 13.101 & 11.290 & 0.315 & 0.272 \\
\hline PPL_06462 & 12.207 & 3.831 & 5.119 & 0.314 & 0.419 \\
\hline PPL_00267 & 41.460 & 12.934 & 12.218 & 0.312 & 0.295 \\
\hline PPL 09564 & 31.736 & 9.859 & 10.958 & 0.311 & 0.345 \\
\hline PPL_06960 & 19.594 & 6.026 & 9.951 & 0.308 & 0.508 \\
\hline PPL 11489 & 232.983 & 71.796 & 79.511 & 0.308 & 0.341 \\
\hline PPL_03260 & 264.638 & 81.631 & 82.869 & 0.308 & 0.313 \\
\hline PPL_03534 & 28.626 & 8.803 & 15.697 & 0.308 & 0.548 \\
\hline PPL_02513 & 131.881 & 40.438 & 38.037 & 0.307 & 0.288 \\
\hline PPL_06940 & 144.822 & 44.212 & 40.690 & 0.305 & 0.281 \\
\hline PPL_06161 & 137.859 & 42.023 & 31.560 & 0.305 & 0.229 \\
\hline PPL_07328 & 13.859 & 4.193 & 5.582 & 0.303 & 0.403 \\
\hline
\end{tabular}




\begin{tabular}{|c|c|c|c|c|c|}
\hline PPL_11171 & 64.661 & 19.474 & 30.459 & 0.301 & 0.471 \\
\hline $\mathrm{PPL} 08205$ & 3.042 & 0.914 & 0.859 & 0.301 & 0.282 \\
\hline PPL_08601 & 57.324 & 17.179 & 16.812 & 0.300 & 0.293 \\
\hline $\mathrm{PPL} 11059$ & 26.396 & 7.923 & 2.476 & 0.300 & 0.094 \\
\hline PPL 03695 & 160.282 & 48.035 & 34.668 & 0.300 & 0.216 \\
\hline $\mathrm{PPL} 11303$ & 8.406 & 2.515 & 4.706 & 0.299 & 0.560 \\
\hline PPL 01924 & 52.183 & 15.512 & 13.704 & 0.297 & 0.263 \\
\hline $\mathrm{PPL} 03891$ & 241.581 & 71.449 & 30.393 & 0.296 & 0.126 \\
\hline PPL_06741 & 127.081 & 37.657 & 39.373 & 0.296 & 0.310 \\
\hline PPL 09463 & 22.121 & 6.542 & 7.000 & 0.296 & 0.316 \\
\hline $\mathrm{PPL} 00478$ & 34.383 & 10.144 & 9.557 & 0.295 & 0.278 \\
\hline $\mathrm{PPL} 12212$ & 19.458 & 5.722 & 6.931 & 0.294 & 0.356 \\
\hline PPL_02831 & 0.604 & 0.177 & 0.269 & 0.293 & 0.445 \\
\hline $\mathrm{PPL} 12112$ & 16.592 & 4.860 & 8.568 & 0.293 & 0.516 \\
\hline PPL_05061 & 22.727 & 6.605 & 7.254 & 0.291 & 0.319 \\
\hline PPL_03988 & 66.203 & 19.168 & 38.925 & 0.290 & 0.588 \\
\hline PPL 02987 & 80.716 & 23.308 & 27.544 & 0.289 & 0.341 \\
\hline PPL_00439 & 29.186 & 8.397 & 8.297 & 0.288 & 0.284 \\
\hline PPL 04183 & 12.259 & 3.535 & 5.355 & 0.288 & 0.437 \\
\hline PPL_06356 & 108.012 & 31.089 & 42.015 & 0.288 & 0.389 \\
\hline PPL 09400 & 4.100 & 1.177 & 1.243 & 0.287 & 0.303 \\
\hline PPL_07051 & 141.394 & 40.646 & 36.604 & 0.287 & 0.259 \\
\hline PPL_02419 & 5.582 & 1.600 & 7.554 & 0.287 & 1.353 \\
\hline PPL_04168 & 0.224 & 0.064 & 0.100 & 0.286 & 0.448 \\
\hline PPL 07752 & 7.525 & 2.155 & 3.337 & 0.286 & 0.443 \\
\hline PPL_10240 & 22.039 & 6.301 & 6.805 & 0.286 & 0.309 \\
\hline PPL_04225 & 105.262 & 29.758 & 35.788 & 0.283 & 0.340 \\
\hline $\mathrm{PPL} 05121$ & 15.852 & 4.478 & 11.091 & 0.283 & 0.700 \\
\hline $\mathrm{PPL} 10465$ & 7.886 & 2.220 & 3.899 & 0.282 & 0.494 \\
\hline PPL_08209 & 10.966 & 3.067 & 2.541 & 0.280 & 0.232 \\
\hline PPL_01355 & 23.940 & 6.712 & 9.997 & 0.280 & 0.418 \\
\hline PPL_06007 & 2.810 & 0.785 & 0.947 & 0.279 & 0.337 \\
\hline PPL_04408 & 45.822 & 12.718 & 15.812 & 0.278 & 0.345 \\
\hline PPL_01187 & 4.076 & 1.129 & 1.345 & 0.277 & 0.330 \\
\hline PPL_09811 & 26.579 & 7.300 & 5.257 & 0.275 & 0.198 \\
\hline PPL_05109 & 49.065 & 13.517 & 9.863 & 0.275 & 0.201 \\
\hline PPL_03567 & 4.191 & 1.154 & 0.640 & 0.275 & 0.153 \\
\hline $\mathrm{PPL} 10616$ & 77.209 & 21.066 & 30.481 & 0.273 & 0.395 \\
\hline PPL_00191 & 31.366 & 8.570 & 11.078 & 0.273 & 0.353 \\
\hline PPL 07822 & 16.621 & 4.504 & 3.196 & 0.271 & 0.192 \\
\hline PPL_11949 & 46.545 & 12.594 & 14.390 & 0.271 & 0.309 \\
\hline PPL_03954 & 72.618 & 19.572 & 18.545 & 0.270 & 0.255 \\
\hline $\mathrm{PPL} 11060$ & 25.606 & 6.918 & 2.707 & 0.270 & 0.106 \\
\hline PPL_09721 & 2.877 & 0.775 & 0.442 & 0.269 & 0.154 \\
\hline PPL 10011 & 54.287 & 14.528 & 12.922 & 0.268 & 0.238 \\
\hline PPL 04616 & 11.020 & 2.946 & 2.926 & 0.267 & 0.265 \\
\hline PPL_05952 & 24.650 & 6.552 & 7.174 & 0.266 & 0.291 \\
\hline PPL_04910 & 66.362 & 17.592 & 14.299 & 0.265 & 0.215 \\
\hline $\mathrm{PPL} 03350$ & 15.844 & 4.191 & 4.997 & 0.265 & 0.315 \\
\hline PPL_07539 & 18.761 & 4.927 & 5.078 & 0.263 & 0.271 \\
\hline PPL_06722 & 21.643 & 5.700 & 5.810 & 0.263 & 0.268 \\
\hline PPL_02238 & 103.006 & 27.082 & 26.527 & 0.263 & 0.258 \\
\hline
\end{tabular}




\begin{tabular}{|c|c|c|c|c|c|}
\hline PPL_09257 & 10.030 & 2.627 & 2.773 & 0.262 & 0.277 \\
\hline PPL_10130 & 108.505 & 28.459 & 25.044 & 0.262 & 0.231 \\
\hline PPL 09126 & 59.840 & 15.603 & 18.192 & 0.261 & 0.304 \\
\hline PPL_09247 & 10.277 & 2.678 & 2.741 & 0.261 & 0.267 \\
\hline PPL_12107 & 7.214 & 1.873 & 2.071 & 0.260 & 0.287 \\
\hline PPL_04587 & 10.452 & 2.722 & 2.970 & 0.260 & 0.284 \\
\hline PPL_05615 & 9.869 & 2.552 & 3.200 & 0.259 & 0.324 \\
\hline PPL 03782 & 69.632 & 17.960 & 27.893 & 0.258 & 0.401 \\
\hline PPL_09097 & 0.148 & 0.038 & 0.055 & 0.258 & 0.375 \\
\hline PPL_11476 & 36.136 & 9.326 & 11.842 & 0.258 & 0.328 \\
\hline PPL_10036 & 15.744 & 4.048 & 3.764 & 0.257 & 0.239 \\
\hline PPL 10504 & 3.818 & 0.978 & 1.327 & 0.256 & 0.348 \\
\hline PPL_10884 & 21.261 & 5.441 & 5.936 & 0.256 & 0.279 \\
\hline PPL_02798 & 16.758 & 4.280 & 5.415 & 0.255 & 0.323 \\
\hline PPL_01544 & 88.059 & 22.442 & 27.100 & 0.255 & 0.308 \\
\hline PPL_00653 & 18.000 & 4.566 & 3.295 & 0.254 & 0.183 \\
\hline PPL 02322 & 3.949 & 1.001 & 1.012 & 0.253 & 0.256 \\
\hline $\mathrm{PPL}=11272$ & 9.362 & 2.371 & 2.327 & 0.253 & 0.249 \\
\hline PPL_06915 & 142.854 & 35.952 & 36.008 & 0.252 & 0.252 \\
\hline PPL_01502 & 8.418 & 2.107 & 3.660 & 0.250 & 0.435 \\
\hline PPL 11277 & 44.349 & 11.074 & 15.550 & 0.250 & 0.351 \\
\hline PPL_07800 & 7.689 & 1.909 & 3.788 & 0.248 & 0.493 \\
\hline $\mathrm{PPL}^{-} 11784$ & 44.601 & 11.071 & 16.910 & 0.248 & 0.379 \\
\hline PPL_02508 & 6.234 & 1.538 & 1.349 & 0.247 & 0.216 \\
\hline PPL_04902 & 2.084 & 0.512 & 1.032 & 0.246 & 0.495 \\
\hline PPL_01110 & 51.411 & 12.579 & 13.924 & 0.245 & 0.271 \\
\hline PPL_09020 & 0.510 & 0.124 & 0.120 & 0.244 & 0.236 \\
\hline PPL_01959 & 90.831 & 22.198 & 18.781 & 0.244 & 0.207 \\
\hline PPL_03739 & 26.842 & 6.562 & 2.826 & 0.244 & 0.105 \\
\hline PPL 04525 & 49.299 & 11.982 & 15.987 & 0.243 & 0.324 \\
\hline PPL_01159 & 8.344 & 2.028 & 1.282 & 0.243 & 0.154 \\
\hline PPL_12334 & 108.530 & 25.957 & 34.957 & 0.239 & 0.322 \\
\hline PPL_00630 & 47.256 & 11.271 & 15.261 & 0.239 & 0.323 \\
\hline PPL_05727 & 59.170 & 14.165 & 18.866 & 0.239 & 0.319 \\
\hline PPL 00735 & 63.217 & 15.036 & 18.239 & 0.238 & 0.289 \\
\hline PPL_03578 & 66.777 & 15.901 & 14.518 & 0.238 & 0.217 \\
\hline PPL_06453 & 165.051 & 39.207 & 52.353 & 0.238 & 0.317 \\
\hline PPL_10008 & 100.002 & 23.784 & 22.715 & 0.238 & 0.227 \\
\hline $\mathrm{PPL} 06710$ & 147.869 & 35.128 & 43.361 & 0.238 & 0.293 \\
\hline PPL_12467 & 168.390 & 39.676 & 30.855 & 0.236 & 0.183 \\
\hline PPL_07318 & 26.866 & 6.340 & 7.334 & 0.236 & 0.273 \\
\hline PPL_02100 & 36.590 & 8.614 & 9.804 & 0.235 & 0.268 \\
\hline $\mathrm{PPL}-11411$ & 1.632 & 0.384 & 0.597 & 0.235 & 0.366 \\
\hline PPL 02110 & 167.508 & 39.396 & 53.837 & 0.235 & 0.321 \\
\hline PPL_09641 & 18.972 & 4.466 & 6.343 & 0.235 & 0.334 \\
\hline PPL_10181 & 67.257 & 15.772 & 12.961 & 0.235 & 0.193 \\
\hline PPL_04384 & 8.610 & 2.011 & 2.627 & 0.234 & 0.305 \\
\hline $\mathrm{PPL} 05551$ & 70.456 & 16.455 & 22.004 & 0.234 & 0.312 \\
\hline PPL_09181 & 71.737 & 16.787 & 19.069 & 0.234 & 0.266 \\
\hline PPL_03898 & 41.428 & 9.645 & 10.113 & 0.233 & 0.244 \\
\hline PPL_07163 & 62.214 & 14.472 & 17.128 & 0.233 & 0.275 \\
\hline PPL_09729 & 19.128 & 4.441 & 5.932 & 0.232 & 0.310 \\
\hline
\end{tabular}




\begin{tabular}{|c|c|c|c|c|c|}
\hline PPL_00904 & 35.080 & 8.153 & 10.570 & 0.232 & 0.301 \\
\hline $\mathrm{PPL} 05418$ & 55.227 & 12.828 & 7.946 & 0.232 & 0.144 \\
\hline PPL_00166 & 34.098 & 7.914 & 7.047 & 0.232 & 0.207 \\
\hline PPL 08485 & 2.256 & 0.522 & 0.486 & 0.231 & 0.215 \\
\hline PPL 03547 & 85.216 & 19.645 & 15.452 & 0.231 & 0.181 \\
\hline PPL 08137 & 10.702 & 2.457 & 1.608 & 0.230 & 0.150 \\
\hline PPL_06004 & 42.355 & 9.758 & 8.414 & 0.230 & 0.199 \\
\hline PPL 08487 & 21.355 & 4.916 & 3.213 & 0.230 & 0.150 \\
\hline PPL_06709 & 39.473 & 9.009 & 11.533 & 0.228 & 0.292 \\
\hline PPL 08863 & 2.710 & 0.616 & 0.711 & 0.227 & 0.262 \\
\hline $\mathrm{PPL} 10206$ & 5.269 & 1.192 & 1.221 & 0.226 & 0.232 \\
\hline PPL 06742 & 35.483 & 8.036 & 7.258 & 0.226 & 0.205 \\
\hline PPL_07175 & 11.364 & 2.553 & 7.495 & 0.225 & 0.660 \\
\hline PPL 08034 & 34.619 & 7.781 & 14.006 & 0.225 & 0.405 \\
\hline PPL_05978 & 0.266 & 0.060 & 0.116 & 0.225 & 0.436 \\
\hline $\mathrm{PPL} 11813$ & 6.546 & 1.474 & 1.673 & 0.225 & 0.255 \\
\hline PPL 04492 & 3.095 & 0.689 & 0.732 & 0.223 & 0.236 \\
\hline PPL_10647 & 22.314 & 4.983 & 3.756 & 0.223 & 0.168 \\
\hline PPL 08207 & 6.207 & 1.378 & 1.244 & 0.222 & 0.200 \\
\hline PPL_09237 & 22.069 & 4.886 & 6.760 & 0.221 & 0.306 \\
\hline PPL_07812 & 1.379 & 0.302 & 0.795 & 0.219 & 0.576 \\
\hline PPL_08516 & 1009.684 & 221.210 & 78.544 & 0.219 & 0.078 \\
\hline PPL_07305 & 72.802 & 15.964 & 26.143 & 0.219 & 0.359 \\
\hline PPL_00222 & 248.940 & 54.533 & 102.483 & 0.219 & 0.412 \\
\hline PPL 10096 & 4.690 & 1.022 & 0.869 & 0.218 & 0.185 \\
\hline PPL_08101 & 4.742 & 1.036 & 2.565 & 0.218 & 0.541 \\
\hline PPL_05060 & 13.205 & 2.872 & 4.887 & 0.217 & 0.370 \\
\hline $\mathrm{PPL} 01252$ & 268.320 & 58.152 & 57.574 & 0.217 & 0.215 \\
\hline PPL_03962 & 39.845 & 8.623 & 13.927 & 0.216 & 0.350 \\
\hline PPL_08293 & 12.945 & 2.756 & 5.269 & 0.213 & 0.407 \\
\hline $\mathrm{PPL}_{-} 12166$ & 5.949 & 1.269 & 0.956 & 0.213 & 0.161 \\
\hline PPL_01032 & 153.398 & 32.525 & 30.928 & 0.212 & 0.202 \\
\hline PPL_05630 & 59.991 & 12.664 & 17.677 & 0.211 & 0.295 \\
\hline PPL_07593 & 2.116 & 0.447 & 0.145 & 0.211 & 0.069 \\
\hline PPL_11385 & 27.007 & 5.679 & 10.675 & 0.210 & 0.395 \\
\hline PPL_02067 & 72.753 & 15.286 & 17.726 & 0.210 & 0.244 \\
\hline PPL_02412 & 66.891 & 14.075 & 22.711 & 0.210 & 0.340 \\
\hline PPL_09829 & 105.889 & 22.252 & 27.169 & 0.210 & 0.257 \\
\hline PPL_04224 & 11.303 & 2.368 & 3.596 & 0.209 & 0.318 \\
\hline $\mathrm{PPL} 06666$ & 18.189 & 3.782 & 3.033 & 0.208 & 0.167 \\
\hline PPL_00295 & 6.881 & 1.430 & 0.985 & 0.208 & 0.143 \\
\hline PPL_08542 & 37.165 & 7.739 & 8.201 & 0.208 & 0.221 \\
\hline PPL_00488 & 65.875 & 13.650 & 13.798 & 0.207 & 0.209 \\
\hline PPL_00888 & 125.297 & 25.858 & 29.152 & 0.206 & 0.233 \\
\hline PPL 11661 & 261.972 & 53.855 & 49.410 & 0.206 & 0.189 \\
\hline PPL_07425 & 9.356 & 1.920 & 2.752 & 0.205 & 0.294 \\
\hline PPL_01927 & 46.770 & 9.553 & 8.569 & 0.204 & 0.183 \\
\hline PPL_00193 & 107.825 & 21.950 & 25.697 & 0.204 & 0.238 \\
\hline $\mathrm{PPL} 03640$ & 1.163 & 0.236 & 0.327 & 0.203 & 0.281 \\
\hline PPL_01054 & 63.423 & 12.851 & 32.702 & 0.203 & 0.516 \\
\hline PPL_00647 & 109.879 & 22.260 & 37.022 & 0.203 & 0.337 \\
\hline PPL_01180 & 20.793 & 4.181 & 4.893 & 0.201 & 0.235 \\
\hline
\end{tabular}




\begin{tabular}{|c|c|c|c|c|c|}
\hline PPL_01600 & 23.011 & 4.618 & 2.077 & 0.201 & 0.090 \\
\hline PPL_04625 & 0.803 & 0.160 & 0.125 & 0.200 & 0.156 \\
\hline PPL_07584 & 22.159 & 4.442 & 4.730 & 0.200 & 0.213 \\
\hline PPL_07392 & 3.504 & 0.697 & 0.712 & 0.199 & 0.203 \\
\hline PPL 02437 & 6.518 & 1.288 & 0.750 & 0.198 & 0.115 \\
\hline $\mathrm{PPL} 11081$ & 95.329 & 18.876 & 13.281 & 0.198 & 0.139 \\
\hline PPL_11129 & 3.082 & 0.606 & 0.513 & 0.197 & 0.166 \\
\hline $\mathrm{PPL} 06942$ & 271.157 & 53.380 & 45.061 & 0.197 & 0.166 \\
\hline PPL_00489 & 91.685 & 18.101 & 35.337 & 0.197 & 0.385 \\
\hline PPL 05895 & 15.190 & 2.980 & 3.249 & 0.196 & 0.214 \\
\hline PPL 08057 & 25.008 & 4.905 & 2.781 & 0.196 & 0.111 \\
\hline PPL 08427 & 4.350 & 0.848 & 1.328 & 0.195 & 0.305 \\
\hline PPL_03914 & 273.744 & 53.144 & 125.752 & 0.194 & 0.459 \\
\hline PPL 06429 & 28.532 & 5.538 & 1.704 & 0.194 & 0.060 \\
\hline PPL_01095 & 56.316 & 10.878 & 13.493 & 0.193 & 0.240 \\
\hline PPL_08849 & 25.131 & 4.830 & 6.880 & 0.192 & 0.274 \\
\hline PPL 04561 & 3.577 & 0.688 & 1.008 & 0.192 & 0.282 \\
\hline PPL_02435 & 53.901 & 10.178 & 9.895 & 0.189 & 0.184 \\
\hline PPL 08928 & 99.289 & 18.680 & 16.476 & 0.188 & 0.166 \\
\hline PPL_00981 & 69.575 & 13.027 & 14.936 & 0.187 & 0.215 \\
\hline PPL_05689 & 179.976 & 33.537 & 41.255 & 0.186 & 0.229 \\
\hline PPL_09484 & 115.434 & 21.469 & 22.899 & 0.186 & 0.198 \\
\hline $\mathrm{PPL} 11091$ & 1.677 & 0.311 & 0.335 & 0.185 & 0.200 \\
\hline PPL_07929 & 37.087 & 6.831 & 6.407 & 0.184 & 0.173 \\
\hline PPL 05205 & 7.660 & 1.403 & 1.806 & 0.183 & 0.236 \\
\hline PPL_12387 & 13.650 & 2.491 & 2.249 & 0.183 & 0.165 \\
\hline PPL_06849 & 27.053 & 4.941 & 6.935 & 0.183 & 0.256 \\
\hline $\mathrm{PPL} 01801$ & 3.374 & 0.605 & 0.459 & 0.179 & 0.136 \\
\hline PPL_04602 & 11.934 & 2.135 & 1.507 & 0.179 & 0.126 \\
\hline PPL_00793 & 1.997 & 0.355 & 0.240 & 0.178 & 0.120 \\
\hline $\mathrm{PPL}_{-} 11461$ & 150.915 & 26.920 & 22.982 & 0.178 & 0.152 \\
\hline PPL_05155 & 288.436 & 51.078 & 27.765 & 0.177 & 0.096 \\
\hline PPL_11092 & 6.956 & 1.224 & 1.706 & 0.176 & 0.245 \\
\hline PPL_07610 & 1.296 & 0.225 & 0.083 & 0.174 & 0.064 \\
\hline PPL_08595 & 4.840 & 0.843 & 1.285 & 0.174 & 0.265 \\
\hline PPL_05076 & 961.923 & 166.664 & 95.529 & 0.173 & 0.099 \\
\hline PPL_03913 & 344.785 & 59.645 & 158.553 & 0.173 & 0.460 \\
\hline $\mathrm{PPL} 10022$ & 332.406 & 57.295 & 41.852 & 0.172 & 0.126 \\
\hline $\mathrm{PPL}=12388$ & 6.595 & 1.132 & 1.264 & 0.172 & 0.192 \\
\hline $\mathrm{PPL} 10122$ & 7.766 & 1.332 & 1.910 & 0.171 & 0.246 \\
\hline PPL_00279 & 27.630 & 4.726 & 7.198 & 0.171 & 0.260 \\
\hline PPL_02493 & 51.890 & 8.880 & 8.197 & 0.171 & 0.158 \\
\hline PPL_04669 & 4.880 & 0.836 & 0.927 & 0.171 & 0.190 \\
\hline PPL_05404 & 1.672 & 0.284 & 0.329 & 0.170 & 0.197 \\
\hline $\mathrm{PPL} 02791$ & 164.277 & 27.933 & 20.111 & 0.170 & 0.122 \\
\hline PPL_05467 & 122.893 & 20.896 & 41.788 & 0.170 & 0.340 \\
\hline PPL_08575 & 74.280 & 12.636 & 17.893 & 0.170 & 0.241 \\
\hline PPL_00782 & 116.401 & 19.650 & 9.085 & 0.169 & 0.078 \\
\hline $\mathrm{PPL} 08711$ & 0.855 & 0.143 & 0.163 & 0.168 & 0.191 \\
\hline PPL_03738 & 6.011 & 1.004 & 0.713 & 0.167 & 0.119 \\
\hline PPL_00690 & 1.519 & 0.254 & 0.341 & 0.167 & 0.224 \\
\hline PPL_00805 & 19.447 & 3.226 & 1.565 & 0.166 & 0.080 \\
\hline
\end{tabular}




\begin{tabular}{|c|c|c|c|c|c|}
\hline PPL_05148 & 0.456 & 0.075 & 0.165 & 0.165 & 0.362 \\
\hline PPL_12359 & 23.650 & 3.828 & 2.123 & 0.162 & 0.090 \\
\hline PPL 08815 & 31.060 & 5.025 & 6.424 & 0.162 & 0.207 \\
\hline PPL_11002 & 70.439 & 11.308 & 9.407 & 0.161 & 0.134 \\
\hline PPL_12127 & 255.371 & 40.747 & 56.948 & 0.160 & 0.223 \\
\hline PPL_00980 & 36.386 & 5.826 & 6.533 & 0.160 & 0.180 \\
\hline PPL_00407 & 32.716 & 5.178 & 6.243 & 0.158 & 0.191 \\
\hline PPL_03667 & 213.539 & 33.450 & 52.269 & 0.157 & 0.245 \\
\hline PPL 01379 & 30.787 & 4.840 & 1.251 & 0.157 & 0.041 \\
\hline PPL_02859 & 44.477 & 6.885 & 3.585 & 0.155 & 0.081 \\
\hline PPL_10279 & 35.216 & 5.468 & 5.894 & 0.155 & 0.167 \\
\hline PPL 05756 & 255.832 & 39.477 & 32.249 & 0.154 & 0.126 \\
\hline PPL_08161 & 137.548 & 21.070 & 78.505 & 0.153 & 0.571 \\
\hline PPL_08655 & 6.212 & 0.951 & 0.906 & 0.153 & 0.146 \\
\hline PPL_01644 & 0.792 & 0.121 & 0.234 & 0.153 & 0.296 \\
\hline PPL_05184 & 571.568 & 87.158 & 157.885 & 0.152 & 0.276 \\
\hline $\mathrm{PPL} 02250$ & 184.639 & 27.473 & 12.307 & 0.149 & 0.067 \\
\hline PPL_11948 & 82.134 & 12.232 & 14.995 & 0.149 & 0.183 \\
\hline PPL_06585 & 31.771 & 4.697 & 5.905 & 0.148 & 0.186 \\
\hline PPL_09142 & 0.917 & 0.135 & 0.458 & 0.147 & 0.500 \\
\hline PPL 00624 & 20.558 & 3.002 & 8.037 & 0.146 & 0.391 \\
\hline PPL_01477 & 347.953 & 50.703 & 55.785 & 0.146 & 0.160 \\
\hline PPL_07801 & 0.534 & 0.078 & 0.163 & 0.146 & 0.305 \\
\hline PPL_09328 & 214.993 & 30.971 & 31.655 & 0.144 & 0.147 \\
\hline PPL_02317 & 19.222 & 2.751 & 1.421 & 0.143 & 0.074 \\
\hline PPL_04500 & 9.082 & 1.277 & 4.126 & 0.141 & 0.454 \\
\hline PPL_04484 & 35.458 & 4.979 & 2.529 & 0.140 & 0.071 \\
\hline PPL_07022 & 4.758 & 0.668 & 0.723 & 0.140 & 0.152 \\
\hline $\mathrm{PPL}^{-11023}$ & 120.871 & 16.890 & 15.702 & 0.140 & 0.130 \\
\hline PPL_07798 & 11.067 & 1.547 & 1.790 & 0.140 & 0.162 \\
\hline PPL_00990 & 118.193 & 16.463 & 84.900 & 0.139 & 0.718 \\
\hline PPL_02174 & 20.870 & 2.881 & 4.434 & 0.138 & 0.212 \\
\hline PPL_10055 & 17.850 & 2.460 & 5.016 & 0.138 & 0.281 \\
\hline PPL_04543 & 81.501 & 11.203 & 9.495 & 0.137 & 0.116 \\
\hline PPL 01369 & 0.277 & 0.038 & 0.129 & 0.137 & 0.465 \\
\hline PPL_09766 & 2.022 & 0.275 & 0.516 & 0.136 & 0.255 \\
\hline PPL_04393 & 38.150 & 5.199 & 7.545 & 0.136 & 0.198 \\
\hline PPL_05087 & 601.117 & 81.956 & 68.011 & 0.136 & 0.113 \\
\hline $\mathrm{PPL} 09250$ & 59.187 & 7.995 & 8.859 & 0.135 & 0.150 \\
\hline PPL_11404 & 6.886 & 0.931 & 0.721 & 0.135 & 0.105 \\
\hline PPL_08816 & 221.274 & 29.368 & 47.199 & 0.133 & 0.213 \\
\hline PPL_02637 & 1034.609 & 137.301 & 52.098 & 0.133 & 0.050 \\
\hline PPL_06262 & 55.275 & 7.377 & 2.742 & 0.133 & 0.050 \\
\hline $\mathrm{PPL} 12106$ & 16.179 & 2.136 & 1.637 & 0.132 & 0.101 \\
\hline PPL_05614 & 53.806 & 7.079 & 11.971 & 0.132 & 0.222 \\
\hline PPL_00979 & 30.880 & 4.036 & 5.290 & 0.131 & 0.171 \\
\hline PPL_12337 & 59.972 & 7.881 & 2.707 & 0.131 & 0.045 \\
\hline $\mathrm{PPL} 01753$ & 47.033 & 6.131 & 7.761 & 0.130 & 0.165 \\
\hline PPL_01008 & 89.967 & 11.604 & 13.724 & 0.129 & 0.153 \\
\hline PPL_07248 & 2.544 & 0.328 & 0.583 & 0.129 & 0.229 \\
\hline PPL_05238 & 18.661 & 2.364 & 1.319 & 0.127 & 0.071 \\
\hline PPL_07756 & 2.864 & 0.363 & 0.495 & 0.127 & 0.173 \\
\hline
\end{tabular}




\begin{tabular}{|c|c|c|c|c|c|}
\hline PPL_09141 & 324.893 & 40.903 & 8.609 & 0.126 & 0.026 \\
\hline $\mathrm{PPL} 11316$ & 25.814 & 3.255 & 4.367 & 0.126 & 0.169 \\
\hline PPL_08574 & 269.162 & 33.524 & 13.830 & 0.125 & 0.051 \\
\hline PPL_04380 & 164.159 & 20.295 & 9.973 & 0.124 & 0.061 \\
\hline PPL 07132 & 317.047 & 39.045 & 37.393 & 0.123 & 0.118 \\
\hline PPL 02514 & 23.053 & 2.844 & 3.299 & 0.123 & 0.143 \\
\hline PPL_01901 & 17.805 & 2.194 & 0.847 & 0.123 & 0.048 \\
\hline $\mathrm{PPL} 10113$ & 107.697 & 13.130 & 11.446 & 0.122 & 0.106 \\
\hline PPL_02881 & 122.029 & 14.880 & 13.749 & 0.122 & 0.113 \\
\hline PPL 04461 & 120.410 & 14.690 & 15.496 & 0.122 & 0.129 \\
\hline $\mathrm{PPL} 00450$ & 23.684 & 2.879 & 1.405 & 0.122 & 0.059 \\
\hline PPL 09155 & 12.198 & 1.494 & 1.301 & 0.122 & 0.107 \\
\hline PPL_08409 & 10.861 & 1.294 & 3.897 & 0.119 & 0.359 \\
\hline PPL 03719 & 43.350 & 5.180 & 6.150 & 0.119 & 0.142 \\
\hline PPL_05163 & 36.108 & 4.261 & 5.760 & 0.118 & 0.160 \\
\hline PPL_01135 & 33.539 & 3.941 & 4.218 & 0.118 & 0.126 \\
\hline PPL 09594 & 2.308 & 0.260 & 0.500 & 0.113 & 0.217 \\
\hline PPL_00861 & 5.291 & 0.594 & 2.834 & 0.112 & 0.536 \\
\hline PPL 09314 & 2.149 & 0.240 & 0.222 & 0.112 & 0.103 \\
\hline PPL_03152 & 45.460 & 5.028 & 5.940 & 0.111 & 0.131 \\
\hline PPL 01999 & 27.045 & 2.995 & 2.371 & 0.111 & 0.088 \\
\hline PPL_07109 & 7.296 & 0.811 & 0.850 & 0.111 & 0.117 \\
\hline PPL_05806 & 56.095 & 5.989 & 4.156 & 0.107 & 0.074 \\
\hline PPL_06967 & 47.132 & 5.042 & 4.895 & 0.107 & 0.104 \\
\hline PPL 07028 & 997.121 & 105.957 & 129.613 & 0.106 & 0.130 \\
\hline PPL_01698 & 10.846 & 1.144 & 0.494 & 0.106 & 0.046 \\
\hline PPL_09707 & 10.349 & 1.088 & 0.921 & 0.105 & 0.089 \\
\hline $\mathrm{PPL} 10755$ & 3.958 & 0.416 & 0.406 & 0.105 & 0.102 \\
\hline PPL_09336 & 52.925 & 5.509 & 5.782 & 0.104 & 0.109 \\
\hline PPL_09983 & 0.864 & 0.090 & 0.271 & 0.104 & 0.314 \\
\hline PPL_05570 & 1.435 & 0.145 & 0.091 & 0.101 & 0.063 \\
\hline PPL_05986 & 98.675 & 9.958 & 11.883 & 0.101 & 0.120 \\
\hline PPL_00658 & 32.418 & 3.083 & 2.774 & 0.095 & 0.086 \\
\hline PPL_08149 & 249.095 & 23.719 & 27.246 & 0.095 & 0.109 \\
\hline PPL_04626 & 29.097 & 2.744 & 3.926 & 0.094 & 0.135 \\
\hline PPL_09422 & 13.238 & 1.240 & 0.776 & 0.094 & 0.059 \\
\hline PPL_02819 & 27.445 & 2.553 & 0.980 & 0.093 & 0.036 \\
\hline PPL_09333 & 4.474 & 0.408 & 0.283 & 0.091 & 0.063 \\
\hline PPL_08970 & 295.722 & 25.940 & 33.955 & 0.088 & 0.115 \\
\hline PPL_07356 & 103.099 & 9.023 & 11.699 & 0.088 & 0.113 \\
\hline PPL_12572 & 9.895 & 0.862 & 0.666 & 0.087 & 0.067 \\
\hline PPL_10553 & 75.388 & 6.582 & 4.168 & 0.087 & 0.055 \\
\hline PPL_08312 & 4.278 & 0.370 & 0.135 & 0.086 & 0.032 \\
\hline PPL_01189 & 45.386 & 3.920 & 6.326 & 0.086 & 0.139 \\
\hline PPL 02737 & 0.594 & 0.050 & 0.236 & 0.084 & 0.397 \\
\hline PPL_11554 & 82.077 & 6.801 & 9.229 & 0.083 & 0.112 \\
\hline PPL_00206 & 30.600 & 2.535 & 4.932 & 0.083 & 0.161 \\
\hline PPL_07247 & 28.969 & 2.365 & 3.839 & 0.082 & 0.133 \\
\hline PPL 04909 & 53.167 & 4.256 & 3.637 & 0.080 & 0.068 \\
\hline PPL_04573 & 455.579 & 36.114 & 293.448 & 0.079 & 0.644 \\
\hline PPL_00802 & 26.914 & 2.117 & 0.778 & 0.079 & 0.029 \\
\hline PPL_05009 & 76.203 & 5.987 & 7.603 & 0.079 & 0.100 \\
\hline
\end{tabular}




\begin{tabular}{|c|c|c|c|c|c|}
\hline PPL_06261 & 112.960 & 8.762 & 4.718 & 0.078 & 0.042 \\
\hline PPL_09489 & 26.633 & 2.069 & 1.780 & 0.078 & 0.067 \\
\hline PPL 07763 & 47.222 & 3.636 & 2.406 & 0.077 & 0.051 \\
\hline PPL_02187 & 7.760 & 0.598 & 1.448 & 0.077 & 0.187 \\
\hline PPL_06345 & 7.732 & 0.580 & 0.644 & 0.075 & 0.083 \\
\hline PPL_09886 & 21.007 & 1.580 & 2.326 & 0.075 & 0.111 \\
\hline PPL_06272 & 29.939 & 2.118 & 2.628 & 0.071 & 0.088 \\
\hline $\mathrm{PPL} 08175$ & 41.067 & 2.810 & 3.930 & 0.068 & 0.096 \\
\hline PPL_06743 & 11.101 & 0.759 & 0.496 & 0.068 & 0.045 \\
\hline PPL_05745 & 47.757 & 3.209 & 2.502 & 0.067 & 0.052 \\
\hline PPL_05149 & 20.800 & 1.398 & 5.051 & 0.067 & 0.243 \\
\hline PPL 01689 & 77.977 & 5.176 & 5.602 & 0.066 & 0.072 \\
\hline PPL_04562 & 66.250 & 4.405 & 7.438 & 0.066 & 0.112 \\
\hline PPL_10596 & 58.794 & 3.807 & 1.981 & 0.065 & 0.034 \\
\hline PPL_01967 & 29.809 & 1.858 & 4.345 & 0.062 & 0.146 \\
\hline PPL_02149 & 54.814 & 3.415 & 5.031 & 0.062 & 0.092 \\
\hline PPL 10446 & 1.901 & 0.117 & 0.183 & 0.061 & 0.096 \\
\hline $\mathrm{PPL}=10174$ & 314.777 & 18.843 & 22.980 & 0.060 & 0.073 \\
\hline PPL_11723 & 115.405 & 6.663 & 1.749 & 0.058 & 0.015 \\
\hline PPL_07244 & 136.905 & 7.725 & 11.207 & 0.056 & 0.082 \\
\hline PPL 10668 & 53.377 & 2.957 & 2.444 & 0.055 & 0.046 \\
\hline PPL_12418 & 9.741 & 0.526 & 0.478 & 0.054 & 0.049 \\
\hline PPL_09184 & 51.382 & 2.647 & 6.428 & 0.052 & 0.125 \\
\hline PPL_11589 & 7.587 & 0.394 & 1.355 & 0.052 & 0.179 \\
\hline PPL_07385 & 178.764 & 8.845 & 7.351 & 0.049 & 0.041 \\
\hline PPL_10987 & 238.076 & 10.848 & 13.687 & 0.046 & 0.057 \\
\hline PPL_00127 & 15.616 & 0.713 & 1.105 & 0.046 & 0.071 \\
\hline PPL_00470 & 13.580 & 0.594 & 0.591 & 0.044 & 0.043 \\
\hline PPL_03581 & 8.791 & 0.366 & 2.313 & 0.042 & 0.263 \\
\hline PPL 07439 & 56.161 & 2.292 & 1.765 & 0.041 & 0.031 \\
\hline PPL_05162 & 270.354 & 11.110 & 5.971 & 0.041 & 0.022 \\
\hline PPL_08455 & 8.588 & 0.338 & 0.533 & 0.039 & 0.062 \\
\hline PPL_07438 & 68.451 & 2.537 & 3.106 & 0.037 & 0.045 \\
\hline PPL_08204 & 709.426 & 24.726 & 46.073 & 0.035 & 0.065 \\
\hline PPL 04929 & 36.297 & 1.280 & 1.032 & 0.035 & 0.028 \\
\hline PPL_09115 & 150.287 & 4.867 & 2.682 & 0.032 & 0.018 \\
\hline PPL_06587 & 7.823 & 0.244 & 0.176 & 0.031 & 0.022 \\
\hline PPL_08654 & 82.353 & 2.519 & 7.757 & 0.031 & 0.094 \\
\hline $\mathrm{PPL} 12417$ & 25.504 & 0.758 & 1.295 & 0.030 & 0.051 \\
\hline PPL_10511 & 35.724 & 1.078 & 0.882 & 0.030 & 0.025 \\
\hline PPL_06854 & 11.664 & 0.334 & 0.665 & 0.029 & 0.057 \\
\hline PPL_07432 & 80.523 & 2.295 & 5.369 & 0.029 & 0.067 \\
\hline PPL_00173 & 37.522 & 0.986 & 2.404 & 0.026 & 0.064 \\
\hline PPL 07303 & 61.442 & 1.546 & 7.047 & 0.025 & 0.115 \\
\hline PPL_05815 & 10.174 & 0.258 & 0.250 & 0.025 & 0.025 \\
\hline PPL_06712 & 68.647 & 1.589 & 1.672 & 0.023 & 0.024 \\
\hline PPL 01859 & 22.737 & 0.467 & 0.774 & 0.021 & 0.034 \\
\hline PPL 09019 & 15.259 & 0.312 & 0.686 & 0.020 & 0.045 \\
\hline PPL_07729 & 61.076 & 1.231 & 4.790 & 0.020 & 0.078 \\
\hline PPL_10669 & 122.081 & 2.435 & 3.067 & 0.020 & 0.025 \\
\hline PPL_06819 & 4.443 & 0.089 & 0.083 & 0.020 & 0.019 \\
\hline PPL_06963 & 219.178 & 4.207 & 5.062 & 0.019 & 0.023 \\
\hline
\end{tabular}




\begin{tabular}{|c|c|c|c|c|c|}
\hline PPL_11179 & 30.275 & 0.412 & 0.555 & 0.014 & 0.018 \\
\hline PPL_06714 & 36.806 & 0.526 & 0.526 & 0.014 & 0.014 \\
\hline PPL_09988 & 255.404 & 2.519 & 6.523 & 0.010 & 0.026 \\
\hline PPL_06545 & 53.452 & 0.453 & 1.013 & 0.008 & 0.019 \\
\hline PPL_07847 & 420.482 & 0.653 & 0.513 & 0.002 & 0.001 \\
\hline
\end{tabular}


Table A4: List of genes regulated by glorin after $P$. pallidum PN500 cells prestarved for $1 \mathrm{hr}$ were stimulated with glorin for an additional $1 \mathrm{hr}$.

\begin{tabular}{|l|c|c|c|}
\hline Gene ID & $\begin{array}{c}\text { RPKM } \\
\text { untreated }\end{array}$ & $\begin{array}{c}\text { RPKM } \\
\text { treated }\end{array}$ & $\begin{array}{c}\text { Fold change } \\
\text { treated/untreated }\end{array}$ \\
\hline PPL_05354 & 17.359 & 987.834 & 56.907 \\
\hline PPL_09347 & 0.497 & 26.789 & 53.915 \\
\hline PPL_12248 & 4.010 & 159.175 & 39.699 \\
\hline PPL_12249 & 16.904 & 494.221 & 29.237 \\
\hline PPL_11763 & 0.927 & 21.002 & 22.667 \\
\hline PPL_03541 & 0.112 & 2.488 & 22.255 \\
\hline PPL_05833 & 3.441 & 72.021 & 20.930 \\
\hline PPL_06644 & 0.500 & 8.704 & 17.404 \\
\hline PPL_07811 & 0.332 & 5.444 & 16.374 \\
\hline PPL_08454 & 0.376 & 5.963 & 15.866 \\
\hline PPL_07812 & 0.302 & 4.675 & 15.490 \\
\hline PPL_00912 & 0.811 & 12.366 & 15.239 \\
\hline PPL_00117 & 93.761 & 1137.095 & 12.128 \\
\hline PPL_08455 & 0.338 & 4.051 & 11.983 \\
\hline PPL_00861 & 0.594 & 6.916 & 11.641 \\
\hline PPL_10324 & 3.096 & 35.963 & 11.616 \\
\hline PPL_04784 & 0.052 & 0.558 & 10.817 \\
\hline PPL_02621 & 25.605 & 239.969 & 9.372 \\
\hline PPL_02620 & 353.455 & 3300.083 & 9.337 \\
\hline PPL_12251 & 1.603 & 14.048 & 8.763 \\
\hline PPL_03564 & 3.124 & 27.046 & 8.656 \\
\hline PPL_07801 & 0.078 & 0.622 & 7.998 \\
\hline PPL_12271 & 11.361 & 87.249 & 7.680 \\
\hline PPL_04587 & 2.722 & 20.715 & 7.610 \\
\hline PPL_07296 & 21.092 & 155.598 & 7.377 \\
\hline PPL_07818 & 3.355 & 24.269 & 7.235 \\
\hline PPL_11303 & 2.515 & 17.436 & 6.932 \\
\hline PPL_07817 & 10.126 & 66.569 & 6.574 \\
\hline PPL_08496 & 5.374 & 34.480 & 6.416 \\
\hline PPL_00973 & 1.829 & 11.723 & 6.411 \\
\hline PPL_12308 & 20.420 & 129.378 & 6.336 \\
\hline PPL_12354 & 37.101 & 234.929 & 6.332 \\
\hline PPL_05452 & 69.272 & 402.077 & 5.804 \\
\hline PPL_08545 & 0.733 & 4.184 & 5.708 \\
\hline PPL_07800 & 1.909 & 10.474 & 5.487 \\
\hline PPL_03784 & 1.026 & 5.620 & 5.478 \\
\hline PPL_05392 & 0.827 & 4.362 & 5.273 \\
\hline PPL_07610 & 0.225 & 1.156 & 5.129 \\
\hline PPL_01618 & 5.703 & 29.001 & 5.085 \\
\hline PPL_07302 & 32.080 & 157.407 & 4.907 \\
\hline PPL_05390 & 0.144 & 0.700 & 4.868 \\
\hline PPL_03785 & 0.440 & 2.143 & 4.865 \\
\hline PPL_12250 & 5.091 & 24.279 & 4.730 \\
\hline PPL_10511 & 1.078 & 5.100 & \\
\hline & & & \\
\hline & & & \\
\hline
\end{tabular}




\begin{tabular}{|c|c|c|c|}
\hline PPL_10193 & 0.279 & 1.289 & 4.625 \\
\hline PPL_10669 & 2.435 & 11.081 & 4.551 \\
\hline PPL 01544 & 22.442 & 99.045 & 4.413 \\
\hline PPL 07432 & 2.295 & 10.085 & 4.394 \\
\hline PPL_09669 & 4.501 & 19.681 & 4.372 \\
\hline PPL_07677 & 0.758 & 3.308 & 4.366 \\
\hline PPL_09729 & 4.441 & 18.623 & 4.194 \\
\hline PPL_05727 & 14.165 & 58.899 & 4.158 \\
\hline PPL 00342 & 8.880 & 36.626 & 4.125 \\
\hline PPL_04384 & 2.011 & 8.277 & 4.116 \\
\hline PPL_02977 & 0.240 & 0.954 & 3.978 \\
\hline PPL 00612 & 136.175 & 538.871 & 3.957 \\
\hline PPL_05027 & 7.782 & 30.380 & 3.904 \\
\hline PPL_12247 & 9.319 & 36.305 & 3.896 \\
\hline PPL_07802 & 1.955 & 7.609 & 3.892 \\
\hline PPL_04550 & 3.574 & 13.362 & 3.739 \\
\hline PPL_08725 & 12.027 & 44.048 & 3.662 \\
\hline PPL_09019 & 0.312 & 1.127 & 3.612 \\
\hline PPL_01689 & 5.176 & 18.299 & 3.535 \\
\hline PPL_01722 & 4.195 & 14.662 & 3.495 \\
\hline PPL 05544 & 11.525 & 38.768 & 3.364 \\
\hline PPL_04108 & 6.79 & 22.55 & 3.32 \\
\hline PPL_02831 & 0.177 & 0.583 & 3.296 \\
\hline PPL_11654 & 2.645 & 8.474 & 3.203 \\
\hline PPL_04393 & 5.199 & 16.529 & 3.179 \\
\hline PPL 07675 & 0.675 & 2.138 & 3.167 \\
\hline PPL_10323 & 1.282 & 3.905 & 3.045 \\
\hline PPL_00902 & 70.05 & 192.55 & 2.74 \\
\hline PPL_00855 & 1.72 & 2.99 & 1.48 \\
\hline PPL_02736 & 5.416 & 1.685 & 0.311 \\
\hline PPL_03602 & 0.516 & 0.158 & 0.306 \\
\hline PPL_00884 & 18.489 & 5.541 & 0.300 \\
\hline PPL_05333 & 15.452 & 4.574 & 0.296 \\
\hline PPL_10516 & 0.362 & 0.106 & 0.294 \\
\hline PPL_04954 & 2.062 & 0.594 & 0.288 \\
\hline PPL_06209 & 93.080 & 26.561 & 0.285 \\
\hline PPL_08648 & 10.447 & 2.953 & 0.283 \\
\hline $\mathrm{PPL}^{-} 10331$ & 3.262 & 0.916 & 0.281 \\
\hline PPL_11619 & 304.579 & 83.717 & 0.275 \\
\hline PPL_03325 & 7.774 & 2.068 & 0.266 \\
\hline PPL_00578 & 16.604 & 4.376 & 0.264 \\
\hline PPL_03341 & 3.669 & 0.964 & 0.263 \\
\hline PPL_04828 & 3.315 & 0.848 & 0.256 \\
\hline PPL 06621 & 5.227 & 1.333 & 0.255 \\
\hline PPL_04238 & 0.616 & 0.155 & 0.251 \\
\hline PPL_02308 & 13.194 & 3.292 & 0.250 \\
\hline PPL_00897 & 1.786 & 0.440 & 0.246 \\
\hline PPL_07908 & 163.753 & 40.183 & 0.245 \\
\hline PPL_08647 & 19.482 & 4.674 & 0.240 \\
\hline PPL_09119 & 22.458 & 5.302 & 0.236 \\
\hline $\mathrm{PPL}=12443$ & 15.613 & 3.634 & 0.233 \\
\hline PPL_01209 & 5.506 & 1.276 & 0.232 \\
\hline
\end{tabular}




\begin{tabular}{|l|c|c|c|}
\hline PPL_10531 & 0.874 & 0.201 & 0.230 \\
\hline PPL_02260 & 205.156 & 46.593 & 0.227 \\
\hline PPL_08055 & 11.058 & 2.505 & 0.227 \\
\hline PPL_12441 & 0.297 & 0.067 & 0.226 \\
\hline PPL_00231 & 2.668 & 0.596 & 0.223 \\
\hline PPL_07208 & 1.653 & 0.361 & 0.218 \\
\hline PPL_02829 & 20.565 & 4.274 & 0.208 \\
\hline PPL_03342 & 47.961 & 9.613 & 0.200 \\
\hline PPL_01921 & 0.971 & 0.193 & 0.199 \\
\hline PPL_02939 & 2.178 & 0.431 & 0.198 \\
\hline PPL_05195 & 69.666 & 13.699 & 0.197 \\
\hline PPL_08935 & 88.024 & 15.984 & 0.182 \\
\hline PPL_05491 & 1.091 & 0.195 & 0.179 \\
\hline PPL_01229 & 1.069 & 0.187 & 0.175 \\
\hline PPL_04307 & 1636.434 & 285.755 & 0.175 \\
\hline PPL_04306 & 1281.763 & 210.234 & 0.164 \\
\hline PPL_05306 & 0.323 & 0.049 & 0.151 \\
\hline PPL_05702 & 23.490 & 3.217 & 0.137 \\
\hline PPL_04465 & 6.978 & 0.900 & 0.129 \\
\hline PPL_05902 & 12.190 & 1.526 & 0.125 \\
\hline PPL_04042 & 37.560 & 3.818 & 0.102 \\
\hline PPL_05194 & 2.799 & 0.160 & 0.057 \\
\hline
\end{tabular}

Table A5: List of genes regulated by glorin after $P$. pallidum PN500 cells prestarved for $1 \mathrm{hr}$ were stimulated with glorin for 2 additional hours.

\begin{tabular}{|l|c|c|c|}
\hline Gene ID & $\begin{array}{c}\text { RPKM } \\
\text { untreated }\end{array}$ & $\begin{array}{c}\text { RPKM } \\
\text { Glorin } \\
\text { treated }\end{array}$ & $\begin{array}{c}\text { Fold change } \\
\text { treated/untrea } \\
\text { ted }\end{array}$ \\
\hline PPL_05354 & 15.810 & 1335.619 & 84.480 \\
\hline PPL_09347 & 0.264 & 21.669 & 82.061 \\
\hline PPL_12248 & 2.667 & 21.660 & 8.123 \\
\hline PPL_12249 & 8.729 & 67.574 & 7.741 \\
\hline PPL_00117 & 101.530 & 702.707 & 6.921 \\
\hline PPL_06644 & 0.587 & 3.173 & 5.408 \\
\hline PPL_00062 & 10.343 & 51.539 & 4.983 \\
\hline PPL_04459 & 4.801 & 22.411 & 4.668 \\
\hline PPL_12354 & 37.449 & 168.014 & 4.486 \\
\hline PPL_04587 & 2.970 & 13.157 & 4.430 \\
\hline PPL_05703 & 0.125 & 0.535 & 4.277 \\
\hline PPL_06407 & 6.709 & 27.641 & 4.120 \\
\hline PPL_02620 & 569.162 & 2267.988 & 3.985 \\
\hline PPL_06262 & 2.742 & 10.433 & 3.805 \\
\hline PPL_07551 & 13.510 & 47.156 & 3.491 \\
\hline PPL_10669 & 3.067 & 10.450 & 3.407 \\
\hline PPL_04328 & 0.114 & 0.389 & 3.405 \\
\hline PPL_06410 & 45.067 & 151.554 & 3.363 \\
\hline PPL_01619 & 68.645 & 228.550 & 3.329 \\
\hline
\end{tabular}




\begin{tabular}{|l|c|c|c|}
\hline PPL_11303 & 4.706 & 15.181 & 3.226 \\
\hline PPL_00902 & 96.22 & 148.75 & 1.54 \\
\hline PPL_00855 & 2.66 & 5.33 & 2.00 \\
\hline PPL_09762 & 31.752 & 10.371 & 0.327 \\
\hline PPL_02638 & 1.959 & 0.639 & 0.326 \\
\hline PPL_05154 & 11.139 & 3.539 & 0.318 \\
\hline PPL_04945 & 8.034 & 2.493 & 0.310 \\
\hline PPL_03339 & 34.276 & 10.616 & 0.310 \\
\hline PPL_04856 & 1.566 & 0.481 & 0.307 \\
\hline PPL_10751 & 3.645 & 1.108 & 0.304 \\
\hline PPL_11455 & 25.184 & 7.547 & 0.300 \\
\hline PPL_06963 & 5.062 & 1.478 & 0.292 \\
\hline PPL_08935 & 235.635 & 68.911 & 0.292 \\
\hline PPL_02856 & 140.397 & 40.876 & 0.291 \\
\hline PPL_03314 & 363.527 & 104.765 & 0.288 \\
\hline PPL_07479 & 27.195 & 7.770 & 0.286 \\
\hline PPL_04882 & 27.333 & 7.654 & 0.280 \\
\hline PPL_10745 & 39.311 & 10.956 & 0.279 \\
\hline PPL_11326 & 5.607 & 1.565 & 0.279 \\
\hline PPL_11401 & 32.889 & 9.128 & 0.278 \\
\hline PPL_08361 & 42.283 & 11.643 & 0.275 \\
\hline PPL_10576 & 212.746 & 57.775 & 0.272 \\
\hline PPL_07249 & 0.623 & 0.169 & 0.271 \\
\hline PPL_02535 & 0.790 & 0.212 & 0.269 \\
\hline PPL_04238 & 0.625 & 0.164 & 0.263 \\
\hline PPL_06004 & 8.414 & 2.213 & 0.263 \\
\hline PPL_06686 & 0.691 & 0.181 & 0.261 \\
\hline PPL_01117 & 5.524 & 1.423 & 0.258 \\
\hline PPL_05486 & 4.564 & 1.168 & 0.256 \\
\hline PPL_01872 & 1.090 & 0.279 & 0.255 \\
\hline PPL_03581 & 2.313 & 0.586 & 0.254 \\
\hline PPL_03364 & 1.715 & 0.426 & 0.248 \\
\hline PPL_04306 & 1049.866 & 259.797 & 0.247 \\
\hline PPL_04071 & 1.920 & 0.472 & 0.246 \\
\hline PPL_01230 & 39.811 & 9.723 & 0.244 \\
\hline PPL_02076 & 2.759 & 0.674 & 0.244 \\
\hline PPL_08030 & 0.297 & 0.072 & 0.243 \\
\hline PPL_04307 & 1296.892 & 314.696 & 0.243 \\
\hline PPL_00769 & 7.213 & 1.752 & 0.243 \\
\hline PPL_11952 & 0.652 & 0.158 & 0.243 \\
\hline PPL_11665 & 0.416 & 0.100 & 0.241 \\
\hline PPL_06751 & 0.575 & 0.139 & 0.241 \\
\hline PPL_05194 & 0.722 & 0.174 & 0.241 \\
\hline PPL_02626 & 0.416 & 0.100 & 0.241 \\
\hline PPL_02829 & 19.170 & 4.555 & 0.238 \\
\hline PPL_04785 & 40.309 & 9.566 & 0.237 \\
\hline PPL_06545 & 1.013 & 0.238 & 0.234 \\
\hline PPL_00136 & 72.238 & 16.741 & 0.232 \\
\hline PPL_08476 & 9.544 & 2.202 & 0.231 \\
\hline PPL_09772 & 0.110 & 0.025 & 0.230 \\
\hline PPL_00897 & 1.495 & 0.326 & 0.218 \\
\hline PPL_09119 & 19.644 & 4.283 & 0.218 \\
\hline
\end{tabular}




\begin{tabular}{|c|c|c|c|}
\hline PPL_04180 & 1.852 & 0.399 & 0.215 \\
\hline PPL_08475 & 10.241 & 2.171 & 0.212 \\
\hline PPL_07775 & 16.341 & 3.450 & 0.211 \\
\hline PPL_04561 & 1.008 & 0.213 & 0.211 \\
\hline PPL_12095 & 1.974 & 0.410 & 0.208 \\
\hline PPL_09571 & 0.491 & 0.101 & 0.207 \\
\hline PPL_08450 & 2.997 & 0.613 & 0.205 \\
\hline PPL_00658 & 2.774 & 0.563 & 0.203 \\
\hline PPL_10422 & 24.566 & 4.936 & 0.201 \\
\hline PPL_00038 & 0.162 & 0.032 & 0.199 \\
\hline PPL_11590 & 0.888 & 0.177 & 0.199 \\
\hline PPL_04774 & 1.921 & 0.372 & 0.194 \\
\hline PPL_08807 & 52.563 & 10.185 & 0.194 \\
\hline PPL_02624 & 5.276 & 1.019 & 0.193 \\
\hline PPL_07908 & 133.324 & 25.640 & 0.192 \\
\hline PPL_00514 & 93.525 & 17.746 & 0.190 \\
\hline PPL_05872 & 1.471 & 0.270 & 0.183 \\
\hline PPL_03343 & 3.365 & 0.606 & 0.180 \\
\hline PPL_00798 & 3.551 & 0.629 & 0.177 \\
\hline PPL_08488 & 15.832 & 2.809 & 0.177 \\
\hline PPL_12096 & 20.680 & 3.635 & 0.176 \\
\hline PPL_05902 & 47.110 & 8.213 & 0.174 \\
\hline PPL_01754 & 28.346 & 4.830 & 0.170 \\
\hline PPL_01394 & 10.409 & 1.723 & 0.166 \\
\hline PPL_08355 & 8.146 & 1.346 & 0.165 \\
\hline PPL_06552 & 74.177 & 12.011 & 0.162 \\
\hline PPL_01209 & 3.153 & 0.484 & 0.154 \\
\hline PPL_05156 & 8.090 & 1.214 & 0.150 \\
\hline PPL_06527 & 1.868 & 0.275 & 0.147 \\
\hline PPL_08477 & 50.321 & 7.318 & 0.145 \\
\hline PPL_02046 & 143.320 & 20.734 & 0.145 \\
\hline PPL_02260 & 242.138 & 34.257 & 0.141 \\
\hline PPL_02367 & 5.327 & 0.744 & 0.140 \\
\hline PPL_04465 & 10.235 & 1.371 & 0.134 \\
\hline PPL_07729 & 4.790 & 0.633 & 0.132 \\
\hline PPL_12441 & 0.418 & 0.054 & 0.130 \\
\hline PPL_03342 & 17.526 & 2.277 & 0.130 \\
\hline PPL_09005 & 35.352 & 4.452 & 0.126 \\
\hline PPL_08409 & 3.897 & 0.467 & 0.120 \\
\hline PPL_08976 & 20.505 & 2.367 & 0.115 \\
\hline PPL_00813 & 12.197 & 1.407 & 0.115 \\
\hline PPL_11308 & 14.053 & 1.583 & 0.113 \\
\hline PPL_00410 & 2.790 & 0.309 & 0.111 \\
\hline PPL_05333 & 32.013 & 3.080 & 0.096 \\
\hline PPL_03638 & 764.683 & 69.983 & 0.092 \\
\hline PPL_09004 & 12.918 & 1.177 & 0.091 \\
\hline PPL_07203 & 126.865 & 11.101 & 0.087 \\
\hline PPL_00519 & 2.116 & 0.179 & 0.084 \\
\hline PPL_01229 & 1.455 & 0.110 & 0.076 \\
\hline PPL_05195 & 117.677 & 8.034 & 0.068 \\
\hline PPL_07303 & 7.047 & 0.480 & 0.068 \\
\hline & & & \\
\hline
\end{tabular}


Table A6: Genes regulated by glorin after $1 \mathrm{hr}$ of pre-starvation plus 1 or 2 hr of glorin treatment

\begin{tabular}{|l|c|c|}
\hline Gene ID & $\begin{array}{c}\text { Fold change } \\
\text { 1 hr glorin- } \\
\text { treated/untreated }\end{array}$ & $\begin{array}{c}\text { Fold change } \\
\text { 2 hr glorin- } \\
\text { treated/untreated }\end{array}$ \\
\hline PPL_05354 & 56.907 & 84.480 \\
\hline PPL_09347 & 53.915 & 82.061 \\
\hline PPL_12248 & 39.699 & 8.123 \\
\hline PPL_12249 & 29.237 & 7.741 \\
\hline PPL_06644 & 17.404 & 5.408 \\
\hline PPL_00117 & 12.128 & 6.921 \\
\hline PPL_02620 & 9.337 & 3.985 \\
\hline PPL_04587 & 7.610 & 4.430 \\
\hline PPL_11303 & 6.932 & 3.226 \\
\hline PPL_12354 & 6.332 & 4.486 \\
\hline PPL_10669 & 4.551 & 3.407 \\
\hline PPL_05333 & 0.296 & 0.096 \\
\hline PPL_04238 & 0.251 & 0.263 \\
\hline PPL_00897 & 0.246 & 0.218 \\
\hline PPL_07908 & 0.245 & 0.192 \\
\hline PPL_09119 & 0.236 & 0.218 \\
\hline PPL_01209 & 0.232 & 0.154 \\
\hline PPL_02260 & 0.227 & 0.141 \\
\hline PPL_12441 & 0.226 & 0.130 \\
\hline PPL_02829 & 0.208 & 0.238 \\
\hline PPL_03342 & 0.200 & 0.130 \\
\hline PPL_05195 & 0.197 & 0.068 \\
\hline PPL_08935 & 0.182 & 0.292 \\
\hline PPL_01229 & 0.175 & 0.076 \\
\hline PPL_04307 & 0.175 & 0.243 \\
\hline PPL_04306 & 0.164 & 0.247 \\
\hline PPL_04465 & 0.129 & 0.134 \\
\hline PPL_05902 & 0.125 & 0.174 \\
\hline PPL_05194 & 0.057 & 0.241 \\
\hline & & \\
\hline
\end{tabular}


Table A7: GO term analysis of genes differentially regulated after 1 hour of pre-starvation plus $1 \mathrm{hr}$ of glorin treatment.

\begin{tabular}{|c|c|}
\hline PPL_00612 & $\begin{array}{l}\text { Molecular Function: catalytic activity (GO:0003824) } \\
\text { Molecular Function: transcription repressor activity } \\
\text { (GO:0016564) } \\
\text { Molecular Function: binding (GO:0005488) } \\
\text { Biological Process: metabolic process (GO:0008152) }\end{array}$ \\
\hline PPL_00861 & $\begin{array}{l}\text { Molecular Function: protein kinase activity (GO:0004672) } \\
\text { Molecular Function: ATP binding (GO:0005524) } \\
\text { Molecular Function: protein serine/threonine kinase activity } \\
\text { (GO:0004674) } \\
\text { Biological Process: protein amino acid phosphorylation } \\
\text { (GO:0006468) }\end{array}$ \\
\hline PPL_02308 & $\begin{array}{l}\text { Molecular Function: ubiquitin-protein ligase activity } \\
\text { (GO:0004842) } \\
\text { Molecular Function: zinc ion binding (GO:0008270) }\end{array}$ \\
\hline PPL_03541 & Biological Process: pathogenesis (GO:0009405) \\
\hline PPL_03564 & $\begin{array}{l}\text { Molecular Function: lipid binding (GO:0008289) } \\
\text { Molecular Function: G-protein coupled receptor activity } \\
\text { (GO:0004930), Biological Process: G-protein coupled receptor } \\
\text { protein signaling pathway (GO:0007186) } \\
\text { Cellular Component: integral to membrane (GO:0016021), }\end{array}$ \\
\hline PPL_04042 & $\begin{array}{l}\text { Molecular Function: transcription factor activity (GO:0003700) } \\
\text { Biological Process: regulation of transcription, DNA-dependent } \\
\text { (GO:0006355) } \\
\text { Cellular Component: transcription factor complex (GO:0005667) }\end{array}$ \\
\hline PPL_04384 & $\begin{array}{l}\text { Molecular Function: signal transducer activity (GO:0004871) } \\
\text { Molecular Function: two-component sensor activity } \\
\text { (GO:0000155) } \\
\text { Molecular Function: two-component response regulator activity } \\
\text { (GO:0000156) } \\
\text { Molecular Function: ATP binding (GO:0005524) } \\
\text { Molecular Function: protein histidine kinase activity } \\
\text { (GO:0004673) } \\
\text { Molecular Function: two-component response regulator activity } \\
\text { (GO:0000156) } \\
\text { Molecular Function: transferase activity, transferring } \\
\text { phosphorus-containing groups (GO:0016772) } \\
\text { Biological Process: signal transduction (GO:0007165) } \\
\text { Biological Process: two-component signal transduction system } \\
\text { (phosphorelay) (GO:0000160) } \\
\text { Biological Process: phosphorylation (GO:0016310) } \\
\text { Biological Process: signal transduction (GO:0007165) } \\
\text { Biological Process: regulation of transcription, DNA-dependent } \\
\text { (GO:0006355) } \\
\text { Biological Process: peptidyl-histidine phosphorylation } \\
\text { (GO:0018106) } \\
\text { Cellular Component: membrane (GO:0016020) }\end{array}$ \\
\hline PPL_04393 & $\begin{array}{l}\text { Molecular Function: GTP binding (GO:0005525) } \\
\text { Biological Process: small GTPase mediated signal transduction } \\
\text { (GO:0007264) } \\
\text { Cellular Component: intracellular (GO:0005622) }\end{array}$ \\
\hline
\end{tabular}




\begin{tabular}{|c|c|}
\hline PPL_04465 & Molecular Function: O-methyltransferase activity (GO:0008171) \\
\hline PPL_04550 & $\begin{array}{l}\text { Molecular Function: glutamate-ammonia ligase activity } \\
\text { (GO:0004356) } \\
\text { Molecular Function: catalytic activity (GO:0003824) } \\
\text { Biological Process: nitrogen compound metabolic process } \\
\text { (GO:0006807) }\end{array}$ \\
\hline PPL_04587 & Cellular Component: viral capsid (GO:0019028) \\
\hline PPL_05027 & $\begin{array}{l}\text { Molecular Function: ubiquitin thiolesterase activity } \\
\text { (GO:0004221) } \\
\text { Biological Process: ubiquitin-dependent protein catabolic } \\
\text { process (GO:0006511) }\end{array}$ \\
\hline PPL_05306 & Molecular Function: O-methyltransferase activity (GO:0008171) \\
\hline PPL_05333 & $\begin{array}{l}\text { Molecular Function: hydrolase activity, hydrolyzing O-glycosyl } \\
\text { compounds (GO:0004553) } \\
\text { Biological Process: carbohydrate metabolic process } \\
\text { (GO:0005975) }\end{array}$ \\
\hline PPL_05390 & $\begin{array}{l}\text { Molecular Function: deoxyribonuclease II activity (GO:0004531) } \\
\text { Biological Process: DNA metabolic process (GO:0006259) }\end{array}$ \\
\hline PPL_05392 & $\begin{array}{l}\text { Molecular Function: deoxyribonuclease II activity (GO:0004531) } \\
\text { Biological Process: DNA metabolic process (GO:0006259) }\end{array}$ \\
\hline PPL_05452 & $\begin{array}{l}\text { Molecular Function: GTP binding (GO:0005525) } \\
\text { Biological Process: small GTPase mediated signal transduction } \\
\text { (GO:0007264) } \\
\text { Cellular Component: intracellular (GO:0005622) }\end{array}$ \\
\hline PPL_05702 & $\begin{array}{l}\text { Biological Process: lipid metabolic process (GO:0006629) } \\
\text { Biological Process: metabolic process (GO:0008152) }\end{array}$ \\
\hline PPL_05727 & $\begin{array}{l}\text { Molecular Function: G-protein coupled receptor activity } \\
\text { (GO:0004930) } \\
\text { Molecular Function: lipid binding (GO:0008289) } \\
\text { Molecular Function: GABA-B receptor activity (GO:0004965) } \\
\text { Biological Process: G-protein coupled receptor protein signaling } \\
\text { pathway (GO:0007186) } \\
\text { Cellular Component: integral to membrane (GO:0016021) }\end{array}$ \\
\hline PPL_06209 & $\begin{array}{l}\text { Molecular Function: hydrolase activity (GO:0016787) } \\
\text { Molecular Function: sphingomyelin phosphodiesterase activity } \\
\text { (GO:0004767), } \\
\text { Biological Process: lipid metabolic process (GO:0006629) } \\
\text { Biological Process: sphingomyelin catabolic process } \\
\text { (GO:0006685) }\end{array}$ \\
\hline PPL_06621 & Molecular Function: catalytic activity (GO:0003824) \\
\hline PPL_07296 & $\begin{array}{l}\text { Molecular Function: GTPase activity (GO:0003924) } \\
\text { Molecular Function: GTP binding (GO:0005525) } \\
\text { Biological Process: signal transduction (GO:0007165) } \\
\text { Biological Process: small GTPase mediated signal transduction } \\
\text { (GO:0007264) } \\
\text { Cellular Component: membrane (GO:0016020) } \\
\text { Cellular Component: intracellular (GO:0005622) }\end{array}$ \\
\hline PPL_07302 & Molecular Function: calcium ion binding (GO:0005509) \\
\hline PPL_07432 & $\begin{array}{l}\text { Molecular Function: ATP binding (GO:0005524) } \\
\text { Molecular Function: nucleotide binding (GO:0000166) } \\
\text { Molecular Function: nucleoside-triphosphatase activity } \\
\text { (GO:0017111) } \\
\text { Molecular Function: ATPase activity (GO:0016887) } \\
\text { Molecular Function: ATPase activity, coupled to }\end{array}$ \\
\hline
\end{tabular}




\begin{tabular}{|c|c|}
\hline & $\begin{array}{l}\text { transmembrane movement of substances (GO:0042626) } \\
\text { Biological Process: transport (GO:0006810) } \\
\text { Cellular Component: membrane (GO:0016020) } \\
\text { Cellular Component: integral to membrane (GO:0016021) }\end{array}$ \\
\hline PPL_07800 & Molecular Function: protein binding (GO:0005515) \\
\hline PPL_07812 & Molecular Function: protein binding (GO:0005515) \\
\hline PPL_07818 & Molecular Function: protein binding (GO:0005515) \\
\hline PPL_08055 & Molecular Function: protein binding (GO:0005515) \\
\hline PPL_08454 & $\begin{array}{l}\text { Molecular Function: lipid binding (GO:0008289) } \\
\text { Molecular Function: G-protein coupled receptor activity } \\
\text { (GO:0004930) } \\
\text { Biological Process: G-protein coupled receptor protein signaling } \\
\text { pathway (GO:0007186) } \\
\text { Cellular Component: integral to membrane (GO:0016021) }\end{array}$ \\
\hline PPL_08455 & $\begin{array}{l}\text { Molecular Function: lipid binding (GO:0008289) } \\
\text { Molecular Function: G-protein coupled receptor activity } \\
\text { (GO:0004930) } \\
\text { Biological Process: G-protein coupled receptor protein signaling } \\
\text { pathway (GO:0007186) } \\
\text { Cellular Component: integral to membrane (GO:0016021) }\end{array}$ \\
\hline PPL_08496 & Biological Process: pathogenesis (GO:0009405) \\
\hline PPL_08648 & Biological Process: pathogenesis (GO:0009405) \\
\hline PPL_08725 & $\begin{array}{l}\text { Molecular Function: protein binding (GO:0005515) } \\
\text { Molecular Function: hydrolase activity (GO:0016787) } \\
\text { Biological Process: metabolic process (GO:0008152) }\end{array}$ \\
\hline PPL_09347 & $\begin{array}{l}\text { Molecular Function: actin binding (GO:0003779) } \\
\text { Biological Process: cytoskeleton organization (GO:0007010) } \\
\text { Biological Process: actin cytoskeleton organization } \\
\text { (GO:0030036) } \\
\text { Cellular Component: actin cytoskeleton (GO:0015629) }\end{array}$ \\
\hline PPL_09669 & $\begin{array}{l}\text { Molecular Function: protein binding (GO:0005515) } \\
\text { Molecular Function: zinc ion binding (GO:0008270) } \\
\text { Cellular Component: intracellular (GO:0005622) }\end{array}$ \\
\hline PPL_09729 & $\begin{array}{l}\text { Molecular Function: iron ion binding (GO:0005506) } \\
\text { Molecular Function: oxidoreductase activity (GO:0016491) } \\
\text { Molecular Function: oxidoreductase activity, acting on paired } \\
\text { donors, with incorporation or reduction of molecular oxygen } \\
\text { (GO:0016705) } \\
\text { Molecular Function: L-ascorbic acid binding (GO:0031418) } \\
\text { Biological Process: oxidation reduction (GO:0055114) }\end{array}$ \\
\hline PPL_10331 & Biological Process: steroid metabolic process (GO:0008202) \\
\hline PPL_10669 & Biological Process: pathogenesis (GO:0009405) \\
\hline PPL_11619 & $\begin{array}{l}\text { Biological Process: metabolic process (GO:0008152) } \\
\text { Molecular Function: oxidoreductase activity (GO:0016491) }\end{array}$ \\
\hline PPL_12248 & $\begin{array}{l}\text { Molecular Function: protein serine/threonine kinase activity } \\
\text { (GO:0004674) } \\
\text { Molecular Function: ATP binding (GO:0005524) } \\
\text { Biological Process: protein amino acid phosphorylation } \\
\text { (GO:0006468) }\end{array}$ \\
\hline PPL_12249 & $\begin{array}{l}\text { Molecular Function: protein serine/threonine kinase activity } \\
\text { (GO:0004674) } \\
\text { Molecular Function: ATP binding (GO:0005524) } \\
\text { Biological Process: protein amino acid phosphorylation }\end{array}$ \\
\hline
\end{tabular}




\begin{tabular}{|l|l|}
\hline & (GO:0006468) \\
\hline PPL_12250 & $\begin{array}{l}\text { Molecular Function: protein serine/threonine kinase activity } \\
\text { (GO:0004674) } \\
\text { Molecular Function: ATP binding (GO:0005524) } \\
\text { Biological Process: protein amino acid phosphorylation } \\
\text { (GO:0006468) }\end{array}$ \\
\hline PPL_12251 & $\begin{array}{l}\text { Molecular Function: protein serine/threonine kinase activity } \\
\text { (GO:0004674) } \\
\text { Molecular Function: ATP binding (GO:0005524) } \\
\text { Biological Process: protein amino acid phosphorylation } \\
\text { (GO:0006468) }\end{array}$ \\
\hline PPL_12271 & $\begin{array}{l}\text { Molecular Function: MAP kinase activity (GO:0004707) } \\
\text { Molecular Function: protein serine/threonine kinase activity } \\
\text { (GO:0004674) } \\
\text { Molecular Function: protein kinase activity (GO:0004672) } \\
\text { Molecular Function: ATP binding (GO:0005524) } \\
\text { Biological Process: protein amino acid phosphorylation } \\
\text { (GO:0006468) }\end{array}$ \\
\hline PPL_12443 & Molecular Function: DNA binding (GO:0003677) \\
\hline
\end{tabular}


Table A8: GO term analysis of genes differentially regulated after 1 hour of pre-starvation plus 2 hours of glorin treatment.

\begin{tabular}{|c|c|}
\hline PPL_00062 & Molecular Function: binding (GO:0005488) \\
\hline PPL_00136 & $\begin{array}{l}\text { Molecular Function: DNA binding (GO:0003677) } \\
\text { Biological Process: nucleosome assembly (GO:0006334) } \\
\text { Cellular Component: nucleosome (GO:0000786) } \\
\text { Cellular Component: nucleus (GO:0005634) }\end{array}$ \\
\hline PPL_00798 & $\begin{array}{l}\text { Molecular Function: sulfotransferase activity (GO:0008146) } \\
\text { Biological Process: carbohydrate biosynthetic process } \\
\text { (GO:0016051) } \\
\text { Cellular Component: integral to membrane (GO:0016021) }\end{array}$ \\
\hline PPL_00813 & Molecular Function: signal transducer activity (GO:0004871) \\
\hline PPL_01117 & $\begin{array}{l}\text { Molecular Function: flavin-containing monooxygenase activity } \\
\text { (GO:0004499) } \\
\text { Molecular Function: FAD binding (GO:0050660) } \\
\text { Molecular Function: NADP or NADPH binding (GO:0050661) } \\
\text { Biological Process: oxidation reduction (GO:0055114) } \\
\text { Cellular Component: intrinsic to endoplasmic reticulum } \\
\text { membrane (GO:0031227) }\end{array}$ \\
\hline PPL_01230 & $\begin{array}{l}\text { Molecular Function: hydrolase activity, acting on ester bonds } \\
\text { (GO:0016788) }\end{array}$ \\
\hline PPL_01394 & $\begin{array}{l}\text { Molecular Function: transporter activity (GO:0005215) } \\
\text { Biological Process: transport (GO:0006810) } \\
\text { Cellular Component: cytoplasm (GO:0005737) } \\
\text { Cellular Component: integral to membrane (GO:0016021) }\end{array}$ \\
\hline PPL_01619 & $\begin{array}{l}\text { Molecular Function: zinc ion binding (GO:0008270) } \\
\text { Molecular Function: hydrolase activity (GO:0016787) } \\
\text { Molecular Function: catalytic activity (GO:0003824) }\end{array}$ \\
\hline PPL_02046 & $\begin{array}{l}\text { Molecular Function: triglyceride lipase activity (GO:0004806) } \\
\text { Biological Process: lipid metabolic process (GO:0006629) }\end{array}$ \\
\hline PPL_02535 & Molecular Function: protein binding (GO:0005515) \\
\hline PPL_02856 & $\begin{array}{l}\text { Molecular Function: triglyceride lipase activity (GO:0004806) } \\
\text { Biological Process: lipid metabolic process (GO:0006629) }\end{array}$ \\
\hline PPL_03364 & $\begin{array}{l}\text { Cellular Component: chromosome, centromeric region } \\
\text { (GO:0000775), Molecular Function: DNA binding (GO:0003677) }\end{array}$ \\
\hline PPL_04071 & $\begin{array}{l}\text { Molecular Function: monooxygenase activity (GO:0004497) } \\
\text { Molecular Function: iron ion binding (GO:0005506) } \\
\text { Molecular Function: electron carrier activity (GO:0009055) } \\
\text { Molecular Function: heme binding (GO:0020037) } \\
\text { Biological Process: oxidation reduction (GO:0055114) }\end{array}$ \\
\hline PPL_04328 & $\begin{array}{l}\text { Molecular Function: deoxyribonuclease II activity (GO:0004531) } \\
\text { Biological Process: DNA metabolic process (GO:0006259) }\end{array}$ \\
\hline PPL_04465 & Molecular Function: O-methyltransferase activity (GO:0008171) \\
\hline PPL_04587 & Cellular Component: viral capsid (GO:0019028) \\
\hline PPL_05154 & $\begin{array}{l}\text { Biological Process: metabolic process (GO:0008152) } \\
\text { Molecular Function: oxidoreductase activity (G0:0016491) }\end{array}$ \\
\hline PPL_05156 & Molecular Function: O-methyltransferase activity (GO:0008171) \\
\hline PPL_05333 & $\begin{array}{l}\text { Molecular Function: hydrolase activity, hydrolyzing O-glycosyl } \\
\text { compounds (GO:0004553) } \\
\text { Biological Process: carbohydrate metabolic process }\end{array}$ \\
\hline
\end{tabular}




\begin{tabular}{|c|c|}
\hline & (GO:0005975) \\
\hline PPL_05703 & $\begin{array}{l}\text { Molecular Function: carbohydrate binding (GO:0030246) } \\
\text { Cellular Component: extracellular region (GO:0005576) }\end{array}$ \\
\hline PPL_06262 & Biological Process: pathogenesis (GO:0009405) \\
\hline PPL_06545 & $\begin{array}{l}\text { Biological Process: sphingolipid metabolic process } \\
\text { (GO:0006665) } \\
\text { Biological Process: lipid metabolic process (GO:0006629) } \\
\text { Cellular Component: Iysosome (GO:0005764) }\end{array}$ \\
\hline PPL_06552 & Biological Process: pathogenesis (GO:0009405) \\
\hline PPL_06686 & $\begin{array}{l}\text { Molecular Function: zinc ion binding (GO:0008270) } \\
\text { Cellular Component: intracellular (GO:0005622) }\end{array}$ \\
\hline PPL_07249 & $\begin{array}{l}\text { Molecular Function: catalytic activity (GO:0003824) } \\
\text { Molecular Function: oxidoreductase activity (GO:0016491) } \\
\text { Molecular Function: binding (GO:0005488) } \\
\text { Biological Process: metabolic process (GO:0008152) }\end{array}$ \\
\hline PPL_07551 & $\begin{array}{l}\text { Molecular Function: ATP binding (GO:0005524) } \\
\text { Molecular Function: ATPase activity (GO:0016887) } \\
\text { Molecular Function: nucleotide binding (GO:0000166) } \\
\text { Molecular Function: nucleoside-triphosphatase activity } \\
\text { (GO:0017111) }\end{array}$ \\
\hline PPL_07729 & $\begin{array}{l}\text { Biological Process: sphingolipid metabolic process } \\
\text { (GO:0006665) } \\
\text { Biological Process: lipid metabolic process (GO:0006629) } \\
\text { Cellular Component: lysosome (GO:0005764) }\end{array}$ \\
\hline PPL_08355 & $\begin{array}{l}\text { Molecular Function: hydrolase activity, hydrolyzing O-glycosyl } \\
\text { compounds (GO:0004553) } \\
\text { Biological Process: carbohydrate metabolic process } \\
\text { (GO:0005975) }\end{array}$ \\
\hline PPL_08361 & $\begin{array}{l}\text { Molecular Function: GTP binding (GO:0005525), Biological } \\
\text { Process: small GTPase mediated signal transduction } \\
\text { (GO:0007264) }\end{array}$ \\
\hline PPL_08450 & $\begin{array}{l}\text { Biological Process: pathogenesis (GO:0009405) } \\
\text { Molecular Function: sugar binding (GO:0005529) }\end{array}$ \\
\hline PPL_08475 & Biological Process: pathogenesis (GO:0009405) \\
\hline PPL_08476 & Biological Process: pathogenesis (GO:0009405) \\
\hline PPL_09347 & $\begin{array}{l}\text { Molecular Function: actin binding (GO:0003779) } \\
\text { Biological Process: cytoskeleton organization (GO:0007010) } \\
\text { Cellular Component: actin cytoskeleton (GO:0015629) }\end{array}$ \\
\hline PPL_09571 & $\begin{array}{l}\text { Molecular Function: acid phosphatase activity (GO:0003993) } \\
\text { Molecular Function: metal ion binding (GO:0046872) } \\
\text { Molecular Function: hydrolase activity (GO:0016787 }\end{array}$ \\
\hline PPL_09762 & $\begin{array}{l}\text { Molecular Function: ATP binding (GO:0005524) } \\
\text { Biological Process: protein import (GO:0017038) } \\
\text { Cellular Component: membrane (GO:0016020) }\end{array}$ \\
\hline PPL_10669 & Biological Process: pathogenesis (GO:0009405) \\
\hline PPL_11326 & $\begin{array}{l}\text { Molecular Function: protein serine/threonine kinase activity } \\
\text { (GO:0004674) } \\
\text { Molecular Function: protein kinase activity (GO:0004672) } \\
\text { Molecular Function: ATP binding (GO:0005524) } \\
\text { Molecular Function: ATP binding (GO:0005524) } \\
\text { Biological Process: protein amino acid phosphorylation } \\
\text { (GO:0006468) }\end{array}$ \\
\hline
\end{tabular}




\begin{tabular}{|l|l|}
\hline PPL_11401 & $\begin{array}{l}\text { Molecular Function: cysteine-type endopeptidase activity } \\
\text { (GO:0004197) } \\
\text { Molecular Function: cysteine-type peptidase activity } \\
\text { (GO:0008234) } \\
\text { Biological Process: proteolysis (GO:0006508) } \\
\text { Cellular Component: extracellular region (GO:0005576) }\end{array}$ \\
\hline PPL_12248 & $\begin{array}{l}\text { Molecular Function: protein serine/threonine kinase activity } \\
\text { (GO:0004674) } \\
\text { Molecular Function: ATP binding (GO:0005524) } \\
\text { Biological Process: protein amino acid phosphorylation } \\
\text { (GO:0006468) }\end{array}$ \\
\hline PPL_12249 & $\begin{array}{l}\text { Molecular Function: protein serine/threonine kinase activity } \\
\text { (GO:0004674) } \\
\text { Molecular Function: ATP binding (GO:0005524) } \\
\text { Biological Process: protein amino acid phosphorylation } \\
\text { (GO:0006468) }\end{array}$ \\
\hline
\end{tabular}




\section{Table A9: Complete List of GPCR genes upregulated by glorin}

Cells were staved for 2 or 3 hours without glorin treatment $(t 2, t 3)$ or pre-starved for 1 hour and treated with glorin for additional 1 or 2 hours $(\mathrm{t} 2+, \mathrm{t} 3+)$. Fold changes are shown that were obtained by comparing gene expression in glorin-treated cells versus untreated cells.

\begin{tabular}{|c|c|c|c|c|c|}
\hline $\begin{array}{l}\text { Locus in } \\
\text { genome }\end{array}$ & Gene Name & $t 2+/ t 2$ & $\mathrm{t} 3+/ \mathrm{t} 3$ & $\begin{array}{l}\text { Putative Identification (IPR } \\
\text { description) }\end{array}$ & Orthologs \\
\hline PPL_03884 & grlE & 1.20755454 & 1.08147317 & $\begin{array}{l}\text { G-protein-coupled receptor (GPCR) } \\
\text { family } 3 \text { protein 5, GABA-B receptor } \\
\text { protein } \\
\text { G-protein coupled receptor activity } \\
\text { (GO:0004930) GABA-B receptor activity } \\
\text { (GO:0004965) triacylglycerol lipase } \\
\text { activity (GO:0004806) }\end{array}$ & $\begin{array}{l}\text { D.discoideum/ } \\
\text { D.fasciculatum }\end{array}$ \\
\hline PPL_00902 & grlL & 2.74841933 & 1.545949343 & $\begin{array}{l}\text { G-protein-coupled receptor (GPCR) } \\
\text { family } 3 \text { protein } 11 \text {, lipid binding } \\
\text { (GO:0008289) G-protein coupled } \\
\text { receptor activity (GO:0004930) GABA-B } \\
\text { receptor activity (GO:0004965) }\end{array}$ & $\begin{array}{l}\text { D.discoideum/ } \\
\text { D.fasciculatum }\end{array}$ \\
\hline PPL_09248 & grlF & 0.825961763 & 0.959042267 & $\begin{array}{l}\text { G-protein-coupled receptor (GPCR) } \\
\text { family } 3 \text { protein } 6 \\
\text { lipid binding (GO:0008289) G-protein } \\
\text { coupled receptor activity (GO:0004930) }\end{array}$ & \\
\hline PPL_05727 & grlH & 4.158168581 & 1.038539037 & $\begin{array}{l}\text { G-protein-coupled receptor (GPCR) } \\
\text { family } 3 \text { protein } 8 \\
\text { lipid binding (GO:0008289) G-protein } \\
\text { coupled receptor activity (GO:0004930) } \\
\text { GABA-B receptor activity (GO:0004965 }\end{array}$ & $\begin{array}{l}\text { D.discoideum/ } \\
\text { D.fasciculatum }\end{array}$ \\
\hline PPL_03564 & & 8.65643913 & 1.696856519 & $\begin{array}{l}\text { G-protein-coupled receptor (GPCR) } \\
\text { family } 3 \text { protein } \\
\text { lipid binding (GO:0008289) G-protein } \\
\text { coupled receptor activity (GO:0004930) }\end{array}$ & $\begin{array}{l}\text { D.discoideum/ } \\
\text { D.fasciculatum }\end{array}$ \\
\hline PPL_10637 & grlM & 1.180710312 & 0.931420984 & $\begin{array}{l}\text { G-protein-coupled receptor (GPCR) } \\
\text { family } 3 \text { protein } 12 \\
\text { G-protein coupled receptor activity } \\
\text { (GO:0004930) lipid binding } \\
\text { (GO:0008289) }\end{array}$ & $\begin{array}{l}\text { D.discoideum/ } \\
\text { D.fasciculatum }\end{array}$ \\
\hline PPL_05022 & grlD & 0.830265822 & 1.749651374 & $\begin{array}{l}\text { G-protein-coupled receptor (GPCR) } \\
\text { family } 3 \text { protein } 4 \\
\text { GABA-B receptor activity (GO:0004965) } \\
\text { lipid binding (GO:0008289) G-protein } \\
\text { coupled receptor activity (GO:0004930) }\end{array}$ & $\begin{array}{l}\text { D.discoideum/ } \\
\text { D.fasciculatum }\end{array}$ \\
\hline PPL_04108 & grlC & 3.321081894 & 0.929070865 & $\begin{array}{l}\text { lipid binding (GO:0008289), G-protein } \\
\text { coupled receptor activity (GO:0004930) }\end{array}$ & $\begin{array}{l}\text { D.discoideum/ } \\
\text { D.fasciculatum }\end{array}$ \\
\hline PPL_08454 & & 15.86575363 & 2.66083276 & $\begin{array}{l}\text { G-protein-coupled receptor (GPCR) } \\
\text { family } 3 \text { protein } \\
\text { G-protein coupled receptor activity } \\
\text { (GO:0004930) lipid binding } \\
\text { (GO:0008289) }\end{array}$ & $\begin{array}{l}\text { D.discoideum/ } \\
\text { D.fasciculatum }\end{array}$ \\
\hline PPL_08455 & $\begin{array}{l}\text { Metabotropic } \\
\text { glutamate }\end{array}$ & 11.98277423 & 2.021828818 & $\begin{array}{l}\text { G-protein-coupled receptor (GPCR) } \\
\text { family } 3 \text { protein, G-protein coupled }\end{array}$ & D.discoideum \\
\hline
\end{tabular}


APPENDIX

\begin{tabular}{|c|c|c|c|c|c|}
\hline & $\begin{array}{l}\text { receptor-like } \\
\text { protein }\end{array}$ & & & $\begin{array}{l}\text { receptor activity (GO:0004930) lipid } \\
\text { binding (GO:0008289) }\end{array}$ & \\
\hline PPL_05762 & grlR & 0.789895954 & 0.800592227 & $\begin{array}{l}\text { G-protein-coupled receptor (GPCR) } \\
\text { family } 3 \text { protein 17, G-protein coupled } \\
\text { receptor activity (GO:0004930) }\end{array}$ & $\begin{array}{l}\text { D.discoideum/ } \\
\text { D.fasciculatum }\end{array}$ \\
\hline PPL_09437 & & 0.416878904 & 0.667195531 & $\begin{array}{l}\text { G-protein-coupled receptor (GPCR) } \\
\text { family } 3 \\
\\
\text { protein } \\
\text { G-protein coupled receptor activity } \\
\text { (GO:0004930) }\end{array}$ & $\begin{array}{l}\text { D. discoideum/ } \\
\text { D.fasciculatum }\end{array}$ \\
\hline PPL_05381 & crlF & 1.047911068 & 0.977542476 & $\begin{array}{l}\text { GPCR, family 2-like } \\
\text { transmembrane receptor activity } \\
\text { (GO:0004888) }\end{array}$ & $\begin{array}{l}\text { D. discoideum/ } \\
\text { D.fasciculatum }\end{array}$ \\
\hline PPL_10136 & $\mathrm{crlC}$ & 1.632033321 & 0.644915034 & $\begin{array}{l}\text { cAMP receptor-like protein, G-protein- } \\
\text { coupled receptor (GPCR) family protein } \\
\text { GPCR, rhodopsin-like superfamily } \\
\text { GPCR, family 2-like transmembrane } \\
\text { receptor activity (GO:0004888) }\end{array}$ & $\begin{array}{l}\text { D.discoideum/ } \\
\text { D.fasciculatum }\end{array}$ \\
\hline PPL_01693 & carB & 0.47333985 & 0.665297373 & $\begin{array}{l}\text { G-protein-coupled receptor, cAMP } \\
\text { receptor 2, G-protein coupled receptor } \\
\text { activity (GO:0004930), cAMP binding } \\
\text { (GO:0030552) transmembrane receptor } \\
\text { activity (GO:0004888) } \\
\end{array}$ & $\begin{array}{l}\text { D.discoideum/ } \\
\text { D.fasciculatum }\end{array}$ \\
\hline PPL_05277 & & 1.148618881 & 0.936387653 & $\begin{array}{l}\text { G-protein coupled receptor activity } \\
\text { (GO:0004930), cAMP binding } \\
\text { (GO:0030552) transmembrane receptor } \\
\text { activity (GO:0004888) } \\
\text { rhodopsin-like superfamily GPCR, family } \\
\text { 2-like } \\
\end{array}$ & $\begin{array}{l}\text { D. discoideum/ } \\
\text { D.fasciculatum }\end{array}$ \\
\hline PPL_00085 & & 0.640793725 & 1.192893791 & $\begin{array}{l}\text { cAMP-type GPCR GPCR, family 2-like } \\
\text { G-protein coupled receptor activity } \\
\text { (GO:0004930), cAMP binding } \\
\text { (GO:0030552) transmembrane receptor } \\
\text { activity (GO:0004888) }\end{array}$ & $\begin{array}{l}\text { D. discoideum/ } \\
\text { D.fasciculatum }\end{array}$ \\
\hline PPL_07940 & crlA & 0.782860038 & 0.790505126 & $\begin{array}{l}\text { G-protein coupled receptor activity } \\
\text { (GO:0004930), cAMP binding } \\
\text { (GO:0030552) } \\
\text { Cellular component: integral to } \\
\text { membrane (GO:0016021) } \\
\end{array}$ & $\begin{array}{l}\text { D. discoideum/ } \\
\text { D.fasciculatum }\end{array}$ \\
\hline PPL_08101 & crlE & 1.119936881 & 0.73910168 & $\begin{array}{l}\text { GPCR, family 2-like } \\
\text { transmembrane receptor activity } \\
\text { (GO:0004888) }\end{array}$ & $\begin{array}{l}\text { D.discoideum/ } \\
\text { D.fasciculatum }\end{array}$ \\
\hline PPL_06402 & $\begin{array}{l}\text { cAMP-type } \\
\text { GPCR }\end{array}$ & 1.30693551 & 1.126307933 & $\begin{array}{l}\text { G-protein coupled receptor activity } \\
\text { (GO:0004930), cAMP binding } \\
\text { (GO:0030552) transmembrane receptor } \\
\text { activity (GO:0004888) }\end{array}$ & $\begin{array}{l}\text { D. discoideum/ } \\
\text { D.fasciculatum }\end{array}$ \\
\hline PPL_03211 & & 0.897006864 & 0.78965748 & $\begin{array}{l}\text { GPCR, family 2-like cAMP-type GPCR } \\
\text { secretin-like receptor, latrophilin } \\
\text { receptor-like protein, transmembrane } \\
\text { receptor activity (GO:0004888) G-protein } \\
\text { coupled receptor activity (GO:0004930), } \\
\text { cAMP binding (GO:0030552) }\end{array}$ & $\begin{array}{l}\text { D.discoideum/ } \\
\text { D.fasciculatum }\end{array}$ \\
\hline PPL_11745 & fscC & 0.378863325 & 0.416001857 & $\begin{array}{l}\text { G-protein-coupled receptor (GPCR) } \\
\text { family protein, frizzled and smoothened- } \\
\text { like sans CRD protein, transmembrane } \\
\text { receptor activity (GO:0004888) }\end{array}$ & $\begin{array}{l}\text { D. discoideum/ } \\
\text { D.fasciculatum }\end{array}$ \\
\hline PPL_09871 & $f s I Q$ & 1.030847142 & 0.773862415 & $\begin{array}{l}\text { G-protein-coupled receptor (GPCR) } \\
\text { family protein, frizzled and smoothened- } \\
\text { like protein }\end{array}$ & $\begin{array}{l}\text { D.discoideum/ } \\
\text { D.fasciculatum }\end{array}$ \\
\hline
\end{tabular}


APPENDIX

\begin{tabular}{|l|l|l|l|l|l|}
\hline & & & & $\begin{array}{l}\text { transmembrane receptor activity } \\
\text { (GO:0004888) }\end{array}$ & \\
\hline PPL_11273 & fscH & 1.58459391 & 1.512767255 & $\begin{array}{l}\text { G-protein-coupled receptor (GPCR) } \\
\text { family protein, frizzled and smothened- } \\
\text { like sans CRD protein, GPCR, family 2- } \\
\text { like } \\
\text { transmembrane receptor activity } \\
\text { (GO:0004888) }\end{array}$ & $\begin{array}{l}\text { D.discoideum/ } \\
\text { D.fasciculatum }\end{array}$ \\
\hline PPL_02857 & & 1.188110538 & 1.158026463 & $\begin{array}{l}\text { ARF GTPase activator activity } \\
\text { (GO:0008060), zinc ion binding } \\
\text { (GO:0008270) transmembrane receptor } \\
\text { activity (GO:0004888) }\end{array}$ & $\begin{array}{l}\text { D.discoideum/ } \\
\text { D.fasciculatum }\end{array}$ \\
\hline PPL_00855 & & 1.48 & 1.89 & $\begin{array}{l}\text { G-protein-coupled receptor (GPCR) } \\
\text { family protein, frizzled and smoothened- } \\
\text { like sans CRD protein }\end{array}$ & $\begin{array}{l}\text { D.discoideum/ } \\
\text { D.fasciculatum }\end{array}$ \\
\hline
\end{tabular}




\section{List of scientific publications and presentations}

$\underline{\text { Asghar A, Groth M, Siol O, Gaube F, Enzensperger C, Glöckner G, Winckler T. }}$ (2012) Developmental gene regulation by an ancient intercellular communication system in social amoebae. Protist. 163(1):25-37.

Asma Asghar, Gernot Glöckner, Oliver Siol, Thomas Winckler (2010).

Glorin-mediated gene expression in Polysphondylium pallidum. The 2010 International Dictyostelium Symposium held in Cardiff, Wales, UK, Aug ${ }^{\text {st }}-$ Aug 6 ${ }^{\text {th }}, 2010$

Asma Asghar, Marco Groth, Oliver Siol, Thomas Winckler (2010).

Preliminary characterization of peptide-based communication in social amoebae. The 2010 International Dictyostelium Symposium held in Cardiff, Wales, UK, Aug ${ }^{\text {st }}-$ Aug $6^{\text {th }}$, 2010

Asma Asghar, Gernot Glöckner, Marco Groth, Thomas Winckler (2009)

Initial characterization of glorin-mediated gene expression in Polysphondylium pallidum. The 2009 International Dictyostelium Symposium held in Estes, Colorado, USA, August $23^{\text {rd }}-28^{\text {th }}, 2009$.

Asma Asghar, Christoph Enzensperger, Thomas Winckler (2010).

Roots of peptide-based communication in social amoebae. The 2010 MiCom conference on microbial interactions held in Jena, Germany. Sep $28^{\text {th }}$ - October $2^{\text {nd }}, 2010$

Asma Asghar, Gernot Glöckner, Christoph Enzensperger, Thomas Winckler (2009) Exploring glorin-mediated cell-cell communication in Polysphondylium pallidum. The 2009 JSMC annual symposium held in Jena, Germany. October $11^{\text {th }}-12^{\text {th }}, 2009$.

Asma Asghar \& Thomas Winckler (2008)

Intercellular communication with peptide derivatives in social amoebae. The 2008 JSMC annual symposium held in Jena, Germany. December $14^{\text {th }}-15^{\text {th }}, 2009$. 


\section{Acknowledgements}

The presented research work was completed between October 2008 and October 2011 at the Department of Pharmaceutical Biology, Institute of Pharmacy, Friedrich Schiller University Jena, Germany. I would like to thank all the people mentioned below who contributed to the success of this project.

First and foremost, I express my heartfelt gratitude and indebtedness to my supervisor Prof. Dr. Thomas Winckler for providing me an opportunity to work under his esteemed supervision. I am grateful to him for his invaluable guidance, creative suggestions and inspiring enthusiasm for science that helped me to develop my scientific skills. I admire the dedication and passion he has for great research. I am thankful to him for being instrumental in planning and shaping up my thesis in an exceptional way; without his superb guidance I stand nowhere in this rapidly growing world of science. Words cannot replace my special thanks for his immense moral support and encouragement when I was struggling between the hard core of my life and the dream to have my $\mathrm{PhD}$ degree.

I am deeply indebted to Prof. Dr. Christian Hertweck (Biomolecular chemistry group, Hans Knöll Institute, Jena), my co-supervisor who was always ready to offer help, guidance and constructive ideas. His direction, assistance and intellectually stimulating discussions have further advanced my appreciation for science in general.

I would especially like to thank Dr. Gernot Glöckner (Berlin Centre for Genomics in Biodiversity research, Berlin) for sharing genome sequencing data of $P$. pallidum PN500 before it was published.

I would also like to acknowledge Prof. Dr. Pauline Schaap (who is a role model for me) and Dr. Christina Schilde from the Univeristy of Dundee, Scotland for their intellectual discussions, fascinating ideas and sharing lab materials and protocols.

I pay my special thanks to Dr. Maria Romerlao and Dicty Stock Center for providing Dictyostelid strains.

I would like to forward my thanks to Dr. Karl-Heinz Gührs (Proteomics facility- Fritz Lipmann Institute Jena, Germany) for helping me in proteome analysis, Dr. Marco Groth (Genome analysis group; Fritz Lipmann Institute Jena) for conducting RNA sequencing (RNA-seq), Dr. Oliver Siol 
for RNA-seq data analysis, and Dr. Christoph Enzensperger (Institute of Pharmaceutical Chemistry, University of Jena) for synthesizing glorin at Institute of Pharmaceutical chemistry.

I would like to thank the team of incredible researchers that I have spent the past three years with. Dr. Friedemann Gaube has been a wonderful colleague, who always extended his help whenever I approached him throughout my stay at the Institute of Pharmaceutical Biology. The fellow PhD scholars, Jana, Anika, Sara, Thomas, Michael and Tilmann have been fun to work with and I have enjoyed being part of such a friendly team. Bärbel, Heide, and Angela, I can never forget your affection!

I would like to acknowledge Jena School for Microbial Communication (JSMC) for offering me a $\mathrm{PhD}$ position and supporting my scientific career. It has contributed greatly to the development of my personality as a young researcher. I extend my special and sincere gratitute to Dr. Carsten Thoms (manager JSMC) and Frau Ulrike Schleier (secretary JSMC) for being so supportive, encouraging, friendly and tolerant.

My acknowledgements would be highly incomplete if I don't mention some nice friends; Stefanie, Chithra Devi and her husband Monickam, Mona (my neighbour), Hanadi, Bushra, Wafa, Karoline, Bita, Alex, and Thobias Koch who always showed me a positive outlook towards life and made my time lively.

Last but not the least, I would like to appreciate the incredible love and support of my family who have always been there and have been the drive that kept me going, always taking interest in the work I have completed. I deeply acknowledge their patience, tolerance, practical advices and sacrifices that boosted me towards my success. A special note of thanks to Ammi, Abo, Shummaila Baji, Shummail Bhai, Yasir (he has been my best childhood friend though we argue alot) and his wife Ana, Bilal, Shani and Usama (for his innocent love towards me). Thanks to all the kids also; Nimrah, Airah, Muaaz, Iman and Mahnoor.

Asma ASGHAR

30 July, 2012

Jena, Germany 


\section{Lebenslauf}

Name, Vorname:

Adresse:

Geburtsdatum:

Nationalität:

Schulausbildung / Ausbildung:

$1986-1996$

$1997-1999$

Universitätsstudium:

$2000-2004$

2005- 2007

$2007-2008$

Promotionsstudium:

$2008-2012$
Asma Asghar

Schlegelstr. 6, D-07747 Jena

08.02.1982

Pakistanisch

Nusrat Gymnasium

Al-Faisal Town Lahore, Pakistan

Pre- medizinische Studien

Lahore Universität für Frauen

Lahore, Pakistan

Bachelor der Pharmazie

Institut der Pharmazie

Universität Punjab, Lahore, Pakistan

Master der Molekularbiologie

Nationales Zentrum der Molekularbiologie Lahore, Pakistan

Forschungsassistentin

Institut der Biochemie

Technische Universität Graz, Österreich

PhD Scholar

Lehrstuhl für pharmazeutische Biologie

Institut der Pharmazie

Friedrich Schiller Universität Jena,

Deutschland 


\section{Curriculum Vitae}

Name:

Address:

Date of birth:

Nationality:

School \& College Education:

$1986-1996$

$1997-1999$

2005- 2007

$2007-2008$

\section{Doctoral Studies:}

$2008-2012$
Asma Asghar

Schlegelstr. 6, D-07747 Jena

08.02.1982

Pakistani

Nusrat High School

Al-Faisal Town Lahore Cantt, Pakistan

Pre- Medical Studies

Lahore College for Women

Lahore, Pakistan

Bachelor of Pharmacy

Institute of Pharmacy

University of the Punjab, Lahore, Pakistan

Master of Molecular Biology

National Centre of Excellence in Molecular

Biology

Lahore, Pakistan

Research Assistant Institute of Biochemistry

Graz University of Technology,

Graz, Austria

PhD Scholar

Department of Pharmaceutical Biology

Institute of Pharmacy

Friedrich Schiller University Jena

Germany 


\section{Ehrenwörtliche Erklärung}

Hiermit erkläre ich,

- dass mir die geltende Promotionsordnung der Biologisch-Pharmazeutischen Fakultät bekannt ist,

- dass ich die Dissertation selbst angefertigt habe, keine Textabschnitte eines Dritten ohne Kennzeichnung übernommen worden sind und alle von mir benutzten Hilfsmittel, persönlichen Mitteilungen und Quellen in meiner Arbeit angegeben sind,

- dass ich die Hilfe eines Promotionsberaters nicht in Anspruch genommen habe und dass Dritte weder unmittelbar noch mittelbar geldwerte Leistungen von mir für Arbeiten erhalten haben, die im Zusammenhang mit dem Inhalt der vorgelegten Dissertation stehen,

- dass ich die Dissertation noch nicht als Prüfungsarbeit für eine staatliche oder andere wissenschaftliche Prüfung eingereicht habe

- dass ich die vorliegende Arbeit weder komplett noch in Form einer in wesentlichen Teilen ähnlichen oder anderen Abhandlung bei der FriederichSchiller-Universität oder einer anderen Hochschule als Dissertation oder Prüfungsarbeit für eine staatliche oder wissenschaftliche Prüfung vorgelegt habe.

Jena, den 30 Juli 2012

Asma Asghar 